\title{
A MEASUREMENT OF THE MUON NEUTRINO CHARGED CURRENT QUASIELASTIC INTERACTION AND A TEST OF LORENTZ VIOLATION WITH THE MINIBOONE EXPERIMENT
}

\author{
Teppei Katori
}

Submitted to the faculty of the University Graduate School in partial fulfillment of the requirements

for the degree

Doctor of Philosophy

in the Department of Physics,

Indiana University

December 2008 
Accepted by the Graduate Faculty, Indiana University, in partial fulfillment of the requirements for the degree of Doctor of Philosophy.

Doctoral Committee

$\begin{array}{r}\text { Rex Tayloe, Ph.D. } \\ \hline\end{array}$

Dobrin P. Bossev, Ph.D.

V. Alan Kostelecký, Ph.D.

Mark Messier, Ph.D.

December 10, 2008 
(C) 2008

Teppei Katori

ALL RIGHTS RESERVED 


\section{Acknowledgments}

I am very fortunate to have had Rex Tayloe for my supervisor. My work during graduate school was successful since I relied $100 \%$ on your support. For FINeSSE, you taught me everything step by step because I didn’t know anything. For Lorentz violation, even though it was a spin-off subject for me and you, but you were so generous, supportive, and patient. And for MiniBooNE, the CCQE work couldn't have been done without your ideas. I am really proud of the amount of work I present in this thesis; it is the evidence of what a good work relationship we have. Finally I want to apologize for my bad English and for how you always had to correct it, including in this thesis. This acknowledgments section is the only one place in all my writings for which you didn’t correct my English! Thank you Rex.

My first three years were supported by the people in Indiana.

I wish to give sincere appreciation to Chris Cox and Nikodem Popławski, I think it was my fate to see you from the very first day in US. I had a hard time (as you know), but because of your help, I not only survived, but also changed my style to be more suitable in this country. No one ever was so influential for me as Chris, I think. And Niko, before I met you, I didn't even know what is Poland (sounds like a country though). You also introduced to me all of Polish culture. Dziekuje bardzo!

I think I never used so-called "office hours", but I usually knocked on the doors of all 
the professors any time I wanted. I wish to thank all professors who made time for me during my sudden visits. I especially want to thank professor Alan Kostelecký, who kindly accepted me all the times I came. Your stories about all kind of physics, from gravity to atomic physics, stimulated me to study as many as possible by myself, and your energetic style of research and teaching reminds me many times how it is fun to study. Thank you.

I also want to thank all the IUCF people. Although I didn't have very much chance to work with Chris Polly, his style of work, as an analysis coordinator of MiniBooNE, was very influential to me. Thank you. I appreciate Shyh Yuan Lee's help when I joined USPSS. What I learned in his class at San Francisco helped me every day of life in this field. Much of my interest in nuclear physics started from Chuck Horowitz, Brian Serot, Tim Landergen, and people at NTC. Thank you. Patrick Ockerse was a sophomore kid but taught me all my electronics and experimental techniques (of course Rex taught me, too), I still have two news articles about you, your ultimate team and your burning house. Aaron Hanson, I always admired your skill at getting along with everyone. We (Japanese SciBooNErs) practiced the pronunciation of your name a lot, I hope I did a good job. I feel I spent time with Sara Walbridge, Melanie Novak, and Crystal Bailey more often outside of IUCF. Thank you for sharing good times with me. I also want to thank Hans-Otto Mayer, Jack Doskow, and Gerard Visser for your help and inspiration for the work on FINeSSE. Thank you everyone.

My friends from the classroom, it is a very strange feeling that now all we are going somewhere to do something, but we have known each other more than six years! Liliana Cabellero, you changed shy Teppei...to an emotional salsa dancer! If the dancing is my mother of energy, then Lily is founding mother of energetic Teppei. Muchas gracias. Leah Welty and Jason Rieger are the first real American students I met. When you described Chicago, I didn't understand very much, but now, yeah, I love this city and want to stay here more. Heechang Na, thank you for talking with me every time I stopped by your office, 
because you are so kind and welcome and easy to talk with! Haiyang "Ocean" Yan, I am proud that I nicknamed you. I think you are a very good physicist. Thank you for teaching me a lot about Chinese history and physics. I always enjoyed it. Masaki (\& Shizuka) Ishitsuka is the first Japanese fellow I met at Indiana even though I left Bloomington before you came. I always enjoyed talking about neutrinos with you, your view of physics is very unique and interesting to me. Thank you. Yun-Chan, Tony, George, Jay, Helber, Jorge, Shinya... I had so much fun in Indiana because of you. Thank you very much.

One of the reasons I wanted to be a physicist is that physicists make friends all over the world so easily. I met many friends at conferences, summer schools, etc. Thank you to everyone who called me a crazy Japanese, even uploading my stupidities on YouTube. That is the biggest honor for me!

During my next three years, I was so influenced by people at Fermilab.

In MiniBooNE, Sam Zeller, it was one of my biggest fortunes during the CCQE analysis that I had a chance to work with you. I was inspired a lot by your impressive ability to do such good physics with so many people. Thank you. The energetic foursome of spokespersons, Janet Conrad, Bill Louis, Steve Brice, and Richard Van de Water, I appreciate your energy in the meetings around five o'clock, when we were supposed to be finished by two o'clock on the agenda. Thank you for driving this difficult experiment and producing lots of good results. The Country House club and Luigi club with people from Los Alamos were always fun. Thank you Bill, Geoff Mills, Gerry Garvey, and Byron Roe. I had more chance to communicate with people who stayed at Fermilab. I really liked the international MiniBooNE coffee break, including: Yong Liu (China), Želimir Djurcic (Serbia), Denis Perevalov (Russia), Serge Ouedraogo (Burkina Faso), Alexis Aguilar-Arevalo (Mexico), Bari Osmanov (Uzbekistan), and Teppei. We can have endless conversation just taking about our coun- 
tries. For the people outside of MiniBooNE, I want to give thanks to Anatoli Butkevich for kindly checking many of my calculations. Spasibo. Žarko Pavlović (and Ana Žanić), you guys are really good friends, from enjoying serious conversations to just having fun drinking. Hvala lijepo! Again, thank you everyone.

The students at MiniBooNE were definitely the students most influential on my physics. MinBooNE has three generations: the first generation (Jocelyn Monroe, Michel Sorel, Ryan Patterson, Chris Cox, Jen Raaf) shared all the ups and downs of the MiniBooNE startup; the second generation (Dave Schmitz, Alexis, Kendall Mahn, Teppei, Serge, Denis, Mike Wilking, Bob Nelson, Ted Laird) was a little strange but had charming people; and the third generation (Van Nguyen, Georgia Karagiorgi, Joe Grange, Joel Mousseau, Steve Linden, Colin Anderson) will complete all physics topics from top to bottom. The first time I joined MiniBooNE, I was just amazed by how students like Jocelyn, Michel, and Ryan are so smart and did so much good work on their own. It was totally impossible for me to imagine I could work like them when I would be a fifth or sixth year. That stimulated me a lot to work like them. Thank you. Alexis is always fun to talk with. It was very fun for me to show you the papers I found on arXiv and asking your opinion. Even if you didn't know the subject, you often gave me really good comments, that reminded me a lot of the stories about Fermi. Muchas gracias Alexis. Kendall ("kawaii Ken-chan") breathed the same air with me more than anybody else (in the office, of course!). If I met such a Japanese supporter like you in Indiana, my life in US would be different. Your enthusiastic style of physics is very vivid and I am often stunned by you. Thank you Kendall, I think we will work together in the future. Dave taught me not only about physics, but also about life in Chicago. It was so impressive for me how you stayed positive through the difficult moments in the oscillation analysis. I am excited that we can work together as postdocs in the new project. Thank you, Dave. Bob Nelson, I saw you more often bartending behind the counter at the User's Center than sitting in front of it. But actually, I remembered lots of good physics conversations with 
you, even though we had ten times more conversations about girls. I also want to thank Chivas Makaroplos here, for handing me beers and life tips. Denis, I didn't imagine we could become such good work partners. I am always impressed with your very steady work. The budyonovka you gave me is so useful at any Russian nationalistic events. Van and Georgia, it was my daily habit to interrupt your job by stopping by your office, thank you very much for listening to my random conversations.

The last two years of my life in Fermilab had yet one more bright spot, SciBooNE. Morgan Wascko brought an extraordinary concordance to this collaboration - it wouldn't work without you. Tsuyoshi Nakaya, the other spokesperson, showed strong leadership, and many of his words were also deeply imprinted in me. And, "everything coordinator" Masashi Yokoyama, thank you for treating me like one of your students. Hide-kazu Tanaka is the hardest working postdoc, really. I am bit scared to become a postdoc after watching you taking care of all details of SciBooNE so carefully. And you are extraordinarily polite! it makes me embarrassed, but I appreciate both. Thank you, Hide-san.

I learned a completely different style of approach to physics from people in SciBooNE; frankly speaking the students at SciBooNE are crazy overworking people. I want to thank all the SciBooNE students, who shared fun times with me. Thank you for accepting me, even though I didn't work on anything on SciBooNE! Katsuki "Bancho" Hiraide, is a super student that SciBooNE is proud to have. I am proud to be your colleague. I saw the maximum amount of work one student can do. I hope you can find good Guinness in Mozumi (or your cleverness may solve this problem, too). Hideyuki Takei, I think you don't have to worry about your skill in English and physics, because you are practically Aaron's mentor. I bestow the position of the president of SBGCJ (SciBooNE gentlemen's club in Japan) to you. Joe Walding, my friend for randomly hanging out around Chicago, is a trendsetter for me. I love copying your London accent and showing off to my other friends. Yasuhiro "Nakaji" Nakajima, a.k.a. "aruku so-go-entertainment", thank you for 
joining in all uncountable stupid stuff with me. I am glad to have such a nice and useful "ko-hai" in Fermilab. Yoshinori "Yoshi" Kurimoto, you are a genuine Japanese boy, you are so cool even with some eccentricities, but that makes you even more adorable. I also enjoyed the random physics conversation we had. I can safely assign you for designing a whole E-coli neutrino detector. Claudio, Camillo, Gaku, Murakami, Jose, Joan, Gary..., you are such good friends and you helped me to expand both my knowledge of physics and my relationships. Thank you very much.

The friends in Chicago always inspire me. Just watching them, they stimulate my creativity, which I believe the most important for the physicists.

My roommate, Tatiana Rodriguez, is the most active and enthusiastic person I have ever seen. I am so lucky to live with you, your stories, knowledge, and activities are so interesting, and we can easily talk until the morning. I am not mad about the milk any more! thank you, Tati. The second roommate, Ekaterina Smirnova, yes, Russian women are scary. I told my mom that Japanese women should be strong like Russian women. I am always amazed by your vitality and strong will. We fought a lot, but somehow we always became closer afterwards. When I saw you for the first time at the User's Center (with Bob, of course), I couldn't imagine all the fun we have now. Spasibo, Katia. If I hadn't met you, Mark Salach, on the street, my life in Chicago would be very different. You gave me the opportunity to meet so many interesting people I may not have had the chance to in my life. Conversations with you are always fresh and (believe it or not) inspire me a lot. Enrique "Kike" De La Cruz, hola chacho, thank you for joining my weekend fun many times, I don't know how much I like Spanish drinking games, but I do like all the people you introduced to me and all the parties you had. Don't forget to take me to get Spanish wine! Gabriella "Gabuchiku" Palencia, thank you for encouraging me the whole time. Sometimes I am very embarrassed because sometimes your appreciation is too much. You are the 
first person to call me a genius! Muchas gracias! Olaf Maleki is my best Polish friend in Chicago. You introduced me to lots of Polish friends in Chicago. I like your relaxed style a lot, all of you are so cool! Andy Crestodina, I always watch you the whole time during the parties...to steal your conversation skill. The way you introduce me to someone makes me so embarrassed that I blush like a little shy girl, but I like it. Mana, Lee, Marta, Adam, Cameron, Lisa, Chuck, Edyta... I really enjoy our conversations and the time we stayed together. Thank you! Finally, Christine Kordiuk, I guess it is my job to tell you when it is Sunday. A large part of this thesis was completed at your cafe "Ballou". Duzhe dyakuyu!

Finally, I want to say thank you to my family. My parents, Kazuyuki and Fumiko Katori, thank you for being patient so many years till Teppei became a reasonable person. I think I am acceptable quality now. And my grandma, Midori Katori, always exactly 60 years older than me, don’t be shy to brag about Teppei in the neighborhood! And to my brother and sister, Kentaro and Aiko Katori, thank you for not forgetting someone between you guys. I am glad we still can have fun or frankly stupid thing, like we did in Paris. Hope it doesn't embarrass Jun-chan too much. My special thanks is given to two of my family members. My grandfather, Shoichi Kimura (1914-2006), you gave me my curiosity for science in my childhood. My journey in science started from the days I investigated the flowers in the field with you. And my uncle, Mineo Kimura (1946-2008). You strongly recommended me to come to the US, that is the only reason why I am here. And now I know you are totally right. I thought it would be possible to work with you in the future. I hope I look like a little more like a physicist now, like you. 
Teppei Katori

\section{A MEASUREMENT OF THE MUON NEUTRINO CHARGED CURRENT QUASIELASTIC INTERACTION AND A TEST OF LORENTZ VIOLATION WITH THE MINIBOONE EXPERIMENT}

The Mini-Booster neutrino experiment (MiniBooNE) at Fermi National Accelerator Laboratory (Fermilab) is designed to search for $\nu_{\mu} \rightarrow \nu_{e}$ appearance neutrino oscillations. Muon neutrino charged-current quasi-elastic (CCQE) interactions $\left(\nu_{\mu}+n \rightarrow \mu+p\right)$ make up roughly $40 \%$ of our data sample, and it is used to constrain the background and cross sections for the oscillation analysis.

Using high-statistics MiniBooNE CCQE data, the muon-neutrino CCQE cross section is measured. The nuclear model is tuned precisely using the MiniBooNE data. The measured total cross section is $\sigma=(1.058 \pm 0.003($ stat $) \pm 0.111($ syst $)) \times 10^{-38} \mathrm{~cm}^{2}$ at the MiniBooNE muon neutrino beam energy $(700-800 \mathrm{MeV})$.

$\nu_{e}$ appearance candidate data is also used to search for Lorentz violation. Lorentz symmetry is one of the most fundamental symmetries in modern physics. Neutrino oscillations offer a new method to test it. We found that the MiniBooNE result is not well-described using Lorentz violation, however further investigation is required for a more conclusive result. 


\section{Contents}

Acknowledgments $\quad$ iv

\begin{tabular}{lll}
\hline I Neutrino physics & 1
\end{tabular}

$\begin{array}{lll}1 & \text { Introduction of this thesis } & 3\end{array}$

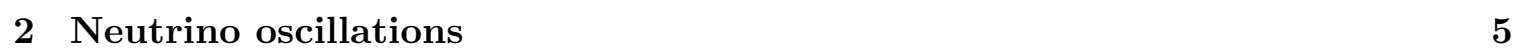

2.1 Neutrino oscillation formula . . . . . . . . . . . . . . . . . . . 5

$2.2 \quad$ Wave packet formalism $\ldots \ldots \ldots \ldots \ldots$

2.2 .1 Group velocity based formalism . . . . . . . . . . . . . . . . . 9

2.2 .2 Time-averaged neutrino oscillations . . . . . . . . . . . . . . 14

2.2 .3 Incoherent sum of neutrino oscillations . . . . . . . . . . . . . . . 14

$2.2 .4 \quad$ Neutrino oscillations with perfect kinematics measurements . . . . . 14

2.2 .5 Neutrino decay $\ldots \ldots \ldots \ldots \ldots$

2.2 .6 Factor 2 ambiguity $\ldots \ldots \ldots \ldots \ldots \ldots$

$2.2 .7 \quad$ False Lorentz violation in neutrino oscillation $\ldots . . . . . .17$ 
$2.3 \quad$ Test of new physics with Neutrino oscillations . . . . . . . . . . . . . . 19

$2.3 .1 \quad$ Neutrino oscillations as natural interferometers . . . . . . . . . . . . 19

2.3 .2 Neutrino oscillations in the search of new physics . . . . . . . . . 21

\begin{tabular}{lll}
\hline 3 & Neutrino interactions & 22
\end{tabular}

$3.1 \quad$ Neutrino-Charged lepton scattering . . . . . . . . . . . . . . . . 23

3.2 Neutrino-Nucleon scattering . . . . . . . . . . . . . . . . . . . . 28

$3.2 .1 \quad$ Llewellyn-Smith formalism for the neutrino experiments . . . . . . . 28

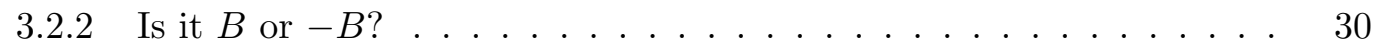

$3.2 .3 \quad$ Llewellyn-Smith formalism for Neutral Current . . . . . . . . . . . . 30

3.2 .4 Conservation of vector current (CVC) . . . . . . . . . . . . 32

$3.2 .5 \quad$ Partial conservation of axial vector current (PCAC) . . . . . . . 34

3.2 .6 The expressions for Llewellyn-Smith's form factors . . . . . . . . . . 36

3.2 .7 Dipole form factor $\ldots \ldots \ldots \ldots$. . . . . . . . . . . . . . 42

$3.2 .8 \quad$ Discrete transformation . . . . . . . . . . . . . . . . . . . . . . . 42

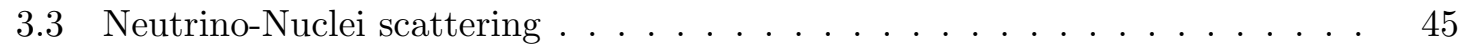

3.3.1 Smith-Moniz formula for the neutrino experiments . . . . . . . . . 45

3.3 .2 Cross section formula comparison with nuance . . . . . . . . . 48

$3.4 \quad$ Neutrino Charged current quasielastic (CCQE) scattering cross section mea-

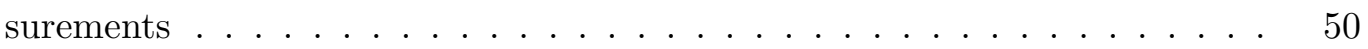

3.4.1 Long baseline accelerator-based neutrino oscillation experiments . . 50

3.4 .2 Neutrino energy reconstruction for $\mathrm{T} 2 \mathrm{~K}$

$3.4 .3 \quad$ CCQE cross section measurement $\ldots \ldots \ldots \ldots$ 
\begin{tabular}{lll}
\hline II Lorentz Violation with Neutrino & 57
\end{tabular}

4 Test of Lorentz violation with neutrino oscillations 59

4.1 Introduction . . . . . . . . . . . . . . . . . . . . . . . 60

$4.2 \quad$ Lorentz violating neutrino oscillation . . . . . . . . . . . . . . . . . . 63

4.3 Test of Lorentz violation with LSND experiment . . . . . . . . . . . . . . . 68

$4.3 .1 \quad$ LSND experiment $\ldots \ldots \ldots \ldots$. . . . . . . . . . . . . . . . 68

4.3 .2 SME formalism for LSND . . . . . . . . . . . . . . . . . . . . . 69

$4.3 .3 \quad$ Analysis and results $\ldots \ldots \ldots$. . . . . . . . . . . . . . 73

5 Global three parameter model for neutrino oscillation with Lorentz vio-

$\begin{array}{ll}\text { lation } & 77\end{array}$

$5.1 \quad$ A global model for neutrino oscillations $\ldots \ldots \ldots$. . . . . . . . . 78

$5.2 \quad$ Analytic and numerical solution of neutrino oscillations . . . . . . . . . 80

$5.2 .1 \quad$ Ferro-Cardano equation $\ldots \ldots \ldots \ldots$. . . . . . . . . . . 81

5.2 .2 Jacobi rotations $\ldots \ldots \ldots \ldots \ldots$. . . . . . . . . . . . . . 82

5.3 Model construction $\ldots \ldots \ldots \ldots \ldots \ldots$

5.3 .1 Bimaximal model . . . . . . . . . . . . . . . . . . 84

5.3 .2 Bicycle model . . . . . . . . . . . . . . 86

5.3 .3 Tandem model $\ldots \ldots \ldots$. . . . . . . . . . . . . . . 89

5.4 Tandem model in MiniBooNE . . . . . . . . . . . . . . . . . . . . . . . 102

6 Summary and outlook for neutrino oscillations with Lorentz violation 105 
III Charged Current Quasielastic scattering measurement in Mini-

BooNE

109

7 MiniBooNE

111

$7.1 \quad$ MiniBooNE, motivation $\ldots \ldots \ldots \ldots \ldots \ldots \ldots$

7.2 Booster neutrino beam . . . . . . . . . . . . . . . . . . . . . . . . . . 112

7.2 .1 Primary proton beam . . . . . . . . . . . . . . . . . . 114

$7.2 .2 \quad$ Secondary meson beam . . . . . . . . . . . . . . . . . . . 115

7.2 .3 Tertiary neutrino beam $\ldots \ldots \ldots \ldots \ldots$

7.2 .4 Neutrino flux error summary $\ldots \ldots \ldots$. . . . . . . . . . . . . . 119

7.3 MiniBoonE detector . . . . . . . . . . . . . . . . . . . . . . . . . 120

7.3.1 $\quad$ Photo multiplier tubes (PMTs) . . . . . . . . . . . . . . . 121

7.3 .2 Mineral oil . . . . . . . . . . . . . . . . . . . . . . . . . . . . . . . 123

7.3 .3 Electronics . . . . . . . . . . . . . . . . . . . . . . . . . . 123

7.3 .4 Energy calibration . . . . . . . . . . . . . . . . . . . 123

7.3 .5 Detector error summary . . . . . . . . . . . . . . . . . . . 124

7.4 MiniBoonE cross section model . . . . . . . . . . . . . . . . . . 125

7.4 .1 The nuance event generator . . . . . . . . . . . . . . . . . 125

$7.4 .2 \quad$ Charged Current Quasi-Elastic (CCQE) scattering . . . . . . . . . 126

$7.4 .3 \quad$ Neutral current elastic (NCE) scattering . . . . . . . . . . . . . . . 128

7.4 .4 Resonance interactions . . . . . . . . . . . . . . . . . 128

7.4 .5 Coherent pion production $\ldots \ldots \ldots \ldots$. . . . . . . . . 129 


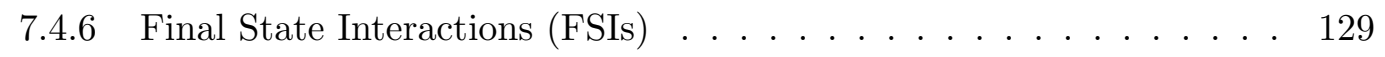

7.4 .7 Re-interactions in the detector . . . . . . . . . . . . . . . . 131

$7.4 .8 \quad$ Cross section error summary $\ldots \ldots \ldots$. . . . . . . . . . . 132

7.5 Event reconstruction $\ldots \ldots \ldots \ldots \ldots \ldots$. . . . . . . . . . . . . . . . . . . .

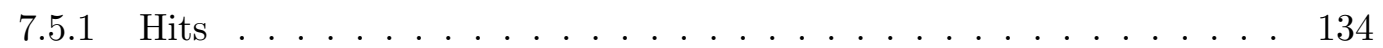

7.5 .2 Subevent . . . . . . . . . . . . . . . . . . . . . . . . . 134

7.5 .3 Particle track fitter $\ldots \ldots \ldots \ldots$. . . . . . . . . . 136

7.5 .4 Particle ID . . . . . . . . . . . . . . . . . . . . . . 140

8 CCQE measurement for MiniBooNE oscillation physics 143

$8.1 \quad \nu_{e}$ appearance oscillation analysis $\ldots \ldots \ldots \ldots \ldots \ldots$

8.1 .1 The blind analysis $\ldots \ldots \ldots \ldots \ldots \ldots$. . . . . . . . . . . . . . 144

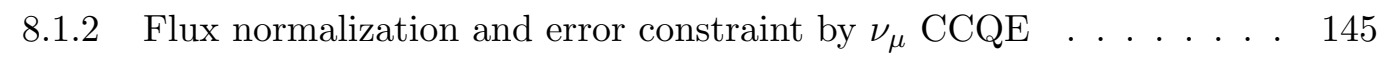

$8.1 .3 \quad$ Kinematic reconstruction test by $\nu_{\mu} \mathrm{CCQE} . \ldots \ldots \ldots$

$8.2 \quad \nu_{\mu}$ CCQE measurement and tuning of relativistic Fermi gas (RFG) model . 148

$8.2 .1 \quad$ CCQE event selection in MiniBooNE . . . . . . . . . . . . . . . 148

8.2 .2 MiniBooNE CCQE events . . . . . . . . . . . . . . . . 150

8.2 .3 Pauli blocking parameter $\kappa \ldots \ldots \ldots \ldots \ldots$. . . . . . . . . 154

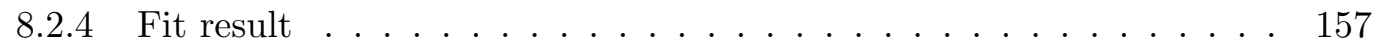

8.2 .5 Conclusions $\ldots \ldots \ldots \ldots \ldots$ 
9 simultaneous CCQE and $\mathrm{CC} 1 \pi Q^{2}$ fit $\quad 164$

$9.1 \quad$ Subevent correction $\ldots \ldots \ldots \ldots$. . . . . . . . . . . . . . . 165

9.2 CC cut . . . . . . . . . . . . . . . . . . . 166

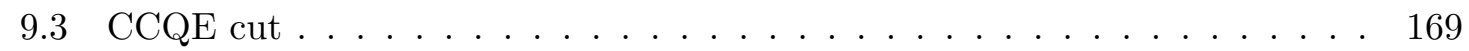

9.4 The theoretical model . . . . . . . . . . . . . . . . . . . . . . 171

$9.5 \quad$ simultaneous fitting of the $1,2,3$ subevent samples $\ldots \ldots \ldots \ldots$

9.6 Pion absorption and coherent fraction tuning $\ldots \ldots \ldots$. . . . . . . . . . 183

$9.7 M_{A}-\kappa \mathrm{fit} \ldots \ldots \ldots \ldots \ldots \ldots \ldots \ldots \ldots$

9.7 .1 the standard fit result $\ldots \ldots \ldots \ldots$. . . . . . . . . . . . . 189

9.7 .2 The systematic errors $\ldots \ldots \ldots \ldots$. . . . . . . . . . . 193

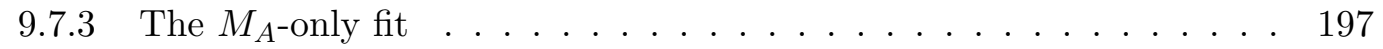

$9.7 .4 \quad Q^{2}$ distributions with the fit $M_{A}$ and $\kappa \ldots \ldots . \ldots 197$

9.7 .5 Conclusion $\ldots \ldots \ldots \ldots \ldots \ldots$

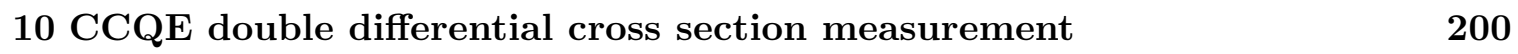

10.1 Introduction of cross section measurement . . . . . . . . . . . . . . . . 201

10.2 Neutrino energy and $Q^{2}$ reconstruction $\ldots \ldots \ldots$. . . . . . . . . 202

10.3 Absolute cross section formula $\ldots \ldots$. . . . . . . . . . . . . . 202

10.3 .1 The absolute cross section formula $\ldots \ldots$. . . . . . . . . . 202

10.3 .2 Three normalization factors . . . . . . . . . . . . . . 206

10.4 Background accounting $\ldots \ldots \ldots \ldots$. . . . . . . . . . . . 207

10.5 Unfolding error . . . . . . . . . . . . . . . . . . . . 210 
10.6 The error matrix formation and error bars . . . . . . . . . . . . . . . . . 213

10.6 .1 The unisim method . . . . . . . . . . . . . . . . . . . 213

10.6 .2 The multisim method . . . . . . . . . . . . . . . . . . 213

10.6 .3 Total and shape-only error bars . . . . . . . . . . . . . . 216

10.7 Flux error estimation $\ldots \ldots \ldots \ldots$

10.8 Cross section error estimation $\ldots \ldots \ldots \ldots$. . . . . . . . . . . . 223

10.9 Detector error estimation $\ldots \ldots \ldots \ldots \ldots$

10.10The CCQE absolute cross section with total error . . . . . . . . . . . . . . 227

10.10.1 The CCQE double differential cross section . . . . . . . . . . . . 227

10.10 .2 The CCQE total cross section . . . . . . . . . . . . . . 232

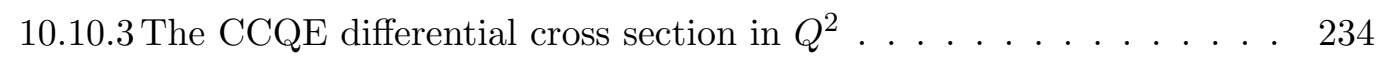

10.10 .4 The energy reconstruction . . . . . . . . . . . . . . 237

10.10 .5 The systematic errors $\ldots \ldots \ldots \ldots$. . . . . . . . . . . 239

11 Summary and outlook for CCQE scattering measurements in MiniBooNE245

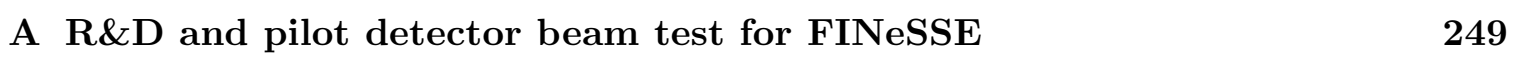

A.1 Neutrino Neutral current elastic (NCE) scattering . . . . . . . . . . . . . . 249

A.2 The scibath pilot detector beam test . . . . . . . . . . . . . 250

\begin{tabular}{ll}
\hline B Gamma matrix algebra & 260
\end{tabular}

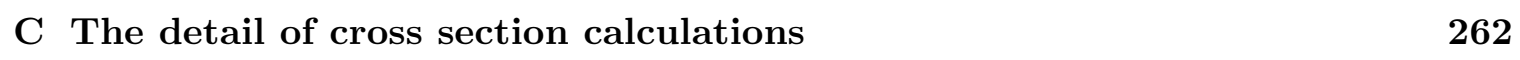

C.1 Neutrino-Nucleon scattering . . . . . . . . . . . . . . . . . . . . . . . 262

xviii 
C.1.1 Kinematics and cross section formula . . . . . . . . . . . . . . . 262

C.1.2 Leptonic tensor term . . . . . . . . . . . . . . . . . . . . 267

C.1.3 Hadronic tensor term . . . . . . . . . . . . . . . . . . 268

C.1.4 Llewellyn Smith formalism . . . . . . . . . . . . . . . . . 274

C.2 Neutrino-Nuclei lepton scattering . . . . . . . . . . . . . . . . . . . . 279

C.2.1 Kinematics and cross section formula . . . . . . . . . . . . . . . . 279

C.2.2 Leptonic and hadronic tensor term . . . . . . . . . . . . . . . . . 282

C.2.3 Hadronic nuclei functions, definitions . . . . . . . . . . . . . . . 285

C.2.4 Analytic solutions for the phase space integrals . . . . . . . . . . . 292

C.2.5 Hadronic nucleon functions, definitions . . . . . . . . . . . . . . 295

\begin{tabular}{|l} 
D Table for Predicted $\nu_{\mu}$ flux for MiniBooNE \\
\hline
\end{tabular} 


\section{List of Tables}

$3.1 \quad$ The comparison of measured axial mass $M_{A} \ldots \ldots \ldots \ldots \ldots \ldots$

$6.1 \quad$ Summary of the test of Lorentz violation with LSND experiment . . . . . . 107

$8.1 \quad$ Uncertainties in $M_{A}$ and $\kappa$ from the fit $\ldots \ldots \ldots \ldots$. . . . . . . . 159

9.1 Data-MC comparison before and after the subevent correction . . . . . . . 166

9.2 Event totals summary after the CCQE cut as determined by the MC . . . . 171

9.3 The systematic error summary for $M_{A}$ and $\kappa \ldots \ldots \ldots$

$9.4 \quad M_{A}$ only fit results with limited $Q^{2} \ldots \ldots \ldots \ldots \ldots$

10.1 The systematic error summary for flux-unfolded total cross section at 700$800 \mathrm{MeV}$ bin . . . . . . . . . . . . . . . . . . . 244

D.1 Predicted $\nu_{\mu}$ flux in MiniBooNE $\ldots \ldots \ldots$. . . . . . . . . . 300 


\section{List of Figures}

$2.1 \quad$ Space time diagram for false Lorentz violation with neutrino oscillation . . 19

$2.2 \quad$ An illustration of neutrino oscillations as interferometers . . . . . . . . . . . 20

$3.1 \quad$ Feynman diagram for neutrino-charged lepton scattering . . . . . . . . . . . 23

3.2 The neutrino-nucleon scattering diagram $\ldots \ldots \ldots \ldots$

$3.3 \quad$ Pseudo scalar contribution for neutrino-nucleon scattering . . . . . . . . . . 35

3.4 The proton electromagnetic current in the Breit frame . . . . . . . . . . . . 39

3.5 The neutrino-nuclei scattering diagram $\ldots \ldots \ldots$. . . . . . . . . 46

3.6 The world data for $\nu_{\mu}$ charged current cross section . . . . . . . . . . . 52

3.7 The CCQE true-reconstruction energy error . . . . . . . . . . . . . . 55

$4.1 \quad$ A cartoon illustrating observer and particle Lorentz violation . . . . . . . . 62

$4.2 \quad$ A sensitivity plot for general neutrino oscillations . . . . . . . . . . . . 67

$4.3 \quad$ The LSND oscillation signal $\ldots \ldots \ldots \ldots \ldots$

4.4 The coordinate system for Lorentz violation . . . . . . . . . . . . . . 71

4.5 The sidereal time distribution of the LSND signal with the results from the fits 74

$4.6 \quad$ Log likelihood value for the 3 -parameter fit of the LSND signal . . . . . . . 75 
5.1 The solution for the bimaximal model . . . . . . . . . . . . . . . . . . . 87

5.2 The oscillation probability from the bimaximal model . . . . . . . . . . 88

5.3 The solution for the bicycle model $\ldots \ldots \ldots$. . . . . . . . . . . . 90

5.4 The solution of neutrino oscillations by the tandem model . . . . . . . . . 93

5.5 The solution of antineutrino oscillations by the tandem model . . . . . . . . 94

$5.6 \quad$ Solar neutrino oscillation probabilities in the tandem model . . . . . . . . . 96

5.7 Survival probabilities for atmospheric neutrinos for the tandem model . . . $\quad 97$

5.8 Survival probabilities for long-baseline reactor antineutrinos for the tandem

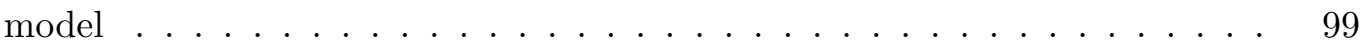

$5.9 \quad$ Oscillation probabilities for short-baseline experiments for the tandem model 101

5.10 Number of candidate $\nu_{e}$ events as a function of neutrino energy $\ldots . . . . .103$

5.11 Candidate $\nu_{e}$ events in MiniBooNE with the tandem model prediction . . . 104

$7.1 \quad$ A picture of Fermilab $\ldots \ldots \ldots \ldots$. . . . . . . . . . . . . . 113

7.2 A schematic overview of the MiniBoonE neutrino beamline . . . . . . . . . 114

7.3 The BNB micro and macro structure . . . . . . . . . . . . . . . . . . 115

7.4 The MiniBooNE magnetic horn $\quad \ldots \ldots \ldots \ldots$

7.5 Predicted $\nu_{\mu}$ flux for MiniBooNE $\ldots \ldots \ldots \ldots \ldots \ldots \ldots$

$7.6 \quad$ A schematic drawing of the MiniBooNE detector $\ldots \ldots \ldots$. . . . . . . 120

$7.7 \quad$ A photo showing the the inner and outer tank regions. . . . . . . . . . . . 122

7.8 Event fractions predicted by nuance for MiniBooNE $\ldots \ldots \ldots \ldots$

7.9 Data and MiniBooNE-tuned nuance comparison for pion absorption and charge exchange cross section $\ldots \ldots \ldots \ldots \ldots$

xxii 
7.10 The data and hadronic interaction packages for the pion absorption and charge exchange total cross section . . . . . . . . . . . . . . . . 132

7.11 Number of tank hits as a function of time for a $\mathrm{CC} 1 \pi$ candidate event $\ldots . .136$

7.12 A cartoon for the particle track fitter . . . . . . . . . . . . . . . . 137

7.13 Emission power from an electron track with function of the solid angle along the track . . . . . . . . . . . . . . . . . . . . . . 138

7.14 The corrected time for the emission from an electron track . . . . . . . . . . 139

7.15 A cartoon illustrating PID . . . . . . . . . . . . . . . . . 141

7.16 An event display for a stopping muon candidate event . . . . . . . . . . . . 142

$8.1 \quad$ A schematic figure illustrating the MiniBooNE blind oscillation analysis. . . 145

8.2 Combined fit result for $\nu_{e}$ oscillation search $\ldots \ldots \ldots$. . . . . . . 147

$8.3 \quad$ A cartoon for CCQE interaction $\ldots \ldots \ldots \ldots$

8.4 Data-MC ratio in two-dimensional kinematic space before fit . . . . . . . . 153

8.5 Schematic view of nucleon momentum space . . . . . . . . . . . . . . 155

$8.6 \quad M_{A}-\kappa$ variation in $Q^{2} \ldots \ldots \ldots \ldots \ldots \ldots \ldots$

$8.7 M_{A}-\kappa$ fit result $\ldots \ldots \ldots \ldots \ldots \ldots \ldots \ldots$

$8.8 \quad \mathrm{CC} 1 \pi$ weighting function used for CC1 $\pi$ background tuning . . . . . . . . . 159

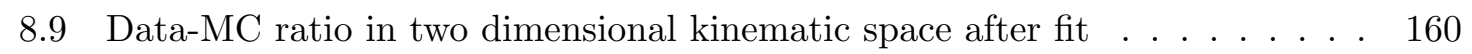

8.10 Neutrino energy plot after fit $\ldots \ldots \ldots 1$

8.11 Muon kinetic energy plot after fit . . . . . . . . . . . . . . . . . 161

8.12 Muon angle plot after fit . . . . . . . . . . . . . . . . . . . 162 
$8.13 M_{A}$ only fit result $\ldots \ldots \ldots \ldots \ldots \ldots \ldots \ldots$

$9.1 \quad$ Data-MC comparison of muon likelihood function . . . . . . . . . . . . 168

$9.2 \quad 2$ vertices distance-muon energy relationship . . . . . . . . . . . . . . . . . 170

$9.3 \quad$ Data-MC comparison for $Q^{2}$ plots before fit . . . . . . . . . . . . . . . 179

$9.4 \quad$ Weighting function of each channel before fit $\ldots \ldots \ldots$. . . . . . . . 180

$9.5 \quad$ Data-MC comparison for $Q^{2}$ plots after fit . . . . . . . . . . . . . . . 181

9.6 Weighting function of each channel after fit $\ldots \ldots \ldots$. . . . . . . 182

9.7 Data-MC ratio of $Q^{2}$ in the 3 subevent sample with various cross section models . . . . . . . . . . . . . . . . . . . . . . . . 184

9.8 Data-MC pull distribution of two dimensional plane in 3 subevents with various cross section models . . . . . . . . . . . . . . . . . . . . . 185

$9.9 \quad$ Data-MC comparison for $Q^{2}$ plots after fit with new cv MC . . . . . . . . 187

9.10 Weighting function of each channel after fit with new cv MC $\ldots . . . . .188$

9.11 The plots for $M_{A}-\kappa$ fit $\ldots \ldots \ldots \ldots$. . . . . . . . . . . . . 191

9.12 The detail of contour plot . . . . . . . . . . . . . . . . . . . 192

9.13 The plots for statistical error only $M_{A}-\kappa$ fit $\ldots \ldots \ldots$. . . . . . . 195

9.14 The plots for flux error only $M_{A}-\kappa$ fit $\ldots \ldots \ldots \ldots \ldots$

9.15 The plots for cross section error only $M_{A}-\kappa$ fit $\ldots \ldots \ldots$

9.16 The plots for detector error only $M_{A}-\kappa$ fit $\ldots \ldots \ldots$. . . . . . . . . . 196

9.17 Data-MC comparison for $Q^{2}$ plots after fit with new $M_{A}$ and $\kappa \ldots . . . \quad 198$

10.1 MC true energy and reconstructed true energy $\ldots \ldots \ldots \ldots \ldots$ 
10.2 The comparison of background subtraction and signal fraction method . . . 210

10.3 The unfolded background removed data for CCQE kinematics . . . . . . . . 212

10.4 A cartoon to show the parameter space $\ldots \ldots \ldots \ldots \ldots$

10.5 An example of multisim . . . . . . . . . . . . . . . . . . . . . 216

$10.6 \pi^{+}$production multisim draws and cv MC for reconstructed neutrino energy 222

10.7 Detector errors for the reconstructed neutrino energy . . . . . . . . . . . 226

10.8 Total error plots for the flux-unfolded double differential cross section and flux-folded total cross section . . . . . . . . . . . . . . . . . . 228

10.9 The flux-folded double differential cross section . . . . . . . . . . . . . . . . 229

10.10The flux-folded double differential cross section with total error . . . . . . . 230

10.11The flux-folded double differential cross section with total error from different angles . . . . . . . . . . . . . . . . . . . . 231

10.12The flux-unfolded total cross section with theoretical prediction . . . . . . . 233

10.13The comparison of $Q^{2}$ distribution of counts and flux-unfolded differential cross section . . . . . . . . . . . . . . . . . . . . . 235

10.14The comparison of new cross section model and flux-unfolded differential cross section . . . . . . . . . . . . . . . . . . . . 236

10.15The flux-unfolded differential cross section with $Q^{2}$ with reconstructed true neutrino energy $700-800 \mathrm{MeV} \ldots \ldots \ldots$. . . . . . . . . . 237

10.16The flux-unfolded total cross section with binding energy $=0 \mathrm{MeV} . \ldots 238$

10.17Flux error on cross sections . . . . . . . . . . . . . . . . . . . 240

10.18 Cross section model error only cross sections . . . . . . . . . . . . 241

10.19Detector model uncertainty only cross sections . . . . . . . . . . . . . 242 
10.20Unfolding error only cross sections . . . . . . . . . . . . . . . . 243

A.1 The FINeSSE detector . . . . . . . . . . . . . . . . . . . . . . . . . . . . 251

A.2 The scibath pilot detector . . . . . . . . . . . . . . . . . . 252

A.3 The scibath pilot detector beam test setup . . . . . . . . . . . . . . . . . 252

A.4 The scibath pilot detector beam test electronics diagram . . . . . . . . . . 253

A.5 The scibath pilot detector beam test principle cartoon . . . . . . . . . . 255

A.6 The scibath pilot detector coordinate resolution . . . . . . . . . . . . 256

A.7 The scibath pilot detector coordinate resolution for UV-to-blue fibers . . . . 257

A.8 The scibath pilot detector signal transformation . . . . . . . . . . . . . . 259

C.1 The neutrino-nucleon scattering diagram . . . . . . . . . . . . . . . 263

C.2 The crossing diagram of neutrino-nucleon scattering $\ldots \ldots \ldots$. . . . . . 278

C.3 The neutrino-nuclei scattering diagram . . . . . . . . . . . . . . . . . . 280

xxvi 


\section{Part I}

Neutrino physics 
An object so small, impalpable, invisible, would seem myth enough, but scientists reached beyond.

\section{- I. Asimov, The Neutrino}




\section{Chapter 1}

\section{Introduction of this thesis}

In this thesis, we present results from two major pieces of work: Tests of Lorentz violation via neutrino oscillations and a measurement of neutrino charged current quasielastic (CCQE) scattering with the MiniBooNE experiment.

In Part I of this thesis, the general features of neutrinos are discussed. This provides introductory material for the remaining parts. In Chapter 2, we discuss the formalism of neutrino oscillations. And we examine the interesting nature of neutrino oscillations. In Chapter 3, we discuss the formalism of neutrino scattering. And we re-derive neutrinocharged lepton, neutrino-nucleon, and neutrino-nuclei scattering cross section formulas. More details of calculations are found in Appendix C.

In Part II, we explain the test of Lorentz violation with neutrino oscillations. This includes three original works: Tests of Lorentz violation in $\bar{\nu}_{\mu} \rightarrow \bar{\nu}_{e}$ oscillations (Chapter 4) [1]; Global three-parameter model for neutrino oscillations using Lorentz violation (Chapter 5) [2]; and Neutrino oscillations and Lorentz violation with MiniBooNE (Chapter 5) [3. In Chapter 4, we provide an introduction to Lorentz violation and briefly discuss the analysis of a test of Lorentz violation in the LSND experiment. In Chapter 5, a model of neutrino oscillations, the "tandem" model, using Lorentz violation is presented. It is 
then compared to existing oscillation data including recent results from the MiniBooNE experiment. In Chapter 6, we summarize Chapters 4 and 5 (Part II), and then discuss the status and outlook of this aspect of Lorentz violation.

In Part III, we present our CCQE measurement in the MiniBooNE experiment. Part III includes two of my original works: Measurement of muon neutrino quasielastic scattering on carbon (Chapter 8) [4]; and Measurement of $\nu_{\mu}$ CCQE double differential cross section (Chapter 10). In Chapter 7, we provide an introduction to the MiniBooNE experiment. We briefly explain the experimental apparatus and the associated systematic uncertainties. The details of the neutrino event model (and errors) used for the neutrino event rate predictions are presented. In Chapter 8, we discuss the CCQE measurement for the MiniBooNE oscillation experiment. The CCQE measurement as was performed for the MiniBooNE $\nu_{e}$ appearance result is presented along with a discussion about the tuning of the nuclear model parameters. Then in Chapter 9, we discuss the simultaneous measurement of the CCQE interaction and the charged current one pion production $(\mathrm{CC} 1 \pi)$ interaction. To improve our CCQE measurement, we need a better description of the background. From this measurement, the CC1 $\pi$ background in the CCQE sample is determined. Finally, in Chapter 10, we present the CCQE differential cross section measurement in MiniBooNE. In this chapter, the differential cross section of the CCQE process is presented with a careful discussion of all systematic errors. In Chapter 11, we summarize from Chapters 8-10 (Part III), and discuss the status and outlook.

In addition to the main text of this thesis, in Appendix A, we introduce the FINeSSE experiment. This Appendix provide a brief introduction of this experiment and results from the pilot detector beam test. This appendix includes one of my original work: A large-volume detector capable of charged-particle tracking [5]. 


\section{Chapter 2}

\section{Neutrino oscillations}

The most distinct feature of neutrinos may be their state definitions. Neutrinos are the only particles in the standard model defined by their flavor eigenstates. All quarks and charged leptons are defined by their mass eigenstates, which are simultaneous eigenstates with the Hamiltonian. So, we can say, "The muon has mass of $105 \mathrm{MeV} / \mathrm{c}^{2}$." but a similar statement does not exist for neutrinos. In addition, because neutrino flavor states are not also eigenstates of the Hamiltonian, neutrino flavor states are not conserved with time evolution.

In this chapter, we derive the equations of neutrino oscillations under the wave packet formalism [6], following Ref. [7]. Then we investigate the interesting nature of the quantum physics of neutrinos.

\subsection{Neutrino oscillation formula}

Since neutrino flavor states are not simultaneous eigenstates of the Hamiltonian, they are not conserved under time evolution. The neutrino flavor states are written as mixtures of 
the Hamiltonian eigenstates,

$$
\left|\nu_{\alpha}>=\sum_{i} U_{\alpha i}^{*}\right| \nu_{i}>
$$

Here, $\mid \nu_{\alpha}>$ are the flavor eigenstates, whereas $\mid \nu_{i}>$ are the Hamiltonian eigenstates, and they are related via the mixing matrix $U_{\alpha i}^{*}$. In the vacuum (= no interactions), the Hamiltonian eigenstate can be identified with the mass eigenstate. Then the mixing matrix is called PMNS matrix. Since the quark-sector analog, the CKM matrix, is defined in order to mix flavor eigenstates to describe mass eigenstates, the PMNS matrix is defined via its conjugate in order to follow the same definition as the CKM matrix.

The time evolution of a flavor eigenstate is,

$$
\left|\nu_{\alpha}(t)>=\sum_{i} U_{\alpha i}^{*} e^{-i \lambda_{i} t}\right| \nu_{i}>
$$

Now, we use $\lambda_{i}$ for the $i^{t h}$ eigenvalue of the Hamiltonian eigenstate $\mid \nu_{i}>$ and $\Delta_{i j}$ for the eigenvalue difference $\left(=\lambda_{i}-\lambda_{j}\right)$.

Then, the the transition amplitude from flavor eigenstate $\alpha$ to flavor eigenstate $\beta$ after time $t$ is,

$$
\begin{aligned}
<\nu_{\beta} \mid \nu_{\alpha}(t)> & =\left(\sum_{j}<\nu_{j} \mid U_{j \beta}^{T}\right)\left(\sum_{i} U_{\alpha i}^{*} e^{-i \lambda_{i} t} \mid \nu_{i}>\right) \\
& =\sum_{j} U_{\alpha i}^{*} e^{-i \lambda_{i} t} U_{\beta i} .
\end{aligned}
$$

Finally, the oscillation transition probability from flavor state $\alpha$ to flavor state $\beta$ after time 
$t$ is,

$$
\begin{aligned}
P_{\nu_{\alpha} \rightarrow \nu_{\beta}}(t)= & \left|<\nu_{\beta}\right| \nu_{\alpha}(t)>\left.\right|^{2}=\left|\left(\sum_{j} U_{\alpha j} e^{i \lambda_{j} t} U_{\beta j}^{*}\right)\left(\sum_{i} U_{\alpha i}^{*} e^{-i \lambda_{i} t} U_{\beta i}\right)\right| \\
= & \left|\sum_{i, j} U_{\alpha i}^{*} U_{\beta i} U_{\alpha j} U_{\beta j}^{*} e^{-i\left(\lambda_{i}-\lambda_{j}\right) t}\right| \\
= & \sum_{i}\left|U_{\alpha i}\right|^{2}\left|U_{\beta i}\right|^{2}+2\left|\sum_{i>j} U_{\alpha i}^{*} U_{\beta i} U_{\alpha j} U_{\beta j}^{*}\left(\cos \left(\Delta_{i j} t\right)+i \sin \left(\Delta_{i j} t\right)\right)\right| \\
= & \sum_{i}\left|U_{\alpha i}\right|^{2}\left|U_{\beta i}\right|^{2}+2 R e \sum_{i>j} U_{\alpha i}^{*} U_{\beta i} U_{\alpha j} U_{\beta j}^{*} \cos \left(\Delta_{i j} t\right)+2 I m \sum_{i>j} U_{\alpha i}^{*} U_{\beta i} U_{\alpha j} U_{\beta j}^{*} \sin \left(\Delta_{i j} t\right) \\
= & \sum_{i}\left|U_{\alpha i}\right|^{2}\left|U_{\beta i}\right|^{2}+2 R e \sum_{i>j} U_{\alpha i}^{*} U_{\beta i} U_{\alpha j} U_{\beta j}^{*}\left(1-2 \sin { }^{2}\left(\frac{\Delta_{i j} t}{2}\right)\right)-\ldots \\
= & \left|U_{\alpha 1}\right|^{2}\left|U_{\beta 1}\right|^{2}+\left|U_{\alpha 2}\right|^{2}\left|U_{\beta 2}\right|^{2}+\left|U_{\alpha 3}\right|^{2}\left|U_{\beta 3}\right|^{2} \\
& +2 R e\left(U_{\alpha 3}^{*} U_{\beta 3} U_{\alpha 1} U_{\beta 1}^{*}\right)+2 R e\left(U_{\alpha 3}^{*} U_{\beta 3} U_{\alpha 2} U_{\beta 2}^{*}+2 R e\left(U_{\alpha 2}^{*} U_{\beta 2} U_{\alpha 1} U_{\beta 1}^{*}\right)\right. \\
& -4 R e \sum_{i>j} U_{\alpha i}^{*} U_{\beta i} U_{\alpha j} U_{\beta j}^{*} \sin ^{2}\left(\frac{\Delta_{i j} t}{2}\right)-\ldots \\
= & \left|\sum_{i} U_{\alpha i}^{*} U_{\beta i}\right|^{2}-4 R e \sum_{i>j} U_{\alpha i}^{*} U_{\beta i} U_{\alpha j} U_{\beta j}^{*} \sin { }^{2}\left(\frac{\Delta_{i j} t}{2}\right)-\cdots
\end{aligned}
$$

Since neutrinos are extremely relativistic particles,

$$
t=L
$$

therefore,

$$
\begin{aligned}
P_{\nu_{\alpha} \rightarrow \nu_{\beta}}(L)= & \delta_{\alpha \beta}-4 \sum_{i>j} \operatorname{Re}\left(U_{\alpha i}^{*} U_{\beta i} U_{\alpha j} U_{\beta j}^{*}\right) \sin ^{2}\left(\frac{\Delta_{i j}}{2} L\right) \\
& +2 \sum_{i>j} \operatorname{Im}\left(U_{\alpha i}^{*} U_{\beta i} U_{\alpha j} U_{\beta j}^{*}\right) \sin \left(\Delta_{i j} L\right)
\end{aligned}
$$

This is the model-independent formula describing neutrino oscillations.

Since the neutrino kinetic term is the only one non-trivial term for the neutrino Hamiltonian eigenstate in the vacuum, under the standard assumption (the so-called "equal mo- 
mentum approximation"), then

$$
\lambda_{i}=E_{i}=\sqrt{p^{2}+m_{i}^{2}} \sim p+\frac{m_{i}^{2}}{2 p} \sim E+\frac{m_{i}^{2}}{2 E} .
$$

Then, using standard units for neutrino oscillation experiments,

$$
\begin{aligned}
\frac{\Delta m_{i j}^{2} L}{4 E} & =\frac{\Delta m_{i j}^{2}\left(M e V^{2}\right) L(f m)}{4 E(M e V) \hbar c(M e V \dot{f} m)} \\
& =1.27 \frac{\Delta m_{i j}^{2}\left(e V^{2}\right) L(m)}{E(M e V)}
\end{aligned}
$$

Equation 2.6 becomes the familiar form of the standard three neutrino massive model in the particle data group [8]:

$$
\begin{aligned}
P_{\nu_{\alpha} \rightarrow \nu_{\beta}}(L)= & \delta_{\alpha \beta}-4 \sum_{i>j} \operatorname{Re}\left(U_{\alpha i}^{*} U_{\beta i} U_{\alpha j} U_{\beta j}^{*}\right) \sin ^{2}\left(1.27 \Delta m_{i j}^{2} \frac{L}{E}\right) \\
& +2 \sum_{i>j} \operatorname{Im}\left(U_{\alpha i}^{*} U_{\beta i} U_{\alpha j} U_{\beta j}^{*}\right) \sin \left(2.54 \Delta m_{i j}^{2} \frac{L}{E} L\right) .
\end{aligned}
$$

Under the assumption that all elements of the mixing matrix are real (equivalent to assumption of no $\mathrm{CP}$ violation in the neutrino sector), the two-neutrino oscillation formula is,

$$
\begin{aligned}
P_{\nu_{\alpha} \rightarrow \nu_{\beta}}(L)= & \delta_{\alpha \beta}-4(\cos \theta) \cdot(\sin \theta) \cdot(\cos \theta) \cdot( \pm \sin \theta) \sin ^{2}\left(1.27 \Delta m_{12}^{2} \frac{L}{E}\right) \\
= & \begin{cases}1-\sin ^{2} 2 \theta \sin ^{2}\left(1.27 \Delta m_{12}^{2} \frac{L}{E}\right) & \text { for } \alpha=\beta \\
\sin ^{2} 2 \theta \sin ^{2}\left(1.27 \Delta m_{12}^{2} \frac{L}{E}\right) & \text { for } \alpha \neq \beta\end{cases}
\end{aligned}
$$

using the Cabibbo-matrix-like mixing matrix, $U=\left(\begin{array}{cc}\cos \theta & \sin \theta \\ -\sin \theta & \cos \theta\end{array}\right)$. 


\subsection{Wave packet formalism}

\subsubsection{Group velocity based formalism}

In this section, we discuss the conditions required for neutrino oscillations to occur. Although the standard formulas above describe neutrino oscillations, in a more rigorous sense, oscillations should be described with wave packets [6]. To see the nature of wave packet formalism, the group velocity based approach [7] is very instructive.

The normalized Hamiltonian eigenstate neutrino wave packet in momentum space is defined

$$
\Psi_{i}(p)=\sqrt{\sqrt{2 \pi} \sigma_{p}} \exp \left(-\frac{\left(p-<p_{i}>\right)^{2}}{4 \sigma_{p}^{2}}\right)
$$

and we have used the mean value of the momentum wave packet $<p_{i}>$, the mean energy $<$ $\left.E_{i}\right\rangle=\sqrt{\left\langle p_{i}\right\rangle^{2}+m_{i}^{2}}$, and the group velocity $v_{i}=\frac{\left\langle p_{i}\right\rangle}{\left\langle E_{i}\right\rangle}$. For simplicity, one-dimensional neutrino propagation is considered but it is easily extended to 3 dimensions. Then, the Fourier transformation of the momentum wave packet is,

$$
\begin{aligned}
\Psi_{i}(x, 0) & =\int_{-\infty}^{\infty} \Psi_{i}(p) e^{i p x} d p \\
& =\frac{1}{\sqrt{\sqrt{2 \pi} \sigma_{x}}} \exp \left(i<p_{i}>x-\frac{x^{2}}{4 \sigma_{x}^{2}}\right) .
\end{aligned}
$$

And, after time $t$, using, $<p_{i}>v_{i}=\frac{\left\langle p_{i}\right\rangle^{2}}{\left\langle E_{i}>\right.}=\frac{\left\langle E_{i}\right\rangle^{2}-m_{i}^{2}}{<E_{i}>} \sim<E_{i}>$,

$$
\begin{aligned}
\Psi_{i}(x, t) & =\frac{1}{\sqrt{\sqrt{2 \pi} \sigma_{x}}} \exp \left(i<p_{i}>\left(x-v_{i} t\right)-\frac{\left(x-v_{i} t\right)^{2}}{4 \sigma_{x}^{2}}\right) \\
& =\frac{1}{\sqrt{\sqrt{2 \pi} \sigma_{x}}} \exp \left(i\left(<p_{i}>x-<E_{i}>t\right)-\frac{\left(x-v_{i} t\right)^{2}}{4 \sigma_{x}^{2}}\right) .
\end{aligned}
$$

Therefore, the transition probability of a flavor eigenstate from $\nu_{\alpha}$ to $\nu_{\beta}$, after propagating 
distance $L$ within time $T$ is,

$$
\begin{aligned}
& P_{\nu_{\alpha} \rightarrow \nu_{\beta}}(L, T)=\left(\Sigma_{j} U_{\alpha j}^{*} \Psi_{j}^{*}(L, T) U_{\beta j}\right) \cdot\left(\Sigma_{i} U_{\alpha i} \Psi_{i}(L, T) U_{\beta i}^{*}\right) \\
& =\frac{1}{\sqrt{2 \pi} \sigma_{x}}\left\{\sum_{i, j} U_{\alpha i} U_{\beta i}^{*} U_{\alpha j}^{*} U_{\beta j}\right. \\
& \times \exp \left[i\left(<p_{i}>-<p_{j}>\right) L-i\left(<E_{i}>-<E_{j}>\right) T\right] \\
& \left.\times \exp \left[-\frac{\left(L-v_{i} T\right)^{2}}{4 \sigma_{x}^{2}}-\frac{\left(L-v_{j} T\right)^{2}}{4 \sigma_{x}^{2}}\right]\right\} \\
& =\frac{1}{\sqrt{2 \pi} \sigma_{x}}\left\{\sum_{i, j} U_{\alpha i} U_{\beta i}^{*} U_{\alpha j}^{*} U_{\beta j}\right. \\
& \exp \left[i\left(<p_{i}>-<p_{j}>\right) L-i\left(<E_{i}>-<E_{j}>\right) T\right] \\
& \left.\times \exp \left[-\frac{L^{2}}{2 \sigma_{x}^{2}}+\frac{L T}{2 \sigma_{x}^{2}}\left(v_{i}+v_{j}\right)-\frac{T^{2}}{4 \sigma_{x}^{2}}\left(v_{i}^{2}+v_{j}^{2}\right)\right]\right\} \\
& =e^{i\left(<p_{i}>-<p_{j}>\right) L} \times \\
& \exp \left\{-\frac{v_{i}^{2}+v_{j}^{2}}{4 \sigma_{x}^{2}}\left[T-\left(\frac{v_{i}+v_{j}}{v_{i}^{2}+v_{j}^{2}} L-i \frac{2 \sigma_{x}^{2}}{v_{i}^{2}+v_{j}^{2}}\left(<E_{i}>-<E_{j}>\right)\right)\right]^{2}\right. \\
& \left.-\frac{L^{2}}{2 \sigma_{x}^{2}}+\frac{v_{i}^{2}+v_{j}^{2}}{4 \sigma_{x}^{2}}\left(\frac{v_{i}+v_{j}}{v_{i}^{2}+v_{j}^{2}} L-i \frac{2 \sigma_{x}^{2}}{v_{i}^{2}+v_{j}^{2}}\left(<E_{i}>-<E_{j}>\right)\right)^{2}\right\}
\end{aligned}
$$

In practice, we know the distance neutrinos propagate, however we do not know the time it takes. So we take the average over $T$ by $\int_{-\infty}^{\infty} e^{\alpha(x-\beta)^{2}} d x=\sqrt{\frac{\pi}{\alpha}}$, and introduce a normalization factor $N_{T}\left(=\sum_{i} \frac{\left|U_{\alpha i}\right|^{2}}{\left|v_{i}\right|}\right)$. Then,

$$
\begin{aligned}
P_{\nu_{\alpha} \rightarrow \nu_{\beta}}(L, T)= & \sqrt{\frac{2}{v_{i}^{2}+v_{j}^{2}}} \cdot \frac{1}{N_{T}} \sum_{i, j} U_{\alpha i} U_{\beta i}^{*} U_{\alpha j}^{*} U_{\beta j} \cdot e^{i\left(<p_{i}>-<p_{j}>\right) L} . \\
& \times \exp \left[-\frac{L^{2}}{2 \sigma_{x}^{2}}+\frac{\left(v_{i}+v_{j}\right)^{2} L^{2}}{4 \sigma_{x}^{2}\left(v_{i}^{2}+v_{j}^{2}\right)}\right. \\
& -i \frac{v_{i}+v_{j}}{v_{i}^{2}+v_{j}^{2}}\left(<E_{i}>-<E_{j}>\right) L \\
& \left.-\frac{\sigma_{x}^{2}}{v_{i}^{2}+v_{j}^{2}}\left(<E_{i}>-<E_{j}>\right)^{2}\right]
\end{aligned}
$$


Using $\sigma_{x} \cdot \sigma_{p} \geq \frac{1}{2}$

$$
\begin{aligned}
& P_{\nu_{\alpha} \rightarrow \nu_{\beta}}(L, T)=\sqrt{\frac{2}{v_{i}^{2}+v_{j}^{2}}} \cdot \frac{1}{N_{T}} \sum_{i, j} U_{\alpha i} U_{\beta i}^{*} U_{\alpha j}^{*} U_{\beta j} \\
& \times \exp \left\{i\left[\left(<p_{i}>-<p_{j}>\right)-\left(<E_{i}>-<E_{j}>\right) \frac{v_{i}+v_{j}}{v_{i}^{2}+v_{j}^{2}}\right] L\right\} \\
& \times \exp \left\{-\frac{L^{2}\left[2\left(v_{i}^{2}+v_{j}^{2}\right)-\left(v_{i}+v_{j}\right)^{2}\right]}{4 \sigma_{x}^{2}\left(v_{i}^{2}+v_{j}^{2}\right)}\right. \\
& \left.-\frac{1}{4 \sigma_{p}^{2}\left(v_{i}^{2}+v_{j}^{2}\right)}\left(<E_{i}>-<E_{j}>\right)^{2}\right\} \\
& =\sqrt{\frac{2}{v_{i}^{2}+v_{j}^{2}}} \cdot \frac{1}{N_{T}} \sum_{i, j} U_{\alpha i} U_{\beta i}^{*} U_{\alpha j}^{*} U_{\beta j} \\
& \left.\times \exp \left\{-i\left[\left(<E_{i}>-<E_{j}>\right) \frac{v_{i}+v_{j}}{v_{i}^{2}+v_{j}^{2}}-<p_{i}>-<p_{j}>\right)\right] L\right\} \\
& \times \exp \left[-\frac{\left(v_{i}-v_{j}\right)^{2} L^{2}}{4 \sigma_{x}^{2}\left(v_{i}^{2}+v_{j}^{2}\right)}\right] \\
& \times \exp \left[-\frac{\left(<E_{i}>-<E_{j}>\right)^{2}}{4 \sigma_{p}^{2}\left(v_{i}^{2}+v_{j}^{2}\right)}\right] .
\end{aligned}
$$

In Eq. 2.15, the first exponential term is imaginary, and so it represents the oscillating coherent nature of neutrinos. The second and third exponential terms are damping terms, and they describe the destructive effects of coherence.

From the first term, we can see that the standard approximation, $T=L$ (Eq. 2.5), corresponds to $T=\left(\frac{v_{i}+v_{j}}{v_{i}^{2}+v_{j}^{2}}\right) L$.

In the first exponential term, we can define the oscillation length,

$$
\begin{gathered}
\exp \left\{-i\left(<E_{i}>-<E_{j}>\right)\left[\frac{v_{i}+v_{j}}{v_{i}^{2}+v_{j}^{2}}-\frac{<p_{i}>-<p_{j}>}{<E_{i}>-<E_{j}>}\right] L \equiv \exp \left(-i \cdot 2 \pi \frac{L}{L_{i j}^{o s c}}\right)\right. \\
\text { with } \quad L_{i j}^{\text {osc }} \equiv \frac{2 \pi}{\left|<E_{i}>-<E_{j}>\right|}\left\{\frac{v_{i}+v_{j}}{v_{i}^{2}+v_{j}^{2}}-\frac{<p_{i}>-<p_{j}>}{<E_{i}>-<E_{j}>}\right\} \stackrel{E R}{\sim} \frac{2 \pi}{\Delta_{i j}} .
\end{gathered}
$$

Here, the oscillation length $L_{i j}^{o s c}$ describes the resonance condition for neutrino oscillations.

In the extremely relativistic case ("ER"), which is always true for neutrinos, the oscillation 
length becomes $\sim \frac{2 \pi}{\Delta_{i j}}$, and, for the standard three neutrino massive model,

$$
L^{o s c} \sim \frac{4 \pi E}{\Delta m_{i j}^{2}}
$$

Next, in the second exponential term,

$$
\begin{aligned}
\exp \left[-\frac{\left(v_{i}-v_{j}\right)^{2} L^{2}}{4 \sigma_{x}^{2}\left(v_{i}^{2}+v_{j}^{2}\right)}\right] \equiv \exp \left[-\frac{1}{4}\left(\frac{L}{L_{i j}^{c o h}}\right)^{2}\right] \\
\text { with } \quad L_{i j}^{c o h} \equiv \sigma_{x} \frac{\sqrt{v_{i}^{2}+v_{j}^{2}}}{\left|v_{i}-v_{j}\right|} \stackrel{E R}{\sim} \frac{\sigma_{x}}{\left|v_{i}-v_{j}\right|},
\end{aligned}
$$

the damping becomes larger when the neutrinos propagate longer distances. Therefore, this term describes the situation when two neutrinos propagate over a long distance and lose coherence. And, the coherence length, $L_{i j}^{c o h}$, is the condition that this damping is sizable. This term is important only for cosmological and astrophysical neutrinos.

Finally, in the third term,

$$
\exp \left[-\frac{\left(<E_{i}>-<E_{j}>\right)^{2}}{4 \sigma_{p}^{2}\left(v_{i}^{2}+v_{j}^{2}\right)}\right] \stackrel{E R}{\sim} \exp \left[-4 \pi^{2}\left(\frac{\sigma_{x}}{L_{i j}^{o s c}}\right)^{2}\right]
$$

the damping is large when energy separation is big. So this term describes the uncertainty principle, that is, the interference happens only when the energy separation (or $\Delta m^{2}$, for the standard three neutrino massive model) is smaller than $\sigma_{p}$. In the extremely relativistic limit, there is another interpretation. If the oscillation length is small compared with the position resolution, the neutrino oscillation is damped. This term is important for the design of realistic long-baseline oscillation experiments.

Finally, the neutrino oscillation probability using the group-velocity based wave packet 
formalism is (again, assuming all mixing matrix elements are real),

$$
\begin{aligned}
P_{\nu_{\alpha} \rightarrow \nu_{\beta}}(L)= & \sum_{i, j} U_{\alpha i} U_{\beta i} U_{\alpha j} U_{\beta j} \times \exp \left(-i 2 \pi \frac{L}{L_{i j}^{\text {osc }}}\right) \\
& \times \exp \left[-\frac{1}{4}\left(\frac{L}{L_{i j}^{c o h}}\right)^{2}\right] \times \exp \left[-4 \pi^{2}\left(\frac{\sigma_{x}}{L_{i j}^{o s c}}\right)^{2}\right] \\
= & \sum_{i}\left|U_{\alpha i}\right|^{2}\left|U_{\beta i}\right|^{2}+2 \sum_{i>j} U_{\alpha i} U_{\beta i} U_{\alpha j} U_{\beta j} \cdot \cos \left(\frac{2 \pi L}{L_{i j}^{o s c}}\right) \\
& \times \exp \left[-\frac{1}{4}\left(\frac{L}{L_{i j}^{c o h}}\right)^{2}\right] \times \exp \left[-4 \pi^{2}\left(\frac{\sigma_{x}}{L_{i j}^{o s c}}\right)^{2}\right] .
\end{aligned}
$$

To further examine the nature of this equation, consider the special case of muon-neutrino disappearance oscillations in the two-neutrino oscillation approximation,

$$
\begin{aligned}
P_{\nu_{\mu} \rightarrow \nu_{\mu}}(L)= & \cos ^{4} \theta+\sin ^{4} \theta+\frac{1}{2} \sin ^{2} 2 \theta \times \cos \left(\frac{2 \pi L}{L_{i j}^{o s c}}\right) \\
& \times \exp \left[-\frac{1}{4}\left(\frac{L}{L_{i j}^{c o h}}\right)\right] \times \exp \left[-4 \pi^{2}\left(\frac{\sigma_{x}}{L_{i j}^{o s c}}\right)^{2}\right] .
\end{aligned}
$$

This formula can be used to quantify the effects of experimental smearing on oscillations. The smearing of the oscillatory shape can be seen in all neutrino oscillation experiments. This happens mainly due to bin migration by poor energy resolution for low-energy events. In a rigorous sense, the bin migration can be treated correctly only by using a simulation of a specific experiment. However, using Eq. 2.21, one can mimic the experimental smearing theoretically.

Interestingly, the first two terms, $\cos ^{4} \theta+\sin ^{4} \theta$, do not depend on any coherent conditions. Thus, when neutrinos lose their coherence by long distance propagation, very good energy resolution, very poor position resolution, etc, this formula reduces to,

$$
P_{\nu_{\mu} \rightarrow \nu_{\mu}} \sim \cos ^{4} \theta+\sin ^{4} \theta
$$




\subsubsection{Time-averaged neutrino oscillations}

For the standard two neutrino oscillation formula (Eq. 2.10), decoherence occurs when the neutrino oscillates many times and the oscillation is averaged over many periods. These are the so-called "time-averaged" neutrino oscillations,

$$
\begin{aligned}
P_{\nu_{\mu} \rightarrow \nu_{\mu}} & =1-\sin ^{2} 2 \theta \sin ^{2}\left(\pi \frac{L}{L_{i j}^{o s c}}\right) \\
& \sim 1-\sin ^{2} 2 \theta \cdot \frac{1}{2}=\cos ^{4} \theta+\sin ^{4} \theta .
\end{aligned}
$$

As expected, we find the same solution as in Eq. 2.22.

\subsubsection{Incoherent sum of neutrino oscillations}

In fact, the $\cos ^{4} \theta+\sin ^{4} \theta$ term can be understood as an incoherent sum of two neutrinos [9]. If any two paths of neutrino transitions are independent, the total oscillation probability will be an incoherent sum of all paths,

$$
\nu_{\mu} \rightarrow\left\{\begin{array}{l}
\stackrel{\cos \theta}{\rightarrow} \nu_{1} \stackrel{\cos \theta}{\rightarrow} \nu_{\mu} \\
\stackrel{\sin \theta}{\rightarrow} \nu_{2} \stackrel{\sin \theta}{\rightarrow} \nu_{\mu}
\end{array} .\right.
$$

Then, the total transition probability is

$$
P_{\nu_{\alpha} \rightarrow \nu_{\alpha}}=\left|\cos ^{2} \theta\right|^{2}+\left|-\sin ^{2} \theta\right|^{2}=\cos ^{4} \theta+\sin ^{4} \theta
$$

and again, we discover the same equation as Eq. 2.22.

\subsubsection{Neutrino oscillations with perfect kinematics measurements}

For accelerator-based neutrino experiments, neutrinos are created by the decay of pions,

$$
\pi^{+} \rightarrow \mu^{+}+\nu_{\mu}
$$


and we consider the following gedanken experiment. If you measure the mass and momentum of the pion and muon with very high precision, you can determine the mass of the neutrino from four-momentum conservation. Then, what do you expect for neutrino oscillations?

When you know the kinematics so precisely, more precise than the mass difference, the neutrino wave function is contracted to either one of the mass eigenstates. This is equivalent to knowing perfectly which mass eigenstate neutrinos take from the production point, as $\nu_{\mu}$, to the detection point, as $\nu_{\mu}$, and the neutrino oscillation probability is again the incoherent sum of all paths, therefore,

$$
P_{\nu_{\mu} \rightarrow \nu_{\mu}}=\cos ^{4} \theta+\sin ^{4} \theta
$$

\subsubsection{Neutrino decay}

Neutrino decay [10] also exhibits the interesting nature of neutrino coherence. If a heavier neutrino, $\nu_{2}$, decays to a lighter, $\nu_{1}$, with decay constant $\Gamma$, then the survival probability of $\nu_{e}$ is,

$$
P_{\nu_{e} \rightarrow \nu_{e}}(t)=\cos ^{4} \theta+e^{-\Gamma t} \sin ^{4} \theta+\frac{1}{2} e^{-\Gamma t} \sin ^{2} 2 \theta \cos \left(\frac{\Delta_{12}}{2} t\right) .
$$

If the decay is very fast,

$$
P_{\nu_{e} \rightarrow \nu_{e}}(t) \sim \cos ^{4} \theta
$$

and this result is equivalent with with an incoherent sum of neutrino oscillation where one of the two paths is eliminated due to the neutrino decay.

\subsubsection{Factor 2 ambiguity}

The group-velocity based understanding of neutrino oscillations has a very famous pitfall, the so-called "factor 2 ambiguity" [11]. Since neutrino oscillations are governed by the 
phase difference of two neutrinos, one can calculate this phase difference from the neutrino wave packet group velocity. For the two-neutrino approximation, the propagation time for $\nu_{1}$ can be written,

$$
t_{1}=\frac{L}{v_{1}}=\frac{<E_{1}>}{<p_{1}>} L
$$

thus, the phase rotation of $\nu_{1}$ after propagating $\mathrm{L}$ is,

$$
\Phi_{1}=p_{1} L-E_{1} t_{1}=\left(p_{1}-\frac{E_{1}^{2}}{p_{1}}\right) L=-\frac{m_{1}^{2}}{p_{1}} L .
$$

Then, the phase difference of $\nu_{1}$ and $\nu_{2}$ after propagating a distance $\mathrm{L}$ is $\left(p_{1} \sim p_{2} \sim E\right)$,

$$
\begin{aligned}
\Phi_{12} & =\left(p_{1}-p_{2}\right) L-\left(E_{1} t_{1}-E_{2} t_{2}\right) \\
& =\left[\left(p_{1}-p_{2}\right)-\left(p_{1}+\frac{m_{1}^{2}}{2 p_{1}}\right) \frac{E_{1}}{p_{1}}+\left(p_{2}+\frac{m_{2}^{2}}{2 p_{2}}\right) \frac{E_{2}}{p_{2}}\right] L \\
& =\left[\left(p_{1}-p_{2}\right)\left(p_{1}+\frac{m_{1}^{2}}{2 p_{1}}\right) \cdot\left(1+\frac{m_{1}^{2}}{2 p_{1}^{2}} L\right)+\left(p_{2}+\frac{m_{2}^{2}}{2 p_{2}}\right) \cdot\left(1+\frac{m_{2}^{2}}{2 p_{2}^{2}}\right)\right] L \\
& \sim-\left[\frac{m_{1}^{2}}{p_{1}}-\frac{m_{2}^{2}}{p_{2}}\right] L \sim-\left[\frac{\Delta m_{12}^{2}}{E}\right] L
\end{aligned}
$$

Therefore the equation of two-neutrino oscillations is,

$$
P_{\nu_{\alpha} \rightarrow \nu_{\beta}}(t)=\sin ^{2} 2 \theta \sin ^{2}\left(2.54 \Delta m_{i j}^{2} \frac{L}{E}\right) \text { for } \alpha \neq \beta
$$

Comparing with the standard formula of Eq. 2.10, the phase term is factor of two bigger!

The problem is that the oscillation is not caused by the interference of two wave packets, but the interference of equal energy (or momentum) plane waves in the wave packets. So, the group velocity cannot be used to describe the phase shift of two neutrinos.

Again, the phase difference for the standard two massive neutrino oscillation is $(t=L)$,

$$
\begin{aligned}
\Phi_{12} & =\left(p_{1}-p_{2}\right) L-\left(E_{1}-E_{2}\right) t \\
& =\left[\left(p_{1}-p_{2}\right)-\left(p_{1}+\frac{m_{1}^{2}}{2 p_{1}}\right)+\left(p_{2}+\frac{m_{2}^{2}}{2 p_{2}}\right)\right] L \\
& \sim-\left[\frac{m_{1}^{2}}{2 p_{1}}-\frac{m_{2}^{2}}{2 p_{2}}\right] L \sim-\left[\frac{\Delta m_{12}^{2}}{2 E}\right] L .
\end{aligned}
$$




\subsubsection{False Lorentz violation in neutrino oscillation}

We start with the following gedanken experiment [11. Twins, Diana and Artemis, perform a $\nu_{\mu}-\nu_{e}$ neutrino oscillation experiment using the following standard two neutrino oscillation formula,

$$
P_{\nu_{\mu} \rightarrow \nu_{e}}=\sin ^{2} 2 \theta \sin ^{2}\left(1.27 \Delta m_{12}^{2} \frac{L}{E}\right)
$$

Diana needs to move to Mars, but since she loves neutrino physics, she decided to keep watching how many neutrinos were detected as she travels on the spaceship. Artemis predicts how many neutrino Diana counts and, of course, the number of neutrino detected is Lorentz invariant - the number of neutrinos detected should be the same in any frame. Since Diana is in a fast moving frame, the baseline $L$ for her is Lorentz contracted,

$$
L^{\prime}=\gamma^{-1} L
$$

and the energy of the neutrinos is shifted by the Lorentz transformation,

$$
E^{\prime}=\gamma(E-\beta p) \sim \gamma E(1-\beta)
$$

Then, she inserts them into the oscillation formula, and finds,

$$
\begin{aligned}
P_{\nu_{\alpha} \rightarrow \nu_{\beta}}^{\prime} & =\sin ^{2} 2 \theta \sin ^{2}\left(1.27 \Delta m_{i j}^{2} \frac{L^{\prime}}{E^{\prime}}\right) \\
& =\sin ^{2} 2 \theta \sin ^{2}\left(1.27 \Delta m_{i j}^{2} \frac{\gamma^{-1} L}{\gamma E(1-\beta)}\right) \neq P_{\nu_{\alpha} \rightarrow \nu_{\beta}}
\end{aligned}
$$

The number of neutrinos is not Lorentz invariant!

The mistake is that Artemis interprets $L$ to be $(L, 0)$ in space-time. Since this $L$ is the space coordinate of the neutrino propagation distance, the space-time point of the neutrino is $(L, T)$. One can solve this problem geometrically (Fig. 2.1). The space coordinate of the space-time point $(L, 0)$ looks like $p^{\prime \prime}$ in Diana's coordinates. This $p^{\prime \prime}$ corresponds to $L^{\prime \prime}$ for the rest frame, or Artemis's coordinates. Here, the space-time point $L^{\prime \prime}$ is found from 
space-time point $p^{\prime \prime}$ by a coordinate transformation, $t^{2}-x^{\prime 2}=t^{2}-x^{2}$. Then, this problem is solved geometrically, and we find,

$$
L^{\prime \prime}=\gamma^{-1} L
$$

This is the so-called Lorentz contraction.

On the other hand, space coordinate of $(L, T)$ in Diana's system is $p^{\prime}$, and it corresponds to $L^{\prime}$ for rest frame, or Artemis's coordinates. We find,

$$
L^{\prime}=\gamma L(1-\beta)
$$

therefore,

$$
P_{\nu_{\alpha} \rightarrow \nu_{\beta}}^{\prime}=\sin ^{2} 2 \theta \sin ^{2}\left(1.27 \Delta m_{i j}^{2} \frac{\gamma L(1-\beta)}{\gamma E(1-\beta)}\right)=P_{\nu_{\alpha} \rightarrow \nu_{\beta}} .
$$

and the oscillation formula is proved to be Lorentz invariant. 


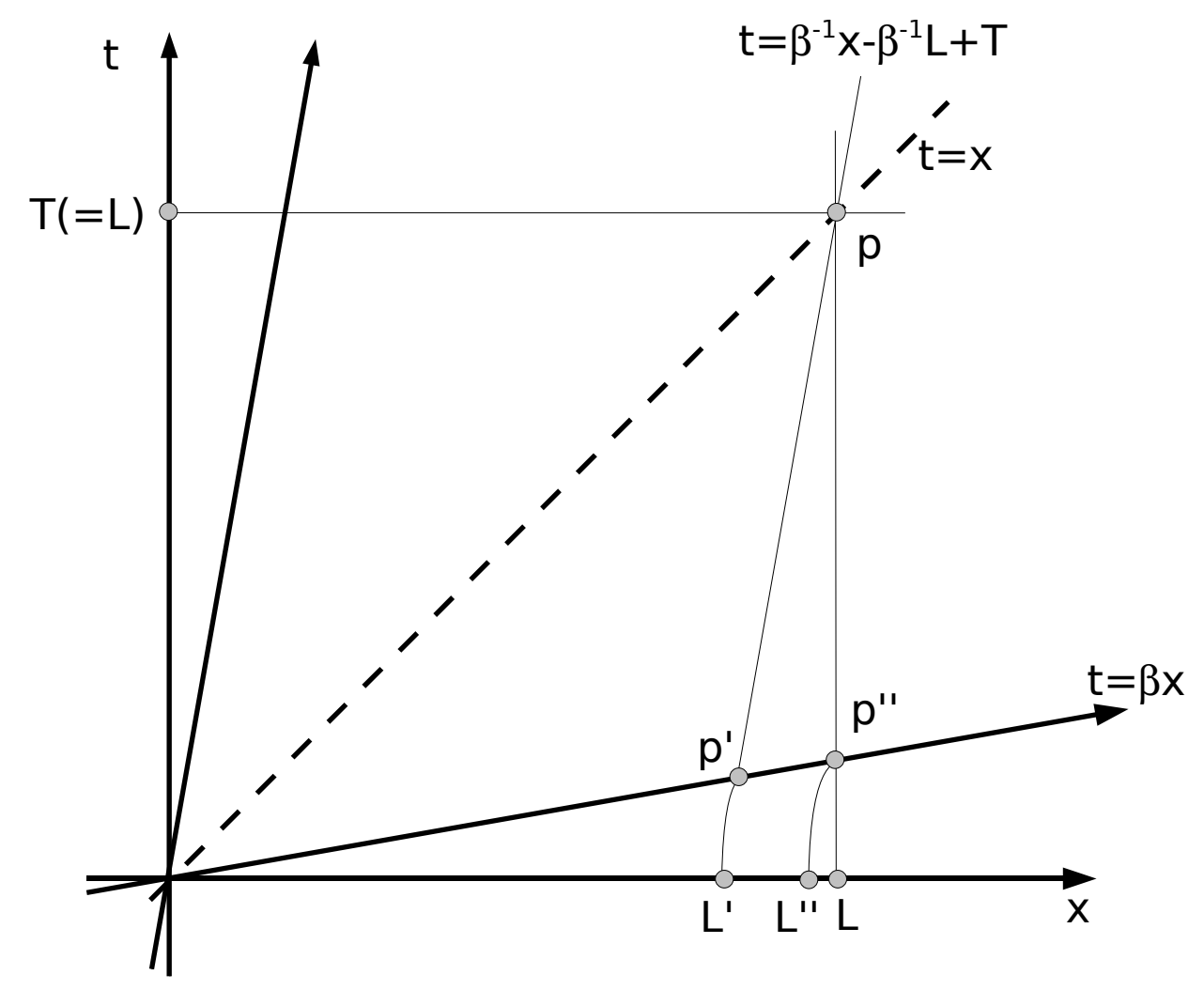

Figure 2.1: Space time diagram for false Lorentz violation with neutrino oscillation.

\subsection{Test of new physics with Neutrino oscillations}

\subsubsection{Neutrino oscillations as natural interferometers}

The oscillation of neutrinos is an interference experiment. Figure 2.2 illustrates shows the concept via an analogy of neutrino oscillations and optical (photon) double-slit experiments.

There is a light source, and we imagine two light paths to reach the screen behind the slits. When light propagates through the slits, the two paths have different light propagation lengths, and hence a different phase rotation, and they create an interference pattern on 


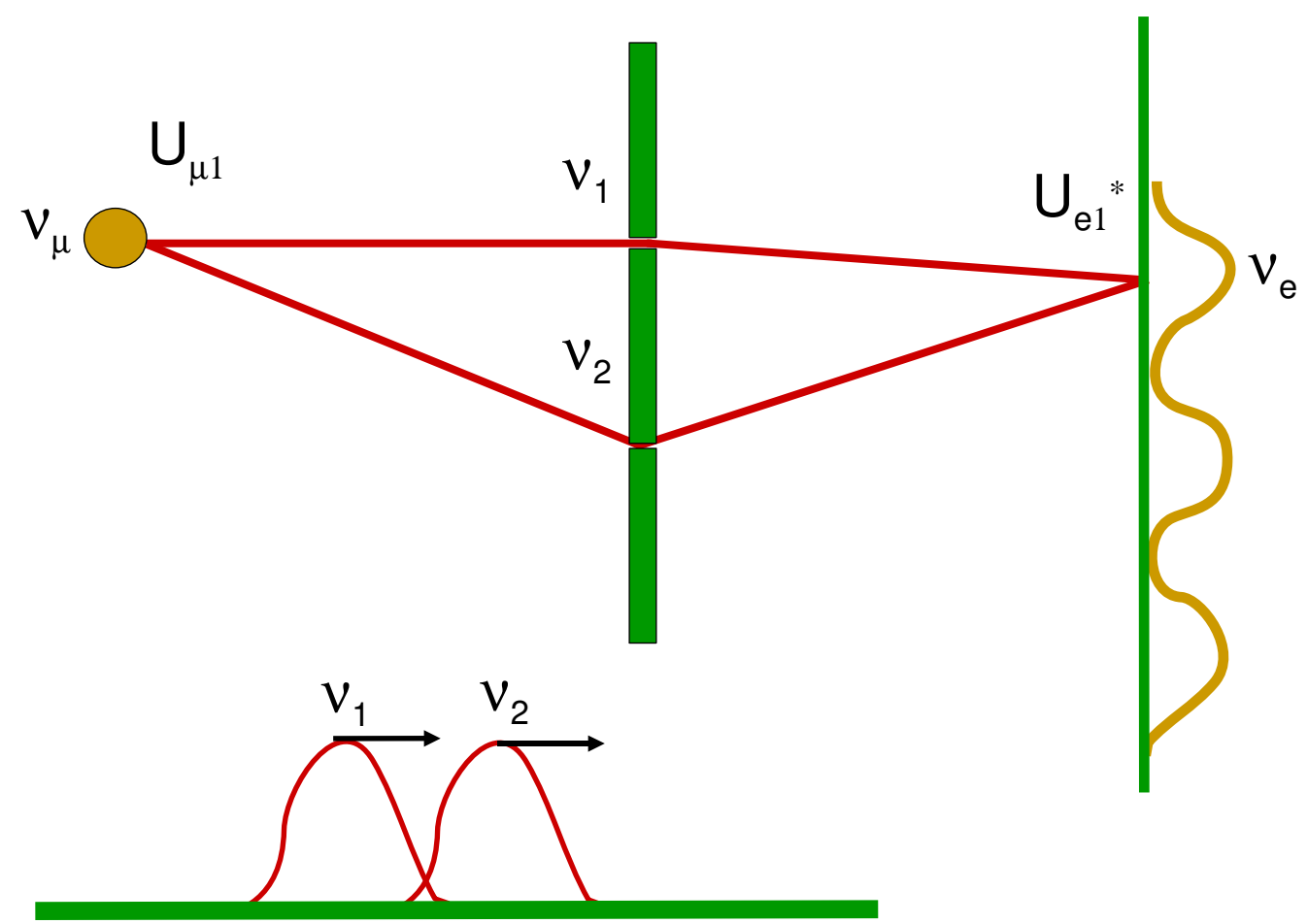

Figure 2.2: An illustration of neutrino oscillations as interferometers. There is an analogy between neutrino oscillations and optical double slit experiments.

the screen.

Connecting to neutrinos, the light source is equivalent to the flavor eigenstates of $\nu_{\mu}$, and the two different light paths are $\nu_{1}$ and $\nu_{2}$, because those two Hamiltonian eigenstates have different phase rotations due to their different Hamiltonian eigenvalues. For example, if two neutrinos have different masses, then $\nu_{1}$ and $\nu_{2}$ have different group velocities, and hence they interfere differently at the detection point. Note, this phase rotation difference does not come from the group velocity difference, as discussed in Sec. 2.2.6. 


\subsubsection{Neutrino oscillations in the search of new physics}

Then, the question is what is the sensitivity of this interferometer? We will use, as an example, the case of atmospheric neutrino oscillations where there is evidence for $\Delta m_{a t m}^{2} \sim$ $10^{-3} \mathrm{eV}^{2}$ [8]. Then the oscillation length (Eq. 2.17) is (the typical atmospheric neutrino energy is $\sim 1 \mathrm{GeV})$,

$$
1 / L^{o s c} \sim \frac{\Delta m^{2}}{E} \sim 10^{-21} \mathrm{GeV}
$$

Now, the natural suppression factor for Planck scale physics in the Standard Model (SM) can be written as a ratio of the electroweak scale $\left(E_{W} \sim 100 \mathrm{GeV}\right)$ to the Planck scale $\left(M_{P} \sim 10^{19} \mathrm{GeV}\right), \lesssim \frac{E_{W}}{M_{P}} \sim 10^{-17}$. Then, the Planck scale physics expected in atmospheric neutrinos may be smaller than $10^{-17} \times 1 \mathrm{GeV}=10^{-17} \mathrm{GeV}$ and the known oscillation length of the atmospheric neutrinos is beyond this naive benchmark scale. Therefore, neutrino oscillations may provide the opportunity to discover high-energy physics that is suppressed in the low energy world and neutrino oscillations are a candidate phenomenon in which to search for physics beyond the Standard Model (BSM).

In Chapter 4, we examine the actual formalism and experimental details in order to use neutrino oscillations to search for a possible signature of new physics, Lorentz violation [1]. It is quite natural to test Lorentz invariance with neutrino oscillations. First, Lorentz invariance is usually tested with high precision interferometers (see, for example [12 14]), and neutrino oscillations are a natural high precision interferometer. Second, Lorentz violation has not been well-investigated with neutrinos. Neutrinos are neutral, fundamental building blocks of the SM, and defined with their flavor eigenstates. These peculiar features of neutrinos encourage people to imagine exciting new discoveries and, perhaps, neutrinos may even shed light on the Planck scale! 


\section{Chapter 3}

\section{Neutrino interactions}

In this chapter, we discuss the topic of neutrino interactions with matter. Since neutrinos only have a weak nuclear charge (the coupling constant of $S U(2)_{L}$ symmetry), they only interact with matter via the weak interaction by exchanging $W^{ \pm}$or $Z^{\circ}$ bosons. However the internal structure of the nucleon and higher order corrections allow more than just $V-A$ interactions, and neutrinos exhibit a rich scattering nature.

We start with a description of the neutrino-charged lepton interaction as it is the most fundamental interaction for neutrinos. Then we move to the neutrino-nucleon interaction, the most fundamental neutrino-hadron interaction. We re-derive the famous LlewellynSmith formalism [15], and discuss the implications for neutrino-nucleon scattering. Finally, we explore the most relevant type of scattering for modern neutrino oscillation experiments, the neutrino-nuclei interaction. We re-derive the cross section formulas of the Smith and Moniz formalism, based on the relativistic Fermi gas (RFG) model [16]. 
(a)

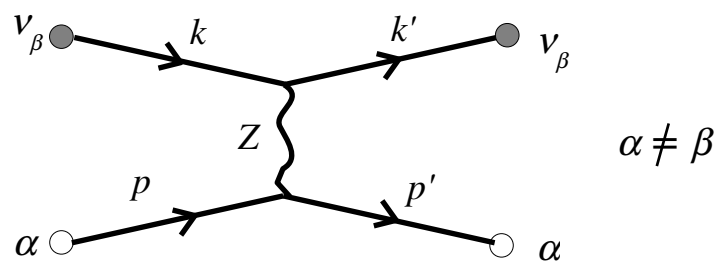

(b)

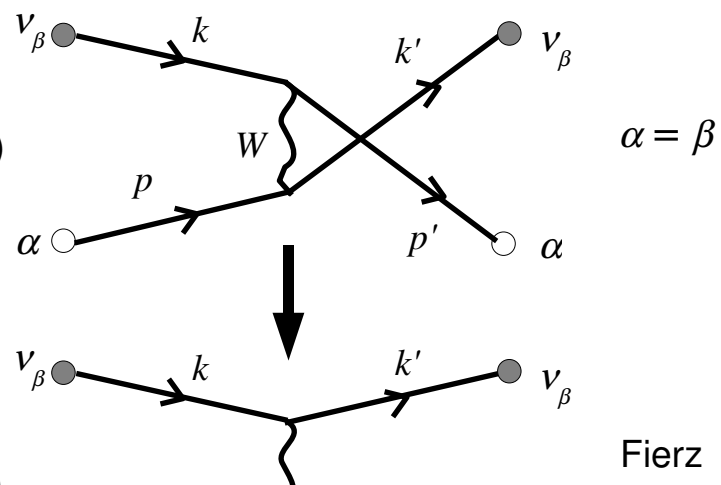

(c)

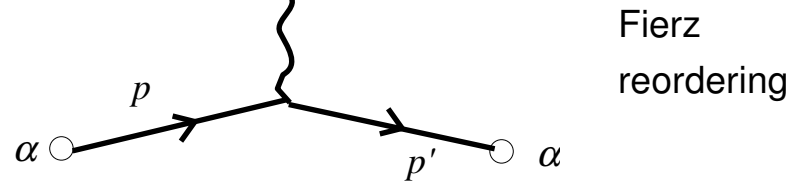

Figure 3.1: Feynman diagram for neutrino-charged lepton scattering.

\subsection{Neutrino-Charged lepton scattering}

Neutrinos interact with any charged lepton via the neutral current (NC) interaction (Fig. 3.1.

$$
\nu_{\alpha}+\beta \stackrel{Z^{o}}{\rightarrow} \nu_{\alpha}+\beta \text { with any } \alpha \text { and } \beta
$$

Neutrinos can also interact via charged current (CC) interaction if the transition connects neutrinos of the same family (Fig. $3.1 \mathrm{~b}$ ),

$$
\nu_{\alpha}+\beta \stackrel{W^{ \pm}}{\rightarrow} \nu_{\alpha}+\beta \text { with } \alpha=\beta
$$

Assume that the charged lepton is an electron $(\alpha=e)$ and the neutrino to be of arbitrary 
flavor $(\beta=e, \mu, \tau)$. We define the incoming and outgoing neutrinos to have 4-momentum $k=\left(E_{\nu}, \vec{k}\right)$ and $k^{\prime}=\left(E_{\nu}^{\prime}, \overrightarrow{k^{\prime}}\right)$, respectively and the incoming and outgoing charged leptons have $p=\left(E_{e}, \vec{p}\right)$ and $p^{\prime}=\left(E_{e}^{\prime}, \overrightarrow{p^{\prime}}\right)$. The general expression for the transition amplitude for neutrino-electron scattering is [9, 17],

$$
\begin{aligned}
\mathcal{M}= & \left.\frac{G_{F}}{\sqrt{2}}\left\{\left[J^{C C}\right)_{\mu}^{\dagger}\left(J^{C C}\right)^{\mu}+2 \rho\left(J^{N C}\right)_{\mu}^{\dagger}\left(J^{N C}\right)^{\mu}\right]\right\} \\
= & \frac{G_{F}}{\sqrt{2}}\left\{\left[\bar{e} \gamma_{\mu}\left(1-\gamma_{5}\right) \nu_{\beta}\right] \cdot\left[\overline{\nu_{\beta}} \gamma^{\mu}\left(1-\gamma_{5}\right) e\right]\right. \\
& \left.+2 \rho\left[\overline{\nu_{\beta}} \gamma_{\mu} \frac{1}{2}\left(1-\gamma_{5}\right) \nu_{\beta}\right] \cdot\left[\bar{e} \gamma^{\mu}\left(g_{V}-g_{A} \gamma_{5}\right) e\right]\right\} .
\end{aligned}
$$

Here $g_{V}$ and $g_{A}$ are the vector and axial vector coupling constants for charged leptons. They depend on the weak mixing angle, $\sin ^{2} \theta_{w}$, and are, in the standard model,

$$
\begin{array}{ccc} 
& e_{L} & e_{R} \\
g_{V}= & -\frac{1}{2}+2 \sin ^{2} \theta_{w} & 2 \sin ^{2} \theta_{w} \\
g_{A}= & -\frac{1}{2} & 0
\end{array}
$$

Here, we use Fierz reordering (Fig. 3.1p to c),

$$
\left[\bar{e} \gamma_{\mu}\left(1-\gamma_{5}\right) \nu_{\beta}\right] \cdot\left[\overline{\nu_{\beta}} \gamma^{\mu}\left(1-\gamma_{5}\right) e\right] \rightarrow\left[\bar{e} \gamma_{\mu}\left(1-\gamma_{5}\right) e\right] \cdot\left[\overline{\nu_{\beta}} \gamma^{\mu}\left(1-\gamma_{5}\right) \nu_{\beta}\right]
$$

Then we can add CC and NC part of the amplitude,

$$
\mathcal{M}=\frac{G_{F}}{\sqrt{2}}\left\{\left[\overline{\nu_{\beta}} \gamma_{\mu} \frac{1}{2}\left(1-\gamma_{5}\right) \nu_{\beta}\right] \cdot\left[\bar{e} \gamma^{\mu}\left(C_{V}-C_{A} \gamma_{5}\right) e\right]\right\}
$$

with

$$
\begin{aligned}
& e=\beta \quad e \neq \beta \\
& C_{V}=\rho g_{V}+1 \quad \rho g_{V} \\
& C_{A}=\rho g_{A}+1 \quad \rho g_{A} \text {. }
\end{aligned}
$$

Then, the spin-averaged square of invariant amplitude ( $\propto$ cross section) is,

$$
\overline{|\mathcal{M}|^{2}}=\frac{1}{\left(2 s_{e}+1\right)\left(2 s_{\nu_{\beta}}+1\right)} \sum_{\text {spin }}|\mathcal{M}|^{2},
$$


where $s_{e}$ and $s_{\nu_{\beta}}$ are the spin degrees of freedom of the electron $(=2)$ and neutrino $(=1)$.

Then,

$$
\begin{aligned}
\overline{|\mathcal{M}|^{2}}= & \frac{G_{F}^{2}}{4} \cdot \operatorname{tr}\left[\overline{\nu_{\beta}}\left(k^{\prime}\right) \gamma_{\mu}\left(1-\gamma_{5}\right) \nu_{\beta}(k)\right]\left[\overline{\nu_{\beta}}(k) \gamma_{\nu}\left(1+\gamma_{5}\right) \nu_{\beta}\left(k^{\prime}\right)\right] \\
& \times \operatorname{tr}\left[\bar{e}\left(p^{\prime}\right) \gamma^{\mu}\left(C_{V}-C_{A} \gamma_{5}\right) e(p)\right]\left[\bar{e}(p) \gamma^{\nu}\left(C_{V}+C_{A} \gamma_{5}\right) e\left(p^{\prime}\right)\right] \\
= & \frac{G_{F}^{2}}{4} \cdot \operatorname{tr}\left[\gamma_{\mu}\left(1-\gamma_{5}\right) \not k \gamma_{\nu}\left(1-\gamma_{5}\right) \not k^{\prime}\right] \\
& \times \operatorname{tr}\left[\gamma^{\mu}\left(C_{V}-C_{A} \gamma_{5}\right)\left(\not p+m_{e}\right) \gamma^{\nu}\left(C_{V}-C_{A} \gamma_{5}\right)\left(\not p^{\prime}+m_{e}\right)\right] \\
= & \frac{G_{F}^{2}}{4} \cdot\left\{\operatorname{tr}\left[2 \gamma_{\mu} \not k \gamma_{\nu} \not k^{\prime}\right]+\operatorname{tr}\left[2 \gamma_{5} \gamma_{\mu} \not k \gamma_{\nu} \not k^{\prime}\right]\right\} \\
& \times\left\{\operatorname{tr}\left(C_{V}^{2}+C_{A}^{2}\right)\left[\gamma^{\mu} \not p \gamma^{\nu} \not p^{\prime}\right]+\operatorname{tr}\left(2 C_{V} C_{A}\right)\left[\gamma_{5} \gamma^{\mu} \not p \gamma^{\nu} \not p^{\prime}\right]\right. \\
& \left.+\operatorname{tr}\left(C_{V}^{2}-C_{A}^{2}\right)\left[\gamma^{\mu} \gamma^{\nu} m_{e}^{2}\right]+\operatorname{tr}\left(2 C_{V} C_{A}\right)\left[m_{e} \gamma_{5} \gamma^{\mu} \gamma^{\nu}\right]\right\} .
\end{aligned}
$$

Using identities of gamma matrix algebra (Appendix B),

$$
\begin{aligned}
\overline{|\mathcal{M}|^{2}=} & \frac{G_{F}^{2}}{4} \cdot\left\{2\left(C_{V}^{2}+C_{A}^{2}\right) \cdot 32\left[(k \cdot p)\left(k^{\prime} \cdot p^{\prime}\right)+\left(k \cdot p^{\prime}\right)\left(k^{\prime} \cdot p\right)\right]\right. \\
& +2\left(2 C_{V} C_{A}\right) \cdot 32\left[(k \cdot p)\left(k^{\prime} \cdot p^{\prime}\right)-\left(k \cdot p^{\prime}\right)\left(k^{\prime} \cdot p\right)\right] \\
& +\left[8\left(k_{\mu} k_{\nu}^{\prime}+k_{\nu} k_{\mu}^{\prime}-\left(k \cdot k^{\prime}\right) g_{\mu \nu}\right)+8 i \epsilon_{\mu \lambda \nu \sigma} k^{\lambda} k^{\prime \sigma}\right] \\
& \left.\times\left[\left(C_{V}^{2}-C_{A}^{2}\right) \cdot 4 m_{e}^{2} g^{\mu \nu}+0\right]\right\} \\
= & \frac{G_{F}^{2}}{4}\left\{64\left(C_{V}+C_{A}\right)^{2}(k \cdot p)\left(k^{\prime} \cdot p^{\prime}\right)+64\left(C_{V}-C_{A}\right)^{2}\left(k \cdot p^{\prime}\right)\left(k^{\prime} \cdot p\right)\right. \\
& \left.+32 m_{e}^{2}\left(C_{V}^{2}-C_{A}^{2}\right) \cdot\left(-2 k \cdot k^{\prime}\right)\right\} \\
= & G_{F}^{2}\left\{16\left(C_{V}+C_{A}\right)^{2}(k \cdot p)\left(k^{\prime} \cdot p^{\prime}\right)+16\left(C_{V}-C_{A}\right)^{2}\left(k \cdot p^{\prime}\right)\left(k^{\prime} \cdot p\right)\right. \\
& \left.-8 m_{e}^{2}\left(C_{V}+C_{A}\right)\left(C_{V}-C_{A}\right)\left(2 k \cdot k^{\prime}\right)\right\} .
\end{aligned}
$$

Now, we define left-handed and right-handed coupling coefficients to simplify the expression above,

$$
\begin{aligned}
& e-\nu_{\beta}(e=\beta) \quad \bar{e}-\nu_{\beta}(e=\beta) \quad e-\nu_{\beta}(e \neq \beta) \quad \bar{e}-\nu_{\beta}(e \neq \beta) \\
& C_{L}=\frac{1}{2}\left(C_{V}+C_{A}\right) \quad \frac{1}{2}+\sin ^{2} \theta_{w} \quad \sin ^{2} \theta_{w} \quad-\frac{1}{2}+\sin ^{2} \theta_{w} \quad \sin ^{2} \theta_{w} \\
& C_{R}=\frac{1}{2}\left(C_{V}+C_{A}\right) \quad \sin ^{2} \theta_{w} \quad \frac{1}{2}+\sin ^{2} \theta_{w} \quad \sin ^{2} \theta_{w} \quad-\frac{1}{2}+\sin ^{2} \theta_{w} \quad,
\end{aligned}
$$


and,

$$
\begin{aligned}
\overline{|\mathcal{M}|^{2}}= & 16 G_{F}^{2}\left[C_{L}^{2}(2 k \cdot p)\left(2 k^{\prime} \cdot p^{\prime}\right)+C_{R}^{2}\left(2 k \cdot p^{\prime}\right)\left(2 k^{\prime} \cdot p\right)\right. \\
& \left.-2 m_{e}^{2} C_{L} C_{R}\left(2 k \cdot k^{\prime}\right)\right] .
\end{aligned}
$$

Using the following Mandelstam variables,

$$
\begin{aligned}
s & =(k+p)^{2}=m_{e}^{2}+2 m_{e} E_{\nu}, \\
u & =\left(p-k^{\prime}\right)^{2}=m_{e}^{2}-2 m_{e} E_{\nu}^{\prime}, \\
t & =\left(k^{\prime}-k\right)^{2}=2 m_{e}\left(E_{\nu}^{\prime}-E_{\nu}\right),
\end{aligned}
$$

Eq. 3.9 can be further simplified,

$$
\overline{|\mathcal{M}|^{2}}=16 G_{F}^{2}\left[C_{L}^{2}\left(s-m_{e}^{2}\right)^{2}+C_{R}^{2}\left(u-m_{e}^{2}\right)^{2}+2 m_{e}^{2} C_{L} C_{R} t\right]
$$

The differential Lorentz-invariant phase space (dLips) is,

$$
\begin{aligned}
d \text { Lips } & =\int \frac{d^{3} k^{\prime}}{(2 \pi)^{3} \cdot 2 E_{\nu}^{\prime}} \cdot \int \frac{d^{3} p^{\prime}}{(2 \pi)^{3} \cdot 2 E_{e}^{\prime}}(2 \pi)^{4} \delta^{4}\left(p+k-p^{\prime}-k^{\prime}\right) \\
& =\int \frac{d^{3} p^{\prime}}{16 \pi^{2} E_{\nu} E_{e}^{\prime}} \delta\left(E_{e}+E_{\nu}-E_{e}^{\prime}-E_{\nu}^{\prime}\right) \\
& =\int \frac{d^{2} p^{\prime}}{16 \pi^{2} E_{\nu} E_{e}^{\prime}}=\int \frac{2 \pi E_{e}^{\prime} d E_{e}^{\prime}}{16 \pi^{2} E_{\nu}^{\prime} E_{e}^{\prime}}=\frac{1}{8 \pi} \int \frac{d E_{e}^{\prime}}{E_{\nu}}
\end{aligned}
$$

Since the differential cross section in the center of mass system is [18],

$$
\begin{aligned}
d \sigma & =\frac{\overline{|\mathcal{M}|^{2}}}{4|\vec{k}| \sqrt{s}} d \text { Lips } \\
\rightarrow \frac{d \sigma}{d E_{e}^{\prime}} & =\frac{1}{32 \pi m_{e} E_{\nu}^{2}} \cdot \overline{|\mathcal{M}|^{2}}
\end{aligned}
$$

Defining the elasticity $y$ and recoil-electron kinetic energy $T_{e}^{\prime}$,

$$
\begin{aligned}
T_{e}^{\prime} & =E_{e}^{\prime}-m_{e}=E_{\nu}-E_{\nu}^{\prime} \\
y & =\frac{T_{e}^{\prime}}{E_{\nu}}=\frac{E_{\nu}-E_{\nu}^{\prime}}{E_{\nu}},
\end{aligned}
$$


gives the differential cross section,

$$
\begin{aligned}
\frac{d \sigma}{d T_{e}^{\prime}} & =\frac{1}{32 \pi m_{e} E_{\nu}^{2}} \cdot 16 G_{F}^{2}\left[C_{L}^{2}\left(2 m_{e} E_{\nu}\right)^{2}+C_{R}^{2}\left(2 m_{e} E_{\nu}^{\prime}\right)^{2}+2 m_{e}^{2} C_{L} C_{R} \cdot 2 m_{e}\left(E_{\nu}^{\prime}-E_{\nu}\right)\right] \\
& =\frac{2 G_{F}^{2} m_{e}}{\pi}\left[C_{L}^{2}+C_{R}^{2}\left(\frac{E_{\nu}^{\prime}}{E_{\nu}}\right)^{2}-C_{L} C_{R} \frac{m_{e}}{E_{\nu}} \cdot \frac{E_{\nu}-E_{\nu}^{\prime}}{E_{\nu}}\right]
\end{aligned}
$$

After integrating over $y$ to find the total cross section,

$$
\begin{aligned}
\sigma & =\frac{2 G_{F}^{2} m_{e}}{\pi} \int_{0}^{1} d y\left[C_{L}^{2}+C_{R}^{2}(1-y)^{2}-C_{L} C_{R} \frac{m_{e}}{E_{\nu}} y\right] \\
& =\frac{2 G_{F}^{2} m_{e} E_{\nu}}{\pi}\left[C_{L}^{2}+\frac{1}{3} C_{R}^{2}-\frac{1}{2} C_{L} C_{R} \frac{m_{e}}{E_{\nu}}\right] \propto E_{\nu}
\end{aligned}
$$

We can see that the total cross section for neutrino-charged lepton scattering monotonically increases with neutrino energy.

If a hypothetical neutrino magnetic moment $\mu_{\nu}$ is included, the differential cross section (Eq. 3.14) can be written,

$$
\begin{aligned}
\frac{d \sigma}{d T_{e}^{\prime}}= & \frac{2 G_{F}^{2} m_{e}}{\pi}\left[C_{L}^{2}+C_{R}^{2}\left(\frac{E_{\nu}^{\prime}}{E_{\nu}}\right)^{2}-C_{L} C_{R} \frac{m+e}{E_{\nu}} \cdot \frac{E_{\nu}-E_{\nu}^{\prime}}{E_{\nu}}\right] \\
& +\frac{\pi \alpha \mu_{\nu}^{2}}{m_{e}^{2}}\left(\frac{1}{T_{e}^{\prime}}-\frac{1}{E_{\nu}}\right) .
\end{aligned}
$$

Thus, a non-zero neutrino magnetic moment implies a significant shape distortion in the low-energy recoil electron spectrum. Using this feature, a number of experimental limits are set for neutrino magnetic moment, although astrophysical limits (cooling of red giants) are usually stronger [9]. The MiniBooNE experiment set an upper limit for the muon neutrino magnetic moment to be $\mu_{\nu_{\mu}}<12.7 \times 10^{-10} \mu_{B}$ with $90 \%$ C.L [19]. 


\subsection{Neutrino-Nucleon scattering}

\subsubsection{Llewellyn-Smith formalism for the neutrino experiments}

The scattering processes under consideration in this section are the following 2 reactions (Fig. 3.2),

$$
\begin{aligned}
& \nu_{l}+n \rightarrow l^{-}+p, \\
& \overline{\nu_{l}}+p \rightarrow l^{+}+n .
\end{aligned}
$$

In Appendix C.1, we derive the expression for neutrino-nucleon differential cross section formula (Eq. C.41),

$$
\begin{aligned}
& \frac{d \sigma}{d Q^{2}}\left(\begin{array}{c}
\nu_{l}+n \rightarrow l^{-}+p \\
\overline{\nu_{l}}+p \rightarrow l^{+}+n
\end{array}\right) \\
& =\frac{M^{2} G_{F}^{2} \cos ^{2} \theta_{c}}{8 \pi E_{\nu}{ }^{2}}\left\{A\left(Q^{2}\right) \pm B\left(Q^{2}\right) \frac{(s-u)}{M^{2}}+C\left(Q^{2}\right) \frac{(s-u)^{2}}{M^{4}}\right\}
\end{aligned}
$$

with the expressions for $A\left(Q^{2}\right), B\left(Q^{2}\right)$, and $C\left(Q^{2}\right)$ given in Eqs. C.38, C.39, and C.40. Here, $E_{\nu}$ is an incident neutrino energy, $M$ is a nucleon mass, and $s$ and $u$ are Mandelstam variables. Now we transform them to the familiar form [20] used in practice. All the contributions to the weak nucleon current other than the vector and axial vector form factors arise from the electromagnetic or strong interaction. However, the electromagnetic and strong interactions are G-parity conserving processes. So one can reasonably omit terms involving G-parity violating second-class-current form factors $\left(F_{V}{ }^{3}\right.$ and $\left.F_{A}{ }^{3}\right)$, which should not exist within the standard model (Sec. 3.2.8). And, we assume all form factors are purely real which mean there is no T-violation in any nucleon weak elastic scattering experiment (Sec. 3.2.8). Also, the $\xi F_{2}$ term may be rewritten as $F_{2}$ which is more standard in this (neutrino) community. This also means $\kappa_{p} F_{2}^{E M, p} \equiv F_{2}^{E M, p}$ and $\kappa_{n} F_{2}^{E M, n} \equiv F_{2}^{E M, n}$. 
(1) Lab frame

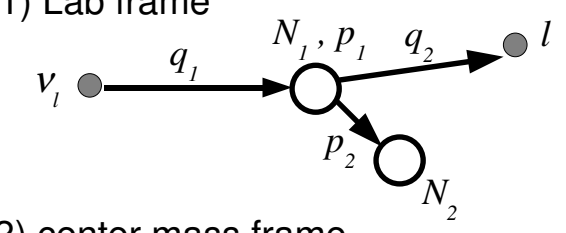

(2) center mass frame

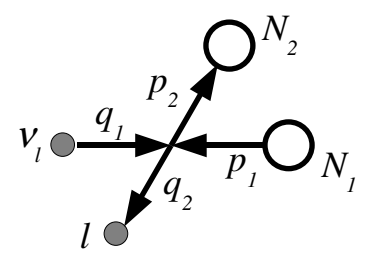

(3) Feynman diagram

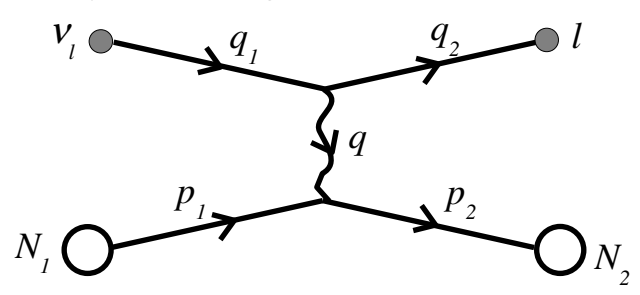

Figure 3.2: The scattering process under consideration, from top to bottom, (1) lab frame view, (2) center-of-mass frame view, and (3) Feynman diagram (t-channel). $\nu_{l}$ is the (anti)neutrino, $l$ is the (positively) negatively charged lepton and $N_{1}$ and $N_{2}$ are the proton or neutron. The charged lepton has mass $m$ and the initial and final state nucleon have the same mass $M$. 
With these procedures, Eqs. C.38, C.39 and C.40 become,

$$
\begin{aligned}
A\left(Q^{2}\right)= & \frac{\left(m^{2}+Q^{2}\right)}{M^{2}}\left[(1+\tau) F_{A}^{2}-(1-\tau) F_{1}^{2}+\tau(1-\tau) F_{2}^{2}+4 \tau F_{1} F_{2}\right. \\
& \left.-\frac{m^{2}}{4 M^{2}}\left(\left(F_{1}+F_{2}\right)^{2}+\left(F_{A}+2 F_{P}\right)^{2}-4\left(1+\frac{Q^{2}}{4 M^{2}}\right) F_{P}^{2}\right)\right] \\
B\left(Q^{2}\right)= & \frac{Q^{2}}{M^{2}} F_{A}\left(F_{1}+F_{2}\right), \\
C\left(Q^{2}\right)= & \frac{1}{4}\left(F_{A}^{2}+F_{1}^{2}+\tau F_{2}^{2}\right) .
\end{aligned}
$$

Here we have used the common abbreviation, $\tau=\frac{Q^{2}}{4 M^{2}}$. Eqs. 3.20, 3.21, and 3.22, as well as Eq. 3.19 agree with [20] except for a missing $\cos ^{2} \theta_{c}$ term in [20].

Next, we are able to eliminate the lepton mass term $\left(\frac{m^{2}}{M^{2}} \ll 1\right)$ for our applications (electron and muon production). In this case, the contribution from the pseudo-scalar form factor $\left(F_{P}\right)$ becomes zero, and these equations agree with those of [21, 22].

\subsubsection{Is it $B$ or $-B ?$}

There exists a sign inconsistency for the $B\left(Q^{2}\right)$-term between many papers (for example [15, 20, 22]). This problem arises from the many possible choices in: (1) the definition of the sign of $g_{A}$ (Eq. 3.62), (2) the sign in front of $g_{A},(3)$ the sign in front of $F_{A}$ (axial vector form factor), and (4) the sign in front of the $B\left(Q^{2}\right)$-term. This problem may be avoided by remembering that $\frac{d \sigma}{d Q^{2}}\left(\nu_{l}+n \rightarrow l^{-}+p\right)>\frac{d \sigma}{d Q^{2}}\left(\bar{\nu}_{l}+p \rightarrow l^{+}+n\right)$.

\subsubsection{Llewellyn-Smith formalism for Neutral Current}

We can modify Eqs. $3.20,3.21$ and 3.22 so that Eq. 3.19 is also correct for the neutral current cross section. Since the neutral weak current is related to the electromagnetic current,

$$
<N\left|J_{Z}^{\mu}\right| N>=<N\left|J_{3}^{\mu}-2 \sin ^{2} \theta_{W} \cdot J_{E M}^{\mu}\right| N>
$$

where $J_{3}$ is the third component of the isospin current and $J_{E M}$ is the electromagnetic current. Then, the nucleon neutral current form factor can be written completely by including 
an isoscalar part of the weak form factor (indicated with $s$ in upper index) [17,

$$
\begin{aligned}
& F_{1}^{Z, p}=\left(\frac{1}{2} F_{1}-\frac{1}{2} F_{1}^{s}\right)-2 \sin ^{2} \theta_{W} F_{1}^{E M, p} \\
& F_{1}^{Z, n}=\left(-\frac{1}{2} F_{1}-\frac{1}{2} F_{1}^{s}\right)-2 \sin ^{2} \theta_{W} F_{1}^{E M, n} \\
& F_{2}^{Z, p}=\left(\frac{1}{2} F_{2}-\frac{1}{2} F_{2}^{s}\right)-2 \sin ^{2} \theta_{W} F_{2}^{E M, p}, \\
& F_{2}^{Z, n}=\left(-\frac{1}{2} F_{2}-\frac{1}{2} F_{2}^{s}\right)-2 \sin ^{2} \theta_{W} F_{2}^{E M, n} \\
& F_{A}^{Z, p}=\frac{1}{2} F_{A}-\frac{1}{2} F_{A}^{s}, \\
& F_{A}^{Z, n}=-\frac{1}{2} F_{A}-\frac{1}{2} F_{A}^{s} .
\end{aligned}
$$

From the conservation of the vector current (CVC, Sec. 3.2.4), the isovector part of electromagnetic currents are related to the weak charged current,

$$
\begin{aligned}
& F_{1}=F_{1}^{E M, p}-F_{1}^{E M, n} \\
& F_{2}=F_{2}^{E M, p}-F_{2}^{E M, n} .
\end{aligned}
$$

Thus, the required replacements in the Llewellyn-Smith formalism (Eqs. C.41, 3.20, 3.21, and 3.22 for neutral current scattering are the following [22]:

$$
\begin{aligned}
G_{F} \cos \theta_{c} & \rightarrow G_{F}, \\
m_{l} & \rightarrow m_{\nu}, \\
F_{1}^{Z, p} & \rightarrow\left(\frac{1}{2}-\sin ^{2} \theta_{W}\right) F_{1}-\sin ^{2} \theta_{W}\left(F_{1}^{E M, p}+F_{1}^{E M, n}\right)-\frac{1}{2} F_{1}^{s}, \\
F_{1}^{Z, n} & \rightarrow-\left(\frac{1}{2}-\sin ^{2} \theta_{W}\right) F_{1}-\sin ^{2} \theta_{W}\left(F_{1}^{E M, p}+F_{1}^{E M, n}\right)-\frac{1}{2} F_{1}^{s}, \\
F_{2}^{Z, p} & \rightarrow\left(\frac{1}{2}-\sin ^{2} \theta_{W}\right) F_{2}-\sin ^{2} \theta_{W}\left(F_{2}^{E M, p}+F_{2}^{E M, n}\right)-\frac{1}{2} F_{2}^{s}, \\
F_{2}^{Z, n} & \rightarrow-\left(\frac{1}{2}-\sin ^{2} \theta_{W}\right) F_{2}-\sin ^{2} \theta_{W}\left(F_{2}^{E M, p}+F_{2}^{E M, n}\right)-\frac{1}{2} F_{2}^{s}, \\
F_{A}^{Z, p} & \rightarrow \frac{1}{2} F_{A}-\frac{1}{2} F_{A}^{s}, \\
F_{A}^{Z, n} & \rightarrow-\frac{1}{2} F_{A}-\frac{1}{2} F_{A}^{s} .
\end{aligned}
$$




\subsubsection{Conservation of vector current (CVC)}

This subsection follows Ref. [17].

The most general expression for the electromagnetic matrix element for the proton is,

$$
<p\left|J_{E M}^{\mu}\right| p>=\bar{p}\left[\gamma_{\mu} F_{1}^{E M, p}+\frac{1}{2 M} i \sigma_{\mu \nu} q^{\nu} F_{2}^{E M, p}\right] p .
$$

Here, we have introduced the proton electromagnetic Dirac and Pauli form factors. Notice

under current conservation, $\partial_{\mu} J_{E M}^{\mu}=0$, the electromagnetic scalar form factor, $F_{V}{ }^{E M, p}$ is naturally zero.

Similarly, using the neutron electromagnetic Dirac and Pauli form factors, the electromagnetic matrix element for the neutron is,

$$
<n\left|J_{E M}^{\mu}\right| n>=\bar{n}\left[\gamma_{\mu} F_{1}^{E M, n}+\frac{1}{2 M} i \sigma_{\mu \nu} q^{\nu} F_{2}^{E M, n}\right] n
$$

Since the proton and neutron comprise the nucleon isodoublet (isospin symmetry or charge symmetry), Eqs. 3.32 and 3.33 can be combined using the projection operators $\tau_{+}, \tau_{-}$and the isodoublet spinor $u$,

$$
\begin{aligned}
& p=\frac{1}{2}\left(1+\tau_{3}\right) u=\tau_{+} u \\
& n=\frac{1}{2}\left(1-\tau_{3}\right) u=\tau_{-} u .
\end{aligned}
$$

Here, $u$ is the eigenspinor of $\tau_{3}$, and the eigenvalue is $+1(-1)$ for the proton (neutron). Then,

$$
\begin{aligned}
& <u\left|J_{E M}^{\mu}\right| u>=\bar{u}\left\{\frac{1}{2}\left[\gamma_{\mu}\left(F_{1}^{E M, p}+F_{1}^{E M, n}\right)+\frac{1}{2 M} i \sigma_{\mu \nu} q^{\nu}\left(F_{2}^{E M, p}+F_{2}^{E M, n}\right)\right]\right. \\
& \left.+\frac{1}{2}\left[\gamma_{\mu}\left(F_{1}^{E M, p}-F_{1}^{E M, n}\right)+\frac{1}{2 M} i \sigma_{\mu \nu} q^{\nu}\left(F_{2}^{E M, p}-F_{2}^{E M, n}\right)\right] \tau_{3}\right\} u
\end{aligned}
$$

The first square bracket term is constant with proton or neutron (isoscalar term), however, the second bracket term changes its sign due to the isospin projection (isovector). Thus one 
can define:

$$
\begin{aligned}
F_{1}^{E M, s} & =F_{1}^{E M, p}+F_{1}^{E M, n}, \text { isoscalar vector form factor, } \\
F_{1}^{E M} & =F_{1}^{E M, p}-F_{1}^{E M, n}, \text { isovector vector form factor, } \\
F_{2}^{E M, s} & =F_{2}^{E M, p}+F_{2}^{E M, n}, \text { isoscalar tensor form factor, } \\
F_{2}^{E M} & =F_{2}^{E M, p}-F_{2}^{E M, n}, \text { isovector tensor form factor. }
\end{aligned}
$$

And Eq. 3.36 may be rewritten,

$$
\begin{aligned}
<u\left|J_{E M}^{\mu}\right| u>= & \bar{u}\left\{\frac{1}{2}\left[\gamma_{\mu} F_{1}^{E M, s}+\frac{1}{2 M} i \sigma_{\mu \nu} q^{\nu} F_{2}^{E M, s}\right]\right. \\
& \left.+\frac{1}{2}\left[\gamma_{\mu} F_{1}^{E M}+\frac{1}{2 M} i \sigma_{\mu \nu} q^{\nu} F_{2}^{E M}\right] \tau_{3}\right\} u
\end{aligned}
$$

Now we go back to weak interaction, the polar part of the matrix element between the proton and neutron is,

$$
\begin{aligned}
<p\left|V^{\dagger \mu}\right| n> & =\bar{u}\left[\gamma_{\mu} F_{1}+\frac{1}{2 M} i \sigma_{\mu \nu} q^{\nu} F_{2}+\frac{q_{\mu}}{M} F_{V}^{3}\right] \tau_{+} u \\
<n\left|V^{\mu}\right| p> & =\bar{u}\left[\gamma_{\mu} F_{1}+\frac{1}{2 M} i \sigma_{\mu \nu} q^{\nu} F_{2}+\frac{q_{\mu}}{M} F_{V}^{3}\right] \tau_{-} u
\end{aligned}
$$

Comparing the second bracket of Eq. 3.37 with Eqs. 3.38 and 3.39 , it can be seen that $J_{E M}$, $V$, and $V^{\dagger}$ make isotriplet (spin 1 representation of $\mathrm{SU}(2)$ ). In order for this symmetry to be perfect, these 2 equations must satisfy,

$$
\begin{aligned}
F_{1} & =F_{1}^{E M}, \\
F_{2} & =F_{2}^{E M}, \\
F_{V}{ }^{3} & =0 .
\end{aligned}
$$

This means that the weak vector form factor, $F_{1}$, and weak tensor form factor, $F_{2}$, are measured from electromagnetic scattering, i.e., electron scattering experiments. In electromagnetic interaction measurements, the current conservation law means the non-existence of the scalar form factor. In weak interaction measurements, CVC means not only the nonexistence of the scalar term, but also it implies a connection between the electromagnetic and the weak vector/tensor form factors [17]. 


\subsubsection{Partial conservation of axial vector current (PCAC)}

This subsection follows Ref. [17].

The general expression for the axial part of the matrix element between the proton and neutron is,

$$
<p\left|A^{\dagger \mu}\right| n>=\bar{p}\left[\gamma_{\mu} F_{A} \gamma_{5}+\frac{q_{\mu}}{M} F_{P} \gamma_{5}\right] n
$$

The origin of the pseudo scalar form factor, $F_{P}$, cannot be of leading order, so must be a second order term (Fig. 3.3). Fig. 3.3a is the so-called "pion vertex correction", and since the interaction with the lepton current is still axial vector, this diagram contributes as a higher order correction to the axial vector coupling. However (Fig. 3.3b), in "one pion exchange", the nucleon current emits a pion, which is a pseudo scalar interaction, and therefore this diagram is the primary contribution to the pseudo scalar form factor.

Then, the axial part of the nucleon-lepton current interaction term consists of 3 parts: pion emission by neutron, subsequent pion propagation, and then pion decay. That is,

$$
\begin{aligned}
& A \sim[n \rightarrow p+\pi] \times[\pi-\text { propagator }] \times[\pi \rightarrow l+\nu] \\
& A \sim\left[i g_{0}\left(\bar{p} \gamma_{5} n\right)\right] \times\left[\frac{1}{q^{2}-m_{\pi}^{2}}\right] \times\left[\frac{G_{F} \cos \theta_{c}}{\sqrt{2}} i f_{\pi} q_{\mu}\left(\bar{l} \gamma^{\mu}\left(1-\gamma_{5}\right) \nu\right)\right]
\end{aligned}
$$

Here, $g_{0}$ and $f_{\pi}$ are the pion-nucleon coupling constant and pion decay constant, respectively. Then, Eq. 3.40 is written,

$$
<p\left|A^{\dagger \mu}\right| n>=\bar{p}\left[\gamma_{\mu} F_{A}-\frac{q_{\mu}}{M} \frac{g_{0} f_{\pi}}{q^{2}-m_{\pi}^{2}}\right] \gamma_{5} n
$$

Although the axial current is not conserved, it may be approximately conserved in $m_{\pi} \rightarrow 0$ limit (partial conservation of axial current),

$$
\lim _{m_{\pi} \rightarrow 0} \partial_{\mu} A^{\mu}=0
$$


(a) pion vertex correction

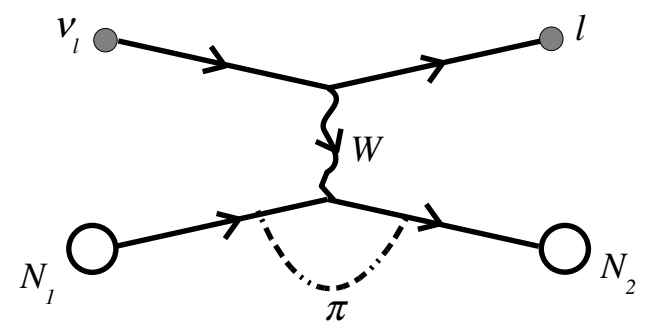

(b) pion One-pion exchange

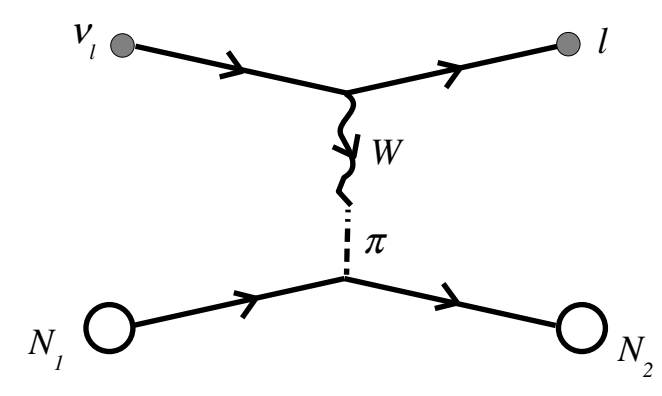

Figure 3.3: Although these two pion diagrams are same order, their contributions are different. (a) pion vertex correction is a higher order correction to the axial vector current, whereas (b) one pion exchange contributes to the pseudo scalar form factor. 
Then, using the Dirac equation, $-i \bar{p} \overleftrightarrow{\not} n=\bar{p}\left(\not p_{p}-\not p_{n}\right) n=p\left(m_{p}+m_{n}\right) n \sim p(2 M) n$ Eq. 3.42 becomes,

$$
\begin{aligned}
0 & =\lim _{m_{\pi} \rightarrow 0} \bar{p}\left[2 M F_{A}-\frac{q^{2}}{M} \frac{g_{0} f_{\pi}}{q^{2}-m_{\pi}^{2}}\right] \gamma_{5} n \\
& =\bar{p}\left[2 M F_{A}-\frac{g_{0} f_{\pi}}{M}\right] \gamma_{5} n .
\end{aligned}
$$

Therefore,

$$
g_{0} f_{\pi}=2 M^{2} F_{A}
$$

Eq. 3.45 is called the Goldberger-Treiman relation [17]. Finally, the pseudo scalar form factor derived from one pion exchange is,

$$
F_{P}=-\frac{g_{0} f_{\pi}}{q^{2}-m_{\pi}^{2}}=\frac{2 M^{2}}{Q^{2}+m_{\pi}^{2}} F_{A}
$$

At our energy scale, $Q^{2}<1.0 \mathrm{GeV}^{2}$, the pseudo scalar form factor $F_{P}$ derived here is bigger than the axial vector form factor $F_{A}$. However, since $F_{P}$ always couples with the muon mass term, $m^{2} / M^{2} \sim 0.01$, the contribution from this term is small (Sec. C.1.4)

\subsubsection{The expressions for Llewellyn-Smith's form factors}

Now, we want to derive expressions for the form factors of Eqs. $3.20,3.21$, and 3.22 . Going back to proton electromagnetic current (Eq. 3.32 and using Gordon decomposition (Eq. C.9),

$$
<p\left|J_{E M}^{\mu}\right| p>=\bar{p}\left[\gamma_{\mu}\left(F_{1}^{E M, p}+F_{2}^{E M, p}\right)-\frac{F_{2}^{E M, p}}{2 M}\left(p_{1}^{\mu}+p_{2}^{\mu}\right)\right] p .
$$

As will be seen in Sec. 3.2.7, we want to provide some physical interpretation of the form factors. When the 3-momentum transfer is low, the scattering should be classical, so the form factors can be interpreted as Fourier transformations of the charge and magnetic moment distribution in configuration space. Therefore, it is good to define form factors 
in 3-dimensions. For this purpose, we need to consider this current in a special Lorentz frame, i.e., the Breit frame (brick wall frame) shown in Fig. 3.4. In the Breit frame, $p_{1}=-p_{2}=(0,0, p)$, there is no energy transfer, so, $F\left(\vec{q}^{2}\right)=F\left(-q^{2}\right)=F\left(Q^{2}\right)$. Now, the proton current is $<p\left|J_{E M}^{\mu}\right| p>\equiv(\rho, \vec{J})$, thus,

$$
\begin{aligned}
& \rho=\bar{p}\left[\gamma^{0}\left(F_{1}^{E M, p}+\kappa_{p} F_{2}^{E M, p}\right)-\frac{E F_{2}^{E M, p}}{M}\right] p, \\
& \vec{J}=\bar{p}\left[\vec{\gamma}\left(F_{1}^{E M, p}+F_{2}^{E M, p}\right)\right] p .
\end{aligned}
$$

Here, we introduce a standard Dirac spinor with Dirac representation for protons with $\chi$ and $\phi$ as spinors for the $z$-direction spin state. Then,

$$
p\left(p_{1}, \lambda_{1}\right)=\sqrt{E+M}\left(\begin{array}{c}
\chi \\
\frac{\vec{\sigma} \cdot \vec{p}_{1}}{E+M} \chi
\end{array}\right)=\sqrt{E+M}\left(\begin{array}{c}
\chi \\
\frac{p}{E+M} \chi
\end{array}\right)
$$

and,

$$
\begin{aligned}
& \bar{p}\left(p_{1}, \lambda_{1}\right) p\left(p_{2}, \lambda_{2}\right)=(E+M)\left(\begin{array}{cc}
\chi^{\dagger} & -\frac{\vec{\sigma} \cdot \vec{p}_{1}}{E+M} \chi^{\dagger}
\end{array}\right) \cdot\left(\begin{array}{c}
\phi \\
\frac{\vec{\sigma} \cdot \vec{p}_{2}}{E+M} \phi
\end{array}\right) \\
& =(E+M)\left(1+\frac{p^{2}}{(E+M)^{2}}\right)=\frac{E^{2}+2 E M+M^{2}+p^{2}}{E+M}=2 E
\end{aligned}
$$

when $\chi=\phi$.

$$
\begin{aligned}
& \bar{p}\left(p_{1}, \lambda_{1}\right) \gamma^{0} p\left(p_{2}, \lambda_{2}\right)=(E+M)\left(\begin{array}{cc}
\chi^{\dagger} & \frac{\vec{\sigma} \cdot \vec{p}_{1}}{E+M} \chi^{\dagger}
\end{array}\right) \cdot\left(\begin{array}{c}
\phi \\
\frac{\vec{\sigma} \cdot \vec{p}_{2}}{E+M} \phi
\end{array}\right) \\
= & (E+M)\left(1-\frac{p^{2}}{(E+M)^{2}}\right)=\frac{E^{2}+2 E M+M^{2}-p^{2}}{E+M}=2 M \\
& \text { when } \chi=\phi
\end{aligned}
$$




$$
\begin{aligned}
& \bar{p}\left(p_{1}, \lambda_{1}\right) \vec{\gamma} p\left(p_{2}, \lambda_{2}\right)=(E+M)\left(\begin{array}{cc}
\chi^{\dagger} & \frac{\vec{\sigma} \cdot \vec{p}_{1}}{E+M} \chi^{\dagger}
\end{array}\right) \cdot\left(\begin{array}{cc}
0 & \vec{\sigma} \\
\vec{\sigma} & 0
\end{array}\right) \cdot\left(\begin{array}{c}
\phi \\
\vec{\sigma} \cdot \vec{p}_{2} \\
E+M
\end{array}\right)
\end{aligned}
$$

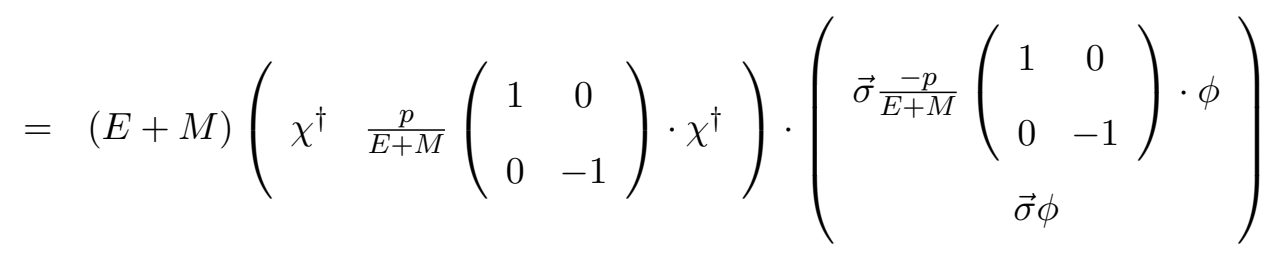

$$
\begin{aligned}
& =\frac{1}{2} q\left[\chi^{\dagger} \vec{\sigma}\left(\begin{array}{cc}
-1 & 0 \\
0 & 1
\end{array}\right) \phi+\chi^{\dagger}\left(\begin{array}{cc}
1 & 0 \\
0 & -1
\end{array}\right) \vec{\sigma} \phi\right]=q \chi^{\dagger}\left(\begin{array}{cc}
0 & \hat{x}+i \hat{y} \\
-\hat{x}+i \hat{y} & 0
\end{array}\right) \phi \\
& \text { when } \chi \neq \phi
\end{aligned}
$$

Thus,

$$
\begin{aligned}
\rho & =\left(2 M F_{1}^{E M, p}+2 M F_{2}^{E M, p}-\frac{2 E^{2} F_{2}^{E M, p}}{M}\right)=2 M\left(F_{1}^{E M, p}+F_{2}^{E M, p}\left(1-\frac{E^{2}}{M^{2}}\right)\right) \\
& =2 M\left(F_{1}^{E M, p}+\frac{q^{2}}{4 M^{2}} F_{2}^{E M, p}\right) \equiv 2 M G_{E}\left(q^{2}\right) \text { when } \chi=\phi .
\end{aligned}
$$

On the other hand, $\vec{J}$ is,

$$
\bar{p} \vec{\gamma} p\left(F_{1}^{E M, p}+F_{2}^{E M, p}\right) \equiv \bar{p} \vec{\gamma} p G_{M}\left(q^{2}\right)=q \chi^{\dagger}\left(\begin{array}{cc}
0 & \hat{x}+i \hat{y} \\
-\hat{x}+i \hat{y} & 0
\end{array}\right) \phi G_{M}\left(q^{2}\right),
$$

and,

$$
J_{1} \pm i J_{2}=q \chi^{\dagger}\left(\begin{array}{cc}
0 & 2(-) \\
-2(+) & 0
\end{array}\right) \phi G_{M}=\mp 2 q G_{M}\left(q^{2}\right) \text { when } \chi=\mp \neq \phi,
$$

Note that $\rho$ is only non-zero for same spin conserving case $(\chi=\phi)$, and $J_{1} \pm i J_{2}$ is only non-zero for spin flipping case $(\chi=\mp \neq \phi)$. Thus,

$$
\begin{aligned}
\rho & =2 M G_{E}\left(Q^{2}\right) \text { for } \lambda_{1}=\lambda_{2}, \\
J_{1} \pm i J_{2} & =\mp 2 q G_{M}\left(Q^{2}\right) \text { for } \lambda_{1}=\mp \frac{1}{2} \neq \lambda_{2} .
\end{aligned}
$$

Therefore, $Q^{2} \rightarrow 0$ limit of Sachs form factors can be interpreted as physical electric charge and magnetic moment [18]. This gives the normalization conditions for the Pauli and Dirac form factors. 


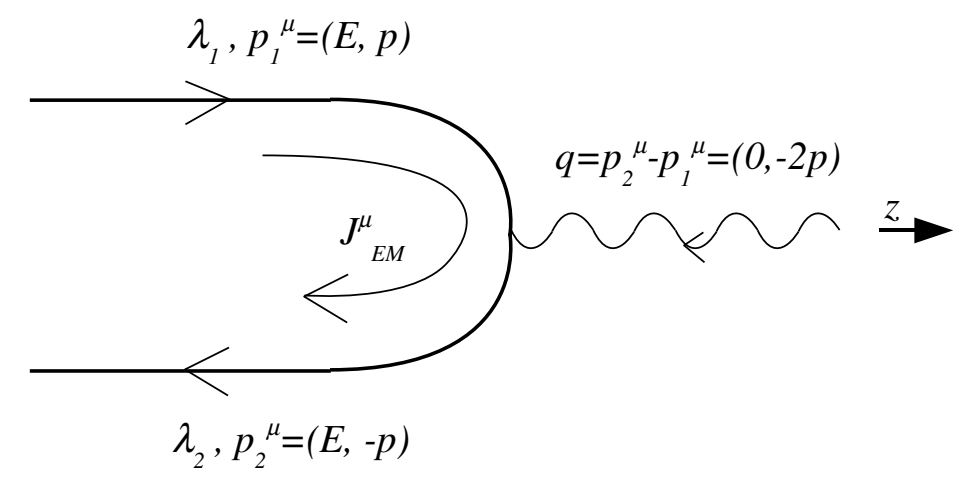

Figure 3.4: Proton electromagnetic current in the Breit frame. In the Breit frame, the energy transfer to the proton is zero.

Now, the $F_{1}$ and $F_{2}$ form factors are defined from the Sachs form factors,

$$
\begin{aligned}
& G_{E}^{p, n}\left(Q^{2}\right)=F_{1}^{E M, p, n}\left(Q^{2}\right)-\frac{Q^{2}}{4 M^{2}} F_{2}^{E M, p, n}\left(Q^{2}\right), \\
& G_{M}^{p, n}\left(Q^{2}\right)=F_{1}^{E M, p, n}\left(Q^{2}\right)+F_{2}^{E M, p, n}\left(Q^{2}\right) .
\end{aligned}
$$

In the $Q^{2} \rightarrow 0$ limit, the Sachs form factors have the physical interpretations,

$$
\begin{aligned}
G_{E}^{p}\left(Q^{2} \rightarrow 0\right) & =1, \text { proton electric charge, } \\
G_{E}^{n}\left(Q^{2} \rightarrow 0\right) & =0, \text { neutron electric charge, } \\
G_{M}^{p}\left(Q^{2} \rightarrow 0\right) & =2.793, \text { proton magnetic moment, } \\
G_{M}^{n}\left(Q^{2} \rightarrow 0\right) & =-1.913, \text { neutron magnetic moment. }
\end{aligned}
$$

Also, the Sachs form factors are assumed to have $Q^{2}$ dipole dependence (equivalent to an 
exponential charge distribution, see Sec. 3.2.7), and we have,

$$
\begin{aligned}
G_{E}^{p, n}\left(Q^{2}\right) & =\frac{G_{E}^{p, n}(0)}{\left(1+\frac{Q^{2}}{M_{V}^{2}}\right)^{2}}, \\
G_{M}^{p, n}\left(Q^{2}\right) & =\frac{G_{M}^{p, n}(0)}{\left(1+\frac{Q^{2}}{M_{V}^{2}}\right)^{2}} .
\end{aligned}
$$

Then the proton/neutron electromagnetic Dirac and Pauli form factors can be defined,

$$
\begin{aligned}
& F_{1}^{E M, p, n}\left(Q^{2}\right)=\frac{G_{E}^{p, n}\left(Q^{2}\right)+\frac{Q^{2}}{4 M^{2}} G_{M}^{p, n}\left(Q^{2}\right)}{\left(1+\frac{Q^{2}}{4 M^{2}}\right)}, \\
& F_{2}^{E M, p, n}\left(Q^{2}\right)=-\frac{G_{E}^{p, n}\left(Q^{2}\right)-G_{M}^{p, n}\left(Q^{2}\right)}{\left(1+\frac{Q^{2}}{4 M^{2}}\right)} .
\end{aligned}
$$

Under CVC, (Sec. 3.2.4, Eqs. 3.24 and 3.25, one can derive expressions for the $F_{1}$ and $F_{2}$ form factors,

$$
\begin{aligned}
F_{1}\left(Q^{2}\right) & =\frac{1+\frac{Q^{2}}{4 M^{2}}(1+\xi)}{\left(1+\frac{Q^{2}}{4 M^{2}}\right)\left(1+\frac{Q^{2}}{M_{V}^{2}}\right)^{2}} \\
F_{2}\left(Q^{2}\right) & =\frac{\xi}{\left(1+\frac{Q^{2}}{4 M^{2}}\right)\left(1+\frac{Q^{2}}{M_{V}^{2}}\right)^{2}}
\end{aligned}
$$

Likewise, for the neutral current, the Sachs form factors can be defined for the isoscalar terms (Eq. 3.26, 3.27, 3.28, and 3.29). The physical interpretation for their $Q^{2} \rightarrow 0$ limit is that the strange quark charge contribution and magnetic moment in the nucleon.

$$
\begin{aligned}
& G_{E}^{s}\left(Q^{2} \rightarrow 0\right)=e_{s}, \text { strange quark contribution for nucleon electric charge, } \\
& G_{M}^{s}\left(Q^{2} \rightarrow 0\right)=\mu_{s}, \text { strange quark contribution for nucleon magnetic moment. }
\end{aligned}
$$

Of course, these are zero in the constituent quark model [18]. However, some measurements indicate small but non-zero contributions [23] and we are waiting for further experiments.

The axial vector form factor is also assumed to have a dipole form (Sec. 3.2.7),

$$
F_{A}\left(Q^{2}\right)=-\frac{g_{A}}{\left(1+\frac{Q^{2}}{M_{A}^{2}}\right)^{2}} .
$$


The $Q^{2} \rightarrow 0$ limit of $F_{A}\left(Q^{2}\right)$ is identified with the axial coupling constant, which is equivalent to the difference of spin contributions from the up and down quarks in the nucleon,

$$
g_{A}=\Delta u-\Delta d=-1.267
$$

The spin contribution to the nucleon from each quark species $q$ is also defined from the longitudinally polarized quark function (denoted by upper "+"). The difference between helicity "+" and "-" (denoted by lower "+" and "-"), and the difference between quark and antiquark functions define $\Delta q(x)$. The integral of $\Delta q(x)$ over all Bjorken $x$ can be identified with the spin contribution of quarks to the nucleon,

$$
\begin{aligned}
\Delta q(x) & \equiv q_{+}^{+}(x)-q_{-}^{+}(x)-\left(\bar{q}_{+}^{+}(x)-\bar{q}_{-}^{+}(x)\right), \\
\Delta q & \equiv \int_{0}^{1} \Delta q(x) d x .
\end{aligned}
$$

Therefore, the axial coupling constant provides an important normalization condition for the quark spin distribution functions.

For neutral-current scattering, (Eq. 3.30 and 3.31), the isoscalar form factors are also assumed to have dipole from,

$$
\begin{aligned}
& F_{A}^{Z, p}\left(Q^{2}\right)=-\frac{g_{A}}{2\left(1+\frac{Q^{2}}{M_{A}^{2}}\right)^{2}}-\frac{\Delta s}{2\left(1+\frac{Q^{2}}{M_{A}^{2}}\right)^{2}}, \\
& F_{A}^{Z, n}\left(Q^{2}\right)=+\frac{g_{A}}{2\left(1+\frac{Q^{2}}{M_{A}^{2}}\right)^{2}}-\frac{\Delta s}{2\left(1+\frac{Q^{2}}{M_{A}^{2}}\right)^{2}},
\end{aligned}
$$

where $\Delta s$ is the spin contribution of strange quark in a nucleon. Here, we can see that a negative $\Delta s$ will enhance the neutrino-nucleon neutral current scattering cross section for protons and decrease the corresponding cross section for neutrons when $g_{A}<0$. We discuss a proposed $\Delta s$ measurement by the neutrino neutral current elastic scatterings, FINeSSE experiment [24], in Appendix A.

And finally, the pseudo scalar form factor can be deduced from PCAC (Sec. 3.2.5),

$$
F_{P}\left(Q^{2}\right)=\frac{2 M^{2}}{m_{\pi}^{2}+Q^{2}} F_{A}\left(Q^{2}\right)
$$




\subsubsection{Dipole form factor}

In scattering theory, the observables from scattering experiments can be interpreted as the Fourier transformation of the charge distribution of the scattering body. For example, the dipole form factor results from an exponential charge distribution,

$$
\rho(r)=\rho_{0} \exp (-M r) \stackrel{\text { Fourier }}{\longleftrightarrow} G\left(|q|^{2}\right) \sim \frac{1}{\left(1+\frac{|q|^{2}}{M^{2}}\right)^{2}} .
$$

The basic motivation for the dipole form of the Sachs form factors (Sec. 3.2.6) is the naive assumption of an exponential electromagnetic charge distribution for the nucleon.

However, this interpretation only works for the static case, where the effective size of

the probe is smaller than the size of scattering body $\left(\frac{1}{|q|}<R\right)$, but big enough compared with the de Broglie wave length of the scattering body $\left(\frac{1}{|q|}>1 / M\right)$. Here, the de Broglie wave length measures the scale of localizability (uncertainty) of the scattering body. All non-relativistic objects satisfy this condition, but this is not always true for the relativistic case. For the relativistic case, for example when $|q|>M \sim 1 \mathrm{GeV}^{2}$, not only the internal structure of a scattering body, but also the dynamical effects contribute to the form factor and the interpretation is complicated. So, the assumption of dipole form factor at high 3 -momentum transfer is necessarily broken [25].

\subsubsection{Discrete transformation}

This subsection follows Ref. [17].

\section{G-parity and second class currents}

G-parity is the combination of isospin rotation and charge transformation,

$$
G=C e^{i \pi I_{2}}
$$


Since the strong interaction is isospin and charge invariant, G-parity is always conserved in those processes. Here isospin rotation only affects the Dirac spinors, and all form factors relatively transform in the same way, so we only consider charge transformation.

Under the charge conjugation operation, $C u(\vec{x}, t)=\gamma^{1} \gamma^{3} \bar{u}(\vec{x}, t)$, bilinear forms transform in the following ways.

$$
\begin{array}{rll}
\bar{p}[F] n & \stackrel{C}{\rightarrow}\left[\gamma_{0} \gamma_{2} F^{T} \gamma_{2} \gamma_{0}\right] p \\
\bar{p}\left[F_{V}^{3}\right] n & \rightarrow & -\bar{n}\left[F_{V}^{3}\right] p \\
\bar{p}\left[\gamma^{\mu} F_{1}\right] n & \rightarrow & \bar{n}\left[\gamma^{\mu} F_{1}\right] p \\
\bar{p}\left[\sigma^{\mu \nu} F_{2}\right] n & \rightarrow & \bar{n}\left[\sigma^{\mu \nu} F_{2}\right] p \\
\bar{p}\left[\gamma_{5} F_{P}\right] n & \rightarrow & -\bar{n}\left[\gamma_{5} F_{P}\right] p \\
\bar{p}\left[\gamma^{\mu} \gamma_{5} F_{A}\right] n & \rightarrow & -\bar{n}\left[g a^{\mu} \gamma_{5} F_{A}\right] p \\
\bar{p}\left[\sigma^{\mu \nu} \gamma_{5} F_{A}^{3}\right] n & \rightarrow & \bar{n}\left[\sigma^{\mu \nu} \gamma_{5} F_{A}^{3}\right] p
\end{array}
$$

Notice that $F_{V}^{3}$ and $F_{A}^{3}$ transform in the relatively opposite way from $F_{1}$ and $F_{A}$, respectively. Now, all interaction types other than $F_{1}$ and $F_{A}$ originate from the strong interaction and are induced from $F_{1}$ and $F_{A}$. So the C-parity of $F_{V}^{3}$ and $F_{2}$ are necessarily the same as that for $F_{1}$, and also, the the C-parity of $F_{P}$ and $F_{A}{ }^{3}$ should be the same as $F_{A}$. Thus, one can prove that there is no $F_{V}{ }^{3}$ and $F_{A}{ }^{3}$ in the nucleon current. Here, $F_{1}, F_{2}, F_{A}$, and $F_{P}$ are called first class, and $F_{V}^{3}$ and $F_{A}{ }^{3}$ are called second class. The second class form factors are known to violate G-parity and do not exist in the standard model [17].

\section{T-invariance and form factors}

All Hamiltonians are hermitian by definition. The CPT transformation replaces the first half with second half, namely hermitian conjugate part. This can be seen in the following expression for a nucleon weak current,

$$
\bar{p}\left[\gamma^{\mu}\left(F_{1}-F_{A} \gamma_{5}\right)+\ldots\right] n \stackrel{C P T}{\longleftrightarrow} \bar{n}\left[\gamma_{\mu}\left(F_{1}^{*}-F_{A}^{*} \gamma_{5}\right)+\ldots\right] p .
$$


This is the famous CPT theorem, and the result is independent from the expression of $\mathrm{C}$, $\mathrm{P}$, and $\mathrm{T}$ transformation [26].

Under the time reversal operation, $T u(\vec{x}, t)=i \gamma^{1} \gamma^{3} u^{*}(\vec{x},-t)$, transformations of bilinear forms are following ways.

$$
\begin{aligned}
\bar{p}[F] n & \stackrel{T}{ } \bar{n}\left[\gamma_{0} \gamma_{3} \gamma_{1} F^{T} \gamma_{1} \gamma_{3} \gamma_{0}\right] p \\
\bar{p}\left[F_{V}^{3}\right] n & \rightarrow \bar{n}\left[F_{V}^{3}\right] p \\
\bar{p}\left[\gamma^{\mu} F_{1}\right] n & \rightarrow \bar{n}\left[\gamma_{\mu} F_{1}\right] p \\
\bar{p}\left[\sigma^{\mu \nu} F_{2}\right] n & \rightarrow-\bar{n}\left[\sigma_{\mu \nu} F_{2}\right] p \\
\bar{p}\left[\gamma_{5} F_{P}\right] n & \rightarrow-\bar{n}\left[\gamma_{5} F_{P}\right] p \\
\bar{p}\left[\gamma^{\mu} \gamma_{5} F_{A}\right] n & \rightarrow \bar{n}\left[g a_{\mu} \gamma_{5} F_{A}\right] p \\
\bar{p}\left[\sigma^{\mu \nu} \gamma_{5} F_{A}^{3}\right] n & \rightarrow \bar{n}\left[\sigma_{\mu \nu} \gamma_{5} F_{A}^{3}\right] p
\end{aligned}
$$

Although CP is violated in weak processes, the amount is small and we can assume CP as a good symmetry. Then, using the result from Sec. 3.2.8 and the parity transformation,

$$
\bar{p}\left[\gamma^{\mu}\left(F_{1}-F_{A} \gamma_{5}\right)+\cdots\right] n \stackrel{C \times P \times T}{\longleftrightarrow} \bar{n}\left[\gamma_{\mu}\left(F_{1}-F_{A} \gamma_{5}\right)+\cdots\right] p .
$$

The required conditions from Eq. 3.67 and Eq. 3.68 are obviously,

$$
F_{1}=F_{1}^{*}, F_{A}=F_{A}^{*}, \cdots
$$

Therefore, T-invariance implies that all the form factors are relatively real [17]. 


\subsection{Neutrino-Nuclei scattering}

\subsubsection{Smith-Moniz formula for the neutrino experiments}

We now want to consider charged-current quasielastic scattering from a bound nucleon, (Fig. 3.5).

$$
\begin{gathered}
\nu_{l}+A \rightarrow l^{-}+A^{\prime}, \\
\overline{\nu_{l}}+A \rightarrow l^{+}+A^{\prime} .
\end{gathered}
$$

The details of the calculation are given in Appendix C.2, In Fig 3.5, initial and final lepton $\nu$ and $l$, initial and final nuclei $A$ and $A^{\prime}$, and initial and final nucleon $N$ and $N^{\prime}$ have 4-momentum $k_{1}, k_{2}, p, p^{\prime}, k$, and $k^{\prime}$ respectively. The charged lepton $l$ has mass $m$, and the nuclear target $A$ has mass $m_{T}$. Also we define nucleons $N$ and $N^{\prime}$ to have mass $M$. We found the expression for the double differential cross section(Eq. C.60 to be,

$$
\begin{aligned}
\frac{d \sigma^{2}}{d k_{2} d \Omega}= & \frac{G_{V}^{2} k_{2}^{2}}{2 \pi^{2} m_{T}}\left\{2 W_{1} \sin ^{2}\left(\frac{1}{2} \chi\right)+W_{2} \cos ^{2}\left(\frac{1}{2} \chi\right)+\sin ^{2}\left(\frac{1}{2} \chi\right) \frac{m^{2}}{m_{T}^{2}} W_{\alpha}+\frac{m^{2}\left(W_{\beta}+W_{8}\right)}{m_{T} \epsilon_{2}}\right. \\
& -\frac{2 W_{8}}{m_{T}} \sin \left(\frac{1}{2} \chi\right) \sqrt{\left.q^{2} \cos ^{2}\left(\frac{1}{2} \chi\right)+|q|^{2} \sin ^{2}\left(\frac{1}{2} \chi\right)+m^{2}\right\},}
\end{aligned}
$$

where $\chi$ is defined through $\frac{k_{2}}{\epsilon_{2}} \cos \theta\left(k_{2}=\left(\epsilon_{2}, \overrightarrow{k_{2}}\right)\right.$, and $\theta$ is a scattering angle of a charged lepton). This formula has five functions, hadronic nuclei functions, $W_{i}, i=1,2, \alpha, \beta, 8$, and we found their expressions in Eqs. C.83, C.84, C.85, C.86, and C.87.

$$
\begin{aligned}
W_{1} & =T_{1}+\frac{1}{2}\left(a_{2}-a_{3}\right) T_{2} \\
W_{2} & =\left[a_{4}+\frac{2 \omega}{|q|} a_{5}+\frac{\omega^{2}}{|q|^{2}} a_{3}+\frac{q^{2}}{2|q|^{2}}\left(a_{2}-a_{3}\right)\right] T_{2} \\
W_{\alpha} & =\frac{m_{T}^{2}}{|q|^{2}}\left(\frac{3}{2} a_{3}-\frac{1}{2} a_{2}\right) T_{2}+\frac{m_{T}^{2} a_{1}}{M^{2}} T_{\alpha}-2 \frac{a_{6} m_{T}^{2}}{M|q|} T_{\beta} \\
W_{\beta} & =\frac{m_{T}}{M}\left(a_{7}+\frac{\omega}{|q|} a_{6}\right) T_{\beta}-\frac{m_{T}}{|q|}\left[a_{5}+\frac{\omega}{|q|}\left(\frac{3}{2} a_{3}-\frac{1}{2} a_{2}\right)\right] T_{2} \\
W_{8} & =\frac{m_{T}}{M}\left(a_{7}+\frac{\omega}{|q|} a_{6}\right)
\end{aligned}
$$


neutrino-bound nucleon scattering

(1) Lab frame

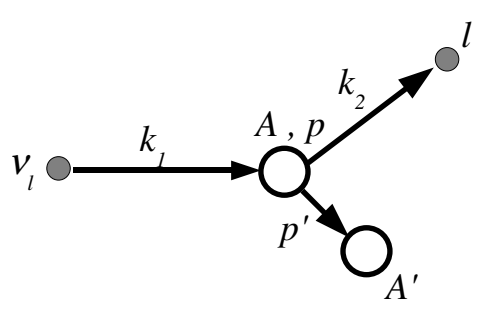

(2) Feynman diagram

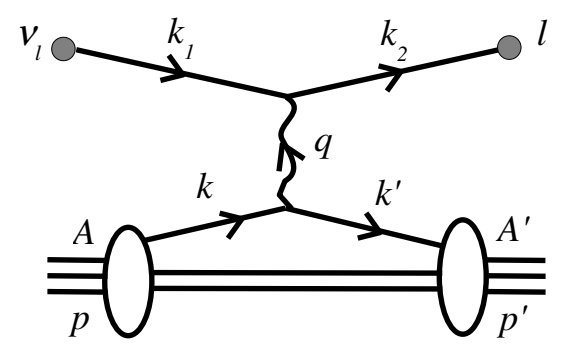

Figure 3.5: The neutrino-nuclei scattering diagram. 


\subsection{NEUTRINO-NUCLEI SCATTERING}

Notice the sign difference in expressions of $W_{\alpha}$ and $W_{\beta}$ compared to the original paper [16, 27 .

The hadronic nuclei functions are made of hadronic nucleon functions, $T_{i}, i=1,2, \alpha, \beta, 8$, and their expressions are found in Eqs. C.105, C.106, C.109, C.108, and C.107 from neutrinonucleon scattering (Eq. C.29 to C.34 which we already know.

$$
\begin{aligned}
T_{1}= & {\left[\frac{1}{2} Q^{2}\left(F_{1}+2 M F_{2}\right)^{2}+\left(2 M^{2}+\frac{1}{2} Q^{2}\right) F_{A}^{2}\right] } \\
T_{2}= & {\left[2 M^{2}\left[F_{1}^{2}+Q^{2} F_{2}^{2}+F_{A}^{2}+Q^{2} F_{A}^{3^{2}}\right]\right] } \\
T_{\alpha}= & \frac{M^{2}}{Q^{2}} T_{1}+\frac{1}{4} T_{2}+4 M^{2} F_{V}^{3}\left[2 M F_{1}-F_{2} Q^{2}+\left(2 M^{2}+\frac{1}{2} Q^{2}\right) F_{V}^{3}\right] \\
& +M^{2}\left(2 M F_{A}-F_{P} Q^{2}\right)\left[F_{A}^{3}+\frac{1}{2 Q^{2}}\left(2 M F_{A}-F_{P} Q^{2}\right)\right] \\
T_{\beta}= & \left.\frac{1}{2} T_{2}+4\left[\left(2 M F_{1}-F_{2} Q^{2}\right) M^{2} F_{V}^{3}+\left(2 M F_{A}-F_{P} Q^{2}\right) M^{2} F_{A}^{3}\right]\right] \\
T_{8}= & {\left[2 M^{2} F_{A}\left(F_{1}+2 M F_{2}\right)\right] }
\end{aligned}
$$

These contain nucleon form factors from Sec. 3.2.6.

The expressions for $a_{i}, i=1, \cdots, 7$ in the hadronic nuclei functions are found in Eq. C.64 to C.70.

$$
\begin{aligned}
& a_{1}=\int d \vec{k} f(\vec{k}, \vec{q}, \omega) \\
& a_{2}=\int d \vec{k} f(\vec{k}, \vec{q}, \omega) \frac{k^{2}}{M^{2}} \\
& a_{3}=\int d \vec{k} f(\vec{k}, \vec{q}, \omega) \frac{k^{2} \cos ^{2} \tau}{M^{2}} \\
& a_{4}=\int d \vec{k} f(\vec{k}, \vec{q}, \omega) \frac{\epsilon_{k}^{2}}{M^{2}} \\
& a_{5}=\int d \vec{k} f(\vec{k}, \vec{q}, \omega) \frac{\epsilon_{k} k \cos \tau}{M^{2}} \\
& a_{6}=\int d \vec{k} f(\vec{k}, \vec{q}, \omega) \frac{k \cos \tau}{M} \\
& a_{7}=\int d \vec{k} f(\vec{k}, \vec{q}, \omega) \frac{\epsilon_{k}}{M}
\end{aligned}
$$


Here $f(\vec{k}, \vec{q}, \omega)$ is a function describing how nucleons distribute in momentum space, and $\cos \tau$ is an angle made between initial nucleon momentum $\vec{k}$ and 3-momentum transfer $\vec{q}$. The analytic solutions are also found for relativistic Fermi gas (RFG) model (Eqs. C.96 to C.102). For RFG model, these integrals, taken within the momentum space of target nucleon, are limited by Pauli blocking. And they are re-written by the integral of target nucleon energy,

$$
\int d \vec{k} f(\vec{k}, \vec{q}, \omega) \sim \int_{k_{\min }}^{k_{\max }} k d k \sim \int_{E_{l o}}^{E_{h i}} \epsilon d \epsilon .
$$

Here, $E_{l o}$ and $E_{h i}$ are written,

$$
\begin{aligned}
& E_{h i}=\sqrt{p_{F}^{2}+M_{n}^{2}} \\
& E_{l o}=\sqrt{p_{F}^{2}+M_{p}^{2}}-\omega+E_{B},
\end{aligned}
$$

where $E_{h i}$ is the energy of an initial nucleon on the Fermi surface and $E_{l o}$ is the lowest energy of an initial nucleon that leads to a final nucleon just above the Fermi surface by adding the energy transfer $\omega$. The Fermi momentum $p_{F}$ and the binding energy $E_{B}$ should be found experimentally. Later, we introduce a new parameter, " $\kappa$ ", to scale $E_{l o}$ to increase Pauli blocking. We discuss this more in Chapter. 8 .

And finally, this results in a cross-check of Smith and Moniz's RFG model.

\subsubsection{Cross section formula comparison with nuance}

The nuance neutrino reaction code (Sec. 7.4) employs the quasi-elastic model of SmithMoniz for both bound and free nucleons. In nuance,

$$
\begin{aligned}
\frac{d \sigma^{2}}{d\left(\ln q^{2}\right) d(\ln \omega)}= & \frac{Q^{2} \omega}{32 \pi m_{T} \epsilon_{1}^{2}} G_{V}^{2} \cdot\left[\begin{array}{c}
\text { isospin } \\
\text { factor }(=1)
\end{array}\right] \cdot\left[\begin{array}{c}
\text { Weakboson } \\
\text { propagator }(\sim 1)
\end{array}\right] \times \\
& 16 \epsilon_{1} \epsilon_{2}\left\{2 W_{1} \sin ^{2}\left(\frac{1}{2} \chi\right)+\cdots\right\}
\end{aligned}
$$


with,

$$
\begin{aligned}
d\left(\ln q^{2}\right) & =\frac{1}{q^{2}} d q^{2}=\frac{2 \epsilon_{1} k_{2}}{q^{2}} d(\cos \theta), \\
d(\ln \omega) & =\frac{1}{\omega} d \omega=-\frac{1}{\omega} d \epsilon_{2}=-\frac{k_{2}}{\omega \epsilon_{2}} d k_{2}, \\
d \Omega & =2 \pi d(\cos \theta) .
\end{aligned}
$$

And remember, in old notation, Eq. C.44 has $q^{2}$ defined as positive, then,

$$
\begin{aligned}
& \quad \frac{d \sigma^{2}}{d\left(\ln q^{2}\right) d(\ln \omega)}=-\frac{q^{2} \omega \epsilon_{2}}{2 \pi m_{T} \epsilon_{1}} G_{V}^{2}\left\{2 W_{1} \sin ^{2}\left(\frac{1}{2} \chi\right)+\cdots\right\} \\
& \rightarrow \quad \frac{d \sigma^{2}}{d q^{2} d \omega}=-\frac{G_{V}^{2} \epsilon_{2}}{2 \pi m_{T} \epsilon_{1}}\left\{2 W_{1} \sin ^{2}\left(\frac{1}{2} \chi\right)+\cdots\right\} \\
& \rightarrow \quad \frac{d \sigma^{2}}{d(\cos \theta) d \epsilon_{2}}=\frac{G_{V}^{2} k_{2} \epsilon_{2}}{\pi m_{T}}\left\{2 W_{1} \sin ^{2}\left(\frac{1}{2} \chi\right)+\cdots\right\} \\
& \rightarrow \quad \frac{d \sigma^{2}}{d k_{2} d \Omega}=\frac{G_{V}^{2} k_{2}^{2}}{2 \pi^{2} m_{T}}\left\{2 W_{1} \sin ^{2}\left(\frac{1}{2} \chi\right)+\cdots\right\} .
\end{aligned}
$$

Therefore, it is verified that nuance is using an identical expression with as that of Smith and Moniz [16]. 


\subsection{Neutrino Charged current quasielastic (CCQE) scatter- ing cross section measurements}

The CCQE cross section has been measured in a number of past experiments. But a precise measurement of the CCQE process has become an urgent program because the CCQE cross section has a tight relationship with neutrino oscillation measurements.

\subsubsection{Long baseline accelerator-based neutrino oscillation experiments}

The goal of next-generation long baseline accelerator-based neutrino oscillation experiments is to measure a non-zero $\theta_{13}$, the last mixing matrix element. The value of $\theta_{13}$ is an important parameter through which to access beyond-the-standard-model (BSM) physics. If it is indeed non-zero, then the hope is to measure leptonic CP violation, which may help in our understanding of leptogenesis, one of the candidate explanations of the baryon asymmetry of the universe [28].

Currently there are two experiments planned, the Tokai-to-Kamioka (T2K) experiment [29] ( $E \sim 800 \mathrm{MeV}, L \sim 300 \mathrm{~km})$ and the NuMI Off-axis $\nu_{e}$ Appearance (NOvA) experiment [30] $(E \sim 2 \mathrm{GeV}, L \sim 800 \mathrm{~km})$. Both experiments will use a $\nu_{\mu}$ beam and search for $\nu_{e}$ appearance events in order to measure $\theta_{13}$ via,

$$
P\left(\nu_{\mu} \rightarrow \nu_{e}\right)=\sin ^{2} \theta_{23} \sin ^{2} 2 \theta_{13} \sin ^{2}\left(1.27 \frac{\Delta m_{32}^{2}\left(e V^{2}\right) L(k m)}{E(G e V)}\right) .
$$

Since a small $P\left(\nu_{\mu} \rightarrow \nu_{e}\right)$ is proportional to $\sin ^{2} \theta_{23}$ and $\sin ^{2}\left(1.27 \frac{\Delta m_{32}^{2} L}{E}\right)$, we also need accurate knowledge of these two oscillation parameters, and can achieve by the measurements of $\nu_{\mu}$ disappearance events,

$$
P\left(\nu_{\mu} \rightarrow \nu_{\mu}\right)=1-\sin ^{2} 2 \theta_{23} \sin ^{2}\left(1.27 \frac{\Delta m_{32}^{2}\left(e V^{2}\right) L(k m)}{E(G e V)}\right) .
$$

These two oscillation parameters are extracted from the shape of $P\left(\nu_{\mu} \rightarrow \nu_{\mu}\right)$ as a function of reconstructed neutrino energy. Therefore, a good extraction of $\sin ^{2} \theta_{23}$ and $\Delta m_{32}^{2}$ relies 


\subsection{NEUTRINO CHARGED CURRENT QUASIELASTIC (CCQE) SCATTERING}

CROSS SECTION MEASUREMENTS

on a good reconstruction of neutrino energy, which requires a better understanding of the signal $\left(\nu_{\mu} \mathrm{CCQE}\right)$ and background interactions, mainly the $\mathrm{CC} 1 \pi$ interaction.

It is important to perform these cross section measurements prior to the oscillation experiments. Although all long baseline accelerator-based neutrino oscillation experiments have near detectors, they exist to constrain neutrino flux uncertainties, and this constraint relies on an accurate knowledge of the neutrino cross sections. Figure 3.6 shows the world's data for neutrino charged current cross sections. As can be seen, the existing data are rather sparse and old. Since the two experiments, T2K and NOvA, span different energy ranges, we need cross section measurements in both energy regions because the dominant interaction types are different in each, and, therefore, the energy reconstruction and backgrounds are different. Fortunately, there is much new input from current and future neutrino cross section measurements: K2K near detector [31] ( 1.2 GeV, completed), MiniBooNE [4] ( $800 \mathrm{MeV}$, ongoing), SciBooNE [32] ( $800 \mathrm{MeV}$, completed), MINOS near detector [33]

( $\sim 2-20 \mathrm{GeV}$, ongoing), MINERvA [34] $(\sim 2-20 \mathrm{GeV}$, approved), and NOMAD [35] ( $5-70 \mathrm{GeV}$, completed). 


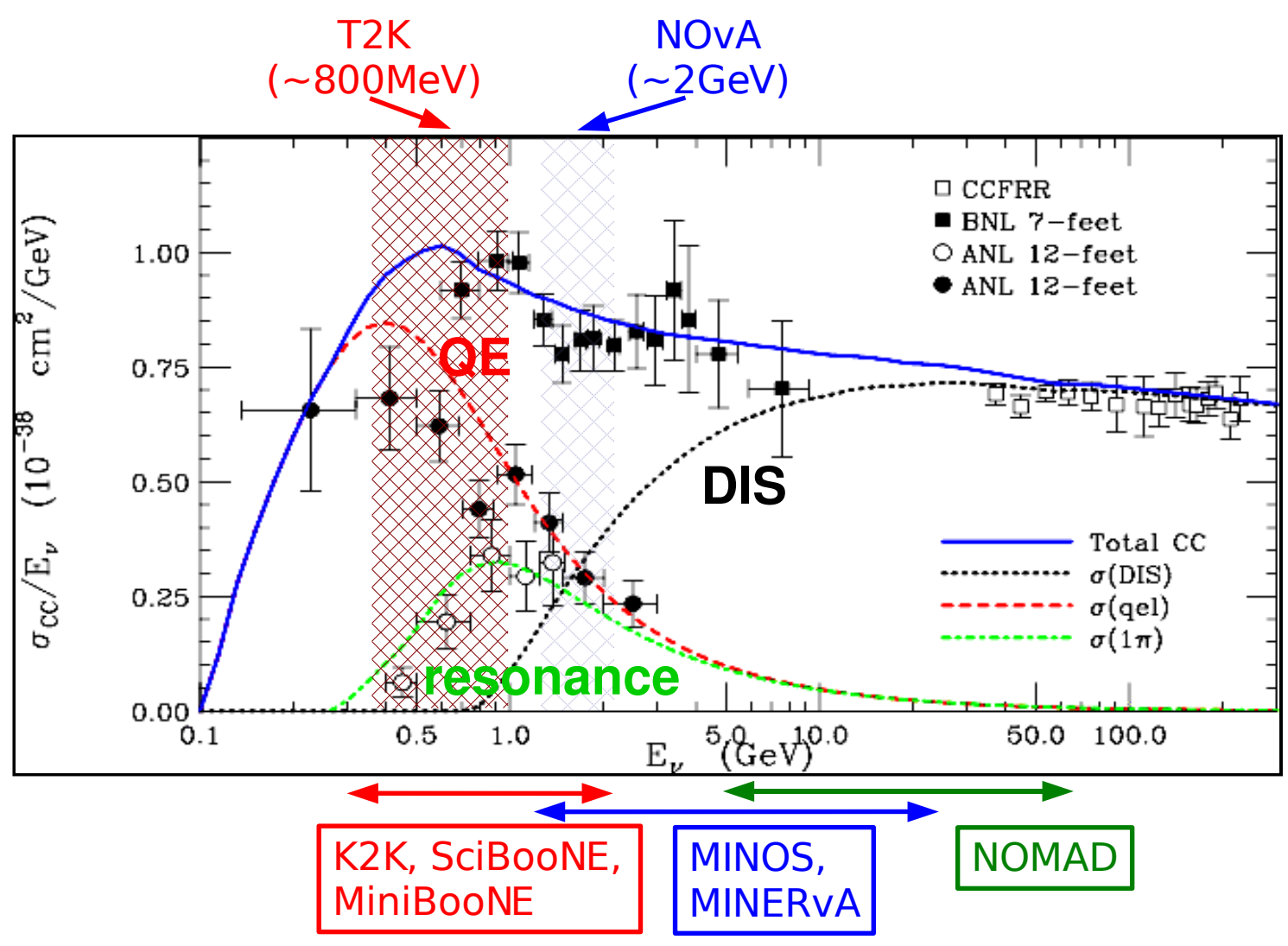

Figure 3.6: The world data for $\nu_{\mu}$ charged current cross section divided by neutrino energy. The figure is taken from [36]. The dominant interaction for T2K and NOvA are quasi-elastic (QE) and deep inelastic scattering (DIS) respectively. The existing data are rather sparse and old, but there will be new input soon from current and future experiments! 


\subsubsection{Neutrino energy reconstruction for T2K}

At the T2K energy scale $(\sim 800 \mathrm{MeV})$, the dominant neutrino reactions are $\nu_{\mu}$ chargedcurrent quasi-elastic (CCQE) interactions,

$$
\nu_{\mu}+n \rightarrow p+\mu^{-}
$$

This channel is used to measure $\nu_{\mu}$ disappearance, and thus the $\nu_{\mu}$ energy reconstruction is critical. Since neutrino oscillation experiments use nuclear targets, understanding of this interaction is not trivial.

Recently K2K [31, 37] and MiniBooNE [4, 39] have reported new measurements of the axial mass, $M_{A}$, which are higher than the historical value (Table 3.1). In this energy range, the axial vector form factor is the dominant contribution to the cross section and controls the $Q^{2}$ dependence. Inconsistency of their results from the world average, and the consistency between K2K and MiniBooNE is best understood in terms of nuclear effects, because most of the past experiments used deuterium targets whereas $\mathrm{K} 2 \mathrm{~K}$ and MiniBooNE used oxygen and carbon. Instead of using the world average, both experiments employ their measured $M_{A}$ values to better simulate CCQE events in their oscillation analyses. After the $M_{A}$ adjustment, both experiments see good agreement between data and simulation [4, 31].

We can only measure the interaction rate, which is the convolution of flux and cross

\begin{tabular}{|l|c|c|}
\hline & $M_{A}(\mathrm{GeV})$ & target \\
\hline $\mathrm{K} 2 \mathrm{~K}(\mathrm{SciFi})$ [31] & $1.20 \pm 0.12$ & oxygen \\
$\mathrm{K} 2 \mathrm{~K}$ (SciBar) [37] & $1.14 \pm 0.11$ & carbon \\
MiniBooNE [4] & $1.23 \pm 0.20$ & carbon \\
world average [38] & $1.026 \pm 0.021$ & deuteron, etc \\
\hline
\end{tabular}

Table 3.1: The comparison of measured axial mass $M_{A}$. 
section $\left(R=\int \Phi \times \sigma\right)$. So, without knowing that the flux prediction is perfect, one cannot tune the cross section model from measured interaction rate. MiniBooNE carefully examined this, and showed that the observed data simulation disagreement is not the effect of mismodeling of the neutrino flux, but is really a cross section model problem. This is discussed in detail in Chapter 8 .

It is not only important to understand the energy reconstruction of signal events (i.e., CCQE interaction), but also for background channels. For Super-K, the neutrino energy is reconstructed from the measured muon energy $E_{\mu}$ and angle $\theta_{\mu}$, assuming a CCQE interaction,

$$
E_{\nu}^{Q E} \sim \frac{M_{N} E_{\mu}-\frac{1}{2} m_{\mu}^{2}}{M_{N}-E_{\mu}+\sqrt{E_{\mu}^{2}-m_{\mu}^{2}} \cos \theta_{\mu}} .
$$

Here, $M_{N}$ and $m_{\mu}$ are nucleon and muon masses. Since this formula assumes a 2-body interaction, any interaction involving more than two particles is a source of neutrino energy mis-reconstruction (Fig 3.7, left). The most notable channel contributing to this is charged current one pion $(\mathrm{CC} 1 \pi)$ production. Especially when the detection of the outgoing pion fails for various reasons (pion absorption, detector effect, etc), $\mathrm{CC} 1 \pi$ events become an irreducible background, and thus they need to understand CC1 $1 \pi$ 's relative contribution rather than rejecting them by cuts (Fig. 3.7, right). 

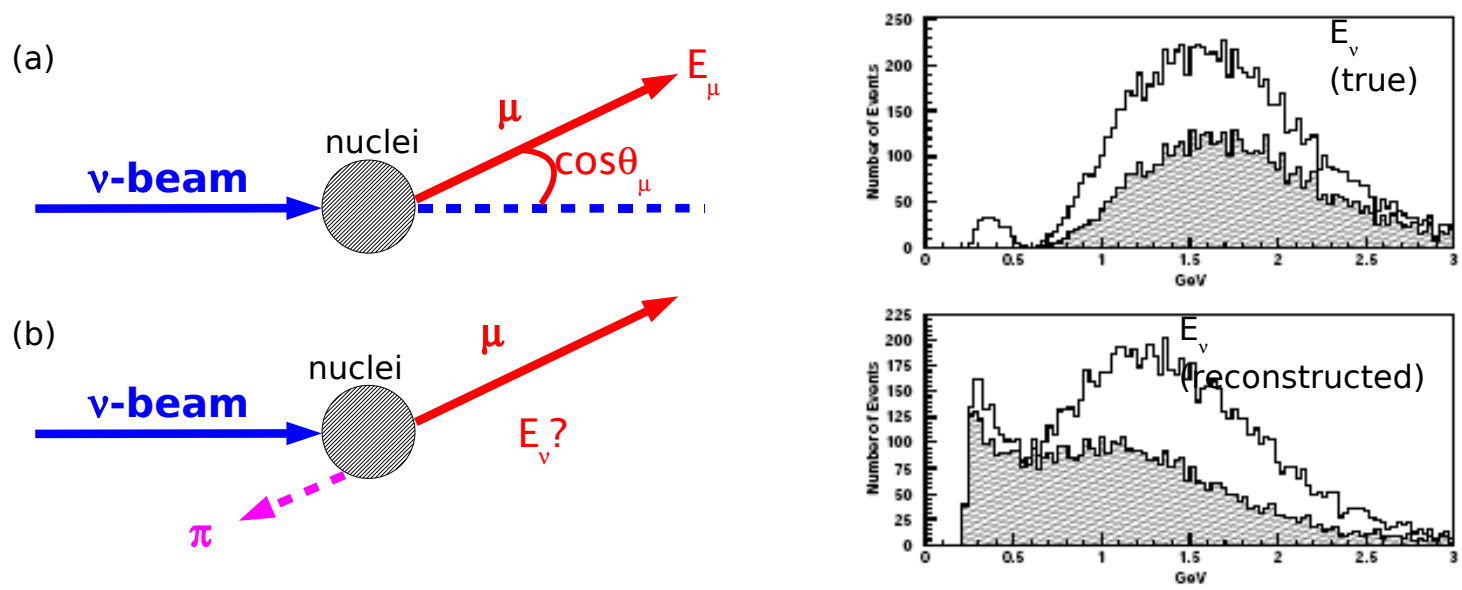

Figure 3.7: (Left) (a) CCQE interaction and (b) CC1 $1 \pi$ interaction. Eq. 3.79 correctly reconstructs neutrino energy only for (a). (b) can be distinguished from (a) by additional pion, however when pion is lost (by pion absorption for example), (b) becomes indistinguishable from intrinsic backgrounds. When (a) and (b) have the same muon kinematics, the reconstructed neutrino energies are the same, however the true neutrino energy for (b) is higher due to the creation of the pion in the event (neutrino energy mis-reconstruction). (Right) true and reconstructed neutrino energy distribution for Super-K predictions with neutrino oscillations. The shaded region is non-QE (mainly $\mathrm{CC} 1 \pi$ ) channels. As can be seen from the bottom plot, $\mathrm{CC} 1 \pi$ background events are misreconstructed at lower neutrino energies and hence can fill out the dip created by neutrino oscillations. Figures are taken from [40]. 


\subsubsection{CCQE cross section measurement}

Currently, most of simulations of CCQE interactions are based on Smith and Moniz's formalism of relativistic Fermi gas (RFG) model [16]. However, to understand nuclear effects correctly, recently developed models for CCQE interactions (for example [41-45]) are more promising. To tune these models, we need to compare with CCQE cross section data. And to obtain precise CCQE cross section measurements, the background should be subtracted carefully. In Chapter 9, we use in situ measurement of $\mathrm{CC} 1 \pi$ events to constraint these $\mathrm{CC} 1 \pi$ background in the CCQE sample, even though most of pions are lost (irreducible backgrounds). Then, in Chapter 10, we report a measured flux-folded differential and double differential cross section, and the flux-unfolded total cross section for the CCQE interaction in MiniBooNE. 


\section{Part II}

\section{Lorentz Violation with Neutrino}


Intellectualism as well as empiricism remains anterior to the problem of orientated space, because it cannot even begin to ask the question. In the case of empiricism, the question was how the image of the world which, in itself, is inverted, can right itself for me. Intellectualism cannot even concede that the image of the world, after the glasses are put on, is inverted.

- M. Merleau-Ponty, Phenomenology of Perception 


\section{Chapter 4}

\section{Test of Lorentz violation with neutrino oscillations}

Lorentz symmetry is one of the most fundamental symmetries of modern physics. Nevertheless, many recently developed theories to describe the most fundamental state of matter and space, such as super strings [46] and quantum gravity [47], do predict a violation of Lorentz symmetry.

Also, a phenomenological model that describes all possible types of Lorentz violation with conventional quantum field theory has been developed. Using this model, the so called Standard-Model Extension (SME) [48 50], one can describe the possible features of neutrino oscillations under the assumption of Lorentz violation. Since the oscillation of neutrinos is effectively a high precision interferometric technique, and neutrinos are the least understood fundamental particles in nature, it is extremely interesting to investigate possible Lorentz violation with neutrinos.

In this chapter, we start with a brief description of the SME formalism, then we discuss a test of Lorentz violation with data from the LSND experiment [1]. This analysis further 
motivates the construction of a global neutrino oscillation model using Lorentz violation [2]. In the next chapter, we discuss this global neutrino oscillation model, and compare the prediction to recent results from the MiniBooNE experiment [3].

\subsection{Introduction}

Lorentz symmetry is a fundamental law in the Standard Model (SM). However, it needs to be supported by experimental observation, and many people have tested this law in various ways over the past 100 years.

Although intrinsic Lorentz violation is very difficult to formulate, Lorentz symmetry could be broken dynamically, namely via spontaneous Lorentz symmetry breaking (SLSB). This is especially relevant after the discovery of the actual process for SLSB in string theory [46], Lorentz violation is widely recognized as a signal of Planck scale physics $\left(M_{P} \sim\right.$ $\left.10^{19} \mathrm{GeV}\right)$. The Planck scale is a very high energy scale, and currently it is impossible to attain. However, if Planck scale physics is suppressed in the low-energy world by its inverse, the natural suppression factor in SM energy scale $\left(E_{W} \sim 100 \mathrm{GeV}\right)$ is, $\lesssim \frac{E_{W}}{M_{P}} \sim 10^{-17}$. Then, precise measurements, for example interference experiments, could observe a signal.

The general effective quantum field theory constructed from the SM and allowing arbitrary coordinate independent Lorentz violation is called the Standard Model Extension (SME) [48 50]. The minimal SME formalism has all the conventional properties of the Standard Model including observer Lorentz covariance, power counting renormalizability, energy momentum conservation, quantized field, micro causality, and spin-statistics with particle Lorentz and CPT violation due to background Lorentz tensor fields of the universe. The minimal SME also has $S U(3)_{C} \times S U(2)_{L} \times U(1)_{Y}$ gauge invariance. Since the background Lorentz tensor fields are fixed in space-time, by definition, they do not transform under an active transformation law. That implies rotation and boost dependence of physics 


\subsection{INTRODUCTION}

in a specific coordinate system.

Here coordinate independence is important for the analysis of Lorentz violation, because Lorentz violation is understood by the coupling of vacuum expectation value (VEV) with Lorentz indices, and the Lagrangian itself is Lorentz invariant with coordinate transformation, or observer Lorentz transformation (passive Lorentz transformation). Thus, Lorentz violation happens when we consider the transformation in a fixed coordinate system, namely particle Lorentz transformation (active Lorentz transformation in a fixed background). This situation is illustrated in Fig. 4.1. Here, a particle is moving in the y-direction in 2-dimensional coordinates, and we consider the rotation in this coordinate system. We introduce a background vector field, which is the source of Lorentz violation. Lorentz violation is the violation of orthochronous Lorentz transformation, where 3 rotation and 3 Lorentz boost generators make the Lorentz group. The rotation violation is considered here.

First, we consider the observer Lorentz transformation (passive Lorentz transformation). This situation is described in Fig. 4.1 $\mathrm{a}$. This is the inverse coordinate transformation to transform the object passively, and as can be seen, the couplings of matter and background fields stay the same, so Lorentz violation does not occur in this transformation.

Next, we consider the particle Lorentz transformation (active Lorentz transformation in fixed background). This situation is described in Fig. 4.1b. This is the active transformation of an object in fixed coordinate space. Now it can be seen that the coupling is not an invariant of the transformation, so Lorentz violation results in physical observables by the active motion in fixed coordinates.

Lorentz symmetry has an intimate relationship with CPT symmetry, namely, CPT violation implies Lorentz violation [51]. This is understood by the following argument. Each Lorentz index has CPT odd parity. Since Lagrangian is Lorentz scalar, this means that the number of Lorentz indices are always even, and any terms in the Lagrangian can be defined as CPT even. However, if odd numbered Lorentz indices are insensitive to particle 


\section{CHAPTER 4. TEST OF LORENTZ VIOLATION WITH NEUTRINO OSCILLATIONS}

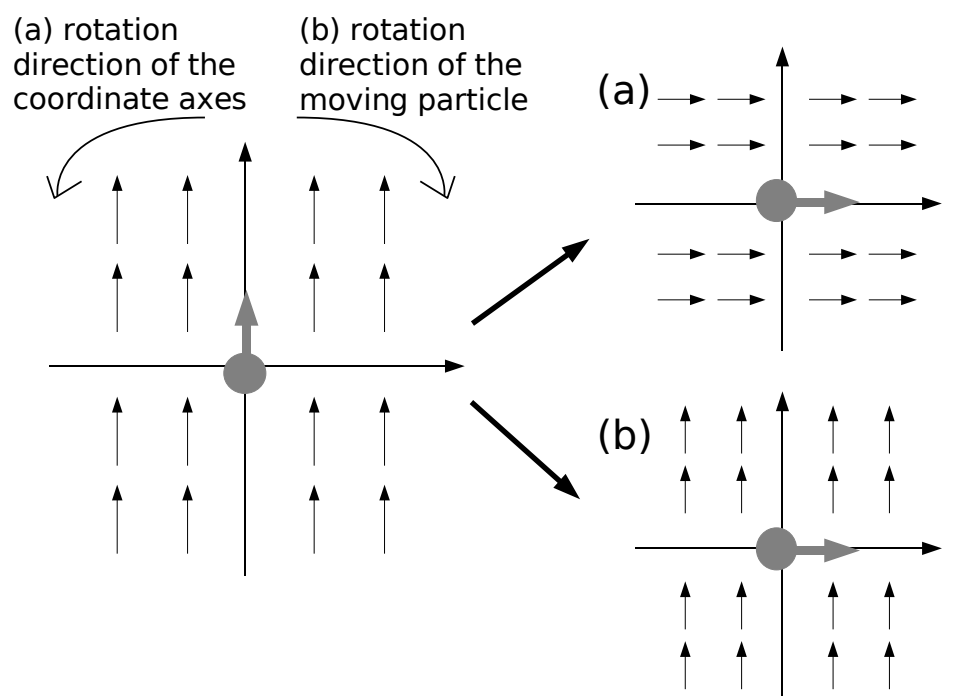

Figure 4.1: A cartoon illustrating observer and particle Lorentz violation.

Lorentz transformation, the phase of CPT is shifted $\pi$ for each Lorentz index. Then, the phase of the CPT transformation is

$$
(-1)^{N}
$$

where $N$ is the number of Lorentz violating Lorentz indices. Rigorously, any interactive field theories with CPT violation always violates Lorentz invariance [51].

Lorentz violation is expected to be smaller than the inverse of Plank scale $\frac{E}{M_{P}}$, where $E$ is the energy scale of the system. Surprisingly, atomic physics has achieved this sensitivity level, and extensive experimental studies have been done (see, for example, Ref. [12, 14, 5254]). A recent experiment [13, 55] of this type reaches a sensitivity to a specific combination of SME coefficients to order $\sim 10^{-31} \mathrm{GeV}$, well beyond a naive estimation of the scale of new physics. However, many of the SME coefficients still have no experimental bounds.

Similarly, quantum interference experiments, such as neutrino oscillations, are also sensitive to the small effect of Lorentz and CPT violation [56]. Tests have been made using 
data from many experiments. Traditionally, a test of Lorentz violation is done via an energy spectrum distortion for neutrino oscillations [57, 58]. This is one of the signals of Lorentz violation in the neutrino sector, which we call "spectrum anomalies" in Sec. 4.2. Using this technique, MACRO [59] and KamLAND [60, 61] data are analyzed. However, the smoking gun of Lorentz violation is direction-dependent physics in a fixed coordinate system. This may be seen in a sidereal time dependence of neutrino oscillation signals, or what we call "periodic variation" in Sec. 4.2, Super-K [62], MINOS [63], LSND [1], AMANDA [64], and MiniBooNE [3] have analyzed their experimental data in this way. For the remainder of this chapter, we focus on tests of Lorentz violation with neutrinos [56, 65, 66].

\subsection{Lorentz violating neutrino oscillation}

For the neutrino sector, Lorentz violation induces additional terms in the Lagrangian. The neutrino free-field Lagrangian is [56],

$$
\begin{aligned}
\mathcal{L} & =\frac{1}{2} i \bar{\psi}_{A} \Gamma_{A B}^{\mu} \overleftrightarrow{D}_{\mu} \psi_{B}-\bar{\psi}_{A} M_{A B} \psi_{B}+h . c . \\
\Gamma_{A B}^{\nu} & \equiv \gamma^{\nu} \delta_{A B}+c_{A B}^{\mu \nu} \gamma_{\mu}+d_{A B}^{\mu \nu} \gamma_{5} \gamma_{\mu}+e_{A B}^{\nu}+i f_{A B}^{\nu} \gamma_{5}+\frac{1}{2} g_{A B}^{\lambda \mu \nu} \sigma_{\lambda \mu}, \\
M_{A B} & \equiv m_{A B}+i m_{5 A B} \gamma_{5}+a_{A B}^{\mu} \gamma_{\mu}+b_{A B}^{\mu}+\frac{1}{2} H_{A B}^{\mu \nu} \sigma_{\mu \nu} .
\end{aligned}
$$

The first term of $\Gamma_{A B}^{\mu}$ and the first and second terms of $M_{A B}$ are the only non-zero terms in the case of conventional neutrino oscillations. The remaining terms in this Lagrangian represent the physics of the background fields. In general, the background Lorentz tensor fields are an infinite series, but if the focus is on a low energy effective theory, these eight additional fields are complete. Here, vacuum expectation values that contain $c_{A B}^{\mu \nu}, d_{A B}^{\mu \nu}$, and $H_{A B}^{\mu \nu}$ are CPT-even (CPT conserving) terms while $e_{A B}^{\mu}, f_{A B}^{\mu}, g_{A B}^{\mu \nu \lambda}, a_{A B}^{\mu}$, and $b_{A B}^{\mu}$ are CPT-odd (CPT non conserving) by definition of the background fields. Notice that each background field has flavor indices (A and B) that, unlike other systems, bring additional complication for the neutrino sector. 
This Lagrangian leads to the modified Dirac equation,

$$
\left(i \Gamma_{A B}^{\mu} \partial_{\mu}-M_{A B}\right) \psi_{B}=0
$$

Neutrino oscillations are a function of eigenvalue differences, so we need to construct an effective Hamiltonian from Eq. 4.5. After some algebra, the effective Hamiltonian within the conventional neutrino flavor basis is,

$$
\begin{gathered}
\left(h_{\mathrm{eff}}\right)_{a b}=|\vec{p}| \delta_{a b}\left(\begin{array}{cc}
1 & 0 \\
0 & 1
\end{array}\right)+\frac{1}{2|\vec{p}|}\left(\begin{array}{cc}
\left(\tilde{m}^{2}\right)_{a b} & 0 \\
0 & \left(\tilde{m}^{2}\right)_{a b}^{*}
\end{array}\right) \\
+\frac{1}{|\vec{p}|}\left(\begin{array}{cc}
{\left[\left(a_{L}\right)^{\mu} p_{\mu}-\left(c_{L}\right)^{\mu \nu} p_{\mu} p_{\nu}\right]_{a b}} & -i \sqrt{2} p_{\mu}\left(\epsilon_{+}\right)_{\nu}\left[\left(g^{\mu \nu \sigma} p_{\sigma}-H^{\mu \nu}\right) \mathcal{C}\right]_{a b} \\
i \sqrt{2} p_{\mu}\left(\epsilon_{+}\right)_{\nu}^{*}\left[\left(g^{\mu \nu \sigma} p_{\sigma}+H^{\mu \nu}\right) \mathcal{C}\right]_{a b}^{*} & {\left[-\left(a_{L}\right)^{\mu} p_{\mu}-\left(c_{L}\right)^{\mu \nu} p_{\mu} p_{\nu}\right]_{a b}^{*}}
\end{array}\right) .
\end{gathered}
$$

Here, the effective Hamiltonian is written in a physical neutrino (three active left-handed neutrinos) and antineutrino (three active right-handed antineutrinos) basis, so the top left block matrix describes $\nu-\nu$ oscillations, top right and bottom left block matrices are for $\nu-\bar{\nu}$ oscillations, and bottom right is for $\bar{\nu}-\bar{\nu}$ oscillations. The first term is the leading energy term and disappears with a phase rotation. The second term is the neutrino mass term. $\tilde{m}_{a b}$ is the light neutrino mass matrix, generally believed to be the solution of the seesaw mechanism [9] and written, using the right-handed $(R)$, left-handed $(L)$, and Dirac (D) mass matrices,

$$
\tilde{m}_{a b}=L-D R^{-1} D^{T}=\left(\begin{array}{ccc}
m_{e e} & m_{e \mu} & m_{\tau e} \\
m_{e \mu} & m_{\mu \mu} & m_{\mu \tau} \\
m_{\tau e} & m_{\mu \tau} & m_{\tau \tau}
\end{array}\right) .
$$

The third term contains all Lorentz violating physics. Here, some SME coefficients only show up in certain combinations, $\left(a_{L}\right)_{a b}^{\mu} \equiv(a+b)_{a b}^{\mu}$ and $\left(c_{L}\right)_{a b}^{\mu \nu} \equiv(c+d)_{a b}^{\mu \nu}$. The parameters $e_{a b}^{\mu}$ and $f_{a b}^{\mu}$ do not contribute to neutrino oscillations. These terms form a Lorentz scalar with the momentum of neutrinos. Neutrino propagation directions are changed with time, but Lorentz violating coefficients are fixed in space (Fig. 4.1(b)). Thus, these Lorentz scalars 
are invariant under observer Lorentz transformation (passive Lorentz transformation) but non-conserving for particle Lorentz transformation (active Lorentz transformation in fixed background). As a consequence, the oscillation signal may exhibit a dependence on the rotation of the earth (sidereal time).

This effective Hamiltonian contains very rich physics, more than just sidereal time variation. The main signals of Lorentz violation can be classified into 6 types [56]

1. Spectral anomalies,

2. L-E conflict,

3. Periodic variation,

4. Compass asymmetries,

5. Neutrino-Antineutrino oscillation,

\section{Classic CPT test.}

Signal (1) is a anomalous energy spectrum distortion, for example the MiniBooNE $\nu_{e}$ lowenergy event excess [67] could be this type of signal. We consider this in Chapter 5 .

Signal (2) is an anomalous mapping of experiment in $\sin ^{2} 2 \theta-\Delta m^{2}$ space. It is possible to have oscillation signals in the region excluded by other experiments in $\sin ^{2} 2 \theta-\Delta m^{2}$ space, because if an oscillation signal is based on an anomalous energy dependence due to Lorentz violation, this signal cannot be mapped correctly in $\sin ^{2} 2 \theta-\Delta m^{2}$ space. The LSND $\bar{\nu}_{e}$ appearance result could be an example. Using $L-E$ space is the model-independent way to show signal sensitivity regions (Fig. 4.2).

Signal (3) is a sidereal variation of an oscillation signal. We focus on this type of signal in this chapter. 
Signal (4) is a special case of rotation violation. Even if an oscillation signal does not exhibit a dependence with sidereal time, time-independent direction dependence could be measurable by actively rotating the neutrino propagation direction on the earth. For example, directional information of atmospheric neutrino data could be used to consider this case.

Signal (5) are helicity-violating neutrino oscillations, and hence Lorentz violating. MiniBooNE $\nu_{e} / \overline{\nu_{e}}$ appearance data could be used to explore this possibility [68], but we do not consider this scenario here.

Signal (6) is the standard test for CPT symmetry by comparing neutrino and antineutrino phenomena.

Some combination of these six types of signal observation would support Lorentz violation even more strongly. The observed signal in the LSND experiment is not understood in $L-E$ plane given results from other experiments [69]. Thus, this can be interpreted as signal type (2). This gives motivation to analyze LSND data under the assumption of Lorentz violation. We search for the signal (4), periodic variation, by analyzing the LSND data, using statistical methods, to find possible variations with sidereal time. Therefore the confirmation of a sidereal variation of LSND oscillation would be very strong evidence for Lorentz violation. 


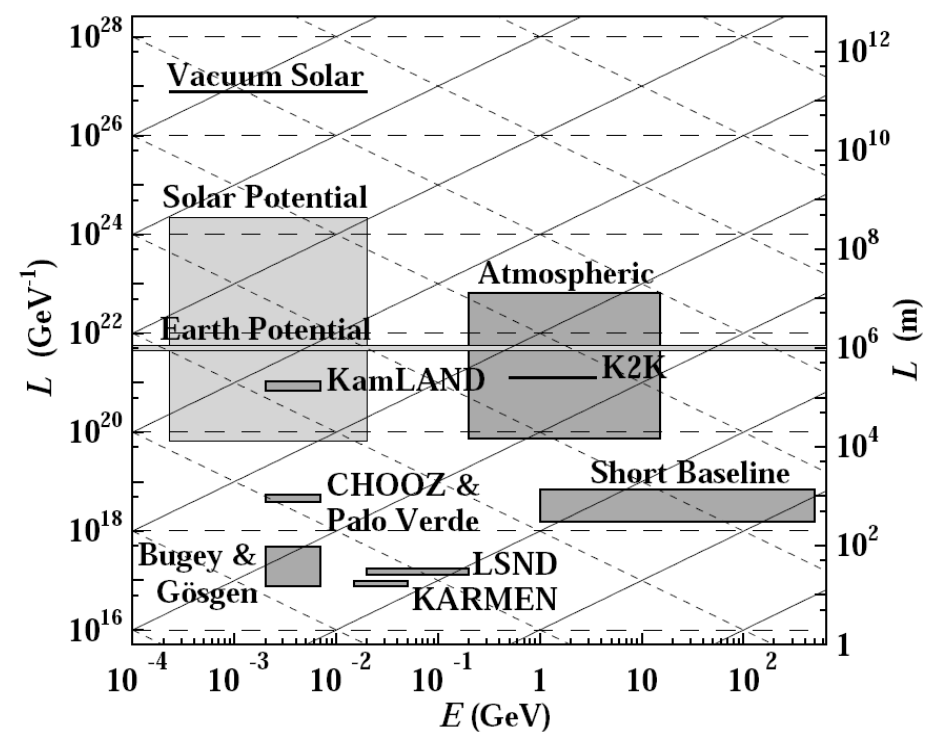

Figure 4.2: A sensitivity plot for general neutrino oscillations illustrating all possible oscillation scenarios taken from [56]. In this space, energy dependence of the neutrino masslike oscillations appear on the solid line, while CPT-odd and CPT-even energy dependences would show occur on the dashed or dotted lines. The square regions show the sensitivity region of various experiment. For example, neutrino oscillations observed by the K2K experiment could be $\Delta m^{2} \sim 10^{-3} \mathrm{eV}^{2}$ or $a_{L} \sim 10^{-21} \mathrm{GeV}$ or $c_{L} \sim 10^{-21}$, but the energy spectrum analysis excludes naive CPT-odd- and CPT-even-like energy dependent solutions. In Chapter 5, a model is presented to create a mass-like oscillatory behavior with CPT-odd and CPT-even terms. 


\subsection{Test of Lorentz violation with LSND experiment}

The details of this analysis are available in [1]. Here, we give a brief summary of a test of Lorentz violation using data from the LSND experiment.

\subsubsection{LSND experiment}

The Liquid Scintillator Neutrino Detector (LSND) experiment [69], completed at the Los Alamos National Laboratory (LANL), observed an excess of $\bar{\nu}_{e}$ in a beam of $\bar{\nu}_{\mu}$ created from $\mu^{+}$decay at rest (Fig. 4.3). The data analysis used the sample of detected $\bar{\nu}_{e} p \rightarrow e^{+} n$ events with positron energy $20<E_{e^{+}}<60 \mathrm{MeV}$. If interpreted as $\bar{\nu}_{\mu}$ to $\bar{\nu}_{e}$ oscillations, this $\bar{\nu}_{e}$ excess implies a two-neutrino oscillation probability of $(0.264 \pm 0.067 \pm 0.045) \%$. Here the first error is statistical and the second error is systematic (neutrino flux, particle detection efficiency, cross sections, etc.). Despite the evidence for neutrino oscillations from solar neutrinos [70 77], atmospheric neutrinos [78-80], accelerator neutrinos [81, 82], and reactor neutrinos [83], the oscillation signal observed at LSND remains a puzzle. Since the neutrino sector is thought as likely to reveal new physics, the LSND anomaly is often explained with new ideas such as sterile neutrino models (see Ref. [84, 85] for a recent example). The MiniBooNE experiment at Fermilab was designed to test the LSND signal (Chapter 7). 


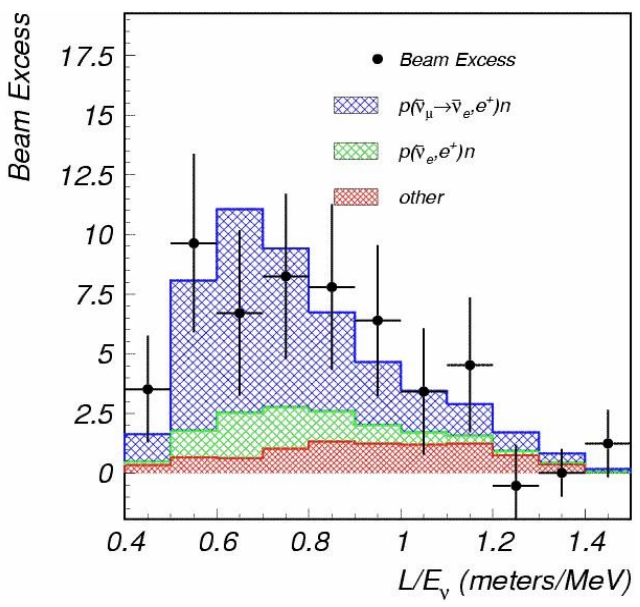

Figure 4.3: The $\bar{\nu}_{e}$ oscillation signal at LSND experiment. The data excess is visible above the predicted background (light cross-hatched area). This excess is consistent with backgrounds plus neutrino oscillations (darker cross-hatched area).

\subsubsection{SME formalism for LSND}

Using an the SME formalism (Eq. 4.6), the relevant effective Hamiltonian is,

$$
\left(h_{\mathrm{eff}}\right)_{a b}=|\vec{p}| \delta_{a b}+\frac{\left(\tilde{m}^{2}\right)_{a b}^{*}}{2|\vec{p}|}+\frac{1}{|\vec{p}|}\left[-\left(a_{L}\right)^{\mu} p_{\mu}-\left(c_{L}\right)^{\mu \nu} p_{\mu} p_{\nu}\right]_{a b}^{*}
$$

The original and complete effective Hamiltonian can describe $\nu-\nu, \bar{\nu}-\bar{\nu}$, and $\nu-\bar{\nu}$ oscillations, but, in this work, lepton-number violating $\nu-\bar{\nu}$ oscillations are not considered. Therefore, the neutrino and antineutrino sectors can be diagonalized separately. For the usual conventional neutrino oscillation case, the effective Hamiltonian (Eq. 4.8) contains only the first two terms. Then, the neutrino oscillation probability depends on $\Delta m^{2}$ and the mixing matrix. But, in this general form, including possible Lorentz and CPT violation, the diagonalization of the effective Hamiltonian is more complicated and, in general, it can not be represented by $\Delta m^{2}$ and the mixing matrix alone.

The effective Hamiltonian of Eq. 4.8 may be used to analyze the LSND $\overline{\nu_{e}}$ appearance data. The problem here is that there are too many free parameters to test. However, under 
the assumption of a short baseline neutrino oscillation experiment, $L$ is small compared with the neutrino oscillation length (Sec. 2.1), and the oscillation probability can be expanded using this effective Hamiltonian [66]. This approximation is especially suitable for the LSND result, because of the small indicated oscillation probability $(\sim 0.26 \%)$. Then, to leading order in $h_{\text {eff }}$, the oscillation probability is

$$
P_{\bar{\nu}_{\mu} \rightarrow \bar{\nu}_{e}} \simeq \frac{\left|\left(h_{\mathrm{eff}}\right) \bar{e} \bar{\mu}\right|^{2} L^{2}}{(\hbar c)^{2}}
$$

Since, in the effective Hamiltonian, $p^{\mu}$ depends on the neutrino propagation direction, this oscillation probability depends on this propagation direction. In order to form a phenomenological expression for the neutrino oscillation probability, it is most convenient to use a coordinate system fixed to the experiment [86, 87]. The standard choice is a Sun-centered system (Fig 4.4 ) that is, to a good approximation, an inertial frame for the experiment.

Recall that the nature of observer Lorentz covariance can guarantee the existence of inertial frame even under particle Lorentz and CPT violation. And, in this coordinate system, the neutrino oscillation transition probability depends on the rotation of the earth with respect to distant star (sidereal time dependence) .

With this choice of coordinates, the neutrino oscillation probability becomes,

$$
\begin{aligned}
P_{\bar{\nu}_{\mu} \rightarrow \bar{\nu}_{e}} \simeq & \frac{L^{2}}{(\hbar c)^{2}} \mid(\mathcal{C})_{\bar{e} \bar{\mu}}+\left(\mathcal{A}_{s}\right)_{\bar{e} \bar{\mu}} \sin \omega_{\oplus} T_{\oplus}+\left(\mathcal{A}_{c}\right)_{\bar{e} \bar{\mu}} \cos \omega_{\oplus} T_{\oplus} \\
& +\left(\mathcal{B}_{s}\right)_{\bar{e} \bar{\mu}} \sin 2 \omega_{\oplus} T_{\oplus}+\left.\left(\mathcal{B}_{c}\right)_{\bar{e} \bar{\mu}} \cos 2 \omega_{\oplus} T_{\oplus}\right|^{2}
\end{aligned}
$$

The parameters, $(\mathcal{C})_{\bar{e} \bar{\mu}},\left(\mathcal{A}_{s}\right)_{\bar{e} \bar{\mu}},\left(\mathcal{A}_{c}\right)_{\bar{e} \bar{\mu}},\left(\mathcal{B}_{s}\right)_{\bar{e} \bar{\mu}}$, and $\left(\mathcal{B}_{c}\right)_{\bar{e} \bar{\mu}}$, depend on the SME coefficients $\left(a_{L}\right)^{\mu}$ and $\left(c_{L}\right)^{\mu \nu}$ and the neutrino propagation direction unit vectors $\hat{N}^{X}, \hat{N}^{Y}$, and $\hat{N}^{Z}$ in the Sun-centered system.

Here $\omega_{\oplus}$ is the sidereal frequency $(=2 \pi / 23 \mathrm{~h} 56 \mathrm{~min} 4.1 \mathrm{sec})$, and $T_{\oplus}$ is the sidereal time which is the time measured from standard origin. The full expressions for each coefficient 

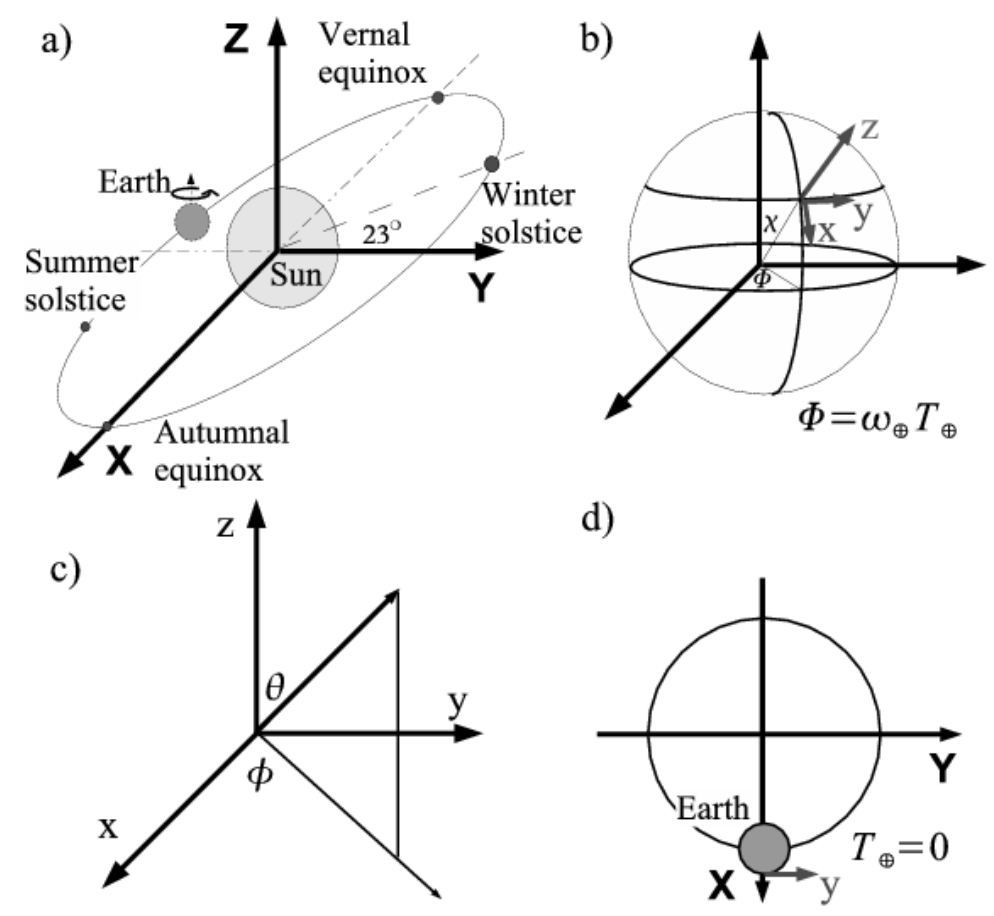

d)

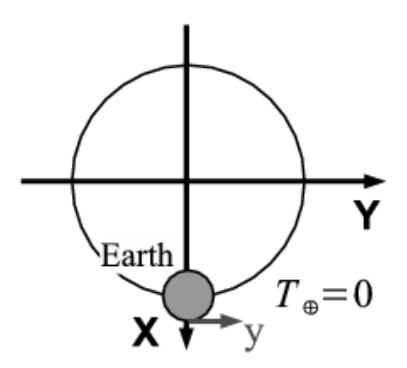

Figure 4.4: The coordinate system for the sidereal time variation analysis of LSND: a) the Sun-centered coordinates, b) the Earth-center coordinates, c) LSND local beam direction coordinates, and d) our definition of $T_{\oplus}=0$. 
are 66

$$
\begin{aligned}
& (\mathcal{C})_{\bar{e} \bar{\mu}}={ }^{\left(\mathcal{C}^{(0)}\right)} \bar{e} \bar{\mu}+E\left(\mathcal{C}^{(1)}\right) \bar{e} \bar{\mu} \\
& \left(\mathcal{A}_{s}\right)_{\bar{e} \bar{\mu}}=\left(\mathcal{A}_{s}^{(0)}\right) \bar{e} \bar{\mu}+E\left(\mathcal{A}_{s}^{(1)}\right) \bar{e} \bar{\mu} \\
& \left(\mathcal{A}_{c}\right)_{\bar{e} \bar{\mu}}=\left(\mathcal{A}_{c}^{(0)}\right) \bar{e} \bar{\mu}+E\left(\mathcal{A}_{c}^{(1)}\right) \bar{e} \bar{\mu} \\
& \left(\mathcal{B}_{s}\right)_{\bar{e} \bar{\mu}}=E\left(\mathcal{B}_{s}^{(1)}\right) \bar{e} \bar{\mu} \\
& \left(\mathcal{B}_{c}\right)_{\bar{e} \bar{\mu}}=E\left(\mathcal{B}_{c}^{(1)}\right) \bar{e} \bar{\mu} \\
& \left(\mathcal{C}^{(0)}\right) \bar{e} \bar{\mu}=\frac{\left(\tilde{m}^{2}\right)_{\bar{e} \bar{\mu}}^{*}}{2 E}-\left(a_{L}\right)_{\bar{e} \bar{\mu}}^{T}+\hat{N}^{Z}\left(a_{L}\right)_{\bar{e} \bar{\mu}}^{Z} \\
& \left(\mathcal{C}^{(1)}\right) \bar{e} \bar{\mu}=-\frac{1}{2}\left(3-\hat{N}^{Z} \hat{N}^{Z}\right)\left(c_{L}\right)_{\bar{e} \bar{\mu}}^{T T}+2 \hat{N}^{Z}\left(c_{L}\right)_{\bar{e}}^{T} \bar{\mu}+\frac{1}{2}\left(1-3 \hat{N}^{Z} \hat{N}^{Z}\right)\left(c_{L}\right) \frac{Z Z}{\bar{e}} \bar{\mu} \\
& \left(\mathcal{A}_{s}^{(0)}\right) \bar{e} \bar{\mu}=-\hat{N}^{Y}\left(a_{L}\right)_{\bar{e} \bar{\mu}}^{X}+\hat{N}^{X}\left(a_{L}\right)_{\bar{e} \bar{\mu}}^{Y} \\
& \left(\mathcal{A}_{s}^{(1)}\right) \bar{e} \bar{\mu}=-2 \hat{N}^{Y}\left(c_{L}\right)_{\bar{e} \bar{\mu}}^{T X}+2 \hat{N}^{X}\left(c_{L}\right)_{\bar{e} \bar{\mu}}^{T Y}+2 \hat{N}^{Y} \hat{N}^{Z}\left(c_{L}\right)_{\bar{e} \bar{\mu}}^{X Z}-2 \hat{N}^{X} \hat{N}^{Z}\left(c_{L}\right)_{\bar{e} \bar{\mu}}^{Y Z} \\
& \left(\mathcal{A}_{c}^{(0)}\right) \bar{e} \bar{\mu}=+\hat{N}^{X}\left(a_{L}\right)_{\bar{e} \bar{\mu}}^{X}+\hat{N}^{Y}\left(a_{L}\right)_{\bar{e} \bar{\mu}}^{Y} \\
& \left(\mathcal{A}_{c}^{(1)}\right) \bar{e} \bar{\mu}=2 \hat{N}^{X}\left(c_{L}\right)_{\bar{e} \bar{\mu}}^{T X}+2 \hat{N}^{Y}\left(c_{L}\right)_{\bar{e} \bar{\mu}}^{T Y}-2 \hat{N}^{X} \hat{N}^{Z}\left(c_{L}\right)_{\bar{e} \bar{\mu}}^{X}-2 \hat{N}^{Y} \hat{N}^{Z}\left(c_{L}\right)_{\bar{e} \bar{\mu}} Z \\
& \left(\mathcal{B}_{s}^{(1)}\right) \bar{e} \bar{\mu}=\hat{N}^{X} \hat{N}^{Y}\left(\left(c_{L}\right)_{\bar{e} \bar{\mu}}^{X X}-\left(c_{L}\right)_{\bar{e} \bar{\mu}}^{Y Y}\right)-\left(\hat{N}^{X} \hat{N}^{X}-\hat{N}^{Y} \hat{N}^{Y}\right)\left(c_{L}\right)_{\bar{e} \bar{\mu}}^{X Y} \\
& \left(\mathcal{B}_{c}^{(1)}\right) \bar{e} \bar{\mu}=-\frac{1}{2}\left(\hat{N}^{X} \hat{N}^{X}-\hat{N}^{Y} \hat{N}^{Y}\right)\left(\left(c_{L}\right)_{\bar{e} \bar{\mu}}^{X X}-\left(c_{L}\right)_{\bar{e} \bar{\mu}}^{Y Y}\right)-2 \hat{N}^{X} \hat{N}^{Y}\left(c_{L}\right)_{\bar{e} \bar{\mu}}^{X Y}
\end{aligned}
$$

The $\hat{N}^{X}, \hat{N}^{Y}$ and $\hat{N}^{Z}$ are the direction vectors of the neutrino beam in the Sun-centered coordinates. The components are further described with a co-latitude $\chi$ of detector location in the Earth-centered system (Fig $4.4 \mathrm{~b}$ ) and the zenith and azimuthal angles $\theta$ and $\phi$ of the local beam system. (Fig 4.4 $)$ :

$$
\left(\begin{array}{c}
\hat{N}^{X} \\
\hat{N}^{Y} \\
\hat{N}^{Z}
\end{array}\right)=\left(\begin{array}{c}
\cos \chi \sin \theta \cos \phi+\sin \chi \cos \theta \\
\sin \theta \sin \phi \\
-\sin \chi \sin \theta \cos \phi+\cos \chi \cos \theta
\end{array}\right)=\left(\begin{array}{c}
-0.053 \\
0.980 \\
-0.194
\end{array}\right) .
$$

Here, we found the LSND beam, that of the Los Alamos Neutron Science Center (LANSCE), direction to be $\chi=54.1^{\circ}, \theta=99.0^{\circ}$, and $\phi=82.6^{\circ}$ [88]. The sidereal time has a specific 
origin $\left(T_{\oplus}=0\right)$ in astronomy, but for simplicity, we chose a relative origin. The sidereal time is defined to start when the local coordinates correspond to the midnight point near the autumnal equinox (Fig 4.4 d). At that time, the $y$-axis of the Earth-centered coordinates coincides with the $Y$ axis of the Sun-centered coordinates. The earth was at autumnal equinox near the start of the LSND experiment on 1993, Sept. 23rd, 00:32 (GMT). The location of LANL is $105^{\circ}$ west of Greenwich, UK, so LANL was at autumnal equinox at 7 hours later on that day. Therefore, the nearest midnight point for LANL near autumnal equinox occurred at 6:28 from the precise autumnal equinox. The revolution angle for the earth (in orbit around the sun) during 6:28 is,

$$
\theta=\frac{\frac{6 \times 60+28}{24 \times 60}}{365.25}=0.27^{\circ} .
$$

This corresponds to approximately 1 minute of earth rotation (about its axis of rotation). Therefore, the estimated difference between the true sun-centered coordinate system and this definition of $T_{\oplus}$ is $\sim 1$ minute, which is sufficiently small compared with the size of time bins used in this analysis.

\subsubsection{Analysis and results}

Since the LSND oscillation candidate sample size is only 186, an unbinned likelihood method was used to maximize statistical power [89, 90] of the data set. The sidereal time distribution of the LSND oscillation sample was found to be consistent with flat (no sidereal time dependence) using Kolmogorov-Smirnov and Pearson's $\chi^{2}$ tests [1]. However, the data did allow (within errors) non-zero Lorentz violation. Figure. 4.5 shows the data compared to 1-parameter $\left((\mathcal{C})_{\bar{e} \bar{\mu}}\right.$ only, no sidereal dependence), 3-parameter $\left((\mathcal{C})_{\bar{e} \bar{\mu}},\left(\mathcal{A}_{s}\right)_{\bar{e} \bar{\mu}}\right.$, and $\left.\left(\mathcal{A}_{c}\right)_{\bar{e} \bar{\mu}}\right)$, and 5-parameter $\left((\mathcal{C})_{\bar{e} \bar{\mu}},\left(\mathcal{A}_{s}\right)_{\bar{e} \bar{\mu}},\left(\mathcal{A}_{c}\right)_{\bar{e} \bar{\mu}},\left(\mathcal{B}_{s}\right)_{\bar{e} \bar{\mu}}\right.$, and $\left.\left(\mathcal{B}_{c}\right)_{\bar{e} \bar{\mu}}\right)$ fits.

Figure 4.6 shows the allowed regions of parameter space resulting from the 3-parameter fit. Since the fitting function is dependent on the square of the parameters, there are always 


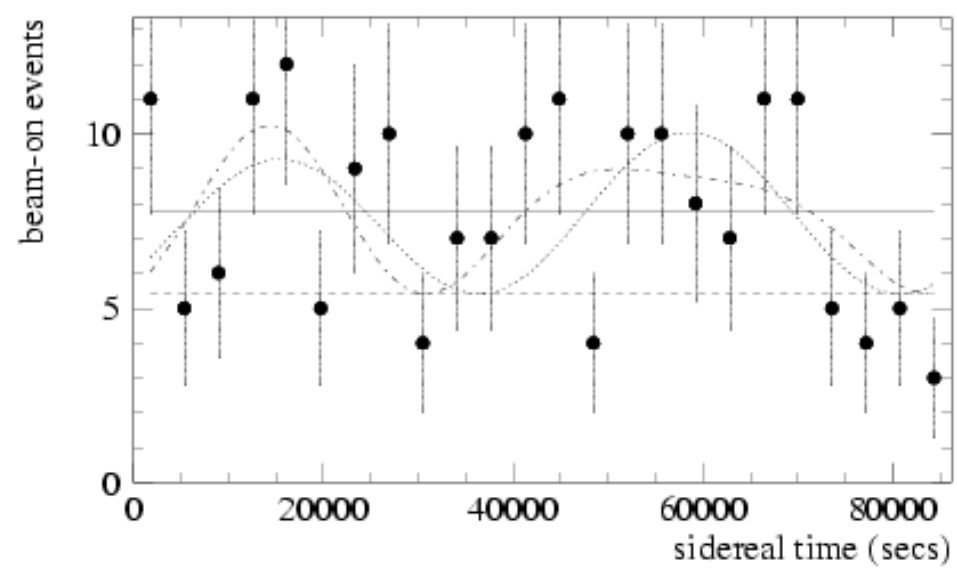

Figure 4.5: Sidereal time distribution of the LSND oscillation data (dots) in 24 time bins together with the maximum- $\ell$ solutions for the 1-parameter (solid line), 3-parameter (dotted), and 5-parameter (dot-dashed) fits. The dashed line indicates the estimated background contribution.

duplicate solutions and positive and negative parameter values. Note that there are two distinct solutions within $1 \sigma$ region.

In summary, we found that the LSND data is consistent with no Lorentz violation, but it can not rule out Lorentz violation as an explanation of neutrino oscillations. This motivates further study.

From here, we can set the scale of possible Lorentz violation as an explanation of LSND. Since the neutrino beam energy is $\sim 40 \mathrm{MeV}$, if the LSND signal is due to Lorentz violation, then the suppression factor of Lorentz violation is $\sim \frac{10^{-19} \mathrm{GeV}}{40 \mathrm{MeV}} \sim 10^{-17}$. And this is the right order of naive expectation for the Standard Model, $\frac{E_{W}}{M_{P}} \sim \frac{100 \mathrm{GeV}}{10^{19} \mathrm{GeV}} \sim 10^{-17}$.

Although this is a small value, $10^{-17}$ is quite large for neutrino oscillations. For example, if atmospheric neutrino oscillations are due to Lorentz violation, that suppression factor for atmospheric neutrinos is $\sim 10^{-21}$ (Sec. 2.3 . This would imply that all Lorentz violation of 


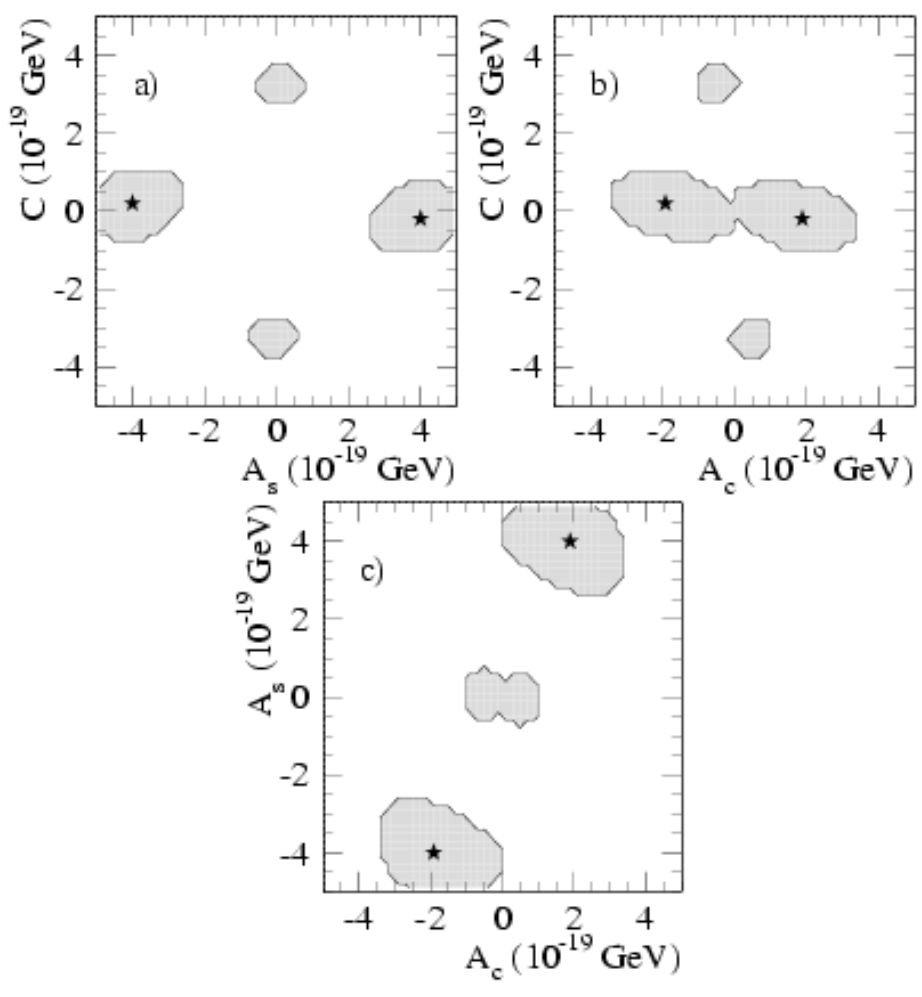

Figure 4.6: Log likelihood value for the 3-parameter fit of the LSND sidereal time distribution: a) $\left(\mathcal{A}_{s}\right)_{\bar{e} \bar{\mu}}$ vs $(\mathcal{C})_{\bar{e} \bar{\mu}}$, b) $\left(\mathcal{A}_{c}\right)_{\bar{e} \bar{\mu}}$ vs $(\mathcal{C})_{\bar{e} \bar{\mu}}$, and c) $\left(\mathcal{A}_{c}\right)_{\bar{e} \bar{\mu}}$ vs $\left(\mathcal{A}_{s}\right)_{\bar{e} \bar{\mu}}$. The contours in a)-c) indicate the 1- $\sigma$ (total error) allowed regions and the stars indicate parameter values for the maximum-log-likelihood parameter values. 
order $10^{-17}$ would wash out all coherent behavior (=L/E dependent oscillatory behavior) for atmospheric neutrino oscillations and the description would become that of time-averaged oscillations, like Eq. 2.22 .

In the next chapter, we consider a possible global model using Lorentz violation to explain the world's data on neutrino oscillations, including the LSND signal. In such a model, LSND can see the Lorentz violation with a scale of $10^{-17}$, and it does not wash out the oscillatory behavior for atmospheric and reactor neutrinos. 


\section{Chapter 5}

\section{Global three parameter model for neutrino oscillation with Lorentz violation}

Neutrino oscillations are described in the solution of the Hamiltonian for neutrinos. Since we are concerned with a $3 \times 3$ Hamiltonian corresponding to the three families of neutrinos, we can find both analytic and numerical solutions from any Hamiltonian unless the solutions do not exist.

Although the energy dependence of Lorentz violation Lagrangian is, in the minimal SME case, $\propto E^{0}$ for the CPT-odd term, and $\propto E^{+1}$ for the CPT-even, some combination of these terms can result in a mass-term-like energy dependence $\propto E^{-1}$ for the solution. The "bicycle model" 65] is the simplest case for this kind of model. This model is very interesting, because it has potential to explain all neutrino oscillation signals, including the LSND signal. Furthermore, this type of model may have a small number of degrees of freedom, which means it is superior as a phenomenological model than the standard 
three massive neutrino model. We have constructed such a model, called the "tandem model" [2]. One of predictions of tandem model was a low energy signal for MiniBooNE. Since MiniBooNE did observe a low-energy excess of $\nu_{e}$ candidate events [67], we compared, in more detail, the tandem model with MiniBooNE data [3]. This tandem model of neutrino oscillations and the comparison with the MiniBooNE data is presented in this chapter.

\subsection{A global model for neutrino oscillations}

We found the equation of neutrino oscillation in Eq. 2.6. We now consider a real $3 \times 3$ Hamiltonian for simplicity. Then, the most general effective Hamiltonian,

$$
h_{e f f}=\left(\begin{array}{ccc}
h_{e e}(E) & h_{e \mu}(E) & h_{e \tau}(E) \\
h_{e \mu}(E) & h_{\mu \mu}(E) & h_{\mu \tau}(E) \\
h_{e \tau}(E) & h_{\mu \tau}(E) & h_{\tau \tau}(E)
\end{array}\right) \text {, }
$$

yields very complicated solutions for neutrino oscillations,

$$
P_{\nu_{\alpha} \rightarrow \nu_{\beta}}(L, E)=\delta_{\alpha \beta}-4 \sum_{i>j} U_{\alpha i}(E) U_{\beta i}(E) U_{\alpha j}(E) U_{\beta j}(E) \sin ^{2}\left(\frac{\Delta_{i j}(E)}{2} L\right) .
$$

Here, both $U(E)$ and $\Delta(E)$ are functions of energy. As far as we are concerned, for a $3 \times 3$ matrix, analytic solutions always exist. But in the more general case, or for dimension greater than four, only numerical solutions exist.

This is the case for Lorentz violating neutrino oscillations. Let us assume that there is no $\nu-\bar{\nu}$ oscillation, then, $\nu-\nu$ oscillation and $\bar{\nu}-\bar{\nu}$ oscillation are described by block diagonal Hamiltonians. For $\nu-\nu$ oscillations, the minimal SME Hamiltonian contains three types of energy dependence, $\propto E^{-1}$ for mass term, $\propto E^{0}$ for CPT-odd term, and $\propto E^{+1}$ for CPT-even term,

$$
\left(h_{e f f}^{\nu}\right)_{a b}=E \delta_{a b}+\frac{\left(m^{2}\right)_{a b}}{2 E}+\frac{1}{E}\left[\left(a_{L}\right)^{\mu} p_{\mu}-\left(c_{L}\right)^{\mu \nu} p_{\mu} p_{\nu}\right]_{a b}
$$


The effective Hamiltonian for $\bar{\nu}-\bar{\nu}$ oscillations is obtained simply by replacing $a_{L} \rightarrow-a_{L}$ according to Eq. 4.6.

Since, we want to focus on the energy dependence, in this chapter we ignore the direction dependence. This can be understood to be plausible for either of two reasons. First, theoretically, if we take the CMB frame as an isotropic, rotationally invariant inertial frame, then the Lorentz violating fields in this frame must be isotropic, or, any directional components must be zero [57]. Although the motion of solar system governs the visible effect against the isotropic Lorentz-violating field, the motion is non-relativistic and suppressed to be order $\sim 10^{-3}$. Second, experimentally, most of the neutrino oscillation data is integrated over long times, and any directional information is simply averaged out.

In either interpretation, it is not unreasonable to start to construct simple model without any directional components in order to reduce the degrees of freedom of the theory. However, it does not preclude the possibility of direction dependence.

Then, Eq. 5.3 becomes,

$$
\begin{aligned}
\left(h_{\mathrm{eff}}^{\nu}\right)_{a b} \approx E \delta_{a b}+\frac{\left(m^{2}\right)_{a b}}{2 E}+\left(a_{L}\right)_{a b}-\frac{4}{3}\left(c_{L}\right)_{a b} E \\
=\left(\begin{array}{ccc}
\frac{\left(m^{2}\right)_{e e}}{2 E}+\left(a_{L}\right)_{e e}-\frac{4}{3}\left(c_{L}\right)_{e e} E & \frac{\left(m^{2}\right)_{e \mu}}{2 E}+\left(a_{L}\right)_{e \mu}-\frac{4}{3}\left(c_{L}\right)_{e \mu} E & \frac{\left(m^{2}\right)_{\tau e}}{2 E}+\left(a_{L}\right)_{e \tau e}-\frac{4}{3}\left(c_{L}\right)_{\tau e} E \\
\frac{\left(m^{2}\right)_{e \mu}}{2 E}+\left(a_{L}\right)_{e \mu}-\frac{4}{3}\left(c_{L}\right)_{e \mu} E & \frac{\left(m^{2}\right)_{\mu \mu}}{2 E}+\left(a_{L}\right)_{\mu \mu}-\frac{4}{3}\left(c_{L}\right)_{\mu \mu} E & \frac{\left(m^{2}\right)_{\mu \tau}}{2 E}+\left(a_{L}\right)_{\mu \tau}-\frac{4}{3}\left(c_{L}\right)_{\mu \tau} E \\
\frac{\left(m^{2}\right)_{\tau e}}{2 E}+\left(a_{L}\right)_{\tau e}-\frac{4}{3}\left(c_{L}\right)_{\tau e} E & \frac{\left(m^{2}\right)_{\mu \tau}}{2 E}+\left(a_{L}\right)_{\mu \tau}-\frac{4}{3}\left(c_{L}\right)_{\mu \tau} E & \frac{\left(m^{2}\right)_{\tau \tau}}{2 E}+\left(a_{L}\right)_{\tau \tau}-\frac{4}{3}\left(c_{L}\right)_{\tau \tau} E
\end{array}\right) .
\end{aligned}
$$

This model has 18 free parameters.

Any alternative models to the standard three massive neutrino model must be more interesting, otherwise there is no motivation to pursue. We require the following seven conditions for alternative models to be more interesting than the standard neutrino oscillation model. Such a model:

1. is based on the quantum field theory; 
2. involves only renormalizable terms;

3. offers acceptable descriptions for solar, atmospheric, and reactor oscillation signals;

4. has a mass term $\lesssim 0.1 \mathrm{eV}$ in order to interpret its origin by the Seesaw mechanism;

5. has Lorentz violating terms $\lesssim 10^{-17}$ to interpret their origin from Plank scale physics;

6. does not have a number of free parameters significantly larger than four;

7. describes the LSND signal.

The condition (1) is met as long as we work under the SME formalism. (2) is satisfied if we use the effective Hamiltonian derived minimal SME (Eq. 5.3. (3) is very challenging because we need $\sim \mathrm{L} / \mathrm{E}$ dependence for the phase of oscillation for high energy (atmospheric neutrinos), even though the CPT-even term dominates at high energy, which has energy dependence $\sim \mathrm{E}$ in the Lagrangian. (4) and (5) add constraints to the model building process. Condition (6), since the standard three massive neutrino model has four free parameters (Sec. 5.3.1), is required for the model to be phenomenologically interesting. Finally, if such a model describes the LSND signal, condition (7), it is very interesting.

\subsection{Analytic and numerical solution of neutrino oscillations}

Since we are working with the $3 \times 3$ effective Hamiltonian matrix, we can find the solution of the cubic equations from the Ferro-Cardano method, or numerical diagonalization by Jacobi rotations 91 . 


\subsubsection{Ferro-Cardano equation}

The eigenvalue solution of an arbitrary $3 \times 3$ matrix (Eq. 5.1) can be found by solving the cubic equation [91],

$$
\lambda^{3}+a \lambda^{2}+b \lambda+c=0
$$

where the $\lambda$ are the eigenvalues of,

$$
h_{\mathrm{eff}}=\left(\begin{array}{ccc}
h_{e e} & h_{e \mu} & h_{\tau e} \\
h_{e \mu} & h_{\mu \mu} & h_{\mu \tau} \\
h_{\tau e} & h_{\mu \tau} & h_{\tau \tau}
\end{array}\right)
$$

The roots and mixing matrix are found to be,

$$
\begin{aligned}
\lambda_{1}= & -2 \sqrt{Q} \cos \left(\frac{\theta}{3}\right)-\frac{a}{3}, \\
\lambda_{2}= & -2 \sqrt{Q} \cos \left(\frac{\theta+2 \pi}{3}\right)-\frac{a}{3}, \\
\lambda_{3}= & -2 \sqrt{Q} \cos \left(\frac{\theta-2 \pi}{3}\right)-\frac{a}{3}, \\
U= & \left(\begin{array}{ccc}
\frac{B_{1} C_{1}}{N_{1}} & \frac{B_{2} C_{2}}{N_{2}} & \frac{B_{3} C_{3}}{N_{3}} \\
\frac{C_{1} A_{1}}{N_{1}} & \frac{C_{2} A_{2}}{N_{2}} & \frac{C_{3} A_{3}}{N_{3}} \\
\frac{A_{1} B_{1}}{N_{1}} & \frac{A_{2} B_{2}}{N_{2}} & \frac{A_{3} B_{3}}{N_{3}}
\end{array}\right),
\end{aligned}
$$


with,

$$
\begin{aligned}
a & =-\left(h_{e e}+h_{\mu \mu}+h_{\tau \tau}\right) \\
b & =\left[\left(h_{e e} h_{\mu \mu}+h_{\mu \mu} h_{\tau \tau}+h_{\tau \tau} h_{e e}\right)-\left(h_{e \mu}^{2}+h_{\mu \tau}^{2}+h_{\tau e}^{2}\right)\right] \\
c & =\left[\left(h_{e e} h_{\mu \tau}^{2}+h_{\mu \mu} h_{\tau e}^{2}+h_{\tau \tau} h_{e \mu}^{2}\right)-\left(h_{e e} h_{\mu \mu} h_{\tau \tau}+2 h_{e \mu} h_{\mu \tau} h_{\tau e}\right)\right] \\
Q & =\frac{1}{9}\left(a^{2}-3 b\right) \\
R & =\frac{1}{54}\left(2 a^{3}-9 a b+27 c\right) \\
\theta & =\cos ^{-1}\left(\frac{R}{\sqrt{Q^{3}}}\right) \\
A_{[1-3]} & =\left[h_{\mu \tau}\left(h_{e e}-\lambda_{[1-3]}\right)-h_{\tau e} h_{e \mu}\right] \\
B_{[1-3]} & =\left[h_{\tau e}\left(h_{\mu \mu}-\lambda_{[1-3]}\right)-h_{e \mu} h_{\mu \tau}\right] \\
C_{[1-3]} & =\left[h_{e \mu}\left(h_{\tau \tau}-\lambda_{[1-3]}\right)-h_{\mu \tau} h_{\tau e}\right] \\
N_{[1-3]} & =\sqrt{A_{[1-3]}^{2} B_{[1-3]}^{2}+B_{[1-3]}^{2} C_{[1-3]}^{2}+C_{[1-3]}^{2} A_{[1-3]}^{2}}
\end{aligned}
$$

In order to have three real roots (eigenvalues),

$$
Q, R \in \mathbf{R} \text { and } R^{2}<Q^{3}
$$

This is always true for the cases of our concern in the next section, and we can always find three eigenvalue differences $\Delta_{i j}=\lambda_{i}-\lambda_{j}$ and mixing matrix element $U_{\alpha i}$. Therefore, we can find solution of neutrino oscillations for any arbitrary Hamiltonian.

\subsubsection{Jacobi rotations}

The eigenvalue problem is always solvable by the numerical methods. Although cubic equation has analytic solutions, we also checked numerically.

The "Jacobi rotation" 91] is the similarity transformation to remove off-diagonal elements of the matrix. Since the real Hamiltonian is symmetric, the first Jacobi rotation can eliminate two off-diagonal elements from Hamiltonian. The next step eliminates other 


\subsection{ANALYTIC AND NUMERICAL SOLUTION OF NEUTRINO OSCILLATIONS}

off-diagonal elements, but the off-diagonal elements eliminated by the first step return. However, the returning values are small. And, the third step eliminates the last off-diagonal elements. These three Jacobi rotations make one "sweep". We usually sweep five times and this is sufficient to make off-diagonal elements to be of negligible size. Then, the remaining diagonal elements, can be understood as roots of the equation:

$$
\begin{aligned}
h_{\mathrm{eff}}= & \left(\begin{array}{ccc}
h_{e e} & h_{e \mu} & h_{\tau e} \\
h_{e \mu} & h_{\mu \mu} & h_{\mu \tau} \\
h_{\tau e} & h_{\mu \tau} & h_{\tau \tau}
\end{array}\right)=O_{e \mu}\left(\begin{array}{ccc}
h_{e e}^{\prime} & 0 & h_{\tau e}^{\prime} \\
0 & h_{\mu \mu}^{\prime} & h_{\mu \tau}^{\prime} \\
h_{\tau e}^{\prime} & h_{\mu \tau}^{\prime} & h_{\tau \tau}^{\prime}
\end{array}\right) O_{e \mu}^{T} \\
= & \underbrace{\cdots O O O}_{U}\left(\begin{array}{ccc}
\lambda_{1} & \sim 0 & \sim 0 \\
\sim 0 & \lambda_{2} & \sim 0 \\
\sim 0 & \sim 0 & \lambda_{3}
\end{array}\right) \underbrace{O^{T} O^{T} O^{T} \ldots}_{U^{T}} .
\end{aligned}
$$

The mixing matrix is calculated from the product of the orthogonal matrices created in each step of the Jacobi rotation. In five sweeps, fifteen orthogonal matrices are created and the product is defined as the mixing matrix.

The order of eigenvalues is in general random, so we also apply the column/row exchanging matrix to remedy the order from small $\left(=\lambda_{1}\right)$ to large $\left(=\lambda_{3}\right)$ after taking the modulus, for example,

$$
\begin{aligned}
& \cdots O\left(\begin{array}{ccc}
\lambda_{2} & \sim 0 & \sim 0 \\
\sim 0 & -\lambda_{1} & \sim 0 \\
\sim 0 & \sim 0 & -\lambda_{3}
\end{array}\right) O^{T} \cdots=\cdots O\left(\begin{array}{ccc}
\lambda_{2} & \sim 0 & \sim 0 \\
\sim 0 & \lambda_{1} & \sim 0 \\
\sim 0 & \sim 0 & \lambda_{3}
\end{array}\right) O^{T} \ldots \\
& =\cdots O R_{12} R_{12}^{T}\left(\begin{array}{ccc}
\lambda_{2} & \sim 0 & \sim 0 \\
\sim 0 & \lambda_{1} & \sim 0 \\
\sim 0 & \sim 0 & \lambda_{3}
\end{array}\right) R_{12} R_{12}^{T} O^{T} \cdots=\underbrace{\cdots O R_{12}}_{U}\left(\begin{array}{ccc}
\lambda_{1} & \sim 0 & \sim 0 \\
\sim 0 & \lambda_{2} & \sim 0 \\
\sim 0 & \sim 0 & \lambda_{3}
\end{array}\right) \underbrace{R_{12}^{T} O^{T} \cdots}_{U^{T}},
\end{aligned}
$$


5 orthogonal matrices are used to change the order of eigenvalues,

$$
\begin{aligned}
& R_{12}=\left(\begin{array}{lll}
0 & 1 & 0 \\
1 & 0 & 0 \\
0 & 0 & 1
\end{array}\right), R_{23}=\left(\begin{array}{lll}
1 & 0 & 0 \\
0 & 0 & 1 \\
0 & 1 & 0
\end{array}\right), R_{31}=\left(\begin{array}{lll}
0 & 0 & 1 \\
0 & 1 & 0 \\
1 & 0 & 0
\end{array}\right) \\
& R_{L H}=\left(\begin{array}{lll}
0 & 0 & 1 \\
1 & 0 & 0 \\
0 & 1 & 0
\end{array}\right), R_{R H}=\left(\begin{array}{lll}
0 & 1 & 0 \\
0 & 0 & 1 \\
1 & 0 & 0
\end{array}\right) .
\end{aligned}
$$

The positivity of eigenvalues is not guaranteed. Since we took the modulus for the eigenvalues, the solution no longer reproduces the original Hamiltonian [92]. Nevertheless, this "new" Hamiltonian satisfies most of the conditions in Sec. 5.1, especially, the model that we discuss in Sec. 5.3 .3 reproduces all experimental results with only three free parameters.

\subsection{Model construction}

\subsubsection{Bimaximal model}

The bimaximal model [93] is the most simple and naive model based on the three neutrino massive model. Since we have two distinct signals, atmospheric neutrino oscillations [7882, 94] and solar neutrino oscillations [70-77, 83, 95, 96], we require four parameters. They are two mass square differences, $\Delta m_{\odot}^{2}$ and $\Delta m_{\text {atm }}^{2}$, and two mixing angles, $\theta_{12}$ and $\theta_{23}$. Then, the effective Hamiltonian is,

$$
h_{\mathrm{eff}}^{\text {bimax }}=\frac{m_{a b}^{2}}{2 E}=\frac{1}{2 E} U_{S M}^{T}\left(\begin{array}{ccc}
0 & 0 & 0 \\
0 & \Delta m_{\odot}^{2} & 0 \\
0 & 0 & \Delta m_{a t m}^{2}
\end{array}\right) U_{S M}
$$


with,

$$
U_{S M}=\left(\begin{array}{ccc}
1 & 0 & 0 \\
0 & \cos \theta_{23} & \sin \theta_{23} \\
0 & -\sin \theta_{23} & \cos \theta_{23}
\end{array}\right) \times\left(\begin{array}{ccc}
\cos \theta_{12} & \sin \theta_{12} & 0 \\
-\sin \theta_{12} & \cos \theta_{12} & 0 \\
0 & 0 & 1
\end{array}\right)
$$

and,

$$
\begin{aligned}
\Delta m_{\odot}^{2} & \simeq 8.0 \times 10^{-5} \mathrm{eV}^{2} ; \\
\Delta m_{a t m}^{2} & \simeq 2.5 \times 10^{-3} \mathrm{eV}^{2} ; \\
\theta_{12} & \simeq 34^{\circ} ; \\
\theta_{23} & \simeq 45^{\circ} .
\end{aligned}
$$

The solution of the bimaximal model is shown in Fig. 5.1. From Eq. 5.2, we see the model is characterized by 3 amplitude terms, $(-4 U U U U)$, and 3 eigenvalue differences $(\Delta)$.

The following are the captions of Fig. 5.1, 5.3, 5.4, and 5.5. From left to right, top to bottom:

1. evolution of $-4 U U U U$ term for $e \leftrightarrow \mu$. Black line is $-4 U_{e 1} U_{e 2} U_{\mu 1} U_{\mu 2}$, which is the coefficient of $\sin ^{2}\left(\Delta_{12} L / 2\right)$. Red is $-4 U_{e 2} U_{e 3} U_{\mu 2} U_{\mu 3}$, the coefficient of $\sin ^{2}\left(\Delta_{23} L / 2\right)$, and blue is $-4 U_{e 3} U_{e 1} U_{\mu 3} U_{\mu 1}$, the coefficient of $\sin ^{2}\left(\Delta_{31} L / 2\right)$;

2. $-4 U U U U$ term for $\mu \leftrightarrow \tau$;

3. $-4 U U U U$ term for $\tau \leftrightarrow e$;

4. evolution of the first row of mixing matrix elements, Black line is $U_{e 1}$, red is $U_{e 2}$ and blue is $U_{e 3}$;

5. the second row mixing matrix elements; Black line is $U_{\mu 1}$, red is $U_{\mu 2}$ and blue is $U_{\mu 3}$;

6. the third row mixing matrix elements. Black line is $U_{\tau 1}$, red is $U_{\tau 2}$ and blue is $U_{\tau 3}$; 
7. evolution of the eigenvalue (positive defined). Black line is $\lambda_{1}$, the smallest eigenvalue. Red is the second smallest eigenvalue $\lambda_{2}$, and blue is the largest eigenvalue $\lambda_{3}$;

8. inverse of $\Delta$ (eigenvalue difference) as a function of energy. Black line is for $\Delta_{12}$, red is $\Delta_{23}$, and blue is $\Delta_{31}$. Note, the $\mathrm{L} / \mathrm{E}$ oscillation dependence shows up as $\log \mathrm{L} \propto \log \mathrm{E}$;

9. Unitarity of mixing matrix. The black lines (3 lines) are elements of diagonal terms of $U^{T} U$ and the red lines (6 lines) are elements of off-diagonal terms of $U^{T} U$. So, black lines should be 1 and red lines should be 0 through all energy regions if the numerical diagonalization works.

Fig. 5.2 shows the neutrino oscillation solutions. Not surprisingly, the model predicts the atmospheric and reactor neutrino oscillations, as well as the solar neutrino oscillation. Here, we use an adiabatic MSW effect to reproduce solar neutrino signals, with the choice of $N_{e}=100\left(/ N_{A} / \mathrm{cm}^{3}\right)$ as electron density in the central region of the Sun [97]. Of course, this model does not predict a signal for LSND and MiniBooNE.

We know this bimaximal model is based on the standard three neutrino massive model and reproduces all known neutrino oscillation data (excepting LSND). So instead of comparing the result of our model with experimental data (and trying to take account of experimental smearing), we compare the result of our model with the bimaximal model as a proxy for data and the three neutrino massive model.

\subsubsection{Bicycle model}

The bicycle model [65] can create an $L / E$ dependence for neutrino oscillations by using only one CPT-odd term $\left(\propto E^{0}\right)$ and one CPT-even term $\left(\propto E^{+1}\right)$. The trick is that, the eigenvalue difference cancels at the leading order contribution, and the second-order term becomes the leading term, like the seesaw mechanism that generates neutrino mass. 
$2006 / 0+/ 17 \quad 19.11$
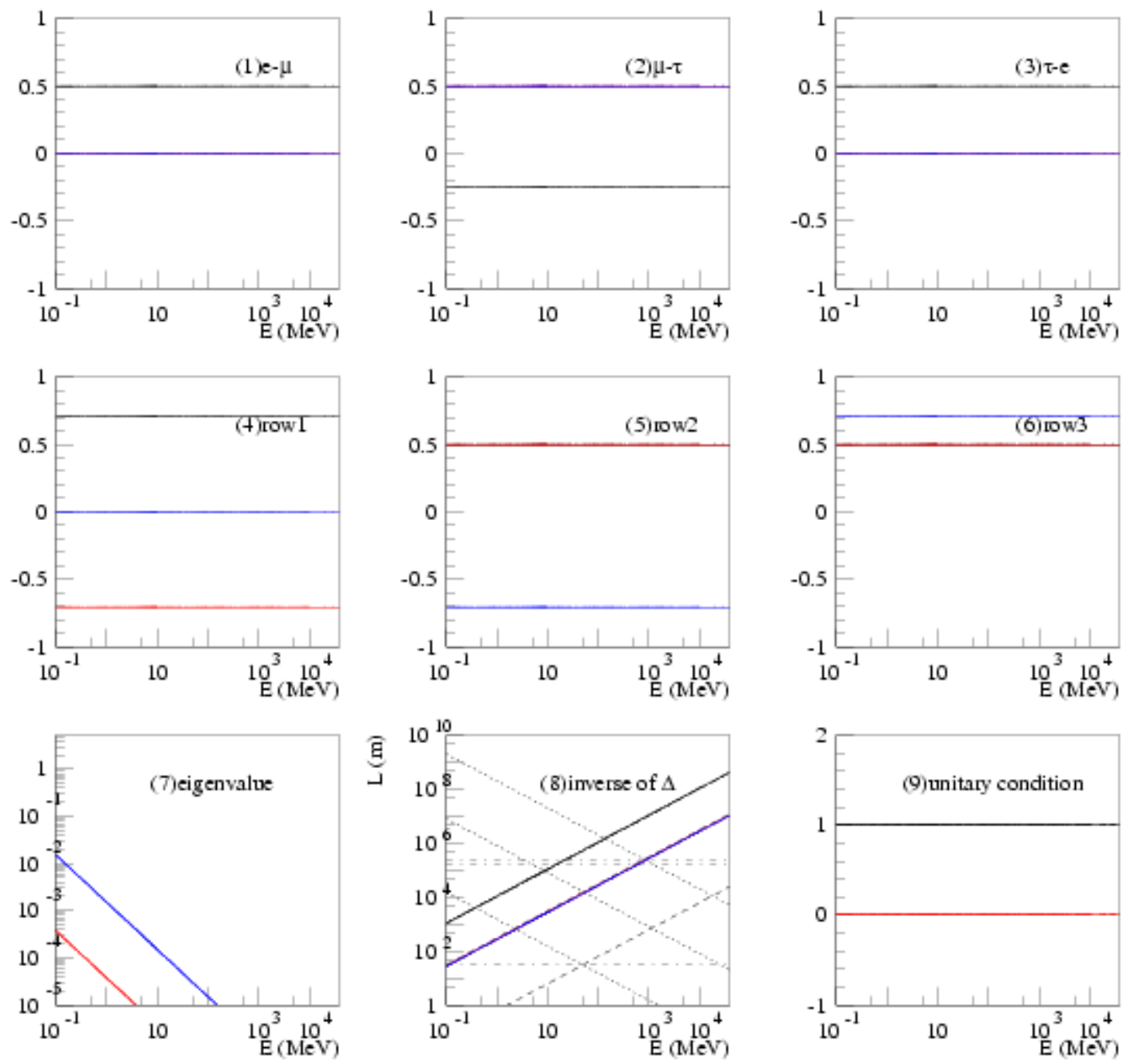

Figure 5.1: The solution for the bimaximal model. The solution is same for $\nu-\nu$ and $\bar{\nu}-\bar{\nu}$ oscillations. The notation is given in the text, Sec. 5.3.1. 

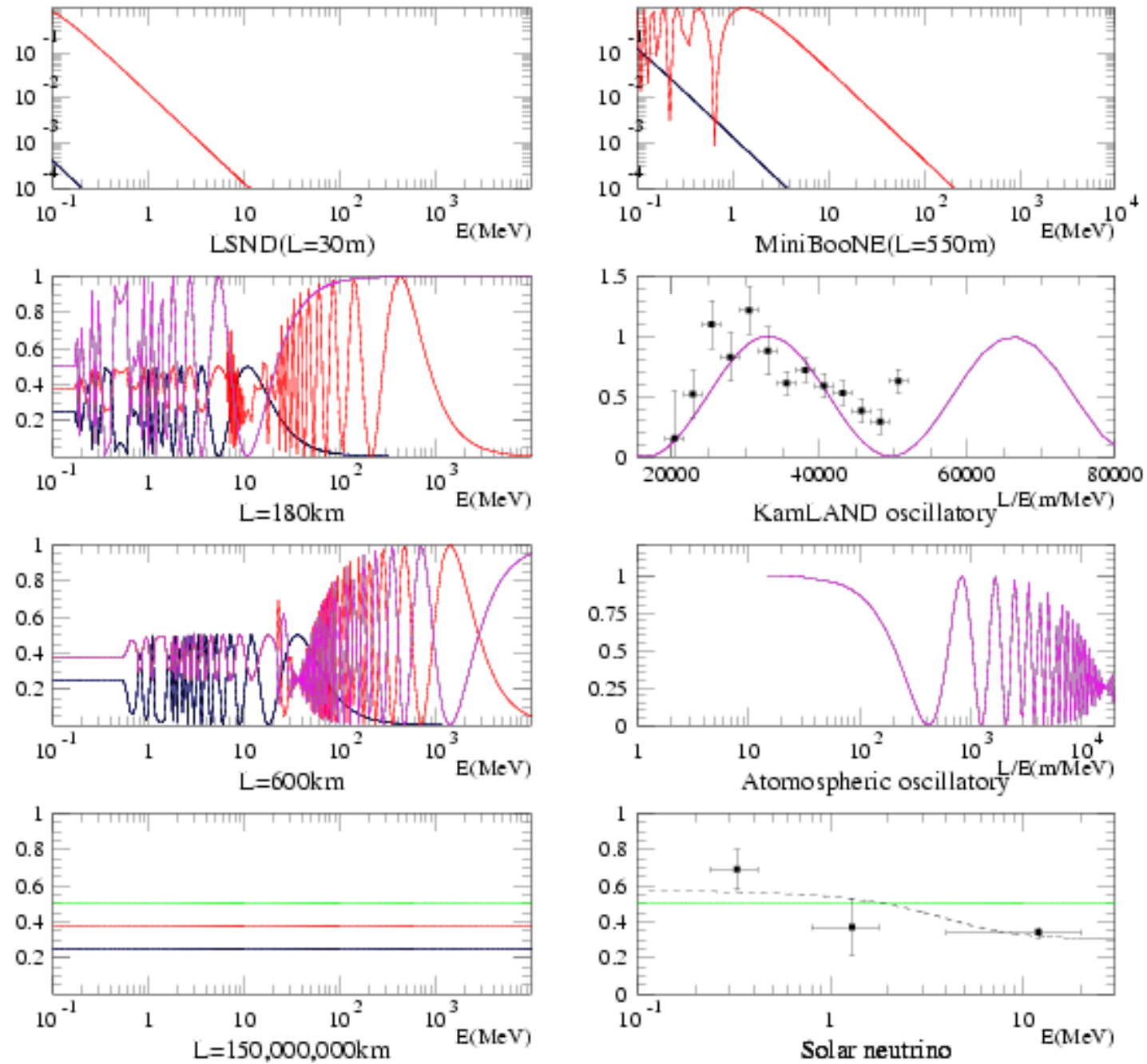

Figure 5.2: The oscillation probability from the bimaximal model. From top to bottom, short baseline experiments (left is LSND, right is for MiniBooNE), KamLAND (with data points from [83]), atmospheric neutrinos, and solar neutrinos (with data points from [97]). The black line is $\nu_{e} \leftrightarrow \nu_{\mu}$, red line is $\nu_{\mu} \leftrightarrow \nu_{\tau}$, green line is $\nu_{e} \leftrightarrow X$, and pink line is $\nu_{\mu} \leftrightarrow X$. Bottom right plot also shows bimaximal model with MSW effect (dashed). The effects of experimental position and energy resolution are not shown. 
The effective Hamiltonian for the bicycle model has the following form,

$$
h_{\mathrm{eff}}^{\text {bicycle }}=\left(\begin{array}{ccc}
c E & a & a \\
a & 0 & 0 \\
a & 0 & 0
\end{array}\right) .
$$

Then, the analytic solution is,

$$
\begin{aligned}
\lambda= & 0, \frac{1}{2}\left[c E \pm \sqrt{(c E)^{2}+8 a^{2}}\right]=\lambda_{0}, \lambda_{ \pm}, \\
U_{\text {bicycle }}= & \left(\begin{array}{lll}
\frac{\lambda_{0}}{\sqrt{2 a^{2}+\lambda_{0}^{2}}} & \frac{\lambda_{-}}{\sqrt{2 a^{2}+\lambda_{-}^{2}}} & \frac{\lambda_{+}}{\sqrt{2 a^{2}+\lambda_{+}}} \\
\frac{a}{\sqrt{2 a^{2}+\lambda_{0}^{2}}} & \frac{a}{\sqrt{2 a^{2}+\lambda_{-}^{2}}} & \frac{a}{\sqrt{2 a^{2}+\lambda_{+}}} \\
\frac{a}{\sqrt{2 a^{2}+\lambda_{0}^{2}}} & \frac{a}{\sqrt{2 a^{2}+\lambda_{-}^{2}}} & \frac{a}{\sqrt{2 a^{2}+\lambda_{+}}}
\end{array}\right)
\end{aligned}
$$

and in the high energy limit,

$$
\begin{aligned}
\lambda & =0, \frac{2 a^{2}}{c E}, c E=\lambda_{1}, \lambda_{2}, \lambda_{3}, \\
\Delta & =\frac{2 a^{2}}{c E}, c E, c E=\Delta_{12}, \Delta_{23}, \Delta_{31}, \\
U_{\text {bicycle }} & =\left(\begin{array}{ccc}
0 & 0 & 1 \\
\frac{1}{\sqrt{2}} & \frac{1}{\sqrt{2}} & 0 \\
\frac{1}{\sqrt{2}} & \frac{1}{\sqrt{2}} & 0
\end{array}\right) .
\end{aligned}
$$

So only the $\nu_{\mu} \leftrightarrow \nu_{\tau}$ channel is non-zero in the high-energy region and this channel couples with $\Delta_{12}$ (Fig. 5.3 , black line of top middle plot). Furthermore, $\Delta_{12}$ has an $L / E$ energy dependence and can create the desired oscillation maximum observed at Super-K. The simple sinusoidal variation model for the bicycle model has also been considered, but provides a poor fit with global data 98 .

\subsubsection{Tandem model}

The tandem model was inspired from and expands upon the bicycle model. Additional details of the tandem model are available in [2]. In order to create a $L / E$ dependence for 
$2006 / 0+/ 2+1+.25$
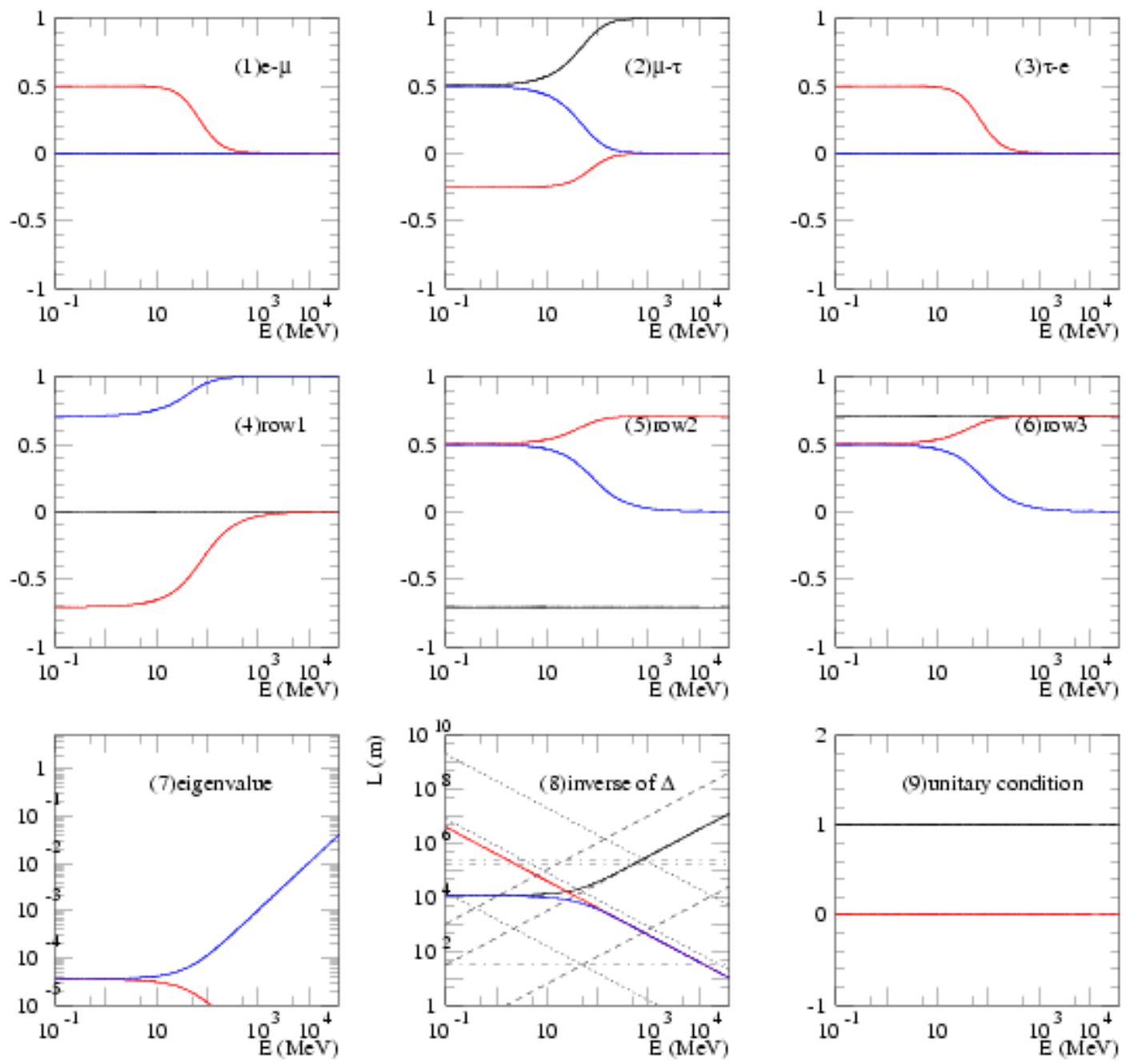

Figure 5.3: The neutrino oscillation solution for the bicycle model. The solution is the same for $\nu-\nu$ and $\bar{\nu}-\bar{\nu}$ oscillations. The plots are described in the text, Sec. 5.3.1. 
KamLAND, we require an additional transition in the oscillation spectrum at low energy. To do this, we introduce a mass term, and the effective Hamiltonian becomes,

$$
h_{\mathrm{eff}}^{\text {tandem }}=\left(\begin{array}{ccc}
\stackrel{\circ}{c} E & \stackrel{\circ}{a} & \stackrel{\circ}{a} \\
\stackrel{\circ}{a} & 0 & \stackrel{\circ}{a} \\
\stackrel{\circ}{a} & \stackrel{\circ}{a} & \frac{\stackrel{\circ}{m}^{2}}{2 E}
\end{array}\right) .
$$

The three parameters here correspond to three terms in Eq. 5.4 .

$$
\begin{aligned}
\stackrel{\circ}{c} & =-\frac{4}{3}\left(c_{L}\right)_{e e} \\
\stackrel{\circ}{a} & =\left(a_{L}\right)_{e \mu}=\left(a_{L}\right)_{\mu \tau}=\left(a_{L}\right)_{\tau e} \\
\frac{\stackrel{m}{m}^{2}}{2 E} & =\frac{\left(m^{2}\right)_{\tau \tau}}{2} .
\end{aligned}
$$

Then, we immediately find the following constraints for these parameters from Sec. 5.1.

- From LSND, $a_{e \mu} \sim 10^{-19} \mathrm{GeV}$, so $\stackrel{\circ}{a} \sim 10^{-19} \mathrm{GeV}$; and

- From seesaw mechanism constraint, $\stackrel{m}{0} 0.1 \mathrm{eV}$.

We can justify why we introduce only a $m_{\tau \tau}$ term. For example, in the simple $\mathrm{SO}(10)$ scenario [9], $m_{\tau \tau}^{2} \propto \mathrm{m}_{t}^{2} \gg$ other mass terms ( $m_{t}$ is the mass of top quark $\sim 174 \mathrm{GeV} / \mathrm{c}^{2}[8]$ ).

After a search through the allowed 3-parameter space, we found that the following parameter set can reasonably reproduce the oscillation data,

$$
\begin{aligned}
\frac{\stackrel{\circ}{ }^{2}}{2} & =5.2 \times 10^{-3} \mathrm{eV}^{2}, \\
\stackrel{\circ}{a} & =-2.4 \times 10^{-19} \mathrm{GeV}, \\
\stackrel{\circ}{c} & =3.4 \times 10^{-17} .
\end{aligned}
$$

Note that these are the parameters to reproduce the $\nu-\nu$ oscillation data. For $\bar{\nu}-\bar{\nu}$ oscillation, $\stackrel{\circ}{a} \rightarrow-\stackrel{\circ}{a}$ according to Eq. 4.6 . 
Figures 5.4 and 5.5 show the tandem model solution for both $\nu-\nu$ and $\bar{\nu}-\bar{\nu}$ oscillations. From the bottom middle plots of Figs. 5.4 and 5.5 (inverse of $\Delta$ ), we can see that the eigenvalue differences make $L / E$-type solutions in the high energy region ( $\gtrsim 100 \mathrm{MeV})$, but it is is not easy to predict from these plots exactly how the oscillations behave because the oscillation amplitudes (-4UUUU terms) also evolve with neutrino energy in a complicated way (Figs. 5.4 and 5.5, top three plots).

Therefore, it is instructive to look more closely at the oscillation signals produced by tandem model. As described above, we use the bimaximal model as a representative for the standard three neutrino massive model and the world neutrino oscillation data. 
$2006 / 0+/ 17 \quad 20.21$
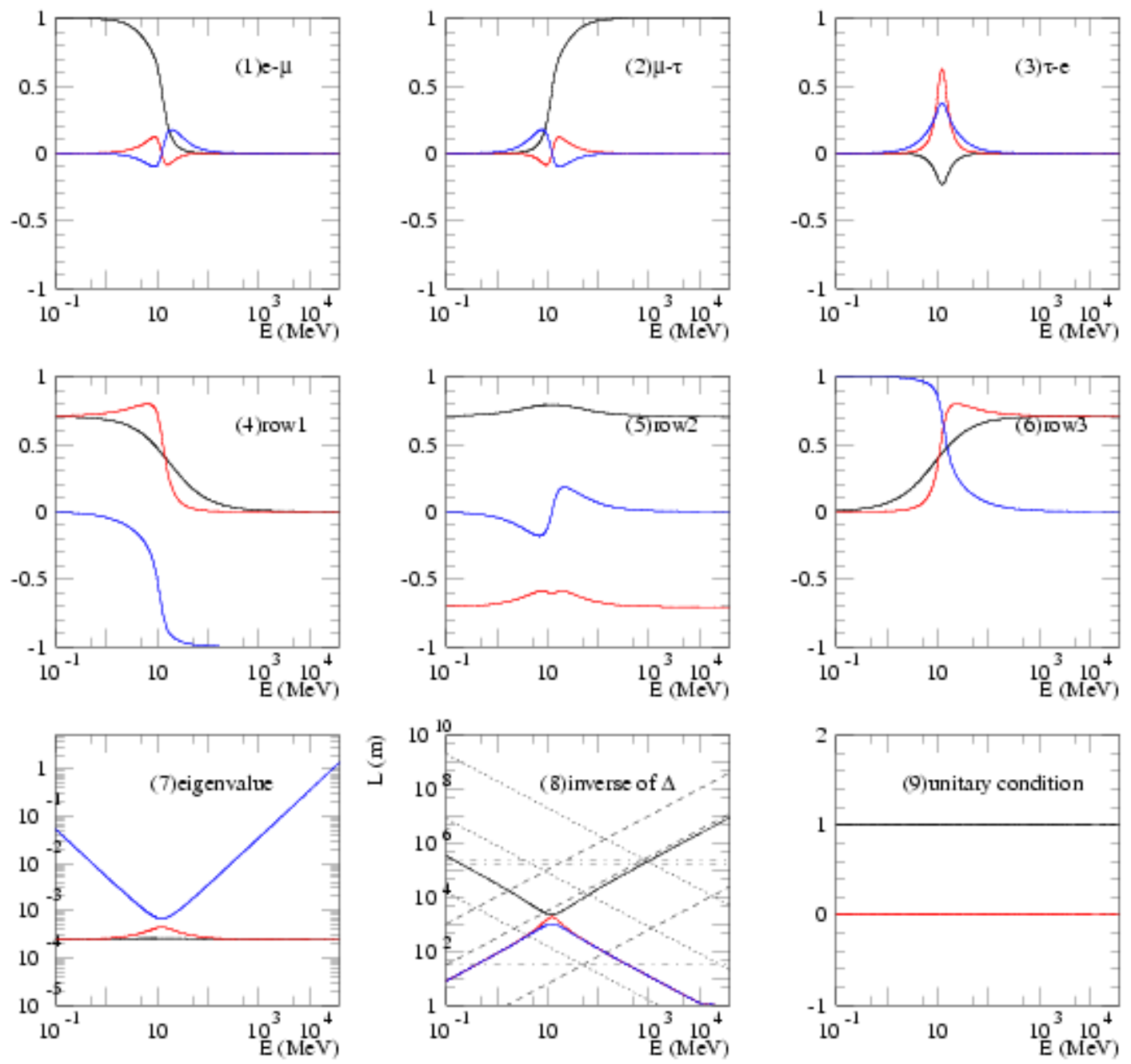

Figure 5.4: The solution of $\nu-\nu$ oscillations by the tandem model. The notation is given in the text, Sec. 5.3.1. 

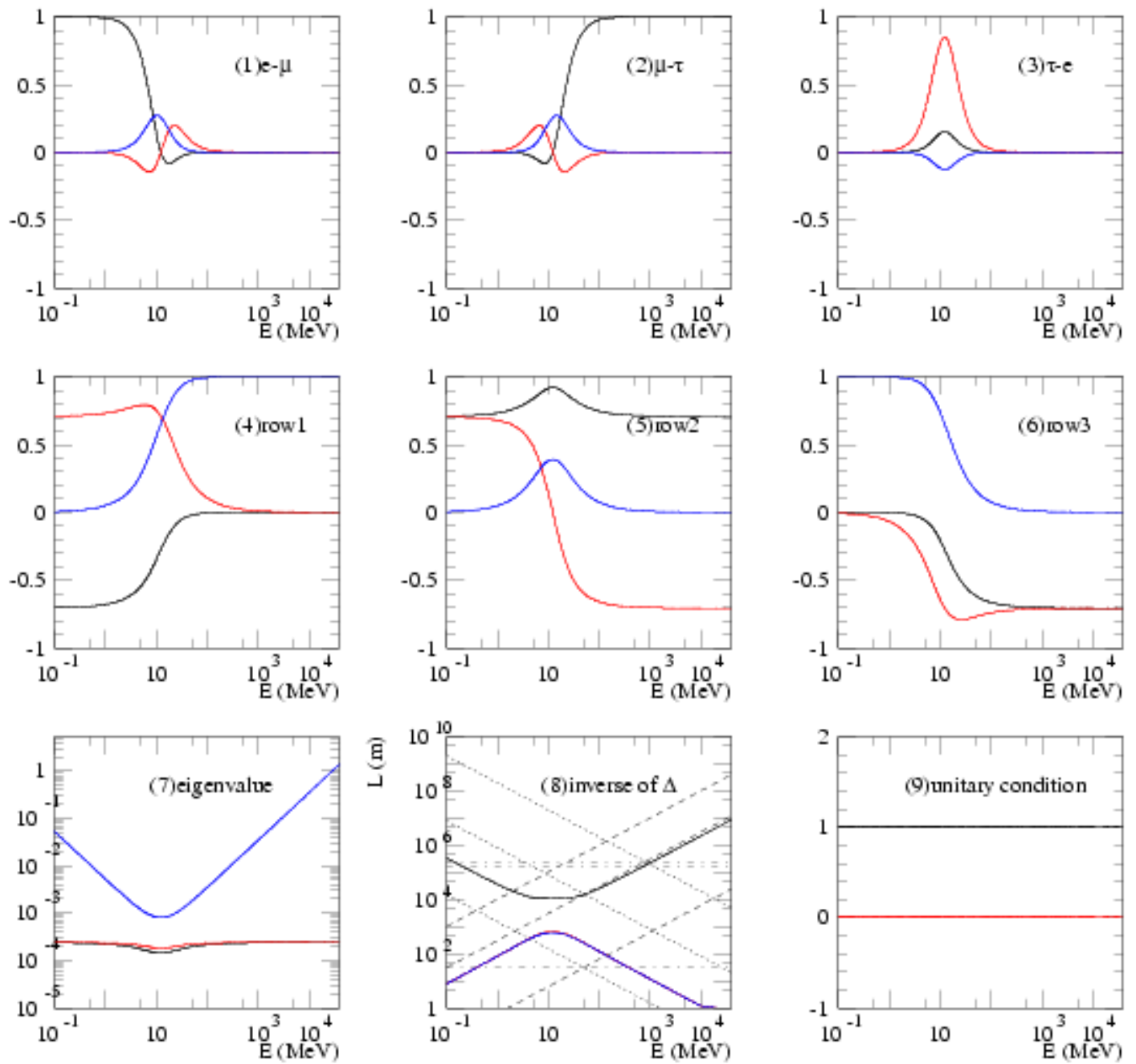

Figure 5.5: The solution of $\bar{\nu}-\bar{\nu}$ oscillations by the tandem model. The notation is given in the text, Sec. 5.3.1. 


\section{Solar neutrinos}

One of the very distinct feature of tandem model is that it does not require the widelybelieved MSW mechanism for solar neutrino oscillation. For general neutrino oscillations, mixing matrix elements are a function of energy (Eq. 5.2). Using this property, the tandem model can produce the energy dependence of neutrino oscillations in the long-baseline limit (time averaged oscillation, Eq. 2.22).

Figure 5.6 shows the result for solar neutrino oscillations. For comparison, standard three neutrino massive model with MSW effect [97] is plotted (Sec. 5.3.1). The tandem model can reproduce the essential nature of the solar neutrino problem, namely $\lesssim 40 \%$ survival probability for ${ }^{8} B$-neutrinos $(15.04 \mathrm{MeV}$ end point energy) and $\gtrsim 50 \%$ for $p p$ neutrino (0.420 MeV end point). Note, since the size of Lorentz violation is bigger (in the tandem model) than the MSW solar potential for the $\nu_{e}$ interaction, this energy dependence of for neutrino oscillations is created via Lorentz violation even though the MSW matter potential may still exist (it just does not manifest itself in this model). Notice the shape of the spectrum is quite different for tandem model compared with the MSW-effect-based model.

\section{Atmospheric neutrinos}

Since the tandem model shows an $L / E$ behavior in the high-energy limit, it can reproduce the observed energy dependence of atmospheric neutrino oscillations (Fig. 5.7). Note, although the solutions are different for $\nu-\nu$ and $\bar{\nu}-\bar{\nu}$ oscillations (Figs. 5.4 and 5.5), $\nu_{\mu}$ disappearance and $\bar{\nu}_{\mu}$ disappearance made by the tandem model are the same at $\gtrsim 100 \mathrm{MeV}$. This is consistent with the atmospheric neutrino charge separated result from MINOS [80]. Because of the parameter we chose, the phase of oscillations are shifted compared to the standard three neutrino massive model. 

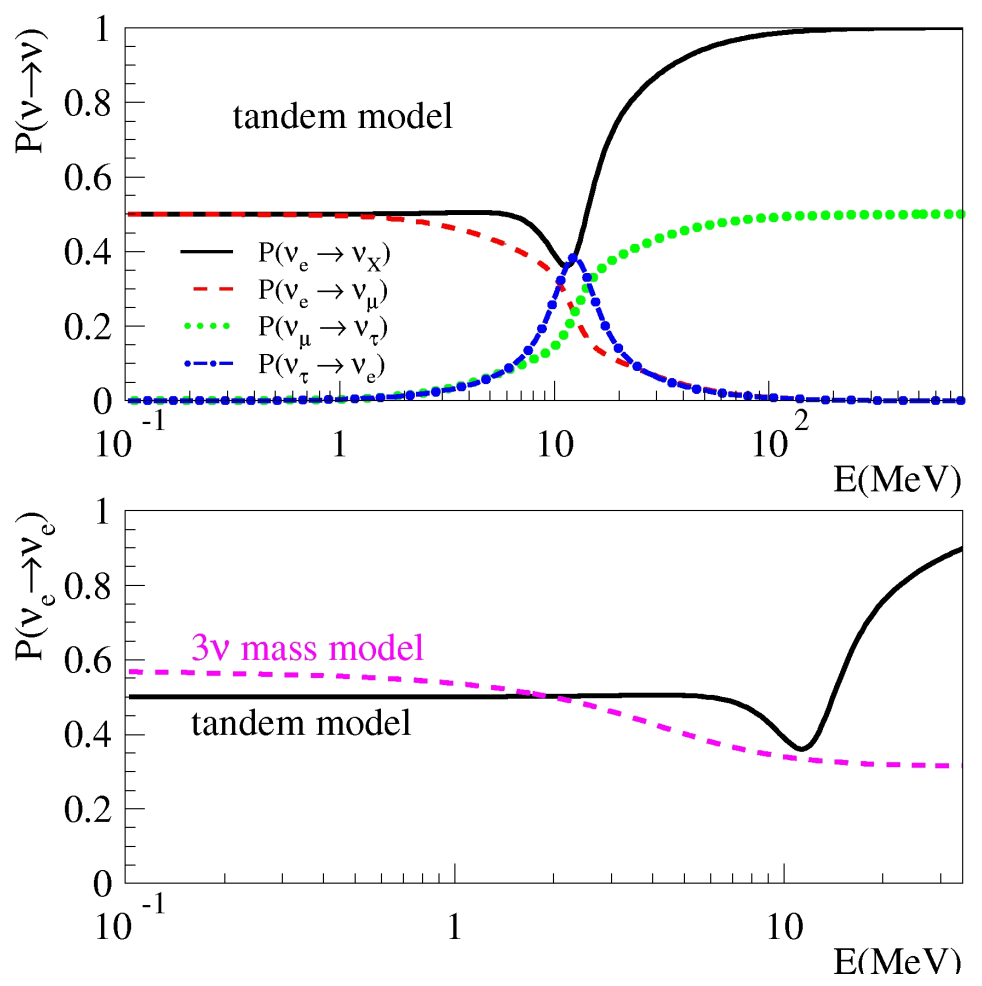

Figure 5.6: Solar-neutrino oscillation probabilities in the tandem model. (a) Curves representing survival probabilities for $\nu_{e}$ (solid), $\nu_{e} \leftrightarrow \nu_{\mu}$ (dashed), $\nu_{\mu} \leftrightarrow \nu_{\tau}$ (dotted), and $\nu_{\tau} \leftrightarrow \nu_{e}$ (dash-dotted). (b) Survival probability of $\nu_{e}$ in the tandem model (solid line) and in the standard three-neutrino massive model with a basic matter-induced effect (dashed) 97. Note the different energy scales. The effects of experimental position and energy resolution are not shown. 

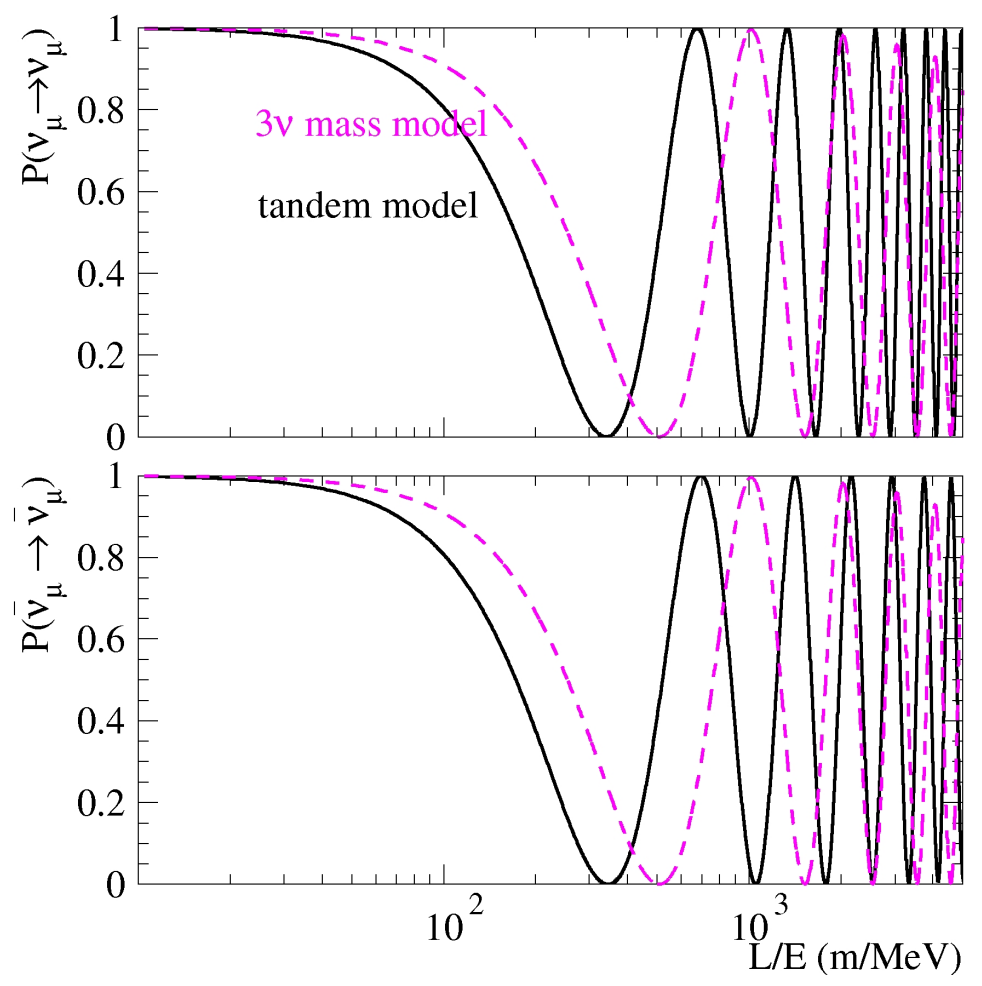

Figure 5.7: Survival probabilities for atmospheric neutrinos as a function of $L / E$ for (a) $\nu_{\mu}$ and (b) $\bar{\nu}_{\mu}$. Curves are shown for the tandem model (solid) and for the standard threeneutrino massive model (dashed). The effects of experimental position and energy resolution are not shown. 


\section{Long-baseline reactor neutrinos}

Figure 5.8 shows the resulting oscillation probability for KamLAND. This $\bar{\nu}_{e}$ disappearance spectrum shape is made by the combination of all three channels around the $1-10 \mathrm{MeV}$ energy region. Notice that all $\bar{\nu}_{e} \leftrightarrow X$ channels die out at $\gtrsim 100 \mathrm{MeV}$. This is also true for $\nu_{e}$. Therefore, this model predicts no $\nu_{e}$ appearance signals for NOvA [30] and T2K [29].

\section{Short-baseline reactor and accelerator neutrinos}

Finally, Fig. 5.9 shows the oscillation probabilities for a few short baseline accelerator experiments. To tune the model parameters, we first constrained the parameter space so as to not create signals for null result short baseline reactor oscillation experiments (baseline $<1 \mathrm{~km}$ ), including, Burgey [99], Gösgen [100], Palo Verde [101, and CHOOZ [102]. In the tandem model, the oscillation probabilities at short baselines are typically small, so they are undetectable by the short baseline reactor experiments. This is because reactor experiments are disappearance experiments and their sensitivities are usually $\sim 10 \%$.

Next, we tested this model for the short baseline accelerator oscillation experiments 69 , 103. As can be seen from Fig. 5.9, the oscillation probability for KARMEN is less than half of LSND at their typical energy $(\sim 40 \mathrm{MeV})$. Therefore, tandem model can make signals for LSND, but not for KARMEN within their experimental sensitivity. The model predicts $\sim 0.1 \%$ level signal for $\bar{\nu}_{e}$ appearance in LSND. This is somewhat smaller than the observation $(0.26 \%)$. Tandem model also predicts $\sim 3$ times larger signal for the proposed OscSNS experiment [104] when compared to LSND.

The final test of this model is the prediction for MiniBooNE. This mode has strong energy dependence in the oscillation probability, and it has a large $\nu_{e}$ appearance signal only in the low energy region. At the time we published the tandem model prediction (June, 2006), MiniBooNE had not yet announced their initial result for $\nu_{e}$ appearance. The 

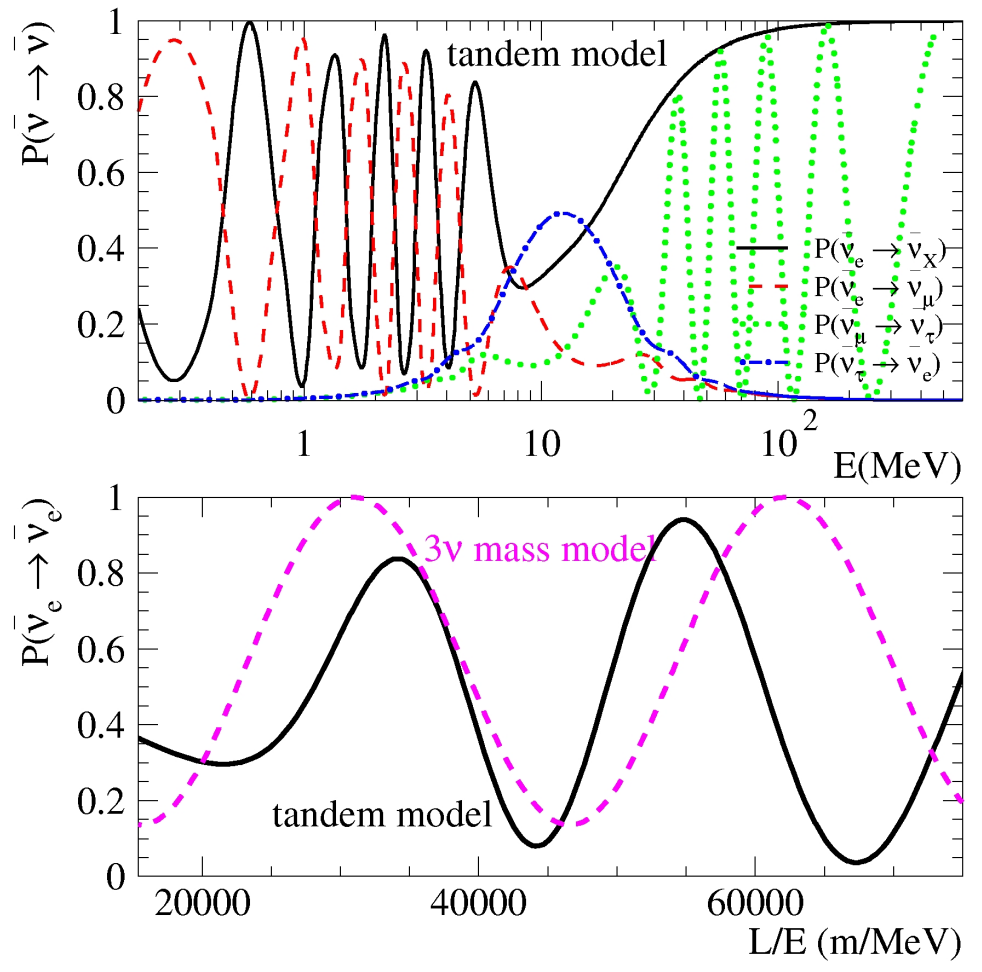

Figure 5.8: Survival probabilities for long-baseline reactor antineutrinos for the tandem model. (a) Survival probabilities as a function of $E$ for $\bar{\nu}_{e}$ (solid), $\bar{\nu}_{e} \leftrightarrow \bar{\nu}_{\mu}$ (dashed), $\bar{\nu}_{\mu} \leftrightarrow \bar{\nu}_{\tau}$ (dotted), and $\bar{\nu}_{\tau} \leftrightarrow \bar{\nu}_{e}$ (dash-dotted). (b) Survival probabilities for $\bar{\nu}_{e}$ as a function of $L / E$ in the tandem model (solid) and in the standard three-neutrino massive model (dashed). The effects of experimental position and energy resolution are not shown. 
CHAPTER 5. GLOBAL THREE PARAMETER MODEL FOR NEUTRINO OSCILLATION WITH LORENTZ VIOLATION

initial oscillation result of MiniBooNE (March, 2007) 67] did see an anomalous excess at low energy $\nu_{e}$ candidate events. Therefore, it is very interesting to examine in further detail, namely the spectrum shape comparison, of tandem model prediction and MiniBooNE data. 


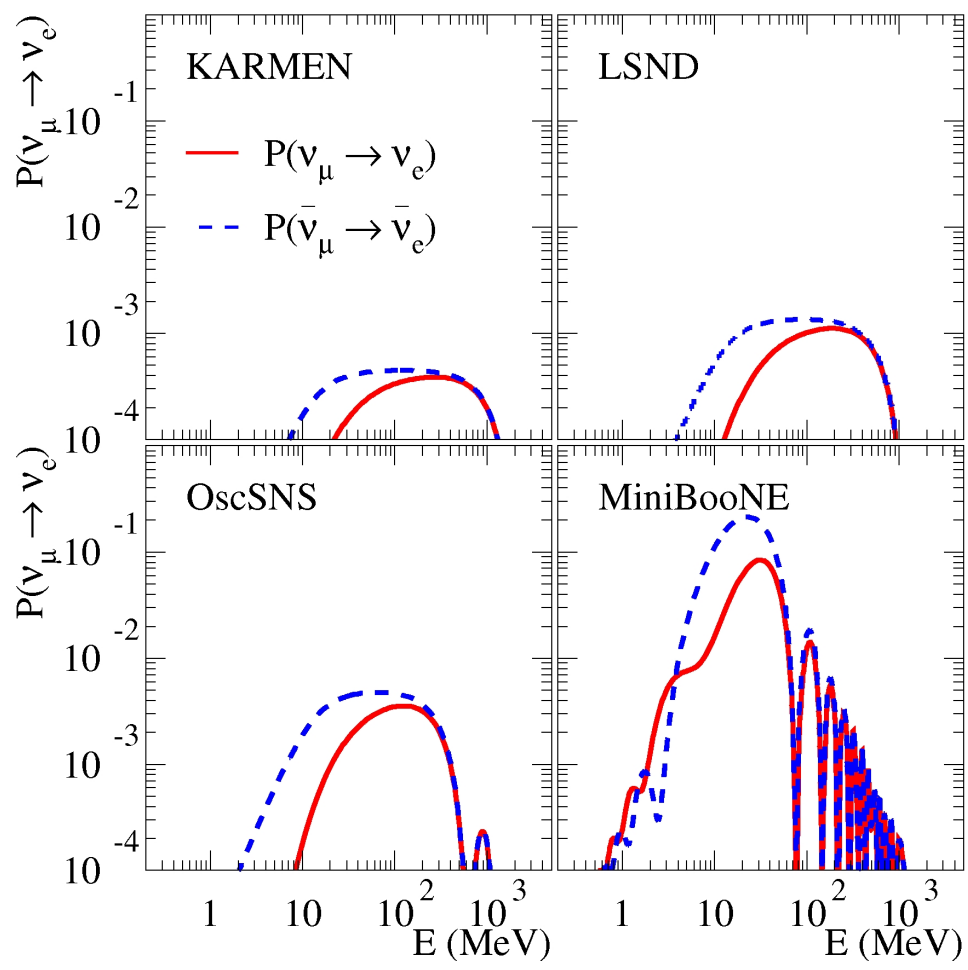

Figure 5.9: Oscillation probabilities for short-baseline experiments for the tandem model. Here, we plot with oscillation probabilities as a function of $E$ for neutrinos (solid) and antineutrinos (dashed) in (a) KARMEN, (b) LSND, (c) the proposed OscSNS experiment, and (d) the currently running MiniBooNE experiment. The effects of experimental position and energy resolution are not shown. 


\subsection{Tandem model in MiniBooNE}

Figure 5.10 shows the neutrino energy spectrum of $\nu_{e}$ oscillation candidate events in MiniBooNE [67]. Notably, the excess of data at low energy does not fit with any standard massive oscillation models in the two neutrino oscillation approximation (Eq. 2.10).

The three parameters of the tandem model (Eq. 5.17) were chosen to maximize the signal yet keep good agreement with all oscillation data. We used the MiniBooNE open database [105] for this study. The open database is in the form of an ntuple with 9934 events. A given "weight" for an event correctly takes account all experimental effect, such as efficiency, energy smearing, etc. Say, the $s^{\text {th }}$ event has weight $w^{s}$, true $\nu_{e}$ energy $E_{\text {true }}^{s}$, and reconstructed $\nu_{e}$ energy $E_{\text {recon }}^{s}$. If a theoretical model predicts $P\left(E_{\text {true }}^{s}\right)$, then the $s^{t h}$ event has an number of event $n^{s}$,

$$
n^{s}=P\left(E_{\text {true }}^{s}\right) \times w^{s} / 9934 .
$$

This event would be binned in energy according to the reconstructed $\nu_{e}$ energy, $E_{\text {recon }}^{s}$. After repeating this process for all events, we can find the model-predicted spectrum of $\nu_{e}$ candidate events with all experimental effect, and we can compare our model result with the data directly. Although the ntuple also included propagation distance for each event, for simplicity, we used a single number, $\sim 520.0 \mathrm{~m}$, resulting from the baseline $(541 \mathrm{~m})$ minus the average pion decay length $(\sim 20 \mathrm{~m}[106])$.

Figure 5.11 shows the result and was presented at the fourth meeting of CPT and Lorentz violation, Bloomington, 9-11 August 2007 [3]. Even though the parameters were tuned to increase the signal size, the predicted signal is smaller as compared to the data. A more sophisticated numerical search in the parameter space is possible for future work. 


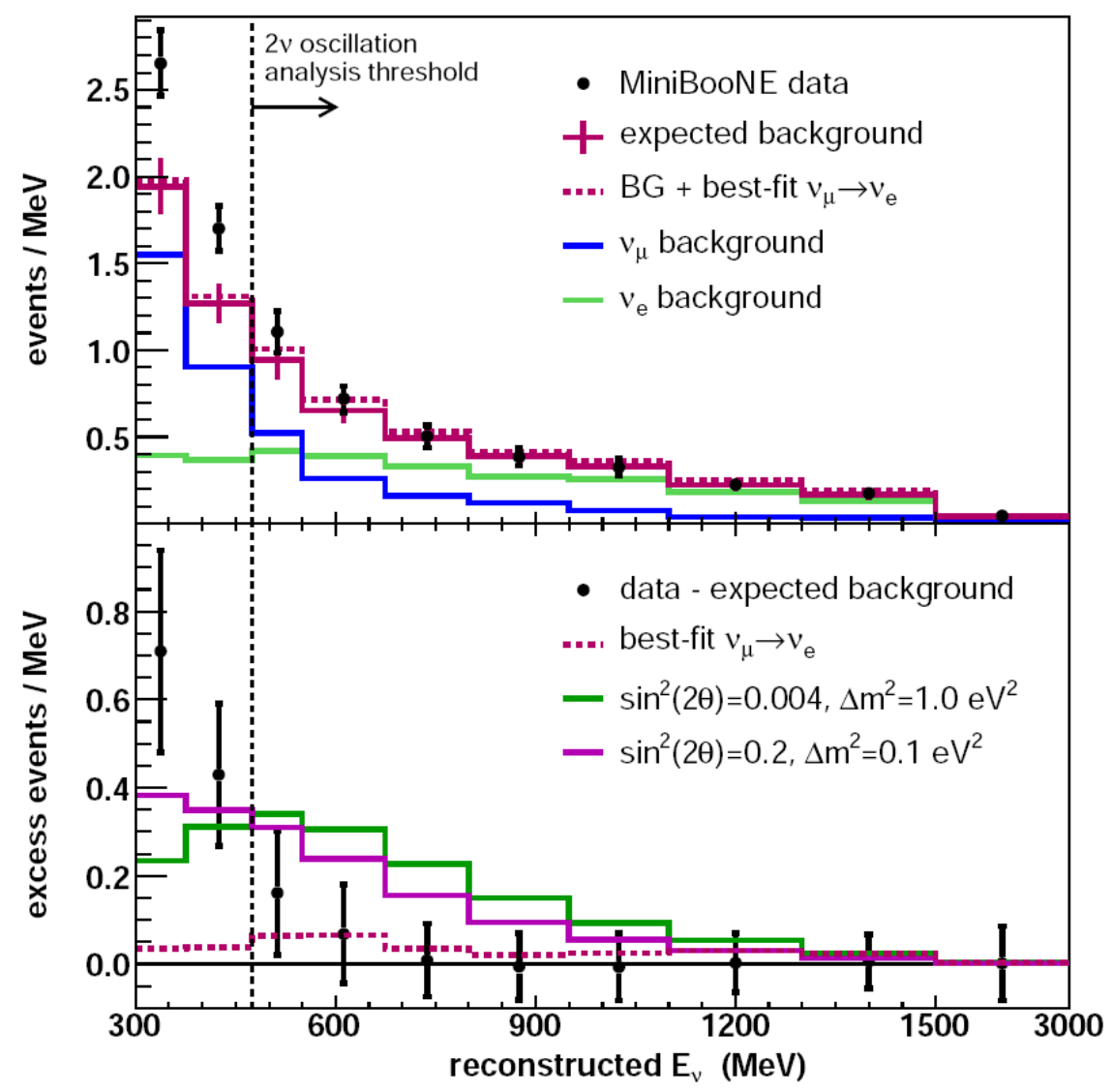

Figure 5.10: Number (per MeV) of candidate $\nu_{e}$ events as a function of reconstructed neutrino energy. In the top plot, the points show the data with statistical error, while the histogram is the expected background with systematic errors from all sources. The vertical dashed line indicates the threshold used in the two-neutrino oscillation analysis. Also shown are the best-fit oscillation spectrum (dashed histogram) and the background contributions from $\nu_{\mu}$ and $\nu_{e}$ events. The bottom plot shows the number of events with the predicted background subtracted as a function of neutrino energy, where the points represent the data with total errors and the two histograms correspond to LSND solutions at high and low $\Delta m^{2}$. 


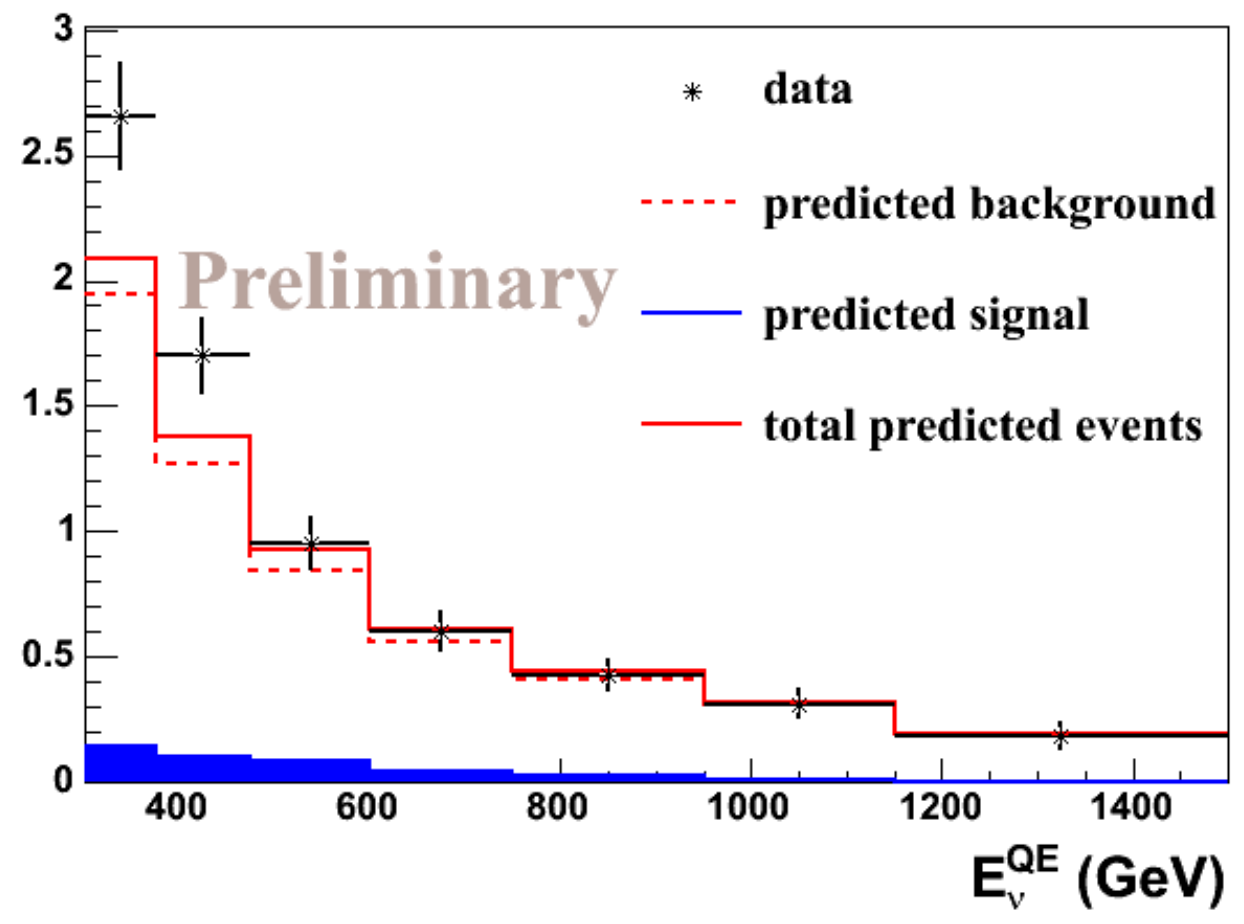

Figure 5.11: Number per $\mathrm{MeV}$ of candidate $\nu_{e}$ events as a function of reconstructed neutrino energy in MiniBooNE. The data is shown (stars) with statistical errors only. The oscillation signal as predicted by the Tandem model is shown in the solid histogram, the predicted background (dashed), and total signal plus background (solid) is also shown. The systematic errors are not shown on this plot but may be seen in Fig. 5.10, The Tandem model prediction is preliminary. 


\section{Chapter 6}

\section{Summary and outlook for neutrino oscillations with Lorentz violation}

In Part II, we have discussed the test of Lorentz violation with neutrino oscillations. Since neutrino oscillations themselves are high precision interferometers, neutrino oscillations are naturally sensitive for small-scale physics, for example, Planck-scale physics suppressed to our energy level.

The motivation for this comes from simple considerations of the sensitivities. For example, in the case of atmospheric neutrino oscillations there is evidence for $\Delta m_{a t m}^{2} \sim$ $10^{-3} \mathrm{eV}^{2}\left[8\right.$. Then the inverse of atmospheric neutrino oscillation length gives $\sim 10^{-21} \mathrm{GeV}$,

and this is beyond the benchmark sensitivity for atmospheric neutrinos $\left(\lesssim 10^{-17} \mathrm{GeV}\right)$ to search the Planck scale physics (Sec. 2.3). Therefore, neutrino oscillations may provide the opportunity to discover high-energy physics that is suppressed in the low energy world and neutrino oscillations are a candidate phenomenon in which to search for physics beyond the Standard Model (BSM).

The anomalous results from the LSND $\bar{\nu}_{e}$ appearance search [69] have motivated alter- 
CHAPTER 6. SUMMARY AND OUTLOOK FOR NEUTRINO OSCILLATIONS WITH

LORENTZ VIOLATION

\begin{tabular}{lcc}
\hline & Combination & fit result \\
\hline 1-parameter fit & $(\mathcal{C})_{\bar{e} \bar{\mu}}$ & $(3.3 \pm 0.4 \pm 0.2) \times\left(10^{-19} \mathrm{GeV}\right)$ \\
& $\left|(\mathcal{C})_{\bar{e} \bar{\mu}}\right|^{2}$ & $(10.7 \pm 2.6 \pm 1.3) \times\left(10^{-19} \mathrm{GeV}\right)^{2}$ \\
\hline 3-parameter fit & & $(-0.2 \pm 1.0 \pm 0.3) \times\left(10^{-19} \mathrm{GeV}\right)$ \\
solution 1 & $(\mathcal{C})_{\bar{e} \bar{\mu}}$ & $(4.0 \pm 1.3 \pm 0.4) \times\left(10^{-19} \mathrm{GeV}\right)$ \\
$($ maximum likelihood $)$ & $\left(\mathcal{A}_{s}\right)_{\bar{e} \bar{\mu}}$ & $(1.9 \pm 1.8 \pm 0.4) \times\left(10^{-19} \mathrm{GeV}\right)$ \\
solution 2 & $\left(\mathcal{B}_{s}\right)_{\bar{e} \bar{\mu}}$ & $(0.1 \pm 0.6 \pm 0.2) \times\left(10^{-19} \mathrm{GeV}\right)$ \\
$($ second maximum $)$ & $(\mathcal{C})_{\bar{e} \bar{\mu}}$ & $(-0.5 \pm 0.6 \pm 0.2) \times\left(10^{-19} \mathrm{GeV}\right)$ \\
$\left|(\mathcal{C})_{\bar{e} \bar{\mu}}\right|^{2}+\frac{1}{2}\left|\left(\mathcal{A}_{s}\right)_{\bar{e} \bar{\mu}}\right|^{2}+\frac{1}{2}\left|\left(\mathcal{A}_{c}\right)_{\bar{e} \bar{\mu}}\right|^{2}$ & $(9.9 \pm 2.3 \pm 1.4) \times\left(10^{-19} \mathrm{GeV}\right)^{2}$ \\
\hline 5-parameter fit & $\left(\mathcal{A}_{s}\right)_{\bar{e} \bar{\mu}}$ & \\
$\left|(\mathcal{C})_{\bar{e} \bar{\mu}}\right|^{2}+\frac{1}{2}\left|\left(\mathcal{A}_{s}\right)_{\bar{e} \bar{\mu}}\right|^{2}+\frac{1}{2}\left|\left(\mathcal{A}_{c}\right)_{\bar{e} \bar{\mu}}\right|^{2}+\frac{1}{2}\left|\left(\mathcal{B}_{s}\right)_{\bar{e} \bar{\mu}}\right|^{2}+\frac{1}{2}\left|\left(\mathcal{B}_{c}\right)_{\bar{e} \bar{\mu}}\right|^{2}$ & $(10.5 \pm 2.4 \pm 1.4) \times\left(10^{-19} \mathrm{GeV}\right)^{2}$ \\
\hline
\end{tabular}

Table 6.1: Summary of the test of Lorentz violation with LSND experiment. The fit result from three different parameter combinations [1]. For 5-parameter fit, only their combination is listed. The definitions of parameter combinations are given in Eqs. 4.11 to 4.23 .

native solutions for neutrino oscillations, such as Lorentz violation. We analyzed the LSND data under the SME formalism to test the allowed sidereal variation [1]. The LSND data can be interpreted as an order $10^{-19} \mathrm{GeV}$ signal of Lorentz violation. We extracted three combinations of SME parameters via a 3-parameter fit, shown in Fig. 4.6 (Eqs. 4.11, 4.11 , and 4.11). The results are summarized in Tab. 6.1 (here, the first and the second errors are statistic and systematic errors). The data are consistent with no sidereal variation, however, they are also well-described within the SME formalism and so, Lorentz violation can not be excluded. 
This result further motivated the construction of a global, "tandem" model for neutrino oscillations using Lorentz violation [2]. The tandem model is motivated from the bicycle model [65], namely, combinations of mass, CPT-odd, and CPT-even parameters were used to produce oscillation signals at certain L-E values to match experimental data. In this way, both mixing angles and eigenvalue differences are complicated functions of energy, but they can mimic solar, atmospheric, and KamLAND oscillation signals, as well as the LSND signal. The model also predicted a signal in the low-energy region of MiniBooNE. However, so far, this tandem model cannot quantitatively reproduce the MiniBooNE result (Fig. 5.11).

The original tandem model also shows limitations when compared to recently published high-statistics neutrino oscillation results. In the solar sector, the recent data from SNO, focusing on low-energy ${ }^{8} B$ neutrinos [107 do prefer an MSW-like energy spectrum more than tandem model. Also, the low-energy neutrino measurement at Borexino combined with results from previous solar neutrino experiments constrain the flux normalization of $p p$-neutrinos [95]. That result also prefers the standard three neutrino massive model with MSW effect. In the atmospheric sector, the oscillation maximum made by tandem model is shifted somewhat from the standard three massive neutrino model, and this shift may be a problem in reproducing the recent high-statistics accelerator based long baseline oscillation data from K2K and MINOS [81, 82]. However, the tandem model does agree well with recent high statistics results from the KamLAND experiment [96].

The SME formalism of neutrino oscillations does have additional possibilities. It looks as though one specific parameter set of a specific model cannot precisely account for the data. However, a small change of the model in the right way can perhaps improve the situation dramatically. Thus, as of now, we cannot make a conclusion about the future of tandem model.

In conclusion, a possibility of Lorentz violation in LSND and MiniBooNE is not yet 
excluded. The simple models, for example those with only a CPT-odd term in the Hamiltonian, can have only a simple energy dependences in the oscillation probability $\left(\sim \sin ^{2}(\Omega L)\right.$, here $\Omega$ is a combination of SME parameters). This type of model is excluded up to a $\sim 10^{-27}$ suppression scale through high energy atmospheric neutrinos from AMANDA 64. However, complications in the diagonalization of Hamiltonians for models which contain more than one type of energy dependence forbids a simple application of this limit to other experiments. Therefore, it is important to test Lorentz violation in all experiments, individually. Among all possible tests, sidereal variation is the "smoking gun" of Lorentz violation.

The sidereal variation analysis for MiniBooNE is an important piece of this program. The fact that the MiniBooNE experiment has an unexplained low energy excess [108] makes the test of Lorentz violation with this data very interesting! A very preliminary sidereal variation test with the MiniBooNE low energy excess has been was presented [109], but needs to be examined further. This is a subject of future work. 


\section{Part III}

\section{Charged Current Quasielastic scattering measurement in MiniBooNE}


We cannot regard it as impossible that integral laws, to use an expression of C. Neumann, will some day take the place of the laws of mathematical elements, or differential laws, that now make up the science of mechanics, and that we shall have direct knowledge of the dependence on one another of the positions of bodies. In such an event, the concept of force will have become superfluous.

- E. Mach, The Science of Mechanics 


\section{Chapter 7}

\section{MiniBooNE}

The mini Booster neutrino experiment (MiniBooNE) is designed to confirm or reject oscillation signals observed by the LSND experiment [69]. The muon neutrino beam created by the Booster Neutrino Beamline (BNB) are observed by the MiniBooNE detector $550 \mathrm{~m}$ from the source. The signature of $\nu_{\mu} \rightarrow \nu_{e}$ oscillation ( $\nu_{e}$ appearance) is a single isolated electron. The first oscillation result from MiniBooNE ruled out the two massive neutrino oscillation hypothesis as an interpretation of the LSND signal [67].

An overview of the MiniBooNE experiment is available elsewhere [19, 106, 110 115]. In this chapter, we briefly describe the main part of the detector [116] and the neutrino beam [117. Then, we discuss the signature of neutrino events in MiniBooNE.

\subsection{MiniBooNE, motivation}

MiniBooNE is designed to test the LSND $\bar{\nu}_{e}$ appearance signal. Since LSND has a baseline $\sim 30 \mathrm{~m}$ and a neutrino energy $\sim 30-60 \mathrm{MeV}, \mathrm{L} / \mathrm{E}$ is $\sim 1$. Under the two massive neutrino oscillation hypothesis (Eq. 2.10), LSND signals correspond to neutrino oscillations with $\Delta m^{2} \sim 1 \mathrm{eV}^{2}$. We have several other well-established oscillation signals. The first kind is 
called atmospheric oscillations [78, 80]. They have been confirmed by long baseline neutrino oscillation experiments [81, 82, 94] and are characterized with $\Delta m_{\text {atm }}^{2} \sim 10^{-3} \mathrm{eV}^{2}$. The second type is called solar neutrino oscillations [70 77, 95]. They have been confirmed by a long baseline reactor neutrino oscillation experiment [83, 96] and are characterized with $\Delta m_{\odot}^{2} \sim 10^{-5} \mathrm{eV}^{2}$. Since the Standard Model (SM) has only three generations of leptons, the confirmation of the LSND signal would suggest the existence of a new type of neutrino around the $1 \mathrm{eV}$ mass scale. These new type of neutrinos do not have a weak charge and hence are sterile for the weak interaction ("sterile" neutrinos). Therefore, the confirmation of the LSND oscillation signal would imply new physics beyond the Standard Model (BSM).

The MiniBooNE experiment was designed to test LSND. MiniBooNE has a $550 \mathrm{~m}$ baseline and $\sim 700 \mathrm{MeV}$ neutrino energy, to have same L/E with LSND so that MiniBooNE is sensitive to the same $\Delta m^{2}$ observed at LSND. However, MiniBooNE has an order higher energy as compared to LSND, and the signal and backgrounds are completely different from LSND.

\subsection{Booster neutrino beam}

Fig. 7.1 shows an overview of Fermilab. For Tevatron operation, the protons accelerated by the Booster are extracted into the Main Injector, and finally injected into the Tevatron to study high energy collider physics. High energy neutrinos are not required but a high-flux of neutrinos are, so protons are extracted directly from the Booster. The Booster Neutrino Beamline (BNB) is separated into three parts (Fig. 7.2),

1. primary proton beam,

2. secondary meson beam,

3. tertiary neutrino beam. 


\subsection{BOOSTER NEUTRINO BEAM}

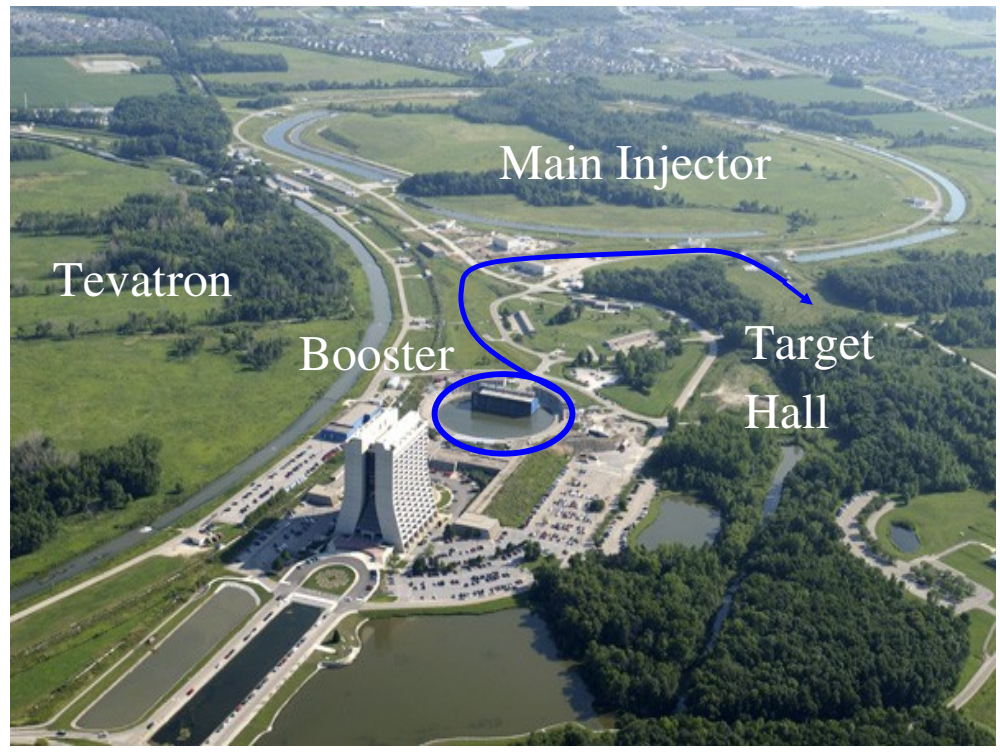

Figure 7.1: A overview of Fermilab. Protons accelerated in the $8 \mathrm{GeV}$ Fermilab Booster are sent to the target hall. The Main Injector and Tevatron are visible in this picture.

The details of the neutrino beam, the flux prediction and errors are available in [106, 112, 117 .

The protons are accelerated to $8 \mathrm{GeV}$ kinetic energy in the Booster synchrotron, then "fast extraction" sends all of protons in the ring to Booster neutrino beamline. The protons collide with the beryllium target in the magnetic focusing horn, and the produced mesons are focused by a toroidal magnetic field to collimate the neutrinos resulting from the decay in flight (DIF) of the mesons. 


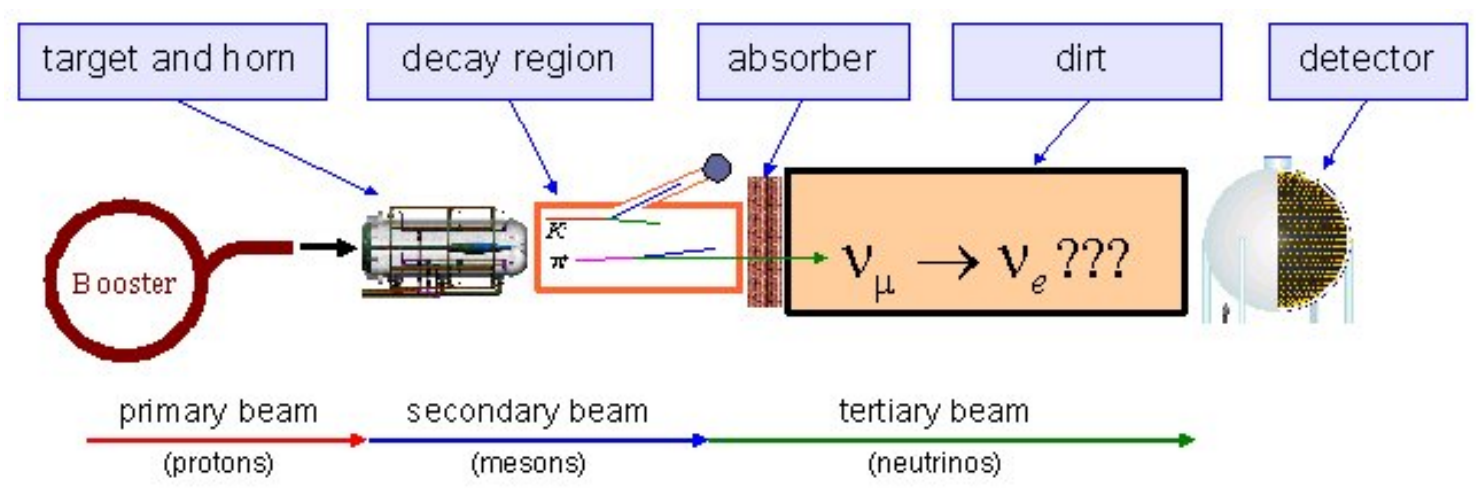

Figure 7.2: A schematic overview of the MiniBooNE neutrino beamline.

\subsubsection{Primary proton beam}

The Fermilab Booster accelerator is a 24 FODO cell synchrotron, but does use combined function magnets for focusing and defocusing [118, 119]. $H^{-}$ions (of $400 \mathrm{MeV}$ kinetic energy) are injected from Fermilab LINAC, then, after passing the electron stripping foil, the protons are accelerated at $200 \mathrm{GeV} / \mathrm{s}$ to $8 \mathrm{GeV}$ kinetic energy, and the fast extraction kicker brings 81 bunches to the Booster Neutrino Beamline. For the first MiniBooNE oscillation data set, (run 3539 to run 12500, or a data-taking period from August 2002 to December 2005), a total of $5.579 \times 10^{20}$ protons were delivered to the target (called "protons on target", or "POT") with $\sim 1.4 \times 10^{8}$ beam triggers. Thus, each spill contains $\sim 4 \times 10^{12}$ POT on average. The spill has a micro structure of 81 bunches in $84 \mathrm{RF}$ buckets. Each bunch has $\sim 6$ ns width with 19 ns separation, distributed over $\sim 1.6 \mu \mathrm{s}$ in each spill (Fig. 7.3a). The MiniBooNE data acquisition system (DAQ) is synchronized with the Booster cycle. Although the Booster is running at $15 \mathrm{~Hz}$, typically the BNB runs at $4-5 \mathrm{~Hz}$, which means the DAQ window opens $4-5$ times every second to cover the 1.6 $\mu$ s spill. In practice, the DAQ window opens on a signal sent via the Fermilab ACNET (Accelerator network) which proceeds the Booster extraction kick by $320 \mu \mathrm{s}$ [111]. The timing is adjusted to open the DAQ window approximately $4.5 \mu$ s before the neutrino beam 
(a)

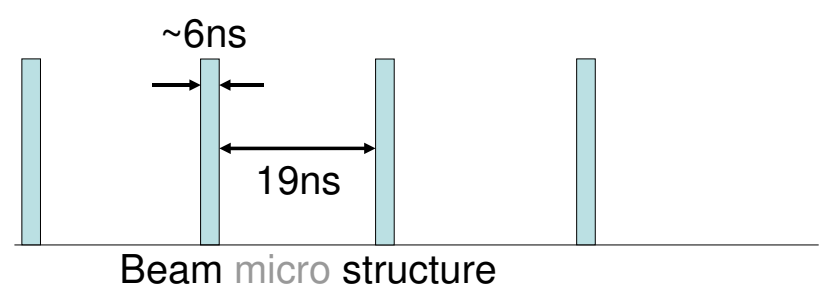

(b)

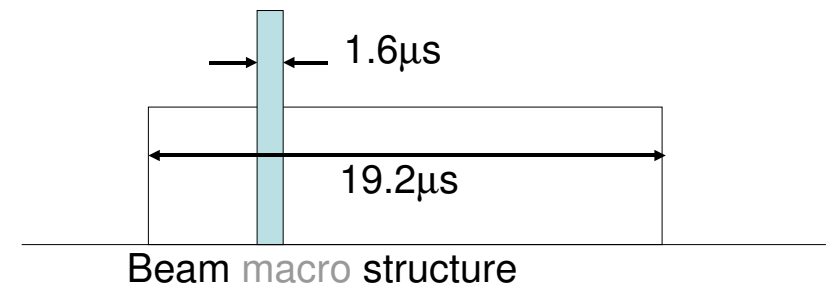

Figure 7.3: The BNB micro and macro structure.

arrives at the detector, and it closes $19.2 \mu$ s later (Fig. 7.3b). The current from the beam protons is measured independently by two toroids, and the difference of these measurement indicates a $2 \%$ absolute error on the POT value. Also, the effects of uncertainty of the beam focal point on the Be target have been simulated, resulting in a less than $1 \%$ absolute POT error.

\subsubsection{Secondary meson beam}

The protons interact with the beryllium target in the magnetic focusing horn to produce mesons. The decay of mesons create the neutrino beam. Uncertainties in this processes result in the dominant errors on the neutrino flux:

$$
\begin{aligned}
& \pi^{+} \rightarrow \mu^{+}+\nu_{\mu}, K^{+} \rightarrow \mu^{+}+\nu_{\mu} \cdots \\
& \mu^{+} \rightarrow e^{+}+\bar{\nu}_{\mu}+\nu_{e}, K^{+} \rightarrow e^{+}+\pi^{\circ}+\nu_{e}, K_{L}^{\circ} \rightarrow e^{ \pm}+\pi^{\mp}+\stackrel{(-)}{\nu_{e}} \cdots
\end{aligned}
$$


The $\nu_{\mu}$ is the dominant neutrino species produced in the BNB (93.5\%). And, the $\nu_{\mu}$ are mainly produced via the $\pi^{+}$decay $(96.7 \%$ of the $93.5 \%$ ). Therefore, the uncertainty in the $\pi^{+}$production model creates the largest systematic error in the neutrino beam predictions [106, 112]. For the $\nu_{e}$ appearance search, $\nu_{e}$ intrinsic to the neutrino beam, are the largest background. Although the dominant contribution in $\nu_{e}$ production is $\mu^{+}$ decay $(51.6 \%)$, since $\mu^{+}$decay is constrained by the $\nu_{\mu}$ CCQE measurement in MiniBooNE (Chapter 8), the next dominant channel for $\stackrel{(-)}{\nu_{e}}$ production, namely $K^{+} / K^{\circ}$ decays, were carefully studied. To calculate the meson production rate for MiniBooNE, high quality external data is required. For this we have used (in addition to other data) the results from the hadron production (HARP) experiment [112, 120] at the European organization for nuclear research (CERN).

For the primary protons and secondary mesons, the uncertainty due to hadronic interactions have been calculated, are less important.

After the mesons are created, they are focused by the toroidal magnetic field created by the magnetic focusing horn (Fig. 7.4), running at $4-5 \mathrm{~Hz}$ with a $\sim 174 \mathrm{kA}$ current. The horn is basically a pulsed coaxial cable, with current flowing from the inner to outer conductors though the end cap, to create a toroidal field between the conductors. Through this process, the neutrino flux is increased by roughly a factor of six over the case with no focusing. The possible variation of the horn current is considered as a systematic error. The more important error is the "skin effect" in the inner conductor. Some portion of the current penetrates inside the conductor surface, creating a magnetic field in the inner conductor. The simulation of this effect predicts a large uncertainty in the high-energy neutrino focusing because small angle mesons (which are higher momentum mesons yielding higher energy neutrinos) feel the magnetic field created inside of the inner conductor. 


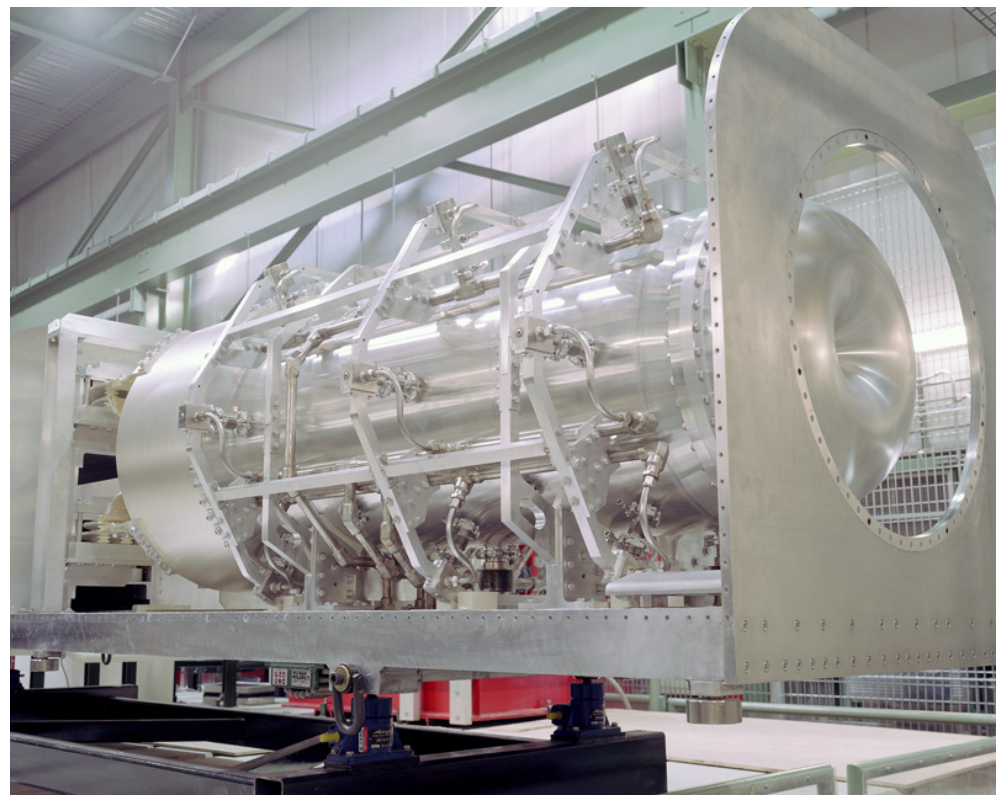

Figure 7.4: The MiniBooNE magnetic horn.

\subsubsection{Tertiary neutrino beam}

After propagating through the $50 \mathrm{~m}$ decay pipe, almost all mesons decay-in-flight (DIF). Figure 7.5 shows the predicted $\nu_{\mu}$ flux as predicted by the MiniBooNE beam simulation. The same $\nu_{\mu}$ flux is used for the $\nu_{e}$ appearance analysis [67], the $\mathrm{NC} \pi^{\circ}$ rate measurement [121], and the CCQE rate measurement [4], as well as all the analysis presented here. The flux values as a function of neutrino energy are provided in Table D.1 of Appendix D. This table can be used for analyzing the MiniBooNE CCQE measurement with interaction models under consideration. The units are neutrinos/POT/50MeV and the integrated value over the entire energy region is $5.167 \times 10^{-10}$ neutrinos/POT. 


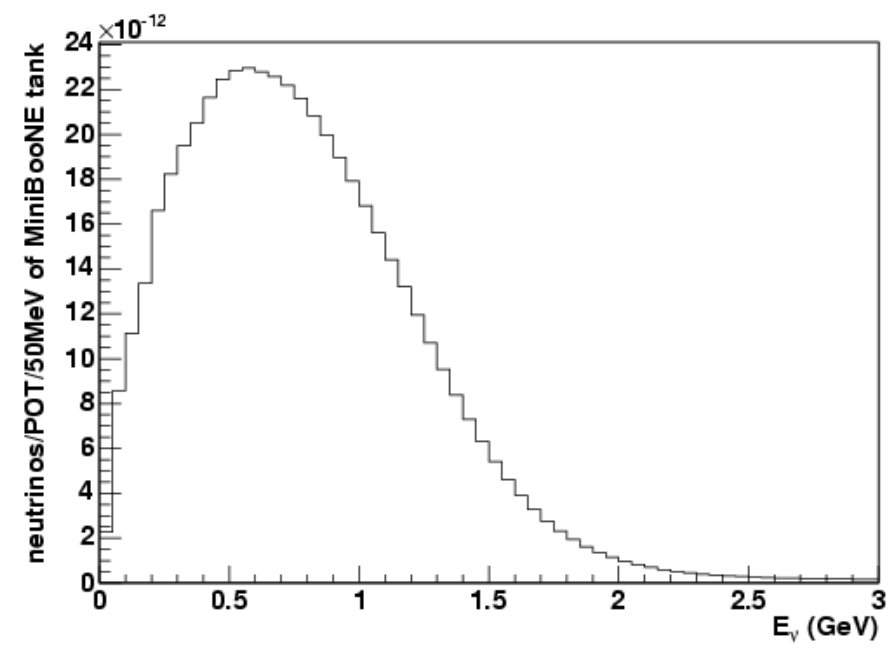

Figure 7.5: Predicted $\nu_{\mu}$ flux at the MiniBooNE detector location. Units are neutrinos/POT/50MeV and the integrated flux is $5.167 \times 10^{-10}$ neutrinos/POT. The table is prepared in Table D.1 of Appendix D. 


\subsubsection{Neutrino flux error summary}

In summary, we consider the following errors in the neutrino flux prediction:

- absolute POT error;

- meson production errors $\left(\pi^{+}, \pi^{-}, K^{+}\right.$, and $\left.K^{o}\right)$;

- hadronic interaction errors (protons and pions total, quasi-elastic, and inelastic scattering with beryllium);

- horn current and skin effect.

Among these errors, the dominant for $\nu_{\mu}$ flux is the $\pi^{+}$production uncertainty. This error is estimated with two methods. First, the error is estimated from the Sanford-Wang fitting model parameters [122] of the HARP and E910 [123] experimental $\pi^{+}$production data. In this way, because of the correlation between seven fitting parameters (one of the eight parameters is fixed), the estimated error from $\pi^{+}$production is around $15 \%$. However most of this error is in the normalization, so any data-MC comparison type analyses, for example a data fit with relatively normalized MC, do not suffer much from this error. This error estimation method was used for the result of Chapter 8

Recently, a second error estimation method was implemented. Since the HARP data were collected with the same energy as the BNB and a replica of the MiniBooNE target was used, one can extract the $\pi^{+}$production rate directly from the HARP result without using a physical parametrization. A spline fit [91] is used to extract a smooth function for the $\pi^{+}$kinetic space from the data. This new method dramatically reduces the error, and estimated error for $\pi^{+}$is now around $5 \%$. This error estimation method was used for the result of Chapter 9 and 10 .

The skin effect in the horn inner conductor is also important because of the effect on the hight energy flux. This error dominates for the neutrino flux error above around $1.2 \mathrm{GeV}$. 


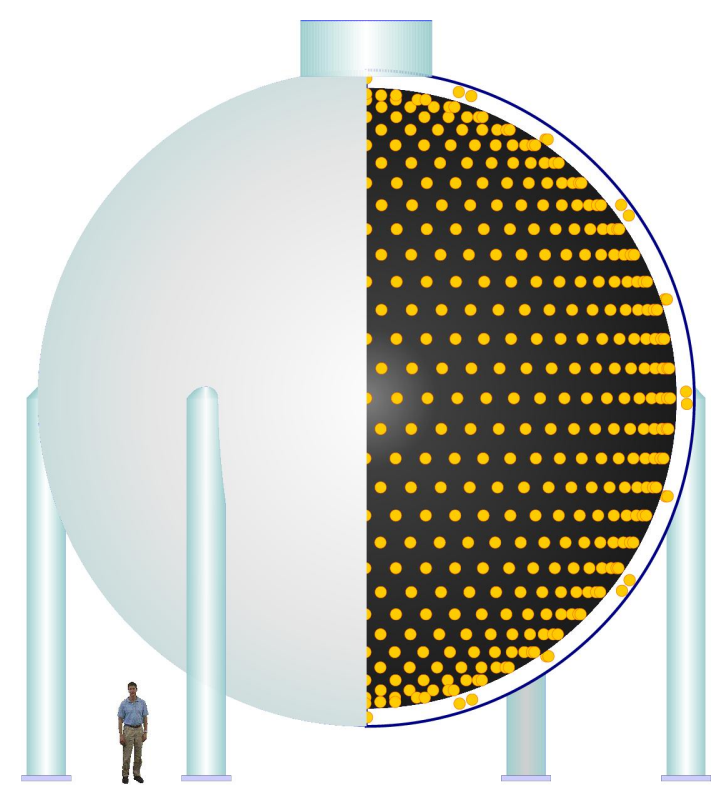

Figure 7.6: A schematic drawing of the MiniBooNE detector showing the signal and veto regions.

Note that the absolute POT error $(\sim 2 \%)$ is only assigned for an absolute measurement, for example the absolute cross section measurement in Chapter 10

\subsection{MiniBooNE detector}

The MiniBooNE detector consists of a spherical $12.2 \mathrm{~m}$ diameter tank filled with mineral oil. Figure 7.6 shows a schematic view. The inside of the tank is separated into two regions via an optical barrier at a radius of $574.6 \mathrm{~cm}$, into inner (signal), and outer (veto) regions. Details of detector structure, simulation, and error analysis are available in [113, 116, 124]. 


\subsubsection{Photo multiplier tubes (PMTs)}

A fiducial region is defined in the inner tank region, where an array of 1280 8" photo multiplier tubes (PMTs) are mounted. The veto region contains an array of 2408 " PMTs. A photo of the PMTs are shown in Fig. 7.7. MiniBooNE uses 1,198 Hamamatsu R1408 PMTs recouped from the LSND experiment and 322 Hamamatsu R5912 PMTs newly purchased. They have different properties (dark currents, timing resolutions, saturation points, etc) and their differences are taken into account in the analysis of the data. The PMTs are low threshold $(\sim 0.2$ photo electron $(\mathrm{PE}))$ and high gain $\left(\sim \times 10^{7}\right)$. This is required for sufficient sensitivity to low-light events. These properties were quantified [124] and in particular, the angular dependence of the photon detection efficiency was measured and implemented in the detector simulation. 


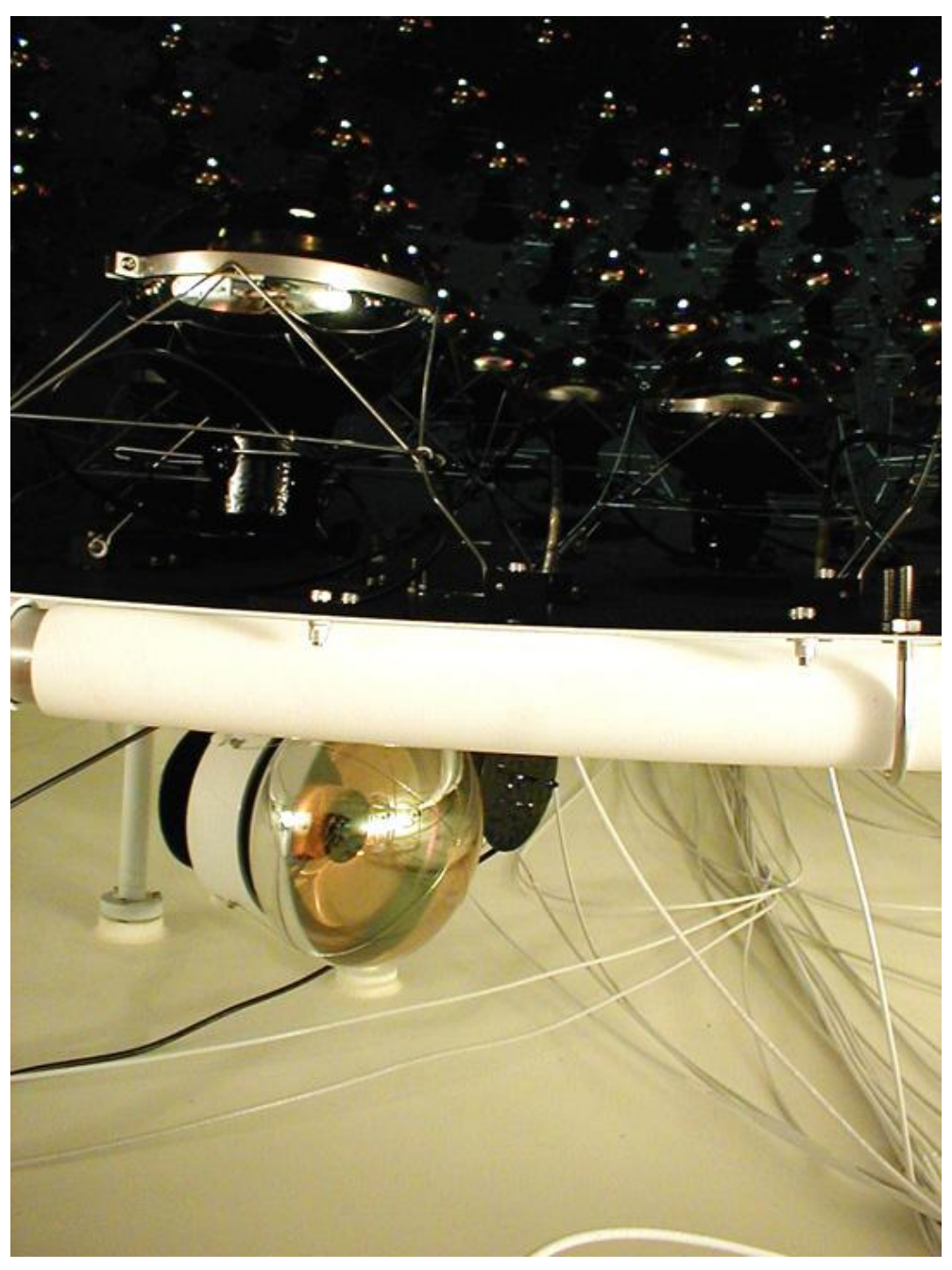

Figure 7.7: A photo showing the the inner (top) and outer (bottom) tank regions. Notice that the inner tank is painted black to reduce reflections which may distort the timing information used to reconstruct interaction vertices. The outer tank is painted white to maximize light collection in the veto in order to reject the particles coming from outside of the detector. 


\subsubsection{Mineral oil}

Cerenkov and scintillation light is emitted from charged particles traversing the detector volume. The optical photons travel through the Marcol 7 light mineral oil (from Exxon/Mobil) Therefore, the properties of mineral oil, especially optical properties (attenuation, Rayleigh/Raman scattering, re-emission spectrum and time constants, etc) are very important to understand. In practice, the uncertainties in the optical properties of mineral oil dominate the detector related errors. The optical properties were measured using a number of methods, and are summarized in [116, 125].

\subsubsection{Electronics}

After traversing the mineral oil, photons impinge on the photo-cathode of the PMTs and create photoelectrons (PEs). The resulting signals are routed to the electronics system where they are amplified and digitized. The PMT signals above a set threshold are digitized and recorded with an 8 bit flash analog-to-digital converter (FADC) sampling at $10 \mathrm{MHz}$ (100 ns). Both time and charge information are recorded within the $19.2 \mu \mathrm{s}$ DAQ window. Since a typical single PE signal results in $\sim 10$ ADC counts, an ADC channel is saturated by 20-30 PE signal. The thresholds of the discriminators are 0.1-0.2 PE, and the associated uncertainty is propagated. Since a larger charge fires the discriminator earlier, the calculated time depends on the charge. This slewing effect is corrected for, as well as an estimated error in the process. The PMTs and electronics are calibrated continuously via a $(3.33 \mathrm{~Hz})$ laser system in the detector tank.

\subsubsection{Energy calibration}

The absolute energy scale is determined from muon-decay ("Michel") electrons, for which decay spectrum is very well known. In addition, using a muon tracker system, consisting of 
an array of scintillator paddles above the detector combined with scintillation cubes hung in the tank, the range of muons is made to correspond with the light seen in the detector.

\subsubsection{Detector error summary}

In summary, the following contributions are considered in the detector error analysis:

- PMT angular efficiency;

- old/new PMT relative efficiency;

- reflections in the tank;

- oil density and thermal expansion;

- oil chemical composition;

- oil optical properties, including extinction length, Rayleigh/Raman scattering, refractive index, scintillation yields, fluorescence yields, UV fluorescence yields, time constant of each fluor, scaling factor for Čerenkov light, Birks' constants;

- discriminator threshold and slewing variation.

The optical properties of oil is the largest contribution to the detector error. Correlations are accounted for in the error analysis and a multi-simulation method ("Multisim") is employed which is described in Sec. 10.6 .

The uncertainties in oil density, thermal expansion, and chemical decomposition propagates to an error in the absolute number of target particles, so these errors are applied for the absolute cross section measurement (Chapter 10 , however, the effect is small $(\sim 0.4 \%)$. 


\subsection{MiniBooNE cross section model}

\subsubsection{The nuance event generator}

In order to model the neutrino signal of interest and to estimate backgrounds, a neutrino event generator is required to estimate the event rates of each neutrino interaction type. MiniBooNE uses the nuance event generator [126] which produces the interaction rates for 99 neutrino interaction types given an input neutrino flux. The original nuance code was written for water Čerenkov detectors, such as Super-Kamiokande, so it has been necessary to modify many parts of this code.

The following models are used for the neutrino interaction calculations in nuance:

- the relativistic Fermi gas (RFG) model by Smith and Moniz's formalism for QE scattering from nucleons [16] (Sec. 3.3),

- the Rein and Sehgal model for resonance interactions [127],

- the GRV98 based parton distribution functions (PDFs) for deep elastic scattering (DIS) cross section [128, 129].

Figure 7.8 shows the breakdown of neutrino interaction types as predicted for MiniBooNE. The major interactions are:

$39 \%$ charged current quasi-elastic (CCQE), $\nu_{\mu}+p \rightarrow \mu^{-}+n$;

$16 \%$ neutral current elastic (NCE), $\nu_{\mu}+p(n) \rightarrow \nu_{\mu}+p(n)$

$25 \%$ charged current one $\pi^{+}$production $\left(\mathrm{CC} 1 \pi^{+}\right), \nu_{\mu}+p(n) \rightarrow \mu^{-}+\pi^{+}+p(n)$;

$4 \%$ charged current one $\pi^{\circ}$ production $\left(\mathrm{CC} 1 \pi^{\circ}\right), \nu_{\mu}+n \rightarrow \mu^{-}+\pi^{\circ}+p$;

$4 \%$ neutral current one $\pi^{ \pm}$production $\left(\mathrm{NC} 1 \pi^{ \pm}\right), \nu_{\mu}+p(n) \rightarrow \mu^{\mp}+\pi^{ \pm}+n(p) ;$ 


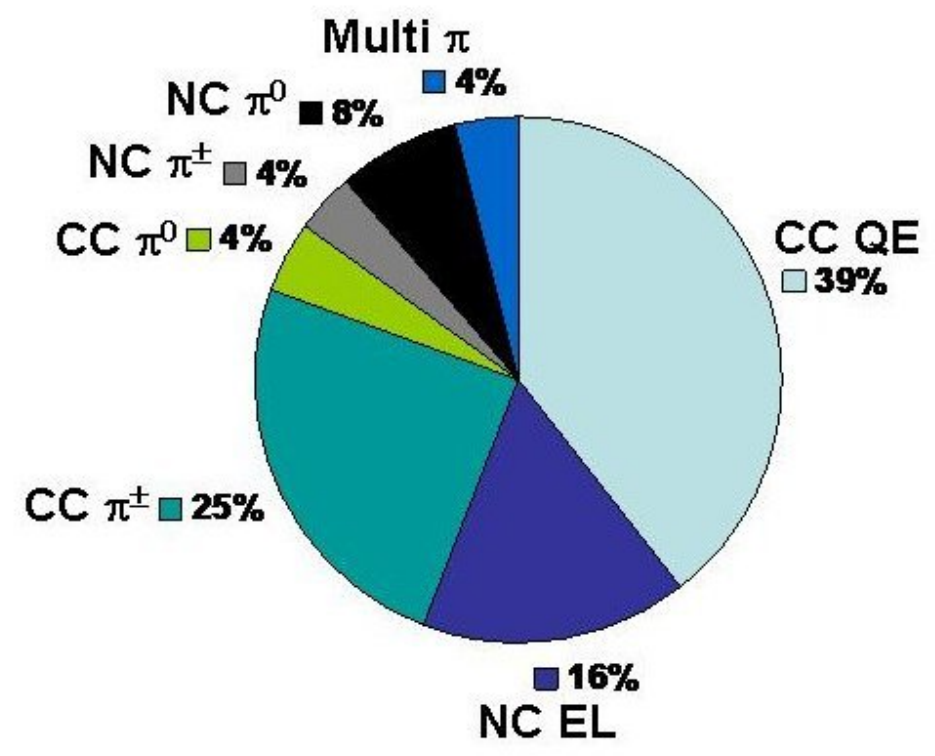

Figure 7.8: Event fractions predicted by nuance for MiniBooNE.

$8 \%$ neutral current one $\pi^{\circ}$ production $\left(\mathrm{NC} 1 \pi^{\circ}\right), \nu_{\mu}+p(n) \rightarrow \mu^{-}+\pi^{\circ}+p(n)$;

$4 \%$ others, multi pion production (multi $\pi$ ), deep inelastic scattering (DIS), etc.

\subsubsection{Charged Current Quasi-Elastic (CCQE) scattering}

CCQE scattering is the dominant neutrino interaction type in MiniBooNE, and comprises roughly $40 \%$ of the total events. The RFG model employed here uses BBA03 form factors [130] instead of simple dipole form for Dirac (Eq. 3.59) and Pauli (Eq. 3.60 form factors. But the axial current form factor is assumed to be of dipole form (Eq. 3.61), with axial mass $M_{A}=1.234 \mathrm{GeV} / \mathrm{c}^{2}$. Although the contribution is small, the pseudo scalar form factor, derived from PCAC (Sec. 3.2.5), is included. The scalar and axial tensor form factors (second class) are set to zero as implied from G-parity conservation (Sec. 3.2.8). We use $220 \mathrm{MeV} / \mathrm{c}$ for the Fermi momentum and $34 \mathrm{MeV}$ for the binding energy of carbon [131, 
as extracted from electron scattering experiments with associated errors of $30 \mathrm{MeV} / c$ and $9 \mathrm{MeV}$, respectively. For free nucleon scattering, the RFG model is used but with the Fermi momentum and binding energy set to zero, therefore, the RFG model employed consistently describes both free and bound nucleon scattering.

The binding energy value of $34 \mathrm{MeV}$ is modified from what is measured from electron scattering $(25 \mathrm{MeV})$, for the following reasons [132]. In electron scattering, the charge of the target nucleon within carbon is not changed, so $\Delta T_{3}=0$, and the nuclear transition is the combination of total isospin changes of 0 or 1 . But in neutrino CCQE scattering the transition occurs through the $\mathrm{A}=12$ isotriplet:

$$
\begin{array}{ccc}
{ }^{12} C(T=0) & \stackrel{\gamma}{\rightarrow} \quad{ }^{12} C^{*}\left(\Delta T=0,1 \Delta T_{3}=0\right) \\
{ }^{12} C(T=0) & \stackrel{W}{\rightarrow} \quad{ }^{12} B\left(\Delta T=1, \Delta T_{3}=-1\right) \\
{ }^{12} C(T=0) & \stackrel{Z}{\rightarrow} \quad{ }^{12} C^{*}\left(\Delta T=1, \Delta T_{3}=0\right) \\
{ }^{12} C(T=0) & \stackrel{W}{\rightarrow} \quad{ }^{12} N\left(\Delta T=1, \Delta T_{3}=+1\right)
\end{array}
$$

CCQE scattering is a $\Delta T_{3}=+1$ transition which means the total isospin changes by 1 , and this is a pure Gamov-Teller (GT) transition if all forbidden corrections are ignored [17]. Since the total isospin 1 state is more repulsive, the binding energy for neutrino CCQE is larger than for electron scattering. The energy difference between $T=0$ and $T=1$ is estimated from the symmetry energy. Including the Coulomb energy, we decided to use $34 \mathrm{MeV}$ for the binding energy of Carbon. We have also introduced an empirical parameter " $\kappa$ ", to increase the effects of Pauli blocking in the model which suppresses low $Q^{2}$ events. More details are provided in Chapter 8.

Since $M_{A}$ and $\kappa$ are measured in the CCQE analysis, errors in these parameters are not included for the CCQE analysis. However, the resultant errors from the fit [4, 39] are considered in other analyses, including the $\nu_{e} / \bar{\nu}_{e}$ appearance oscillation analysis [67, 68, 133] and the $\nu_{\mu} / \bar{\nu}_{\mu}$ disappearance oscillation analysis [134]. 


\subsubsection{Neutral current elastic (NCE) scattering}

NCE scattering is governed by identical expressions as for CCQE after the replacement of appropriate form factors (Sec. 3.2.3). The notable difference is that NCE scattering is sensitive to isoscalar form factors, as can be seen in Eq. 3.26 to 3.31 . Especially, the $Q^{2} \rightarrow 0$ limit of the isoscalar terms of axial form factors (Eq. 3.64 and 3.63) are proportional to the strange quark contribution to the nucleon spin $(\Delta s)$. This is discussed in Appendix A. The error in $\Delta s$ is accounted for in this analysis. However, since contamination of NCE in the CCQE sample is very small $<1 \%$, the error from $\Delta s$ is negligible.

\subsubsection{Resonance interactions}

The baryonic resonance interaction is the primary source of one pion production for MiniBooNE,

$$
\begin{aligned}
& \nu_{\mu}+p \rightarrow \Delta^{++} \rightarrow \mu^{-}+\pi^{+}+p \\
& \nu_{\mu}+n \rightarrow \Delta^{+} \rightarrow \mu^{-}+\pi^{+}+n, \text { etc } .
\end{aligned}
$$

The nuance model employs a relativistic harmonic oscillator quark model of Rein and Sehgal [127]. The original code of nuance was modified to take into account the pion angular distribution due to the spin structure of the resonance states [121]. In total, 18 resonances are used to contribute to the invariant mass $W<2 \mathrm{GeV}$, but the $\Delta(1232)$ resonance dominates at this energy scale. For reactions with bound nucleons, a uniform Fermi momentum and constant binding energy are given. Therefore, Pauli blocking is implemented. In-medium effects for the width of resonances are not taken into account. A different (from CCQE) axial mass and error for one pion production are used, $M_{A}^{1 \pi}=1.10 \mathrm{GeV} / \mathrm{c}^{2}$. The uncertainty on $M_{A}^{1 \pi}$ is one of the dominant contributions to the cross section error for the CCQE exclusive measurement (Chapter 8), because CC1 $\pi$ is the dominant background. This problem has been (partially) surmounted with a simultaneous measurement of CCQE 
and $\mathrm{CC} 1 \pi$ (Chapter 9 and 10 ). A different axial mass is assumed for multi pion production processes, $M_{A}^{N \pi}=1.30 \mathrm{GeV} / \mathrm{c}^{2}$, but the contribution from these channels are small.

\subsubsection{Coherent pion production}

Pions are also produced in the coherent interaction of neutrino with carbon nuclei,

$$
\begin{aligned}
& \nu_{\mu}+X \quad \rightarrow \mu^{-}+\pi^{+}+X^{\prime} \\
& \nu_{\mu}+X \rightarrow \nu_{\mu}+\pi^{o}+X^{\prime} .
\end{aligned}
$$

Coherent scattering has a distinct features in the angular distribution of both muons and pions. The KEK-to-Kamioka (K2K) experiment in Japan [135], and MiniBooNE [121] have measured the fraction of pions produced coherently. $\mathrm{K} 2 \mathrm{~K}$ found zero contribution for coherent $C C \pi^{\circ}$ production. MiniBooNE found a non-zero value in coherent $N C \pi^{\circ}$ production but $\sim 33 \%$ smaller than model prediction. The latest result from the SciBar Booster Neutrino Experiment (SciBooNE) supports the non existence of $C C \pi^{o}$ coherent production [32]. The original model of Rein and Sehgal predict a sizable amount of coherent pion production, however some models predict much smaller cross sections, for example Fig. 2 of Ref. [32]. Because of the current confusion of both theory and the experiment, a large error is assumed for the coherent fraction. The coherent event fraction is tuned from data (Sec. 9.6) by changing the axial mass parameter for coherent pion production, $M_{A}^{c o h}=1.03 \mathrm{GeV} / \mathrm{c}^{2}$.

\subsubsection{Final State Interactions (FSIs)}

A neutrino interaction is modeled having instantaneous contact with an incoherent nucleon (impulse approximation, or IA). Then the products of the interaction are propagated through the nucleus in the event model. The starting point is based on the measured density distribution of ${ }^{12} C$ [136], and the nucleons have a radially-dependent density distribution 
and Fermi momentum. Through this process, hadrons in the final state may re-interact in the nucleus (FSI). To simulate this, nuance calculates the interaction probabilities for various processes every $0.3 \mathrm{fm}$ step until the particles leave the $\sim 2.5 \mathrm{fm}$ radius spherical carbon atom [136]. The interaction probabilities are based on external data of $\pi-N$ and $N-N$ cross sections and angular distributions [137-139], as well as the carbon nuclear density. To mimic $\Delta$ de-excitation by $\Delta-N$ interactions,

$$
\Delta+N \rightarrow N+N
$$

a constant probability of de-excitation is applied for every $\Delta-N$ interaction. We use $20 \%$ for $\Delta^{+}+N$ and $\Delta^{\circ}+N$, and $10 \%$ for $\Delta^{++}+N$ and $\Delta^{-}+N$. These values were chosen from data-MC comparisons in the $\mathrm{K} 2 \mathrm{~K}$ experiment [140]. Thus there is no energy dependence of the $\Delta-N$ cross section for the de-excitation considered. After the re-interaction, the first step is changed to $1.0 \mathrm{fm}$ and the density distribution is modified to prevent too much re-scatterings [141, 142].

Among all the FSI, pion absorption and pion charge exchange are the important processes contributing to the uncertainty in the CCQE analysis:

$$
\begin{aligned}
& \pi^{+}+X \quad \rightarrow X^{\prime} \\
& \pi^{+}+X \rightarrow \pi^{o}+X^{\prime} .
\end{aligned}
$$

Because they result in the same final state as CCQE, CC1 $\pi$ interaction with pion absorption and pion charge exchange are intrinsic backgrounds. Figures 7.9 show a comparison of data and the MiniBooNE-tuned nuance model for pion absorption and charge exchange total cross sections as a function of pion momentum. As is evident from the figure, nuance describes data within its errors. These errors are taken into account for the all analysis. 

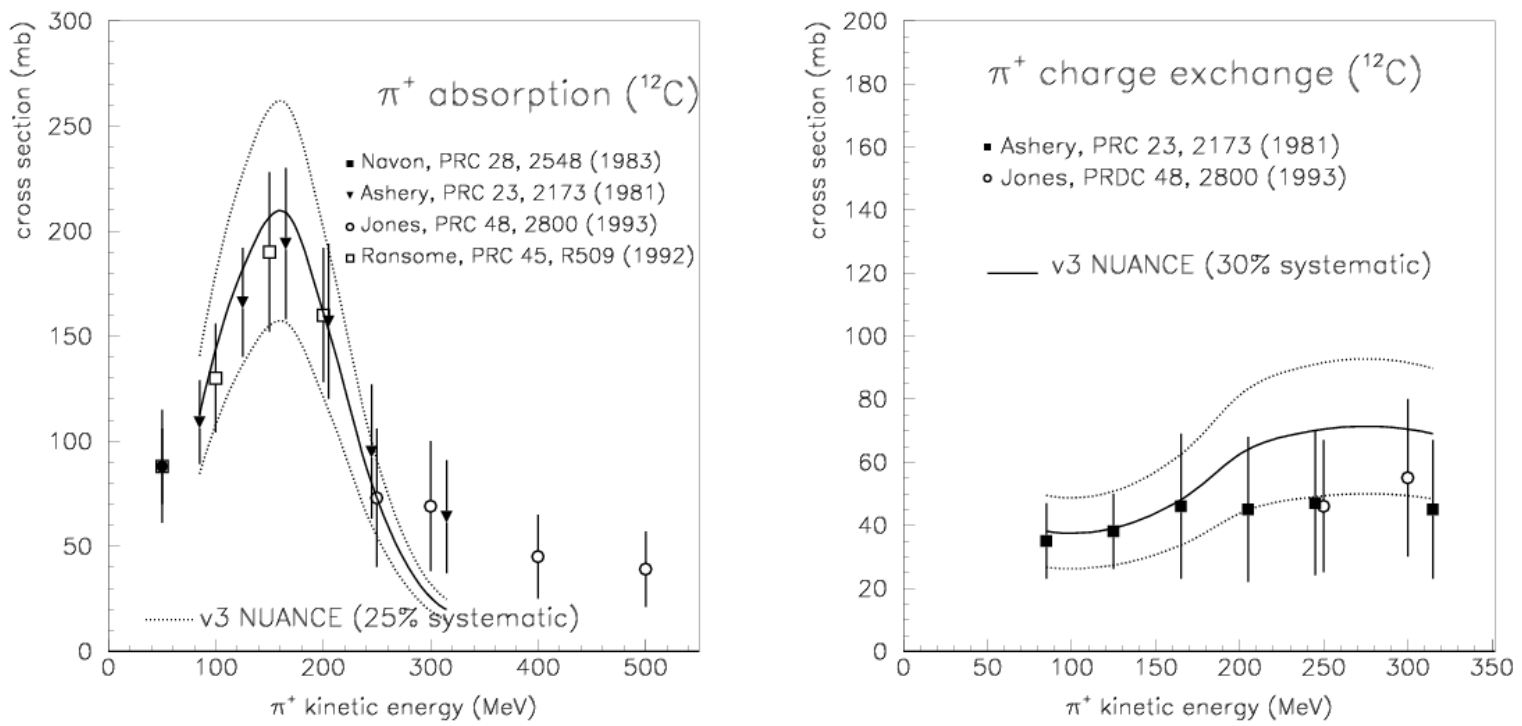

Figure 7.9: Data [143-146] and MiniBooNE-tuned nuance comparison for pion absorption (left) and pion charge exchange (right) cross section as a function of the pion kinetic energy.

\subsubsection{Re-interactions in the detector}

Although nuance is used to quantify errors from pion absorption and charge exchange in the nuclei, we need to take into account additional errors from these processes in the propagation of pions in the detector media. Figure 7.10 shows a comparisons of data [143] with results from two different hadronic interaction packages used in the GEANT3 [147] based detector simulation. Although the default hadronic interaction package is GFLUKA [148], we decided to use GCALOR [149], because it utilizes more realistic total cross sections for these processes for almost all pion energies. The errors from pion absorption and charge exchange uncertainties in the detector media were estimated from the difference of data and the GCALOR model. 

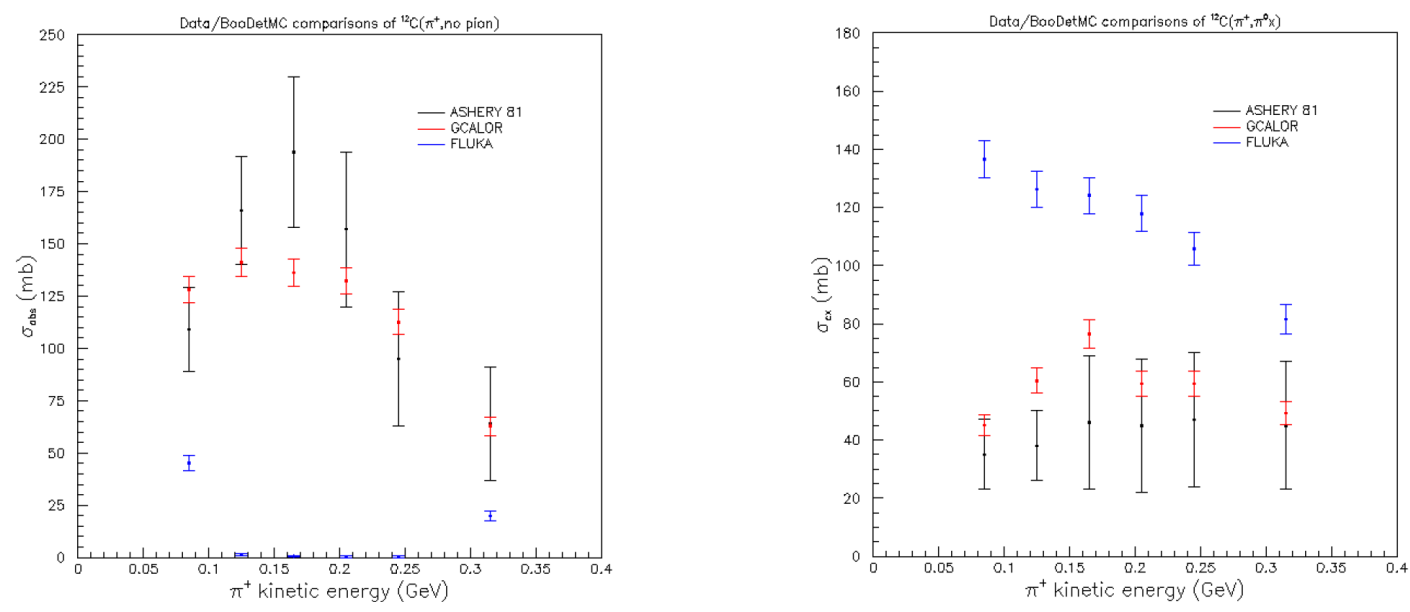

Figure 7.10: Data [143] compared to two hadronic interaction packages result for pion absorption (left) and charge exchange (right) cross section.

\subsubsection{Cross section error summary}

In summary, the following parameters and processes were considered for cross section errors:

- CCQE axial mass $\left(M_{A}\right)$ and Pauli blocking parameter $(\kappa)$;

- Fermi momentum $\left(P_{F}\right)$ and binding energy $\left(E_{B}\right)$ of carbon;

- strange quark spin contribution in a nucleon $(\Delta s)$;

- axial mass for one pion $\left(M_{A}^{1 \pi}\right)$ and multi pion $\left(M_{A}^{N \pi}\right)$ resonance production;

- $\mathrm{CC} 1 \pi$ shape error;

- pion absorption, charge exchange, and $\Delta$ de-excitation in the carbon nuclei;

- pion absorption and charge exchange in the detector media;

- axial mass for $\mathrm{CC}$ and $\mathrm{NC}$ coherent pion production $\left(M_{A}^{c o h}\right)$;

- DIS scale parameter; 
- $\nu_{e}$ flux estimation error from $\nu_{\mu} \mathrm{CCQE}$ measurement;

- neutral current $\pi^{o}$ production related error (coherent fraction, $\Delta$ radiative decay, etc).

The errors resulting from $M_{A}$ and $\kappa$ are important for other analyses, but they do not contribute here because they result from the measurements. The errors on $P_{F}$ and $E_{B}$ are rather large, $14 \%$ and $26 \%$, but the contribution is relatively small. The $25 \%$ error on $M_{A}^{1 \pi}$ gives large variation in the background of CCQE. This is the biggest cross section error for CCQE analysis in Chapter 8, but was not applied for the analysis of Chapters 9 and 10. Since the $M_{A}^{1 \pi}$ error practically effects only normalization of pion production events, an additional error for the shape of $\mathrm{CC} 1 \pi$ background to $\mathrm{CCQE}$ was applied in the analysis of Chapter 8 , but not in that of Chapters 9 and 10 since the background was determined from the measurement.

We assume $25 \%$ and $30 \%$ error for pion absorption and charge exchange, as well as $100 \%$ for $\Delta$ de-excitation in the carbon nuclei. Strictly speaking, some part of the error of pion absorption and $\Delta$ de-excitation are double-counted, the the conservative approach was chosen. In addition, the error from pion absorption and charge exchange was included in the detector simulation at $35 \%$ and $50 \%$ respectively.

The error on $M_{A}^{c o h}$ is $14 \%$ for the methods of Chapter 8 , but was increased to $100 \%$ for Chapter 9 and 10 to take into account recent results from K2K, MiniBooNE and SciBooNE [32, 121, 135]

MiniBooNE used a blind analysis for the $\nu_{e}$ appearance oscillation results. Therefore, the error on $\nu_{e}$ was minimized as much as possible without using actual data. Since the $\nu_{e}$ flux or $\nu_{e} \mathrm{CCQE}$ measurement is constrained from the measurement of $\nu_{\mu} \mathrm{CCQE}$ (Chapter 8), any disconnection between those two channels must be accounted for with additional errors. An energy-dependent difference error between $\nu_{e} \mathrm{CCQE}$ and $\nu_{\mu} \mathrm{CCQE}$ and an energy spectrum 
error for $\nu_{e} \mathrm{CCQE}$ itself were employed. Of course, these errors are not applied to any cross section analyses.

Since neutral current $\pi^{\circ}$ production $\left(\mathrm{NC} \pi^{\circ}\right)$ events are the single biggest error for the when identifying isolated electrons (the signal for $\nu_{e}$ appearance oscillation), errors on this process must be carefully set. These are determined from the measured $\pi^{\circ}$ spectrum [121, but again, these errors are not applied (and are negligible anyway) for the CCQE analysis.

\subsection{Event reconstruction}

The details of event reconstruction are available in [113.

\subsubsection{Hits}

Although the information about particle energies is conveyed by the charge measured by each PMT, the number of PMT fired in each event, called hits, is a simple yet very powerful proxy for the total energy deposited in the tank. For example, by requiring that the number of hits in the veto region (veto hits) is less than 6 rejects $99.99 \%$ of the particles that leave the fiducial volume for the CCQE analysis. And, since the end point of Michel spectrum is $\sim 53 \mathrm{MeV}$ corresponding to $\sim 100$ hits in the signal region (tank hits), requiring tank hits greater than 200 eliminates most of the Michel electrons and is a powerful cut to select muons.

\subsubsection{Subevent}

One event in the data stream corresponds to one beam trigger, or the sequence of the time and charge information from all PMTs in the 19.2 $\mu$ s DA window. Since individual particle events in the tank create a group of hits with clustered time values, it is convenient 


\subsection{EVENT RECONSTRUCTION}

to define a "subevent", or well-separated timing cluster, to sort out a particular data event. Figure 7.11 shows an example where three subevents can be observed. This is a typical charged current one pion production $(\mathrm{CC} 1 \pi)$ event candidate:

$\nu_{\mu}+p(n) \underset{\mathbf{1}}{\rightarrow}+\mu^{-}+\pi^{+}+p(n) \rightarrow \mu^{-}+\mu^{+}+\nu_{\mu}+p(n) \stackrel{\mathbf{2}}{\rightarrow} e^{-}+\bar{\nu}_{e}+\nu_{\mu}+e^{+}+\nu_{e}+\bar{\nu}_{\mu}+\nu_{\mu}+p(n)$

The first subevent corresponds to the primary $\mu^{-}$Čerenkov emission. Then there are two subsequent subevents, corresponding to Čerenkov emission by either $e^{-}$or $e^{+}$from a $\mu^{-}$ or $\mu^{+}$decay. Also notice that the first subevent contains more than 200 tank hits - this means this subevent is likely to be made by an energetic charged particle, like a muon. The second and third subevents have less than 200 tank hits, likely to be made by low energy charged particles, for example, Michel electrons. For the analysis of MiniBooNE data, the total number of subevents is used to classify events into physics categories. For example, an event with 3 subevents, like that shown in Fig. 7.11, is a $\mathrm{CC} 1 \pi$ event candidate. If it contained 2 subevents, most likely, is was a charged current quasielastic (CCQE) event candidate,

$$
\nu_{\mu}+n \rightarrow \mu^{-}+p \rightarrow e^{-}+\bar{\nu}_{e}+\nu_{\mu}+p
$$

Events are mis-classified when subevents are lost. For example, the $\mu^{-}$capture [150] process reduces the total number of subevents in a particular event, because a captured muon decayin-orbit (DIO) does not emit a Michel electron. This process is well known and measured to occur for approximately $\sim 8 \%$ of $\mu^{-}$stopping in mineral oil. Other example is the pion absorption process. Again, the absorbed pion does not decay to a muon, and hence there is no Michel electron emission. This process is also modeled in the detector simulation, however large errors on pion absorption results in large errors on the estimated number of 2-and 3-subevent events. This problem is considered in Chapter 9 


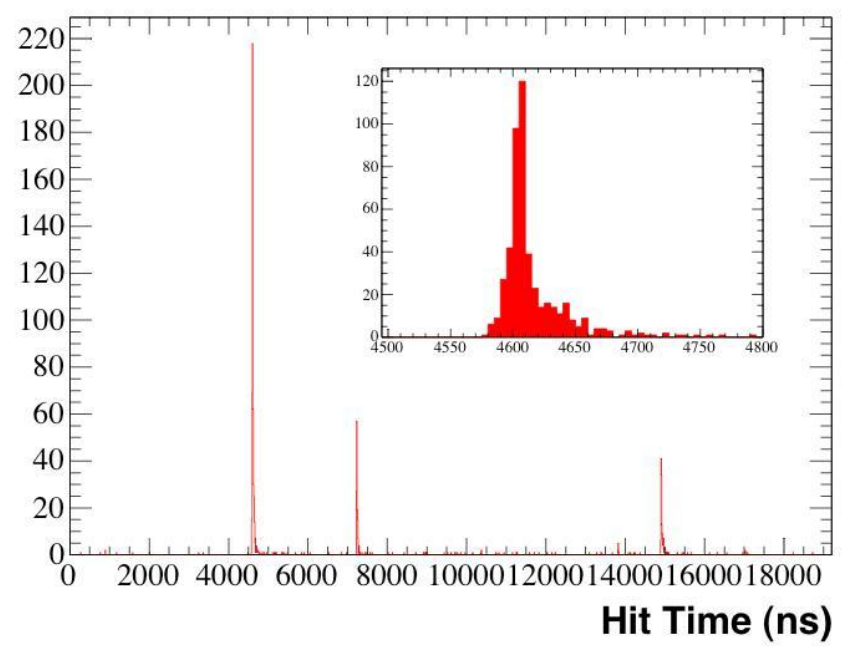

Figure 7.11: Number of tank hits as a function of time in a $\mathrm{CC} 1 \pi$ candidate event.

\subsubsection{Particle track fitter}

So far, the MiniBooNE detector has been explained as a calorimeter. However the charge and time information as well as topological information from individual PMT hits provides particle track information as well. Each PMT provides five quantities for each event which can be used to find particle tracks,

$$
\left\{\left(x^{k}, y^{k}, z^{k}\right), t^{k}, q^{k}\right\}, k=1, \cdots, 1280 \text { (number of PMTs). }
$$

To specify a single particle track, we need the following parameters,

- $\operatorname{position}(x, y, z)$;

- time $(t)$;

- direction $(u x, u y, u z)$;

- $\operatorname{energy}(E)$. 


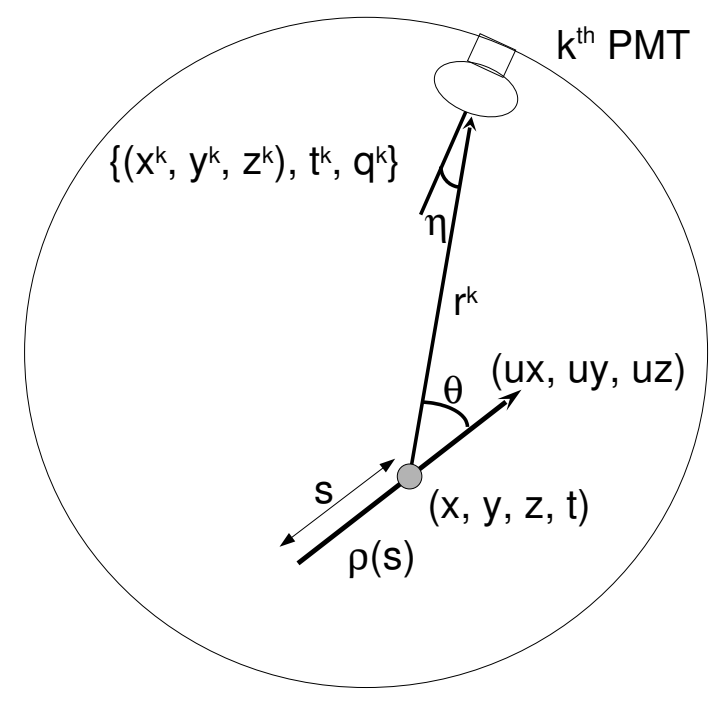

Figure 7.12: An illustration of the particle track fitter. A particle is moving in direction (ux,uy,uz). Čerenkov light emitted from the point $(\mathrm{x}, \mathrm{y}, \mathrm{z})$ at time $\mathrm{t}$, in direction $\theta$, is observed by $k^{t h}$ PMT with angle $\eta$ with respect to the normal vector. Under the point-like approximation, this $(\mathrm{x}, \mathrm{y}, \mathrm{z}, \mathrm{t})$ can be regarded the track center. However, in the reality, emission density $\rho(\mathrm{s})$ is a function of s along the track.

We use the vector $\mathbf{x}$ to denote this parameter set. Since the charge and time information predicted for the $k^{\text {th }}$ PMT is a function of $\mathbf{x}$, one can form a likelihood function to find $\mathbf{x}$ from PMT charge and time information. Figure 7.12 shows the situation for a simulated track. Since Čerenkov emission is directional, we can use this information from all PMTs to find a particle position, time, direction, and energy.

The surprising fact is that most of Čerenkov light goes into a limited solid angle with respect to the particle track. Furthermore, the Čerenkov emission is an instantaneous process, so most of emission goes into this narrow solid angle in a limited time period. Figures 7.13 and 7.14 show the solid angle and time distributions of Čerenkov light. This implies that one can approximate an event with all Čerenkov light emitted from single point, 


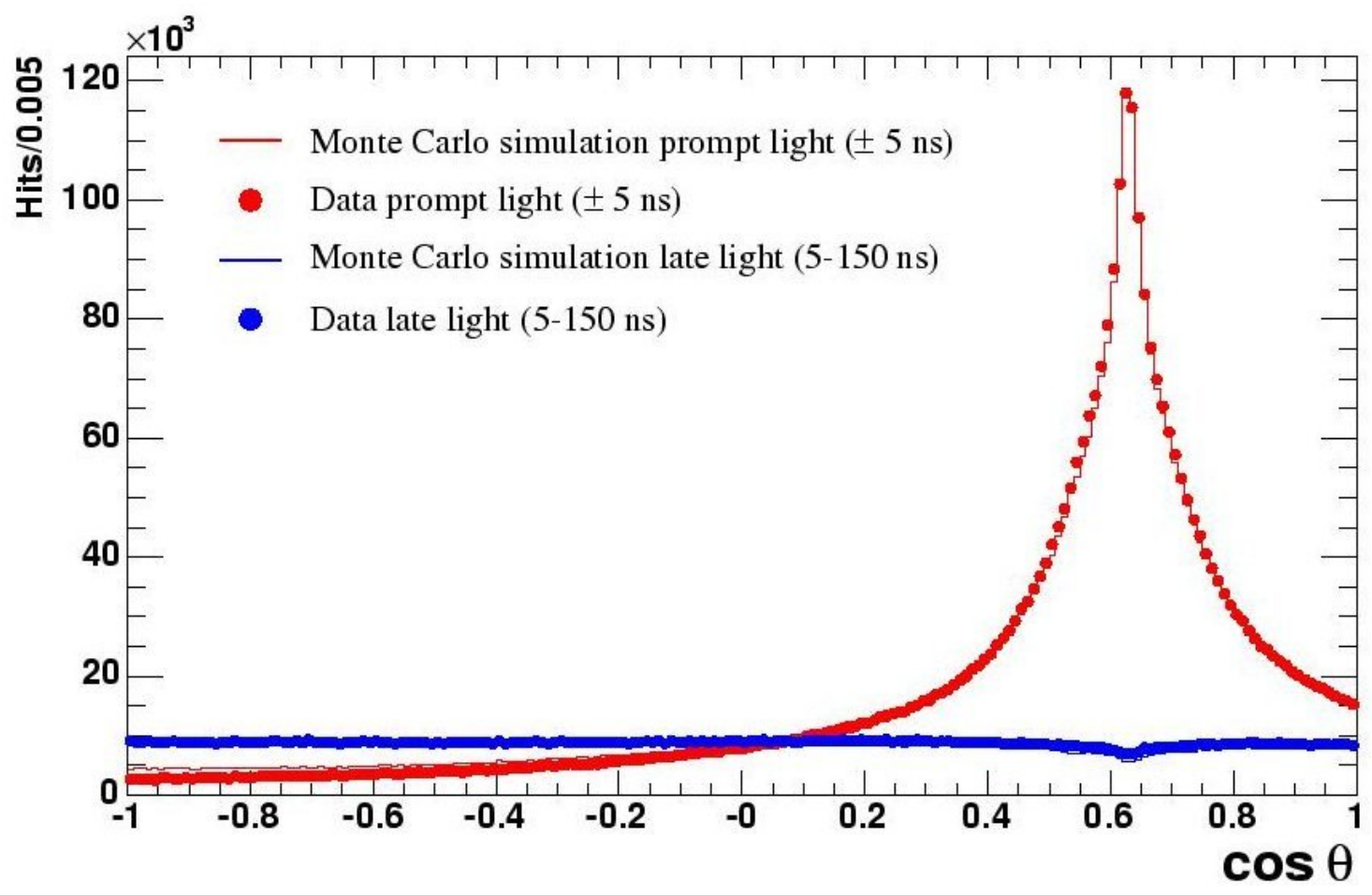

Figure 7.13: Emission power from an electron track as a function of the solid angle along the track. The red curve shows the power of the strong directional Cerenkov emission, and the blue curve shows the power of the isotropic scintillation emission.

represented by the track center. From this, the particle track position (called track center), time, direction, and energy, may be determined ( after some corrections about delayed and isotropic scintillation light).

Of course this point-like approximation is broken at some level, instead, we can introduce the emission density $\rho(\mathrm{s})$ as a function of s along the particle track. This track-based fitter dramatically improves angular resolution [113.

The point-like approximation fitter was used in the analysis of Chapter 8 , and trackbased fitter for that of Chapter 9 and 10. 


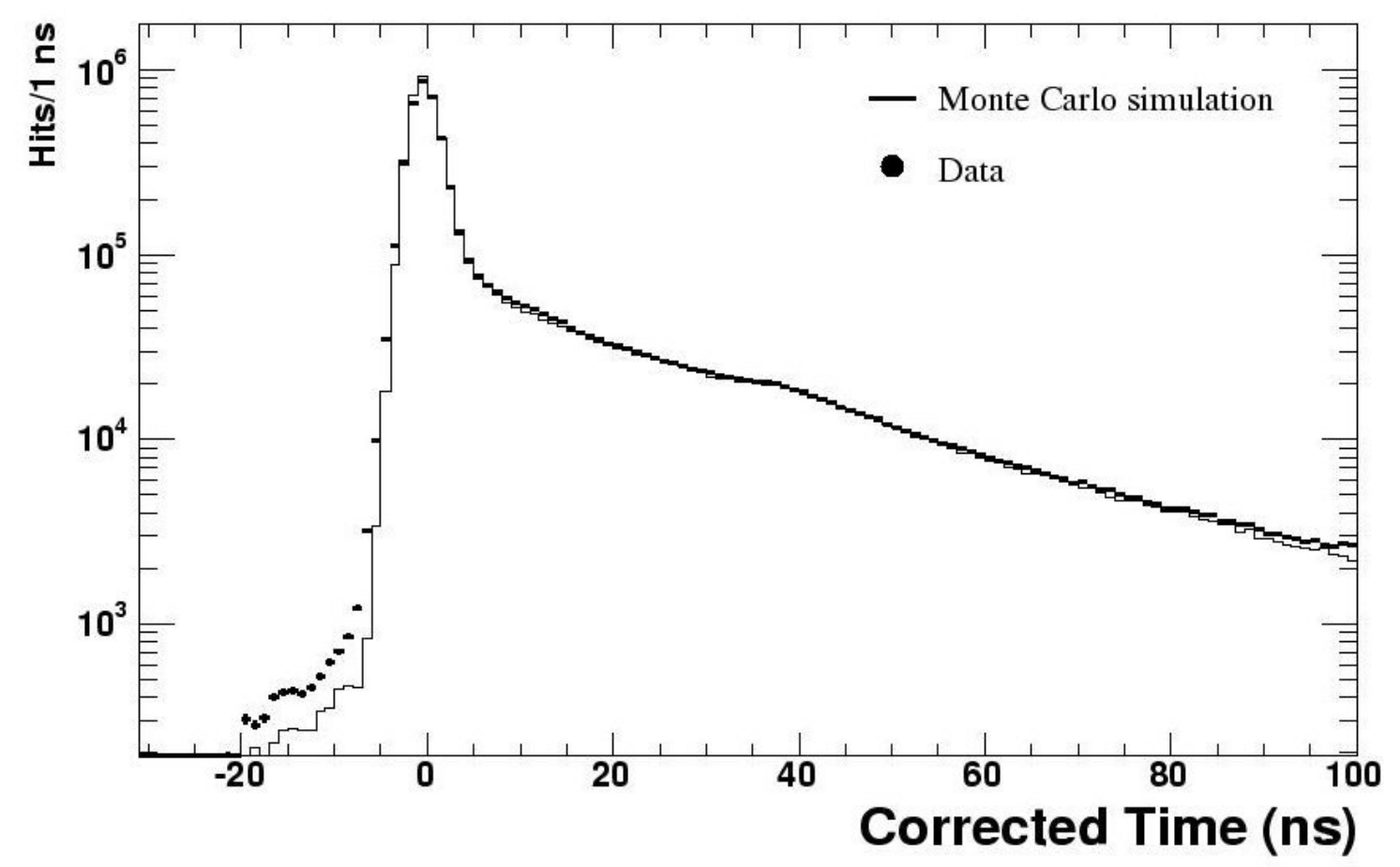

Figure 7.14: The corrected time for the emission of light from an electron track. The corrected time $t^{c}$ is defined by measured time $t^{k}$, emission time $t$, and photon propagation time $\frac{r^{k}}{c_{n}}$, (here $c_{n}$ is the speed of light in the oil $(=19.5 \mathrm{~cm} / \mathrm{ns})$ ). Then, $t^{c}=t^{k}-t-\frac{r^{k}}{c_{n}}$. The peak corresponds to the Čerenkov emission, and the tail is delayed scintillation light. 


\subsubsection{Particle ID}

The particle identification (PID) is done by using track features, which is the integrated information from all PMTs (Fig. 7.15). Fig. 7.16 is an example of actual event display of a stopping muon candidate event.

In particular, the likelihood ratio is simple, yet powerful estimator for particle ID under a hypothesis. The electron-to-muon $(\mathcal{L}(e / \mu))$ and electron-to- $\pi^{\circ}\left(\mathcal{L}\left(e / \pi^{\circ}\right)\right)$ likelihood ratio are constructed [113] for $\nu_{e}$ appearance oscillation analysis to reject muon and $\pi^{\circ}$ like tracks. In this thesis, we used the negative of $\log \mathcal{L}(e / \mu)$ to select muon like tracks (Chapter 9). 


\section{Cerenkov Light...}

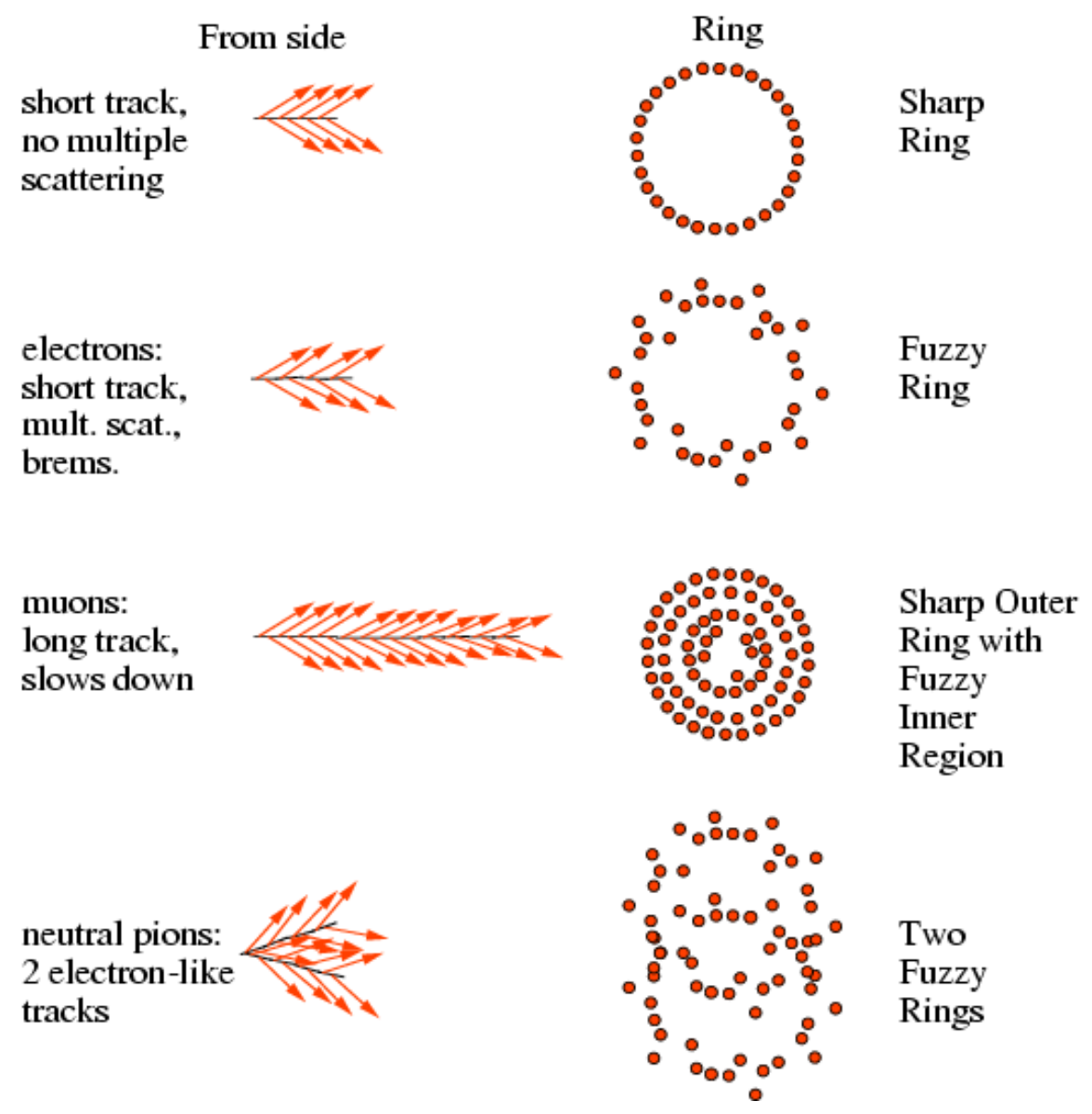

Figure 7.15: A cartoon illustrating PID. PID is done utilizing the features of tracks, originated in the charge, time, and the topological information from all PMTs. 


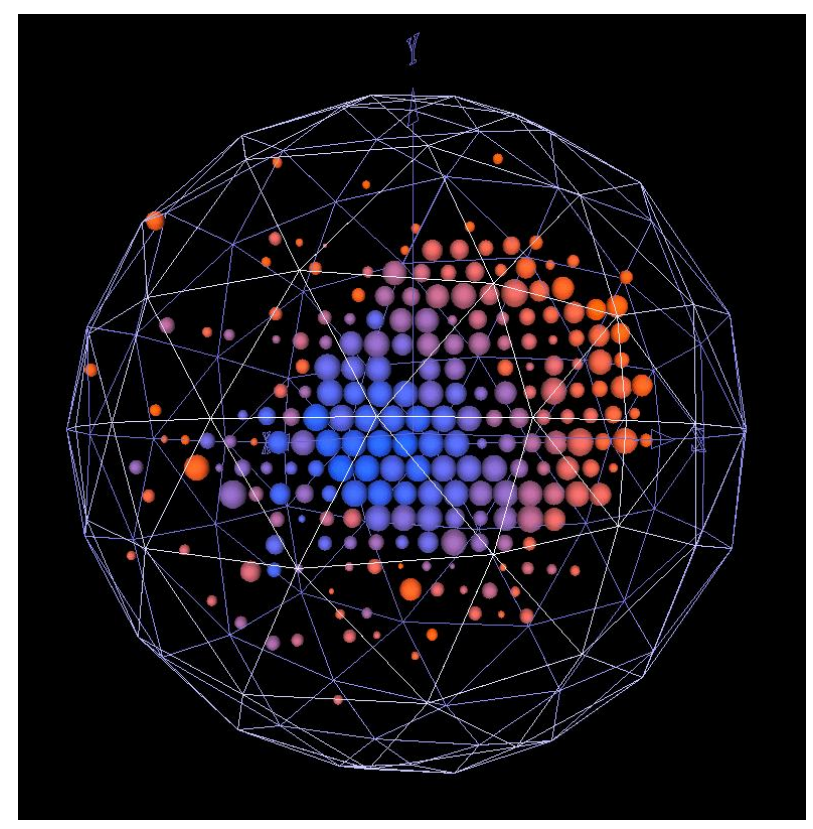

Figure 7.16: An event display for a muon candidate event. Each sphere represents a hit from a PMT, and the size and the color show charge and time information. As described in Fig. 7.15, a stopping muon is characterized with sharp edge and filled circle shape hits. 


\section{Chapter 8}

\section{CCQE measurement for}

\section{MiniBooNE oscillation physics}

\section{1 $\nu_{e}$ appearance oscillation analysis}

The results from and the details of the oscillation analysis are available in [67, 112 114].

The goal of MiniBooNE experiment is to test the observed LSND $\bar{\nu}_{e}$ appearance signal [69]. However, because of the blind analysis constraint, $\nu_{e}$ appearance signal candidates are forbidden to be analyzed. The muon neutrino charged current quasielastic $\left(\nu_{\mu} \mathrm{CCQE}\right)$ sample is a completely exclusive set from the electron neutrino CCQE ( $\nu_{e} \mathrm{CCQE}$ ) sample, which is the signal of $\nu_{e}$ appearance search. Thus, $\nu_{\mu}$ CCQE sample is very useful to check our machinery under the the blindness constraint and, therefore it is important for the $\nu_{e}$ appearance search. 


\subsubsection{The blind analysis}

MiniBooNE employed a blind analysis for the $\nu_{e}$ appearance oscillation analysis. Figure 8.1 shows schematically the concept of the blind analysis. The data are points in an $N$-dimensional space. For example, each event has associated quantities for number of veto hits, average time of hits, estimated particle energy, etc. Here, the data is shown in 3-dimensional space for the illustration. Then, the complete data set is divided into data subsets with various cuts. Each subset is called a "box". These boxes, specifically called "open boxes" contain various event types (CCQE candidates, $\mathrm{NC} \pi^{\circ}$ candidates, $\operatorname{cosmic}$ muons, etc.) and are designed to not contain oscillation candidate events. Then, the "open box" data are analyzed to understand and to tune the simulation and analysis tools. These results are then used for the analysis of the "closed box" events. This "closed box" uses cuts to select oscillation candidates, and has been defined as a signal box from the beginning of the experiment. This works well for MiniBooNE, because the definition of signal events are single isolated electrons,

$$
\nu_{\mu} \stackrel{?}{\rightarrow} \nu_{e}+n \rightarrow e^{-}+p
$$

So, the oscillation candidate events are exclusive and easily separated from other event types. Note, the boxes are not exclusive in general, so open boxes often overlap each other, but the closed signal box is completely exclusive. In the end, MiniBooNE used more than $99 \%$ of the data for these studies. In order to double-check the final results from the blind analysis, two independent oscillation analyses were used based on different particle reconstruction and identification algorithms. The closed box was finally opened in March, 2007 [67. 


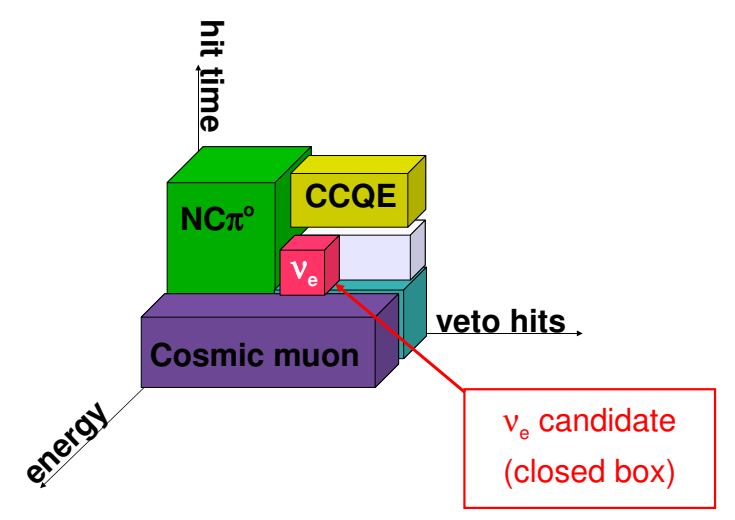

Figure 8.1: A schematic figure illustrating the MiniBooNE blind oscillation analysis. Note, the open boxes are not exclusive set in general, but the closed box is completely exclusive.

\subsubsection{Flux normalization and error constraint by $\nu_{\mu}$ CCQE}

The $\nu_{e}$ oscillation signal was determined with a simultaneous fit of $\nu_{e}$ and $\nu_{\mu}$ CCQE candidate events. This combined fit method [112 115] has great power for two reasons.

The first is flux normalization. Since MiniBooNE is single detector oscillation experiment, unlike K2K [81] or MINOS [82], we have a large uncertainty in the beam neutrino flux, large enough to hide at the $\sim 1 \%$ level any oscillation signal. This problem is solved by using the high statistics sample of $\nu_{\mu} \mathrm{CCQE}$ candidates to make an independent flux measurement. Figure 8.2 shows the general scheme. Since the combined fit technique fits both $\nu_{e} \mathrm{CCQE}$ and $\nu_{\mu} \mathrm{CCQE}$ simultaneously, $\nu_{e}$ oscillation candidate events $\left(\nu_{\mu} \rightarrow \nu_{e}\right)$ cannot be interpreted as an incorrect $\nu_{\mu}$ flux prediction. If events were interpreted as $\nu_{e}$ oscillation events but were due to an incorrect $\nu_{\mu}$ flux prediction, we would not see a good fit for $\nu_{\mu} \mathrm{CCQE}$ events. Therefore, a measurement of the $\nu_{\mu} \mathrm{CCQE}$ rate works as a flux monitor for the oscillation analysis.

The second reason is that the fit constrains the number of intrinsic $\nu_{e}$ events. The 
possible $\nu_{e}$ backgrounds are either misidentification of events or an incorrect estimation of (intrinsic) $\nu_{e}$ created in the beam. And, the majority $(\sim 50 \%)$ of intrinsic $\nu_{e}$ arise in $\mu^{+}$ decay,

$$
\pi^{+} \rightarrow \mu^{+}+\nu_{\mu} \rightarrow e^{+}+\nu_{e}+\bar{\nu}_{\mu}+\nu_{\mu}
$$

The fact that every $\nu_{e}$ is accompanies by a $\nu_{\mu}$ connects the $\nu_{\mu} \mathrm{CCQE}$ measurement to the $\nu_{e}$ prediction. Again, an incorrect prediction of intrinsic $\nu_{e}$ would imply an incorrect $\nu_{\mu}$ flux prediction, or an incorrect prediction of the $\nu_{\mu} \mathrm{CCQE}$ rate. Therefore, the intrinsic $\nu_{e}$ flux from $\mu^{+}$decay is measured via the high-statistics $\nu_{\mu}$ CCQE event sample. Then, the intrinsic $\nu_{e}$ background with the largest uncertainty is that from $K^{+}$decay.

\subsubsection{Kinematic reconstruction test by $\nu_{\mu} \mathrm{CCQE}$}

To understand neutrino oscillations, it is necessary to know the energy of the candidate neutrinos. Since $\nu_{e}$ are measured via $\nu_{e} \mathrm{CCQE}$, the neutrino energy must be reconstructed from CCQE kinematics. However, because of the blindness procedure, it was not possible to look at $\nu_{e} \mathrm{CCQE}$ sample before the analysis was complete.

The $\nu_{\mu} \mathrm{CCQE}$ sample is the most abundant sample ( $40 \%$ of total neutrino events) and was used to understand various features of the data. For example, the kinematic variables are important for the selection of $\nu_{e}$ candidate events, and so the validity of kinematic reconstruction must be demonstrated using $\nu_{\mu} \mathrm{CCQE}$ events. Especially, the study of $\nu_{\mu} \mathrm{CCQE}$ revealed a number of inadequacies in the nuclear model used, and the relativistic Fermi gas (RFG) model was tuned using the $\nu_{\mu}$ CCQE box. This (tuned) model was then used for $\nu_{e}$ oscillation search [67] for better $\nu_{e}$ kinematic reconstruction. 


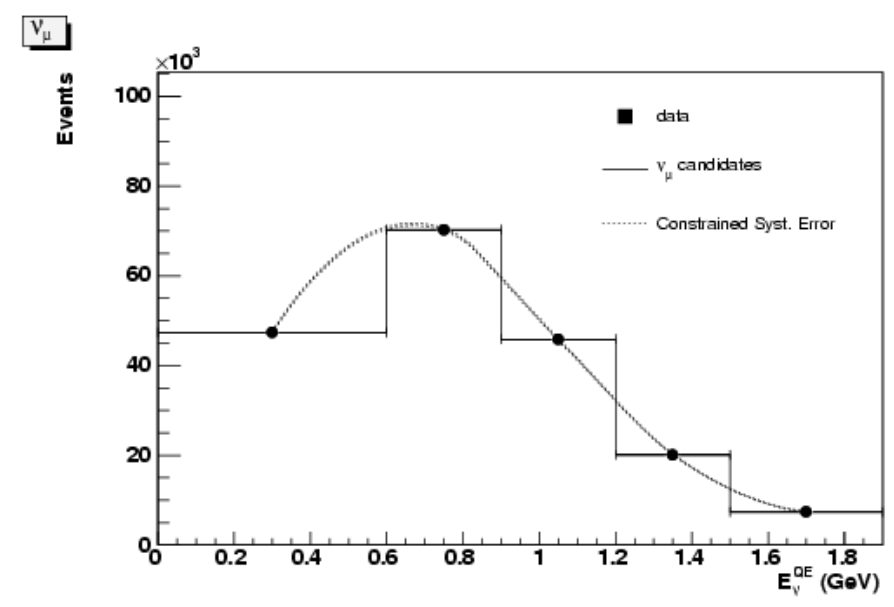

ve

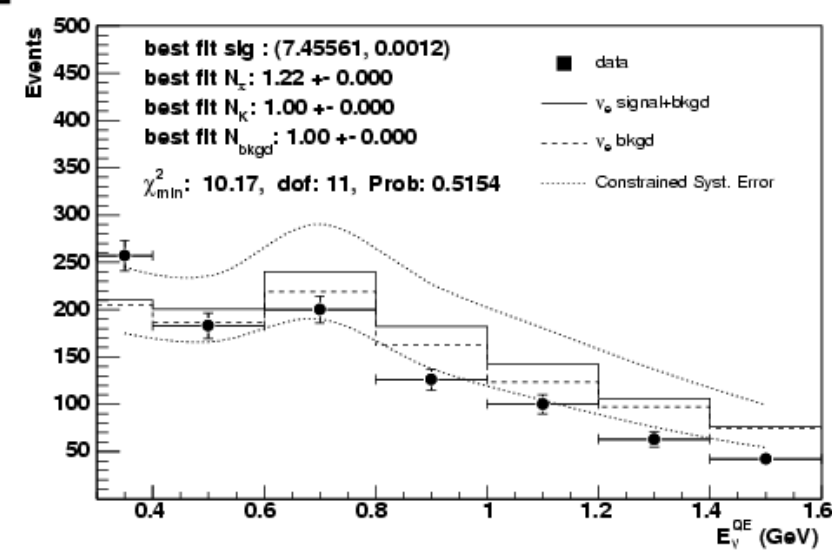

Figure 8.2: An example plot from the combined fit. The top plot shows $\nu_{\mu} \mathrm{CCQE}$ candidate data and the prediction. The bottom plot shows the $\nu_{e}$ CCQE candidate data along with signal and background predictions, including the oscillation hypothesis. A good fit with high-statistics $\nu_{\mu}$ candidates does not allow large variations in the $\nu_{\mu}$ flux. Thus, (1) a large variation of $\nu_{e}$ appearance from $\nu_{\mu}$ variation $\left(\nu_{\mu} \rightarrow \nu_{e}\right)$ is not allowed, and (2) a large variation of intrinsic $\nu_{e}$ from $\mu^{+}$decay is not allowed. 


\section{2 $\nu_{\mu}$ CCQE measurement and tuning of relativistic Fermi gas (RFG) model}

In this section, we present the details of $\nu_{\mu} \mathrm{CCQE}$ box and the tuning of the RFG model which took place before $\nu_{e}$ box opening.

\subsubsection{CCQE event selection in MiniBooNE}

The MiniBooNE detector, a spherical tank filled with mineral oil, detects Cerenkov light from charged particles. The identification of $\nu_{\mu} \mathrm{CCQE}$ interactions relies solely on the detection of the primary muon Čerenkov light and the associated decay electron Čerenkov light in these events (Fig. 8.3):

$$
\nu_{\mu}+n \rightarrow \mu^{-}+p \rightarrow e^{-}+\nu_{\mu}+\overline{\nu_{e}}+p .
$$

By avoiding requirements on the outgoing proton kinematics, the selection is less dependent on nuclear models. The scintillation light from the proton, although not used directly in the $\nu_{\mu} \mathrm{CCQE}$ analysis, was studied using neutral current elastic scattering events in MiniBooNE [110].

To define CCQE candidates, we applied the following cuts:

1. $(54.2 \%) 2$ total subevents;

2. (52.9\%) 1st subevent average time $\mathrm{T}$ is $4400<\mathrm{T}(\mathrm{ns})<6400$;

3. $(46.4 \%)$ veto hits for both 1 st and 2 nd subevent $<6$;

4. (41.6\%) tank hits for the 1 st subevent $>200$, and the 2 nd subevent $<200$;

5. $(41.3 \%)$ reconstructed track center $<500 \mathrm{~cm}$; 


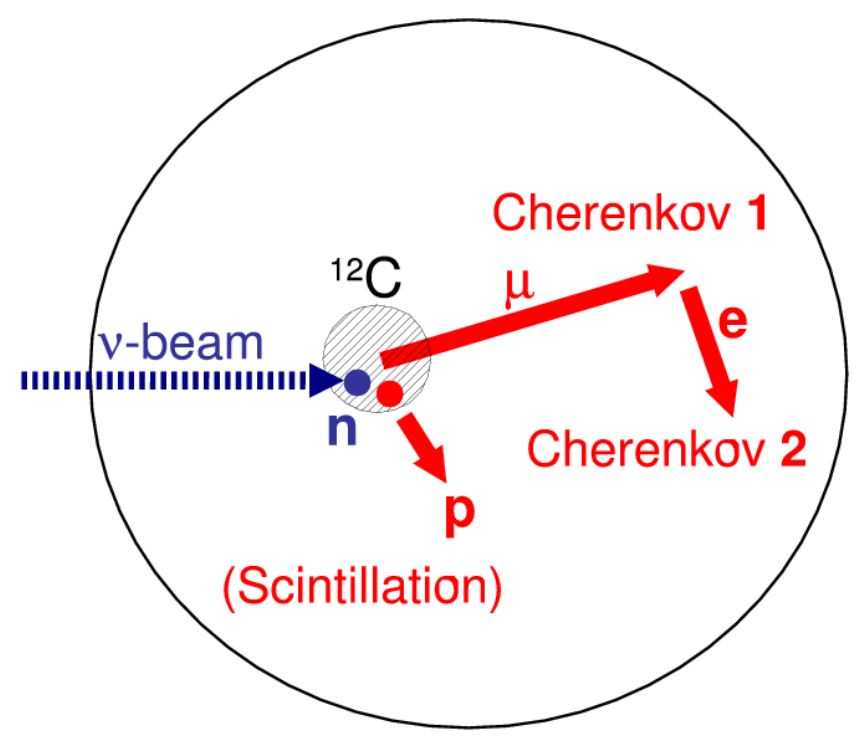

Figure 8.3: Schematic figure of a CCQE interaction. The primary Čerenkov light from the muon (Čerenkov 1, first subevent) and subsequent Čerenkov light from the decayed electron (Čerenkov 2, second subevent) are used to tag the CCQE event. For most events, protons only emit scintillation light, and our selection is insensitive to this information. 
6. (35.0\%) Michel distance $\Delta \mathrm{R}<100 \mathrm{~cm}$.

The numbers in parentheses are the calculated efficiency after each cut. Cut (1) selects 2 timing cluster as CCQE candidate, (2) is the beam window cut, (3) rejects incoming particles, (4) requires the 1st subevent to be MIP-like and the 2nd subevent to be Michel electron-like, (5) is the fiducial volume cut, and (6) requires the Michel electron to be near the endpoint of the muon track.

The Michel distance $\Delta \mathrm{R}$ is defined [115] by,

$$
\Delta R=\sqrt{\left(X_{1}-X_{2}\right)^{2}+\left(Y_{1}-Y_{2}\right)^{2}+\left(Z_{1}-Z_{2}\right)^{2}} .
$$

Here, $\left(X_{2}, Y_{2}, Z_{2}\right)$ is the electron track center, and the muon track endpoint $\left(X_{1}, Y_{1}, Z_{1}\right)$ is defined from the muon track center $X^{R}$, and direction $U X^{R}$, and the $\frac{d E}{d x}$ measured from polyethylene Range $\left(E^{R}\right)$,

$$
X_{1}=X^{R}+U X^{R} \times \frac{\operatorname{Range}\left(E^{R}\right)}{2}, Y_{1} \cdots
$$

A total of 193,709 events pass these cuts from $5.58 \times 10^{20}$ protons on target collected between August 2002 and December 2005. The cuts are estimated to be $35 \%$ efficient at selecting $\nu_{\mu} \mathrm{CCQE}$ events in a $500 \mathrm{~cm}$ radius, with a CCQE purity of $74 \%$. The predicted backgrounds are: $74.8 \%$ CC $1 \pi^{+}, 15.0 \%$ CC $1 \pi^{0}, 4.0 \%$ NC $1 \pi^{ \pm}, 2.6 \%$ CC multi- $\pi, 0.9 \%$ NC elastic, $0.8 \% \bar{\nu}_{\mu} \mathrm{CC} 1 \pi^{-}, 0.8 \% \mathrm{NC} 1 \pi^{0}, 0.6 \% \eta / \rho / K$ production, and $0.5 \%$ deep inelastic scattering and other events. Because pions can be absorbed via final state interactions in the target nucleus, a large fraction of the background events look like CCQE events in the MiniBooNE detector. "CCQE-like" events, all events with a muon and no pions in the final state, are predicted to be $84 \%$ of the sample after cuts.

\subsubsection{MiniBooNE CCQE events}

Figure 8.4 shows the ratio of data to Monte Carlo (MC) CCQE events as a function of muon kinetic energy $T_{\mu}(\mathrm{GeV})$ and muon scattering angle $\cos \theta_{\mu}$. Note the muon energy and muon 


\section{2. $\nu_{\mu}$ CCQE MEASUREMENT AND TUNING OF RELATIVISTIC FERMI GAS} (RFG) MODEL

scattering angle are the observables and the basis of all reconstructed kinematic variables in the $\nu_{\mu} \mathrm{CCQE}$ channel. One can immediately see that the data-MC agreement is poor.

We can only measure the interaction rate, which is the convolution of flux and cross section $\left(R=\int \Phi \times \sigma\right)$. So, when data-MC agreement is poor, without knowing flux prediction is perfect, one cannot tune the cross section model from the measured interaction rate. We show our observed data-MC mismatching is not the effect of mismodeling of neutrino flux, but is really a cross section model problem, by the following approach.

There are 6 auxiliary lines: (a), (b), and (c) are equal neutrino energy lines, 0.4, 0.8, and $1.2 \mathrm{GeV}$ respectively, while (d), (e), and (f) are equal $Q^{2}$ lines of $0.2,0.6$, and $1.0 \mathrm{GeV}^{2}$ each. The data-MC disagreement follows the lines of equal $Q^{2}$, not equal neutrino energy. This indicates that the data-MC disagreement is not due to the neutrino flux prediction, but due to the neutrino interaction prediction, because the former is a function of neutrino energy and the latter is a function of $Q^{2}$.

$$
R=\int \Phi \times \sigma \rightarrow R\left[E_{\nu}, Q^{2}\right]=\int \Phi\left[E_{\nu}\right] \times \sigma\left[Q^{2}\right]
$$

So we assume that the data-MC disagreement comes from our neutrino interaction model and we adjust the model to describe the data. This is a critical task for MiniBooNE since the goal is to measure $\nu_{e} \mathrm{CCQE}$ events, but the $\mathrm{MC}$ and all reconstruction tools must be reliable and tested with the copious $\nu_{\mu} \mathrm{CCQE}$ events due to the blind analysis constraint on the $\nu_{e}$ CCQE channel.

The data-MC disagreement is classified in 2 regions in this plane (Fig. 8.4),

1. data deficit at low $Q^{2}$ region, light gray band near the top left corner

2. data excess at high $Q^{2}$ region, black band from the top right to the bottom left

Since we are employing the relativistic Fermi gas (RFG) model [16] in our MC, we wish to fix these problems within the RFG model. The low $Q^{2}$ physics is controlled by the 
nuclear model, so we decided to tune the model, especially the strength of Pauli blocking, to fix region (1). This is justified because electron scattering data has not provided precise information for Pauli blocking in the low $Q^{2}$ region in terms of the RFG model [45]. For (2), we need to increase axial mass $M_{A}$, to increase the cross section at high $Q^{2}$. Here, the axial mass is understood to be an experimental parameter in the axial form factor (Eq. 3.61). This treatment is also justified because elastic electron scattering cannot measure the axial mass precisely. Interestingly, this higher axial mass than world average $\left(=1.03 \mathrm{GeV} / \mathrm{c}^{2}[38]\right)$ is also observed by the K2K experiment in Japan [31, 37, as we see in Sec. 3.4 . 


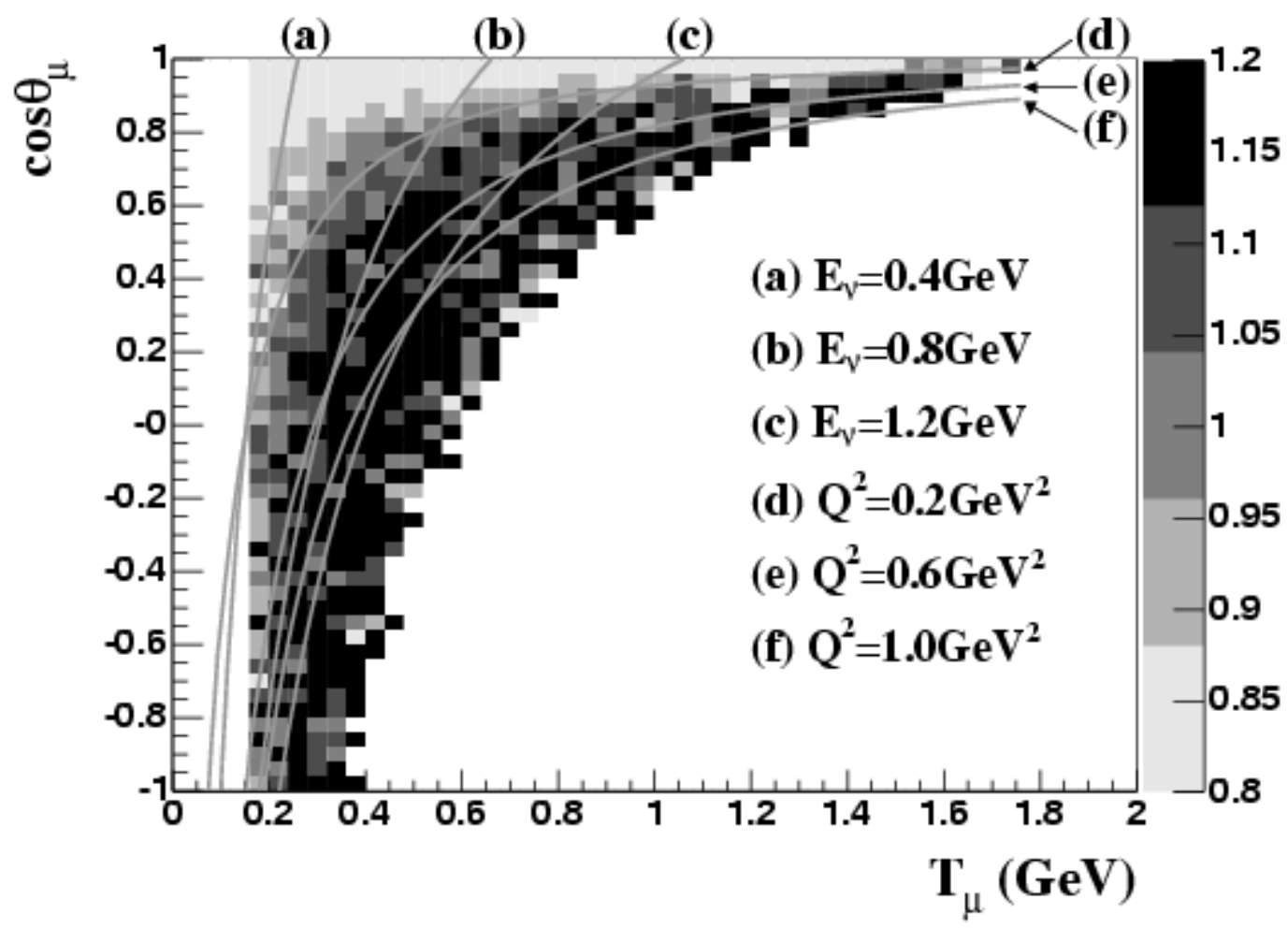

Figure 8.4: Ratio of MiniBooNE $\nu_{\mu}$ CCQE data/simulation as a function of reconstructed muon angle and kinetic energy. The prediction is prior to any CCQE model adjustments; the $\chi^{2} /$ dof $=79.5 / 53$. The ratio forms a $2 \mathrm{D}$ surface whose values are represented by the gray scale, shown on the right. If the simulation modeled the data perfectly, the ratio would be unity everywhere. Contours of constant $E_{\nu}$ and $Q^{2}$ are overlaid. 


\subsubsection{Pauli blocking parameter $\kappa$}

Currently, MiniBooNE is using the nuance neutrino interaction generator [126]. In nuance, CCQE interactions on carbon are modeled by the Relativistic Fermi Gas (RFG) model [16]. To achieve our goal within the RFG model, we introduced a new parameter, $\kappa$, in the phase space integral of nucleon distribution (Eq. 3.72, 3.73, and 3.74,

$$
\begin{aligned}
& E_{h i}=\sqrt{k_{F}^{2}+M_{n}^{2}}, \\
& E_{l o}=\kappa\left(\sqrt{k_{F}^{2}+M_{p}^{2}}-\omega+E_{B}\right),
\end{aligned}
$$

where $M_{n}$ is the target neutron mass, $M_{p}$ is the outgoing proton mass, $k_{F}$ is Fermi momentum $(=220 \mathrm{MeV}), E_{B}$ is binding energy $(=34 \mathrm{MeV})$, and $\omega$ is the energy transfer. In the RFG model, $E_{h i}$ is the energy of an initial nucleon on the Fermi surface and $E_{l o}$ is the lowest energy of an initial nucleon that leads to a final nucleon just above the Fermi momentum. The function of parameter $\kappa$ is to reduce the phase space of the nucleon Fermi sea, especially when the energy transfer is small (Fig. 8.5).

Figure 8.5 shows how this works. Here, $E_{h i}$ is the upper limit of Fermi sea, and $E_{l o}$ is the bottom of Fermi sea relevant for a particular scattering event. Increasing $\kappa$ reduces the nucleon momentum space, and the scattering rate when $Q^{2}$ is low $\left(\lesssim 0.3 \mathrm{GeV}^{2}\right)$.

Figure 8.6, it can be seen that this parameter controls the $Q^{2}$ distribution only in the low $Q^{2}$ region. This is complementary to the role of $M_{A}$, since $M_{A}$ mainly controls the $Q^{2}$ distribution in the high $Q^{2}$ region.

We vary these 2 parameters in a grid search to find the values that minimize $\chi^{2}$. We discuss the detail of fit procedure (Sec. 9.7) and error matrix construction (Sec. 10.6) in later chapters. 


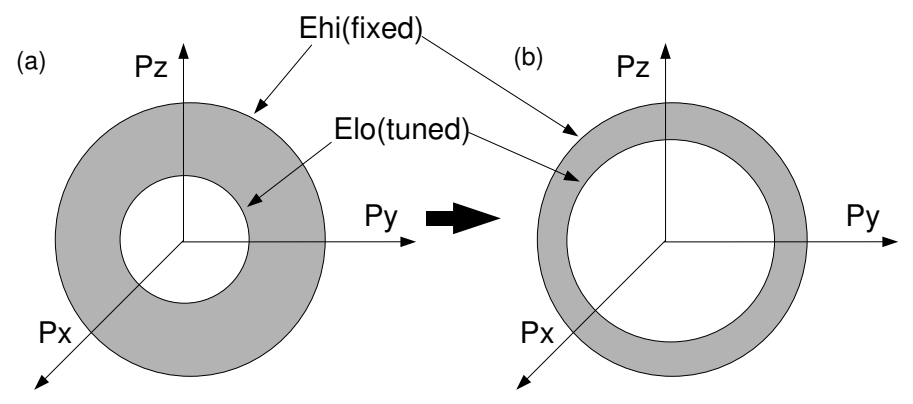

Figure 8.5: Schematic view of nucleon momentum space. In the RFG model, nucleon states are filled from $E_{l o}$ to $E_{h i}$. The parameter $\kappa$ reduces available momentum space to reduce the interaction rate for low $Q^{2}$ interactions. 

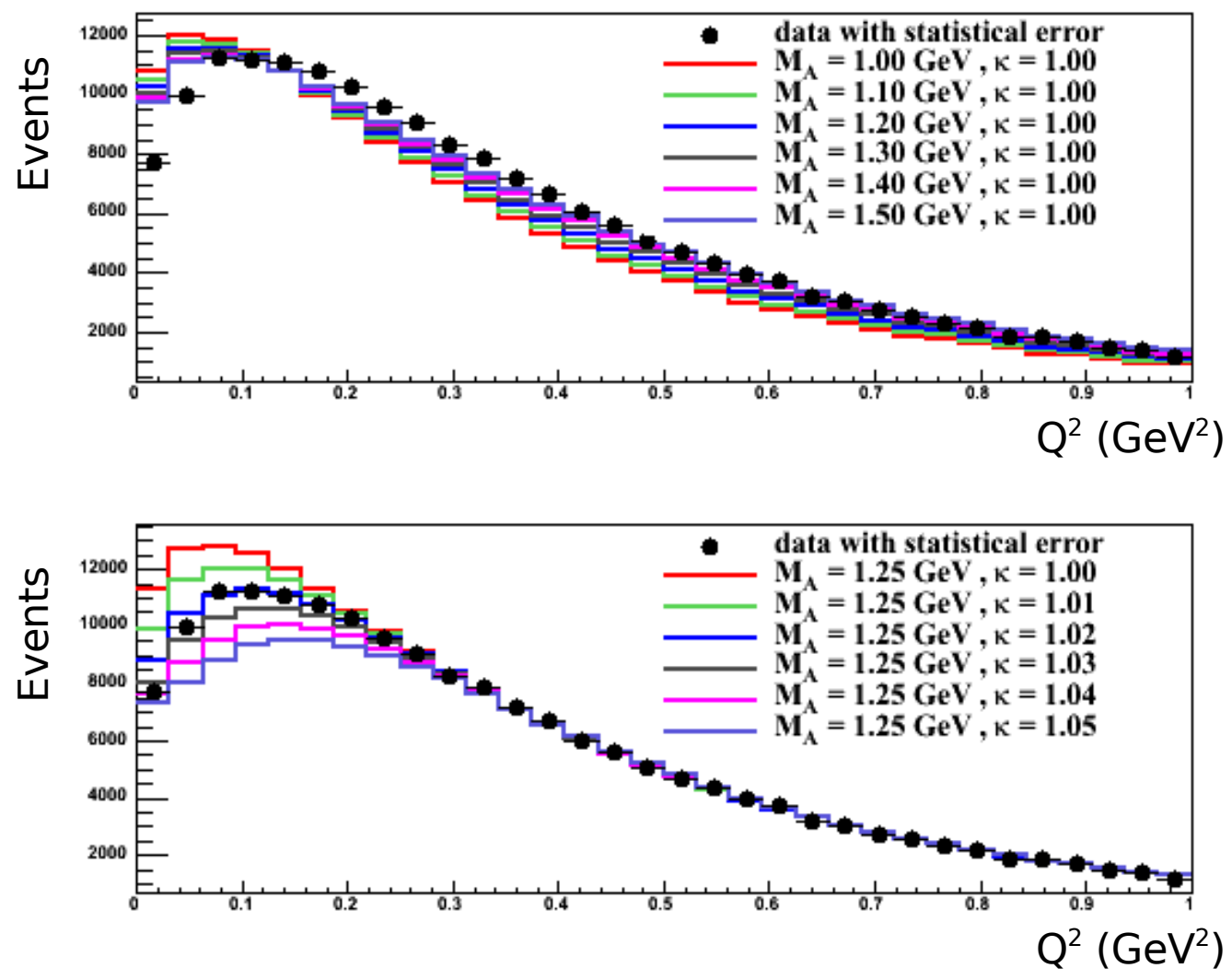

Figure 8.6: Effect of $M_{A}$ and $\kappa$ variations on the MiniBooNE, flux integrated $Q^{2}$ distribution. The top plot shows various $M_{A}$ with fixed $\kappa$, and bottom plot shows various $\kappa$ with fixed $M_{A}$. Note, the $M_{A}$ variation has large impact at high $Q^{2}$ while the $\kappa$ variation has a significant impact only for $Q^{2}$ below $\lesssim 0.2 \mathrm{GeV}^{2}$. 


\subsubsection{Fit result}

\section{$M_{A}-\kappa$ fit}

Finally, the parameters extracted from the MiniBooNE $\nu_{\mu}$ CCQE data are:

$$
\begin{aligned}
M_{A} & =1.23 \pm 0.20 \mathrm{GeV} / \mathrm{c}^{2} ; \\
\kappa & =1.019 \pm 0.011 ; \\
\chi^{2} / \text { dof } & =32.8 / 30 .
\end{aligned}
$$

Table 8.1 shows the contributions to the systematic uncertainties on $M_{A}$ and $\kappa$. The detector model uncertainties dominate the error in $M_{A}$ due to their impact on the energy and angular reconstruction of CCQE events in the MiniBooNE detector. The dominant error on $\kappa$ is the uncertainty in the $Q^{2}$ shape of background events.

The result of this fitting, including all sources of systematic uncertainty, is shown in Fig. 8.7. Since the background error dominates at low $Q^{2}$, and it drives the large error bars at low $Q^{2}$. Note that, the shape uncertainty of the background, namely the $Q^{2}$ distribution shape uncertainty of $C C 1 \pi^{+}$events, is included in these error bands not in the $1-\sigma$ contour. From the data, we found the predicted $Q^{2}$ shape of $C C 1 \pi^{+}$events has a large uncertainty. To quantify this uncertainty, we extract a $Q^{2}$ shape weighting function for $C C 1 \pi^{+}$events (Fig. 8.8). The extracted shape information from the data is implemented in the MC, and the fit is performed again. The result of those 2 fits, one using the MC predicted $C C 1 \pi^{+}$ distribution, and the other using $\mathrm{MC}$ tuned on $C C 1 \pi^{+}$data, are shown with the star and the triangle in the inset plot of Fig. 8.7. The difference is interpreted as the background shape error and added to the error on the extracted parameters.

Figure 8.9 shows the agreement between data and simulation after incorporating the $M_{A}$ and $\kappa$ values from the $Q^{2}$ fit to MiniBooNE $\nu_{\mu}$ CCQE data into the nuclear model. Comparing to Fig. 8.4, the improvement is substantial and the data are well-described throughout 


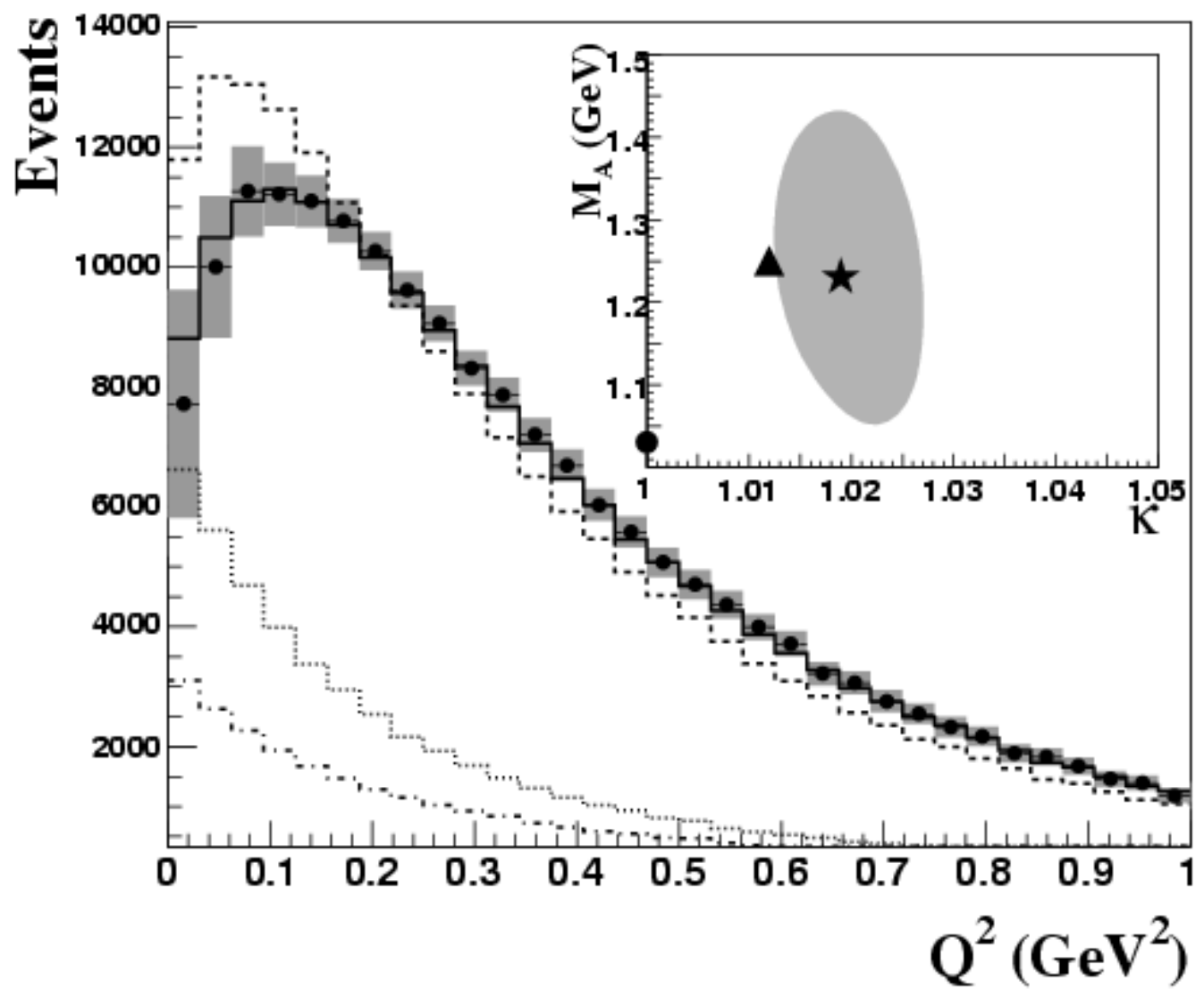

Figure 8.7: Reconstructed $Q^{2}$ for $\nu_{\mu} \mathrm{CCQE}$ events including systematic errors. The simulation, before (dashed) and after (solid) the fit, is normalized to data. The dotted (dot-dash) curve shows backgrounds that are not CCQE (not "CCQE-like"). The inset shows the $1 \sigma$ CL contour for the best-fit parameters (star), along with the starting values (circle), and fit results after varying the background shape (triangle). 


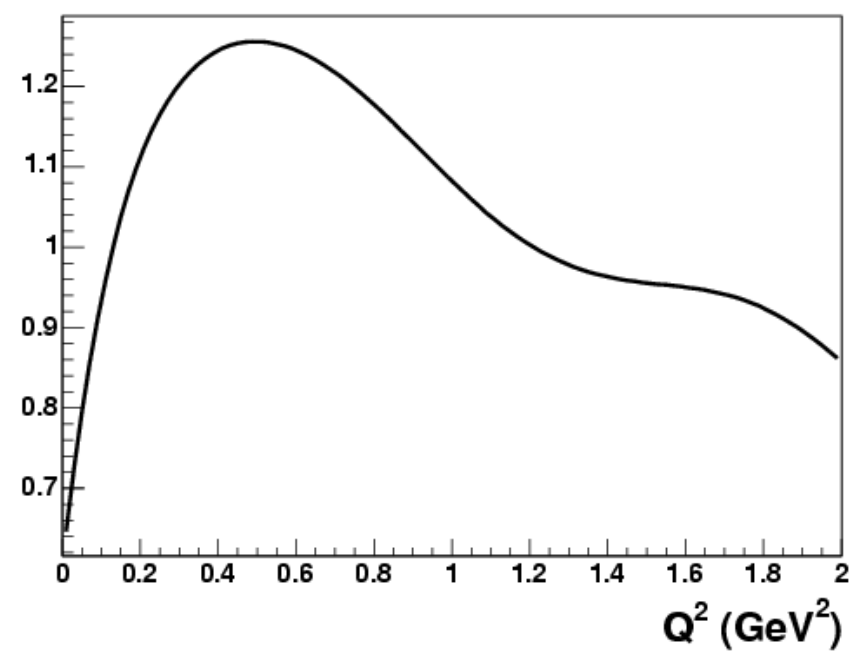

Figure 8.8: $\mathrm{CC} 1 \pi$ weighting function used for $\mathrm{CC} 1 \pi$ background tuning. The data-MC ratio of $\mathrm{CC} 1 \pi$ event is used to extract the weighting function for correct the shape of $\mathrm{CC} 1 \pi$ background for CCQE. Note, the extracted weighting function is normalized, so we only take into account the shape of measured $\mathrm{CC} 1 \pi$ event.

\begin{tabular}{ccc}
\hline error source & $\delta M_{A}$ & $\delta \kappa$ \\
\hline data statistics & 0.03 & 0.003 \\
neutrino flux & 0.04 & 0.003 \\
neutrino cross sections & 0.06 & 0.004 \\
detector model & 0.10 & 0.003 \\
$\mathrm{CC} \pi^{+}$background shape & 0.02 & 0.007 \\
\hline total error & 0.20 & 0.011 \\
\hline
\end{tabular}

Table 8.1: Uncertainties in $M_{A}$ and $\kappa$ from the fit to MiniBooNE $\nu_{\mu}$ CCQE data. The total error is not a simple quadrature sum because of the correlation between the two parameters. 


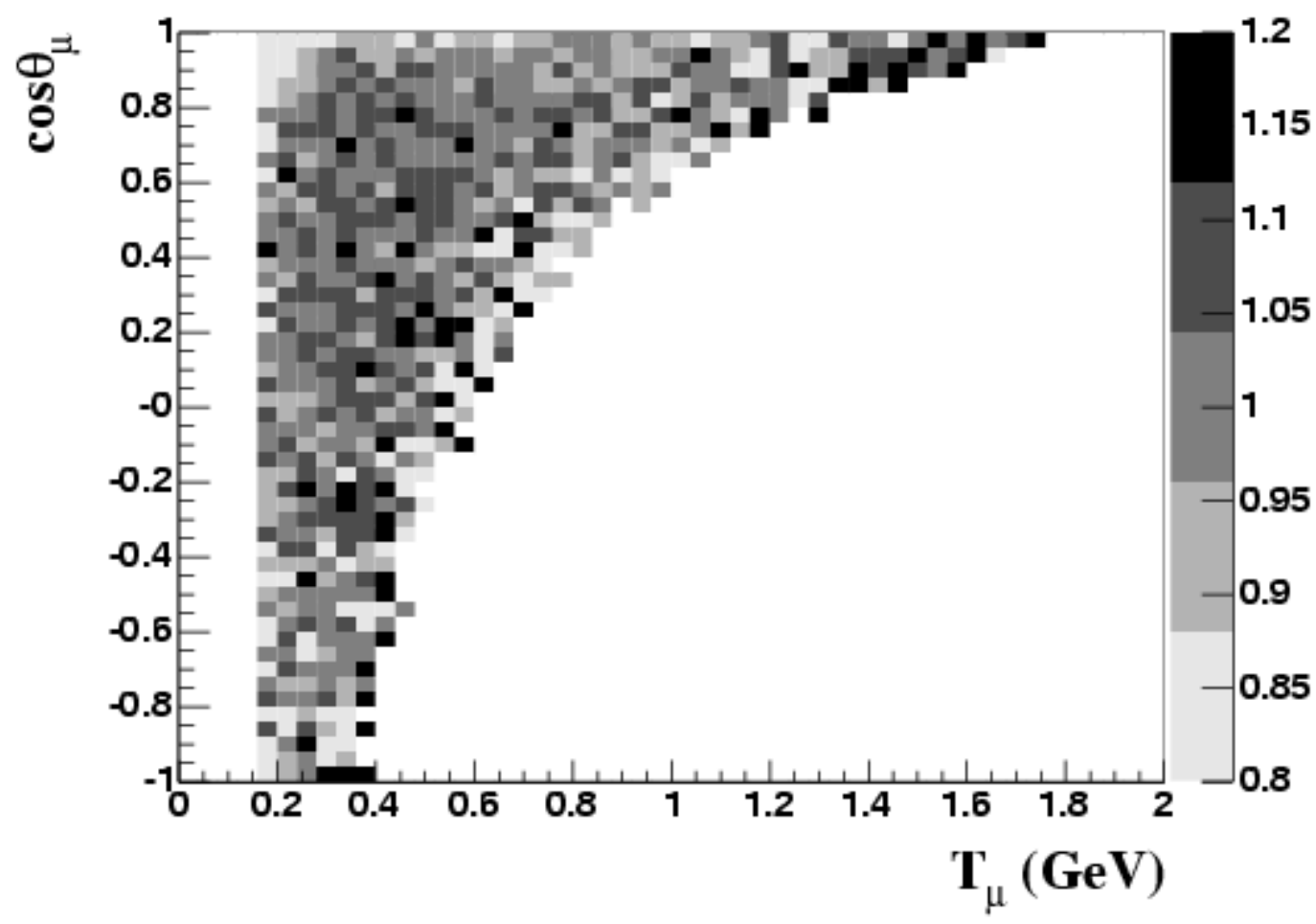

Figure 8.9: Ratio of data/simulation as a function of muon kinetic energy and angle after the CCQE model adjustments; the $\chi^{2} /$ dof $=45.1 / 53$. Compare to Figure 8.4 .

the kinematic phase space. Since the whole kinematic space is fixed, not surprisingly, all of the individual kinematic variables exhibit good data-MC agreement. Figures 8.10, 8.11, and 8.12 show that data and MC agree well within error bars for reconstructed muon neutrino energy, muon kinetic energy and muon scattering angle. 
8.2. $\nu_{\mu}$ CCQE MEASUREMENT AND TUNING OF RELATIVISTIC FERMI GAS (RFG) MODEL

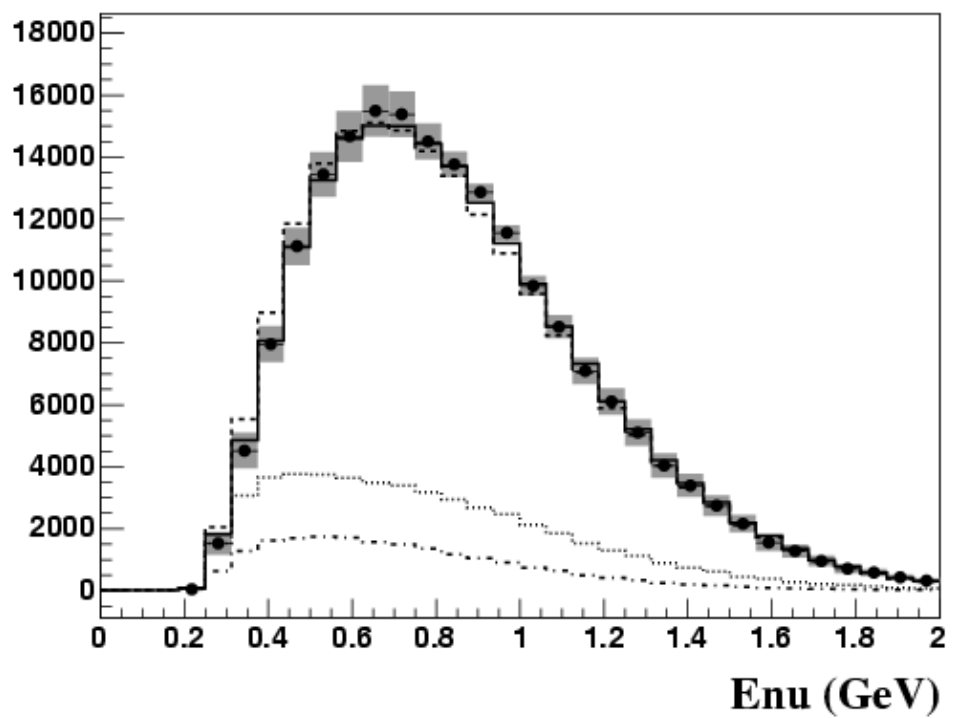

Figure 8.10: Reconstructed muon neutrino energy, line notations are the same as Fig 8.7

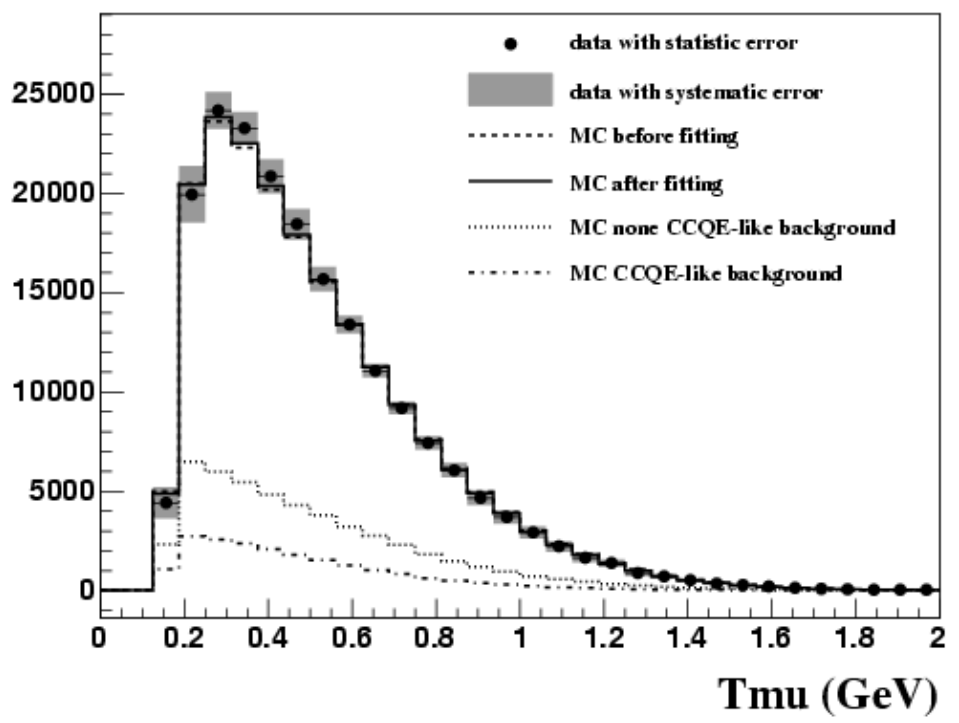

Figure 8.11: Muon kinetic energy, line notations are the same as Fig 8.7 . 


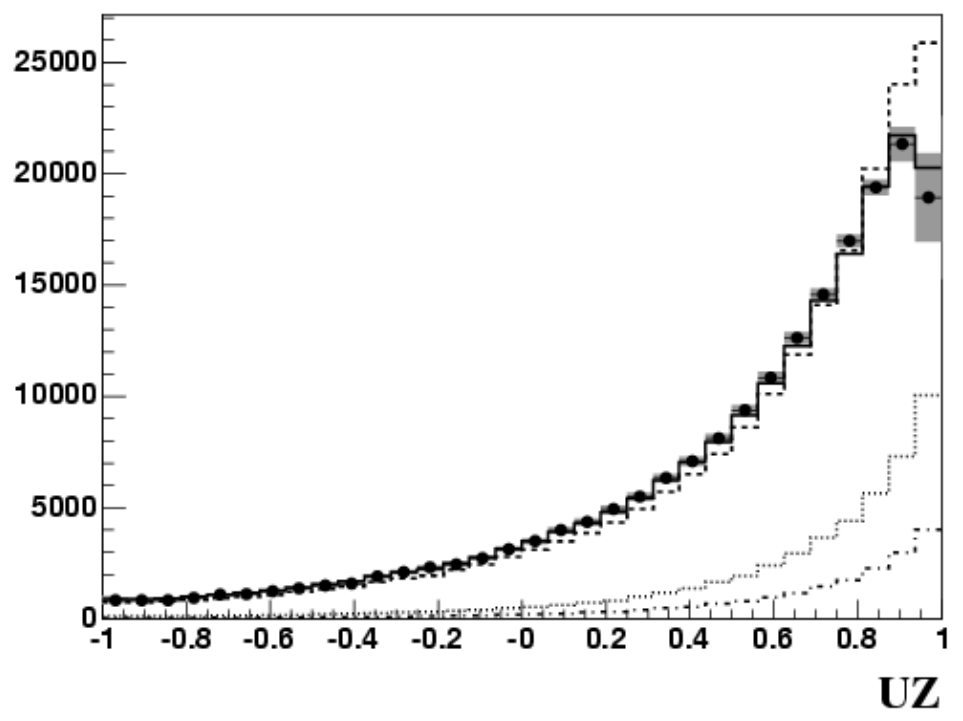

Figure 8.12: $\quad$ Measured muon angle, line notations are the same as Fig 8.7 .

\section{$M_{A}$ only fit}

In general, varying $M_{A}$ allows us to reproduce the high $Q^{2}$ behavior of the observed data events. A fit for $M_{A}$ above $Q^{2}>0.25 \mathrm{GeV}^{2}$ yields consistent results, $M_{A}=1.25 \pm$ $0.12 \mathrm{GeV} / \mathrm{c}^{2}$ (Figs. 8.13). However, fits varying only $M_{A}$ across the entire $Q^{2}$ range leave considerable disagreement at low $Q^{2}$. This data-MC disagreement at low $Q^{2}$ would eventually reflect in data-MC disagreement in reconstructed neutrino energy, because data-MC disagreement in $Q^{2}$ spreads out in the kinematic plane and would affect the energy reconstruction across a wide region. The Pauli-blocking parameter $\kappa$ is instrumental here, enabling this model to match the behavior of the data down to $Q^{2}=0$. 

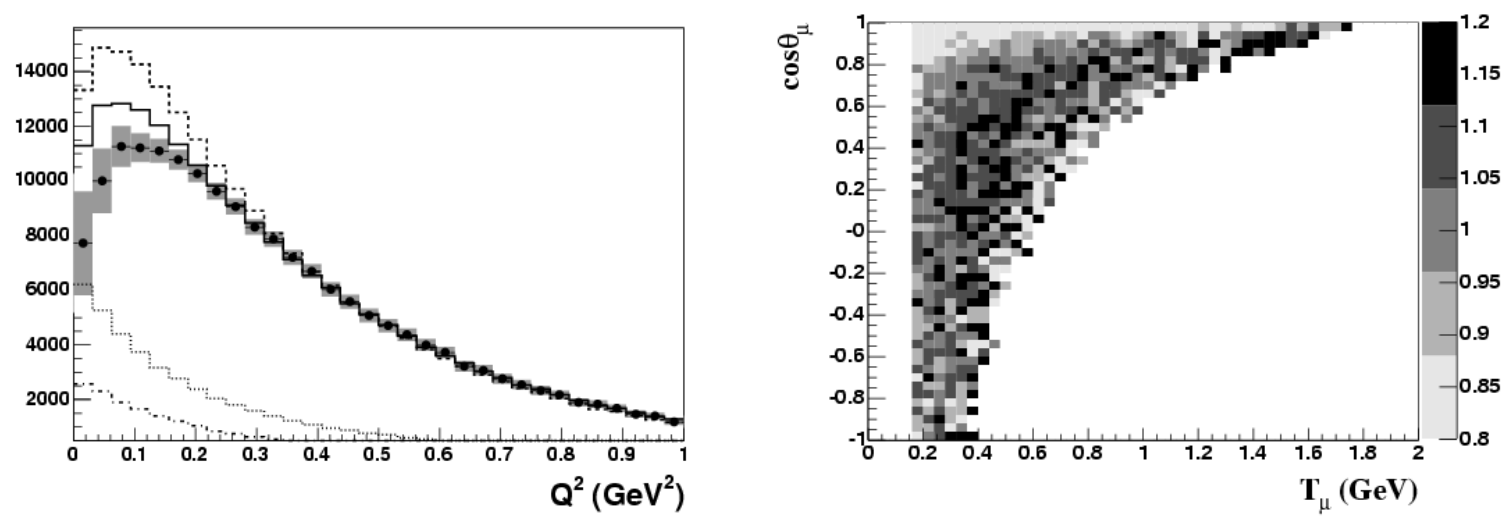

Figure 8.13: Reconstructed $Q^{2}$ for $\nu_{\mu}$ CCQE events and data-MC ratio in the kinematic plane. The analogous plots with Fig. 8.7 and 8.9 , but the fit is performed using $M_{A}$ only, with fixed $\kappa(=1.0$, no enhanced Pauli blocking).

\subsubsection{Conclusions}

As described above, we tuned the RFG model to yield a good description of the MiniBooNE

$\nu_{\mu} \mathrm{CCQE}$ data. This tuned RFG model was then used for the $\nu_{e} \mathrm{CCQE}$ interaction in the search for $\nu_{\mu} \rightarrow \nu_{e}$ oscillation [67]. 


\section{Chapter 9}

\section{simultaneous CCQE and $\mathrm{CC} 1 \pi Q^{2}$ fit}

Charged current quasielastic (CCQE) event candidates are charged current (CC) candidate events with 2 subevents. Charged current one pion production $(\mathrm{CC} 1 \pi)$ candidates are $\mathrm{CC}$ candidate events with 3 subevents. Since $\mathrm{CC} 1 \pi$ is the biggest background contribution to the CCQE candidate sample, a simultaneous measurement of both CCQE and CC1 $\pi$ is used to constrain the $\mathrm{CC} 1 \pi$ background in the CCQE sample.

The challenge is that nearly $90 \%$ of $\mathrm{CC} 1 \pi$ background events in the CCQE sample are "CCQE-like" events for MiniBooNE. That is, the second Michel electron resulting from the pion-decay muon are absent, mainly due to pion absorption (or pion charge exchange) in the nuclei or in the detector media. Also, the uncertainty on the pion absorption is large ( $\sim 20 \%$ ), and hence it is not immediately clear how to apply the CC1 $\pi$ candidate information measured in the 3 -subevent sample to the 2 -subevent sample.

The goal of this part of the CCQE analysis was to determine the CC1 $\pi$ background in the CCQE sample via a measurement in four-momentum transfer, $Q^{2}$. The basic principle 
is that the shape and normalization measurement of the 3 -subevent sample in $Q^{2}$ determines the background for 2 subevents. A measurement of the 1- and 2-subevent samples determines the normalization for the CCQE sample, which has only a small impact for 3 subevents. The shape of CCQE events in $Q^{2}$ is found in a later fit for $M_{A}$ and $\kappa$. In this way, we maintain self-consistency.

The extracted $\mathrm{CC} 1 \pi Q^{2}$ distribution is used to re-weight all events to produce new central value (cv) MC. This new cv MC is used to estimate background contents from all kinetic measurements in CCQE sample. This new background prediction is used in the next chapter, to measure the CCQE absolute cross sections in MiniBooNE.

\subsection{Subevent correction}

In this chapter, we are only interested in the muon kinematics, so when we say " 2 subevents", we mean not the information from the second subevent, but the event information for the muon when the total number of subevents is 2, which are CCQE candidates. Similarly, "3 subevents" selects a muon with two additional Michel electrons, i.e., CC1 $\pi$ candidates. However, when we say the ordinal number, for example the second subevent, it does means the second subevent information, for example, the energy spectrum of Michel electron.

The total number of subevents is one plus the number of Michel electrons, and this is the most crucial information for this analysis. Since we need good selection criteria for the total number of subevent, or Michel electrons, we need to remove as many subevents as possible that are caused not by Michel electrons but by other detector activities. In general, there is much activity in the tank and sometimes this activity can exceed the time and charge threshold for the default subevent algorithm to count these as subevents. To eliminate these subevents, we apply a "subevent correction". That is, when tank hits $<20$, this subevent is eliminated as a candidate Michel electron and removed as a subevent. For 
CHAPTER 9. SIMULTANEOUS CCQE AND CC1 $\pi Q^{2}$ FIT

\begin{tabular}{|l|c|c|c|c|c|c|}
\hline & \multicolumn{3}{|c|}{ data } & \multicolumn{3}{c|}{ MC } \\
\cline { 2 - 7 } & before & after & change & before & after & change \\
\hline 1st subevent & 77470 & 82564 & $6.58 \%$ & 96489 & 103531 & $7.30 \%$ \\
2nd subevent & 217540 & 215908 & $-0.74 \%$ & 408451 & 405098 & $-0.82 \%$ \\
3rd subevent & 53748 & 50883 & $-5.33 \%$ & 72158 & 68910 & $-4.50 \%$ \\
4th subevent & 2036 & 1478 & $-27.41 \%$ & 1283 & 951 & $-31.24 \%$ \\
\hline
\end{tabular}

Table 9.1: Data-MC comparison before and after the subevent correction.

example, 3-subevent candidates can become 2-subevent candidates. Table 9.1 shows the number of events for the data and MC samples before and after the subevent correction. The fraction of events migrated before and after the subevent correction are consistent between data and MC. So we can validate that our MC correctly simulate low hits events.

\subsection{CC cut}

For this analysis, we used the track-based fitter for the reconstruction of the muon kinematics (Sec. 7.5.3). Then, we defined the following "CC cut" to select CC inclusive events with 1,2 , and 3 subevents total:

1. (58.1\%) number of veto hits for 1 st, 2 nd, and 3 rd subevent $<6$;

2. $(57.7 \%) 1$ st subevent average time $\mathrm{T}$ is $4400<\mathrm{T}(\mathrm{ns})<6400$;

3. (56.4\%) reconstructed track center $<500 \mathrm{~cm}$ for the 1 st subevent;

4. (49.9\%) kinetic energy of the 1st subevent particle (muon hypothesis) is $>200 \mathrm{MeV}$;

5. $(45.6 \%)-\log \mathcal{L}(e / \mu)$ is $>0.02$ for 1 subevent, and $>0.0$ for 2 and 3 subevents. 
The numbers indicate the efficiency (as calculated by the simulation) after these cuts. Here, (1) is a veto cut to reject incoming particles, (2) is the beam window cut, (3) is the fiducial volume cut, (4) requires the 1st subevent particle to have enough energy, and (5) is the likelihood cut to select muons (Sec. 7.5.3).

To avoid the region where data is poorly described and the background contribution is expected to be large, the likelihood cut has different criteria when the subevent total is 1 (Fig. 9.1). The red line is CCQE, blue line is $\mathrm{CC} 1 \pi$, and other colors show other background channels. Notice that this cut is not designed to remove $\mathrm{CC} 1 \pi$ events, since we want to determine the $\mathrm{CC} 1 \pi$ background in the CCQE sample from the data. Instead, this simultaneous fit assumes that we understand the other background channels, and it is important to remove these. 

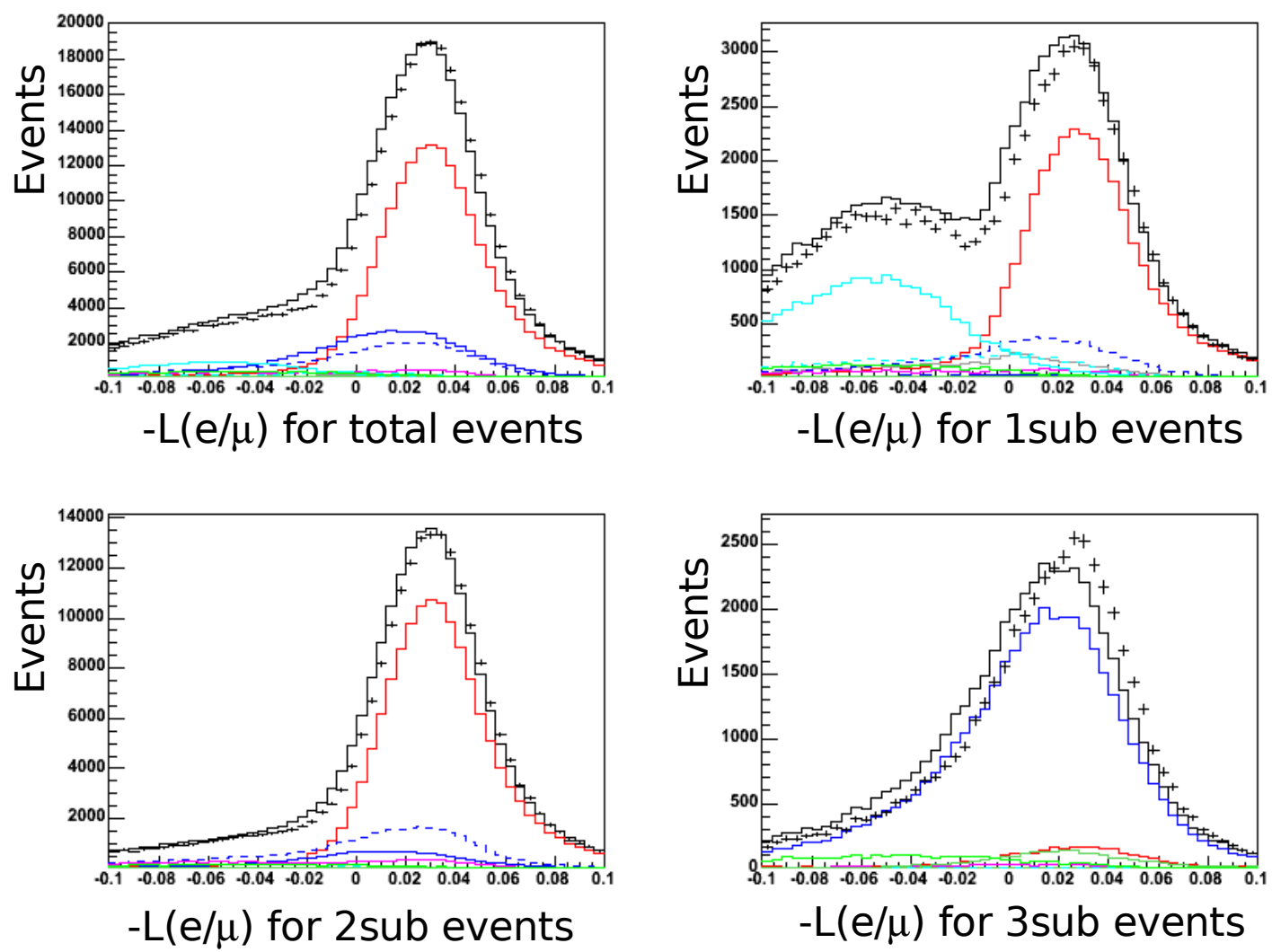

Figure 9.1: Data-MC comparison of muon likelihood function. The positive $\mathrm{x}$ direction is the muon likelihood. From top left to bottom right, total events, 1 subevent, 2 subevents, and 3 subevents. Notice all likelihood here is defined for the first subevent. The red line is CCQE, blue lines are resonance $\mathrm{CC} 1 \pi$ with pion (solid), and without pion (dashed), green lines are coherent CC1 $\pi$ with pion (solid), and without pion (dashed), turquoise line is for $\mathrm{NC} \pi$ and gray line is for other channels. 


\subsection{CCQE cut}

From the CC inclusive sample, one can find the background in the 2 subevent sample. Then this background-subtracted 2 subevent sample is the final sample used for the CCQE analysis. For this purpose, we apply further cuts (only) to the 2 subevent sample to define CCQE sample:

6. (36.7\%) 2 subevents total;

7. $(33.5 \%)$ the distance between the first and second subevent vertices, $d(\mathrm{~cm})$, and the muon kinetic energy $E(\mathrm{MeV})$ satisfy the following relationship, $d>100.0$ and $d>0.5 \times E-100.0$.

This CCQE sample is a subset of the CC inclusive sample, and used in the simultaneous fit (Sec. 9.6). The region selected by this cut is illustrated in Fig. 9.2. Again, this cut is not designed to reject $\mathrm{CC} 1 \pi$ events. Instead, this cut removes other event types and $\mathrm{CC} 1 \pi$ events where the muon reconstruction has failed.

After these cuts, 142948 events survived in the data corresponding to $5.58 \times 10^{20}$ protons on target collected between August 2002 and December 2005 within $0.0<Q^{2}\left(\mathrm{GeV}^{2}\right)<2.0$. Table 9.2 shows a summary of event totals from the standard MC. The CCQE cut has an $82 \%$ purity and $33.5 \%$ efficiency at selecting $\nu_{\mu} \mathrm{CCQE}$ events in a $500 \mathrm{~cm}$ radius. The events are classified for later use. Here, the $\mathrm{CC} 1 \pi$ events are split into "with $\pi$ " and "without $\pi$ " samples based on the existence of a second Michel electron. CC1 $\pi$ events with pion absorption by either the nuclei or by the detector are classified as "without $\pi$ ". "res" and "coh" indicate the the pion is created by the resonance reaction or by coherent scattering. More specifically, resonance pion production occurs in nuance channels 3 and 5 , and coherent pion production from nuance channel 97. Other backgrounds, neutral current elastic (NCE), $\mathrm{CC} \pi^{\circ}$, neutral current $\pi^{+} / \pi^{\circ}$ productions are less important in this analysis. 


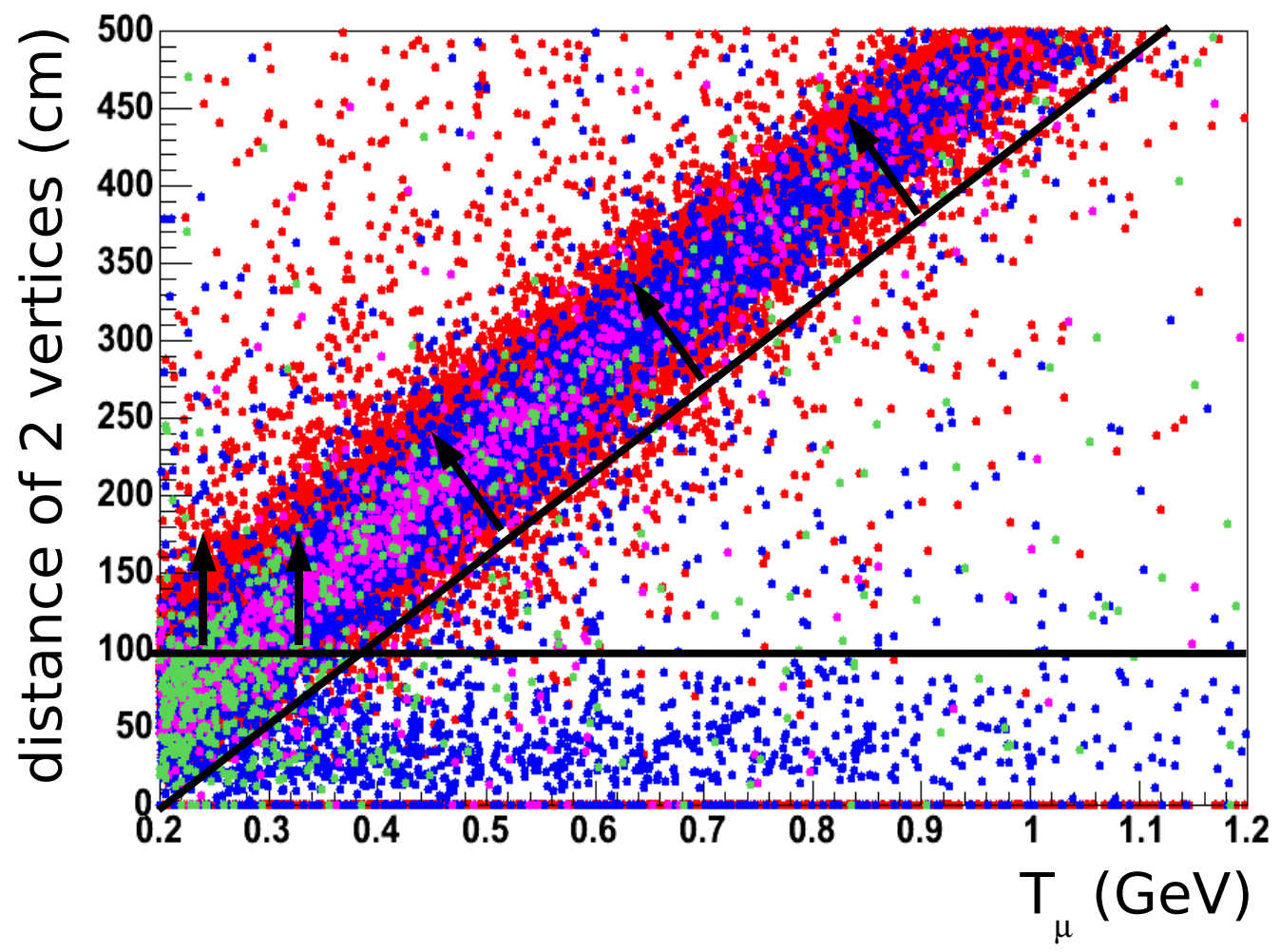

Figure 9.2: Distance-muon energy relationship. The $\mathrm{x}$-axis is the muon kinetic energy $(\mathrm{GeV})$ and the $\mathrm{y}$-axis is the distance between muon vertex and electron vertex. Red dots are CCQE, blue dots are $\mathrm{CC} 1 \pi$, pink dots are charged current $\pi^{\circ}\left(\mathrm{CC} \pi^{\circ}\right)$ events, and green dots are other channels. The arrows show the region selected by this cut. 


\begin{tabular}{|l|c|c|c|c|}
\hline & total & 1 subevent & 2 subevents & 3 subevents \\
\hline data & 204171 & 27959 & 142948 & 33264 \\
\hline MC total & 516262 & 72338 & 365799 & 78125 \\
CCQE & 360544 & 54883 & 300076 & 5586 \\
NCE & 2833 & 2668 & 134 & 30 \\
CC1 $\pi$ with $\pi$ (res) & 71391 & 659 & 6507 & 64225 \\
CC1 $\pi$ without $\pi(\mathrm{res})$ & 50681 & 7332 & 43274 & 74 \\
CC1 $\pi$ with $\pi$ (coh) & 6402 & 78 & 580 & 5744 \\
CC1 $\pi$ without $\pi(\mathrm{coh})$ & 3307 & 502 & 2794 & 11 \\
$\mathrm{CC} \pi^{\circ}$ & 11683 & 1695 & 9045 & 943 \\
$\mathrm{NC} \pi^{+}$ & 1632 & 1455 & 162 & 15 \\
$\mathrm{NC} \pi^{\circ}$ & 3820 & 2445 & 1311 & 64 \\
others & 3969 & 622 & 1915 & 1432 \\
\hline
\end{tabular}

Table 9.2: Event totals summary after the CCQE cut as determined by the MC with an arbitrary normalization

Here, we can see a clear dominance of the CCQE channel in 2 subevents and CC1 $\pi$ with $\pi$ (res) in 3 subevents. Next, we need to determine the amount of $\mathrm{CC} 1 \pi$ without $\pi$ in 2 subevents sample, which is the biggest intrinsic background for MiniBooNE CCQE analysis.

\subsection{The theoretical model}

If good muon reconstruction is available in all 1, 2, and 3 subevent candidates, the difference between the three samples is only the number of Michel electrons. The following argument is applied to any given $i^{\text {th }}$ bin of muon kinematics, and we consider the connection of a given bin beyond each subevent. Naively, this exercise precisely determines the background 
contribution to the 2 subevent sample from 1 and 3 subevent samples, and we can avoid all errors from associated background. This is not completely true as we will see later.

In the perfect world (where all Michel electrons are detected with $100 \%$ efficiency - no muon capture, no pion absorption, etc), 1, 2, and 3 subevent samples are described by the $\mathrm{CCQE}, \mathrm{CC} 1 \pi$, and other channels. In a given bin of muon kinematics, we can apply the following simultaneous equations,

$$
\begin{aligned}
S 1 & =G 1, \\
S 2 & =A+G 2, \\
S 3 & =B+G 3 .
\end{aligned}
$$

Here, $S 1, S 2$, and $S 3$ represent 1, 2, and 3 subevent CC data, $A$ is CCQE prediction, $B$ is $\mathrm{CC} 1 \pi$ prediction, and $G 1, G 2$, and $G 3$ are other (background) channels. Since we do not have enough information to determine the number of events from other channels, we simply use the MC predictions and subtract from the data with scaling factor $(\sim 1.4$, Sec. 9.5 ,

$$
\begin{aligned}
& S 1^{\prime}=0, \\
& S 2^{\prime}=A, \\
& S 3^{\prime}=B .
\end{aligned}
$$

Now, in the real world, we have many processes that can cause the migration of events into different subevent candidates. The key is that the probability for this to occur is either known or small, excepting pion absorption and pion charge exchange.

We introduce the following five processes to migrate an event to different subevent sample:

a muon capture $(\sim 8 \%)$;

$b$ Michel electron detection inefficiency $(\sim 10 \%)$; 
$c$ accidental electron from cosmic rays and other processes (small);

$d$ pion absorption and pion charge exchange (large and not well known);

$e$ pion production in the detector (small).

For two set $s$ and $t$, we write union and intersection by $s \cup t$ and $s \cap t$. Then, Eq. 9.1 becomes,

$$
\begin{aligned}
& S 1^{\prime}=(a \cup b) \cdot A+(a \cup b) \cap(b \cup d) \cdot B, \\
& S 2^{\prime}=[1-(a \cup b)-(c \cup e)] \cdot A+(a \cup b \cup d) \cdot B-(a \cup b) \cap(b \cup d) \cdot B, \\
& S 3^{\prime}=(c \cup e) \cdot A+[1-(a \cup b \cup d)] \cdot B .
\end{aligned}
$$

We then assume all the physics processes resulting in the migration of CCQE are correctly modeled with the MC,

$$
\begin{aligned}
& A_{1} \equiv(a \cup b) \cdot A, \\
& A_{2} \equiv[1-(a \cup b)-(c \cup e)] \cdot A, \\
& A_{3} \equiv(c \cup e) \cdot A .
\end{aligned}
$$

We can check this a simple numerical check. From Tab. 9.2, $A_{1} \sim 55000, A_{2} \sim 300000$, and $A_{3} \sim 5500$. Since $a \cup b=a+b-a \cdot b \sim 17 \%$, number of $A_{1}$ is roughly correct. Also, $c \cup e \sim 2 \%$, so $c$ and $e$ are both of order $1 \%$. 
Next, we can rewrite the $\mathrm{CC} 1 \pi$ parts,

$$
\begin{aligned}
& (a \cup b) \cap(b \cup d) \cdot B=(a \cup b) \cdot(b+d-b d) \cdot B=(a \cup b) b \cdot B+(a \cup b)(1-b) \cdot d B \\
& \equiv B_{1}+d D 1 \\
& (a \cup b \cup d) \cdot B-(a \cup b) \cap(b \cup d) \cdot B=[(a \cup b) \cup d] \cdot B-B_{1}-d D_{1}, \\
& =[(a \cup b)+d-(a \cup b) d] \cdot B-B_{1}-d D_{1} \\
& =(a \cup b) \cdot B-B_{1}+[1-(a \cup b)] \cdot d B-d D_{1} \\
& \equiv B_{2}+d D_{2}-d D_{1} \\
& {[1-(a \cup b \cup d)] \cdot B=[1-(a \cup b)] \cdot B-[1-(a \cup b)] \cdot d B} \\
& \equiv B_{3}-d D_{2} .
\end{aligned}
$$

Then, combine Eqs. 9.3 and 9.4 .

$$
\begin{aligned}
S 1^{\prime} & =A_{1}+B_{1}+d D_{1}, \\
S 2^{\prime} & =A_{2}+B_{2}+d D_{2}-d D_{1}, \\
S 3^{\prime} & =A_{3}+B_{3}-d D_{2} .
\end{aligned}
$$

Thus, the contribution from $\mathrm{CC} 1 \pi$ can be split into two parts, the first part is independent of pion absorption $\left(B_{1}, B_{2}\right.$, and $\left.B_{3}\right)$, and the second part is caused by pion absorption $\left(D_{1}\right.$ and $\left.D_{2}\right)$.

Since we want to determine the shape (in $Q^{2}$ ) of CCQE later, we introduce a scaling factor $\alpha$ for CCQE events in order to take account the normalization without modifying the shape. For $\mathrm{CC} 1 \pi$, we scale and modify the shape with a $5^{t h}$ order polynomial function $\beta$. Finally, we also let the pion absorption rate, $d$, vary:

$$
A \rightarrow \alpha[1] A, B \rightarrow \beta[5] B, D \rightarrow \beta[5] D, d \rightarrow \delta[1]
$$

The number in the bracket shows the number of parameters contained in each function. 
Then,

$$
\begin{aligned}
S 1^{\prime} & =\alpha A_{1}+\beta B_{1}+\delta \beta D_{1}, \\
S 2^{\prime} & =\alpha A_{2}+\beta B_{2}+\delta \beta D_{2}-\delta \beta D_{1}, \\
S 3^{\prime} & =\alpha A_{3}+\beta B_{3}-\delta \beta D_{2} .
\end{aligned}
$$

An additional complication is that the fraction of coherent events in $\mathrm{CC} 1 \pi$ is not well known. Then coherent fraction changes the connection between the 1, 2, and 3 subevent samples in a way we cannot achieve with only $\beta$ and $\delta$. To account for this, we separate coherent fraction of $\mathrm{CC} 1 \pi$ from the resonance $\mathrm{CC} 1 \pi$, and we introduce new parameter $\epsilon$ to change the fraction of coherent event,

$$
B \rightarrow B+\epsilon E, D \rightarrow D+\epsilon F
$$

Here, $B$ and $D$ are newly defined to be the resonance $\mathrm{CC} 1 \pi$ events with and without a pion, and $E$ and $F$ represent the coherent $\mathrm{CC} 1 \pi$ events with and without a pion. Finally, Eqs. 9.6 become,

$$
\begin{aligned}
S 1^{\prime} & =\alpha A_{1}+\beta B_{1}+\epsilon \beta E_{1}+\delta \beta D_{1}+\epsilon \delta \beta F_{1} \\
S 2^{\prime} & =\alpha A_{2}+\beta B_{2}+\epsilon \beta E_{2}+\delta \beta D_{2}+\epsilon \delta \beta F_{2}-\delta \beta D_{1}-\epsilon \delta \beta F_{1} \\
S 3^{\prime} & =\alpha A_{3}+\beta B_{3}+\epsilon \beta E_{3}-\delta \beta D_{2}-\epsilon \delta \beta F_{2} .
\end{aligned}
$$

Remember that these are simultaneous equations for a given bin of any muon kinematic variables. There are four parameters among three coupled equations, so the solution is not possible analytically. In fact, even if $\delta$ and $\epsilon$ are fixed, the ambiguities in the background (denoted as, $G_{1}, G_{2}$, and $G_{3}$ ) and shape uncertainty of CCQE (which we will measure later) give additional freedom and the equations are not analytically solvable. Therefore, instead of an analytical solution, a fitting technique is used that minimizes the data-MC disagreement to find parameters $\alpha$ and $\beta$, with fixed $\delta$ and $\epsilon$. 


\section{5 simultaneous fitting of the $1,2,3$ subevent samples}

In this section, we describe the technique to find the $\mathrm{CC} 1 \pi$ background in the $\mathrm{CCQE}$ sample via a simultaneous fit of the reconstructed $Q^{2}$ distribution for the 1,2 , and 3 subevent samples. The result of this fit is a $\mathrm{CC} 1 \pi$ weighting function in reconstructed $Q^{2}$ and a new cv MC sample is created by weighting the events with this function. This new cv MC may be used to estimate background in other kinematic variables, for example, muon kinetic energy, muon angle, etc. Finally, the background is subtracted properly to estimate the absolute cross section. The cross section measurement is described in detail in Chapter 10 .

Under the assumption that the muon kinematic reconstruction is perfect, all errors associated to $\mathrm{CC} 1 \pi$ event prediction for $\mathrm{CCQE}$ measurement are zero, except pion absorption and pion charge exchange. The errors which disconnect the 1, 2, and 3 subevent samples are the systematic errors which do not cancel. All errors are summarized in Chapter 10.

Here, we apply a normalization factor of 0.94 for neutral current elastic (NCE) events extracted from NCE analysis in MiniBooNE [151, and 1.4 for other channels (which is roughly the same normalization of $\mathrm{CC} 1 \pi \sim 1.4$ after the fit). We use the absolute normalization measured from $\mathrm{NC} \pi^{\circ}$ sample to our $\mathrm{NC} \pi^{\circ}$ events which is based on the $\mathrm{NC} \pi^{\circ}$ rate measurement in MiniBooNE [121.

Figs. 9.3 and 9.4 show the data-MC agreement before the simultaneous fit. Fig. 9.3 shows the $Q^{2}$ distributions for data and $\mathrm{MC}$ broken into physics channels. Here we apply a normalization factor of 1.3 for the $\mathrm{CCQE}$ and $\mathrm{CC} 1 \pi$ channels (Fig. 9.4), and obtain good data-MC agreement for the 1 and 2 subevent samples, but not for 3 subevents.

Note that:

1. CCQE is dominant in the 1,2 subevent samples;

2. $\mathrm{CC} 1 \pi$ resonance is dominant in 3 subevent sample; 
3. $\mathrm{CC} 1 \pi$ resonance without pion is the dominant CCQE background in 2 subevent sample;

4. CC1 $1 \pi$ coherent makes sharp peak at low $Q^{2}$ in 3 subevent sample.

From bottom left plot in Fig. 9.3 , we can determine the shape in $Q^{2}$ of the correction needed for $\mathrm{CC} 1 \pi$ resonance events. Then, under the assumption that our pion absorption rate and $Q^{2}$ reconstruction is correct, we can find the background to 2 subevent events, both in shape and normalization, even though the dominant background $\mathrm{CC} 1 \pi$ events have undetected pions.

Figs. 9.5 and 9.6 shows the situation after the fit. Now, all 1, 2, and 3 subevent samples show good data-MC agreement except for the lowest $Q^{2}$ bins. This can be interpreted that our choice of kappa (=1.022) from the previous analysis is too large and we need to re-fit to determine a new value. The shape of the $\mathrm{CC} 1 \pi$ weighting function (Fig. 9.6, top right plot) extracted from the 3 subevent sample is similar to that used to determine the $\mathrm{CC} 1 \pi$ shape uncertainty [4] (Fig. 8.8). However, the weighting function determined here also contains normalization information that was ignored in the previous analysis. And hence, this fit result changes the normalization for both CCQE and CC1 $1 \pi$. This change increases the $\mathrm{CC} 1 \pi$ fraction by about $15 \%$, and it corresponds to a $3 \%$ increase in the background to the CCQE sample. Therefore, the CCQE fraction decreases by $3 \%$ from our standard nuance prediction (Sec. 7.4).

The fit is performed by minimizing $\chi^{2}$ considering statistical error only. The fit is performed by considering the different subevent samples iteratively. Two iterations is sufficient to find a solution. The first four $Q^{2}$ bins are omitted (corresponding to $Q^{2}\left(\mathrm{GeV}^{2}\right)<0.1$ ) to avoid the low $Q^{2}$ data-MC disagreement as this will be rectified by determining the best values for $M_{A}$ and $\kappa$ with the new background contribution. So, effectively, the 3 subevent sample is used to find the $Q^{2}$ shape and normalization for CC1 $1 \pi$ events, and, the 1 and 2 
subevent samples are used to determine the CCQE normalization, which has a little impact on the $\mathrm{CC} 1 \pi$ shape and normalization because of the small CCQE fraction in the 3 subevent sample. 

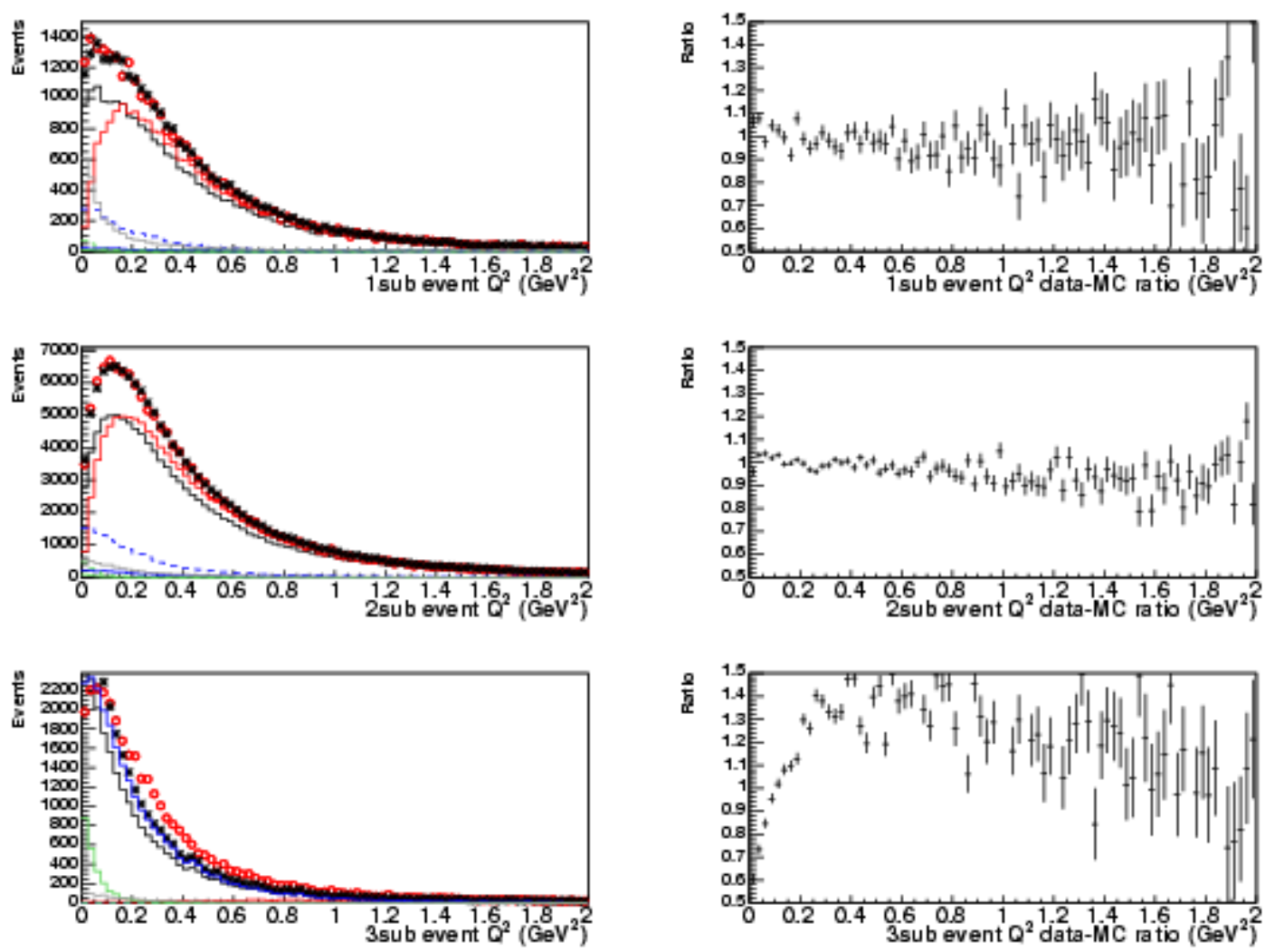

Figure 9.3: Data-MC comparison for $Q^{2}$ plots before fit. From top to bottom, 1, 2, and 3 subevents (left) and data to MC ratio (right). The red circles are data, and black dots are MC after fit, and the black lines are MC before fit. For colored lines, red is CCQE, blue is $\mathrm{CC} 1 \pi$ resonance, with pion (solid), and without pion (dashed), green lines are $\mathrm{CC} 1 \pi$ coherent, with pion (solid), and without pion (dashed), and gray lines for for others. All colored lines are after fit. 

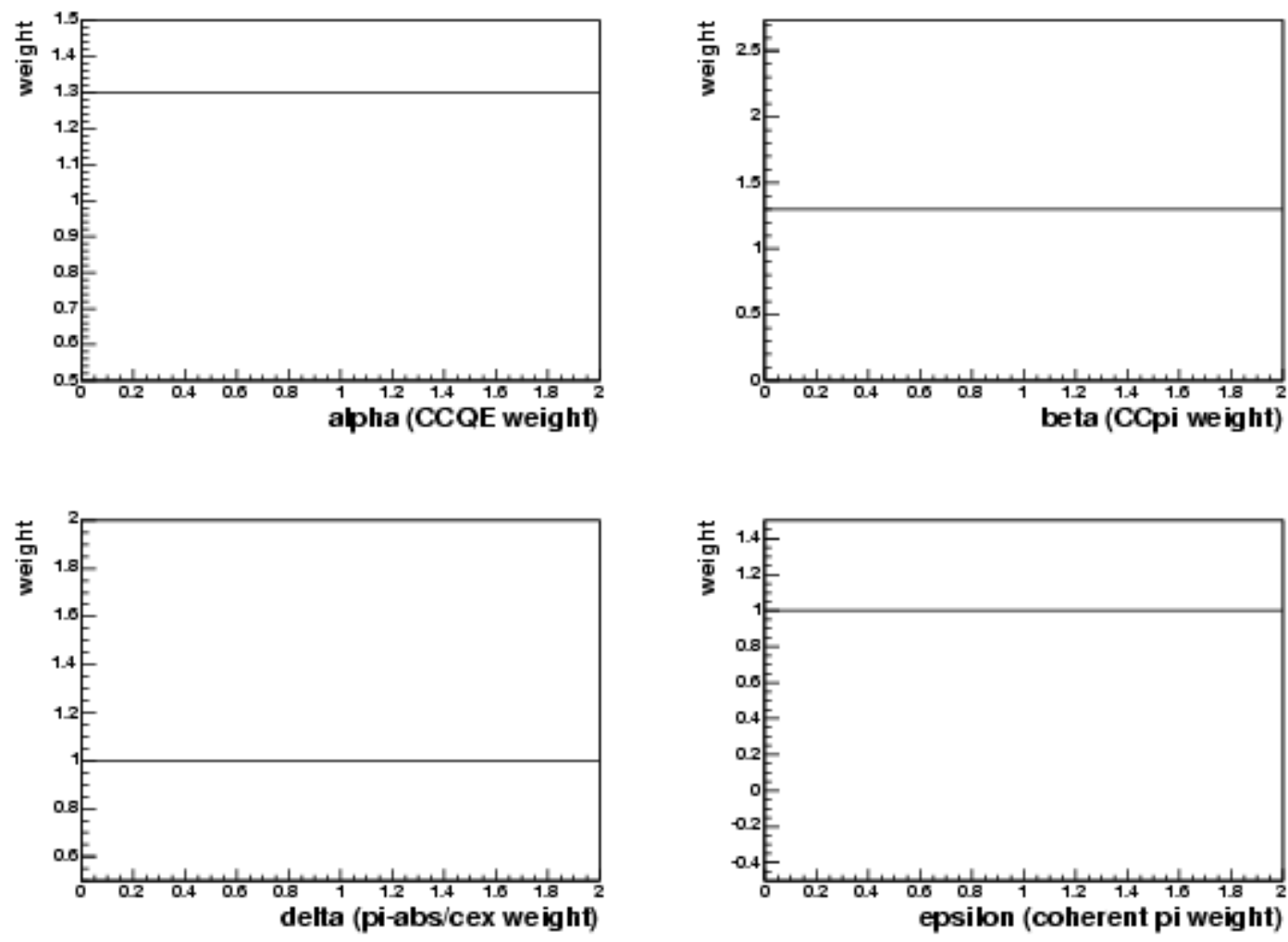

Figure 9.4: Weighting function of each channel before fit. From top left to bottom right, CCQE, $\mathrm{CC} 1 \pi$, pion absorption, and coherent fraction of $\mathrm{CC} 1 \pi$. 

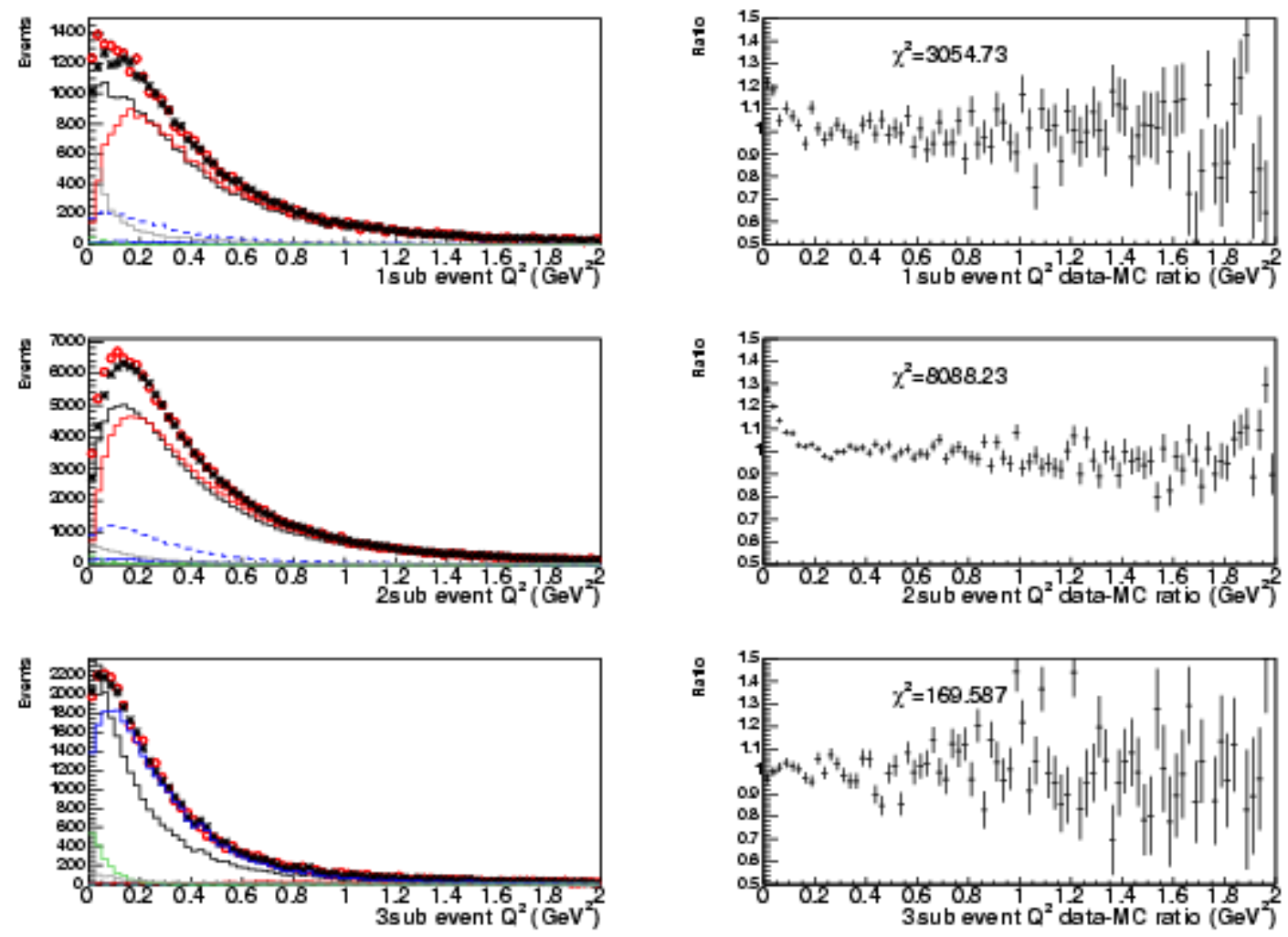

Figure 9.5: Data-MC comparison for $Q^{2}$ plots after fit. The notations are that same as Fig. 9.3. Note $\chi^{2}$ s shown here come from all $Q^{2}$ bins, but for 1 and 2 subevents, the first four bins are not used in fit. 

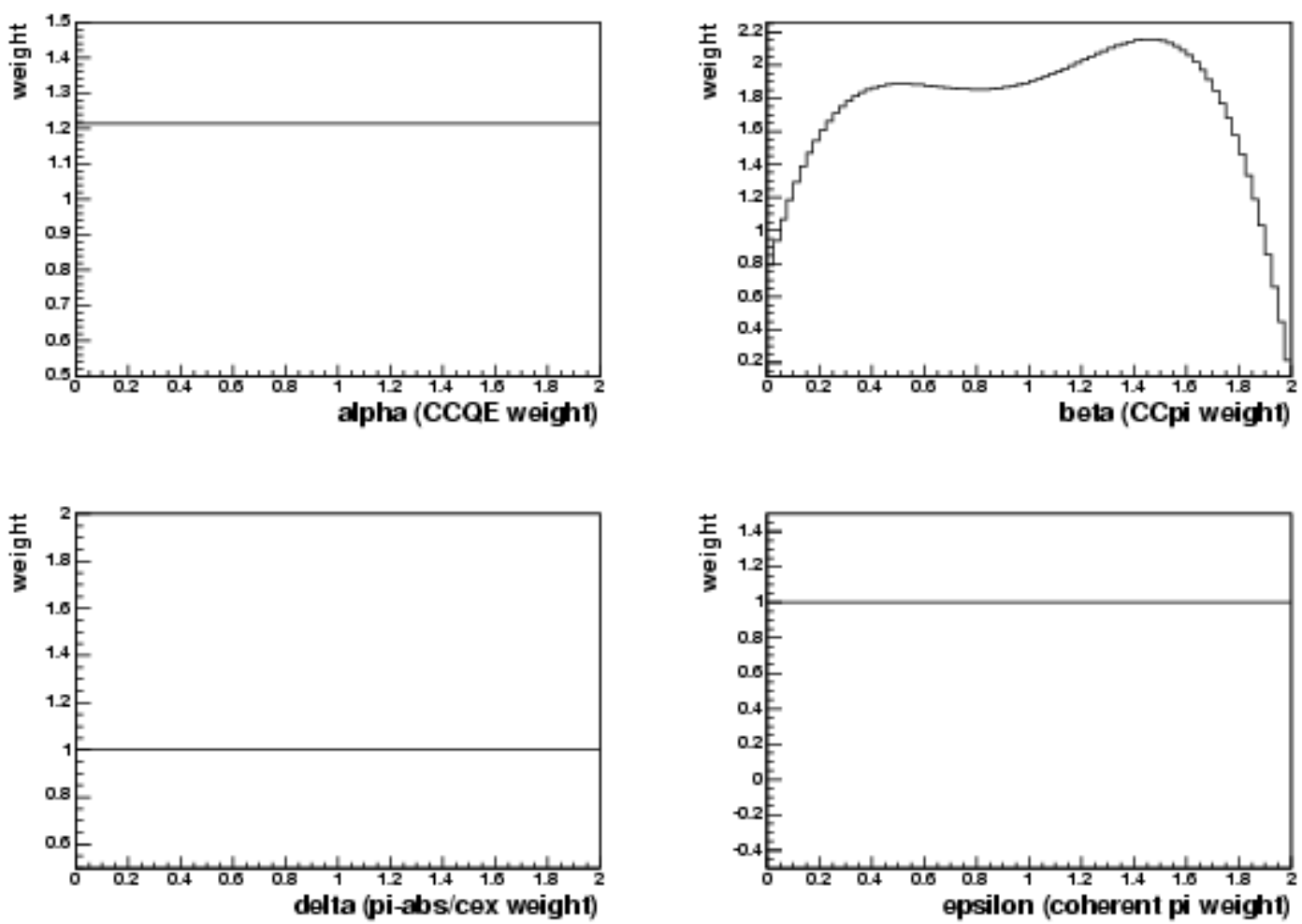

Figure 9.6: Weighting function of each channel after fit. The notations are the same as Fig. 9.4 


\subsection{Pion absorption and coherent fraction tuning}

Although the work of the previous section resulted in good data-MC agreement in $Q^{2}$, unfortunately that is not the end of story. Since different hypotheses for pion absorption and pion coherent fraction also provide good fits, we need these uncertainties into account in the errors. For example, if the pion absorption scaling factor is one of the fitting parameters, the entire $Q^{2}$ region may be well-fit by increasing pion absorption by $\sim 30 \%$. Another example is, if the fraction of coherent $\mathrm{CC} 1 \pi$ events is zero as suggested by results from $\mathrm{K} 2 \mathrm{~K}$ and SciBooNE [32, 135], the fit then provides a different CC1 $\pi$ background shape for the 2 subevent sample.

Fig. 9.7 shows the data-MC ratio for the 3 subevent sample as a function of $Q^{2}$ with various cross section models. These plots correspond to bottom right plots of Fig. 9.5. From top to bottom, pion absorption is increased $0 \%, 15 \%$, and $30 \%$, and from left to right, the coherent fraction is decreased from default (100\%), 50\%, and $0 \%$. Top left plot is our default solution. As we see, any of these parameter combinations nicely reproduce data in $Q^{2}$. However, if data-MC agreement is examined in the two dimensional muon kinetic energy $\left(T_{\mu}\right)$ and muon angle $\left(\cos \theta_{\mu}\right)$ plane (Fig. 9.8), the models may be differentiated. This plot is analogous with Fig. 8.4, although Fig. 8.4 is the data-MC ratio, however here is

the distribution of pull $\left(=\frac{\text { data-MC }}{\text { statistical error }}\right)$ in all bins. Clearly, the $\chi^{2}$ value shows that some of the parameter choices are better than others to reproduce data. The general trends are:

1. Larger pion absorption is preferred, but not too large;

2. Lower coherent fraction gives better fit.

We chose to use a compromise solution, that of the central plot in Figs. 9.7 and 9.8, where the pion absorption is increased by $15 \%$ and coherent fraction decreased by $50 \%$. This was selected as our new central value (cv) MC, with the remaining parameter values as possible 

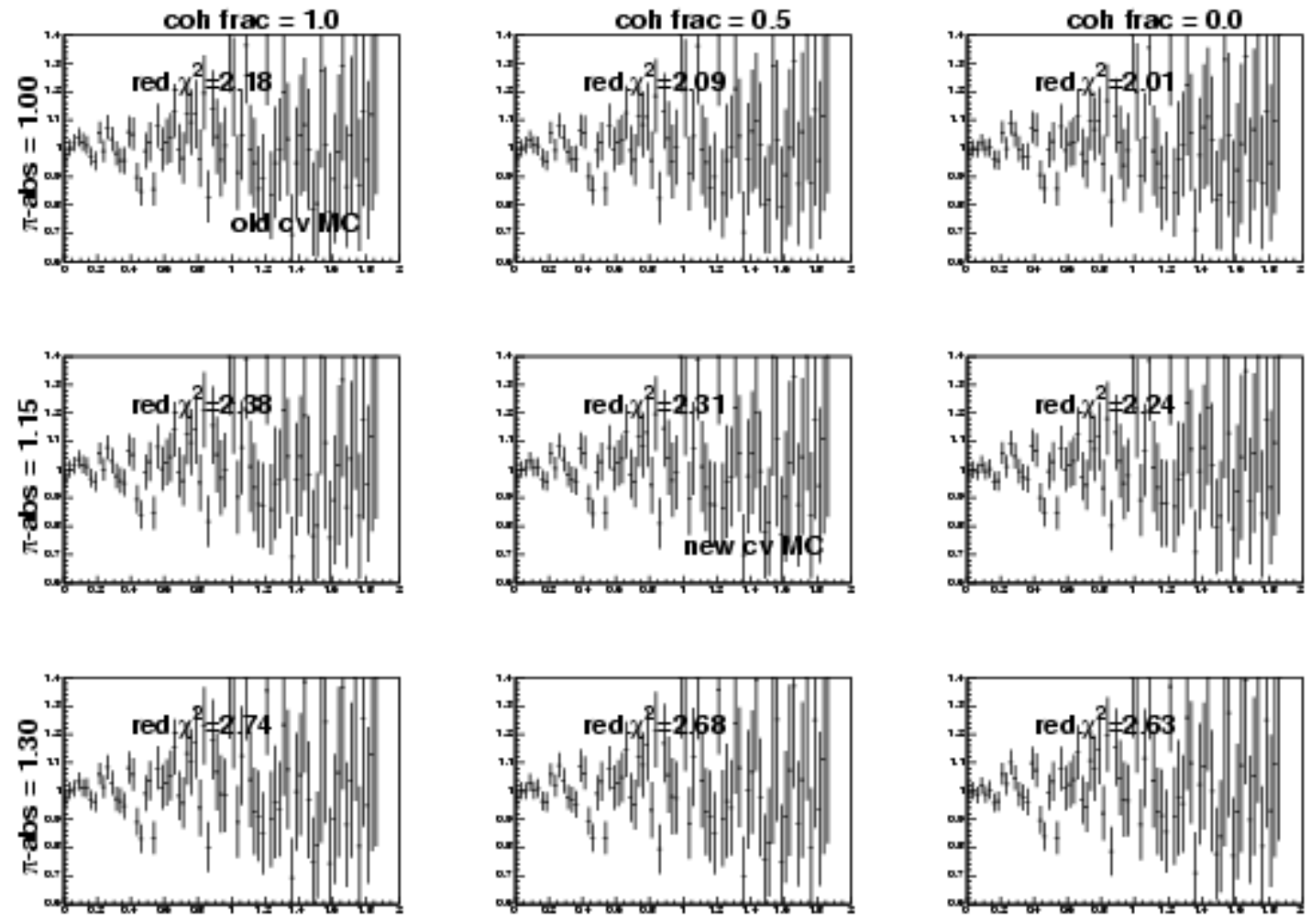

Figure 9.7: Data-MC ratio of $Q^{2}\left(0-2 \mathrm{GeV}^{2}\right)$ in the 3 subevent sample with various cross section models. Numbers in the plots show statistical error only data-MC reduced $\chi^{2}$. From top to bottom, pion absorption is increased $0 \%, 15 \%$, and $30 \%$. From left to right, coherent fraction is decreased from default (100\%), 50\%, and $0 \%$. Top left plot is old cv, and the middle plot is the new cv. 

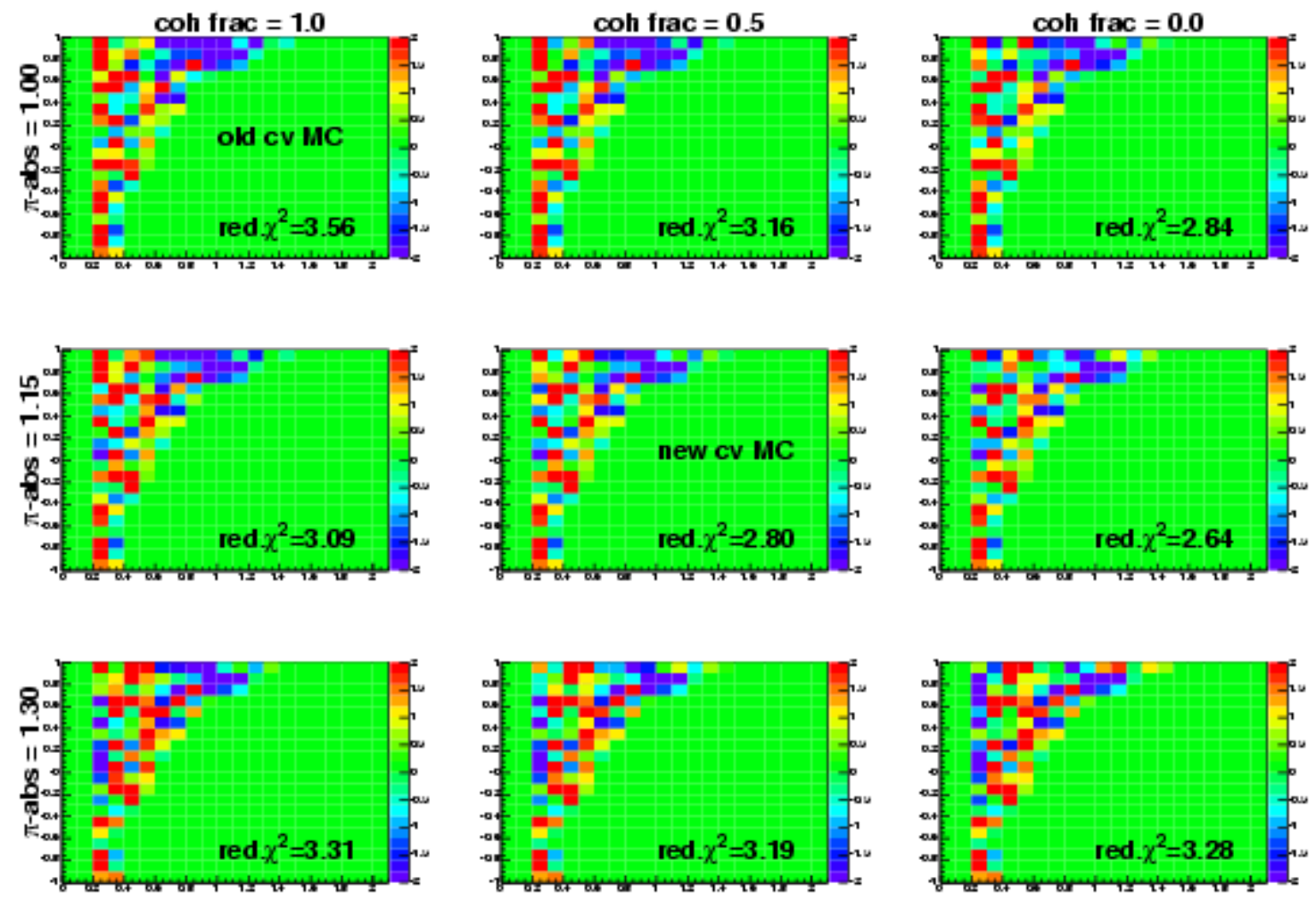

Figure 9.8: Data-MC pull $\left(=\frac{\text { data-MC }}{\text { statistical error }}\right)$ distribution in two dimensional muon kinematics $\left(0<T_{\mu}(\mathrm{GeV})<2\right.$ and $\left.-1<\cos \theta_{\mu}<1\right)$ for 3 subevent sample for the various cross section models. Numbers in the plots show statistical error only data-MC reduced $\chi^{2}$. From top to bottom, pion absorption is increased $0 \%, 15 \%$, and $30 \%$. From left to right, coherent fraction is decreased from default (100\%), 50\%, and 0\%. Top left plot is old cv, and the middle plot is our new cv. 
excursions accounted for in the error. Trend (1) above suggests the a $15 \%$ increase of pion absorption is a reasonable choice. Also the magnitude of this change is within the range of both nuclear pion absorption (25\% error, Fig. 7.9) and detector pion absorption (35\% error, Fig. 7.10). Trend (2) suggests zero coherent fraction is the best choice, but to make a more conservative choice, a $50 \%$ decrease of coherent fraction was selected with an error of $100 \%$ on this value (Sec. 10.6). This value is rather close to the MiniBooNE-measured $\mathrm{NC} \pi^{\circ}$ coherent fraction ( $25 \%$ of the predicted value [121]). This democratic choice covers both $0 \%$ (the K2K/SciBooNE prefered value) and 100\%(the MiniBooNE default value) coherent fraction within the error.

With the pion absorption increased by $15 \%$, the background in the 2 subevent sample increases the signal in the 3 subevent sample decreases. This last change further increases the $\mathrm{CC} 1 \pi$ fraction in the CCQE sample to around $21 \%$, for a total increase of $39 \%$. This corresponds to $6 \%$ increase in the background of the CCQE sample and the CCQE fraction decreases by $6 \%$ from our standard nuance prediction (Sec. 7.4).

Figs. 9.9 and 9.10 show the prediction of this new cv MC compared to data. The low $Q^{2}$ data-MC disagreement is left to be understood with the CCQE model (parameters $M_{A}$ and $\kappa)$. 

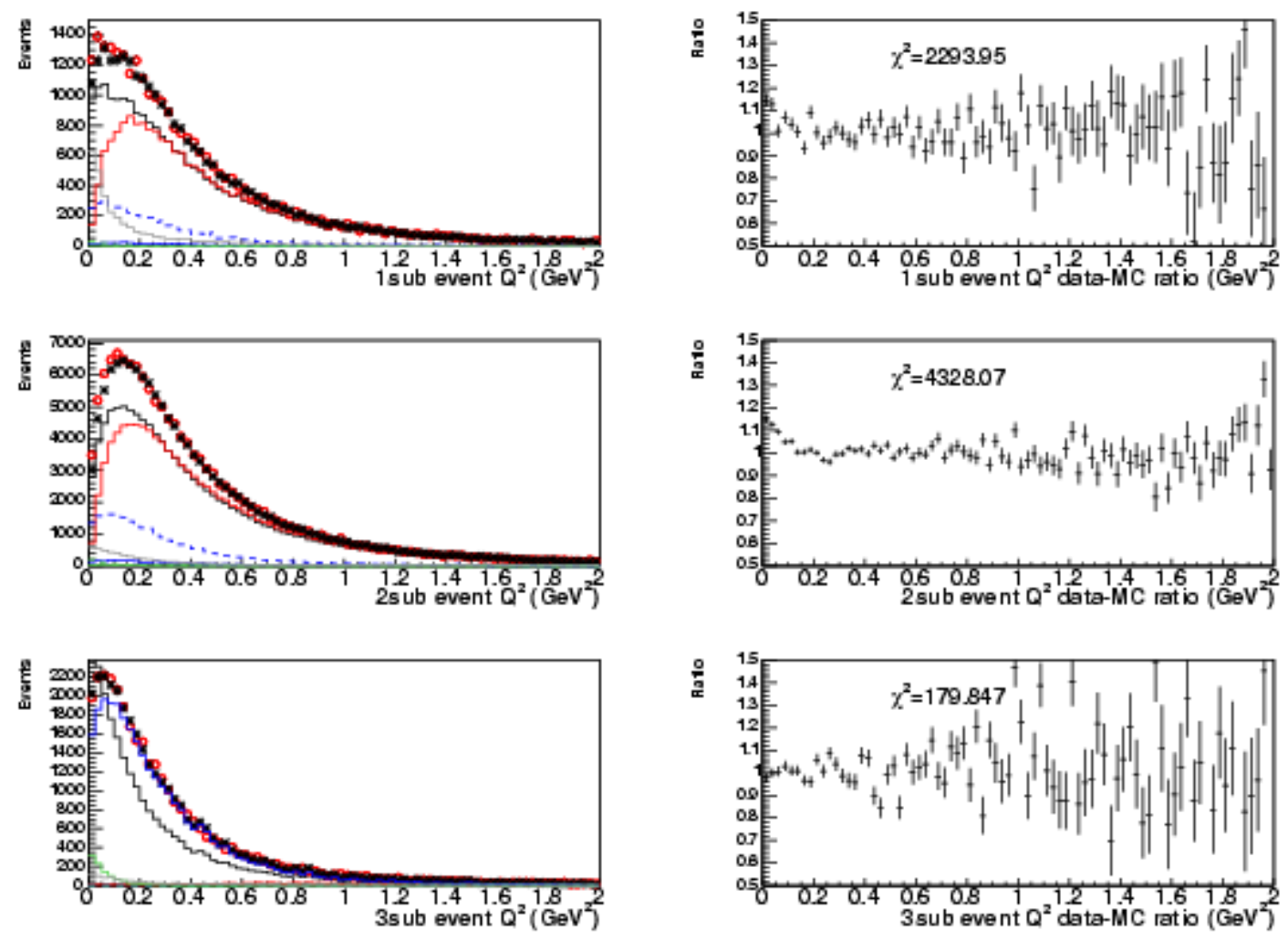

Figure 9.9: Data-MC comparison for $Q^{2}$ plots after fit with new cv MC. The notations are same with Fig. 9.3. Note $\chi^{2}$ s shown here come from all $Q^{2}$ bins, but for 1 and 2 subevents, the first four bins are not used in fit. 

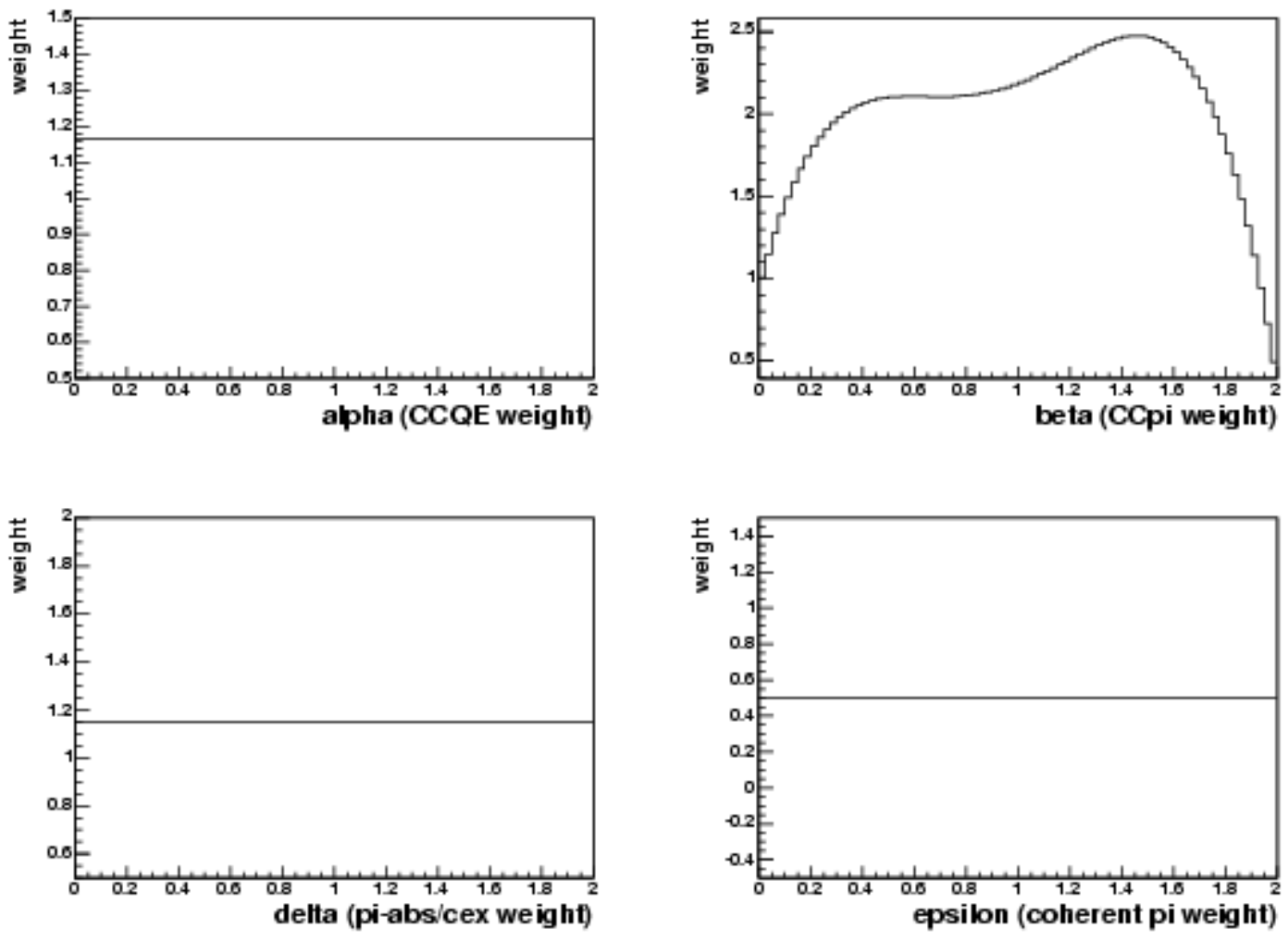

Figure 9.10: Weighting function of each channel after fit with new cv MC. The notations are same with Fig. 9.4 . 


\section{$9.7 \quad M_{A}-\kappa$ fit}

\subsection{1 the standard fit result}

We vary two parameters, $M_{A}$ and $\kappa$ in a grid search to find the values that minimize $\chi^{2}$ of data compared to the normalized model prediction as a function of $Q^{2}$. This fit uses the inverse of error matrix including all systematic errors. Here, we take into account all possible correlations between systematics by using the inverse of the full error matrix, not by adding systematics as pull terms. All fitting MC samples with different $M_{A}$ and $\kappa$ are normalized with data (shape-only fit). We use 40 bins, from 0 to $1 \mathrm{GeV}^{2}$ (38 d.o.f.) for the standard fit.

$$
\begin{aligned}
\chi^{2} & =(\text { data }-M C)^{T} M_{\text {total }}^{-1}(\text { data }-M C), \\
M^{\text {total }} & =M^{p p}+M^{p m}+M^{k p}+M^{k 0}+M^{b e}+M^{x s}+M^{p 0}+M^{o m}+M^{u n}+M^{p i}+M^{d t}+M^{m c} .
\end{aligned}
$$

Where the $M$ are the error matrices resulting from various sources of uncertainty,

$$
\begin{aligned}
& M^{p p}(4.5 \%) \pi^{+} \text {production spline fit, } \\
& M^{p m}(0.3 \%) \pi^{-} \text {production Sanford-Wang fit, } \\
& M^{k p}(0.2 \%) K^{+} \text {production Feynman scaling fit, } \\
& M^{k 0}(0.0 \%) K^{o} \text { production Feynman scaling fit, } \\
& M^{b e}(4.5 \%) \text { beamline and horn models, } \\
& M^{x s}(3.1 \%) \text { cross section models, } \\
& M^{p 0}(0.0 \%) \pi^{\circ} \text { yield, } \\
& M^{o m}(3.3 \%) \text { detector models, } \\
& M^{u n}(2.5 \%) \text { electronics models, }
\end{aligned}
$$


$M^{p i}(1.0 \%)$ pion absorption and coherent fraction model error,

$M^{d t}(0.3 \%)$ data statistics,

$M^{m c}(0.2 \%)$ MC statistics.

Each error matrix is created using either a multisim or unisim method, as described in Sec. 10.6. The numbers in the parentheses show the resulting total normalization error, also defined in Sec. 10.6.

The total normalization error is equivalent with the error on a single bin histogram. Note that this number contains no information about the $Q^{2}$ shape uncertainty, so a "big" error matrix does not necessarily have to a large effect on the uncertainty in the fit. But, nevertheless, it shows the rough size of each error matrix.

The result of the final fit is shown in Fig. 9.11. The best-fit values are:

$$
\begin{aligned}
M_{A} & =1.32 \pm 0.17 \mathrm{GeV} / \mathrm{c}^{2} ; \\
\kappa & =1.007 \pm 0.009 ; \\
\chi^{2} / d o f & =44.3 / 38 .
\end{aligned}
$$

Fig. 9.12 shows in more detail the best fit point and associated contour. Since the new fit result (solid star) is barely the outside of the $1-\sigma$ contour of the old fit (olive contour). We conclude the new fit value of $M_{A}$ and $\kappa$ are consistent with our previous fit. 

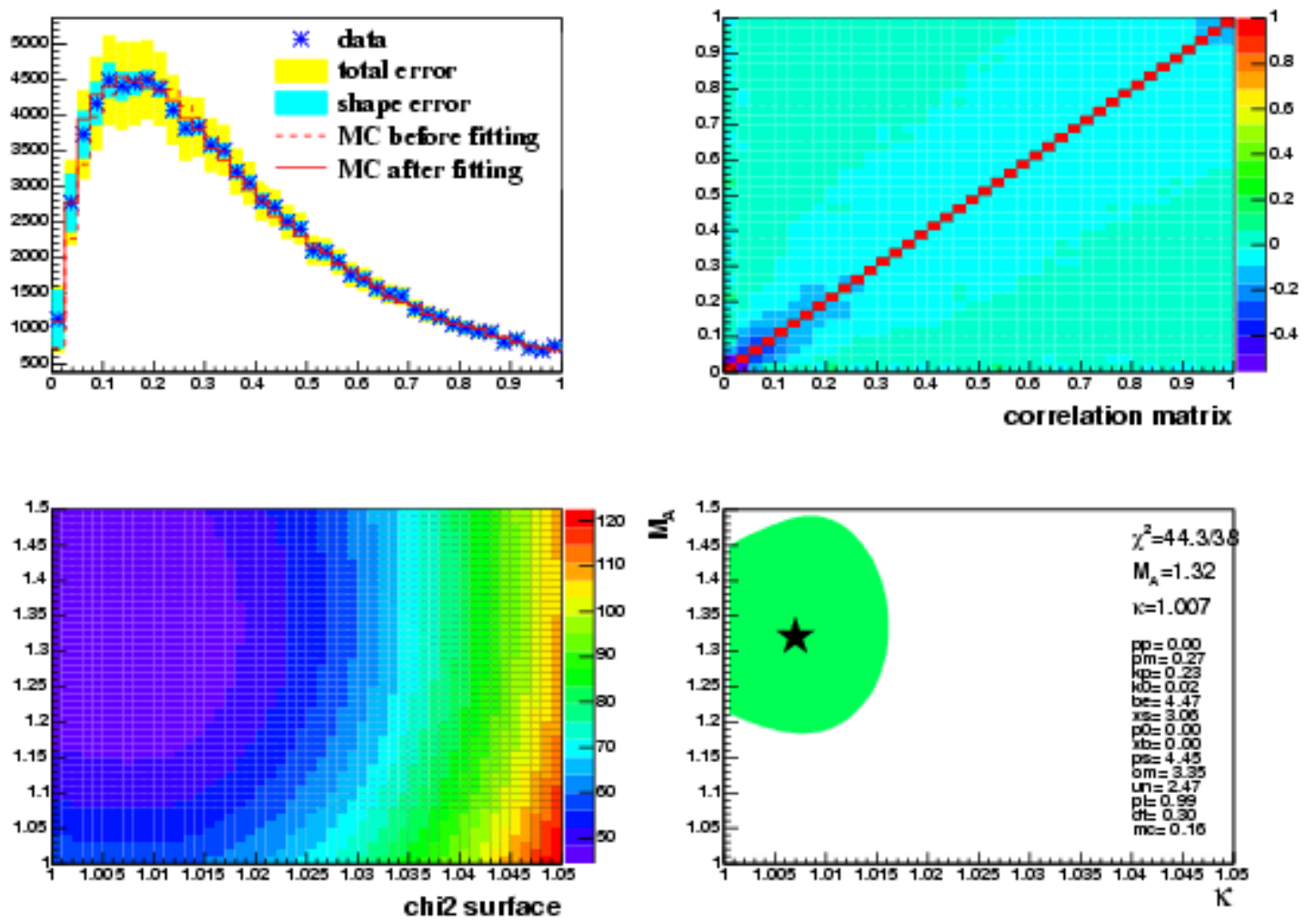

Figure 9.11: The plots for $M_{A}-\kappa$ fit. Top left plot shows data-MC comparison before and after the fit. Blue dots are data with total error (yellow) and shape only error (turquoise), and red dashed line and solid lines shows before fit and after fit. Top right plot is correlation matrix within fitting region of $Q^{2}$ plot, bottom left plot shows $\chi^{2}$ surface in $M_{A}-\kappa$ space. Bottom right plot shows the best fit point and $1-\sigma$ contour, defined $\chi_{\min }^{2}+2.3$, here "2.3" is the $\chi^{2}$ value to have $68.3 \%$ cumlative probability for $\chi^{2}$ distribution with 2 d.o.f [89, 90]. 


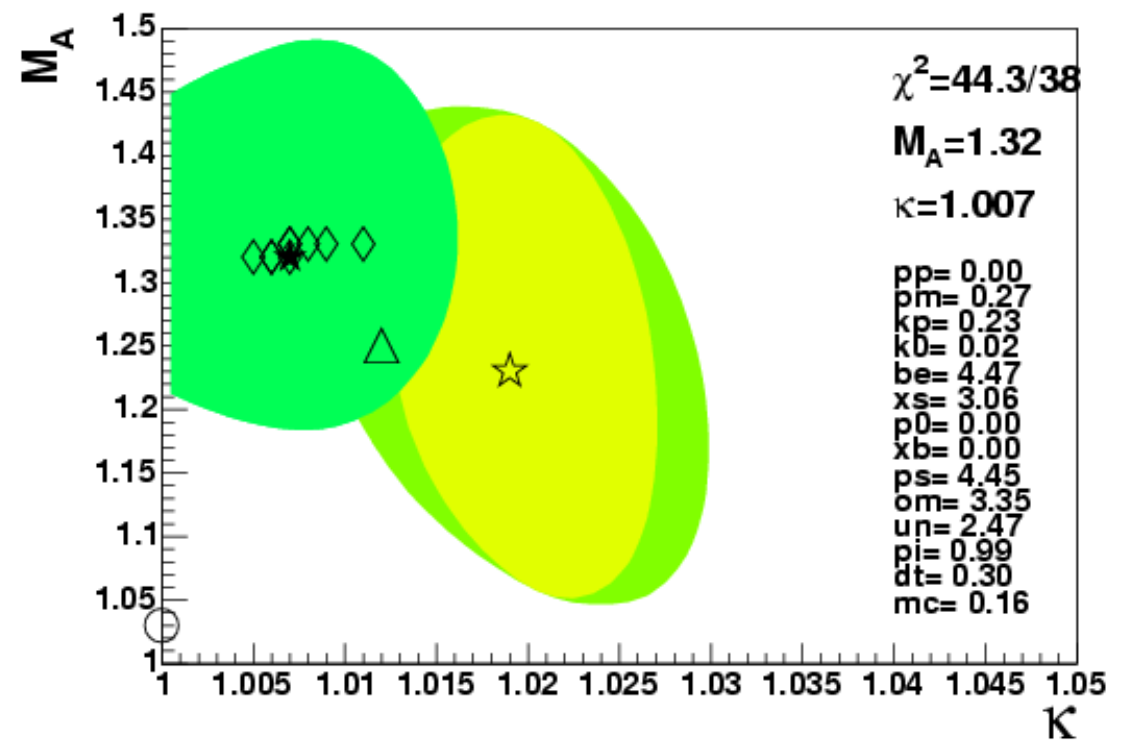

Figure 9.12: The detail of contour plot. Here the solid star marker represents the best fit point and the green region is the $1-\sigma$ contour. There are nine open diamond markers, they represent the best fit points of $\mathrm{cv} \mathrm{MC}$ and other eight cross section models without pion absorption model error $\left(M^{p i}\right)$. The $1-\sigma$ contour covers all these possible solutions. The open circle marker indicates the original parameter set from nuance. The open star marker and ocher contour is the best fit point and $1-\sigma$ contour from Chapter 8. In Chapter 8, the $\mathrm{CC} 1 \pi$ background shape error was treated slightly differently from the other errors. The result, open triangle marker, is added in quadrature for the final $M_{A}$ and $\kappa$ errors. In this plot, to indicate a contour that takes into account all errors, the $1-\sigma$ contour has been expanded such that $\kappa$ error becomes the size of total error in our previous work. Then this olive contour is better representative of $1-\sigma$ contour with total systematic error in Chapter 8. Right column shows the best fit $\chi^{2}$ and the best fit parameter values for $M_{A}$ and $\kappa$, as well as systematic contributions (total normalization error) described in Sec. 9.7 . 


\subsubsection{The systematic errors}

Figs. 9.13, 9.14, 9.15, and 9.16 show the fit results with (respectively) statistical error only, flux error only, cross section only, and detector error only.

The statistical error only fit (Fig. 9.13) and flux error only fit (Fig. 9.14) plots have a same structure, which means that, although the flux error contributes strongly to the normalization error, its contribution to the shape, which is important for the shape-only fit, is weak, and gives same result with statistical error only fit.

The cross section error only fit (Fig. 9.15) plot shows an elongated contour along the $\kappa$ direction. This is due to the ambiguity at low $Q^{2}$, for example from the coherent fraction, and yields a large error on $\kappa$. For practical reasons, we simulate the pion absorption and the pion charge exchange in the nuclei within the detector model simulation, so they are not included in this plot, however, the number shown in Tab. 9.3 includes all cross section model errors.

Fig. 9.16 shows the error from the detector modeling. This plot has not only a large contour, but also has a different best fit point from the others. Note that all of the optical model multisim histograms and detector unisim histograms are numerically smoothed to eliminate MC-statistics noise [112, 113. To do this, we take a ratio of each histogram

with cv MC histogram, then this ratio is fit with $4^{\text {th }}$ order polynomial function. The products of this extracted function and cv MC histogram replaces each original histogram. The large errors of both $M_{A}$ and $\kappa$ are explained by the large background fractions. 1,2,3 subevent fitting (Sec. 9.5), together with the pion absorption correction (Sec. 9.6), increases the background fraction by around $6 \%$, or the $\mathrm{CC} 1 \pi$ fraction in the background channels increases by nearly $40 \%$. Since $90 \%$ are pion-absorbed events, the error on pion absorption gives large uncertainties for both $M_{A}$ and $\kappa$. Note, because of a technical reason, we include the nuclear pion absorption error in the detector model error. 
CHAPTER 9. SIMULTANEOUS CCQE AND CC1 $\pi Q^{2}$ FIT

\begin{tabular}{ccc}
\hline error source & $\delta M_{A}$ & $\delta \kappa$ \\
\hline data statistics & 0.05 & 0.004 \\
neutrino flux & 0.04 & 0.003 \\
neutrino cross sections & 0.03 & 0.007 \\
detector model & 0.13 & 0.007 \\
\hline total error & 0.17 & 0.009 \\
\hline
\end{tabular}

Table 9.3: The systematic error summary for $M_{A}$ and $\kappa$. The total error is not a simple quadrature sum because of the correlation between the two parameters.

Tab. 9.3 summarizes the contribution from each of the major systematic errors. As expected, the detector model uncertainty is the largest contribution to the $M_{A}$ error, and the cross section model and the detector model give the largest error on $\kappa$. 

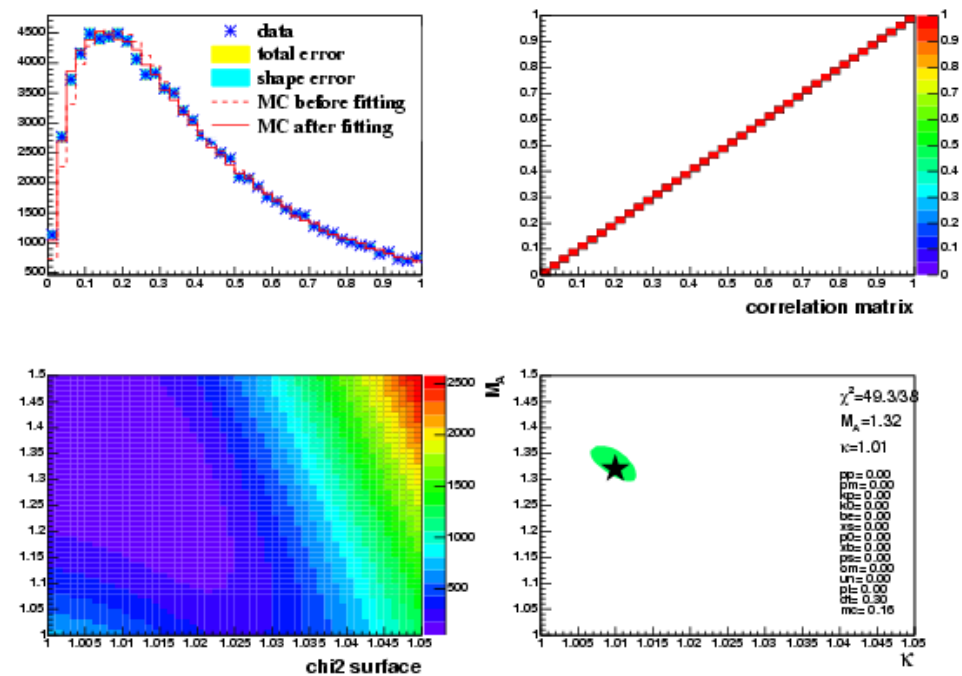

Figure 9.13: The plots for statistical error only $M_{A}-\kappa$ fit. The notations are same with Fig. 9.11
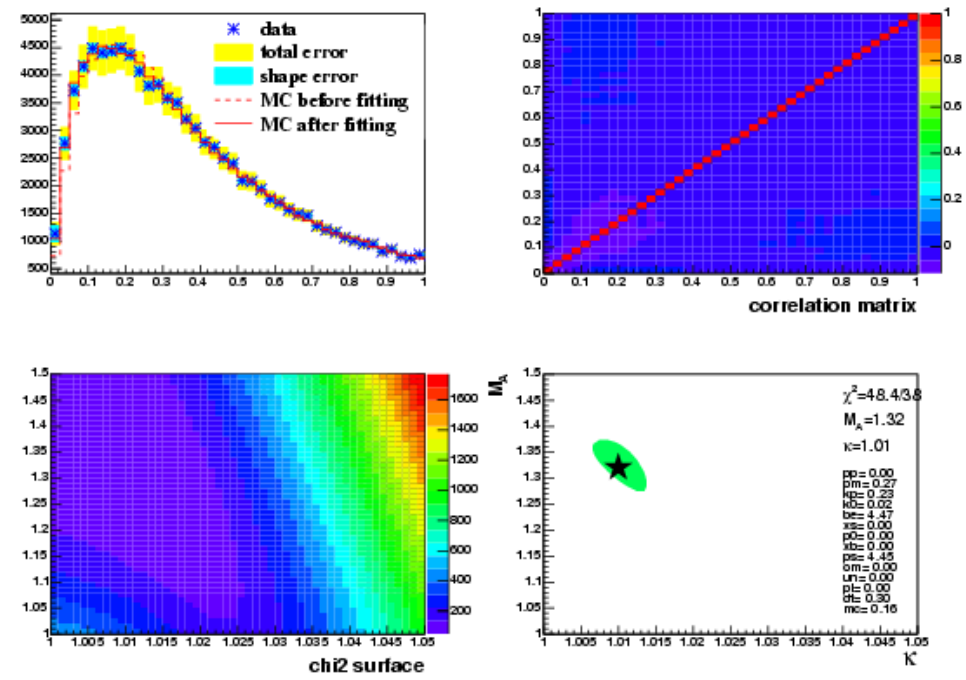

Figure 9.14: The plots for flux error only $M_{A}-\kappa$ fit. The notations are same with Fig. 9.11 

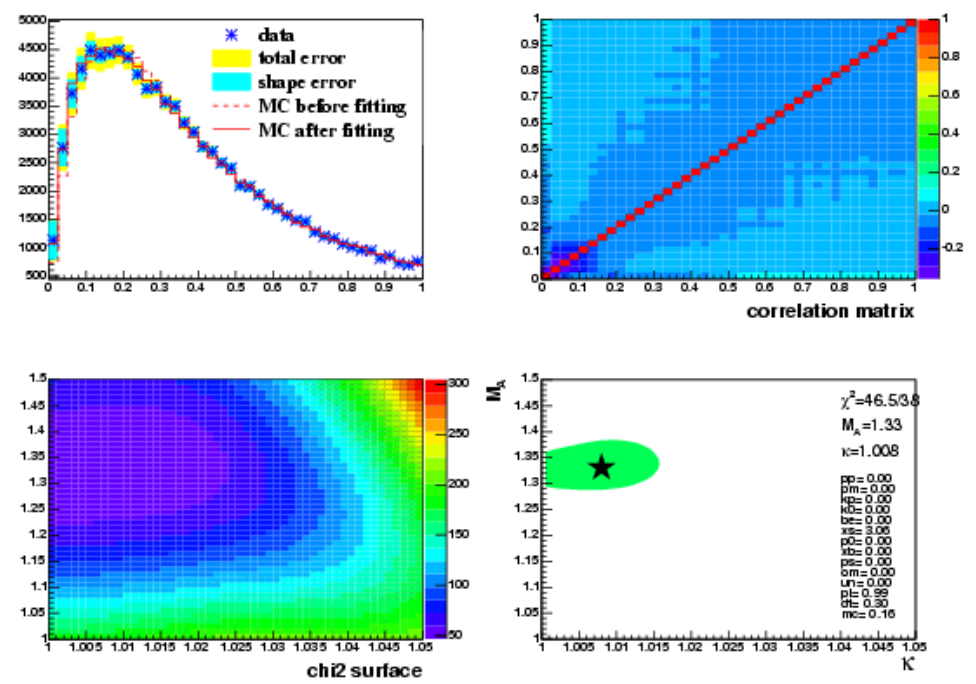

Figure 9.15: The plots for cross section error only $M_{A}-\kappa$ fit. The notations are same with Fig. 9.11
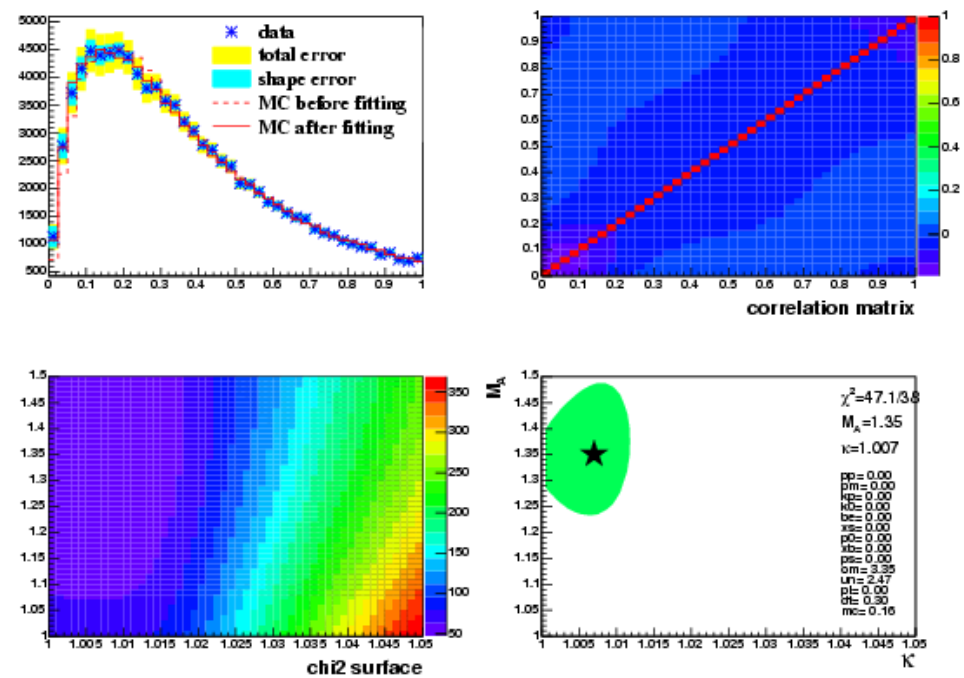

Figure 9.16: The plots for detector error only $M_{A}-\kappa$ fit. The notations are same with Fig. 9.11 


\subsubsection{The $M_{A}$-only fit}

Table 9.4 shows the fit result with $M_{A}$ as the only free parameter. Unlike the fit from Chapter 8 (Fig. 8.13), the fit is reasonable over $0.0<Q^{2}\left(\mathrm{GeV}^{2}\right)<1.0$ without $\kappa$. Also, the change of reduced $\chi^{2}$ from the fit with $\kappa$ is very small. This is due to the new background measurement (as compared to that used in Chapter 8). The shape modification of the $\mathrm{CC} 1 \pi$ measured background to the 2 subevent sample better fits the data in the low $Q^{2}$ region.

\subsection{4 $Q^{2}$ distributions with the fit $M_{A}$ and $\kappa$}

Figure 9.17 is the analog Fig. 9.9 but with the fit $M_{A}(=1.32 \mathrm{GeV})$ and $\kappa(=1.007)$ from the procedure described in this chapter. As expected, all of 1,2 , and 3 subevent sample $Q^{2}$ plots show good data-MC agreement.

\subsubsection{Conclusion}

In this chapter, we measured the $\mathrm{CC} 1 \pi$ background in CCQE sample using data. The majority of the background events are $\mathrm{CC} 1 \pi$ events without pions. We determined their $Q^{2}$ behavior using a $\mathrm{CC} 1 \pi$ sample with pions and applied to CCQE sample. This background-

\begin{tabular}{lccc}
\hline$Q^{2}\left(\mathrm{GeV}^{2}\right)$ & d.o.f & $\chi^{2}$ & $M_{A}\left(\mathrm{GeV} / \mathrm{c}^{2}\right)$ \\
\hline $0.00<Q^{2}<1.00$ & 39 & 45.2 & $1.32 \pm 0.12$ \\
$0.05<Q^{2}<1.00$ & 37 & 43.5 & $1.26 \pm 0.13$ \\
$0.10<Q^{2}<1.00$ & 35 & 40.8 & $1.31 \pm 0.14$ \\
$0.15<Q^{2}<1.00$ & 33 & 38.6 & $1.31 \pm 0.14$ \\
$0.20<Q^{2}<1.00$ & 31 & 36.7 & $1.31 \pm 0.14$ \\
\hline
\end{tabular}

Table 9.4: $M_{A}$ only fit results with limited $Q^{2}$. 

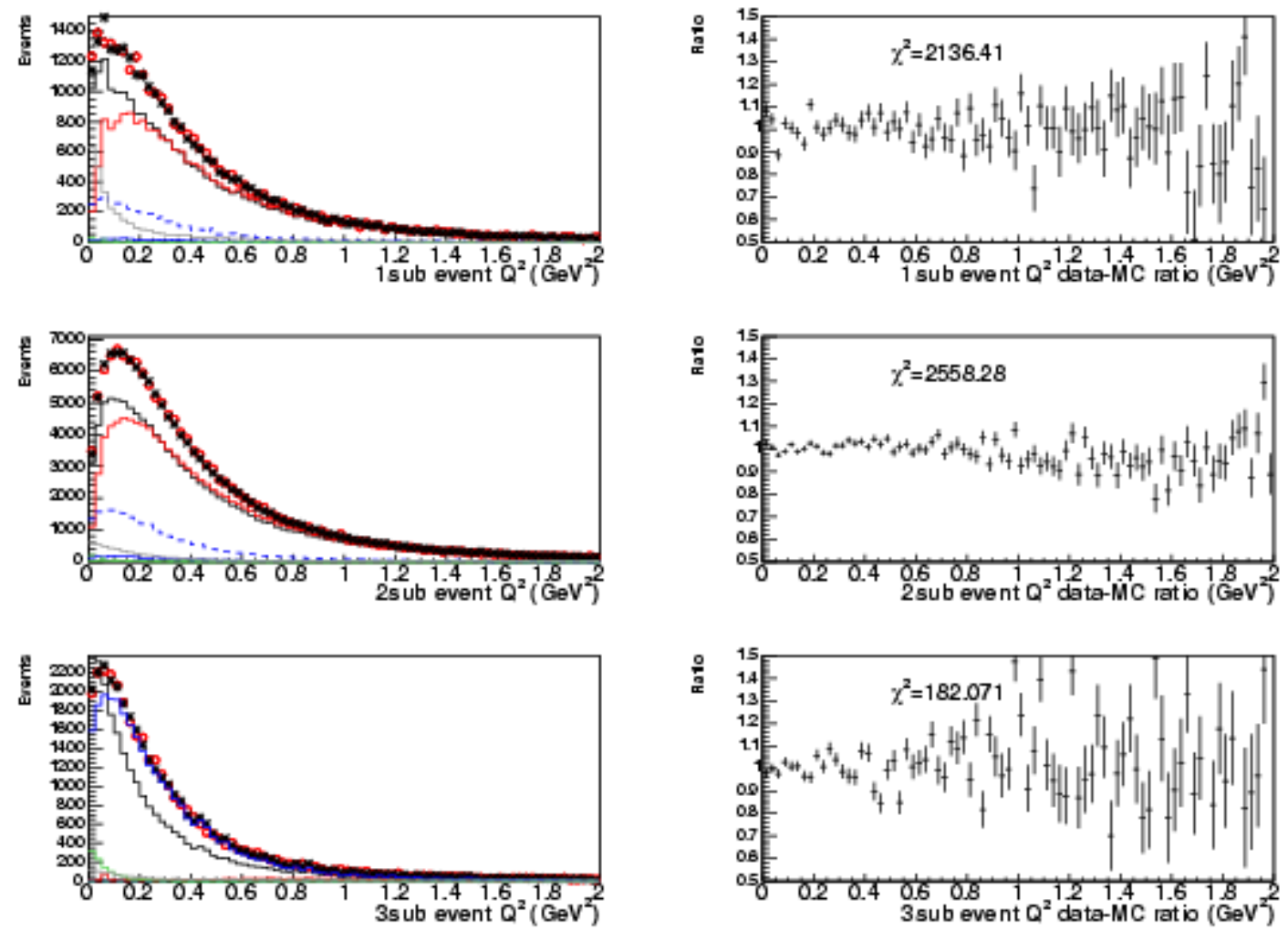

Figure 9.17: Data-MC comparison for $Q^{2}$ plots after fit with new $M_{A}$ and $\kappa$. The notations are same with Fig. 9.3 . 
9.7. $M_{A}-\kappa$ FIT

subtracted CCQE sample is used to measure the absolute cross section, as described in the next chapter. 


\section{Chapter 10}

\section{CCQE double differential cross}

\section{section measurement}

In this chapter, we present the absolute CCQE cross section measurement in MiniBooNE. First, we define our cross section formula and all systematic errors carefully. Then, using the result from Chapter 9 , the backgrounds are subtracted from the data. This backgroundsubtracted data is unfolded by the direct transformation matrix from reconstructed variables to true variables. We use iteration to improve our result. Finally, efficiency and target numbers are corrected.

The cross sections are shown in three ways, (1) flux-folded double differential cross section $\left(\frac{d^{2} \sigma}{d T_{\mu} d \cos \theta_{\mu}}\left(\mathrm{cm}^{2} / 0.1 / 0.1 \mathrm{GeV}\right)\right),(2)$ flux-folded differential cross section $\left(\frac{d \sigma}{d Q^{2}}\left(\mathrm{~cm}^{2} / 0.1 \mathrm{GeV}^{2}\right)\right)$, and (3) flux-unfolded total cross section $\left(\sigma\left(\mathrm{cm}^{2}\right)\right)$. The measured total cross section at flux peak is, $\sigma_{700-800 \mathrm{MeV} \text { bin }}^{C C Q E}=1.058 \pm 0.111 \times 10^{-38} \mathrm{~cm}^{2}$. The error is dominated by the systematic error. The measured error is $\sim 20 \%$ higher than the original nuance prediction, however, it agrees with the new $M_{A}\left(1.32 \mathrm{GeV} / \mathrm{c}^{2}\right)$ and $\kappa(1.007)$ found in Chapter 9 . 


\subsection{Introduction of cross section measurement}

Theoretical physicists want experimental data to test theories. Since the effects of experimental smearing are not easily calculable by the theorists, they need data after experimental effects are removed. We formulate the cross section formula rigorously in next section, instead, here, we want to show how experimental effects are unfolded to produce the final results. We call $D$ our measurement, namely all experimental-effect-corrected data, and this is binned with variable $T$ (muon energy, neutrino energy, interaction vertex, etc), then $i^{\text {th }}$ bin of data $\left(\frac{d D}{d T}\right)_{i}$ is written,

$$
\int\left(\frac{d \sigma\left(E_{\nu}\right)}{d T}\right)_{i} \cdot \phi\left(E_{\nu}\right) d E_{\nu}=\left(\frac{d D}{d T}\right)_{i} \pm \delta\left(\frac{d D}{d T}\right)_{i}
$$

The left side is calculated by theorists, and they compare their calculation with the right side, that is the result from the experimentalists. The data points have an associated error for each bin, and there are correlations between bins. These errors include all experimental effects such as uncertainties in flux, background cross section model, detector effects, etc. The various source of errors contribute to both shape and normalization errors.

Traditionally, experimentalists provide their results in the form of a so called "fluxfolded differential cross section". The flux-folded cross section is defined as a cross section averaged over the neutrino flux $\left(E_{\nu} \equiv E_{\nu}^{c}\right.$ : const), then,

$$
\left(\frac{d \sigma\left(E_{\nu}^{c}\right)}{d T}\right)_{i}=\frac{1}{\Phi\left(E_{\nu}^{c}\right)} \cdot\left(\frac{d D}{d T}\right)_{i}
$$

Here, $\Phi\left(E_{\nu}^{c}\right)=\int_{0}^{\infty} \phi\left(E_{\nu}\right) d E_{\nu}$ is the integral of neutrino flux with mean energy $E_{\nu}^{c}$. The special case is considered when the experimental measurement is a function of neutrino energy. Then we can calculate the total cross section for each neutrino energy bin,

$$
\sigma\left(E_{\nu}\right)_{i}=\left(\frac{D}{\phi\left(E_{\nu}\right)}\right)_{i}
$$

We call this the "flux-unfolded total cross section". As we see in Sec. 10.3, this expression depends on nuclear models and reconstruction method. We provide our data in a nuclear 
model independent way, however it still suffers dependence on the energy reconstruction (Fig. 10.12).

\subsection{Neutrino energy and $Q^{2}$ reconstruction}

Throughout this chapter, the neutrino energy, $E_{\nu}$, and the 4-momentum transfer, $Q^{2}$, are always defined as "reconstructed" $E_{\nu}$ and $Q^{2}$. For example, for the unfolding process, we defined true neutrino energy as, not MC true information neutrino energy, but the neutrino energy reconstructed from MC true muon energy and MC true muon angle. In this way, true neutrino energy is nuclear model independent. Reconstructed neutrino energy $E_{\nu}$ and $Q^{2}$ are defined as followings,

$$
\begin{aligned}
& E_{\nu}(\text { recon })=\frac{1}{2} \cdot \frac{2\left(M_{n}-B\right) E_{\mu}-\left(\left(M_{n}-B\right)^{2}+m_{\mu}^{2}-M_{p}^{2}\right)}{\left(M_{n}-B\right)-E_{\mu}+\sqrt{E_{\mu}^{2}-m_{\mu}^{2}} \cos \theta_{\mu}} \\
& Q^{2}(\text { recon })=-m_{\mu}^{2}+2 E_{\nu}\left(E_{\mu}-\sqrt{E_{\mu}^{2}-m_{\mu}^{2}} \cos \theta_{\mu}\right) .
\end{aligned}
$$

And, we use $=34 \mathrm{MeV}$ as a binding energy (Sec. 7.4.

\subsection{Absolute cross section formula}

\subsubsection{The absolute cross section formula}

For a given data set, the amount of background is estimated using MC. Using the central value (cv) MC background prediction, one can subtract background content from the $j^{\text {th }}$ bin of data,

$$
d_{j}-b_{j}^{c v}
$$

Here, the central value (cv) MC is the our standard MC used to compare with data. $d$ and $b^{c v}$ are data and $\mathrm{cv} \mathrm{MC}$ background histograms (in bin $\mathrm{j}$ ) and are, generally, functions 


\subsection{ABSOLUTE CROSS SECTION FORMULA}

of reconstructed variables, for example visible energy, measured angle, etc. Since the data are always a function of reconstructed variables, which is the experimentalist's language, we need to transform to function of physical true variables. This process is generally called unfolding. Unfolding has two parts, unsmearing, and efficiency correction. For unsmearing, our standard method is to use the normalized reconstructed to true transformation matrix, instead of inverting the smearing matrix, which is true-to-reconstructed transformation. The corrected data are calculated with

$$
\sum_{j}^{n} u_{i j}^{c v} \cdot\left(d_{j}-b_{j}^{c v}\right) .
$$

For a given reconstructed to true transformation matrix $U_{i j}^{c v}$, the normalized matrix $u_{i j}$ is defined by normalizing in true index $i$,

$$
u_{i j}^{c v}=\frac{U_{i j}^{c v}}{\sum_{k} U_{k j}^{c v}},
$$

then, $u_{i j}^{c v}$ is the probability of events in the $j^{\text {th }}$ reconstructed bin to come from $i^{\text {th }}$ true bin and,

$$
\sum_{i}^{n} u_{i j}^{c v}=1
$$

After this procedure, the data may be binned using the "true" variables. However, unfolding is not finished. Since the detector efficiency biases the data, we need a correction for that. The meaning of this process is to "recover" the events eliminated by series of cuts using the MC generated event information. The efficiency is corrected by the number of events after cut (accepted) divided by the number before the cut (generated). Then,

$$
\frac{\sum_{j}^{n} u_{i j}^{c v} \cdot\left(d_{j}-b_{j}^{c v}\right)}{\frac{N_{i}^{a c c, c v}}{N_{i}^{g e n, c v}}} .
$$

Finally we need to correct for flux, the number of protons on target (POT), and the target number by dividing by the flux factor $\Phi$, the POT delivered in data taking period $P$, and 
the number of target nucleon in the fiducial volume $T$, Therefore,

$$
\sigma_{i}^{\text {flux-folded }}=\frac{\sum_{j}^{n} u_{i j}^{c v} \cdot\left(d_{j}-b_{j}^{c v}\right)}{\frac{N_{i}^{a c c, c v}}{N_{i}^{g e n, c v}} \cdot \Phi^{c v} \cdot P^{c v} \cdot T^{c v}} .
$$

This is the standard flux-folded differential cross section formula as function of some true variables.

The special case is considered when the cross section is written as a function of neutrino energy. First of all, neutrino energy is a reconstructed variable. For the unfolding process, we defined the true neutrino energy as, not the MC true information neutrino energy, but the neutrino energy reconstructed from MC true muon energy and MC true muon angle. In this way, the true neutrino energy is nuclear model independent.

Unfortunately this choice is not perfect neither. As can be seen from Fig. 10.1, reconstructed neutrino energy is never the same (with scattering from carbon) with the true neutrino energy across the entire energy region. True neutrino energy defined from true muon energy and true muon angle suffers (1) a bias from the nuclear binding, and (2) a smearing due to Fermi motion. To take account the bias of neutrino energy reconstruction, we measured systematic shift and smearing from the difference between MC true neutrino energy and reconstructed true neutrino energy for each $0.1 \mathrm{GeV}$ energy bin. Then, we assign horizontal error bars for each flux-unfolded total cross section bin. We can divide this by predicted neutrino flux histogram $\phi_{i}^{c v}$ to remove neutrino flux shape, bin by bin of neutrino energy, and the difference of reconstructed true neutrino energy and MC true neutrino energy are within vertical and horizontal error bars. Finally, the flux-unfolded total cross section formula is,

$$
\sigma_{i}^{f l u x-\text { unfolded }}=\frac{\sum_{j}^{n} u_{i j}^{c v} \cdot\left(d_{j}-b_{j}^{c v}\right)}{\frac{N_{i}^{a c c, c v}}{N_{i}^{g e n, c v}} \cdot \phi_{i}^{c v} \cdot P^{c v} \cdot T^{c v}} .
$$

For our case, the effect of neutrino energy reconstruction bias is within the error (Fig. 10.12), so it is not a big problem. 

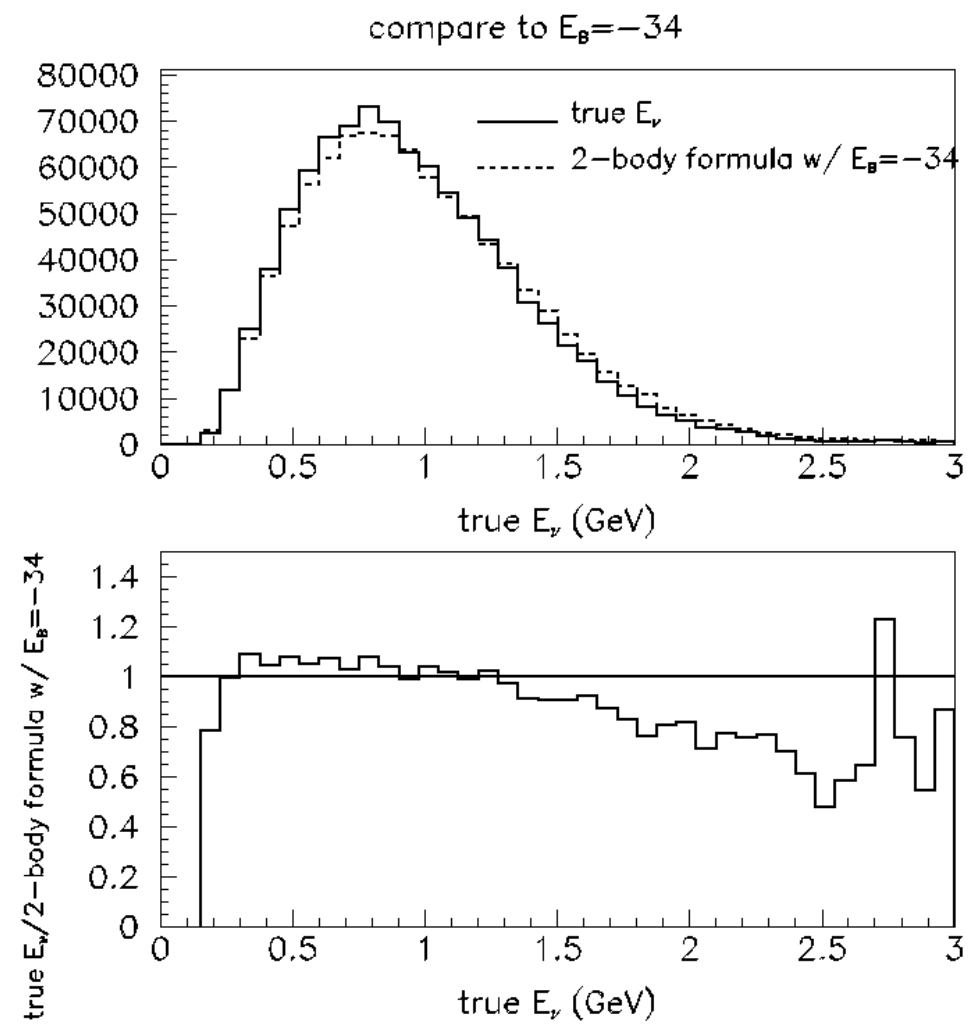

Figure 10.1: Plot of MC true neutrino energy and reconstructed true neutrino energy. The top plot shows MC true neutrino energy (solid) and reconstructed true neutrino energy (dashed) from Eq. 10.4 with $B=34 \mathrm{MeV}$, with MiniBooNE flux prediction. The bottom plot shows their ratio. The ratio is flat in the region our data is presented (0.1 to $1.4 \mathrm{GeV}$, see Fig. 10.12). 


\subsubsection{Three normalization factors}

The cross section formulas (Eq.10.6 and 10.7) have the following three normalization terms:

- $\Phi=5.167 \times 10^{-10}($ neutrinos $/ \mathrm{POT})$, POT normalized integrated $\nu_{\mu}$ flux;

- $P=5.58 \times 10^{20}($ POT), POT collected between August 2002 and December 2005

- $T=1.5134 \times 10^{32}$ (nucleon), $\frac{4}{3} \pi(550 \mathrm{~cm})^{3} \cdot 0.845 \mathrm{~g} / \mathrm{cm}^{3} \cdot 6.02214 \times 10^{23} \cdot \frac{6.0}{14.06}$.

The $\Phi$ error is accounted for in the fluctuations on number of generated events (Sec. 10.7). The error on $P$ is $2 \%$ from the absolute POT number and $1 \%$ from the beam optics [117].

Finally, the error on $T$ is calculated from its components; volume, density, Avogadro's number, and chemical composition. The error associated with volume is important to correctly estimate the uncertainty in target number. The relevant considerations are:

1. error on the reconstructed data vertex and the reconstructed MC vertex;

2. error on the reconstructed MC vertex and the true MC vertex;

3. error on the true MC vertex and absolute calibration.

Here, we do not quantify (1), because this is the topic of reconstruction performance difference between data and $\mathrm{MC}$, and that is taken care by the detector simulation error in Sec. 10.9,

For (2), the inherent difference between reconstructed $\mathrm{MC}$ vertex and true MC vertex is corrected for in the efficiency correction, so we do not assign any additional errors.

Number (3) depends on the choice of target region. We decided to use a $550 \mathrm{~cm}$ sphere as a target volume. Since we use $550 \mathrm{~cm}$ radius sphere to generate MC event, there is no uncertainty related with the detector edge effect (optical separation, PMT configuration, 
eccentricity of the tank, etc), and we can safely trust that the $550 \mathrm{~cm}$ sphere in $\mathrm{MC}$ is $550 \mathrm{~cm}$ in the real world. For example, a $2 \mathrm{~cm}$ uncertainty of the wall location $(610.6 \mathrm{~cm} \rightarrow 608.6 \mathrm{~cm})$ results in a $1 \%$ uncertainty in volume, so it is better to avoid this kind of error. Also, events with $\mathrm{MC}$ true vertex $>550 \mathrm{~cm}$ make $0 \%$ contribution to reconstructed events within the $500 \mathrm{~cm}$ fiducial volume, so limiting the region to generate $\mathrm{MC}$ events to $<550 \mathrm{~cm}$ removes no events and results in no change of efficiency. So, no additional errors are assigned for this consideration.

The density error $\left( \pm 0.001 \mathrm{~g} / \mathrm{cm}^{3}\right)$ is around $0.1 \%$. The thermal expansion $\left(\sim 10^{-5} / \mathrm{K}\right)$ is negligible [116], and the error on Avogadro's number is also negligible [8]. For the chemical composition of the mineral oil, we used $\left(\mathrm{CH}_{2.06}\right)_{n}$ as a model of oil [152], another model is $\left(C_{n} H_{2 n+2}\right)$ with $n \sim 30$ [153]. They are consistent and the difference with simple model $\left(\mathrm{CH}_{2}\right)_{n}$ is $0.3 \%$.

From these studies, we decided to assign $2.26 \%$ total error matrix on top of all other errors $(2 \%+1 \%+0.1 \%+0.3 \%$ in quadrature sum). This error is only applicable for the absolute cross section measurement, thus we did not use this error contribution for the $M_{A}-\kappa$ fit (Chapter 9).

\subsection{Background accounting}

Given data and MC, one can estimate the number of background and signal events. However, the MC prediction always has some discrepancies with data, and so, ideally speaking, our prediction for background should not be sensitive to the details of the background subtraction process. However this must be checked.

Let's assume that our background prediction is perfect, for example when some reliable external measurements are available. This is true for the CCQE interaction, because the background to CCQE is almost all charged current one pion production ( $\mathrm{CC} 1 \pi)$ with 
pion absorption (and pion charge exchange), and this is measured from $\mathrm{CC} 1 \pi$ data sample (Chapter 9).

Then, there are two ways to remove background from $j^{\text {th }}$ bin of data: a background subtraction method, Eq. 10.8, and a signal fraction method, Eq. 10.9.

$$
\begin{aligned}
\sigma_{i}^{s u b} & =\frac{\sum_{j}^{n} u_{i j}^{c v} \cdot\left(d_{j}-b_{j}^{c v}\right)}{\frac{N_{i}^{a c c, c v}}{N_{i}^{g e n, c v}} \cdot \Phi^{c v} \cdot P^{c v} \cdot T^{c v}}, \\
\sigma_{i}^{f r a c} & =\frac{\sum_{j}^{n} u_{i j}^{c v} \cdot\left(d_{j} \cdot \frac{s_{j}^{c v}}{s_{j}^{c v}+b_{j}^{c v}}\right)}{\frac{N_{i}^{a c c, c v}}{N_{i}^{g e n, c v}} \cdot \Phi^{c v} \cdot P^{c v} \cdot T^{c v}} .
\end{aligned}
$$

Notice that the background subtraction method is independent of cv MC signal histogram $s_{j}^{c v}$, and only depends on cv MC background histogram $b_{j}^{c v}$.

To compare the difference of these two methods, we define the difference of data and MC prediction, both for signal and background [151],

$$
\begin{aligned}
d_{j} & =s_{j}^{\text {true }}+b_{j}^{\text {true }}, \\
s_{j}^{\text {true }} & =s_{j}^{c v}+\delta s_{j}, \\
b_{j}^{\text {true }} & =b_{j}^{c v}+\delta b_{j} .
\end{aligned}
$$

Here, the data histogram $d$ is split into a true signal histogram $s^{\text {true }}$ and a true background histogram $b^{\text {true }}$. Then, the discrepancy between the $j^{\text {th }}$ bin of the true signal histogram and the cv MC signal histogram is defined to be $\delta s_{j}$. For background, the similar discrepancy is defined to be $\delta b_{j}$.

Now, Eq. 10.8 is rewritten,

$$
\begin{aligned}
\sigma_{i}^{s u b} & =\frac{\sum_{j}^{n} u_{i j}^{c v} \cdot\left(d_{j}-b_{j}^{c v}\right)}{\frac{N_{i}^{a c c, c v}}{N_{i}^{g e n, c v}} \cdot \Phi^{c v} \cdot P^{c v} \cdot T^{c v}}=\frac{\sum_{j}^{n} u_{i j}^{c v} \cdot\left(s_{j}^{\text {true }}\right)}{\frac{N_{i}^{a c c, c v}}{N_{i}^{g e n, c v}} \cdot \Phi^{c v} \cdot P^{c v} \cdot T^{c v}}+\frac{\sum_{j}^{n} u_{i j}^{c v} \cdot \delta b_{j}}{\frac{N_{i}^{a c c, c v}}{N_{i}^{g e n, c v}} \cdot \Phi^{c v} \cdot P^{c v} \cdot T^{c v}} \\
& =\sigma_{i}^{\text {true }}+\frac{\sum_{j}^{n} u_{i j}^{c v} \cdot \delta b_{j}}{\frac{N_{i}^{a c c, c v}}{N_{i}^{g e n, c v}} \cdot \Phi^{c v} \cdot P^{c v} \cdot T^{c v}} .
\end{aligned}
$$


Similarly, Eq. 10.9 is rewritten,

$$
\begin{aligned}
& \sigma_{i}^{f r a c}=\frac{\sum_{j}^{n} u_{i j}^{c v} \cdot\left(d_{j} \cdot \frac{s_{j}^{c v}}{s_{j}^{c v}+b_{j}^{c v}}\right)}{\frac{N_{i}^{a c c, c v}}{N_{i}^{g e n, c v}} \cdot \Phi^{c v} \cdot P^{c v} \cdot T^{c v}} \\
& =\frac{\sum_{j}^{n} u_{i j}^{c v} \cdot\left(s_{j}^{c v}\right)}{\frac{N_{i}^{a c c, c v}}{N_{i}^{g e n, c v}} \cdot \Phi^{c v} \cdot P^{c v} \cdot T^{c v}}+\frac{\sum_{j}^{n} u_{i j}^{c v} \cdot\left(\frac{s_{j}^{c v} \cdot\left(\delta s_{j}+\delta b_{j}\right)}{s_{j}^{c v}+b_{j}^{c v}}\right)}{\frac{N_{i}^{a c c, c v}}{N_{i}^{g e n, c v}} \cdot \Phi^{c v} \cdot P^{c v} \cdot T^{c v}} \\
& =\frac{\sum_{j}^{n} u_{i j}^{c v} \cdot\left(s_{j}^{t r u e}\right)}{\frac{N_{i}^{a c c, c v}}{N_{i}^{g e n, c v}} \cdot \Phi^{c v} \cdot P^{c v} \cdot T^{c v}}+\frac{\sum_{j}^{n} u_{i j}^{c v} \cdot\left(\frac{s_{j}^{c v} \cdot\left(\delta s_{j}+\delta b_{j}\right)-\delta s_{j}\left(s_{j}^{c v}+b_{j}^{c v}\right)}{s_{j}^{c v}+b_{j}^{c v}}\right)}{\frac{N_{i}^{a c c, c v}}{N_{i}^{g e n, c v}} \cdot \Phi^{c v} \cdot P^{c v} \cdot T^{c v}} \\
& =\sigma_{i}^{t r u e}+\frac{\sum_{j}^{n} u_{i j}^{c v} \cdot\left(\frac{s_{j}^{c v} \cdot \delta b_{j}-b_{j}^{c v} \cdot \delta s_{j}}{s_{j}^{c v}+b_{j}^{c v}}\right)}{\frac{N_{i}^{a c c, c v}}{N_{i}^{g e n, c v}} \cdot \Phi^{c v} \cdot P^{c v} \cdot T^{c v}} .
\end{aligned}
$$

Note that Eq. 10.10 has no bias from cv MC signal prediction, while Eq. 10.11 does depend on cv MC signal prediction. Clearly, Eq. 10.10 gives less biased result when we are confident with our background prediction, but not for our signal prediction.

From Fig. 10.2, you can see the differences of these two methods applied to that CCQE data are largest at low $Q^{2}$ or high UZ, where the cv MC signal prediction is poor. Here, red lines are for background subtraction method and blue lines are for signal fraction method. For the CCQE sample, since our Pauli blocking parameter $\kappa$ in the default MC $(=1.022)$ is stronger than the new preferred value $(=1.007)$, found after $\mathrm{CC} 1 \pi$ background correction (Chapter 9), we expect that our cr MC signal has lower values than data at high UZ and low $Q^{2}$. As you can see from Fig. 10.2 , blue lines have lower values for high $\mathrm{UZ}$ and low $Q^{2}$ than the red lines, which is an indication that signal fraction method biases data to agree with cv MC signal. Since data-MC disagreement may be a discovery, it shouldn't be biased.

However, if one is not confident with the background prediction, the situation is different. In this case, Eq. 10.8 may make negative values in some bins, which is obviously unphysical. On the other hand, Eq. 10.9 is positively defined for all bins. Therefore, the choice of background accounting is based on our knowledge of signal and background channels. 

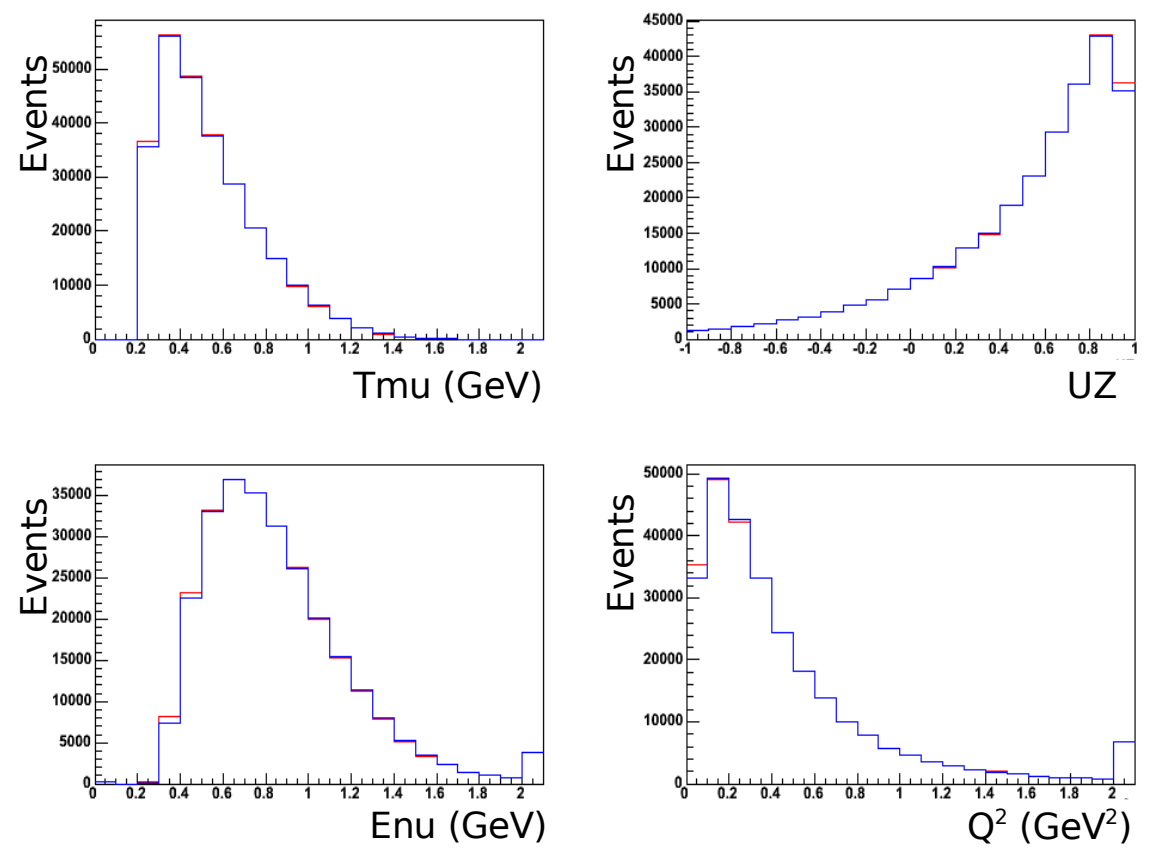

Figure 10.2: The background-removed data comparison for CCQE kinematics. The red lines are for background subtraction method, and the blue lines are for signal fraction method. From top left to bottom right plot, muon kinetic energy, muon angle, reconstructed $E_{\nu}$, and reconstructed $Q^{2}$.

For our CCQE case, since we have reliable background prediction based on our measurement (Chapter 9), we use the background subtraction method in order to keep the background subtracted data independent from the signal prediction as much as is possible.

\subsection{Unfolding error}

Unfolding is based on a given $\mathrm{cv}$ MC signal. By construction, the transformation from reconstructed variables to true variables is perfect for cv MC signal histograms. However, in any realistic situation, unfolding process suffers from bias, because background removed 
data are never exactly equal with cv MC signal. In such case, the unfolded result can be improved in an iterative process.

The unfolding process is based on the G-matrix,

$$
G_{i j}^{0 t h}=\frac{u_{i j}^{c v}}{\frac{N_{i}^{a c c, c v}}{N_{i}^{g e n, c v}}}=\frac{U_{i j}^{0 t h}}{\sum_{k} U_{k j}^{0 t h} \cdot \frac{N_{i}^{a c c, c v}}{N_{i}^{g e n, c v}}} .
$$

Here we explicitly write the transformation matrix and we define this as the $0^{\text {th }}$ iteration of G-matrix.

This G-matrix transforms background subtracted data histogram $d_{j}-b_{j}$ to the $0^{t h}$ iterated background subtracted data $\left(d_{i}-b_{i}\right)^{0 \text { th }}$,

$$
\left(d_{i}-b_{i}\right)^{0 t h}=\sum_{j} G_{i j}^{0 t h}\left(d_{j}-b_{j}\right) .
$$

Here, our unfolding process "restores" the background subtracted data $d_{j}-b_{j}$ to the unknown true distribution. If we assume that our unfolded background subtracted data $\left(d_{i}-b_{i}\right)^{0 t h}$ is closer to the unknown true distribution more than the generated cv MC signal prediction $N_{i}^{g e n, c v}$, we can correct the G-matrix so that it transforms the reconstructed distribution of MC to $\left(d_{i}-b_{i}\right)^{0 t h}$ where the reconstructed MC was originally transformed back to generated cv MC signal $N_{i}^{g e n, c v}$. After this correction, the background subtracted data $d_{j}-b_{j}$ will be unfolded to a distribution closer to the unknown true distribution. The corrected transformation matrix is defined,

$$
U_{i j}^{1 s t}=\left(\frac{d_{i}^{0 t h}}{N_{i}^{g e n, c v}}\right)^{-1} U_{i j}^{0 t h},
$$

then, the $1^{s t}$ iterated G-matrix, $G_{i j}^{1 s t}$ is defined,

$$
G_{i j}^{1 s t}=\frac{U_{i j}^{1 s t}}{\sum_{k} U_{k j}^{1 s t} \cdot \frac{N_{i}^{a c c, c v}}{N_{i}^{g e n, c v}} .}
$$

And then, the $1^{\text {st }}$ iterated unfolded background subtracted data is,

$$
\left(d_{i}-b_{i}\right)^{1 s t}=\sum_{j} G_{i j}^{1 s t}\left(d_{j}-b_{j}\right)
$$



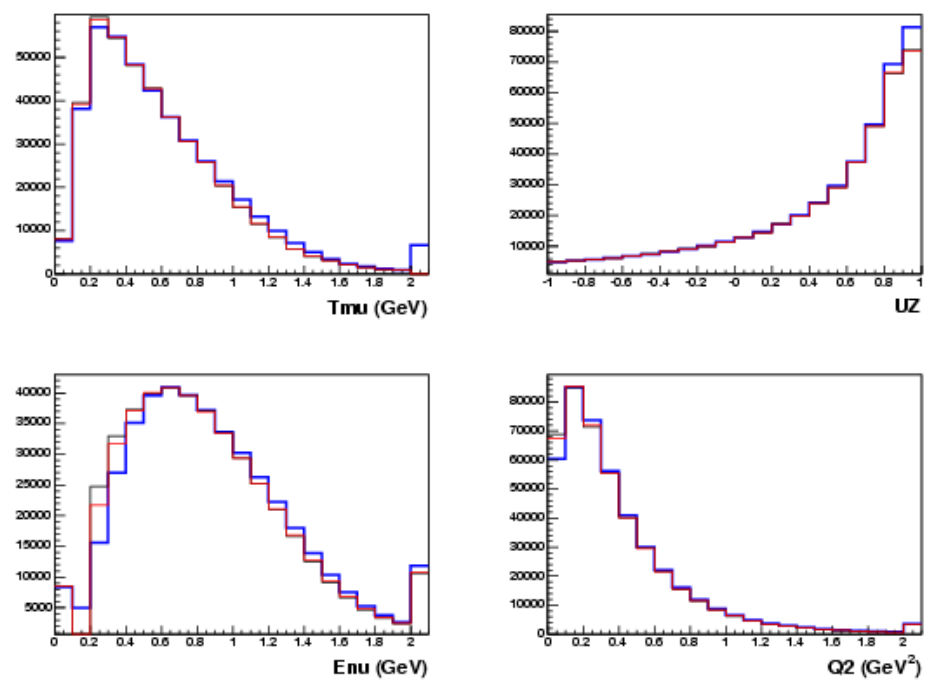

Figure 10.3: The unfolded background removed data for CCQE kinematics (same with Fig. 10.2). The black lines are for $0^{\text {th }}$ iteration, the red lines are for $8^{\text {th }}$ iteration, and the blue lines show cr MC signal generated. The $8^{\text {th }}$ iterated data is our result, and the difference of these two histograms gives the unfolding error.

The iteration process for G-matrix is based on the assumption that the solution is converging to the true distribution. However, there is some uncertainty in this and we introduce an unfolding error to quantify. It is estimated from the difference between the $0^{\text {th }}$ and the $n^{\text {th }}$ iterated background subtracted data.

Fig. 10.3 shows unfolded kinematic distributions for $0^{\text {th }}$ (black) and $8^{\text {th }}$ (red) iteration, with cv MC signal generated (blue). Although higher order iterations were considered, it was determined that the $8^{\text {th }}$ iteration has converged. Thus, we chose the $8^{\text {th }}$ iterated data as our official central values and the difference of $0^{\text {th }}$ and $8^{\text {th }}$ iterated data as the unfolding error. 


\subsection{The error matrix formation and error bars}

Before explaining each source of systematic error, our method to calculate the error matrices and the error bars is presented.

\subsubsection{The unisim method}

In MiniBooNE, a systematic error for a given uncertainty is calculated with either a unisim or a multisim method [112 114]. For the unisim method, a new set of MC events "MC unisim" for particular systematic is created by changing a particular parameter to a $1-\sigma$ excursion value, and an error matrix may be constructed by combining with the central value $\mathrm{MC}$ data set, " $M C^{c v ",}$

$$
M_{i j}^{\text {unisim }}=\left(N_{i}^{\text {unisim }}-N_{i}^{c v}\right)\left(N_{j}^{u n i s i m}-N_{j}^{c v}\right)
$$

Here, $N_{i}^{\text {unisim }}$ and $N_{i}^{c v}$ are number of events in the $i^{\text {th }}$ bin of a given histogram from the unisim $\mathrm{MC}\left(M C^{\text {unisim }}\right)$ and $\mathrm{cv} \mathrm{MC}\left(M C^{c v}\right)$, respectively. All unisim parameters are assumed independent, so the total error matrix considering all unisim parameters is the simple sum of all unisim error matrices.

\subsubsection{The multisim method}

The unisim method cannot estimate the systematic error correctly if there are correlations between different parameters. Some systematic errors are highly correlated, for example, the errors of $M_{A}$ and $\kappa$ are negatively correlated. So when $M_{A}$ is fluctuated to larger value within its error, $\kappa$ needs to be changed to a smaller value within its error, to be consistent with what the data tells us.

The multisim method correctly handles this effect. First, we classify parameters into $G$ groups. The $g^{t h}$ group consists of $u$ parameters. These parameters are dependent upon 
each other, but independent from the parameters in other groups. So, in the end, we can form $G$ independent multisim error matrices and the final total error matrix will be the simple sum of all matrices. For our case, first eight error matrices in Sec. 9.7 are based on multisim $(\mathrm{G}=8)$.

We will use the cross section error matrix for an example of one of $\mathrm{G}$ groups. To simplify the explanation, we assume only three systematic parameters, $M_{A}, \kappa$, and $\Delta s$ (Sec. 7.4). Since $M_{A}$ and $\kappa$ are simultaneously extracted by fit, they have covariance values (actually negative correlations), not only variances. However, the error of $\Delta s$ is assumed to be uncorrelated with the other two parameters. Then, we can define the following cross section input error matrix,

$$
M_{x s e c}^{\text {input }}=\left(\begin{array}{ccc}
\operatorname{var}\left(M_{A}\right) & \operatorname{cov}\left(M_{A}, \kappa\right) & 0 \\
\operatorname{cov}\left(M_{A}, \kappa\right) & \operatorname{var}(\kappa) & 0 \\
0 & 0 & \operatorname{var}(\Delta s)
\end{array}\right) .
$$

Fig. 10.4 illustrates the parameter space defined by this cross section input error matrix. Since parameter distributions are assumed Gaussian, the parameter space has an ellipsoid shape with a fuzzy edge. If there were no correlations, ellipsoid aligns symmetrically on the three axes. Here, since $M_{A}$ and $\kappa$ are correlated, the ellipsoid is tilted in the $M_{A}-\kappa$ plane. Then, we randomly pick a point from this parameter space, for example $M_{A}=$ $1.1 \mathrm{GeV} / \mathrm{c}^{2}, \kappa=1.001, \Delta s=0.01$, etc. Then, a new MC data set is created with this parameter set. We do this $\sim 100$ times. If we then want to form the error matrix for the reconstructed neutrino energy, $E_{\nu}^{Q E}$, we make $\sim 100$ histograms from each MC set, each made with a specific set of input parameters. (Fig. 10.5, left plot, black lines). They are distributed around the histogram made by cv MC (Fig. 10.5, left plot, red lines). Finally, the average of all histograms create the cross section output error matrix,

$$
M_{i j}^{\text {multisim }}=\frac{1}{100} \sum_{s=1}^{100}\left(N_{i}^{s}-N_{i}^{c v}\right)\left(N_{j}^{s}-N_{j}^{c v}\right) .
$$




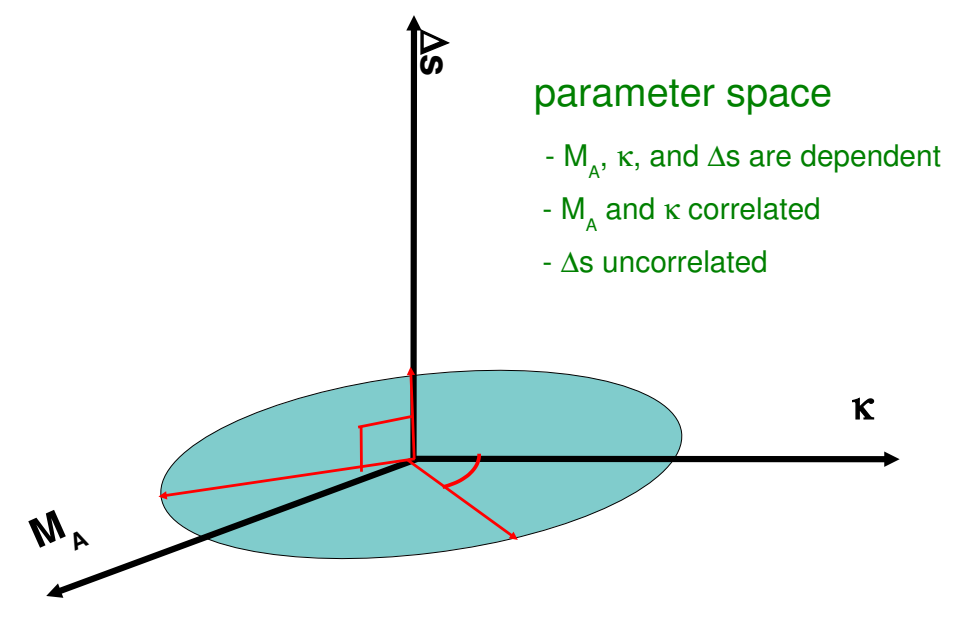

Figure 10.4: An illustration of the multisim input parameter space.

Here, $N_{i}^{s}$ is the number of $i^{\text {th }}$ bin of $s^{\text {th }}$ histogram from the $s^{\text {th }}$ MC run from within the set of multisims (100 total MCs).

In Fig. 10.5, the right plot shows the error bars resulting from an example multisim. The turquoise and red error bars are called the total and shape-only error bars, defined in next section.

Practically it is very difficult to create hundreds of MCs with adequate statistics. Instead, we utilize an event weight method, namely we only apply the ratio of parameter change (weight) to each event, so that we do not have to regenerate new MC data sets for every multisim. Also, in this method, because we re-weight the same event many times, we do not have to worry about MC statistics in forming an error matrix. However, the detector model multisims must be made by generating entirely new MC sets, because systematic parameters for the detector simulation affect the nature of events rather than the likelihood of their occurring which we can control by weight the events [113]. 

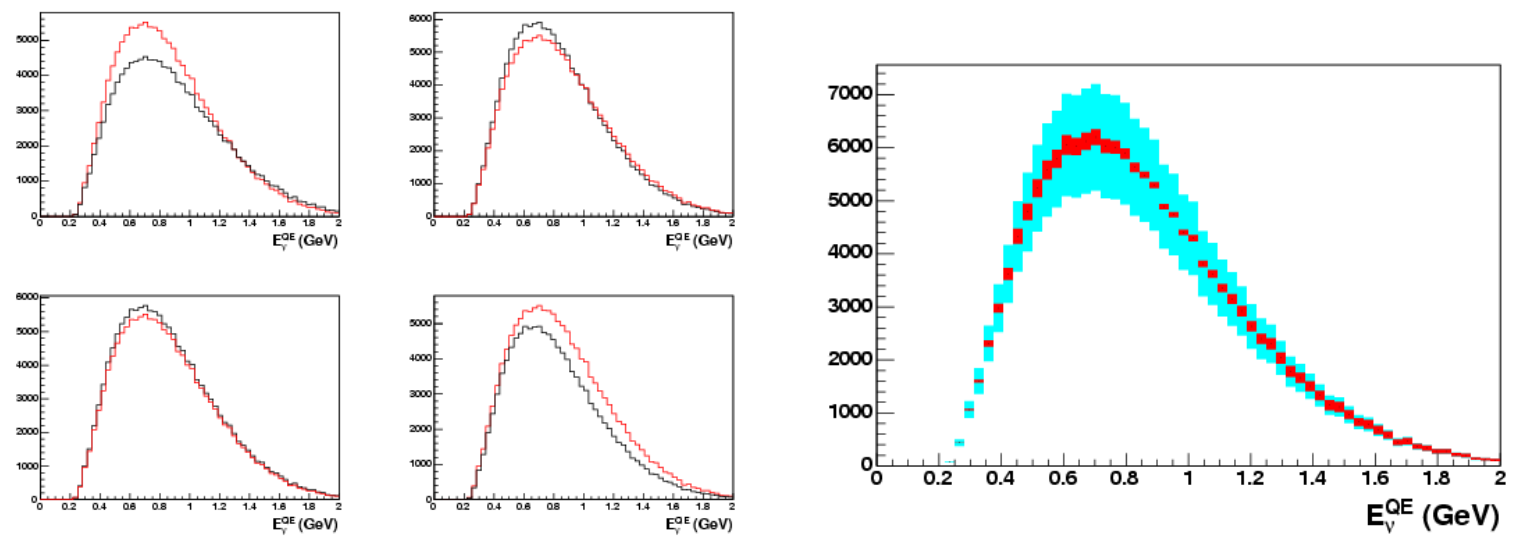

Figure 10.5: An example of the multisim error process. The left plot shows the cv MC (red) and each histogram made by a different parameter set (black). The right plot shows the resulting bands for the total (turquoise) and shape-only (red) errors.

\subsubsection{Total and shape-only error bars}

For a given histogram, $N_{i}, i=1, \cdots, n$, we can form the $n \times n$ error matrix $M_{i j}$. The diagonal element of the error matrix, $M_{i i}$, is called the variance of $N_{i}, \operatorname{var}\left(N_{i}\right)$, and an off-diagonal element, $M_{i j}(i \neq j)$, is called the covariance of $N_{i}$ and $N_{j}, \operatorname{cov}\left(N_{i}, N_{j}\right)$. Then, the total error for the $i^{\text {th }}$ bin $\delta N_{i}$ is,

$$
\delta N_{i}=\sqrt{M_{i i}}
$$

Notice that if the histogram is combined into only one bin, $N_{T}=N_{1}+\cdots+N_{n}$, total error for the total number of events, $N_{T}$, becomes,

$$
\delta N_{T}=\sqrt{\operatorname{var}\left(N_{1}+\cdots N_{n}\right)}=\sqrt{\sum_{i j} M_{i j}},
$$

where we have used the sum rule of variance and covariance, $\operatorname{var}\left(N_{1}+N_{2}\right)=\operatorname{var}\left(N_{1}\right)+$ $\operatorname{var}\left(N_{2}\right)+2 \operatorname{cov}\left(N_{1}, N_{2}\right)$. We call $\frac{\delta N_{T}}{N_{T}}$ the "total normalization error", because this is handy expression to show the size of error matrix in single number, as we have seen in Sec. 9.7 . 
Since the total error does not quantify the correlations between bins, people are often interested in a shape-only error, which includes information about not only the variance but also covariance (Fig. 2 in [4]).

Consider a new histogram, $V_{i}$, with

$$
V_{i}=\left\{V_{1}, \cdots, V_{n}, V_{n+1}\right\}=\left\{\frac{N_{1}}{N_{T}}, \cdots, \frac{N_{n}}{N_{T}}, N_{T}\right\}
$$

The error matrix, $U_{k l}$, for this histogram, is defined via the Jacobian $S_{k i}$,

$$
\begin{aligned}
U(V)_{k l} & =\sum_{i j}^{n} S_{k i} M(N)_{i j} S_{l j}, \\
S_{k i} & \equiv \frac{\partial V_{k}}{\partial N_{i}}=\left\{\begin{array}{ll}
\frac{\partial}{\partial N_{i}}\left(\frac{N_{k}}{N_{T}}\right)=\frac{1}{N_{T}}\left(\delta_{i k}-\frac{N_{k}}{N_{T}}\right) & \text { for } 1<k<n \\
\frac{\partial}{\partial N_{i}}\left(N_{T}\right) & \text { for } k=n+1
\end{array} .\right.
\end{aligned}
$$

Then the variance extracted from the new error matrix is,

$$
U_{k k}=\left\{\begin{array}{lc}
\frac{1}{N_{T}^{2}}\left(M_{k k}-2 \frac{N_{k}}{N_{T}} \sum_{i}^{n} M_{k i}+\frac{N_{k}^{2}}{N_{T}^{2}} \sum_{i j}^{n} M_{i j}\right) & \text { for } 1<k<n \\
\sum_{i j}^{n} M_{i j} & \text { for } k=n+1
\end{array} .\right.
$$

Notice the variation of total number mentioned above is contained in the $(n+1)^{t h}$ diagonal element. Finally, the shape-only error for the $k^{\text {th }}$ bin is defined,

$$
\delta N_{k}^{\text {shape }} \equiv \sqrt{M_{k k}-2 \frac{N_{k}}{N_{T}} \sum_{i}^{n} M_{k i}+\frac{N_{k}^{2}}{N_{T}^{2}} \sum_{i j}^{n} M_{i j}} .
$$

A more complete analysis shows that any arbitrary error matrix can be separated into three terms [154], $M^{\text {shape }}, M^{\text {mixed }}$, and $M^{\text {norm }}$.

$$
\begin{aligned}
M_{i j} & =\left[M_{i j}-\frac{N_{i}}{N_{T}} \sum_{k}^{n} M_{k j}-\frac{N_{j}}{N_{T}} \sum_{k}^{n} M_{i k}+\frac{N_{i} N_{j}}{N_{T}^{2}} \sum_{k l}^{n} M_{k l}\right] \rightarrow M^{\text {shape }} \\
+ & {\left[N_{i}\left(\frac{1}{N_{T}} \sum_{k}^{n} M_{k j}-\frac{N_{j}}{N_{T}^{2}} \sum_{k l}^{n} M_{k l}\right)+N_{j}\left(\frac{1}{N_{T}} \sum_{k}^{n} M_{i k}-\frac{N_{i}}{N_{T}^{2}} \sum_{k l}^{n} M_{k l}\right)\right] \rightarrow M^{\text {mixed }} } \\
+ & {\left[\frac{N_{i} N_{j}}{N_{T}^{2}} \sum_{k l} M_{k l}\right] \rightarrow M^{\text {norm }} }
\end{aligned}
$$


However, this subtlety is not used for the analysis presented here.

We now proceed to a description of the of the error calculations for the cross section analysis.

\subsection{Flux error estimation}

The error on the neutrino flux includes the following five type of errors calculated from event weight multisims:

$M^{p p}(6.1 \%) \pi^{+}$production spline fit;

$M^{p m}(0.3 \%) \pi^{-}$production Sanford-Wang fit;

$M^{k p}(0.2 \%) K^{+}$production Feynman scaling fit;

$M^{k 0}(0.0 \%) K^{o}$ production Feynman scaling fit;

$M^{b e}(5.1 \%)$ beamline and horn models.

The numbers in the parentheses show the contributions to the normalization error of the flux-unfolded total cross section in the $700-800 \mathrm{MeV}$ bin. Of course, they are related with the total normalization error, shown in Sec. 9.7. For a given multisim, the cross section formula of the $s^{\text {th }}$ flux excursion is used to create the $s^{\text {th }}$ cross section histogram. Then, the $i^{\text {th }}$ bin of this histogram is given by,

$$
\sigma_{i}^{s}=\frac{\sum_{j}^{n} u_{i j}^{s} \cdot\left(d_{j}-b_{j}^{s}\right)}{\frac{N_{i}^{a c c, s}}{N_{i}^{g e n, s}} \cdot \Phi^{s} \cdot P^{s} \cdot T^{s}} .
$$

To take account of the error correctly, we consider the multisim variation before flux-folded cross section approximation (Eq. 10.1). Since the generated number of events is proportional 
to the flux times cross section, the denominator in the above expression is subject to the following approximations,

$$
\frac{\left(\int \sigma\left(E_{\nu}\right) \cdot \phi^{s}\right) \cdot P^{s}}{N_{i}^{g e n, s}} \sim \frac{\left(\int \sigma\left(E_{\nu}\right) \cdot \phi^{c v}\right) \cdot P^{c v}}{N_{i}^{g e n, c v}} .
$$

Then we define the flux-folded cross section (Eq. 10.2),

$$
\frac{\left(\int \sigma\left(E_{\nu}\right) \cdot \phi^{c v}\right) \cdot P^{c v}}{N_{i}^{g e n, c v}} \equiv \frac{\sigma\left(E_{\nu}^{c}\right) \cdot \Phi^{c v} \cdot P^{c v}}{N_{i}^{g e n, c v}} .
$$

For the flux multisims, the target number is not fluctuated, so,

$$
T^{s}=T^{c v}
$$

These imply that we do not have to fluctuate flux factor $\Phi$, which is not currently possible with our simulation machinery, instead, we can use same flux factor $\Phi^{c v}$ and the same generated number of event $N^{g e n, c v}$, for all $\sigma^{s}$. This approximation states all flux systematic error comes from $N_{i}^{a c c, c v}$. This is somewhat strange comparing with the naive multisim variation $\frac{N_{i}^{a c c, s}}{N_{i}^{a c c, s} \Phi^{s}}$. But this term cancels most of flux shape variation in the ratio, and net effect ends up as a normalization variation $\Phi^{s}$. But since our measurement is not $\sigma \cdot\left(\int \Phi\right)$, but $\left(\int \sigma \cdot \Phi\right)$, it is more appropriate that the final flux systematic error includes flux shape variation.

Thus, the cross section formula for the $s^{\text {th }}$ flux multisim is,

$$
\sigma_{i}^{s, f l u x-\text { folded }}=\frac{\sum_{j}^{n} u_{i j}^{s} \cdot\left(d_{j}-b_{j}^{s}\right)}{\frac{N_{i}^{a c c, s}}{N_{i}^{g e n, c v}} \cdot \Phi^{c v} \cdot P^{c v} \cdot T^{c v}}
$$

The neutrino flux error contributes via this formula in two ways. First, the number of background events $b_{j}^{s}$ fluctuate, and then second, the number of accepted signal events $N_{i}^{a c c, s}$ fluctuate. Although these two fluctuations of background and signal are correlated, this formula does not account for the correlation. For example, if the $s^{\text {th }}$ flux model increases 
the $j^{\text {th }}$ bin background $\delta b_{j}$, and increase $i^{\text {th }}$ bin accepted signal $\delta N_{i}$,

$$
\begin{aligned}
\sigma_{i}^{c v} \rightarrow \sigma_{i}^{s} & =\frac{\sum_{j}^{n} u_{i j}^{s} \cdot\left(d_{j}-b_{j}^{s}\right)}{\frac{N_{i}^{a c c, s}}{N_{i}^{g e n, c v}} \cdot \Phi^{c v} \cdot P^{c v} \cdot T^{c v}} \\
& =\frac{\sum_{j}^{n} u_{i j}^{s} \cdot\left(d_{j}-b_{j}^{c v}-\delta b_{j}\right)}{\frac{N_{i}^{a c c, c v}+\delta N_{i}}{N_{i}^{g e n, c v}} \cdot \Phi^{c v} \cdot P^{c v} \cdot T^{c v}} .
\end{aligned}
$$

Then data is further subtracted by $\delta b_{j}$, and divided by a larger number $N_{i}^{a c c, c v}+\delta N_{i}$, thus the data receives twice flux normalization effect. It may cause a significant over counting of flux error if data have a large background.

One way to avoid this problem is to use the signal fraction method to calculate flux error. Then, fluctuation of signal and background cancel in the numerator and the only contribution of error is from the fluctuation of accepted signal events. This cancellation is true up to shape error contribution from flux error, and it prevents a double counting of normalization error:

$$
\begin{aligned}
\sigma_{i}^{c v} \rightarrow \sigma_{i}^{s} & =\frac{\sum_{j}^{n} u_{i j}^{s} \cdot\left(d_{j} \cdot \frac{s_{j}^{s}}{s_{j}^{s}+b_{j}^{s}}\right)}{\frac{N_{i}^{a c c, s} \cdot \Phi_{i}^{c e n, c v}}{N_{i}^{g e v}} \cdot P^{c v} \cdot T^{c v}} \\
& =\frac{\sum_{j}^{n} u_{i j}^{s} \cdot\left(d_{j} \cdot \frac{s_{j}^{s}+\delta s_{j}}{s_{j}^{s}+\delta s_{j}+b_{j}^{s}+\delta b_{j}}\right)}{\frac{N_{i}^{a c c, s}+\delta N_{i}}{N_{i}^{g e n, c v}} \cdot \Phi^{c v} \cdot P^{c v} \cdot T^{c v}} \\
& \sim \frac{\sum_{j}^{n} u_{i j}^{s} \cdot\left(d_{j} \cdot \frac{s_{j}^{s}}{s_{j}^{s}+b_{j}^{s}}\right)}{\frac{N_{i}^{a c c, s}+\delta N_{i}}{N_{i}^{g e n, c v}} \cdot \Phi^{c v} \cdot P^{c v} \cdot T^{c v}} .
\end{aligned}
$$

There is another way to avoid double counting of flux normalization error. One can normalize background variations to cv MC background, then the contribution for background subtraction method is due to shape variations of background,

$$
\begin{aligned}
\sigma_{i}^{c v} \rightarrow \sigma_{i}^{s} & =\frac{\sum_{j}^{n} u_{i j}^{s} \cdot\left(d_{j}-b_{j}^{s, s h a p e}\right)}{\frac{N_{i}^{a c c, s}}{N_{i}^{g e n, c v}} \cdot \Phi^{c v} \cdot P^{c v} \cdot T^{c v}} \\
& =\frac{\sum_{j}^{n} u_{i j}^{s} \cdot\left(d_{j}-b_{j}^{c v}-\delta b_{j}^{s h a p e}\right)}{\frac{N_{i}^{a c c, c v}+\delta N_{i}}{N_{i}^{g e n, c v}} \cdot \Phi^{c v} \cdot P^{c v} \cdot T^{c v}} .
\end{aligned}
$$


Now, $\sigma^{s}$ contains the background variation $b_{j}^{s, \text { shape }}$, that is due to shape variation, and the contribution to the normalization is very small.

These three methods are illustrated in Fig. 10.6. They show $\pi^{+}$production multisim draws and cv MC. The top row shows that the background subtraction method creates a large variation in the multisim draws due to the normalization variation, which is absent in the signal fraction and shape-only background subtraction methods. However, as you can see from middle row, since CCQE is not a background-dominated sample, this background variation is totally overwhelmed by the signal variation that enters via the efficiency correction. As a result, error bar for these three methods are very similar. Therefore, for simplicity, we decided to use the background subtraction method for the flux error calculation. 

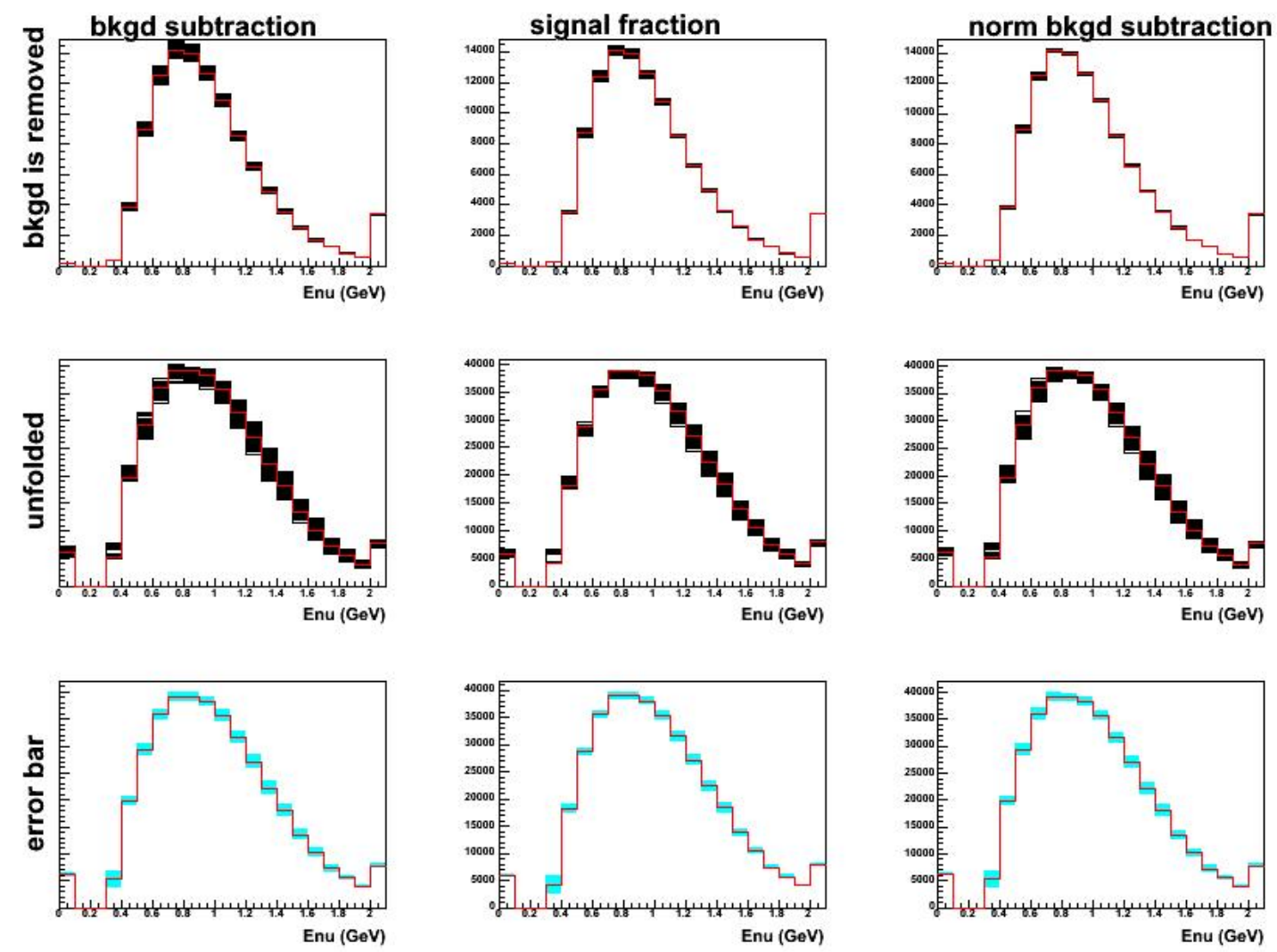

Figure 10.6: $\pi^{+}$production multisim draws and cv MC for reconstructed neutrino energy. From left to right, background subtraction method, signal fraction method, shape-only background subtraction method, and from top to bottom, after removing background, after unfolding, and after all calculated errors. All three methods yield similar error bars. 


\subsection{Cross section error estimation}

The cross section errors include the following three type of errors calculated from the event weight multisims:

$M^{x s}(3.9 \%)$ cross section models;

$M^{p 0}(0.0 \%) \pi^{\circ}$ yield;

$M^{p i}(1.2 \%)$ pion absorption and coherent fraction model error.

These errors only effect the background channels and so they do not change the number of generated (signal) events. And, of course, they do not vary the flux factor or the POT and target numbers,

$$
N_{i}^{g e n, s}=N_{i}^{g e n, c v}, \Phi^{s}=\Phi^{c v}, P^{s}=P^{c v}, T^{s}=T^{c v}
$$

Therefore, the cross section formula for $s^{\text {th }}$ cross section multisim is,

$$
\sigma_{i}^{s, x s}=\frac{\sum_{j}^{n} u_{i j}^{s} \cdot\left(d_{j}-b_{j}^{s}\right)}{\frac{N_{i}^{a c c, s}}{N_{i}^{g e n, c v}} \cdot \Phi^{c v} \cdot P^{c v} \cdot T^{c v}} .
$$

Notice that this is the same formula as that for the flux variations, Eq. 10.14).

Basically, the cross section error is the error on the prediction of background. Since we are using the straight prediction for interactions other than the CCQE and CC1 $\pi$ channels, we keep all errors in cross section input error matrix in multisim for the "other" channels.

For CCQE, since this is the channel to be measured, we do not need any errors except those on pion absorption and pion charge exchange that change the CCQE detection efficiency. We define our signal to be CCQE interaction per nucleon, so the pion-absorbed $\mathrm{CC} 1 \pi$ channel is not signal but background.

For $\mathrm{CC} 1 \pi$, we need to take care because we measure it from data (Chapter 9). There are two types of cross section errors, the first type is the cross section error in 2 subevent 
sample, and the second type is the error associated with the measurement in 3 subevents and the subtraction from the 2 subevents sample.

For the first type of error, since background measurement corresponds to both $\mathrm{CC} 1 \pi$ shape and normalization, we only need the errors from pion absorption and pion charge exchange.

The second type of error is the error about the background subtraction. Since we measured the background from the 3 subevents sample and applied it to 2 subevents, We need to take account the error associated with this process. In Chapter 9, we found that the there are multiple choices for pion absorption and coherent fraction that satisfy the data-MC agreement in 3 subevents sample. To take account this as an error, we measured $\mathrm{CC} 1 \pi$ from the 3 subevents sample data with different hypothesis on pion absorption and coherent fraction. The excursion of these variations are chosen from the data for pion absorption (Sec. 9.5). We used three different values, default, $15 \%$ increase, and $30 \%$ increase. For coherent fraction, we used three different values, default (100\%), 50\% and $0 \%$. In the meantime, we chose a new central value, $15 \%$ increase pion absorption and $50 \%$ decrease coherent fraction. This new central value $\mathrm{MC}$ and eight other combinations of pion absorption and coherent fraction make an error matrix. Fig. 10.7, right column plots shows this error.

\subsection{Detector error estimation}

The detector error calculation includes the following errors, one type are from generated multisims and the type are from generated unisim:

$M^{o m}(4.4 \%)$ optical model;

$M^{u n}(2.1 \%)$ detector unisims. 
Again, like the cross section error, the detector error has no variations in flux factor, POT, generated number of events, or target number.

$$
N_{i}^{g e n, s}=N^{g e n, c v}, \Phi^{s}=\Phi^{c v}, P^{s}=P^{c v}, T^{s}=T^{c v},
$$

and the cross section formula for the $s^{\text {th }}$ detector errors is the same as Eqs. 10.14) and (10.17),

$$
\sigma_{i}^{s, \text { detec }}=\frac{\sum_{j}^{n} u_{i j}^{s} \cdot\left(d_{j}-b_{j}^{s}\right)}{\frac{N_{i}^{a c c, s}}{N_{i}^{g e n, c v}} \cdot \Phi^{c v} \cdot P^{c v} \cdot T^{c v}} .
$$

The detector errors are shown in Fig. 10.7 (left and middle columns). Since the statistical noise in the detector simulation has a significant effect for the covariance terms in the error matrix, we used $4^{\text {th }}$ order polynomial smoothing to the detector model error matrix for $M_{A}-\kappa$ fit (Sec. ??). However, this smoothing has very small impact for the variance terms. So, we do not use smoothing for any cross section measurement results. 

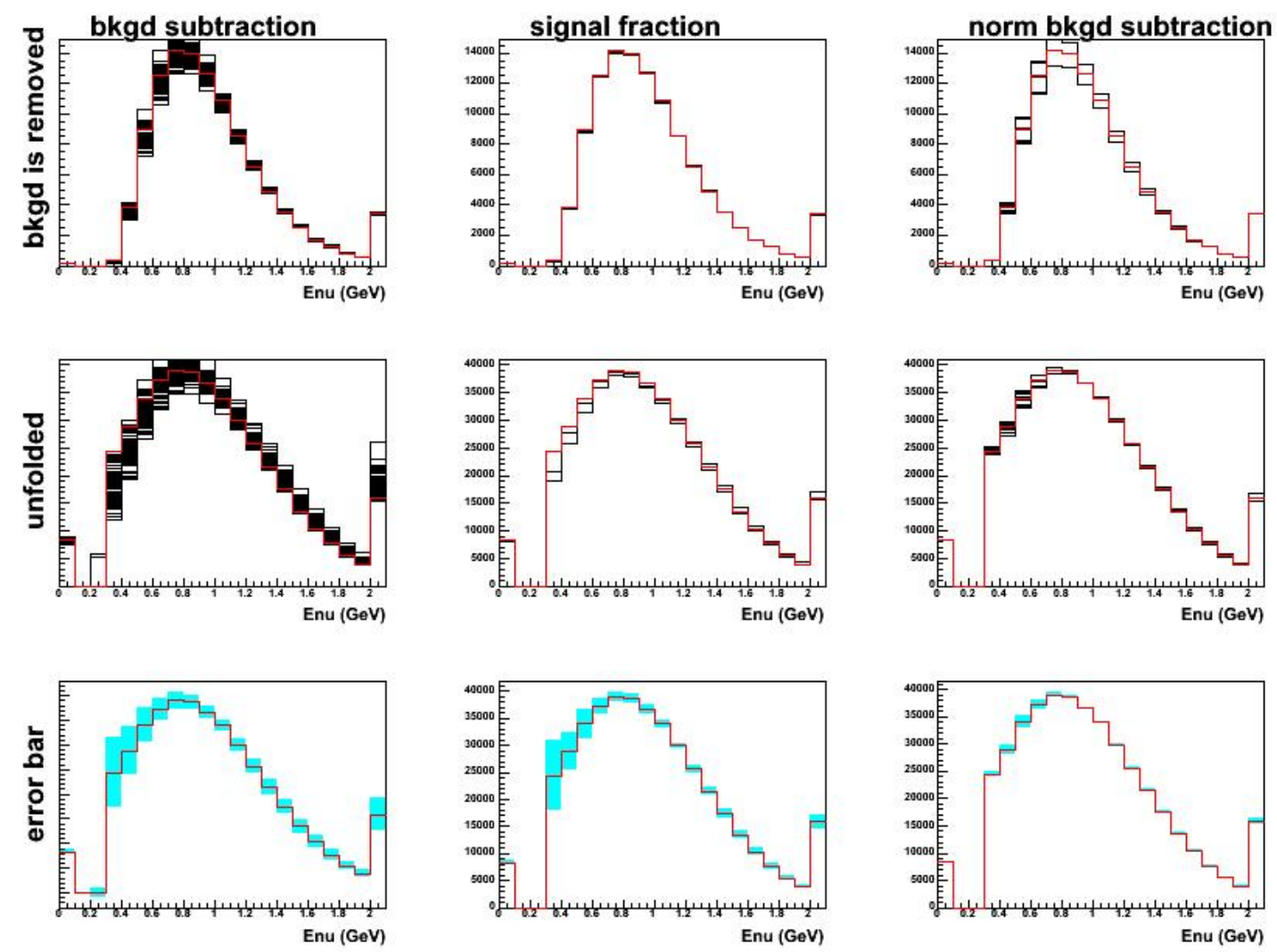

Figure 10.7: Detector errors for the reconstructed neutrino energy. From left to right: optical model, detector unisim, and pion absorption unisim (which is not detector error but listed here for convention). From top to bottom: after removing background, after unfolding, and calculated error bar plot. 


\subsection{The CCQE absolute cross section with total error}

In this section, the muon neutrino CCQE absolute cross sections are presented. The cross sections are measured on carbon and presented as "per nucleon" values.

\subsubsection{The CCQE double differential cross section}

The flux-folded double-differential cross section and the flux-unfolded total cross section are summarized in Fig. 10.8. The top left plot is the total error of the flux-folded double differential cross section in the $T_{\mu}-\cos \theta_{\mu}$ two-dimensional plane. The flux-folded total cross section and the number of events after cuts are indicated as well. Fig. 10.9 shows the value of the flux-folded double differential cross section as a function of $T_{\mu}$ and $\cos \theta_{\mu}$.

The top right plot of Fig. 10.8 shows the double differential cross section values (red) in $T_{\mu}-\cos \theta_{\mu}$ with total error bar (yellow). Figures 10.10 and 10.11 offer larger plots and multiple views from different angles.

The bottom left plot of Fig. 10.8 displays the flux-unfolded total cross section as a function of true neutrino energy. In this plot, turquoise error boxes show total error, the error bars show the shape error (vertical) and the true neutrino energy reconstruction model dependence error (horizontal). The flux-unfolded total cross section at $E_{\nu}=700-800 \mathrm{MeV}$ and its error are printed on the plot.

Finally, the bottom right plot of Fig. 10.8 shows the flux-folded differential cross section as function of $Q^{2}$. Here, $Q^{2}$ is unfolded not by the true MC (nuclear) $Q^{2}$, but by the reconstructed true $Q^{2}$ with same sense of true $E_{\nu}$ to avoid model dependencies (Sec. 10.3). The turquoise error boxes show total error, and error bars show shape error. The integral of the flux-folded total cross section with averaged neutrino energy $\left(E_{\nu} \sim 700 \mathrm{MeV}\right)$ and its error are printed. 

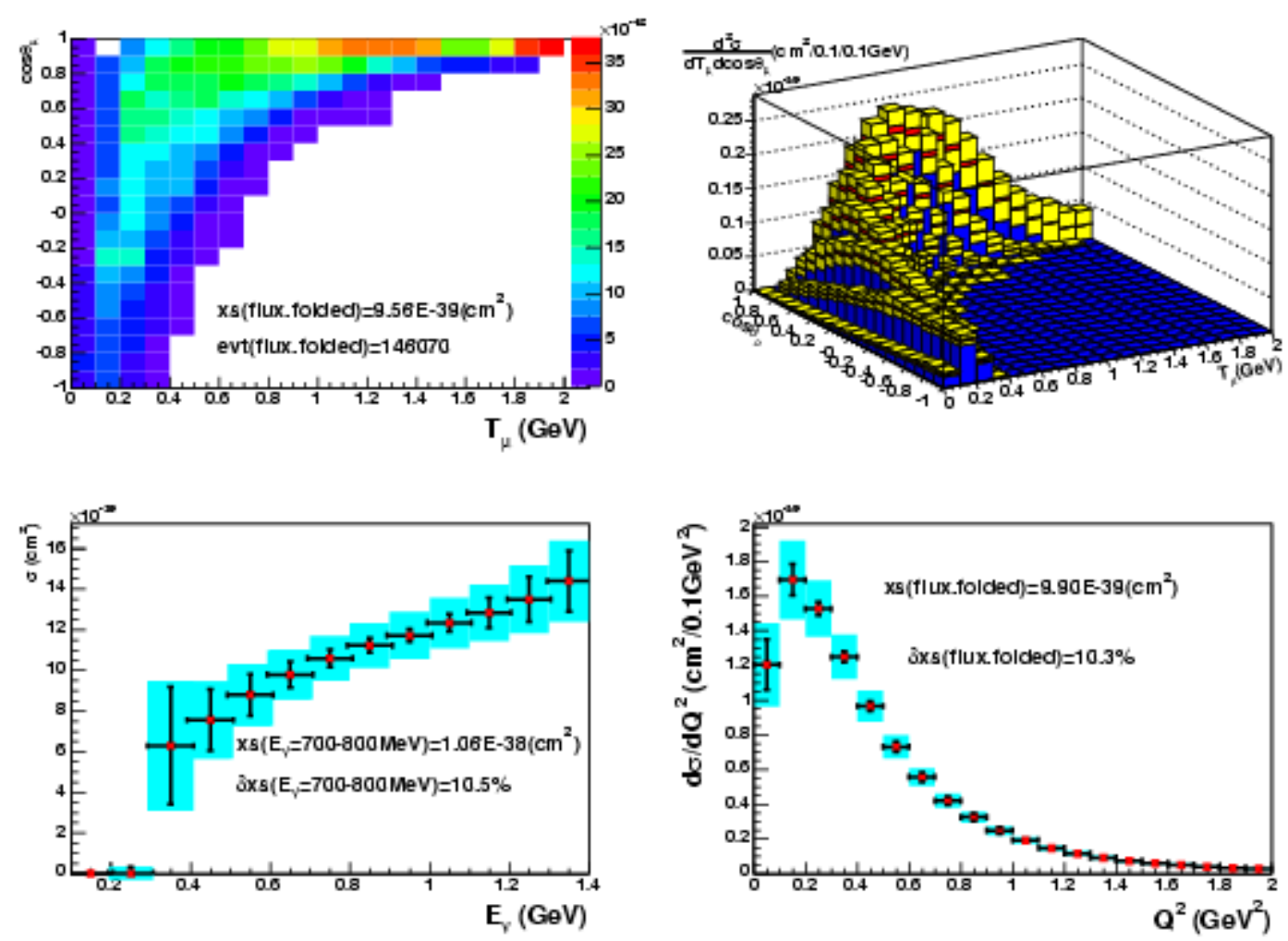

Figure 10.8: Total error plots for the flux-unfolded double differential cross section and flux-folded total cross section. The notation of plots is found in Sec. 10.10.1.

The measurement of flux-unfolded CCQE cross section in the 700-800 MeV bin in MiniBooNE is,

$$
\sigma_{700-800 \text { MeVbin }}^{C C Q E}=1.058 \pm 0.111 \times 10^{-38} \mathrm{~cm}^{2} .
$$

Note, the error is dominated by systematics. 


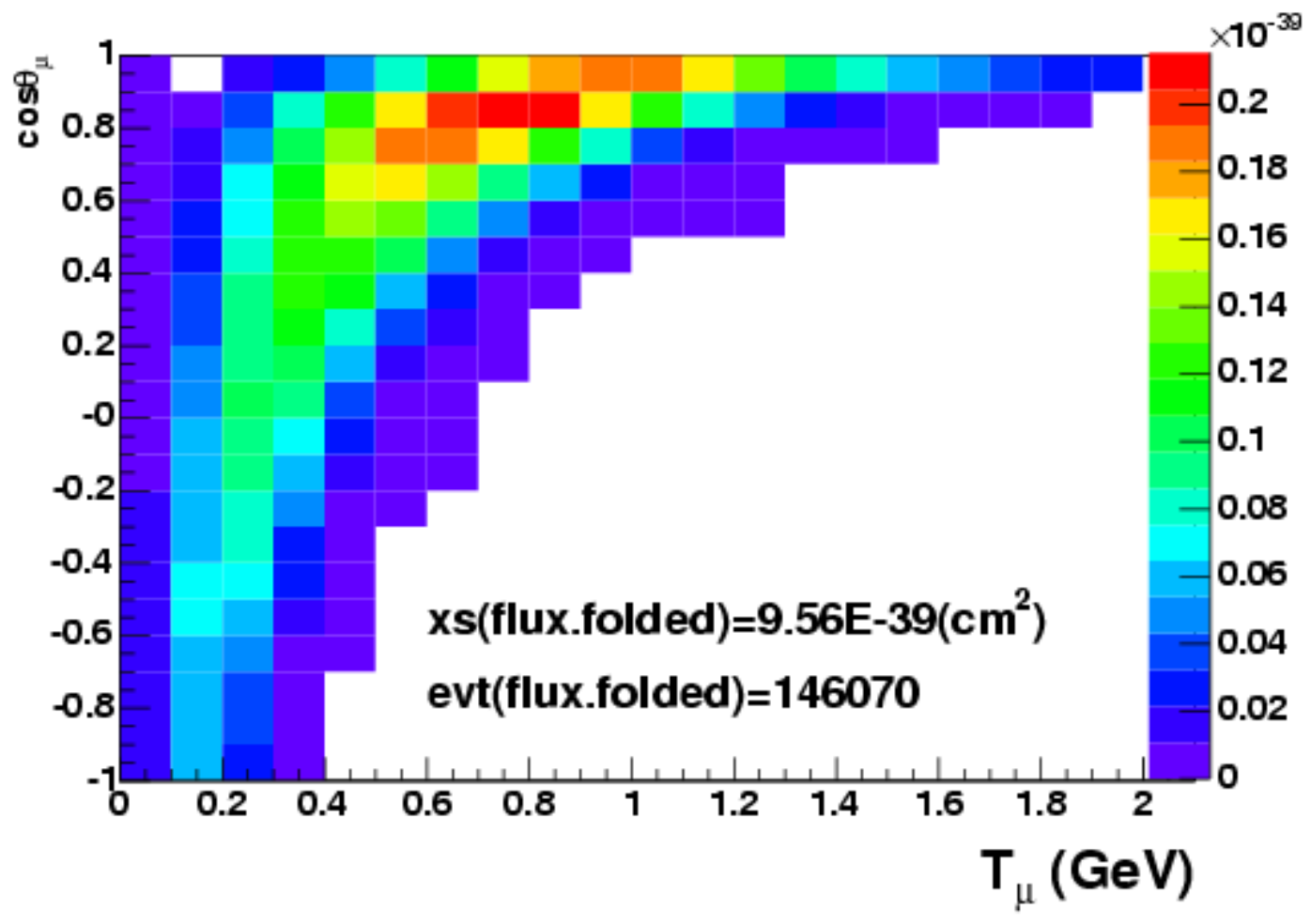

Figure 10.9: The flux-folded double differential cross section. 


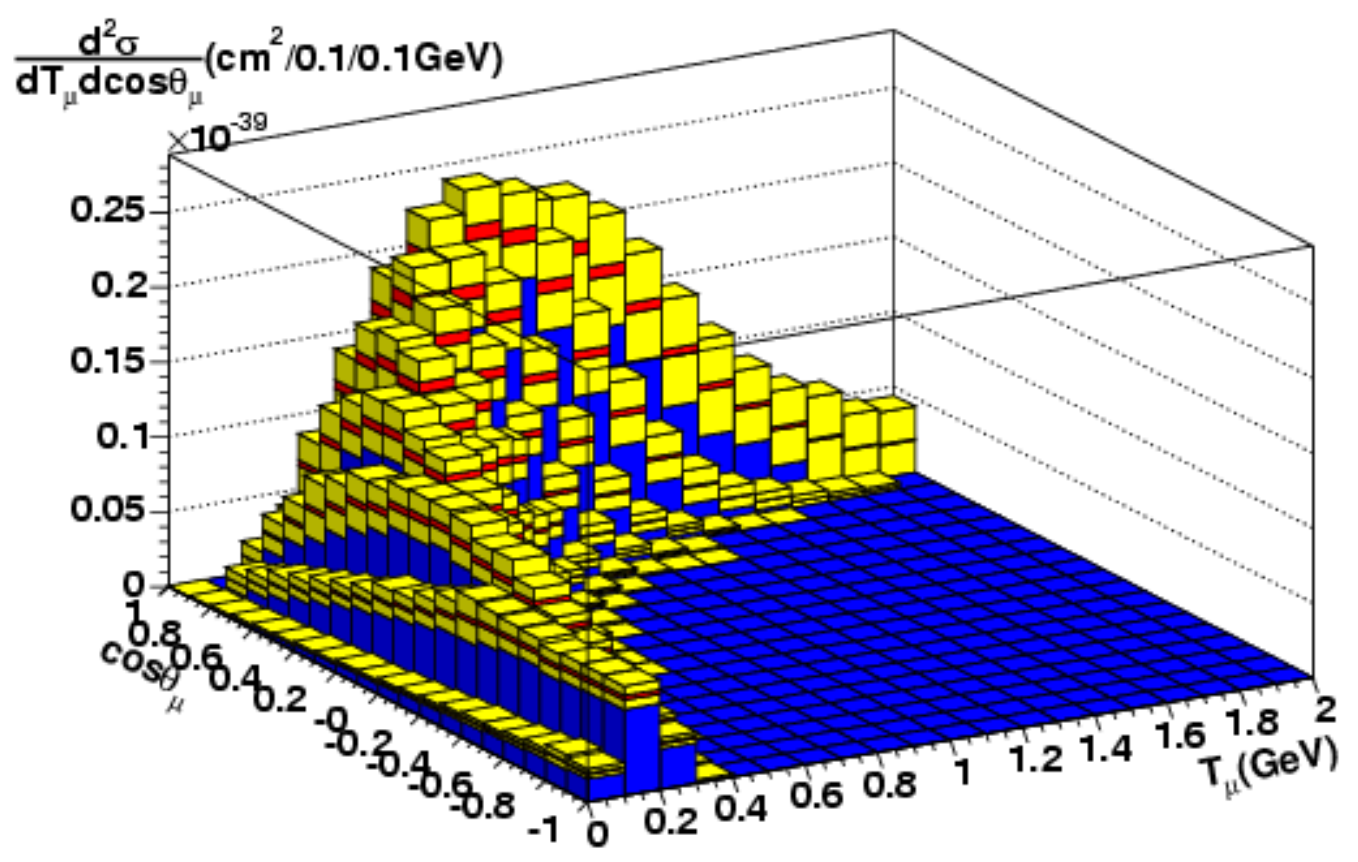

Figure 10.10: The flux-folded double differential cross section with total error. 

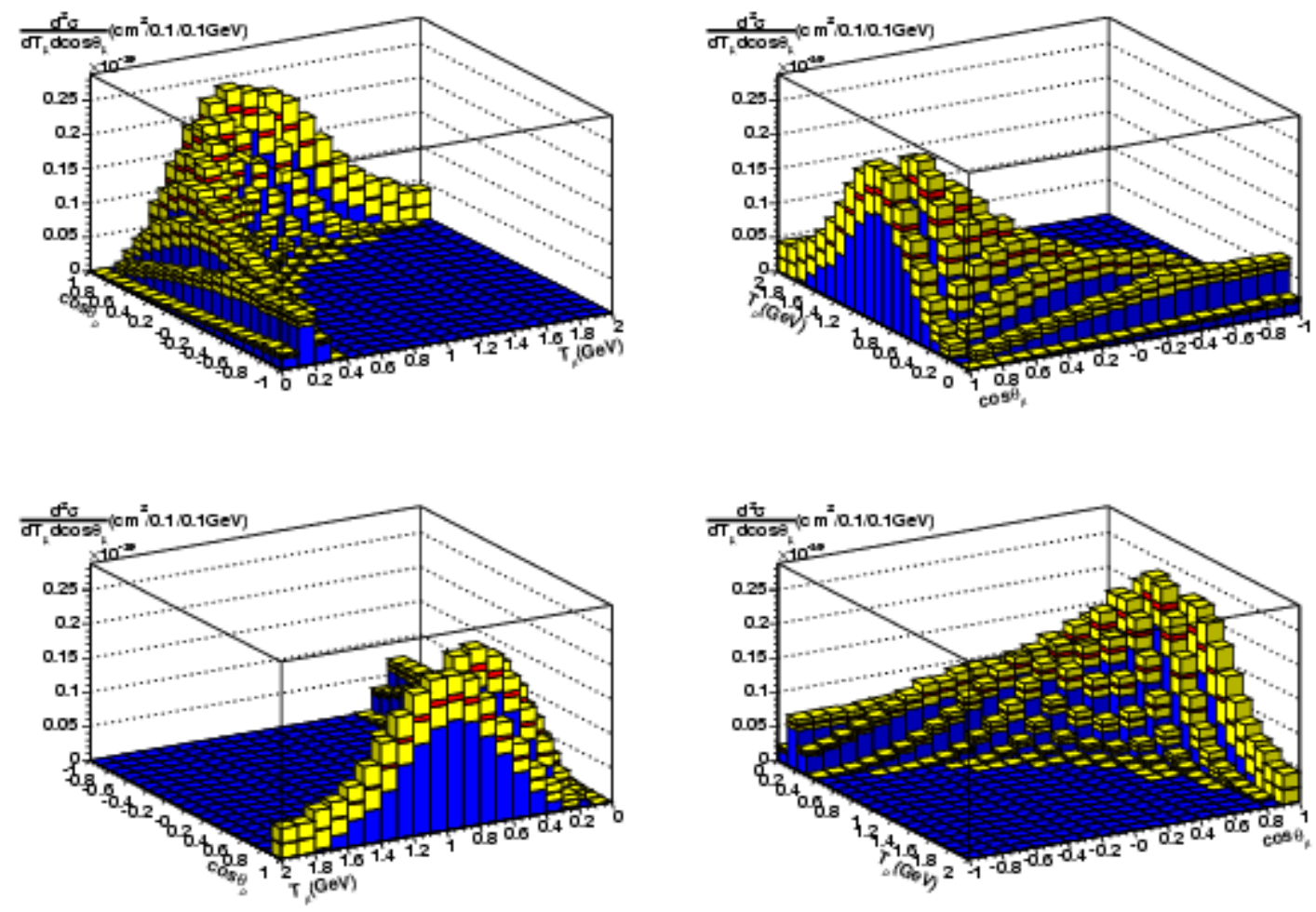

Figure 10.11: The flux-folded double differential cross section with total error from different angles. 


\subsubsection{The CCQE total cross section}

Fig. 10.12 is the same as the bottom left plot of Fig. 10.8, namely the flux-unfolded total cross section with total error bars (turquoise), shape only error bars (vertical on markers), and the energy reconstruction model dependence error bars (horizontal on marker). Fig. 10.12 shows the comparison with relativistic Fermi gas (RFG) model based on Smith and Moniz [16]. Three lines show nuance predictions, red $\left(M_{A}=1.03 \mathrm{GeV} / \mathrm{c}^{2}, \kappa=1.000\right)$, green $\left(M_{A}=1.23 \mathrm{GeV} / \mathrm{c}^{2}, \kappa=1.022\right)$, and blue $\left(M_{A}=1.32 \mathrm{GeV} / \mathrm{c}^{2}, \kappa=1.007\right)$ with Fermi momentum $=220 \mathrm{MeV} / \mathrm{c}$ and binding energy $=34 \mathrm{MeV}$ (Sec. 7.4.2). Note, our fluxunfolded total cross section is a function of reconstructed true neutrino energy, whereas both neutrino flux and theoretical curves are plotted as a function of MC true neutrino energy (Sec. 10.3). To describe the effect of this, we also show the blue and magenta histogram (scaled to data). The blue histogram is the cv MC signal generated events, as a function of reconstructed true neutrino energy, and then divided by neutrino flux. On the other hand, the magenta histogram is the cv MC signal generated events, as a function of MC true neutrino energy, and then divided by neutrino flux. Notice that the neutrino flux is a function of MC true neutrino energy. If the neutrino energy reconstruction is perfect and the smearing by the Fermi motion is negligible, then blue and magenta histograms would be identical. The difference of blue and magenta histograms are the effect of energy reconstruction between reconstructed true neutrino energy and $\mathrm{MC}$ true neutrino energy. We find both magenta and blue histograms agree with data, so the bias from the energy reconstruction is not significant with binding energy of $34 \mathrm{MeV}$ in 0.1 to $1.4 \mathrm{GeV}$.

Comparing with the theoretical value $\left(\sim 0.9 \times 10^{-38} \mathrm{~cm}^{2}\right.$, from red line $)$, the measured cross section is almost $\sim 20 \%$ higher in the flux peak region $\left(E_{\nu}=700-800 \mathrm{MeV}\right)$. However, rather surprisingly, the measured cross section agrees with new cross section model found in Chapter $9\left(M_{A}=1.32 \mathrm{GeV} / \mathrm{c}^{2}, \kappa=1.007\right)$. 


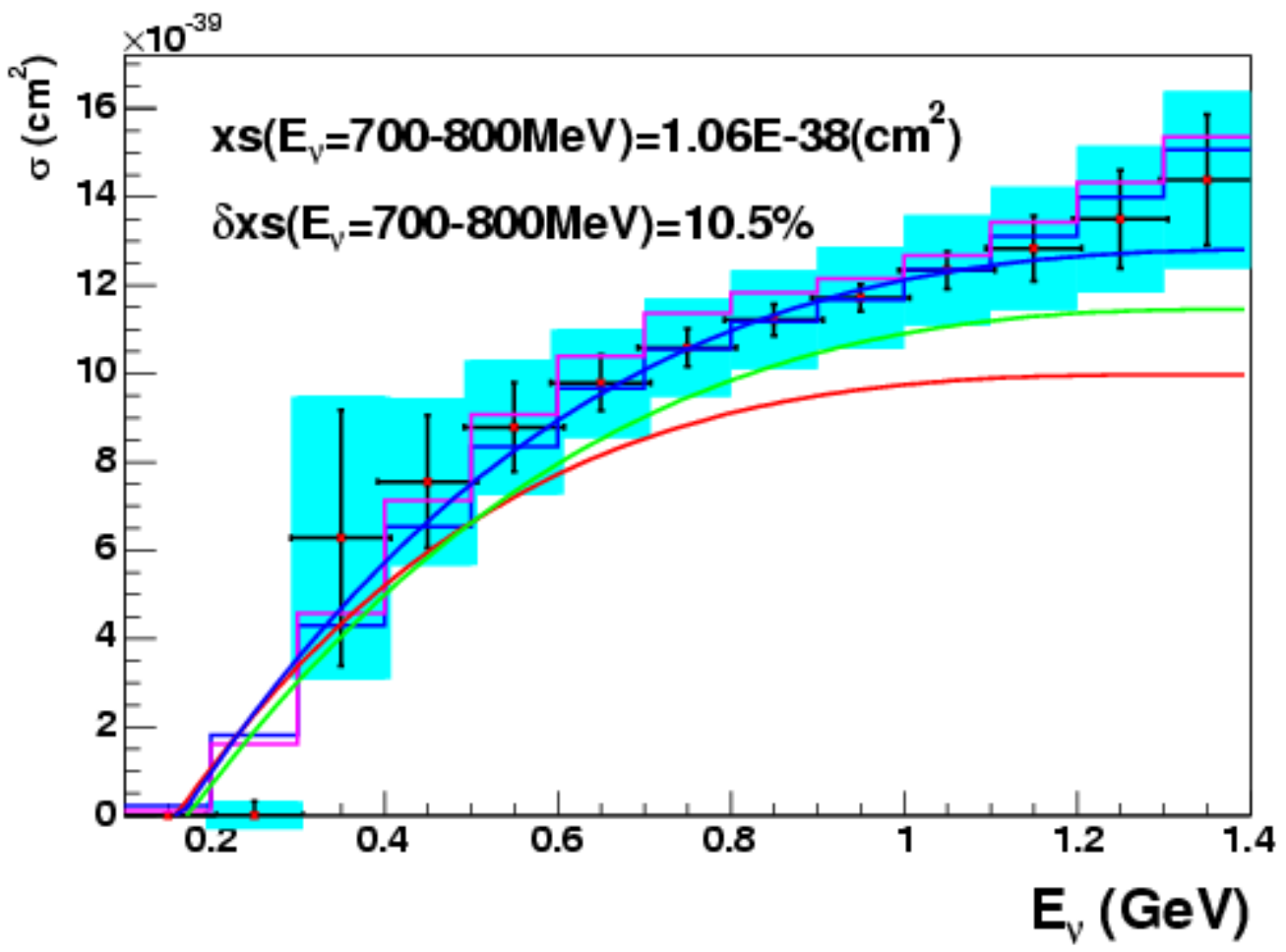

Figure 10.12: The flux-unfolded total cross section with theoretical prediction. The notations are given in the text, Sec. 10.10.2. 


\subsubsection{The CCQE differential cross section in $Q^{2}$}

Fig. 10.13 shows a comparison of the $Q^{2}$ distribution of counts and flux-unfolded differential cross section with reconstructed true $Q^{2}$. Top plot shows number of events and total normalization error. This plot is used for $M_{A}-\kappa$ fit (Chapter 9). The bottom plot is identical with the bottom right plot of Fig 10.8 .

In Chapter 9, we found a new CCQE cross section model via a measurement of the distribution of events in $Q^{2}$. In principle, we should find the same solution from the differential cross section, $\frac{d \sigma}{d Q^{2}}$. In Fig. 10.14 , we compare our $\frac{d \sigma}{d Q^{2}}$ from the data (Fig. 10.8, bottom right) and the nuance prediction for CCQE RFG model with new parameters integrated with MiniBooNE flux. As should be, they agree well.

Finally, Fig. 10.15 shows the flux-folded differential cross section of $Q^{2}$ with reconstructed true neutrino energy in the range $700-800 \mathrm{MeV}$. This corresponds to the differential cross section for a slice of reconstructed true neutrino energy of 700-800 MeV, and the integral corresponds to $7^{\text {th }}$ bin of Fig. 10.12 . 

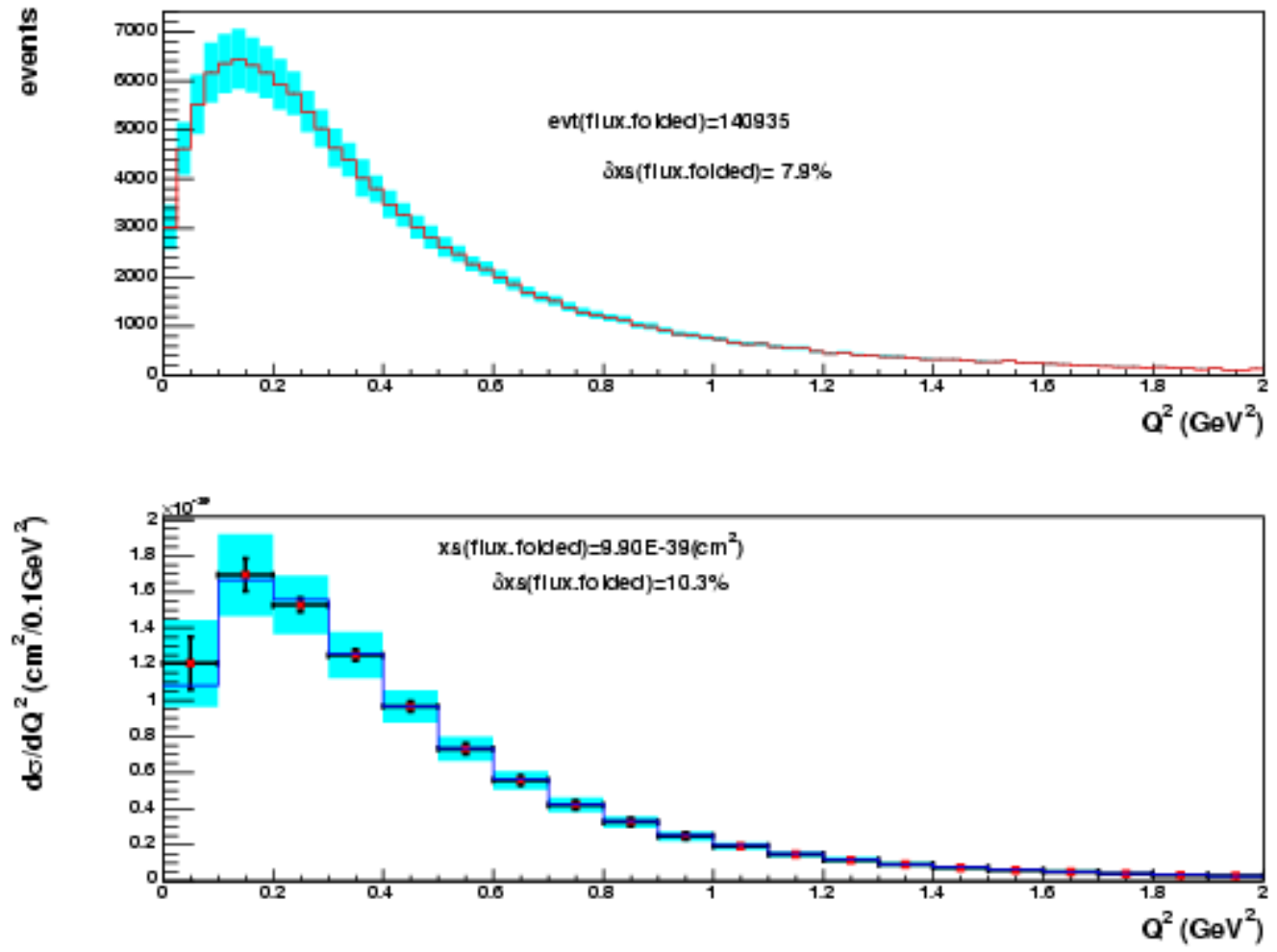

Figure 10.13: The comparison of $Q^{2}$ distribution of counts and flux-unfolded differential cross section. The notations are given in the text, Sec. 10.10.1. 


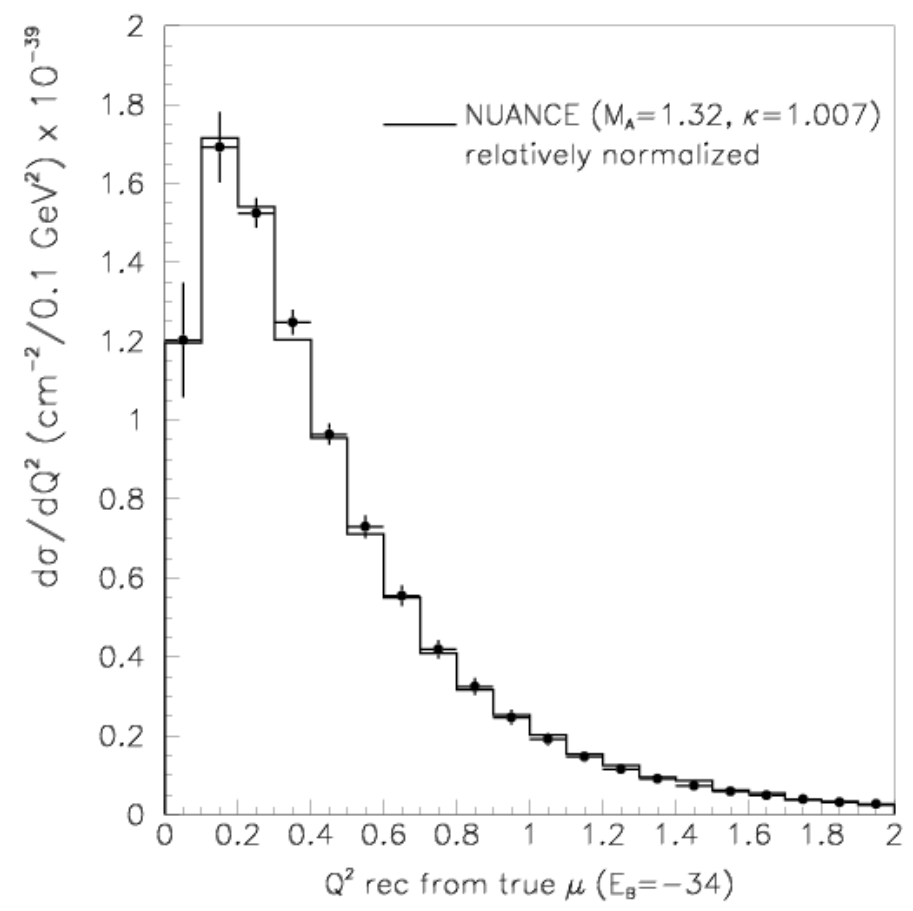

Figure 10.14: The comparison of new cross section model (solid line) and flux-unfolded differential cross section (dots) from the bottom right plot of Fig. 10.8 or the bottom plot of Fig. 10.13 . 


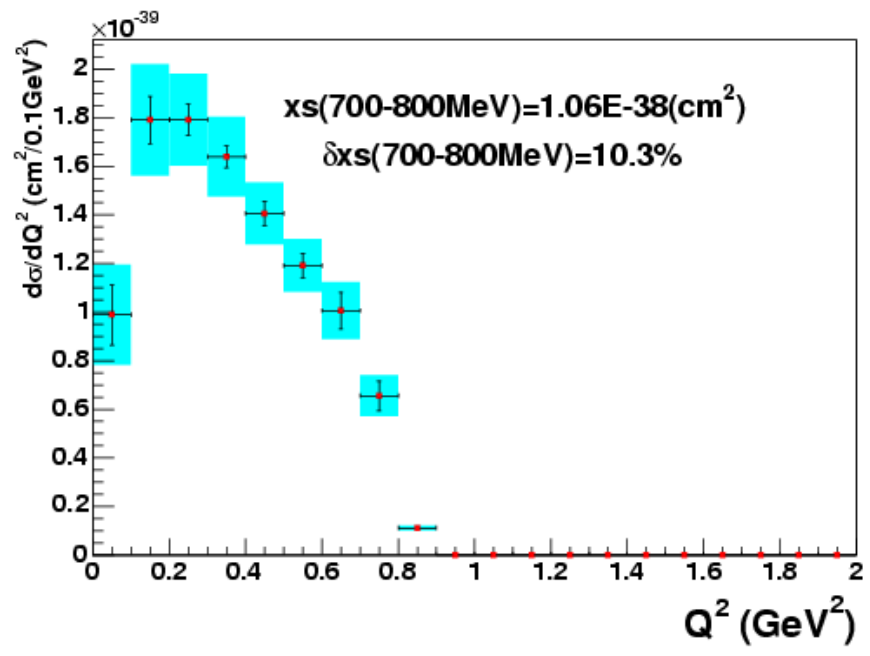

Figure 10.15: The flux-unfolded differential cross section with $Q^{2}$ with reconstructed true neutrino energy 700-800 MeV. The notations are given in the text, Sec. 10.10.1.

\subsubsection{The energy reconstruction}

As discussed in Sec. 10.3, if we try to avoid nuclear model dependency, the true neutrino energy needs to be defined from true muon energy and true muon angle. Then, the fluxunfolded total cross section suffers reconstruction biases. We use the standard quasielastic formula to reconstruct neutrino energy (Eq. 10.4) and $Q^{2}$ (Eq. 10.5), however the choice of binding energy is a visible effect.

In Fig. 10.16 a plot identical to Fig. 10.12 with the exception of the binding energy changed to $B=0 \mathrm{MeV}$ is shown. Now, the magenta and blue histograms defined in Sec. 10.10 .2 do not agree. Notice, however, that the data and blue histogram agrees, as long as the neutrino energy is reconstructed with the same binding energy. Therefore we can conclude that the choice of $34 \mathrm{MeV}$ for the binding energy is less biased. This study clearly shows that the data is best (least model dependent) presented as a double differential cross section (in $T_{\mu}-\cos \theta_{\mu}$ ) when only the muon kinematic reconstruction is available. 


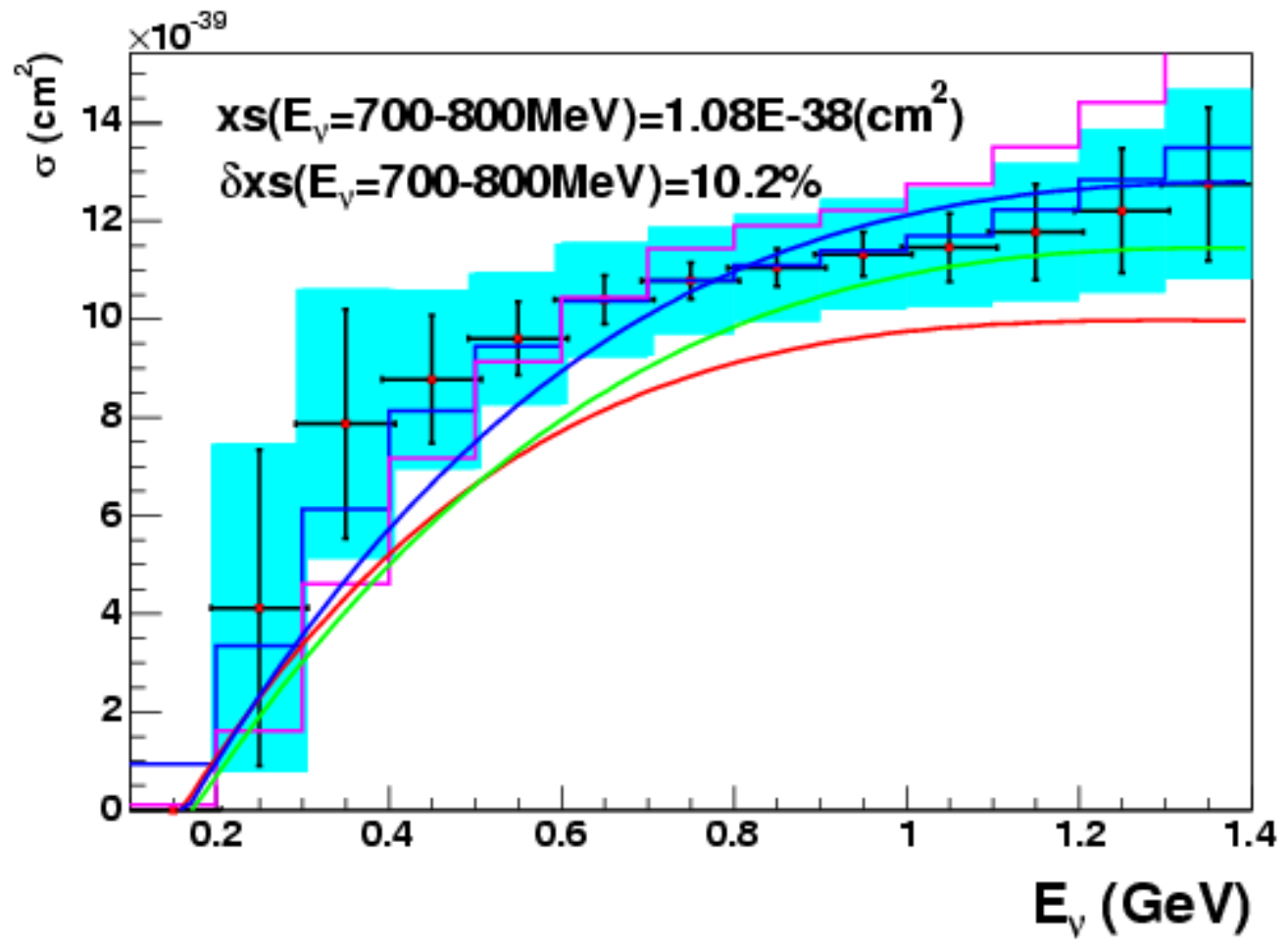

Figure 10.16: The flux-unfolded total cross section with theoretical prediction. The neutrino energy is reconstructed with binding energy $=0 \mathrm{MeV}$. The notations are given in the text, Sec. 10.10.3. 


\subsubsection{The systematic errors}

The breakdown of systematic errors are shown in Table 10.1. Figures 10.17, 10.18, 10.19. and 10.20 , show each of the systematic errors on the differential cross section individually.

Figure 10.17 shows the flux error. The flux error results in the largest normalization error. The increase of flux error at high energy results from the horn skin effect (Sec. 7.2). The uncertainty in $\pi^{+}$production results in the biggest normalization error contribution among all the systematics, and it is relatively uniform across the entire energy region.

Figure 10.18 shows the cross section model error. It is large at where background contamination is large, as can be seen from top left plot. Therefore, it is also large in the low neutrino-energy region (bottom left) where background channels are a big contribution and where pion absorption and pion charge exchange uncertainties are important.

Figure 10.19 shows the detector uncertainty error. Since this error causes a shift of

energy spectrum, it is large at both low and high energy regions. The large shape errors show that the correlations are also large (bottom left).

Finally, the unfolding error is shown in Fig. 10.20 

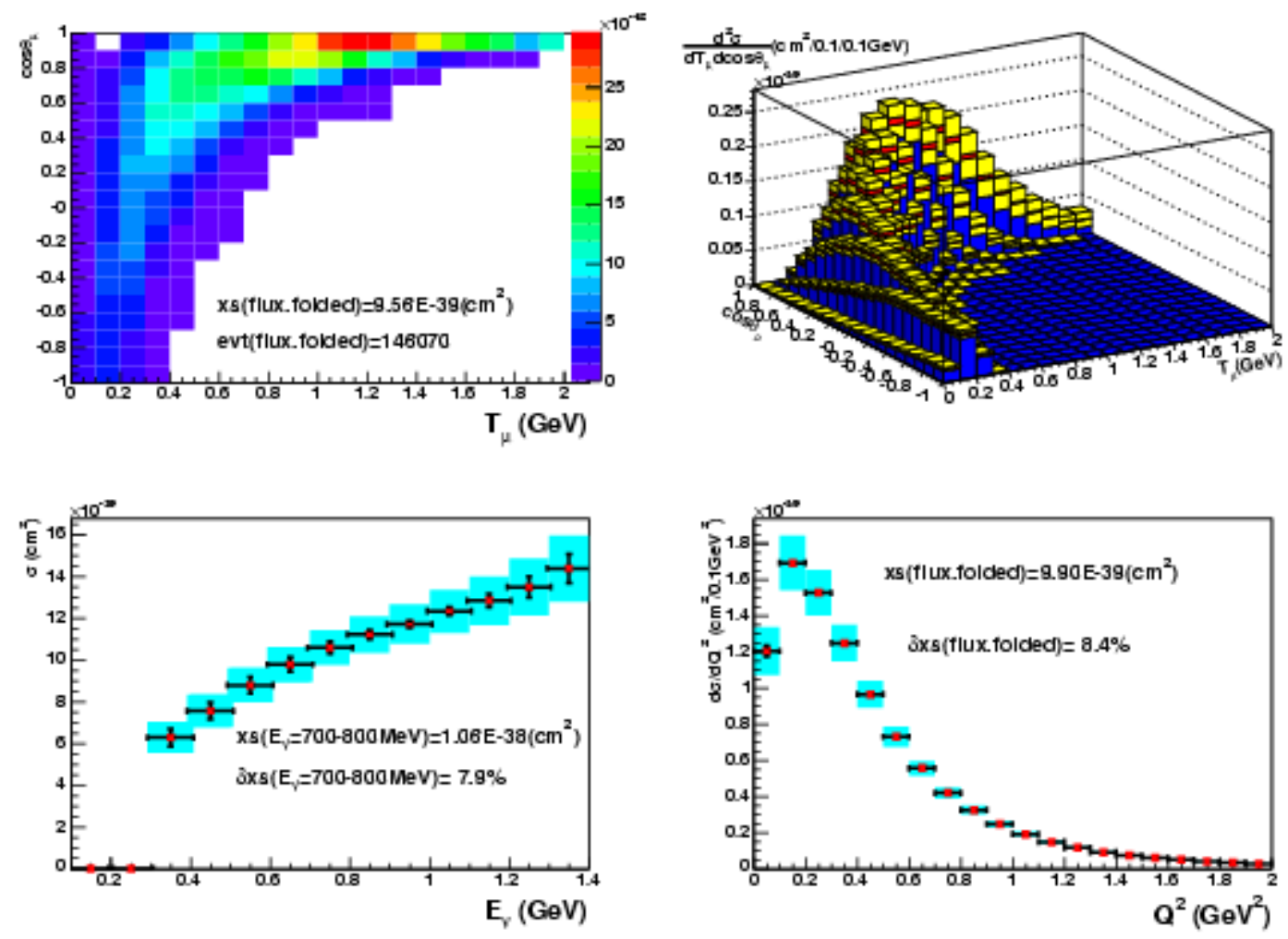

Figure 10.17: Flux error only plots for the flux-unfolded double differential cross section and flux-folded total cross section. The notation of plots are found in Sec. 10.10.1 

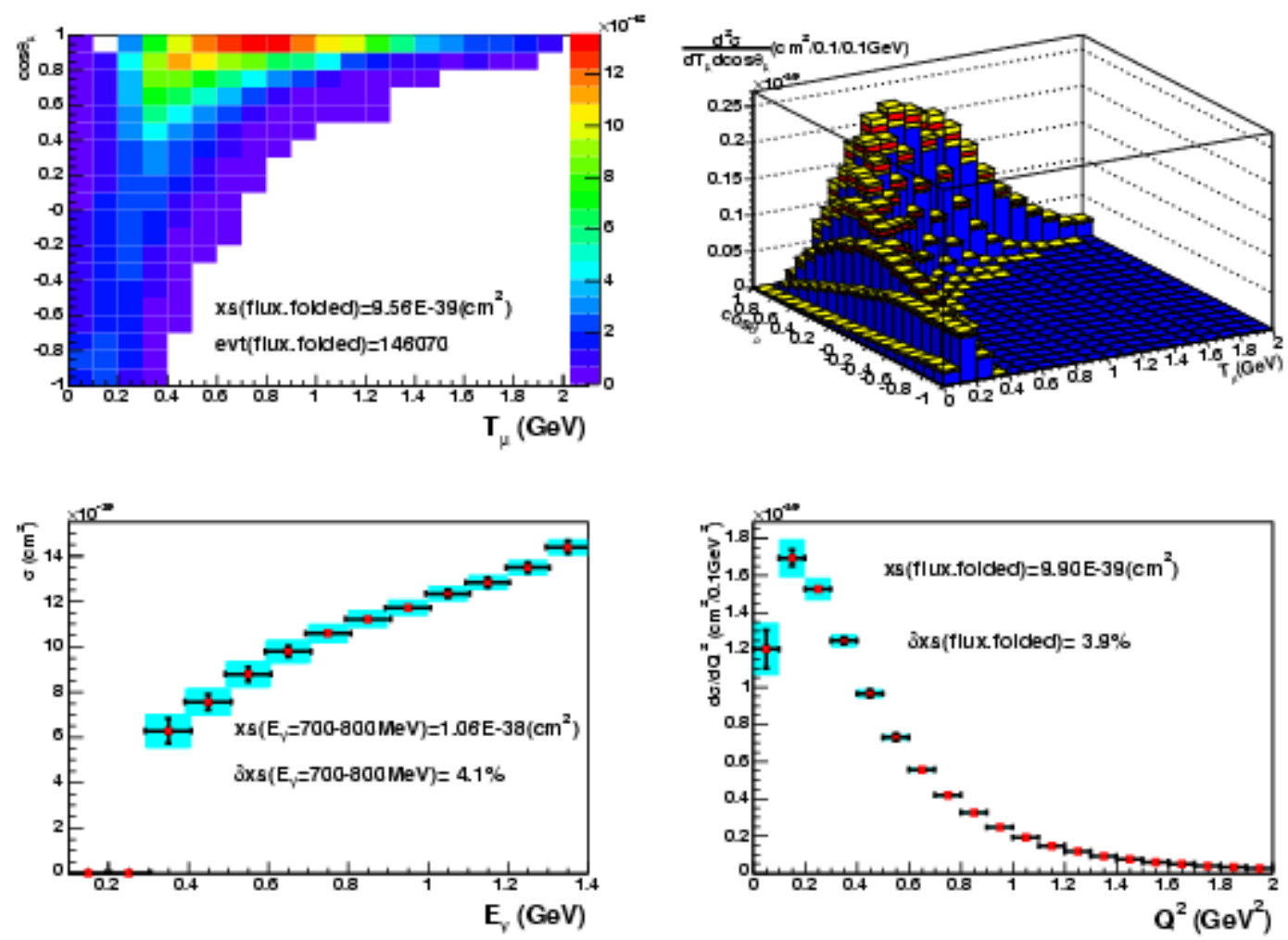

Figure 10.18: Cross section model error only plots for the flux-unfolded double differential cross section and flux-folded total cross section. The notation of plots are found in Sec. 10.10 .1 . 

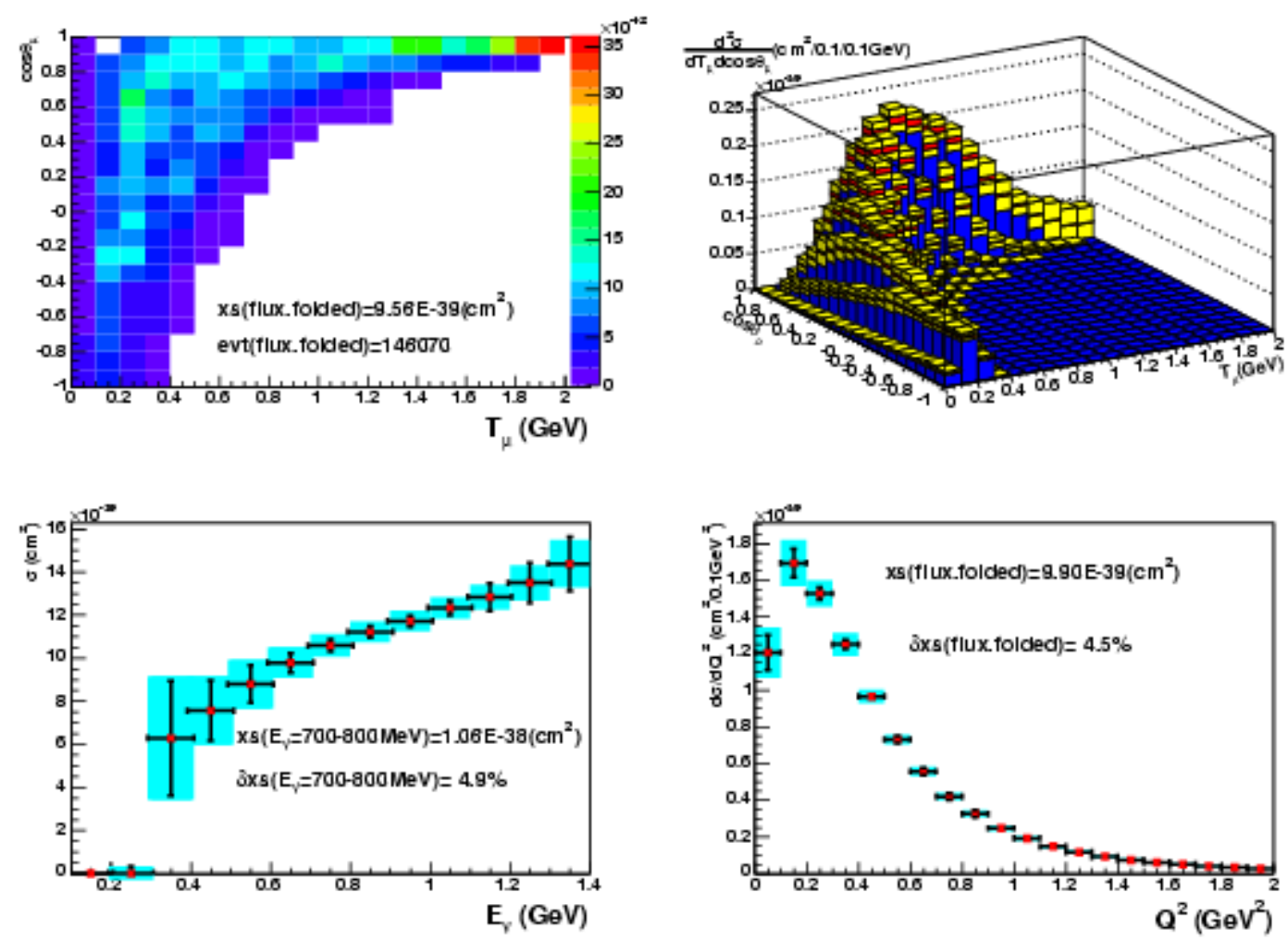

Figure 10.19: Detector model uncertainty only plots for the flux-unfolded double differential cross section and flux-folded total cross section. The notation of plots are found in Sec. 10.10 .1 . 

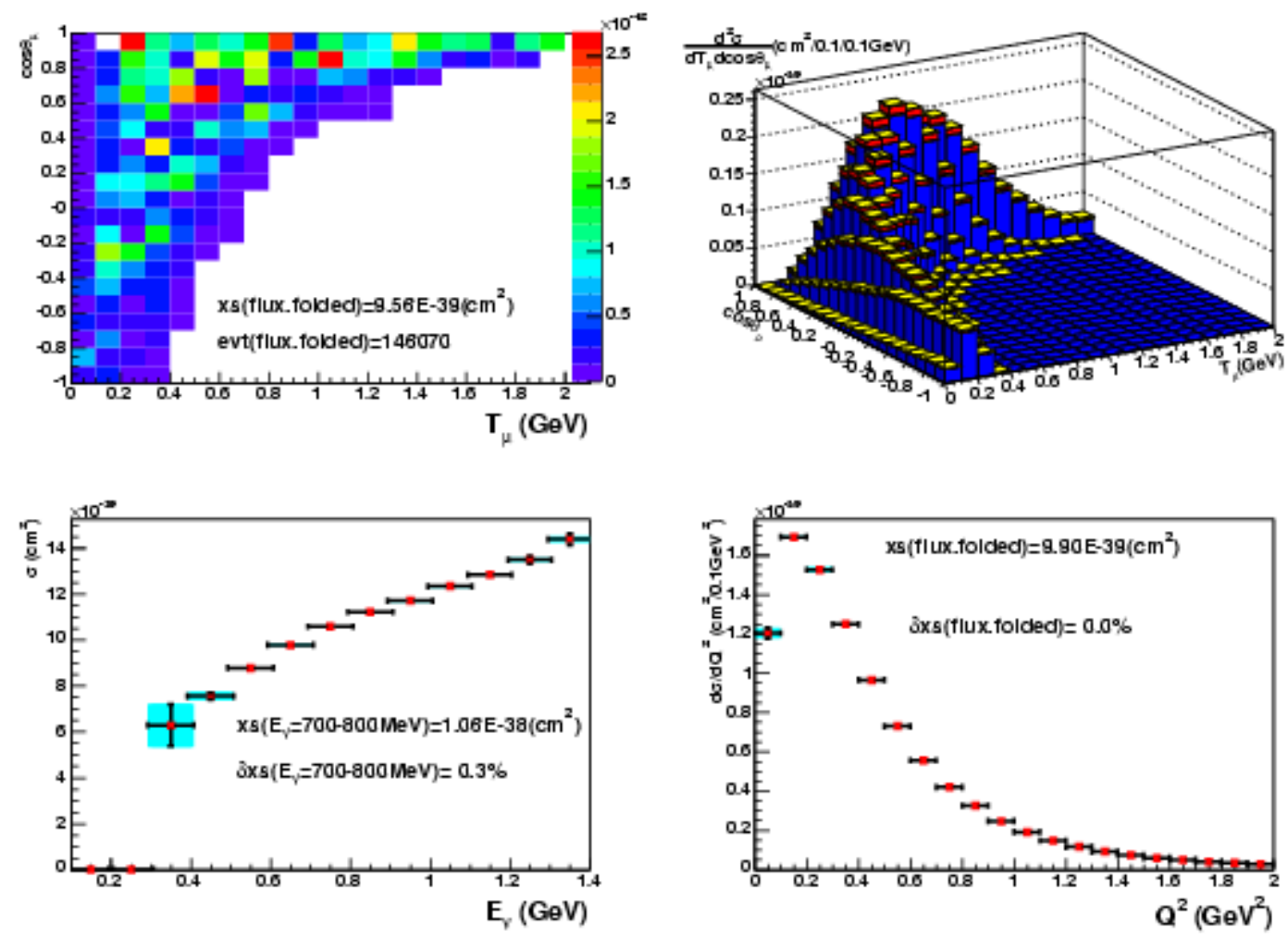

Figure 10.20: Unfolding error only plots for the flux-unfolded double differential cross section and flux-folded total cross section. The notation of plots are found in Sec. 10.10.1. 
CHAPTER 10. CCQE DOUBLE DIFFERENTIAL CROSS SECTION MEASUREMENT

\begin{tabular}{|l|c|}
\hline & cross section error \\
\hline statistics and unfolding & $0.3 \%$ \\
flux & $7.9 \%$ \\
cross section & $4.1 \%$ \\
detector & $4.9 \%$ \\
POT and target normalization & $2.3 \%$ \\
total & $10.5 \%$ \\
\hline
\end{tabular}

Table 10.1: The systematic error summary for flux-unfolded total cross section at 700$800 \mathrm{MeV}$ bin. 


\section{Chapter 11}

\section{Summary and outlook for CCQE scattering measurements in MiniBooNE}

In Part III of this thesis, we have presented a measurement of the charged current quasielastic (CCQE) interaction in MiniBooNE. The CCQE interaction is the most abundant neutrino interaction ( $\sim 40 \%$ of the sample) in the MiniBooNE energy range $(\sim 700 \mathrm{MeV})$, therefore, this reaction channel has high statistical precision. The required cuts to isolate the CCQE sample are relatively model independent yet of high efficiency $(\sim 35 \%)$, and high purity $(\sim 75 \%)$. This channel was used for various checks of the MiniBooNE experiment. In particular, MiniBooNE relies on the CCQE measurement in the neutrino oscillation search for two reasons: to constrain the $\nu_{\mu}$ flux normalization and the intrinsic $\nu_{e}$ background; and to test the kinematic energy reconstruction of $\nu_{e}$ [67].

The combined fit method employed for the neutrino oscillation search successfully used the $\nu_{\mu} \mathrm{CCQE}$ data. The details of this technique are discussed in other theses [112 115], 
and the results were published [67].

An initial disagreement between data and $\mathrm{MC}$ in the muon kinematic variables was determined to be caused by incorrect parameters used in the relativistic Fermi gas (RFG) model. After further investigation, we performed a shape-only fit with two parameters of the RFG model, and we found that the data was better described with an adjustment of two parameters,

$$
\begin{aligned}
M_{A} & =1.23 \pm 0.20 \mathrm{GeV} / \mathrm{c}^{2} ; \\
\kappa & =1.019 \pm 0.011
\end{aligned}
$$

and these results were published [4].

Then, we proceeded with a measurement of the absolute cross section of the CCQE interaction. First, we subtracted the background based on a measurement of that background, instead of using the predictions from an event generator. The in situ measurement of the background is more reliable than predictions for the background. As a consequence, we successfully removed the (best estimate of the) background from the data, although the size of the uncertainties on background remains approximately the same as in the previous analysis [4, due to ambiguities in pion absorption and pion coherent scattering. At this stage, we have extracted new values for the RFG parameters through a two-parameter shape-only fit. We found,

$$
\begin{aligned}
M_{A} & =1.32 \pm 0.17 \mathrm{GeV} / \mathrm{c}^{2} ; \\
\kappa & =1.007 \pm 0.009
\end{aligned}
$$

The extracted value for $M_{A}$ is approximately $30 \%$ higher than the world average value (=1.03 GeV/c ${ }^{2}$ ). The extracted $M_{A}$ is inconsistent with the world-averaged value and the new value for $\kappa$ is consistent with zero. The change in the value for $\kappa$ is because of the change in the $\mathrm{CC} 1 \pi$ background, especially in the low $Q^{2}$ region. 
We have carefully examined all systematic errors and have obtained the flux-folded (or "flux-averaged") double differential cross section for the muon neutrino CCQE interaction. This is a model-independent result based only upon the measured kinematics of the finalstate muon. This is an important experimental result that may be used to develop and tune theoretical models of nuclear structure relevant for neutrino-nuclei scattering.

Further, we have calculated a flux-unfolded total cross section as a function of reconstructed neutrino energy. We have carefully defined this measurement, so that it is as modelindependent as possible in the extraction of a reconstructed neutrino energy. However, there are inevitable limitations in the muon-based kinematic reconstruction of neutrino energy and special care is needed to compare this data with that of others, for example [155]. The resulting cross section at the peak energy of the MiniBooNE neutrino flux (700-800 MeV) is

$$
\sigma_{700-800 \text { MeVbin }}^{C C Q E}=1.058 \pm 0.111 \times 10^{-38} \mathrm{~cm}^{2} .
$$

This agrees with the prediction of the RFG model with new $M_{A}\left(=1.32 \mathrm{GeV} / \mathrm{c}^{2}\right)$ and $\kappa$ (=1.007). However, this agreement is with an $M_{A}$ in the RFG that is $\sim 30 \%$ higher than the world average. The total cross section in the $700-800 \mathrm{MeV}$ bin is $\sim 20 \%$ higher than the RFG model prediction using the world-average $M_{A}$.

The MiniBooNE data points for total cross section are rather high compared to previous measurements. For example, recently the NOMAD experiment [35, 155] extracted $M_{A} \sim 1.05 \mathrm{GeV} / \mathrm{c}^{2}$, consistent with the world-average value. In the NOMAD analysis, the interaction rate is normalized with the well-known rate for deep-inelastic scattering and so, it may be argued that the flux normalization is well-known. Since NOMAD shows excellent agreement with the world average above $\sim 4 \mathrm{GeV}$, the value where the neutrinonucleon CCQE scattering total cross section "plateaus" seems well-known. Although the plateau of the total cross section starts at about $2 \mathrm{GeV}$ and the MiniBooNE data span 
$0.1<E_{\nu}(\mathrm{GeV})<1.4$, it still seems evident that the MiniBooNE data disagree with the world average data.

It is generally thought that the discrepancy in $M_{A}$ as measured by $\mathrm{K} 2 \mathrm{~K}$, MiniBooNE, and SciBooNE compared to the world average may be explained by nuclear effects of carbon [156] because the world average is set by old bubble chamber experiments using mainly deuteron targets where nuclear effects are small. And, modern oscillation experiments use heavier targets, such as carbon or water. However, NOMAD, using a active carbon nuclear target in their drift chambers, measured a $M_{A}$ consistent with the world average. Also, recent studies show nuclear effects are negligible in a limited $Q^{2}$ region (for example $\left.0.5<Q^{2}\left(\mathrm{GeV}^{2}\right)<1.0\right)$ [27]. A fit within $0.5<Q^{2}\left(\mathrm{GeV}^{2}\right)<1.0$ using MiniBooNE data was performed (Sec. 9.7.3), but a similar $M_{A}$ value resulted (as compared to $\left.0.0<Q^{2}\left(\mathrm{GeV}^{2}\right)<1.0\right)$. This indicates that the origin of the high $M_{A}$ value is not likely a nuclear effect.

Nevertheless, the $M_{A}$ extracted from a $\left(Q^{2}\right)$ shape-only fit, predicts about the correct overall rate for the CCQE interaction, as seen in Fig. 10.12. These disparate results for $M_{A}$ between low and high energy regions must be understood in further work. For example, the newly-proposed liquid Argon time projection chamber experiment, MicroBooNE [157], will use $A r$ for an active nuclear target. And this detector technology provides excellent tracking and will allow the reconstruction of the recoil proton in CCQE neutrino energy reconstruction. These two features will provide additional information to resolve some of these exciting mysteries in CCQE cross section measurements. 


\section{Appendix A}

\section{R\&D and pilot detector beam test for FINeSSE}

\section{A.1 Neutrino Neutral current elastic (NCE) scattering}

Neutrino neutral current elastic (NCE) scattering is identical with charged-current quasielastic (CCQE) scattering in the cross section formulas, by replacing the CCQE form factors with those for NCE (see Sec. 3.2.3). The notable difference is that NCE is sensitive to isoscalar form factors in addition to isovector form factors. Since the dominant contribution expected for the isoscalar term is that from any strange quarks present in the nucleus, the measurement of isoscalar Dirac and Pauli form factors are active field for modern electron scattering experiments, such as SAMPLE [158, PVA4 [159], G0 [23], and HAPPEX [160].

Of course, these strange form factors are zero in the constituent quark model [18]. However, some measurements indicate small but non-zero contributions [23] and we are waiting for further experiments. However, a small coupling and large radiative correction makes very difficult to measure isoscalar axial form factor in electron scattering experiments [161].

The zero- $Q^{2}$ limit of the isoscalar axial form factor $F_{A}^{s}\left(Q^{2}\right)$ is related to the spin con- 
tribution of the strange quark in a nucleon, and is called $\Delta s$ (Eqs. 3.63 and 3.64), and may be connected with longitudinally polarized PDFs of strange quarks $\Delta s(x)$,

$$
\Delta s(x) \equiv s_{+}^{+}(x)-s_{-}^{+}(x)-\left(\bar{s}_{+}^{+}(x)-\bar{s}_{-}^{+}(x)\right)
$$

Here, $\Delta s(x)$ is defined from the difference of longitudinally polarized (denoted upper "+") strange quark PDF, helicity + and - (lower "+" and "-"), from quarks and anti quarks. Then, $\Delta s$ connects the elastic nucleon form factor and longitudinally polarized $\mathrm{PDF}$,

$$
F_{A}^{s}\left(Q^{2} \rightarrow 0\right) \equiv \Delta s \equiv \int_{0}^{1} \Delta s(x) d x
$$

It is an important check to verify the connection between low- $Q^{2}$ elastic world and high $-Q^{2}$ DIS world. Currently, there is a disagreement known between HERMES Semiinclusive DIS measurement [162] at Deutsches Elektronen-Synchrotron (DESY) and BNL E734 NCE measurement [163] at Brookhaven National Laboratory (BNL) which is not yet understood. To better understand the right side of the equation, a new semi-inclusive DIS analysis using only kaons is performed [164]. This result is consistent with previous HERMES result, which implies that the inconsistency with BNL734 remains. However, the extraction of $\Delta s$ from inclusive DIS measurement, such as SMC [165], under the assumption of $\mathrm{SU}(3)$ flavor symmetry, does agree with BNL734. So the situation is rather confused and we also need to test the left side of this equation. For this purpose, a new NCE scattering experiment, FINeSSE [24, is planned. The NCE analysis from MiniBooNE [110, 151, 166] and SciBooNE [167] are also awaited.

\section{A.2 The scibath pilot detector beam test}

The Fermilab Intense Neutrino Scintillator Scattering Experiment, FINeSSE [24], is a proposed experiment to focus on the NCE measurement. FINeSSE is planned to use the 

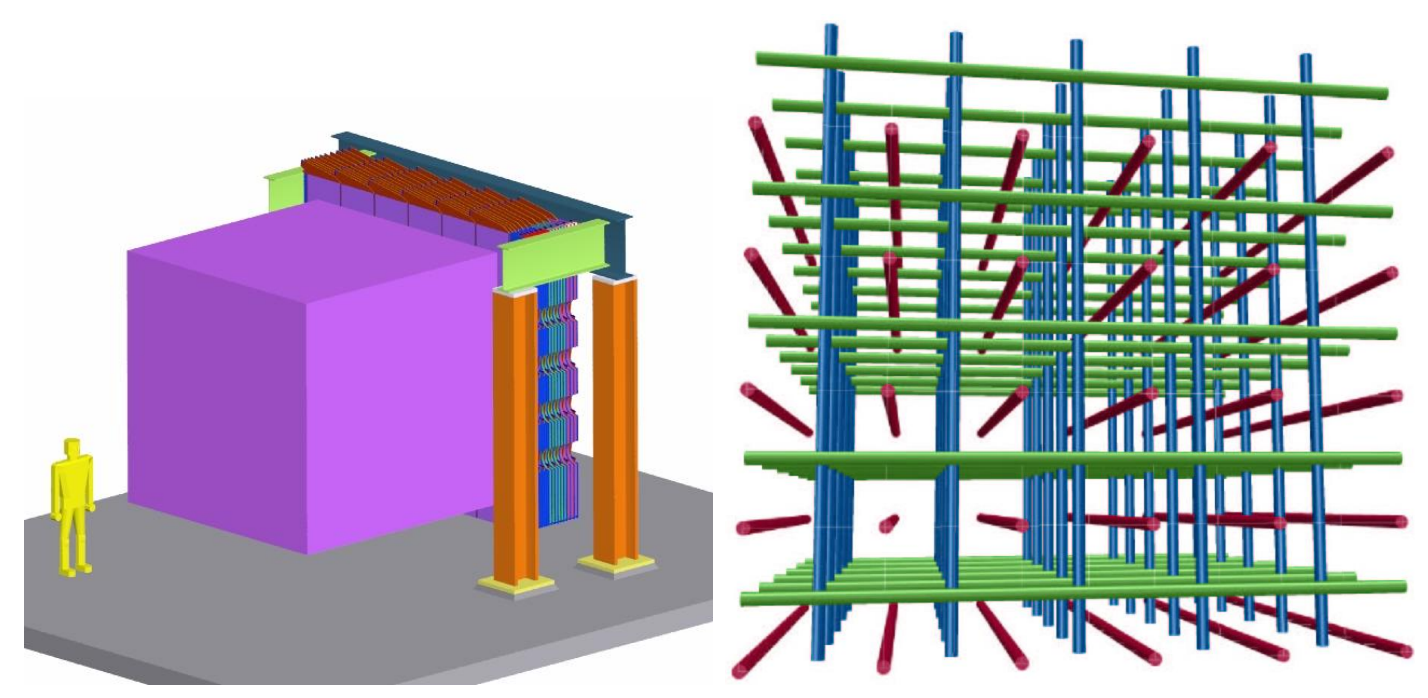

Figure A.1: The FINeSSE detector and the inside of the scibath detector.

detector called "scibath", which consists of an array of wave length shifting (WLS) fibers immersed in liquid scintillator. With this detector, it is possible to reconstruct charged particle tracks three-dimensionally. Figure A.1 shows cartoons of FINeSSE detector. The left figure shows FINeSSE vertex and tracking detector "scibath", which has $(2.5 \mathrm{~m})^{3}$ active liquid scintillator volume with over 10,000 WLS fibers, with muon range stack behind to measure muon energies. The right figure is a cartoon of the inside of scibath detector.

To test the principle of scibath detector, we performed beam tests with a scibath pilot detector. Figure A.2 shows the inside of the scibath pilot detector. The pilot detector consists with 30 of blue-to-green WLS fibers in the liquid scintillator. The pilot detector of scibath was built and beam test was perform at Indiana University Cyclotron Facility (IUCF). The detail of this beam test is available in [5].

Figure A.3 shows the picture of experimental setup. Figure A.4 shows the electronics diagram for scibath prototype beam test. The test was done at radiation effects research program (RERP) at IUCF. using a $200 \mathrm{MeV}$ kinetic energy proton beam.

Figure A.5 shows the principle of this detector and the beam test. Fig. A.5.a) shows the 


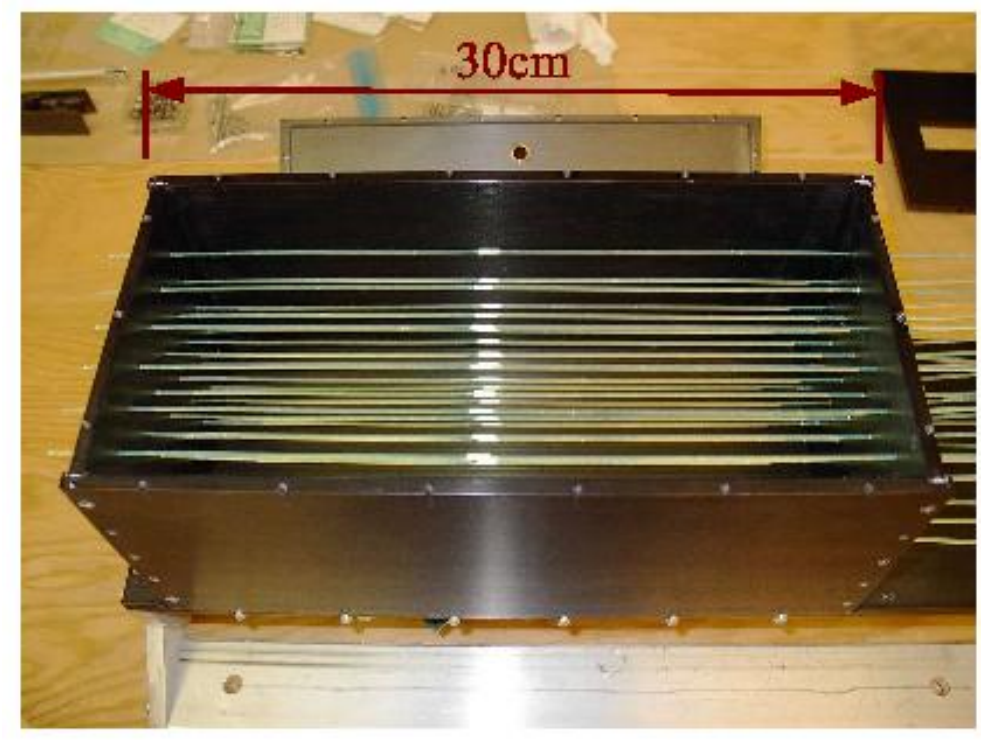

Figure A.2: The scibath pilot detector. 30 WLS fibers in the liquid scintillator make one-dimensional array to reconstruct charged particle tracks.

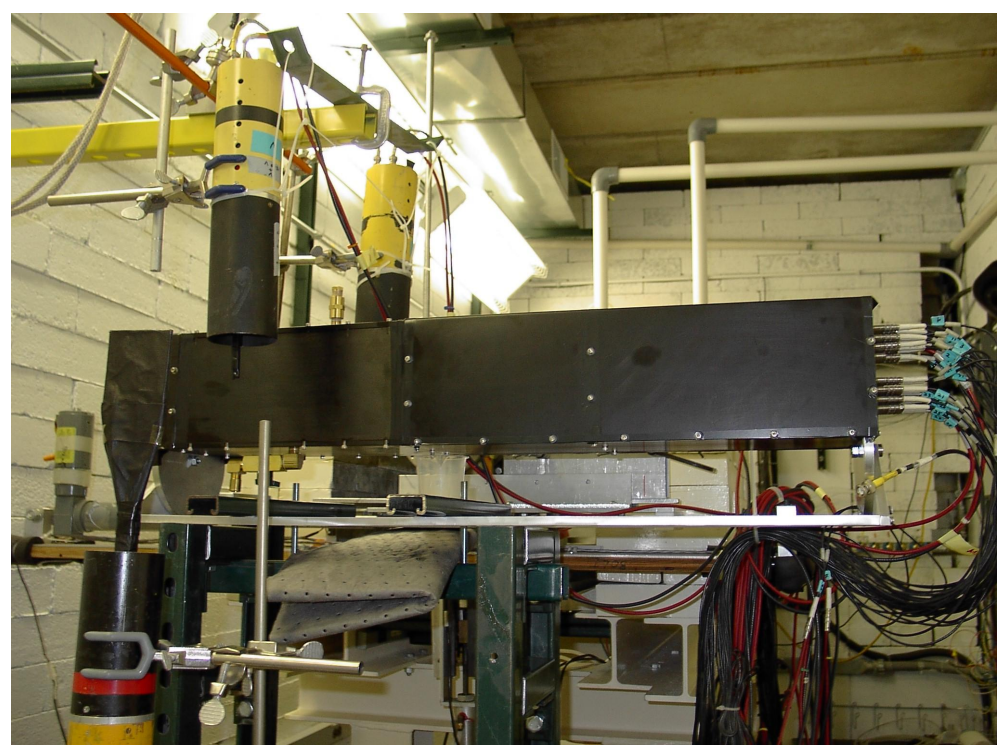

Figure A.3: The scibath pilot detector beam test setup at RERP at IUCF. The detector is sandwiched by three trigger paddles although we used only two of them. 


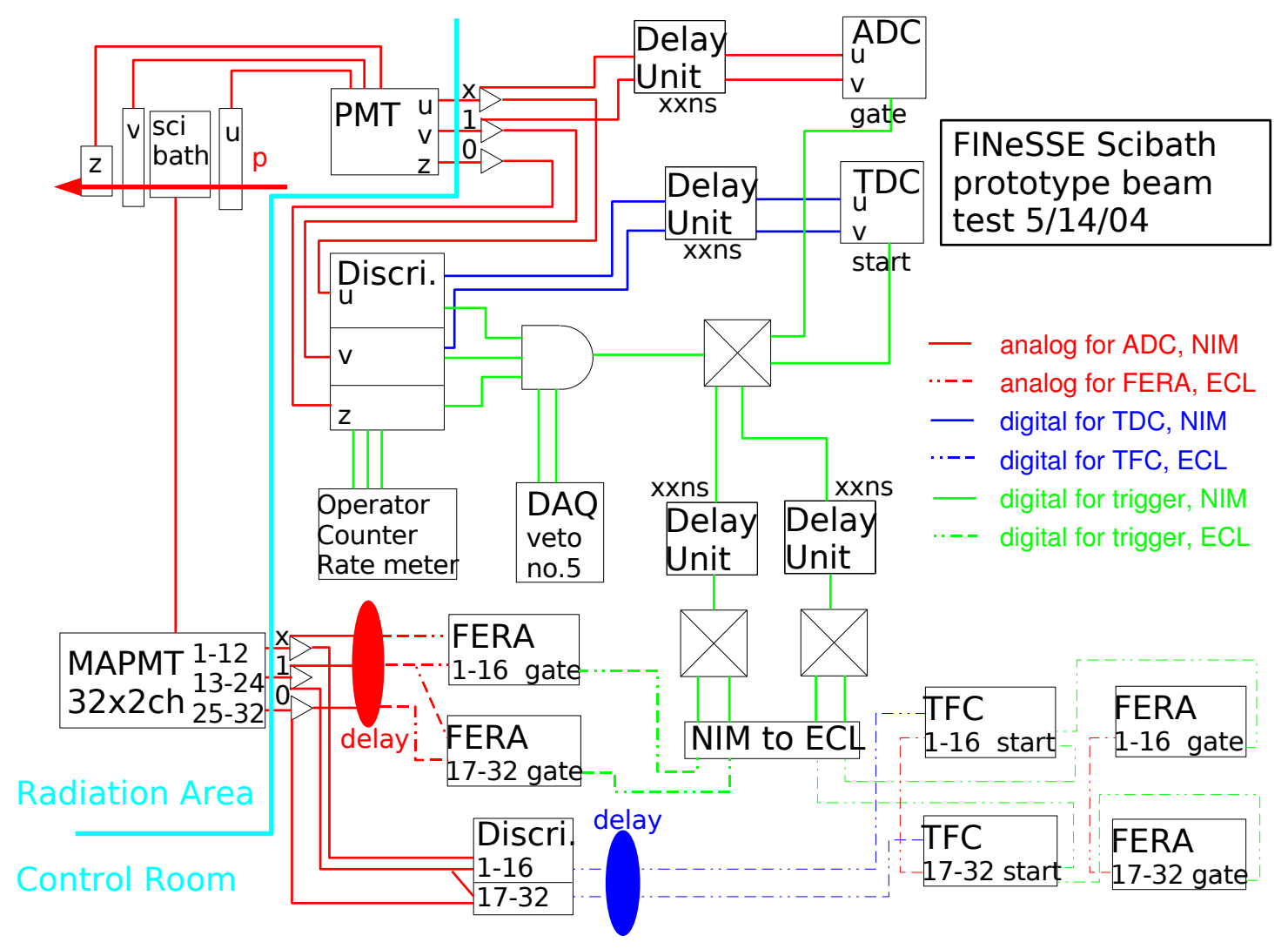

Figure A.4: The scibath pilot detector beam test electronics diagram at RERP at IUCF. 
situation for one arbitrary fiber. When a charged particle passes the detector, a fiber sees the integrated charge information from the scintillation emission in the track of a charged particle. For this beam test, Fig. A.5.b), a particle track is defined from two trigger paddles, and $200 \mathrm{MeV}$ protons penetrate the detector from the side. From the nearest fiber to a track, we found $17 \pm 2$ photoelectrons (PEs). This number is different depending on the combination of different types of fibers and liquid scintillators [5]. We also tried to tilt or rotate the detector to simulate angled tracks [5]. Fig. A.5.c) shows a realistic situation of this experiment. Light from a track can be collected by all fibers in the detector. In other word, track information are shared by all fibers, unlike typical tracking detector where particle information exist only where particle passes in the detector. However, Fig. A.5.d), collected charges are different from each fiber depending on their distance. This information allow us to reconstruct particle tracks with the coordinate resolution smaller than the fiber separations.

Figure A.6 shows the result of the proton track measurement. Tracks are found by least square fit with the straight lines. We found,

$$
\begin{aligned}
\text { coordinate resolution } & =0.50 \mathrm{~cm}, \\
\text { angular resolution } & =6.2^{\circ},
\end{aligned}
$$

after removing the width contribution of a trigger paddle ( 0.5 in). Notably, we achieved a better resolution than the fiber separation $(2 \mathrm{~cm})$. The resolution is even better to use UVto-blue fibers and non WLS liquid scintillator, instead of using typical blue-to-green fibers and liquid scintillator (Fig. A.7). Because short attenuation of non WLS liquid scintillator helps to localize light and sending blue light by UV-to-blue fibers has advantage for the quantum efficiency of typical bi-alkali photo-cathode PMTs. In this case, we found,

$$
\begin{aligned}
\text { coordinate resolution } & =0.28 \mathrm{~cm}, \\
\text { angular resolution } & =3.5^{\circ} .
\end{aligned}
$$




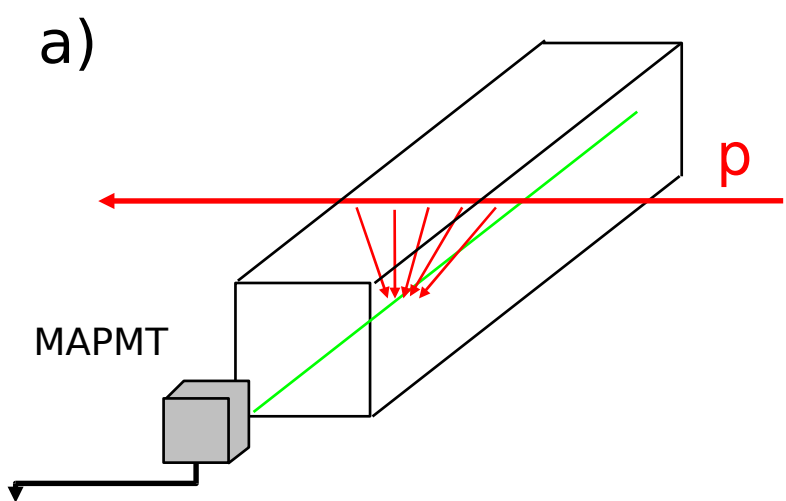

b)

C)

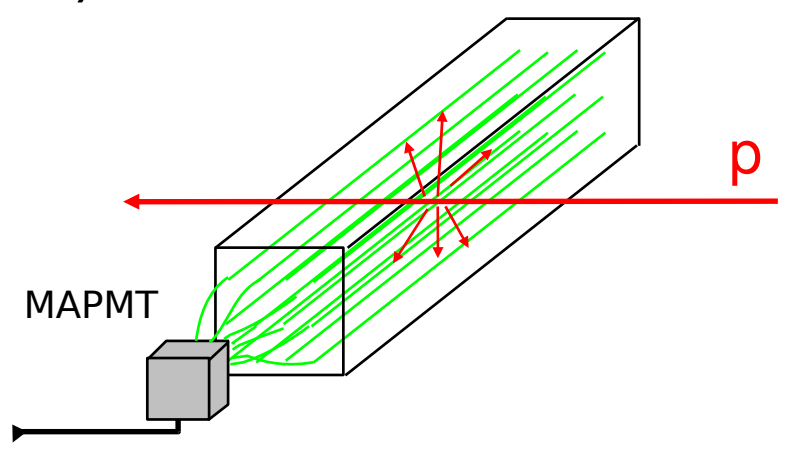

d)
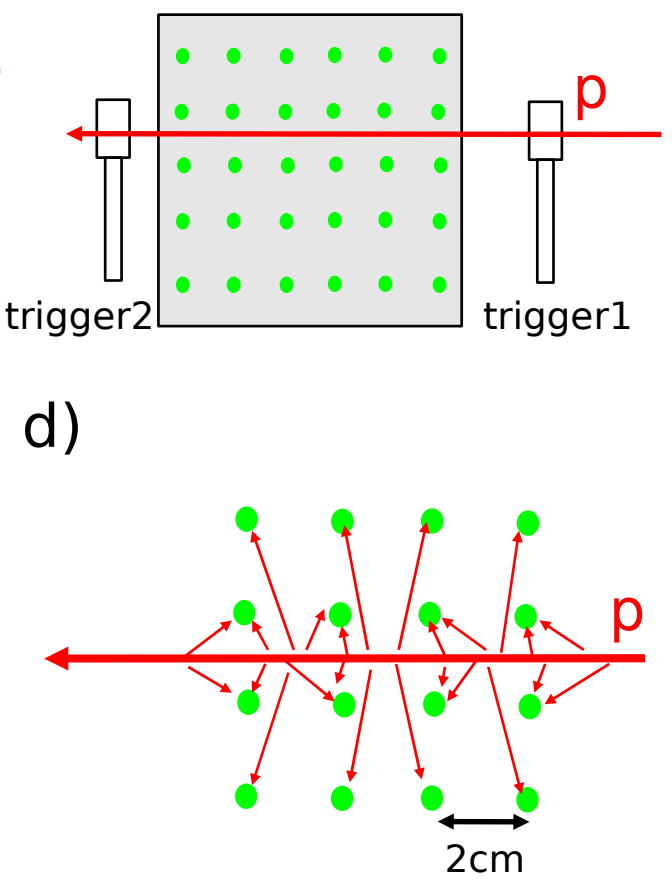

Figure A.5: The scibath pilot detector beam test principle cartoon. They are explained in the text. 

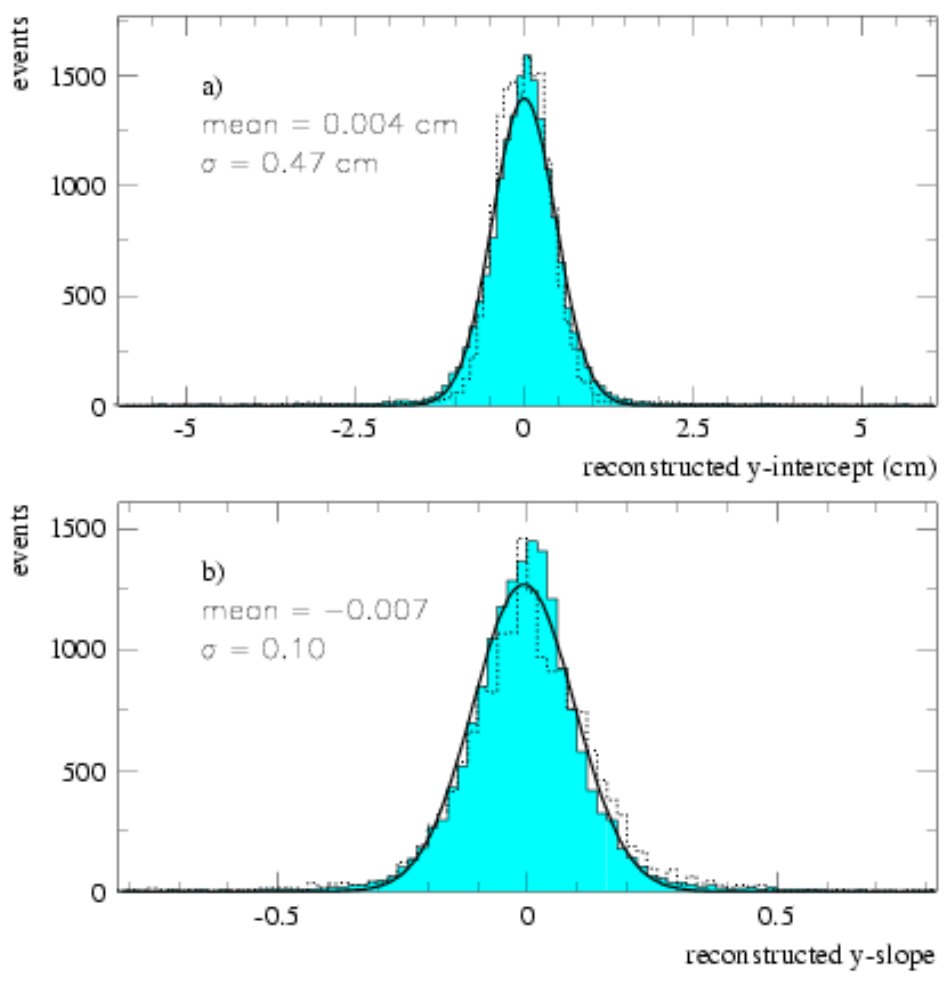

Figure A.6: The scibath pilot detector coordinate and angular resolutions. Note, measurement smearing by the trigger width (0.5in) is subtracted from the measured RMS. 
a)
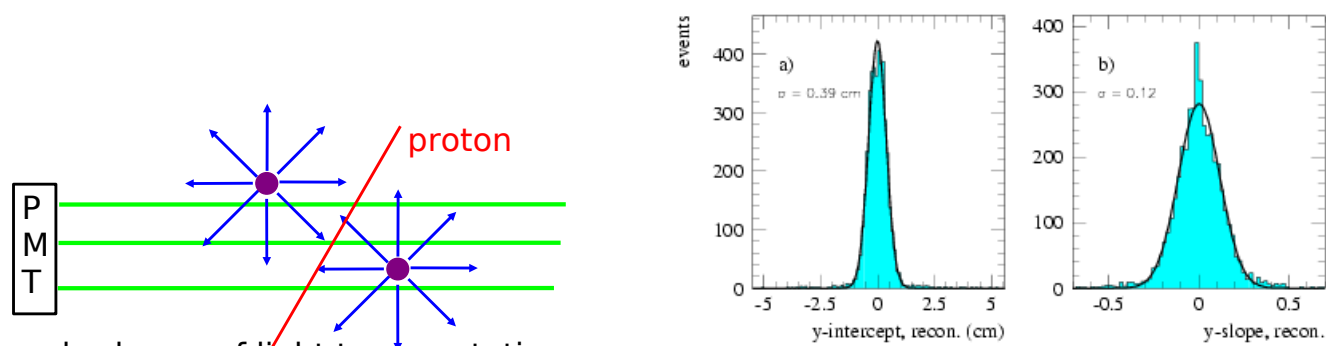

traditional scheme of light transportation $\mathrm{p} \rightarrow \mathrm{UV} \rightarrow$ Blue $\rightarrow$ Green $\rightarrow$ PMT

b)
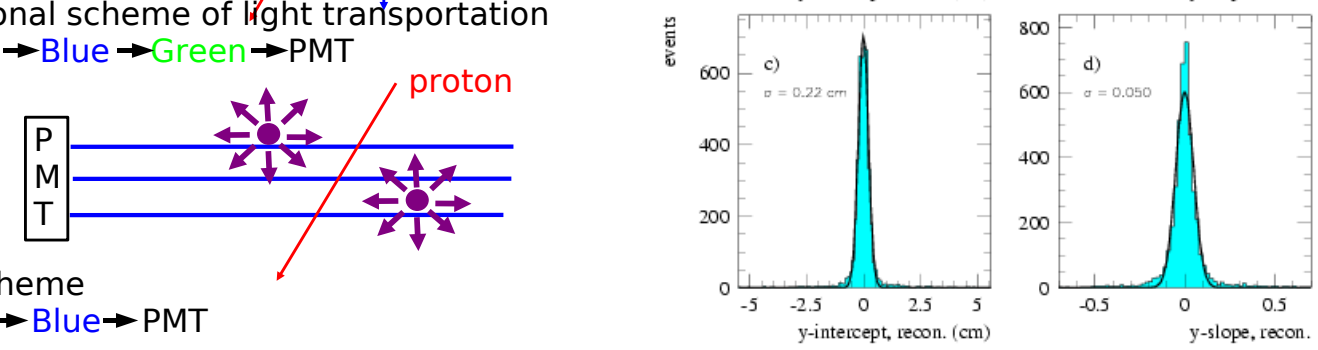

Figure A.7: The left cartoon illustrates two experimental settings, (a) blue-to-green WLS fiber with liquid scintillator, and (b) UV-to-blue WLS fiber with non WLS liquid scintillator. In the case of (b), the short attenuation of non WLS liquid scintillator localizes scintillation emission and improve the coordinate resolution. On top of that, the sending blue light to PMT has advantage for the quantum efficiency of typical bi-alkali photo-cathode. The right plot shows the result. for scibath pilot detector coordinate and angular resolutions for various fibers and liquid scintillator combinations. Top two plots are for UV-to-green fibers and non WLS liquid scintillator. Bottom two plots are for UV-to-blue fibers and non WLS liquid scintillator. The later combination has better coordinate resolution than the standard blue-to-green fibers and liquid scintillator. 
We also considered a signal transformation method for finding particle tracks. Say, light generated at $j^{\text {th }}$ location in the detector is seen by $i^{t h}$ fiber. Then we can form a response matrix $R^{i j}$, because total light seen by $i^{\text {th }}$ fiber is an integral of all light generated at all locations in the detector. Once you make such response matrix, then the inverse of that unveils a particle track from integrated light information from all fibers. The response matrix has a special shape,

$R^{i j}=\left(\begin{array}{cccc}R_{0} & R_{1} & R_{2} & \cdots \\ R_{1} & R_{0} & R_{1} & \cdots \\ R_{2} & R_{1} & R_{0} & \cdots \\ \vdots & \vdots & \vdots & \ddots\end{array}\right), R_{0}^{i j}=\left(\begin{array}{cccc}a & b & c & \cdots \\ b & a & b & \cdots \\ c & b & a & \cdots \\ \vdots & \vdots & \vdots & \ddots\end{array}\right), R_{1}^{i j}=\left(\begin{array}{cccc}d & e & f & \cdots \\ e & d & e & \cdots \\ f & e & d & \cdots \\ \vdots & \vdots & \vdots & \ddots\end{array}\right), \cdots$

and it turns out this is the special case of so called block Toeplitz matrix, which has known fast inversion algorithm [168]. Since full scale of scibath detector has over 10,000 fibers, this kind of special algorithm may help the CPU time. Figure A.8 shows the result. Before the transformation, a track is already visible as ADC count maximum, but we can find the accurate track by a fit. However, after the transformation, there is a sharper peak on the track and we know the location of track without fit. Therefore, this method can be used for the cross check of track finding fit. 


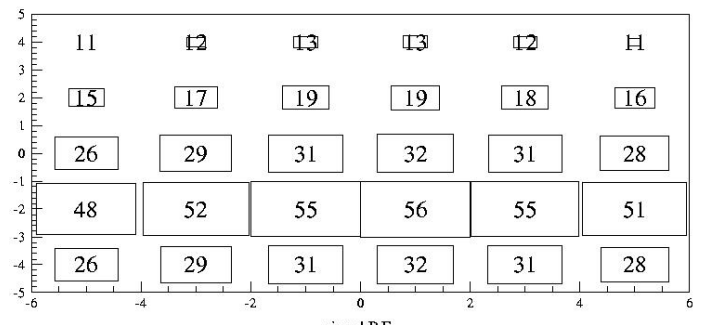

\begin{tabular}{|c|c|c|c|c|c|c|}
\hline 情 & -328 & -162 & -195 & -188 & -11311 & -247 \\
\hline 2 & 552 & -161 & 17 & $\mathrm{E2D}$ & $E 32$ & 887 \\
\hline 㖩 & -22 & $-[1007$ & -41 & -153 & -20 & 68 \\
\hline-2 & 4333 & 3682 & 3544 & 4400 & 3686 & 4568 \\
\hline$-4 \mid$ & -160 & -269 & -5 & -342 & -39 & $=28$ \\
\hline
\end{tabular}

Figure A.8: The scibath pilot detector signal transformation. Top plot shows ADC count for all fibers when a proton track is on the $4^{\text {th }}$ fiber array. Bottom plot shows after the transformation using the inverse of response matrix. Now, track is visible without any fitting procedures. 


\section{Appendix B}

\section{Gamma matrix algebra}

Following relationships are often found in literatures, for example p.123 and p.261 of [18].

$$
\begin{aligned}
\operatorname{tr}(1) & =4 \\
\operatorname{tr}\left(\text { odd number of } \gamma_{\mu}\right) & =0 \\
\operatorname{tr}(\phi \phi \phi \phi d) & =4[(a \cdot b)(c \cdot d)-(a \cdot c)(b \cdot d)+(a \cdot d)(b \cdot c)] \\
\operatorname{tr}\left(\gamma_{5}\right) & =0 \\
\operatorname{tr}\left(\gamma_{5} \phi \phi \phi\right) & =0 \\
\operatorname{tr}\left(\gamma_{5} \phi \phi \phi \phi d\right) & =4 i \epsilon_{\mu \nu \lambda \sigma} a^{\mu} b^{\nu} c^{\lambda} d^{\sigma} \\
\gamma_{\mu} \gamma^{\mu} & =4 \\
\gamma_{\mu} \phi \gamma^{\mu} & =-2 \not \phi \\
\gamma_{\mu} \phi \phi \phi \gamma^{\mu} & =4 a \cdot b \\
\gamma_{\mu} \phi \phi \phi \phi \gamma^{\mu} & =-2 \phi \phi \phi
\end{aligned}
$$




$$
\begin{aligned}
\operatorname{tr}\left(\gamma^{\mu} \not p_{1} \gamma^{\nu} \not p_{2}\right) & =4\left[p_{1}^{\mu} p_{2}^{\nu}+p_{1}^{\nu} p_{2}^{\mu}-\left(p_{1} \cdot p_{2}\right) g^{\mu \nu}\right] \\
\operatorname{tr}\left[\gamma^{\mu}\left(1-\gamma_{5}\right) p_{1} \gamma^{\nu}\left(1-\gamma_{5}\right) \not p_{2}\right] & =2 \operatorname{tr}\left(\gamma^{\mu} \not p_{1} \gamma^{\nu} \not p_{2}\right)-8 i \epsilon^{\mu \nu \lambda \sigma} p_{1 \lambda} p_{2 \sigma} \\
\operatorname{tr}\left(\gamma^{\mu} \not p_{1} \gamma^{\nu} \not p_{2}\right) \operatorname{tr}\left(\gamma_{\mu} \not p_{3} \gamma_{\nu} \not p_{4}\right) & =32\left[\left(p_{1} \cdot p_{3}\right)\left(p_{2} \cdot p_{4}\right)+\left(p_{1} \cdot p_{4}\right)\left(p_{2} \cdot p_{3}\right)\right] \\
\operatorname{tr}\left(\gamma^{\mu} \not p_{1} \gamma^{\nu} \gamma_{5} \not p_{2}\right) \operatorname{tr}\left(\gamma_{\mu} \not p_{3} \gamma_{\nu} \gamma_{5} \not p_{4}\right) & =32\left[\left(p_{1} \cdot p_{3}\right)\left(p_{2} \cdot p_{4}\right)-\left(p_{1} \cdot p_{4}\right)\left(p_{2} \cdot p_{3}\right)\right]
\end{aligned}
$$

$\operatorname{tr}\left[\gamma^{\mu}\left(1-\gamma_{5}\right) p_{1} \gamma^{\nu}\left(1-\gamma_{5}\right) p_{2}\right) \operatorname{tr}\left(\gamma_{\mu}\left(1-\gamma_{5}\right) p_{3} \gamma_{\nu}\left(1-\gamma_{5}\right) p_{4}\right]=256\left(p_{1} \cdot p_{3}\right)\left(p_{2} \cdot p_{4}\right)$ 


\section{Appendix C}

\section{The detail of cross section calculations}

This Appendix describes the further details of the calculation of neutrino-nucleon scattering (Sec. 3.2) and neutrino-nuclei scattering (Sec. 3.3).

\section{C.1 Neutrino-Nucleon scattering}

\section{C.1.1 Kinematics and cross section formula}

The scattering process under consideration are the following 2 reactions,

$$
\begin{aligned}
& \nu_{l}+n \rightarrow l^{-}+p, \\
& \bar{\nu}_{l}+p \rightarrow l^{+}+n .
\end{aligned}
$$


(1) Lab frame

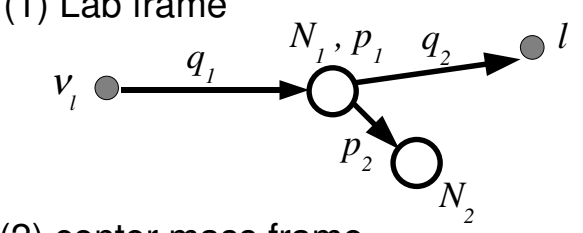

(2) center mass frame

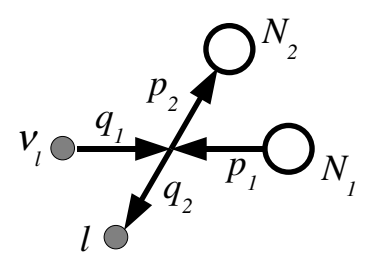

(3) Feynman diagram

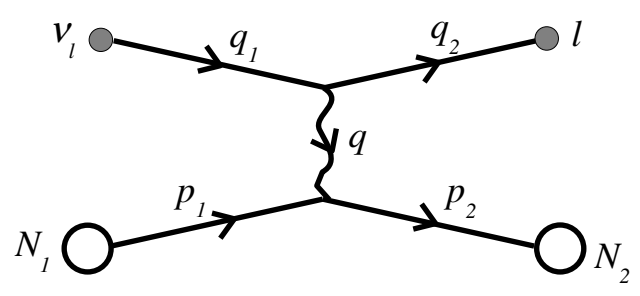

Figure C.1: Scattering under consideration, from top to bottom, (1) lab frame, (2) centerof-mass frame, and (3) Feynman diagram (t-channel). $\nu_{l}$ stands for (anti)neutrino, $l$ stands for (positively) negatively charged lepton and $N_{1}$ and $N_{2}$ stands for proton or neutron. The charged lepton has mass $m$ and both initial and final state nucleon have equal mass $M$. 
In Fig C.1, $\nu, l, N_{1}$ and $N_{2}$ have 4 -momentum $q_{1}, q_{2}, p_{1}$ and $p_{2}$ respectively. The charged lepton $l$ has mass $m$, and nucleon $N_{1}$ and $N_{2}$ have equal mass $M$. These 4-momenta are,

$$
\begin{aligned}
& q_{1}=\left(E_{\nu}, \overrightarrow{p_{\nu}}\right), \\
& q_{2}=\left(E_{l}, \overrightarrow{p_{l}}\right), \\
& p_{1}=(M, 0), \\
& p_{2}=(E, \vec{p}) .
\end{aligned}
$$

In this Appendix, we don't write the index of 4-momentum explicitly.

These momenta satisfy the following relations,

$$
\begin{aligned}
P & =p_{1}+p_{2}, \\
q & =p_{2}-p_{1}=q_{1}-q_{2}, \\
n & =q_{1}+q_{2}, \\
s & =\left(p_{1}+q_{1}\right)^{2}=\left(p_{2}+q_{2}\right)^{2}=M^{2}+2 M E_{\nu}, \\
u & =\left(q_{2}-p_{1}\right)^{2}=\left(p_{2}-q_{1}\right)^{2}=M^{2}+m^{2}-2 M E_{l}, \\
t & =q^{2}=\sum_{\text {all }}(\text { mass })^{2}-(s+u)=2 M^{2}+m^{2}-(s+u)=2 M\left(E_{l}-E_{\nu}\right), \\
\nu & =\frac{q \cdot p_{1}}{M}=\frac{M E_{\nu}-M E_{l}}{M}=E_{\nu}-E_{l},
\end{aligned}
$$


further more,

$$
\begin{aligned}
q^{2} & =\left(p_{2}-p_{1}\right)^{2}=2 M^{2}-2 p_{1} \cdot p_{2}=\left(q_{1}-q_{2}\right)^{2}=m^{2}-2 q_{1} \cdot q_{2}, \\
P^{2} & =\left(p_{1}+p_{2}\right)^{2}=2 M^{2}+2 p_{1} \cdot p_{2}=4 M^{2}-q^{2}, \\
n^{2} & =\left(q_{1}+q_{2}\right)^{2}=m^{2}+2 q_{1} \cdot q_{2}=2 m^{2}-q^{2}, \\
s-u & =4 M E_{\nu}+q^{2}-m^{2}=P \cdot n, \\
P \cdot n & =\left(p_{1}+p_{2}\right) \cdot\left(q_{1}+q_{2}\right)=\left(p_{2}-p_{1}\right) \cdot\left(q_{1}-q_{2}\right)+2 p_{1} \cdot q_{1}+2 p_{2} \cdot q_{2} \\
& =q^{2}+2 M E_{\nu}+\left(2 M E_{\nu}-m^{2}\right)=q^{2}-m^{2}+4 M E_{\nu}=-m^{2}+2 M\left(E_{\nu}+E_{l}\right) \\
& =-2 M \nu-m^{2}+4 M E_{\nu}, \\
q \cdot n & =\left(q_{1}-q_{2}\right) \cdot\left(q_{1}+q_{2}\right)=\left(q_{1}-q_{2}\right) \cdot\left(q_{1}-q_{2}\right)+2 q_{1} \cdot q_{2}-2 q_{2}^{2}=-m^{2}, \\
q \cdot P & =\left(p_{2}-p_{1}\right) \cdot\left(p_{2}+p_{1}\right)=\left(p_{2}-p_{1}\right) \cdot\left(p_{2}-p_{1}\right)+2 p_{1} \cdot p_{2}-2 p_{1}^{2}=0 .
\end{aligned}
$$

In Llewellyn Smith's paper [15], the general nucleon weak interaction current operator is defined,

$$
\begin{gathered}
<p\left|J_{\mu}^{+}\right| n>=\cos \theta_{c}\left(\bar{p}\left(p_{2}\right) \Gamma_{\mu} n\left(p_{1}\right)\right) \\
\Gamma_{\mu}=\gamma_{\mu}\left(F_{1}+F_{A} \gamma_{5}\right)+\frac{1}{2 M} i \sigma_{\mu \nu} q^{\nu} \xi F_{2}+\frac{1}{M} P_{\mu} F_{A}^{3} \gamma_{5}+\frac{q_{\mu}}{M}\left(F_{V}^{3}+F_{P} \gamma_{5}\right),
\end{gathered}
$$

here $\xi$ is defined as a difference of the relative anomalous magnetic moment,

$$
\xi=\kappa_{p}-\kappa_{n}=\left(\frac{\mu_{p}}{\mu_{N}}-1\right)-\left(\frac{\mu_{n}}{\mu_{N}}-0\right)=1.793-(-1.913)=3.706 .
$$

On the other hand, in Pais's paper [169], the general nucleon weak current is defined,

$$
\Gamma_{\mu}=\gamma_{\mu}\left(g_{V}-g_{A} \gamma_{5}\right)-P_{\mu}\left(f_{V}-f_{A} \gamma_{5}\right)+q_{\mu}\left(h_{V}-h_{A} \gamma_{5}\right)
$$

note, $h_{V}$ and $f_{A}$ are G-parity violating second class currents (Sec. 3.2.8). Using Gordon's decomposition,

$$
\overline{u\left(p_{2}\right)} \gamma_{\mu} u\left(p_{1}\right)=\frac{1}{2 M} \overline{u\left(p_{2}\right)}\left[\left(p_{2}+p_{1}\right)_{\mu}+i \sigma_{\mu \nu}\left(p_{2}-p_{1}\right)^{\nu}\right] u\left(p_{1}\right)
$$


one can see the relationship of these 2 definitions.

$$
\begin{aligned}
\text { Pais } & \leftrightarrow \text { Llewellyn Smith } \\
g_{V} & \leftrightarrow F_{1}+\xi F_{2} \\
f_{V} & \leftrightarrow \frac{1}{2 M} \xi F_{2} \\
h_{V} & \leftrightarrow \frac{1}{M} F_{V}^{3} \\
g_{A} & \leftrightarrow-F_{A} \\
f_{A} & \leftrightarrow \frac{1}{M} F_{A}^{3} \\
h_{A} & \leftrightarrow-\frac{1}{M} F_{P}
\end{aligned}
$$

In the following, We will use Pais's notation of general nucleon weak form factors.

The differential Lorentz-invariant phase space (dLips) is,

$$
\begin{aligned}
d \text { Lips } & =\frac{p_{l}^{2} d p_{l} d(\cos \theta)}{8 \pi E_{l} E} \delta\left(E_{l}+E-E_{\nu}-M\right)=\frac{p_{l} E_{l} d E_{l} d q^{2}}{8 \pi E_{l} E \cdot 2 p_{l} E_{\nu}} \delta\left(E_{l}+E-E_{\nu}-M\right) \\
& =\frac{d q^{2}}{16 \pi M E_{\nu}} .
\end{aligned}
$$

Here, we use $d q^{2}=2 p_{l} E_{\nu} d(\cos \theta)$ and $p_{l} d p_{l}=E_{l} d E_{l}$. Then, we have the following differential cross section is,

$$
\begin{aligned}
\frac{d \sigma}{d Q^{2}}= & \frac{1}{64 \pi E_{\nu}^{2} M^{2}} \frac{G_{F}^{2}}{2} \frac{1}{\left(2 s_{p}+1\right)\left(2 s_{\nu}+1\right)} \\
& \times \sum_{\operatorname{spin}}\left[\cos \theta_{c}\left(\bar{p} \Gamma_{\mu} n\right)\right]\left[\cos \theta_{c}\left(\bar{p} \Gamma_{\nu} n\right)\right]^{\dagger}\left[\bar{l} \gamma^{\mu}\left(1-\gamma_{5}\right) \nu\right]\left[\bar{l} \gamma^{\nu}\left(1-\gamma_{5}\right) \nu\right]^{\dagger} \\
= & \frac{1}{64 \pi E_{\nu}{ }^{2} M^{2}} \frac{G_{F}{ }^{2} \cos \theta_{c}{ }^{2}}{2} \frac{1}{2} w_{\mu \nu} \tau^{\mu \nu}
\end{aligned}
$$

Here, $w_{\mu \nu}$ and $\tau^{\mu \nu}$ are the hadronic and leptonic tensors. We would especially like to calculate Eq. C.12 for neutrino-neutron charge current reaction (Eq.C.1), then other results are straight forward from there. Let us start from the easiest part, the leptonic tensor term calculation. 


\section{C.1. NEUTRINO-NUCLEON SCATTERING}

\section{C.1.2 Leptonic tensor term}

The leptonic tensor is defined with the Dirac spinors $\nu\left(p_{1}\right)$ and $l\left(p_{2}\right)$, and using gamma matrix algebra (Appendix B],

$$
\begin{aligned}
\tau_{\mu \nu} & =\operatorname{Tr}\left[\bar{l} \gamma_{\mu}\left(1-\gamma_{5}\right) \nu\right]\left[\bar{\nu} \gamma_{\nu}\left(1-\gamma_{5}\right) l\right] \\
& =\operatorname{Tr}\left[\gamma_{\mu}\left(1-\gamma_{5}\right) \phi_{1} \gamma_{\nu}\left(1-\gamma_{5}\right)\left(q_{2}+m\right)\right] \\
& =\operatorname{Tr}\left[\left\{\gamma_{\mu}\left(1-\gamma_{5}\right) \phi_{1} \gamma_{\nu}\left(1-\gamma_{5}\right) q_{2}\right\}+m \gamma_{\mu}\left(1-\gamma_{5}\right) \phi_{1} \gamma_{\nu}\left(1-\gamma_{5}\right)\right] \\
& =2 \operatorname{Tr}\left[\gamma_{\mu} q_{1} \gamma_{\nu} q_{2}\right]+8 i \epsilon_{\mu \alpha \nu \beta} q_{1 \alpha} q_{2 \beta} \\
& =8\left[q_{1 \mu} q_{2 \nu}-\left(q_{1} \cdot q_{2}\right) g_{\mu \nu}+q_{2 \mu} q_{1 \nu}\right]+8 i \epsilon_{\mu \alpha \nu \beta} q_{1 \alpha} q_{2 \beta},
\end{aligned}
$$

where we have following relations,

$$
\begin{aligned}
n_{\mu} n_{\nu} & =\left(q_{1}+q_{2}\right)_{\mu}\left(q_{1}+q_{2}\right)_{\nu}=q_{1 \mu} q_{1 \nu}+q_{1 \mu} q_{2 \nu}+q_{2 \mu} q_{1 \nu}+q_{2 \mu} q_{2 \nu} \\
q_{\mu} q_{\nu} & =\left(q_{1}-q_{2}\right)_{\mu}\left(q_{1}-q_{2}\right)_{\nu}=q_{1 \mu} q_{1 \nu}-q_{1 \mu} q_{2 \nu}-q_{2 \mu} q_{1 \nu}+q_{2 \mu} q_{2 \nu} \\
n_{\mu} q_{\nu} & =\left(q_{1}+q_{2}\right)_{\mu}\left(q_{1}-q_{2}\right)_{\nu}=q_{1 \mu} q_{1 \nu}-q_{1 \mu} q_{2 \nu}+q_{2 \mu} q_{1 \nu}-q_{2 \mu} q_{2 \nu} .
\end{aligned}
$$

Using Eq. C.16 and anti-symmetric property of $\epsilon_{\mu \alpha \nu \beta}$,

$$
\begin{aligned}
8 i \epsilon_{\mu \alpha \nu \beta} q_{1 \alpha} q_{2 \beta} & =4 i \epsilon_{\mu \alpha \nu \beta} q_{1 \alpha} q_{2 \beta}+4 i \epsilon_{\mu \alpha \nu \beta} q_{1 \alpha} q_{2 \beta} \\
& =4 i \epsilon_{\mu \alpha \nu \beta} q_{1 \alpha} q_{2 \beta}-4 i \epsilon_{\mu \alpha \nu \beta} q_{2 \alpha} q_{1 \beta} \\
& =-4 i \epsilon_{\mu \alpha \nu \beta}\left(q_{1 \alpha} q_{1 \beta}-q_{1 \alpha} q_{2 \beta}+q_{2 \alpha} q_{1 \beta}-q_{2 \alpha} q_{2 \beta}\right) \\
& =-4 i \epsilon_{\mu \alpha \nu \beta} n_{\alpha} q_{\beta} .
\end{aligned}
$$

Finally From Eq. C.14 and C.15, the leptonic tensor term becomes,

$$
\tau_{\mu \nu}=4\left[n_{\mu} n_{\nu}-q_{\mu} q_{\nu}+\left(q^{2}-m^{2}\right) g_{\mu \nu}+i \epsilon_{\mu \nu \alpha \beta} n_{\alpha} q_{\beta}\right]
$$




\section{C.1.3 Hadronic tensor term}

Hadronic tensor is, using Dirac spinors $n\left(p_{1}\right)$ and $p\left(p_{2}\right)$,

$$
\begin{aligned}
& w_{\mu \nu}=\operatorname{Tr}\left[\bar{p}\left\{\gamma_{\mu}\left(g_{V}-g_{A} \gamma_{5}\right)-P_{\mu}\left(f_{V}-f_{A} \gamma_{5}\right)+q_{\mu}\left(h_{V}-h_{A} \gamma_{5}\right)\right\} n\right] \times \\
& {\left[\bar{n}\left\{\gamma_{\nu}\left(g_{V}^{*}-g_{A}^{*} \gamma_{5}\right)-P_{\nu}\left(f_{V}^{*}+f_{A}^{*} \gamma_{5}\right)+q_{\nu}\left(h_{V}{ }^{*}+h_{A}{ }^{*} \gamma_{5}\right)\right\} p\right]} \\
& =\operatorname{Tr}\left[\left(\not_{2}+M\right)\left\{\gamma_{\mu}\left(g_{V}-g_{A} \gamma_{5}\right)-P_{\mu}\left(f_{V}-f_{A} \gamma_{5}\right)+q_{\mu}\left(h_{V}-h_{A} \gamma_{5}\right)\right\}\right] \times \\
& {\left[\left(p_{1}+M\right)\left\{\gamma_{\nu}\left(g_{V}{ }^{*}-g_{A}{ }^{*} \gamma_{5}\right)-P_{\nu}\left(f_{V}{ }^{*}+f_{A}^{*} \gamma_{5}\right)+q_{\nu}\left(h_{V}{ }^{*}+h_{A}{ }^{*} \gamma_{5}\right)\right\}\right]} \\
& =\operatorname{Tr}\left[p_{2}\left\{\gamma_{\nu}\left(g_{V}-g_{A} \gamma_{5}\right)-P_{\mu}\left(f_{V}-f_{A} \gamma_{5}\right)+q_{\mu}\left(h_{V}-h_{A} \gamma_{5}\right)\right\}\right] \times \\
& {\left[\not p_{1}\left\{\gamma_{\nu}\left(g_{V}{ }^{*}-g_{A}{ }^{*} \gamma_{5}\right)-P_{\nu}\left(f_{V}^{*}+f_{A}^{*} \gamma_{5}\right)+q_{\nu}\left(h_{V}{ }^{*}+h_{A}{ }^{*} \gamma_{5}\right)\right\}\right]} \\
& +\operatorname{MTr}\left[\left\{\gamma_{\mu}\left(g_{V}-g_{A} \gamma_{5}\right)-P_{\mu}\left(f_{V}-f_{A} \gamma_{5}\right)+q_{\mu}\left(h_{V}-h_{A} \gamma_{5}\right)\right\}\right] \times \\
& {\left[\not \not_{1}\left\{\gamma_{\nu}\left(g_{V}^{*}-g_{A}^{*} \gamma_{5}\right)-P_{\nu}\left(f_{V}^{*}+f_{A}^{*} \gamma_{5}\right)+q_{\nu}\left(h_{V}^{*}+h_{A}^{*} \gamma_{5}\right)\right\}\right]} \\
& +\operatorname{MTr}\left[\not p_{2}\left\{\gamma_{\mu}\left(g_{V}-g_{A} \gamma_{5}\right)-P_{\mu}\left(f_{V}-f_{A} \gamma_{5}\right)+q_{\mu}\left(h_{V}-h_{A} \gamma_{5}\right)\right\}\right] \times \\
& {\left[\left\{\gamma_{\nu}\left(g_{V}{ }^{*}-g_{A}{ }^{*} \gamma_{5}\right)-P_{\nu}\left(f_{V}{ }^{*}+f_{A}{ }^{*} \gamma_{5}\right)+q_{\nu}\left(h_{V}{ }^{*}+h_{A}{ }^{*} \gamma_{5}\right)\right\}\right]} \\
& +M^{2} \operatorname{Tr}\left[\gamma_{\mu}\left(g_{V}-g_{A} \gamma_{5}\right)-P_{\mu}\left(f_{V}-f_{A} \gamma_{5}\right)+q_{\mu}\left(h_{V}-h_{A} \gamma_{5}\right)\right] \times \\
& {\left[\gamma_{\nu}\left(g_{V}{ }^{*}-g_{A}{ }^{*} \gamma_{5}\right)-P_{\nu}\left(f_{V}{ }^{*}+f_{A}{ }^{*} \gamma_{5}\right)+q_{\nu}\left(h_{V}{ }^{*}+h_{A}{ }^{*} \gamma_{5}\right)\right] .}
\end{aligned}
$$




\section{1st term}

The 1st term in Eq. C.19 is,

$$
\begin{aligned}
& \operatorname{Tr}\left[p_{2}\left\{\gamma_{\mu}\left(g_{V}-g_{A} \gamma_{5}\right)-P_{\mu}\left(f_{V}-f_{A} \gamma_{5}\right)+q_{\mu}\left(h_{V}-h_{A} \gamma_{5}\right)\right\}\right] \times \\
& {\left[p_{1}\left\{\gamma_{\nu}\left(g_{V}^{*}-g_{A}^{*} \gamma_{5}\right)-P_{\nu}\left(f_{V}^{*}+f_{A}^{*} \gamma_{5}\right)+q_{\nu}\left(h_{V}^{*}+h_{A}^{*} \gamma_{5}\right)\right\}\right] } \\
= & \operatorname{Tr}\left[p_{2} \gamma_{\mu}\left(g_{V}-g_{A} \gamma_{5}\right) \not p_{1} \gamma_{\nu}\left(g_{V}^{*}-g_{A}^{*} \gamma_{5}\right)\right] \\
& +\operatorname{Tr}\left[\not p_{2} P_{\mu}\left(f_{V}-f_{A} \gamma_{5}\right) \not p_{1} P_{\nu}\left(f_{V}^{*}+f_{A}^{*} \gamma_{5}\right)\right]+\operatorname{Tr}\left[\not p_{2} q_{\mu}\left(h_{V}-h_{A} \gamma_{5}\right) \not p_{1} q_{\nu}\left(h_{V}^{*}+h_{A}^{*} \gamma_{5}\right)\right] \\
& -\operatorname{Tr}\left[\not p_{2} P_{\mu}\left(f_{V}-f_{A} \gamma_{5}\right) \not p_{1} q_{\nu}\left(h_{V}^{*}+h_{A}^{*} \gamma_{5}\right)\right]-\operatorname{Tr}\left[\not p_{2} q_{\mu}\left(h_{V}-h_{A} \gamma_{5}\right) \not p_{1} P_{\nu}\left(f_{V}^{*}+f_{A}^{*} \gamma_{5}\right)\right] \\
= & \left(g_{V}^{2}+g_{A}^{2}\right) \operatorname{Tr}\left[p_{2} \gamma_{\mu} \not p_{1} \gamma_{\nu}\right]-\left(g_{V} g_{A}^{*}+g_{A} g_{V}^{*}\right) \operatorname{Tr}\left[\gamma_{5} \not p_{2} \gamma_{\mu} \not p_{1} \gamma_{\nu}\right] \\
& +P_{\mu} P_{\nu}\left(f_{V}^{2}+f_{A}^{2}\right) \operatorname{Tr}\left[\not p_{2} \not p_{1}\right]+q_{\mu} q_{\nu}\left(h_{V}^{2}+h_{A}^{2}\right) \operatorname{Tr}\left[\not p_{2} \not p_{1}\right] \\
& -P_{\mu} q_{\nu}\left(f_{V} h_{V}^{*}+f_{A} h_{A}^{*}\right) \operatorname{Tr}\left[\not p_{2} \not p_{1}\right]-q_{\mu} P_{\nu}\left(h_{V} f_{V}^{*}+h_{A} f_{A}^{*}\right) \operatorname{Tr}\left[p_{2} \not p_{1}\right],
\end{aligned}
$$

then,

$$
\begin{aligned}
= & 4\left(g_{V}^{2}+g_{A}^{2}\right)\left[p_{1 \mu} p_{2 \nu}+p_{1 \nu} p_{2 \mu}-\left(p_{1} \cdot p_{2}\right) g_{\mu \nu}\right]-4 i\left(g_{V} g_{A}^{*}+g_{A} g_{V}{ }^{*}\right) \epsilon_{\mu \alpha \nu \beta} p_{2 \alpha} p_{1 \beta} \\
& +4\left(f_{V}^{2}+f_{A}^{2}\right)\left(p_{1} \cdot p_{2}\right) P_{\mu} P_{\nu}+4\left(h_{V}^{2}+h_{A}^{2}\right)\left(p_{1} \cdot p_{2}\right) q_{\mu} q_{\nu} \\
& -4\left(f_{V} h_{V}^{*}+f_{A} h_{A}^{*}\right)\left(p_{1} \cdot p_{2}\right) P_{\mu} q_{\nu}-4\left(h_{V} f_{V}^{*}+h_{A} f_{A}^{*}\right)\left(p_{1} \cdot p_{2}\right) q_{\mu} P_{\nu} \\
= & 2\left(g_{V}^{2}+g_{A}^{2}\right)\left(P_{\mu} P_{\nu}-q_{\mu} q_{\nu}\right)-2\left(g_{V}^{2}+g_{A}^{2}\right)\left(2 M^{2}-q^{2}\right) g_{\mu \nu}+2 i\left(g_{V} g_{A}^{*}+g_{A} g_{V}^{*}\right) \epsilon_{\mu \alpha \nu \beta} P_{\alpha} q_{\beta} \\
& +2\left(2 M^{2}-q^{2}\right)\left\{\left(f_{V}^{2}+f_{A}^{2}\right) P_{\mu} P_{\nu}+\left(h_{V}^{2}+h_{A}^{2}\right) q_{\mu} q_{\nu}\right. \\
& \left.-\left(f_{V} h_{V}^{*}+f_{A} h_{A}^{*}\right) P_{\mu} q_{\nu}-\left(h_{V} f_{V}^{*}+h_{A} f_{A}^{*}\right) q_{\mu} P_{\nu}\right\},
\end{aligned}
$$


and finally,

$$
\begin{aligned}
& =-2\left(g_{V}^{2}+g_{A}^{2}\right)\left(2 M^{2}-q^{2}\right) g_{\mu \nu}+2\left[\left(g_{V}^{2}+g_{A}^{2}\right)+\left(2 M^{2}-q^{2}\right)\left(f_{V}^{2}+f_{A}^{2}\right)\right] P_{\mu} P_{\nu} \\
& -4 i \operatorname{Re}\left(g_{A}{ }^{*} g_{V}\right) \epsilon_{\mu \nu \alpha \beta} q_{\alpha} P_{\beta} \\
& +2\left[-\left(g_{V}^{2}+g_{A}^{2}\right)+\left(2 M^{2}-q^{2}\right)\left(h_{V}^{2}+h_{A}^{2}\right)\right] q_{\mu} q_{\nu} \\
& -2\left(2 M^{2}-q^{2}\right)\left\{\left[\operatorname{Re}\left(f_{V} h_{V}{ }^{*}\right)+i \operatorname{Im}\left(f_{V} h_{V}{ }^{*}\right)+\operatorname{Re}\left(f_{A} h_{A}{ }^{*}\right)+i \operatorname{Im}\left(f_{A} h_{A}{ }^{*}\right)\right] P_{\mu} q_{\nu}\right. \\
& \left.+\left[\operatorname{Re}\left(f_{V} h_{V}^{*}\right)-i \operatorname{Im}\left(f_{V} h_{V}{ }^{*}\right)+\operatorname{Re}\left(f_{A} h_{A}{ }^{*}\right)-i \operatorname{Im}\left(f_{A} h_{A}{ }^{*}\right)\right] q_{\mu} P_{\nu}\right\} \\
& =-2\left(g_{V}^{2}+g_{A}^{2}\right)\left(2 M^{2}-q^{2}\right) g_{\mu \nu}+2\left[\left(g_{V}^{2}+g_{A}^{2}\right)+\left(2 M^{2}-q^{2}\right)\left(f_{V}^{2}+f_{A}^{2}\right)\right] P_{\mu} P_{\nu} \\
& -4 i \operatorname{Re}\left(g_{A}{ }^{*} g_{V}\right) \epsilon_{\mu \nu \alpha \beta} q_{\alpha} P_{\beta}+2\left[-\left(g_{V}^{2}+g_{A}{ }^{2}\right)+\left(2 M^{2}-q^{2}\right)\left(h_{V}^{2}+h_{A}^{2}\right)\right] q_{\mu} q_{\nu}-2\left(2 M^{2}-q^{2}\right) \\
& \times\left\{\left[\operatorname{Re}\left(f_{V} h_{V}{ }^{*}\right)+\operatorname{Re}\left(f_{A} h_{A}{ }^{*}\right)\right]\left(P_{\mu} q_{\nu}+q_{\mu} P_{\nu}\right)\right. \\
& \left.+i\left[\operatorname{Im}\left(f_{V} h_{V}{ }^{*}\right)+\operatorname{Im}\left(f_{A} h_{A}{ }^{*}\right)\right]\left(P_{\mu} q_{\nu}-q_{\mu} P_{\nu}\right)\right\} .
\end{aligned}
$$

\section{2nd and 3rd term}

The 2nd term in Eq. C.20 and 3rd term in Eq. C.21 have symmetric forms. The 2nd term is,

$$
\begin{aligned}
& \operatorname{MTr}\left[\left\{\gamma_{\mu}\left(g_{V}-g_{A} \gamma_{5}\right)-P_{\mu}\left(f_{V}-f_{A} \gamma_{5}\right)+q_{\mu}\left(h_{V}-h_{A} \gamma_{5}\right)\right\}\right] \times \\
& {\left[p_{1}\left\{\gamma_{\nu}\left(g_{V}^{*}-g_{A}^{*} \gamma_{5}\right)-P_{\nu}\left(f_{V}^{*}+f_{A}^{*} \gamma_{5}\right)+q_{\nu}\left(h_{V}^{*}+h_{A}^{*} \gamma_{5}\right)\right\}\right] } \\
= & \operatorname{MTr}\left[\gamma_{\mu}\left(g_{V}-g_{A} \gamma_{5}\right) p_{1}\left(-P_{\nu}\right)\left(f_{V}^{*}+f_{A}^{*} \gamma_{5}\right)\right]+\operatorname{MTr}\left[\gamma_{\mu}\left(g_{V}-g_{A} \gamma_{5}\right) p_{1} q_{\nu}\left(h_{V}^{*}+h_{A}^{*} \gamma_{5}\right)\right] \\
& -M \operatorname{Tr}\left[P_{\mu}\left(f_{V}-f_{A} \gamma_{5}\right) p_{1} \gamma_{\nu}\left(g_{V}^{*}-g_{A}^{*} \gamma_{5}\right)\right]+\operatorname{MTr}\left[q_{\mu}\left(h_{V}-h_{A} \gamma_{5}\right) p_{1} \gamma_{\nu}\left(g_{V}^{*}-g_{A}^{*} \gamma_{5}\right)\right] \\
= & -M P_{\nu}\left(g_{V} f_{V}^{*}+g_{A} f_{A}^{*}\right) \operatorname{Tr}\left[\gamma_{\mu} \not_{1}\right]+M q_{\nu}\left(g_{V} h_{V}^{*}+g_{A} h_{A}^{*}\right) \operatorname{Tr}\left[\gamma_{\mu} p_{1}\right] \\
& -M P_{\mu}\left(f_{V} g_{V}^{*}+f_{A} g_{A}^{*}\right) \operatorname{Tr}\left[\not p_{1} \gamma_{\nu}\right]+M q_{\mu}\left(h_{V} g_{V}^{*}+h_{A} g_{A}^{*}\right) \operatorname{Tr}\left[\not p_{1} \gamma_{\nu}\right] \\
= & -M P_{\nu}\left(g_{V} f_{V}^{*}+g_{A} f_{A}^{*}\right) \cdot 4 p_{1 \mu}+M q_{\nu}\left(g_{V} h_{V}^{*}+g_{A} h_{A}^{*}\right) \cdot 4 p_{1 \mu} \\
& -M P_{\mu}\left(f_{V} g_{V}^{*}+f_{A} g_{A}^{*}\right) \cdot 4 p_{1 \nu}+M q_{\mu}\left(h_{V} g_{V}^{*}+h_{A} g_{A}^{*}\right) \cdot 4 p_{1 \nu} .
\end{aligned}
$$


Next, the 3rd term Eq. C.21,

$$
\begin{aligned}
& \operatorname{MTr}\left[p_{2}\left\{\gamma_{\mu}\left(g_{V}-g_{A} \gamma_{5}\right)-P_{\mu}\left(f_{V}-f_{A} \gamma_{5}\right)+q_{\mu}\left(h_{V}-h_{A} \gamma_{5}\right)\right\}\right] \times \\
& {\left[\left\{\gamma_{\nu}\left(g_{V}^{*}-g_{A}^{*} \gamma_{5}\right)-P_{\nu}\left(f_{V}^{*}+f_{A}^{*} \gamma_{5}\right)+q_{\nu}\left(h_{V}^{*}+h_{A}^{*} \gamma_{5}\right)\right\}\right] } \\
= & M \operatorname{Mr}\left[p_{2} \gamma_{\mu}\left(g_{V}-g_{A} \gamma_{5}\right)\left(-P_{\nu}\right)\left(f_{V}^{*}+f_{A}^{*} \gamma_{5}\right)\right]+\operatorname{MTr}\left[p_{2} \gamma_{\mu}\left(g_{V}-g_{A} \gamma_{5}\right) q_{\nu}\left(h_{V}^{*}+h_{A}^{*} \gamma_{5}\right)\right] \\
& -M \operatorname{Mr}\left[\not p_{2} P_{\mu}\left(f_{V}-f_{A} \gamma_{5}\right) \gamma_{\nu}\left(g_{V}^{*}-g_{A}^{*} \gamma_{5}\right)\right]+\operatorname{MTr}\left[p_{2} q_{\mu}\left(h_{V}-h_{A} \gamma_{5}\right) \gamma_{\nu}\left(g_{V}^{*}-g_{A}^{*} \gamma_{5}\right)\right] \\
= & -M P_{\nu}\left(g_{V} f_{V}^{*}-g_{A} f_{A}^{*}\right) \cdot 4 p_{2 \mu}+M q_{\nu}\left(g_{V} h_{V}^{*}-g_{A} h_{A}^{*}\right) \cdot 4 p_{2 \mu} \\
& -M P_{\mu}\left(f_{V} g_{V}^{*}-f_{A} g_{A}^{*}\right) \cdot 4 p_{2 \nu}+M q_{\mu}\left(h_{V} g_{V}^{*}-h_{A} g_{A}^{*}\right) \cdot 4 p_{2 \nu} .
\end{aligned}
$$

If we combine both results Eq. C.24 and EqC.25,

$$
\begin{aligned}
& -4 M P_{\nu} P_{\mu}\left(g_{V} f_{V}^{*}\right)+4 M P_{\nu} q_{\mu}\left(g_{A} f_{A}^{*}\right)+4 M q_{\nu} P_{\mu}\left(g_{V} h_{V}^{*}\right)-4 M q_{\nu} q_{\mu}\left(g_{A} h_{A}^{*}\right) \\
& -4 M P_{\mu} P_{\nu}\left(f_{V} g_{V}^{*}\right)+4 M P_{\mu} q_{\nu}\left(f_{A} g_{A}^{*}\right)+4 M q_{\mu} P_{\nu}\left(h_{V} g_{V}^{*}\right)-4 M q_{\mu} q_{\nu}\left(h_{A} g_{A}^{*}\right) \\
= & -4 M P_{\mu} P_{\nu}\left(g_{V} f_{V}^{*}+f_{V} g_{V}^{*}\right)-4 M q_{\mu} q_{\nu}\left(g_{A} h_{A}^{*}+h_{A} g_{A}^{*}\right) \\
& +4 M\left\{P_{\nu} q_{\mu}\left[\operatorname{Re}\left(f_{A} g_{A}^{*}\right)-i \operatorname{Im}\left(f_{A} g_{A}^{*}\right)\right]+q_{\nu} P_{\mu}\left[\operatorname{Re}\left(g_{V} h_{V}^{*}\right)+i \operatorname{Im}\left(g_{V} h_{V}^{*}\right)\right]\right. \\
& \left.+P_{\mu} q_{\nu}\left[\operatorname{Re}\left(f_{A} g_{A}^{*}\right)+i \operatorname{Im}\left(f_{A} g_{A}^{*}\right)\right]+q_{\mu} P_{\nu}\left[\operatorname{Re}\left(g_{V} h_{V}^{*}\right)-i \operatorname{Im}\left(g_{V} h_{V}^{*}\right)\right]\right\} \\
= & -4 M P_{\mu} P_{\nu}\left(g_{V} f_{V}^{*}+f_{V} g_{V}^{*}\right)-4 M q_{\mu} q_{\nu}\left(g_{A} h_{A}^{*}+h_{A} g_{A}^{*}\right) \\
& +4 M\left(P_{\mu} q_{\nu}+P_{\nu} q_{\mu}\right)\left[\operatorname{Re}\left(f_{A} g_{A}^{*}\right)+\operatorname{Re}\left(g_{V} h_{V}^{*}\right)\right] \\
& +4 M\left(P_{\mu} q_{\nu}-P_{\nu} q_{\mu}\right)\left[i \operatorname{Im}\left(f_{A} g_{A}^{*}\right)+i \operatorname{Im}\left(g_{V} h_{V}^{*}\right)\right] .
\end{aligned}
$$




\section{4th term}

Finally, the 4th term in Eq. C.22,

$$
\begin{aligned}
& M^{2} \operatorname{Tr}\left[\gamma_{\mu}\left(g_{V}-g_{A} \gamma_{5}\right)-P_{\mu}\left(f_{V}-f_{A} \gamma_{5}\right)+q_{\mu}\left(h_{V}-h_{A} \gamma_{5}\right)\right] \times \\
& {\left[\gamma_{\nu}\left(g_{V}^{*}-g_{A}^{*} \gamma_{5}\right)-P_{\nu}\left(f_{V}^{*}+f_{A}^{*} \gamma_{5}\right)+q_{\nu}\left(h_{V}^{*}+h_{A}^{*} \gamma_{5}\right)\right] } \\
= & M^{2} \operatorname{Tr}\left[\gamma_{\mu}\left(g_{V}-g_{A} \gamma_{5}\right) \gamma_{\nu}\left(g_{V}^{*}-g_{A}^{*} \gamma_{5}\right)\right] \\
& -M^{2} P_{\mu} P_{\nu} \operatorname{Tr}\left[\left(f_{V}-f_{A} \gamma_{5}\right)\left(f_{V}^{*}+f_{A}^{*} \gamma_{5}\right)\right]+M^{2} q_{\mu} q_{\nu} \operatorname{Tr}\left[\left(h_{V}-h_{A} \gamma_{5}\right)\left(h_{V}^{*}+h_{A}^{*} \gamma_{5}\right)\right] \\
& -M^{2} P_{\mu} q_{\nu} \operatorname{Tr}\left[\left(f_{V}-f_{A} \gamma_{5}\right)\left(h_{V}^{*}+h_{A}^{*} \gamma_{5}\right)\right]-M^{2} q_{\mu} P_{\nu} \operatorname{Tr}\left[\left(h_{V}-h_{A} \gamma_{5}\right)\left(f_{V}^{*}+f_{A}^{*} \gamma_{5}\right)\right] \\
= & 4 M^{2}\left(g_{V}^{2}-g_{A}^{2}\right) \operatorname{Tr}\left[\gamma_{\mu} \gamma_{\nu}\right]+4 M^{2} P_{\mu} P_{\nu}\left(f_{V}^{2}-f_{A}^{2}\right)+4 M^{2} q_{\mu} q_{\nu}\left(h_{V}{ }^{2}-h_{A}^{2}\right) \\
& -4 M^{2} P_{\mu} q_{\nu}\left(f_{V} h_{V}^{*}-f_{A} h_{A}^{*}\right)-4 M^{2} q_{\mu} P_{\nu}\left(h_{V} f_{V}^{*}-h_{A} f_{A}^{*}\right) \\
= & 4 M^{2}\left\{\left(g_{V}^{2}-g_{A}^{2}\right) g_{\mu \nu}+\left(f_{V}^{2}-f_{A}^{2}\right) P_{\mu} P_{\nu}+\left(h_{V}^{2}-h_{A}^{2}\right) q_{\mu} q_{\nu}\right. \\
& -\left(P_{\mu} q_{\nu}+P_{\nu} q_{\mu}\right)\left[\operatorname{Re}\left(f_{V} h_{V}^{*}\right)-\operatorname{Re}\left(f_{A} h_{A}^{*}\right)\right] \\
& \left.-\left(P_{\mu} q_{\nu}-P_{\nu} q_{\mu}\right)\left[i \operatorname{Im}\left(f_{V} h_{V}^{*}\right)-i \operatorname{Im}\left(f_{A} h_{A}^{*}\right)\right]\right\} .
\end{aligned}
$$

Now, we check our result with Pais's paper [169]. If we add all terms Eq.C.23, C.26 and C.27. the hadronic tensor can be written,

$$
\begin{aligned}
w_{\mu \nu}= & w_{1} g_{\mu \nu}+w_{2} P_{\mu} P_{\nu}+w_{3} i \epsilon_{\mu \nu \alpha \beta} q_{\alpha} P_{\beta}+w_{4} q_{\mu} q_{\nu} \\
& +w_{5}\left(P_{\mu} q_{\nu}+P_{\nu} q_{\mu}\right)+w_{6}\left(P_{\mu} q_{\nu}-P_{\nu} q_{\mu}\right),
\end{aligned}
$$

with,

$$
\begin{gathered}
w_{1}=-2\left(g_{V}^{2}+g_{A}^{2}\right)\left(2 M^{2}-q^{2}\right)+4 M^{2}\left(g_{V}^{2}-g_{A}^{2}\right) \\
=-4 M^{2} g_{V}^{2}+2 g_{V}^{2} q^{2}-4 M^{2} g_{A}^{2}+2 g_{A}^{2} q^{2}+4 M^{2} g_{V}^{2}-4 M^{2} g_{A}^{2} \\
=-8 M^{2} g_{A}^{2}+2 q^{2}\left(g_{V}^{2}+g_{A}^{2}\right), \\
w_{2}=2\left[\left(g_{V}^{2}+g_{A}^{2}\right)+\left(2 M^{2}-q^{2}\right)\left(f_{V}^{2}+f_{A}^{2}\right)\right]-4 M\left(g_{V} f_{V}^{*}+f_{V} g_{V}^{*}\right)+4 M^{2}\left(f_{V}^{2}-f_{A}^{2}\right) \\
=2\left(g_{V}-2 M f_{V}\right)\left(g_{V}^{*}-2 M f_{V}^{*}\right)+2 g_{A}^{2}-2 q^{2}\left(f_{V}^{2}+f_{A}^{2}\right) \\
=2 g_{A}^{2}+2\left|g_{V}-2 M f_{V}\right|^{2}-2 q^{2}\left(f_{V}^{2}+f_{A}^{2}\right),
\end{gathered}
$$




$$
\begin{gathered}
w_{3}=-4 \operatorname{Re}\left(g_{A}{ }^{*} g_{V}\right), \\
w_{4}=2\left[-\left(g_{V}^{2}+g_{A}^{2}\right)+\left(2 M^{2}-q^{2}\right)\left(h_{V}^{2}+h_{A}^{2}\right)\right]-4 M\left(g_{A} h_{A}^{*}+h_{A} g_{A}^{*}\right)+4 M^{2}\left(h_{V}^{2}-h_{A}^{2}\right) \\
=-2 g_{V}^{2}-2\left(g_{A}+2 M h_{A}\right)\left(g_{A}^{*}+2 M h_{A}^{*}\right)-2 q^{2}\left(h_{V}^{2}+h_{A}^{2}\right)+8 M^{2} h_{V}^{2}+8 M^{2} h_{A}^{2} \\
=-2 g_{V}^{2}-2\left|g_{A}+2 M h_{A}\right|^{2}+2\left(-q^{2}+4 M^{2}\right)\left(h_{V}^{2}+h_{A}^{2}\right),
\end{gathered}
$$$$
w_{5}=-2\left(2 M^{2}-q^{2}\right)\left[\operatorname{Re}\left(f_{V} h_{V}^{*}\right)+\operatorname{Re}\left(f_{A} h_{A}^{*}\right)\right]
$$$$
+4 M\left[\operatorname{Re}\left(f_{A} g_{A}^{*}\right)+\operatorname{Re}\left(g_{V} h_{V}{ }^{*}\right)\right]-4 M^{2}\left[\operatorname{Re}\left(f_{V} h_{V}{ }^{*}\right)-\operatorname{Re}\left(f_{A} h_{A}{ }^{*}\right)\right]
$$$$
=2 \operatorname{Re}\left\{2 M \left[\left(f_{A} g_{A}{ }^{*}+g_{V} h_{V}{ }^{*}\right)-M\left(f_{V} h_{V}{ }^{*}+f_{A} h_{A}{ }^{*}\right)\right.\right.
$$$$
\left.\left.+\frac{q^{2}}{2 M}\left(f_{V} h_{V}^{*}+f_{A} h_{A}^{*}\right)-M\left(f_{V} h_{V}^{*}-f_{A} h_{A}^{*}\right)\right]\right\}
$$$$
=2 \operatorname{Re}\left\{2 M\left[\left(g_{V}-2 M f_{V}\left(1-\frac{q^{2}}{4 M^{2}}\right)\right) h_{V}^{*}+\left(g_{A}^{*}+\frac{q^{2}}{2 M} h_{A}^{*}\right) f_{A}\right]\right\},
$$

$$
\begin{aligned}
w_{6}= & -2\left(2 M^{2}-q^{2}\right)\left[i \operatorname{Im}\left(f_{V} h_{V}{ }^{*}\right)+i \operatorname{Im}\left(f_{A} h_{A}{ }^{*}\right)\right]+4 M\left[i \operatorname{Im}\left(f_{A} g_{A}{ }^{*}\right)+i \operatorname{Im}\left(g_{V} h_{V}{ }^{*}\right)\right] \\
& -4 M^{2}\left[i \operatorname{Im}\left(f_{V} h_{V}{ }^{*}\right)-i \operatorname{Im}\left(f_{A} h_{A}{ }^{*}\right)\right] \\
= & 2 i \operatorname{Im}\left\{2 M \left[\left(f_{A} g_{A}{ }^{*}+h_{V}{ }^{*} g_{V}\right)-M\left(f_{V} h_{V}{ }^{*}+f_{A} h_{A}{ }^{*}\right)\right.\right. \\
& \left.\left.+\frac{q^{2}}{2 M}\left(f_{V} h_{V}^{*}+f_{A} h_{A}^{*}\right)-M\left(f_{V} h_{V}^{*}-f_{A} h_{A}^{*}\right)\right]\right\} \\
= & 2 i \operatorname{Im}\left\{2 M\left[\left(g_{V}-2 M f_{V}\left(1-\frac{q^{2}}{4 M^{2}}\right)\right) h_{V}^{*}+\left(g_{A}{ }^{*}+\frac{q^{2}}{2 M} h_{A}^{*}\right) f_{A}\right]\right\} .
\end{aligned}
$$

These results agree with [169] except some definition differences and typos. Note that all interference with second class currents is contained in $w_{5}$ and $w_{6}$. Also note that $w_{6}$ is purely imaginary and it is responsible for T-violation for neutrino-nucleon elastic scattering. 


\section{C.1.4 Llewellyn Smith formalism}

Finally, we get the following equation from Eq. C.18 and Eq. C.28,

$$
\begin{aligned}
\frac{1}{4} w_{\mu \nu} \tau_{\mu \nu}= & {\left[w_{1} g_{\mu \nu}+w_{2} P_{\mu} P_{\nu}+w_{3} i \epsilon_{\mu \nu \alpha \beta} q_{\alpha} P_{\beta}+w_{4} q_{\mu} q_{\nu}+w_{5}\left(P_{\mu} q_{\nu}+P_{\nu} q_{\mu}\right)+w_{6}\left(P_{\mu} q_{\nu}-P_{\nu} q_{\mu}\right)\right] } \\
& \times\left[n_{\mu} n_{\nu}-q_{\mu} q_{\nu}+\left(q^{2}-m^{2}\right) g_{\mu \nu}+i \epsilon_{\mu \nu \alpha \beta} n_{\alpha} q_{\beta}\right] \\
= & w_{1} n^{2}-w_{1} q^{2}+4 w_{1}\left(q^{2}-m^{2}\right)+w_{2}(P \cdot n)^{2}-w_{2}(P \cdot q)^{2}+w_{2}\left(q^{2}-m^{2}\right) P^{2} \\
& +2 w_{3}\left(\delta_{\alpha \gamma} \delta_{\beta \delta}-\delta_{\alpha \delta} \delta_{\gamma \beta}\right) q_{\alpha} P_{\beta} n_{\gamma} q_{\delta}+w_{4}(q \cdot n)^{2}-w_{4} q^{4}+w_{4}\left(q^{2}-m^{2}\right) q^{2} \\
& 2 w_{5}(P \cdot n)(q \cdot n)-2 w_{5}(P \cdot q) q^{2}+2 w_{5}\left(q^{2}-m^{2}\right)(P \cdot q) .
\end{aligned}
$$

Notice that the $w_{6}$ term disappears by symmetry, which means differential cross section of elastic neutrino-nucleon scattering is T-invariance, and therefore no $\mathrm{CP}$ violating physics. Using Eq. C.6 to organize the formula in $(s-u)$,

$$
\begin{aligned}
\frac{1}{4} w_{\mu \nu} \tau_{\mu \nu}= & w_{1}\left(2 m^{2}-q^{2}-q^{2}+4 q^{2}-4 m^{2}\right)+w_{2}(P \cdot n)^{2}+w_{2}\left(q^{2}-m^{2}\right)\left(4 M^{2}-q^{2}\right)+2 w_{3}\left(-q^{2} P \cdot n\right) \\
& +w_{4}\left(m^{4}-q^{4}+q^{4}-m^{2} q^{2}\right)-2 m^{2} w_{5}(P \cdot n) \\
= & (P \cdot n)^{2} w_{2}+(P \cdot n)\left(-2 q^{2} w_{3}-2 m^{2} w_{5}\right)+2 w_{1}\left(q^{2}-m^{2}\right) \\
& +w_{2}\left(q^{2}-m^{2}\right)\left(4 M^{2}-q^{2}\right)-m^{2} w_{4}\left(q^{2}-m^{2}\right) \\
= & (s-u)^{2} w_{2}-(s-u)\left(2 q^{2} w_{3}+2 m^{2} w_{5}\right)+\left(q^{2}-m^{2}\right)\left[2 w_{1}+\left(4 M^{2}-q^{2}\right) w_{2}-m^{2} w_{4}\right] .(\text { C. } .36)
\end{aligned}
$$

Thus, the differential cross section Eq. C.12 becomes,

$$
\begin{aligned}
\frac{d \sigma}{d Q^{2}} & =\frac{G_{F}^{2} \cos \theta_{c}^{2}}{64 \pi E_{\nu}{ }^{2} M^{2}} \frac{1}{4} w_{\mu \nu} \tau^{\mu \nu} \\
& =\frac{M^{2} G_{F}^{2} \cos ^{2} \theta_{c}}{8 \pi E_{\nu}{ }^{2}}\left\{A\left(Q^{2}\right)+B\left(Q^{2}\right) \frac{(s-u)}{M^{2}}+C\left(Q^{2}\right) \frac{(s-u)^{2}}{M^{4}}\right\}
\end{aligned}
$$




\section{C.1. NEUTRINO-NUCLEON SCATTERING}

here, with a substitution of Pais to Llewellyn Smith form factors (Eq. C.10), $A\left(Q^{2}\right), B\left(Q^{2}\right)$ and $C\left(Q^{2}\right)$ are written,

$$
\begin{aligned}
A\left(Q^{2}\right)= & \frac{\left(q^{2}-m^{2}\right)}{8 M^{4}}\left\{2\left[-8 M^{2} g_{A}^{2}+2 q^{2}\left(g_{V}^{2}+g_{A}^{2}\right)\right]\right. \\
& +2\left(4 M^{2}-q^{2}\right)\left[g_{A}^{2}+\left|g_{V}-2 M f_{V}\right|^{2}-q^{2}\left(f_{V}^{2}+f_{A}^{2}\right)\right] \\
& \left.+2 m^{2}\left[g_{V}^{2}+\left|g_{A}+2 M h_{A}\right|^{2}-\left(-q^{2}+4 M^{2}\right)\left(h_{V}^{2}+h_{A}^{2}\right)\right]\right\} \\
= & \frac{\left(q^{2}-m^{2}\right)}{M^{2}}\left\{-2 g_{A}^{2}+\frac{q^{2}}{2 M^{2}}\left(g_{V}^{2}+g_{A}^{2}\right)\right. \\
& +\left(1-\frac{q^{2}}{4 M^{2}}\right)\left[g_{A}^{2}+g_{V}^{2}+4 M^{2} f_{V}^{2}-4 M \operatorname{Re}\left(g_{V}^{*} f_{V}\right)\right. \\
& \left.\left.-q^{2}\left(f_{V}^{2}+f_{A}^{2}\right)\right]+\frac{m^{2}}{4 M^{2}}\left[g_{V}^{2}+\left|g_{A}+2 M h_{A}\right|^{2}-\left(4 M^{2}-q^{2}\right)\left(h_{V}^{2}+h_{A}^{2}\right)\right]\right\},
\end{aligned}
$$

using Eq. C.10 to rewrite all form factors with Llewellyn Smith's notation,

$$
\begin{aligned}
& =\frac{\left(q^{2}-m^{2}\right)}{M^{2}}\left\{-2 F_{A}^{2}+\frac{q^{2}}{2 M^{2}}\left(F_{A}^{2}+\left(F_{1}+\xi F_{2}\right)^{2}\right)+\left(1-\frac{q^{2}}{4 M^{2}}\right) \times\right. \\
& {\left[F_{A}{ }^{2}+\left(F_{1}+\xi F_{2}\right)^{2}+\left(\xi F_{2}\right)^{2}-4 M R e\left(\left(F_{1}^{*}+\xi F_{2}{ }^{*}\right) \frac{\xi F_{2}}{2 M}\right)-q^{2}\left(\frac{1}{4 M^{2}}\left(\xi F_{2}^{2}\right)^{2}+\frac{1}{M^{2}}\left(F_{A}^{3}\right)^{2}\right)\right]} \\
& \left.+\frac{m^{2}}{4 M^{2}}\left[\left(F_{1}+\xi F_{2}\right)^{2}+\left|-F_{A}-2 F_{P}\right|^{2}-\left(4 M^{2}-q^{2}\right)\left(\frac{\left(F_{V}^{3}\right)^{2}}{M^{2}}+\frac{F_{P}^{2}}{M^{2}}\right)\right]\right\} \\
& =\frac{\left(q^{2}-m^{2}\right)}{M^{2}}\left\{-\left(1-\frac{q^{2}}{4 M^{2}}\right) F_{A}^{2}+\left(1+\frac{q^{2}}{4 M^{2}}\right)\left(F_{1}\right)^{2}+\left[\frac{q^{2}}{2 M^{2}}+\left(1-\frac{q^{2}}{4 M^{2}}\right)\left(2-2-\frac{q^{2}}{4 M^{2}}\right)\right]\left(\xi F_{2}\right)^{2}\right. \\
& +\left[2 \frac{q^{2}}{2 M^{2}}+\left(1-\frac{q^{2}}{4 M^{2}}\right)(2-2)\right] \operatorname{Re}\left(F_{1}^{*} \xi F_{2}\right)-\frac{q^{2}}{M^{2}}\left(1-\frac{q^{2}}{4 M^{2}}\right)\left(F_{A}{ }^{3}\right)^{2} \\
& \left.+\frac{m^{2}}{4 M^{2}}\left[\left|F_{1}+\xi F_{2}\right|^{2}+\left|F_{A}+2 F_{P}\right|^{2}-4\left(1-\frac{q^{2}}{4 M^{2}}\right)\left(\left(F_{V}^{3}\right)^{2}+F_{P}^{2}\right)\right]\right\}
\end{aligned}
$$

and finally,

$$
\begin{aligned}
= & \frac{\left(m^{2}-q^{2}\right)}{M^{2}}\left\{\left(1-\frac{q^{2}}{4 M^{2}}\right) F_{A}^{2}-\left(1+\frac{q^{2}}{4 M^{2}}\right)\left(F_{1}\right)^{2}\right. \\
& -\frac{q^{2}}{4 M^{2}}\left(1+\frac{q^{2}}{4 M^{2}}\right)\left(\xi F_{2}\right)^{2}-\frac{q^{2}}{M^{2}} \operatorname{Re}\left(F_{1}^{*} \xi F_{2}\right)+\frac{q^{2}}{M^{2}}\left(1-\frac{q^{2}}{4 M^{2}}\right)\left(F_{A}^{3}\right)^{2} \\
& \left.-\frac{m^{2}}{4 M^{2}}\left[\left|F_{1}+\xi F_{2}\right|^{2}+\left|F_{A}+2 F_{P}\right|^{2}-4\left(1-\frac{q^{2}}{4 M^{2}}\right)\left(\left(F_{V}^{3}\right)^{2}+F_{P}^{2}\right)\right]\right\} \\
= & \frac{\left(m^{2}+Q^{2}\right)}{M^{2}}\left\{\left(1+\frac{Q^{2}}{4 M^{2}}\right) F_{A}^{2}-\left(1-\frac{Q^{2}}{4 M^{2}}\right)\left(F_{1}\right)^{2}\right. \\
& +\frac{Q^{2}}{4 M^{2}}\left(1-\frac{Q^{2}}{4 M^{2}}\right)\left(\xi F_{2}\right)^{2}+\frac{Q^{2}}{M^{2}} \operatorname{Re}\left(F_{1}^{*} \xi F_{2}\right)-\frac{Q^{2}}{M^{2}}\left(1+\frac{Q^{2}}{4 M^{2}}\right)\left(F_{A}^{3}\right)^{2} \\
& \left.-\frac{m^{2}}{4 M^{2}}\left[\left|F_{1}+\xi F_{2}\right|^{2}+\left|F_{A}+2 F_{P}\right|^{2}-4\left(1+\frac{Q^{2}}{4 M^{2}}\right)\left(\left(F_{V}^{3}\right)^{2}+F_{P}^{2}\right)\right]\right\} .
\end{aligned}
$$


Similarly, $B\left(Q^{2}\right)$ term and $C\left(Q^{2}\right)$ term are,

$$
\begin{aligned}
& B\left(Q^{2}\right)=\frac{1}{8 M^{2}}\left\{-2 q^{2}\left(-4 R e\left(g_{A}{ }^{*} g_{V}\right)\right)-2 m^{2}\right. \\
& \left..4 \operatorname{Re} M\left[\left(g_{V}-2 M f_{V}\left(1-\frac{q^{2}}{4 M^{2}}\right)\right) h_{V}{ }^{*}+\left(g_{A}{ }^{*}+\frac{q^{2}}{2 M} h_{A}{ }^{*}\right) f_{A}\right]\right\} \\
& =\frac{q^{2}}{M^{2}} \operatorname{Re}\left[-F_{A}^{*}\left(F_{1}+\xi F_{2}\right)\right]-\frac{m^{2}}{M} \operatorname{Re}\left[\left(F_{1}+\frac{q^{2}}{4 M^{2}} \xi F_{2}\right) \frac{F_{V}^{3 *}}{M}+\left(-F_{A}^{*}-\frac{q^{2}}{2 M^{2}} F_{P}\right) \frac{F_{A}{ }^{3}}{M}\right] \\
& =-\left\{\frac{q^{2}}{M^{2}} \operatorname{Re}\left[F_{A}^{*}\left(F_{1}+\xi F_{2}\right)\right]+\frac{m^{2}}{M^{2}} \operatorname{Re}\left[\left(F_{1}+\frac{q^{2}}{4 M^{2}} \xi F_{2}\right) F_{V}^{3 *}\right.\right. \\
& \left.\left.-\left(F_{A}^{*}+\frac{q^{2}}{2 M^{2}} F_{P}\right) F_{A}^{3}\right]\right\} \\
& =\left\{\frac{Q^{2}}{M^{2}} \operatorname{Re}\left[F_{A}{ }^{*}\left(F_{1}+\xi F_{2}\right)\right]-\frac{m^{2}}{M^{2}} \operatorname{Re}\left[\left(F_{1}-\frac{Q^{2}}{4 M^{2}} \xi F_{2}\right) F_{V}^{3 *}\right.\right. \\
& \left.\left.-\left(F_{A}^{*}-\frac{Q^{2}}{2 M^{2}} F_{P}\right) F_{A}^{3}\right]\right\} \text {. } \\
& C\left(Q^{2}\right)=\frac{1}{8} \cdot 2\left[g_{A}^{2}+\left|g_{V}-2 M f_{V}\right|^{2}-q^{2}\left(f_{V}^{2}+f_{A}^{2}\right)\right] \\
& =\frac{1}{4}\left\{F_{A}^{2}+\left|F_{1}+\xi F_{2}-\xi F_{2}\right|^{2}-q^{2}\left[\left(\frac{1}{2 M} \xi F_{2}\right)^{2}+\left(\frac{1}{M} F_{A}^{3}\right)^{2}\right]\right\} \\
& =\frac{1}{4}\left\{F_{A}^{2}+F_{1}^{2}-\frac{q^{2}}{4 M^{2}}\left(\xi F_{2}\right)^{2}-\frac{q^{2}}{M^{2}}\left(F_{A}^{3}\right)^{2}\right\} \\
& =\frac{1}{4}\left\{F_{A}^{2}+F_{1}^{2}+\frac{Q^{2}}{4 M^{2}}\left(\xi F_{2}\right)^{2}+\frac{Q^{2}}{M^{2}}\left(F_{A}^{3}\right)^{2}\right\} \text {. }
\end{aligned}
$$

These results agree with [15] except the location of “*” in $\left(F_{1}-\frac{Q^{2}}{4 M^{2}} \xi F_{2}\right) F_{V}{ }^{3 *}$ at B-term. Note here that contribution from $F_{P}$ and $F_{V}^{3}$ are very small since they always couple with $\frac{m^{2}}{M^{2}} \ll 1$.

Now, the expression for Eq. C.2 is easily deduced from the crossing diagram with replacing $q_{2} \rightarrow-q_{1}$ and $q_{1} \rightarrow-q_{2}$ (Fig. C.2). This replacement corresponds to exchange $s$ and $u$.

$$
\begin{aligned}
\frac{d \sigma}{d Q^{2}}(s, t, u) & \rightarrow \frac{d \sigma}{d Q^{2}}(u, t, s) \\
B\left(Q^{2}\right) & \rightarrow-B\left(Q^{2}\right)
\end{aligned}
$$


Therefore,

$$
\begin{aligned}
& \frac{d \sigma}{d Q^{2}}\left(\begin{array}{c}
\nu_{l}+n \rightarrow l^{-}+p \\
\overline{\nu_{l}}+p \rightarrow l^{+}+n
\end{array}\right) \\
& =\frac{M^{2} G_{F}^{2} \cos ^{2} \theta_{c}}{8 \pi E_{\nu}{ }^{2}}\left\{A\left(Q^{2}\right) \pm B\left(Q^{2}\right) \frac{(s-u)}{M^{2}}+C\left(Q^{2}\right) \frac{(s-u)^{2}}{M^{4}}\right\}
\end{aligned}
$$


(a)

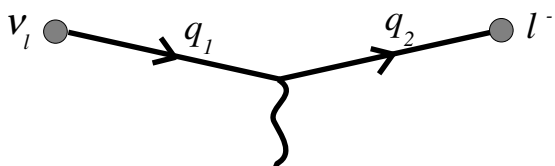

(b)

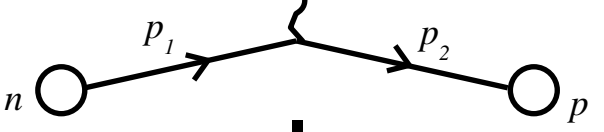

(c)

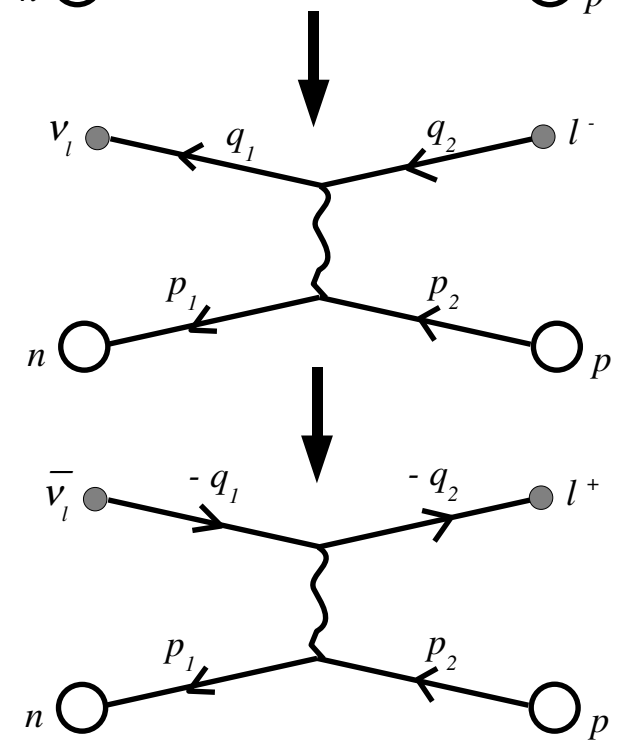

Figure C.2: (a) is the Feynman diagram of Eq. C.1, after the time reversal operation, one can get (b), then exchange of neutrino and lepton gives (3), which is the Feynman diagram of Eq. C.2. 


\section{C.2 Neutrino-Nuclei lepton scattering}

\section{C.2.1 Kinematics and cross section formula}

Now, we want to consider charge current quasi elastic scattering with bound nucleon.

$$
\begin{gathered}
\nu_{l}+A \rightarrow l^{-}+A^{\prime}, \\
\overline{\nu_{l}}+A \rightarrow l^{+}+A^{\prime} .
\end{gathered}
$$

In Fig C.3, initial and final lepton $\nu$ and $l$, initial and final nuclei $A$ and $A^{\prime}$, initial and final nucleon $N$ and $N^{\prime}$ have 4-momentum $k_{1}, k_{2}, p, p^{\prime}, k$, and $k^{\prime}$ respectively. The charged lepton $l$ has mass $m$, and nuclear target $A$ has mass $m_{T}$, also we define nucleons $N$ and $N^{\prime}$ to have mass $M$.

In modern notation, lab frame kinematics are defined in the following way.

$$
\begin{aligned}
k_{1} & =\left(\epsilon_{1}, 0,0, \epsilon_{1}\right) \\
k_{2} & =\left(\epsilon_{2}, \overrightarrow{k_{2}}\right) \\
p & =\left(m_{T}, 0,0,0\right) \\
k^{2} & =\epsilon^{2}-|\vec{k}|^{2} \equiv \epsilon^{2}-k^{2} \\
q^{2} & =w^{2}-|\vec{q}|^{2} \equiv w^{2}-q^{2}<0 \\
k \cdot q & =w \epsilon-\vec{k} \cdot \vec{q}=w \epsilon-k q \cos \tau
\end{aligned}
$$

Here, we use $k$ and $q$ as the abbreviations of $|\vec{k}|$ and $|\vec{q}|$, and $\tau$ is the angle defined between $\vec{k}$ and $\vec{q}$. These expressions are used for modern papers, for example [155]. 
neutrino-bound nucleon scattering

(1) Lab frame

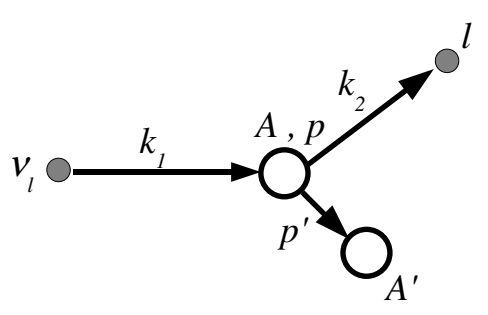

(2) Feynman diagram

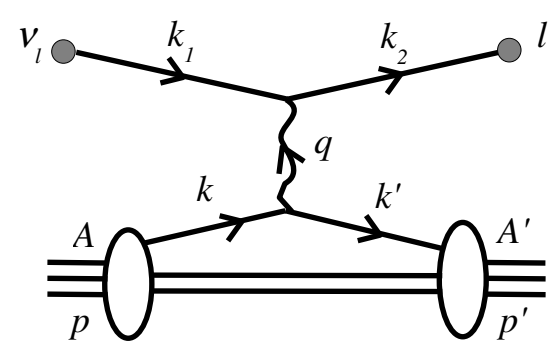

Figure C.3: The neutrino-nuclei scattering diagram. 
For old papers, including the original Smith-Moniz paper [16], use different notations.

$$
\begin{aligned}
k_{1} & =\left(\epsilon_{1}, 0,0, i \epsilon_{1}\right) \\
k_{2} & =\left(\overrightarrow{k_{2}}, i \epsilon_{2}\right) \\
p & =\left(0,0,0, i m_{T}\right) \\
k^{2} & =-\epsilon^{2}+|k|^{2} \equiv k^{2}-\epsilon^{2} \\
q^{2} & =-w^{2}+|q|^{2} \equiv q^{2}-w^{2}>0 \\
k \cdot q & =-w \epsilon+\vec{k} \cdot \vec{q}=-w \epsilon+k q \cos \tau
\end{aligned}
$$

One needs a special care for the original paper written by Smith and Moniz, because they define $q=k_{2}-k_{1}$, then,

$$
k \cdot q=-w \epsilon+\vec{k} \cdot \vec{q}=-w \epsilon-k q \cos \tau
$$

these are the expressions used in the original paper [16].

For neutrino-bound nucleon weak interaction, the cross section is,

$$
\begin{aligned}
d \sigma & =\frac{\overline{|\mathcal{M}|^{2}}}{\text { flux }} d \text { Lips }=\frac{\overline{|\mathcal{M}|^{2}}}{4\left|k_{1} \cdot p\right|} \frac{d^{3} k_{2}}{(2 \pi)^{3} \cdot 2 \epsilon_{2}} \frac{d^{3} p^{\prime}}{(2 \pi)^{3} \cdot 2 E_{p}}(2 \pi)^{4} \delta^{4}\left(\mathrm{p}^{\prime}-\mathrm{p}-\mathrm{q}\right) \\
& =\frac{\overline{|\mathcal{M}|^{2}}}{4\left|k_{1} \cdot p\right|} \frac{d^{3} k_{2}}{(2 \pi)^{2} \cdot 2 \epsilon_{2}} \underbrace{\frac{d^{3} p^{\prime}}{2 E_{p}} \delta^{4}\left(\mathrm{p}^{\prime}-\mathrm{p}-\mathrm{q}\right)}_{\text {put into hadronic tensor }} \\
& =\frac{1}{4 \epsilon_{1} \cdot m_{T}} \frac{k_{2}^{2} d k_{2} d \Omega}{(2 \pi)^{2} \cdot 2 \epsilon_{2}} \frac{\left(G_{F} \cos \theta_{c}\right)^{2}}{2} \frac{1}{2} \tau_{\mu \nu} W^{\mu \nu}
\end{aligned}
$$

therefore, double differential cross section for neutrino-bound nucleon scattering can be written,

$$
\frac{d \sigma^{2}}{d k_{2} d \Omega}=\frac{\left(G_{F} \cos \theta_{c}\right)^{2} k_{2}^{2}}{2 \pi^{2} m_{T}}\left(\frac{1}{16 \epsilon_{1} \epsilon_{2}}\right)\left(\frac{1}{4}\right) \tau_{\mu \nu} W^{\mu \nu} .
$$

This expression looks similar with Eq. (3) of the original paper [16], excepting many coefficient differences. We will see later, that factor $\frac{1}{16 \epsilon_{1} \epsilon_{2}}$ cancels with $\tau_{\mu \nu} W^{\mu \nu}$, and factor $\frac{1}{4}$ cancels with the nucleon tensor definition difference between Smith-Moniz and LlewellynSmith. 


\section{C.2.2 Leptonic and hadronic tensor term}

The leptonic tensor is of the usual form (Eq. C.13,

$$
\tau_{\mu \nu}=8\left[k_{1 \mu} k_{2 \nu}+k_{2 \mu} k_{1 \nu}-\left(q_{1} \cdot q_{2}\right) g_{\mu \nu}+q_{2 \mu}-i \epsilon_{\mu \nu \alpha \beta} k_{1 \alpha} k_{2 \beta}\right]
$$

The hadronic tensor is defined in the original paper (Eq. (2) of Ref. [16]),

$$
W_{\mu \nu}=W_{1} \delta_{\mu \nu}+\frac{W_{2}}{m_{T}^{2}} p_{\mu} p_{\nu}+\frac{W_{\alpha}}{m_{T}^{2}} q_{\mu} q_{\nu}+\frac{W_{\beta}}{m_{T}^{2}}\left(p_{\mu} q_{\nu}+p_{\nu} q_{\mu}\right)+\frac{W_{8}}{m_{T}^{2}} \epsilon_{\mu \nu \alpha \beta} p_{\alpha} q_{\beta}
$$

and we re-write this in modern notation,

$$
W_{\mu \nu}=-W_{1} g_{\mu \nu}+\frac{W_{2}}{m_{T}^{2}} p_{\mu} p_{\nu}+\frac{W_{\alpha}}{m_{T}^{2}} q_{\mu} q_{\nu}+\frac{W_{\beta}}{m_{T}^{2}}\left(p_{\mu} q_{\nu}+p_{\nu} q_{\mu}\right)+i \frac{W_{8}}{m_{T}^{2}} \epsilon_{\mu \nu \alpha \beta} p_{\alpha} q_{\beta} .
$$

This is more standard expression in modern articles, for example [45, 155]. We ignore $W_{6}$ term because it will disappear if one takes contraction with leptonic tensor as we see in Eq. C.35. Although Eq. C.48 and C.49 are quite similar, they show more difference with their lab frame expression.

In the Lab frame, the old notation is,

$$
W_{\mu \nu}=W_{1} \delta_{\mu \nu}-W_{2} \delta_{\mu 4} \delta_{\nu 4}+\frac{W_{\alpha}}{m_{T}^{2}} q_{\mu} q_{\nu}+i \frac{W_{\beta}}{m_{T}}\left(\delta_{\mu 4} q_{\nu}+\delta_{\nu 4} q_{\mu}\right)-i \frac{W_{8}}{m_{T}} \epsilon_{\mu \nu \alpha 4} q_{\alpha} \cdot(\mathrm{C} .50)
$$

On the other hand, modern notation,

$$
W_{\mu \nu}=-W_{1} g_{\mu \nu}+W_{2} g_{\mu 0} g_{\nu 0}+\frac{W_{\alpha}}{m_{T}^{2}} q_{\mu} q_{\nu}+\frac{W_{\beta}}{m_{T}}\left(g_{\mu 0} q_{\nu}+g_{\nu 0} q_{\mu}\right)-i \frac{W_{8}}{m_{T}} \epsilon_{\mu \nu \alpha 0} q_{\alpha}(\mathrm{C} .51)
$$

We adopt Eq. C.51 for later calculations. If we take the contraction with Leptonic tensor, using $\frac{k_{2}}{\epsilon_{2}} \cos \theta \equiv \cos \chi$, the coefficient for $W_{i}$ term becomes,

$$
\begin{gathered}
W_{1} \rightarrow-\left[2\left(k_{1} \cdot k_{2}\right)-4\left(k_{1} \cdot k_{2}\right)\right]=2\left(\begin{array}{c}
\epsilon_{1} \\
0 \\
0 \\
\epsilon_{1}
\end{array}\right) \cdot\left(\begin{array}{c}
\epsilon_{2} \\
\overrightarrow{k_{2}}
\end{array}\right)=2 \epsilon_{1} \epsilon_{2}\left(1-\frac{k_{2}}{\epsilon_{2}} \cos \theta\right) \\
=2 \epsilon_{1} \epsilon_{2}(1-\cos \chi)=4 \epsilon_{1} \epsilon_{2} \sin ^{2}\left(\frac{1}{2} \chi\right)
\end{gathered}
$$




$$
\begin{aligned}
& W_{2} \rightarrow 2 \epsilon_{1} \epsilon_{2}-\left(k_{1} \cdot k_{2}\right)=\epsilon_{1}\left(\epsilon_{2}+k_{2} \cos \theta\right)=\epsilon_{1} \epsilon_{2}(1+\cos \chi) \\
& =2 \epsilon_{1} \epsilon_{2} \cos ^{2}\left(\frac{1}{2} \chi\right) \text {, } \\
& W_{\alpha} \rightarrow \frac{1}{m_{T}^{2}}\left[2\left(k_{1} \cdot q\right)\left(k_{2} \cdot q\right)-\left(k_{1} \cdot k_{2}\right) q^{2}\right] \\
& =\frac{1}{m_{T}^{2}}\left[2\left(k_{2}^{2}-k_{1} \cdot k_{2}\right)\left(k_{1} \cdot k_{2}-k_{1}^{2}\right)-\left(k_{1} \cdot k_{2}\right)\left(k_{2}-k_{1}\right)^{2}\right] \\
& =\frac{1}{m_{T}^{2}}\left[2 k_{2}^{2}\left(k_{1} \cdot k_{2}\right)-2\left(k_{1} \cdot k_{2}\right)^{2}-\left(k_{1} \cdot k_{2}\right)\left(k_{2}^{2}-2 k_{1} \cdot k_{2}\right)\right] \\
& =\frac{1}{m_{T}^{2}} k_{2}^{2}\left(k_{1} \cdot k_{2}\right)=\frac{m^{2}}{m_{T}^{2}} \cdot 2 \epsilon_{1} \epsilon_{2} \sin ^{2}\left(\frac{1}{2} \chi\right), \\
& W_{\beta} \rightarrow \frac{1}{m_{T}}\left[2 \epsilon_{1}\left(k_{2} \cdot q\right)+2 \epsilon_{2}\left(k_{1} \cdot q\right)-2\left(k_{1} \cdot k_{2}\right)\left(\epsilon_{2}-\epsilon_{1}\right)\right] \\
& =\frac{1}{m_{T}}\left[2 \epsilon_{1}\left(k_{2}^{2}-k_{1} \cdot k_{2}\right)+2 \epsilon_{2}\left(k_{1} \cdot k_{2}-k_{1}^{2}\right)-2\left(k_{1} \cdot k_{2}\right)\left(\epsilon_{2}-\epsilon_{1}\right)\right] \\
& =\frac{1}{m_{T}} 2 \epsilon_{1} k_{2}^{2}=\frac{m^{2}}{m_{T}} 2 \epsilon_{1}, \\
& W_{8} \rightarrow-\frac{1}{m_{T}} \epsilon^{\mu \nu \sigma}{ }^{0} q_{\sigma} \epsilon_{\mu \nu \alpha \beta} k_{1}^{\alpha} k_{2}^{\beta}=-\frac{1}{m_{T}}\left|\begin{array}{cccc}
\delta_{\mu}^{\mu} & \delta_{\mu}^{\nu} & \delta_{\mu}^{\sigma} & \delta_{\mu}^{0} \\
\delta_{\nu}^{\mu} & \delta_{\nu}^{\nu} & \delta_{\nu}^{\sigma} & \delta_{\nu}^{0} \\
\delta_{\alpha}^{\mu} & \delta_{\alpha}^{\nu} & \delta_{\alpha}^{\sigma} & \delta_{\alpha}^{0} \\
\delta_{\beta}^{\mu} & \delta_{\beta}^{\nu} & \delta_{\beta}^{\sigma} & \delta_{\beta}^{0}
\end{array}\right| q_{\sigma} k_{1}^{\alpha} k_{2}^{\beta}=-\frac{2}{m_{T}}\left(\delta_{\alpha}^{\sigma} \delta_{\beta}^{0}-\delta_{\alpha}^{0} \delta_{\beta}^{\sigma}\right) q_{\sigma} k_{1}^{\alpha} k_{2}^{\beta} \\
& =-\frac{2}{m_{T}}\left[\epsilon_{2}\left(k_{1} \cdot q\right)-\epsilon_{1}\left(k_{2} \cdot q\right)\right]=-\frac{2}{m_{T}}\left[\epsilon_{2}\left(k_{1} \cdot k_{2}-k_{1}^{2}\right)-\epsilon_{1}\left(k_{2}^{2}-k_{1} \cdot k_{2}\right)\right] \\
& =-\frac{2}{m_{T}}\left[\left(k_{1} \cdot k_{2}\right)\left(\epsilon_{1}+\epsilon_{2}\right)-m^{2} \epsilon_{1}\right]=\frac{2}{m_{T}}\left[-2 \epsilon_{1} \epsilon_{2}\left(\epsilon_{1}+\epsilon_{2}\right) \sin ^{2}\left(\frac{1}{2} \chi\right)+m^{2} \epsilon_{1}\right] \text {. }
\end{aligned}
$$

In total,

$$
\begin{aligned}
\tau^{\mu \nu} W_{\mu \nu}= & 16 \epsilon_{1} \epsilon_{2}\left\{2 \sin ^{2}\left(\frac{1}{2} \chi\right) W_{1}+\cos ^{2}\left(\frac{1}{2} \chi\right) W_{2}+\frac{m^{2}}{m_{T}^{2}} \sin ^{2}\left(\frac{1}{2} \chi\right) W_{\alpha}+\frac{m^{2}}{m_{T} \epsilon_{2}}\left(W_{\beta}+W_{8}\right)\right. \\
& \left.-\frac{2}{m_{T}} W_{8}\left(\epsilon_{1}+\epsilon_{2}\right) \sin ^{2}\left(\frac{1}{2} \chi\right)\right\} .
\end{aligned}
$$


We use the following transformation for the second line of Eq. C.57.

$$
\begin{aligned}
\sin \left(\frac{1}{2} \chi\right)\left(\epsilon_{1}+\epsilon_{2}\right) & =\sqrt{\sin ^{2}\left(\frac{1}{2} \chi\right)\left(\epsilon_{1}+\epsilon_{2}\right)^{2}}=\sqrt{\frac{1}{2}\left(\epsilon_{1}^{2}+2 \epsilon_{1} \epsilon_{2}+\epsilon_{2}^{2}\right)\left(1-\frac{k_{2}}{\epsilon_{2}} \cos \theta\right)} \\
& =\sqrt{\frac{1}{2}\left[\epsilon_{1}^{2}\left(1-\frac{k_{2}}{\epsilon_{2}} \cos \theta\right)+\epsilon_{2}^{2}\left(1-\frac{k_{2}}{\epsilon_{2}} \cos \theta\right)+2 \epsilon_{1} \epsilon_{2}-2 \epsilon_{1} \epsilon_{2} \cos \theta\right]} \\
& =\sqrt{\epsilon_{1}^{2} \sin ^{2}\left(\frac{1}{2} \chi\right)+\epsilon_{2}^{2} \cos ^{2}\left(\frac{1}{2} \chi\right)+\frac{1}{2}\left(q^{2}+m^{2}\right)} \\
& =\sqrt{\left(-q^{2}+|q|^{2}+2 \epsilon_{1} \epsilon_{2}\right) \sin ^{2}\left(\frac{1}{2} \chi\right)+\frac{1}{2}\left(q^{2}+m^{2}\right)} \\
& =\sqrt{q^{2} \cos ^{2}\left(\frac{1}{2} \chi\right)+|q|^{2} \sin ^{2}\left(\frac{1}{2} \chi\right)+\epsilon_{1} \epsilon_{2}\left(1-\frac{k_{2}}{\epsilon_{2}} \cos \theta\right)+\frac{1}{2}\left(-q^{2}+m^{2}\right)} \\
& =\sqrt{q^{2} \cos ^{2}\left(\frac{1}{2} \chi\right)+|q|^{2} \sin ^{2}\left(\frac{1}{2} \chi\right)+\frac{1}{2}\left(q^{2}+m^{2}\right)+\frac{1}{2}\left(-q^{2}+m^{2}\right)}
\end{aligned}
$$

Putting Eq. C.58 together into Eq. C.57,

$$
\begin{aligned}
\tau^{\mu \nu} W_{\mu \nu}= & 16 \epsilon_{1} \epsilon_{2}\left\{2 W_{1} \sin ^{2}\left(\frac{1}{2} \chi\right)+W_{2} \cos ^{2}\left(\frac{1}{2} \chi\right)+\sin ^{2}\left(\frac{1}{2} \chi\right) \frac{m^{2}}{m_{T}^{2}} W_{\alpha}+\frac{m^{2}\left(W_{\beta}+W_{8}\right)}{m_{T} \epsilon_{2}}\right. \\
& \left.-\frac{2 W_{8}}{m_{T}} \sin \left(\frac{1}{2} \chi\right) \sqrt{q^{2} \cos ^{2}\left(\frac{1}{2} \chi\right)+|q|^{2} \sin ^{2}\left(\frac{1}{2} \chi\right)+m^{2}}\right\} .
\end{aligned}
$$

Finally combining Eq. C.47 and Eq. C.59, we can get Eq. (3) from Smith and Moniz paper [16] except for a factor of $\frac{1}{4}$. We will see this factor $\frac{1}{4}$ cancels with the definition of $W_{i}$. The lab frame expression for double differential cross section,

$$
\begin{aligned}
\frac{d \sigma^{2}}{d k_{2} d \Omega}= & \frac{G_{V}^{2} k_{2}^{2}}{2 \pi^{2} m_{T}}\left(\frac{1}{4}\right)\left\{2 W_{1} \sin ^{2}\left(\frac{1}{2} \chi\right)+W_{2} \cos ^{2}\left(\frac{1}{2} \chi\right)+\sin ^{2}\left(\frac{1}{2} \chi\right) \frac{m^{2}}{m_{T}^{2}} W_{\alpha}+\frac{m^{2}\left(W_{\beta}+W_{8}\right)}{m_{T} \epsilon_{2}}\right. \\
& \left.-\frac{2 W_{8}}{m_{T}} \sin \left(\frac{1}{2} \chi\right) \sqrt{q^{2} \cos ^{2}\left(\frac{1}{2} \chi\right)+|q|^{2} \sin ^{2}\left(\frac{1}{2} \chi\right)+m^{2}}\right\} .
\end{aligned}
$$




\section{C.2. NEUTRINO-NUCLEI LEPTON SCATTERING}

\section{C.2.3 Hadronic nuclei functions, definitions}

The Llewellyn-Smith formalism uses hadronic nucleon tensor to contract with leptonic tensor. Since the Smith and Moniz formalism describes the interaction of nuclei and lepton, it uses hadronic nuclei tensor to contract with leptonic tensor. Each nuclei tensor has hadronic nuclei function, and it is described by the combination of hadronic nucleon functions.

For the lab frame nuclei hadronic tensor, we have Eq. C.51. For nucleon tensor, again, we modified to modern notation,

$$
T_{\mu \nu}=-T_{1} g_{\mu \nu}+\frac{T_{2}}{M^{2}} p_{\mu} p_{\nu}+\frac{T_{\alpha}}{M^{2}} q_{\mu} q_{\nu}+\frac{T_{\beta}}{M^{2}}\left(p_{\mu} q_{\nu}+p_{\nu} q_{\mu}\right)+i \frac{T_{8}}{M^{2}} \epsilon_{\mu \nu \alpha \beta} p_{\alpha} q_{\beta}
$$

and they are related with the integral of nucleon phase space,

$$
W_{\mu \nu}=\int d \vec{k} f(\vec{k}, \vec{q}, \omega) T_{\mu \nu}
$$

Here $f(\vec{k}, \vec{q}, \omega)$ is a function describing how nucleons distribute in momentum space,

$$
f(\vec{k}, \vec{q}, \omega)=\frac{m_{T} \Omega}{(2 \pi)^{3}} \frac{\delta\left(\epsilon_{k}-\epsilon_{k-q}+\omega\right)}{\epsilon_{k} \epsilon_{k-q}} \cdot n_{i}(|\vec{k}|)\left(1-n_{f}(|\vec{k}-\vec{q}|)\right),
$$

- $\Omega=$ quantization volume, for RFG model, $\Omega=\frac{3 \pi^{2} N}{p_{F}^{3}}$, where $N$ is the number of neutrons in the nucleus,

- $\epsilon_{k}=$ nucleon energy before the scattering, using the initial binding energy $\epsilon_{1}\left(=E_{B}\right.$ for our case), $\epsilon_{k}=\sqrt{|\vec{k}|^{2}+m^{2}}-\epsilon_{1}=\epsilon-\epsilon_{1}$,

- $\epsilon_{k-q}=$ nucleon energy after the scattering, using the final binding energy $\epsilon_{2}(=0$ for our case) and the effective energy transfer $\omega_{\text {eff }}=\omega+\epsilon_{2}-\epsilon_{1}, \epsilon_{k-q}=\sqrt{|\vec{k}-\vec{q}|^{2}+m^{2}}-\epsilon_{2}=$ $\epsilon+\omega_{e f f}-\epsilon_{2}=\epsilon+\omega-\epsilon_{1}$,

- $\delta\left(\epsilon-\epsilon_{k-q}+\omega\right)=$ energy conservation,

- $n_{i}(|\vec{k}|)=$ a function describes the Fermi momentum distribution for the target nucleon in the nuclei. For RFG model, $n_{i}(|\vec{k}|)=\theta\left(p_{F}-|\vec{k}|\right)$, 
- $n_{f}(|\vec{k}-\vec{q}|)=$ a function describes the final nucleon states after scattering, When the states are already occupied, interactions do not happen (called Pauli blocking). For RFG model, $n_{f}(|\vec{k}-\vec{q}|)=\theta\left(p_{F}-|\vec{k}-\vec{q}|\right)$.

We will consider all integrals in the next subsection, now, we define the following 7 functions.

$$
\begin{aligned}
& a_{1}=\int d \vec{k} f(\vec{k}, \vec{q}, \omega) \\
& a_{2}=\int d \vec{k} f(\vec{k}, \vec{q}, \omega) \frac{k^{2}}{M^{2}} \\
& a_{3}=\int d \vec{k} f(\vec{k}, \vec{q}, \omega) \frac{k^{2} \cos ^{2} \tau}{M^{2}} \\
& a_{4}=\int d \vec{k} f(\vec{k}, \vec{q}, \omega) \frac{\epsilon_{k}^{2}}{M^{2}} \\
& a_{5}=\int d \vec{k} f(\vec{k}, \vec{q}, \omega) \frac{\epsilon_{k} k \cos \tau}{M^{2}} \\
& a_{6}=\int d \vec{k} f(\vec{k}, \vec{q}, \omega) \frac{k \cos \tau}{M} \\
& a_{7}=\int d \vec{k} f(\vec{k}, \vec{q}, \omega) \frac{\epsilon_{k}}{M}
\end{aligned}
$$

For the RFG model, these integrals, taken within the momentum space of target nucleon available for the interaction, can be re-written by the integral of target nucleon energy. Then, the highest energy nucleons available for the scattering are ones located on the Fermi surface, and the lowest energy nucleons join the scattering are ones who can escape from Fermi surface by adding energy transfer,

$$
\int d \vec{k} f(\vec{k}, \vec{q}, \omega) \sim \int_{k_{\min }}^{k_{\max }} k d k \sim \int_{E_{l o}}^{E_{h i}} \epsilon d \epsilon
$$

here, $E_{l o}$ and $E_{h i}$ are written,

$$
\begin{aligned}
E_{h i} & =\sqrt{p_{F}^{2}+M_{n}^{2}}, \\
E_{l o} & =\sqrt{p_{F}^{2}+M_{p}^{2}}-\omega+E_{B} .
\end{aligned}
$$

Then, we want to find the expressions for nuclei hadronic tensors in terms of nucleon hadronic tensors. Let's contract both hadronic tensors by 4 tensors, (1) $g_{\mu \nu}$, (2) $q_{\mu} q_{\nu},(3)$ 
$g_{\mu 4} g_{\nu 4}$, and (4) $g_{\mu 4} q_{\nu}$.

$$
\begin{aligned}
g_{\mu \nu} T^{\mu \nu} & =-4 T_{1}+\frac{k^{2}}{M^{2}} T_{2}+\frac{q^{2}}{M^{2}} T_{\alpha}+\frac{2 k \cdot q}{M^{2}} T_{\beta} \\
q_{\mu} q_{\nu} T^{\mu \nu} & =-q^{2} T_{1}+\frac{(k \cdot q)^{2}}{M^{2}} T_{2}+\frac{q^{4}}{M^{2}} T_{\alpha}+\frac{2 q^{2}(k \cdot q)}{M^{2}} T_{\beta} \\
g_{\mu 0} g_{\nu 0} T^{\mu \nu} & =-T_{1}+\frac{\epsilon_{k}^{2}}{M^{2}} T_{2}+\frac{\omega^{2}}{M^{2}} T_{\alpha}+\frac{2 \epsilon_{k} \omega}{M^{2}} T_{\beta} \\
g_{\mu 0} q_{\nu} T^{\mu \nu} & =-\omega T_{1}+\frac{\epsilon_{k}(k \cdot q)}{M^{2}} T_{2}+\frac{\omega q^{2}}{M^{2}} T_{\alpha}+\frac{\epsilon_{k} q^{2}+\omega(k \cdot q)}{M^{2}} T_{\beta} \\
g_{\mu \nu} W^{\mu \nu} & =-4 W_{1}+W_{2}+\frac{q^{2}}{m_{T}^{2}} W_{\alpha}+\frac{2 \omega}{m_{T}} W_{\beta} \\
q_{\mu} q_{\nu} W^{\mu \nu} & =-q^{2} W_{1}+\omega^{2} W_{2}+\frac{q^{4}}{m_{T}^{2}} W_{\alpha}+\frac{2 \omega q^{2}}{m_{T}} W_{\beta} \\
g_{\mu 0} g_{\nu 0} W^{\mu \nu} & =-W_{1}+W_{2}+\frac{\omega^{2}}{m_{T}^{2}} W_{\alpha}+\frac{2 \omega}{m_{T}} W_{\beta} \\
g_{\mu 0} q_{\nu} W^{\mu \nu} & =-\omega W_{1}+\omega W_{2}+\frac{\omega q^{2}}{m_{T}^{2}} W_{\alpha}+\frac{\left(q^{2}+\omega^{2}\right)}{m_{T}} W_{\beta}
\end{aligned}
$$

Eqs. C.71 to C.74 and Eqs. C.75 to C.78 are related through Eq. C.62, thus,

$$
\begin{gathered}
-4 W_{1}+W_{2}+\frac{q^{2}}{m_{T}^{2}} W_{\alpha}+\frac{2 \omega}{m_{T}} W_{\beta} \\
=\int f d \vec{k}\left[-4 T_{1}+\frac{k^{2}}{M^{2}} T_{2}+\frac{q^{2}}{M^{2}} T_{\alpha}+\frac{2 k \cdot q}{M^{2}} T_{\beta}\right] \\
-q^{2} W_{1}+\omega^{2} W_{2}+\frac{q^{4}}{m_{T}^{2}} W_{\alpha}-\frac{2 \omega q^{2}}{m_{T}} W_{\beta} \\
=\int f d \vec{k}\left[-q^{2} T_{1}+\frac{(k \cdot q)^{2}}{M^{2}} T_{2}+\frac{q^{4}}{M^{2}} T_{\alpha}+\frac{2 q^{2}(k \cdot q)}{M^{2}} T_{\beta}\right] \\
-W_{1}+W_{2}+\frac{\omega^{2}}{m_{T}^{2}} W_{\alpha}+\frac{2 \omega}{m_{T}} W_{\beta} \\
=\int f d \vec{k}\left[-T_{1}+\frac{\epsilon_{k}^{2}}{M^{2}} T_{2}+\frac{\omega^{2}}{M^{2}} T_{\alpha}+\frac{2 \epsilon_{k} \omega}{M^{2}} T_{\beta}\right] \\
-W_{1}+W_{2}+\frac{q^{2}}{m_{T}^{2}} W_{\alpha}+\left(\frac{q^{2}+\omega^{2}}{\omega m_{T}}\right) W_{\beta} \\
=\int f d \vec{k}\left[-T_{1}+\frac{\epsilon_{k}(k \cdot q)}{\omega M^{2}} T_{2}+\frac{q^{2}}{M^{2}} T_{\alpha}+\left(\frac{\epsilon_{k} q^{2}}{\omega M^{2}}+\frac{(k \cdot q)}{M^{2}}\right) T_{\beta}\right] .
\end{gathered}
$$




\section{APPENDIX C. THE DETAIL OF CROSS SECTION CALCULATIONS}

Then we calculate the following 4 combinations, (i) (Eq. C.79) - (Eq. C.80), (ii) (Eq. C.79) - (Eq. C.81), (iii) (Eq. C.79 - (Eq. C.82), and (iv) $\frac{q^{2}}{\omega^{2}} \times($ Eq. C.80 $+($ Eq. C.81).

$$
\begin{aligned}
& -3 W_{1}+\left(1-\frac{\omega^{2}}{q^{2}}\right) W_{2}=\int f d \vec{k}\left[-3 T_{1}+\left(\frac{k^{2}}{M^{2}}-\frac{(k \cdot q)^{2}}{q^{2} M^{2}}\right) T_{2}\right] \\
& -3 W_{1}+\frac{q^{2}-\omega^{2}}{m_{T}^{2}} W_{\alpha}=\int f d \vec{k}\left[-3 T_{1}+\frac{k^{2}-\epsilon_{k}^{2}}{M^{2}} T_{2}+\frac{q^{2}-\omega^{2}}{M^{2}} T_{\alpha}+2 \frac{(k \cdot q)-\epsilon_{k} \omega}{M^{2}} T_{\beta}\right] \\
& -3 W_{1}+\left(\frac{2 \omega}{m_{T}}-\frac{q^{2}+\omega^{2}}{\omega m_{T}}\right) W_{\beta} \\
& =\int f d \vec{k}\left[-3 T_{1}+\left(\frac{k^{2}}{M^{2}}-\frac{\epsilon_{k}(k \cdot q)}{\omega M^{2}}\right) T_{2}+\left(\frac{(k \cdot q)}{M^{2}}-\frac{\epsilon_{k} q^{2}}{\omega M^{2}}\right) T_{\beta}\right] \\
& \left(1-\frac{q^{2}}{\omega^{2}}\right) W_{1}+\frac{q^{4}-\omega^{4}}{m_{T}^{2} \omega^{2}} W_{\alpha}+2 \frac{q^{2}-\omega^{2}}{m_{T} \omega} W_{\beta} \\
& =\int f d \vec{k}\left[\left(1-\frac{q^{2}}{\omega^{2}}\right) T_{1}+\left(\frac{(k \cdot q)^{2}}{M^{2} \omega^{2}}-\frac{\epsilon_{k}^{2}}{M^{2}}\right) T_{2}+\frac{q^{4}-\omega^{4}}{M^{2} \omega^{2}} T_{\alpha}+2\left(\frac{q^{2}(k \cdot q)}{M^{2} \omega^{2}}-\frac{\epsilon_{k} \omega}{M^{2}}\right) T_{\beta}\right]
\end{aligned}
$$

Using Eq. C.44. (i), (ii), (iii), and (iv) can be re-written as the following.

$$
\begin{aligned}
& -3 W_{1}-\frac{|q|^{2}}{q^{2}} W_{2}=\int d \vec{k}\left[-3 T_{1}+\frac{1}{M^{2} q^{2}}\left[|k|^{2}|q|^{2}-\epsilon_{k}^{2}|q|^{2}-\omega^{2}|k|^{2}+2 \epsilon_{k} \omega(\vec{k} \cdot \vec{q})-(\vec{k} \cdot \vec{q})^{2}\right] T_{2}\right] \\
& 3 W_{1}+\frac{|q|^{2}}{m_{T}^{2}} W_{\alpha}=\int d \vec{k}\left[3 T_{1}+\frac{|k|^{2}}{M^{2}} T_{2}+\frac{|q|^{2}}{M^{2}} T_{\alpha}+2 \frac{(\vec{k} \cdot \vec{q})}{M^{2}} T_{\beta}\right] \\
& 3 W_{1}-\frac{|q|^{2}}{m_{T} \omega} W_{\beta}=\int d \vec{k}\left[3 T_{1}+\left(\frac{|k|^{2}}{M^{2}}-\frac{\epsilon_{k}(\vec{k} \cdot \vec{q})}{\omega M^{2}}\right) T_{2}+\left(\frac{(\vec{k} \cdot \vec{q})}{M^{2}}-\frac{\epsilon_{k}|q|^{2}}{\omega M^{2}}\right) T_{\beta}\right] \\
& \frac{|q|^{2}}{\omega^{2}} W_{1}+\frac{|q|^{2}}{m_{T}^{2}}\left(\frac{|q|^{2}}{\omega^{2}}-2\right) W_{\alpha}-2 \frac{|q|^{2}}{m_{T} \omega} W_{\beta} \\
& =\int d \vec{k}\left[\frac{|q|^{2}}{\omega^{2}} T_{1}+\frac{(\vec{k} \cdot \vec{q})}{M^{2}}\left(\frac{(\vec{k} \cdot \vec{q})}{\omega^{2}}-\frac{2 \epsilon_{k}}{\omega}\right) T_{2}+\frac{|q|^{2}}{M^{2}}\left(\frac{|q|^{2}}{\omega^{2}}-2\right) T_{\alpha}\right. \\
& \left.+\frac{2}{M^{2}}\left(-\frac{\epsilon_{k}|q|^{2}}{\omega}+\frac{|q|^{2}(\vec{k} \cdot \vec{q})}{\omega^{2}}-(\vec{k} \cdot \vec{q})\right) T_{\beta}\right]
\end{aligned}
$$


Then, calculate the combination, $2 \times(\mathrm{iii})+(\mathrm{iv})-\left(\frac{|q|^{2}}{\omega^{2}}-2\right) \times(\mathrm{ii})$,

$$
\begin{aligned}
& 2\left(-3 W_{1}+\frac{|q|^{2}}{m_{T} \omega} W_{\beta}\right)+\left(\frac{|q|^{2}}{\omega^{2}} W_{1}+\frac{|q|^{2}}{m_{T}^{2}}\left(\frac{|q|^{2}}{\omega^{2}}-2\right) W_{\alpha}-2 \frac{|q|^{2}}{m_{T} \omega} W_{\beta}\right) \\
& -\left(\frac{|q|^{2}}{\omega^{2}}-2\right)\left(3 W_{1}+\frac{|q|^{2}}{m_{T}^{2}} W_{\alpha}\right) \\
= & \int d \vec{k}\left[2\left(-3 T_{1}+\left(-\frac{|k|^{2}}{M^{2}}+\frac{\epsilon_{k}(\vec{k} \cdot \vec{q})}{\omega M^{2}}\right) T_{2}+\left(-\frac{(\vec{k} \cdot \vec{q})}{M^{2}}+\frac{\epsilon_{k}|q|^{2}}{\omega M^{2}}\right) T_{\beta}\right)\right. \\
& +\left(\frac{|q|^{2}}{\omega^{2}} T_{1}+\frac{(\vec{k} \cdot \vec{q})}{M^{2}}\left(\frac{(\vec{k} \cdot \vec{q})}{\omega^{2}}-\frac{2 \epsilon_{k}}{\omega}\right) T_{2}+\frac{|q|^{2}}{M^{2}}\left(\frac{|q|^{2}}{\omega^{2}}-2\right) T_{\alpha}\right. \\
& \left.\left.+\frac{2}{M^{2}}\left(-\frac{\epsilon_{k}|q|^{2}}{\omega}+\frac{|q|^{2}(\vec{k} \cdot \vec{q})}{\omega^{2}}-(\vec{k} \cdot \vec{q})\right) T_{\beta}\right)-\left(\frac{|q|^{2}}{\omega^{2}}-2\right) \cdot(\mathrm{ii})\right],
\end{aligned}
$$

then,

$$
\begin{aligned}
\rightarrow & -2 \frac{|q|^{2}}{\omega^{2}} W_{1}=\int d \vec{k}\left[\left(-6+\frac{|q|^{2}}{\omega^{2}}\right) T_{1}+\left(-\frac{2|k|^{2}}{M^{2}}+\frac{(\vec{k} \cdot \vec{q})}{M^{2} \omega^{2}}\right) T_{2}+\frac{|q|^{2}}{M^{2}}\left(\frac{|q|^{2}}{\omega^{2}}-2\right) T_{\alpha}\right. \\
& +\frac{2(\vec{k} \cdot \vec{q})}{M^{2}}\left(\frac{|q|^{2}}{\omega^{2}}-2\right) T_{\beta} \\
& \left.-\left(\frac{|q|^{2}}{\omega^{2}}-2\right) \cdot\left\{3 T_{1}+\frac{|k|^{2}}{M^{2}} T_{2}+\frac{|q|^{2}}{M^{2}} T_{\alpha}+2 \frac{(\vec{k} \cdot \vec{q})}{M^{2}} T_{\beta}\right\}\right] \\
\rightarrow & 2 \frac{|q|^{2}}{\omega^{2}} W_{1}=\int d \vec{k}\left[2 \frac{|q|^{2}}{\omega^{2}} T_{1}+\frac{1}{M^{2}}\left(\frac{|k|^{2}|q|^{2}}{\omega^{2}}-\frac{(\vec{k} \cdot \vec{q})}{\omega^{2}}\right) T_{2}\right] \\
\rightarrow & W_{1}=\int d \vec{k}\left[T_{1}+\frac{1}{2 M^{2}}\left(|k|^{2}-\frac{(-|k||q| \cos \tau)^{2}}{|q|^{2}}\right) T_{2}\right] \\
\rightarrow & W_{1}=T_{1}+\frac{1}{2}\left(a_{2}-a_{3}\right) T_{2} .
\end{aligned}
$$


$W_{2}$ can be found from (i) and Eq. C.83.

$$
\begin{aligned}
W_{2}= & -\frac{3 q^{2}}{2|q|^{2}}\left(a_{2}-a_{3}\right) T_{2} \\
& -\int d \vec{k}\left[\frac{1}{M^{2}|q|^{2}}\left(|k|^{2}|q|^{2}-\epsilon_{k}^{2}|q|^{2}-\omega^{2}|k|^{2}-2 \epsilon_{k} \omega|k||q| \cos \tau-|k|^{2}|q|^{2} \cos ^{2} \tau\right) T_{2}\right] \\
= & \frac{3}{2}\left(-\frac{\omega^{2}}{|q|^{2}}+1\right)\left(a_{2}-a_{3}\right) T_{2} \\
& -\int d \vec{k}\left[\frac{1}{M^{2}}\left(|k|^{2}-\epsilon_{k}^{2}-\frac{\omega^{2}|k|^{2}}{|q|^{2}}-2 \frac{\epsilon_{k} \omega|k|}{|q|} \cos \tau-|k|^{2} \cos ^{2} \tau\right) T_{2}\right] \\
= & {\left.\left[\frac{3}{2}\left(-\frac{\omega^{2}}{|q|^{2}}+1\right)\left(a_{2}-a_{3}\right)-a_{2}+a_{4}+\frac{\omega^{2}}{|q|^{2}} a_{2}+\frac{2 \omega}{|q|} a_{5}-a_{3}\right)\right] T_{2} } \\
= & {\left[a_{4}+\frac{2 \omega}{|q|} a_{5}+\frac{\omega^{2}}{|q|^{2}} a_{3}-\frac{q^{2}}{2|q|^{2}}\left(a_{2}-a_{3}\right)\right] T_{2} } \\
q^{2} \rightarrow-q^{2} & {\left[a_{4}+\frac{2 \omega}{|q|} a_{5}+\frac{\omega^{2}}{|q|^{2}} a_{3}+\frac{q^{2}}{2|q|^{2}}\left(a_{2}-a_{3}\right)\right] T_{2} . }
\end{aligned}
$$

The last step comes from the fact that $q^{2}$ is positive defined in old, including Smith and Moniz's, papers.

From (ii),

$$
\begin{aligned}
W_{\alpha} & =-\frac{3 m_{T}^{2}}{2|q|^{2}}\left(a_{2}-a_{3}\right) T_{2}+f r m_{T}^{2}|q|^{2} \int d \vec{k}\left[\frac{|k|^{2}}{M^{2}} T_{2}+\frac{|q|^{2}}{M^{2}} T_{\alpha}-2 \frac{|k||q| \cos \tau}{M^{2}} T_{\beta}\right] \\
& =\frac{m_{T}^{2}}{|q|^{2}}\left[\frac{3}{2}\left(a_{3}-a_{2}\right) T_{2}+a_{2} T_{2}+\frac{|q|^{2} a_{1}}{M^{2}} T_{\alpha}-2 \frac{a_{6}|q|}{M} T_{\beta}\right] \\
& =\frac{m_{T}^{2}}{|q|^{2}}\left(\frac{3}{2} a_{3}-\frac{1}{2} a_{2}\right) T_{2}+\frac{m_{T}^{2} a_{1}}{M^{2}} T_{\alpha}-2 \frac{a_{6} m_{T}^{2}}{M|q|} T_{\beta} .
\end{aligned}
$$

Notice sign in front of $a_{6}$ term is minus. This sign is plus in original Smith and Moniz's paper [27]. 
Finally from (iii),

$$
\begin{aligned}
W_{\beta}= & -\frac{3 m_{T} \omega}{2|q|^{2}}\left(a_{3}-a_{2}\right) T_{2} \\
& +\frac{m_{T} \omega}{|q|^{2}} \int d \vec{k}\left[\left(-\frac{|k|^{2}}{M^{2}}-\frac{\epsilon_{k}|k||q| \cos \tau}{\omega M^{2}}\right) T_{2}+\left(\frac{|k||q| \cos \tau}{M^{2}}+\frac{\epsilon_{k}|q|^{2}}{\omega M^{2}}\right) T_{\beta}\right] \\
= & -\frac{3 m_{T} \omega}{2|q|^{2}}\left(a_{3}-a_{2}\right) T_{2} \\
& +\int d \vec{k}\left[\left(-\frac{m_{T} \omega|k|^{2}}{|q|^{2} M^{2}}-\frac{m_{T} \epsilon_{k}|k| \cos \tau}{|q| M^{2}}\right) T_{2}+\left(\frac{m_{T} \omega|k| \cos \tau}{|q| M^{2}}+\frac{m_{T} \epsilon_{k}}{M^{2}}\right) T_{\beta}\right] \\
= & -\frac{3 m_{T} \omega}{2|q|^{2}}\left(a_{3}-a_{2}\right) T_{2}+\left(-\frac{m_{T} \omega}{|q|^{2}} a_{2}-\frac{m_{T} a_{5}}{|q|}\right) T_{2}+\frac{m_{T}}{M}\left(a_{7}+\frac{\omega}{|q|} a_{6}\right) T_{\beta} \\
= & \frac{m_{T}}{M}\left(a_{7}+\frac{\omega}{|q|} a_{6}\right) T_{\beta}-\frac{m_{T}}{|q|}\left[a_{5}+\frac{\omega}{|q|}\left(\frac{3}{2} a_{3}-\frac{1}{2} a_{2}\right)\right] T_{2} .
\end{aligned}
$$

Notice, the sign in front of $T_{2}$ is minus. This sign is plus in original Smith and Moniz's paper [27].

The last term, $W_{8}$, is antisymmetric and disappears for all contractions with the symmetric tensor. Now we go back to Eq. C.62, and take the contraction with the antisymmetric tensor $\epsilon_{\mu \nu \alpha \beta}$, then take contraction with $g^{\alpha 4} q^{\beta}$,

$$
\begin{array}{ll} 
& -\frac{1}{m_{T}} \epsilon^{\mu \nu \alpha \beta} \epsilon_{\mu \nu \sigma 0} q^{\sigma} W_{8}=\frac{1}{M^{2}} \int d \vec{k}\left[\epsilon^{\mu \nu \alpha \beta} \epsilon_{\mu \nu \sigma \tau} k^{\sigma} q^{\tau} T_{8}\right] \\
\rightarrow \quad & -\frac{1}{m_{T}}\left(g_{\sigma \alpha} g_{4 \beta}-g_{\sigma \beta} g_{4 \alpha}\right) q^{\sigma} W_{8}=\frac{1}{M^{2}} \int d \vec{k}\left[\left(\delta_{\sigma}^{\alpha} \delta_{\tau}^{\beta}-\delta_{\sigma}^{\beta} \delta_{\tau}^{\alpha}\right) k^{\sigma} q^{\tau} T_{8}\right] \\
\rightarrow & -\frac{i}{m_{T}}\left(\omega^{2}-q^{2}\right) W_{8}=\frac{1}{M^{2}} \int d \vec{k}\left[\left(\epsilon_{k} q^{2}-(k \cdot q) \omega\right) T_{8}\right] \\
\rightarrow & W_{8}=\frac{m_{T}}{|q|^{2} M^{2}} \int d \vec{k}\left[\left(\epsilon_{k}|q|^{2}+|k \| q| \cos \tau\right) T_{8}\right] \\
\rightarrow & W_{8}=\frac{m_{T}}{M}\left(a_{7}+\frac{\omega}{|q|} a_{6}\right) .
\end{array}
$$

Therefore, Eq. C.83, C.84, C.85, C.86, and C.87 are the expressions of hadronic nuclei functions, $W_{1}, W_{2}, W_{\alpha}, W_{\beta}$, and $W_{8}$ in terms of hadronic nucleon functions, $T_{1}, T_{2}, T_{\alpha}, T_{\beta}$, and $T_{8}$. 


\section{C.2.4 Analytic solutions for the phase space integrals}

For RFG model, Eqs. C.64 to C.70 have analytic solutions. To calculate them, we first define the following.

$$
\begin{aligned}
q_{e f f}^{2} & =|\vec{q}|^{2}-\omega_{e f f}^{2} \\
a & =\epsilon_{1}\left(1+\frac{e p_{2}}{\omega}\right) \\
b & =\epsilon_{2}\left(1-\frac{e p_{1}}{\omega}\right) \\
c & =-\frac{\omega_{e f f}}{|\vec{q}|} \\
d & =\frac{q_{e f f}^{2}}{2|\vec{q}| M} \\
b_{j} & =\int d \vec{k} f(\vec{k}, \vec{q}, \omega)\left(\frac{\epsilon}{M}\right)^{j} \\
& =\int d \vec{k} \frac{m_{T} \Omega}{(2 \pi)^{3}} \frac{\delta\left(\epsilon_{k}-\epsilon_{k-q}+\omega\right)}{\epsilon \epsilon_{k-q}} \cdot n_{i}(|\vec{k}|)\left[1-n_{f}(|\vec{k}-\vec{q}|)\right] \cdot\left(\frac{\epsilon}{M}\right)^{j} \\
& =\int_{k_{\min }}^{k_{\max }} k^{2} d k d \cos \tau \frac{m_{T} \Omega}{(2 \pi)^{2}} \frac{\delta\left(\epsilon_{k}-\epsilon_{k-q}+\omega\right)}{\epsilon \epsilon_{k-q}} \operatorname{cdot}\left(\frac{\epsilon}{M}\right)^{j}
\end{aligned}
$$

Since $\epsilon_{k-q}=\sqrt{|\vec{k}-\vec{q}|+M^{2}}-\epsilon_{2}=\sqrt{|\vec{k}|^{2}+|\vec{q}|^{2}-2|\vec{k}||\vec{q}| \cos \tau+M^{2}}-\epsilon_{2}$, we can re-write energy conservation with function of $\cos \tau$, by using a standard formula, $\delta(f(x))=\sum_{i} \frac{\delta\left(x-x_{i}\right)}{f^{\prime}\left(x_{i}\right)}$. For our case,

$$
\begin{aligned}
f(\cos \tau) & =\epsilon-\sqrt{|\vec{k}|^{2}+|\vec{q}|^{2}-2|\vec{k}||\vec{q}| \cos \tau+M^{2}}+\omega_{e f f}, \\
f\left(\cos \tau_{0}\right) & =\epsilon-\sqrt{|\vec{k}|^{2}+|\vec{q}|^{2}-2|\vec{k}||\vec{q}| \cos \tau_{0}+M^{2}}+\omega_{e f f}=0 \\
& \rightarrow \cos \tau_{0}=\frac{|\vec{q}|^{2}-\omega_{\text {eff }}^{2}-2 \epsilon \omega_{e f f}}{2|\vec{k}||\vec{q}|}, \\
f^{\prime}(\cos \tau) & =\frac{|\vec{k}||\vec{q}|}{\sqrt{\epsilon^{2}+|\vec{q}|^{2}-2|\vec{k}||\vec{q}| \cos \tau}}, \\
f^{\prime}\left(\cos \tau_{0}\right) & =\frac{|\vec{k}||\vec{q}|}{\left(\epsilon+\omega_{e f f}\right)} .
\end{aligned}
$$


then,

$$
\begin{aligned}
\delta\left(\epsilon_{k}-\epsilon_{k-q}+\omega\right) & =\delta\left(\epsilon-\epsilon_{1}-\sqrt{|\vec{k}-\vec{q}|+M^{2}}+\epsilon_{2}+\omega\right) \\
& =\delta\left(\epsilon-\sqrt{|\vec{k}|^{2}+|\vec{q}|^{2}-2|\vec{k}||\vec{q}| \cos \tau+M^{2}}+\omega_{e f f}\right) \\
& =\frac{\epsilon+\omega_{e f f}}{|\vec{k}||\vec{q}|} \delta\left[\cos \tau+\frac{2 \epsilon \omega_{e f f}+\omega_{\text {eff }}^{2}-|\vec{q}|^{2}}{2|\vec{k}||\vec{q}|}\right] .
\end{aligned}
$$

And $b_{0}$ becomes,

$$
\begin{aligned}
b_{0} & =\int_{k_{\min }}^{k_{\max }} k^{2} d k d \cos \tau \frac{m_{T} \Omega}{(2 \pi)^{2}} \frac{1}{\epsilon_{k} \epsilon_{k-q}} \cdot \frac{\epsilon+\omega_{\text {eff }}}{|\vec{k}||\vec{q}|} \delta\left[\cos \tau+\frac{2 \epsilon \omega_{\text {eff }}+\omega_{\text {eff }}^{2}-|\vec{q}|^{2}}{2|\vec{k}||\vec{q}|}\right] \\
& =\frac{m_{T} \Omega}{(2 \pi)^{2}|\vec{q}|} \int_{k_{\min }}^{k_{\max }} k d k \frac{\epsilon+\omega_{\text {eff }}}{\epsilon_{k} \epsilon_{k-q}} \\
& =\frac{m_{T} \Omega}{(2 \pi)^{2}|\vec{q}|} \int_{E_{l o}}^{E_{h i}} \epsilon d \epsilon \frac{\epsilon+\omega_{\text {eff }}}{\left(\epsilon-\epsilon_{1}\right)\left(\epsilon-\epsilon_{1}+\omega\right)} .
\end{aligned}
$$

Let's focus on the inside of this integral,

$$
\begin{aligned}
\frac{\epsilon\left(\epsilon+\omega_{e f f}\right)}{e p_{k} \epsilon_{k-q}} & =\frac{\epsilon\left(\epsilon+\omega-\epsilon_{1}+\epsilon_{2}\right)}{\left(\epsilon-\epsilon_{1}\right)\left(\epsilon-\epsilon_{1}+\omega\right)} \\
& =\frac{\left(\epsilon-\epsilon_{1}\right)\left(\epsilon-\epsilon_{1}+\omega\right)+\epsilon \epsilon_{1}-\epsilon_{1}^{2}+\epsilon_{1} \omega+\epsilon \epsilon_{2}}{\left(\epsilon-\epsilon_{1}\right)\left(\epsilon-\epsilon_{1}+\omega\right)} \\
& =1+\frac{\omega \epsilon \epsilon_{1}-\omega \epsilon_{1}^{2}+\epsilon_{1} \omega^{2}+\omega \epsilon \epsilon_{2}+\omega \epsilon_{1} \epsilon_{2}(1-1)+\epsilon \epsilon_{1} \epsilon_{2}(1-1)+\epsilon_{1}^{2} \epsilon_{2}(1-1)}{\omega\left(\epsilon-\epsilon_{1}\right)\left(\epsilon-\epsilon_{1}+\omega\right)} \\
& =1+\frac{\epsilon_{1}\left(\omega \epsilon-\omega \epsilon_{1}+\omega^{2}+\epsilon_{2} \epsilon-\epsilon_{1} \epsilon_{2}+\omega \epsilon_{2}\right)+\epsilon_{2}\left(\omega \epsilon-\omega \epsilon_{1} \epsilon \epsilon_{1}+\epsilon_{1}^{2}\right)}{\left(\epsilon-\epsilon_{1}\right)\left(\epsilon-\epsilon_{1}+\omega\right)} \\
& =1+\frac{\epsilon_{1}\left(\omega+\epsilon_{2}\right)\left(\epsilon-\epsilon_{1}+\omega\right)+\epsilon_{2}\left(\omega-\epsilon_{1}\right)\left(\epsilon-\epsilon_{1}\right)}{\left(\epsilon-\epsilon_{1}\right)\left(\epsilon-\epsilon_{1}+\omega\right)} \\
& =1+\frac{a}{\epsilon-\epsilon_{1}}+\frac{b}{\epsilon-\epsilon_{1}+\omega} .
\end{aligned}
$$

Therefore,

$$
b_{0}=\left.\frac{m_{T} \Omega}{(2 \pi)^{2}|\vec{q}|}\left[\epsilon+a \ln \left(\epsilon-\epsilon_{1}\right)+b \ln \left(\epsilon-\epsilon_{1}+\omega\right)\right]\right|_{E_{l o}} ^{E_{h i}} .
$$


Similarly, we can get expressions for $b_{1}$ and $b_{2}$.

$$
\begin{aligned}
& \frac{\epsilon^{2}\left(\epsilon+\omega_{e f f}\right)}{e p_{k} \epsilon_{k-q}}=\epsilon\left(1+\frac{a}{\epsilon-\epsilon_{1}}+\frac{b}{\epsilon-\epsilon_{1}+\omega}\right) \\
& =\epsilon+a\left(1+\frac{\epsilon_{1}}{\epsilon-\epsilon_{1}}\right)+b\left(1+\frac{\epsilon_{1}-\omega}{\epsilon-\epsilon_{1}+\omega}\right) \\
& \rightarrow b_{1}=\frac{m_{T} \Omega}{(2 \pi)^{2}|\vec{q}| M}\left[\frac{\epsilon^{2}}{2}+a\left\{\epsilon+\epsilon_{1} \ln \left(\epsilon-\epsilon_{1}\right)\right\}\right. \\
& \left.+b\left\{\epsilon+\left(\epsilon_{1}-\omega\right) \ln \left(\epsilon-\epsilon_{1}+\omega\right)\right\}\right]||_{E_{l o}}^{E_{h i}} \\
& \frac{\epsilon^{3}\left(\epsilon+\omega_{e f f}\right)}{e p_{k} \epsilon_{k-q}}=\epsilon^{2}\left(1+\frac{a}{\epsilon-\epsilon_{1}}+\frac{b}{\epsilon-\epsilon_{1}+\omega}\right) \\
& =\epsilon^{2}+a \frac{\epsilon^{2}-\epsilon_{1}^{2}+\epsilon_{1}^{2}}{\epsilon-\epsilon_{1}}+b \frac{\epsilon^{2}-\left(\epsilon_{1}-\omega\right)^{2}+\left(\epsilon_{1}-\omega\right)^{2}}{\epsilon-\epsilon_{1}+\omega} \\
& =\epsilon^{2}+a\left(\epsilon+\epsilon_{1}+\frac{\epsilon_{1}^{2}}{\epsilon-\epsilon_{1}}\right)+b\left(\epsilon+\left(\epsilon_{1}-\omega\right)+\frac{\left(\epsilon_{1}-\omega\right)^{2}}{\epsilon-\epsilon_{1}+\omega}\right) \\
& \rightarrow b_{2}=\frac{m_{T} \Omega}{(2 \pi)^{2}|\vec{q}| M}\left[\frac{\epsilon^{3}}{3}+a\left\{\frac{\epsilon^{2}}{2}+\epsilon \epsilon_{1}+\epsilon_{1}^{2} \ln \left(\epsilon-\epsilon_{1}\right)\right\}\right. \\
& \left.+b\left\{\frac{\epsilon^{2}}{2}+\epsilon\left(\epsilon_{1}-\omega\right)+\left(\epsilon_{1}-\omega\right)^{2} \ln \left(\epsilon-\epsilon_{1}+\omega\right)\right\}\right]||_{E_{l o}}^{E_{h i}}
\end{aligned}
$$

Now, we go back to Eq. C.64 to C.70, using Eq. C.89,

$$
\begin{gathered}
a_{1}=\int d \vec{k} f=b_{0} \\
a_{2}=\int d \vec{k} f \frac{k^{2}}{M^{2}}=\int d \vec{k} f\left(\frac{\epsilon^{2}}{M^{2}}-1\right)=b_{2}-b_{0} \\
a_{3}=\int d \vec{k} f \frac{k^{2} \cos ^{2} \tau}{M^{2}}=\int d \vec{k} f \frac{4 \omega_{e f f}^{2} \epsilon^{2}-4 \epsilon \omega_{e f f}\left(|\vec{q}|^{2}-\omega_{e f f}^{2}\right)+|\vec{q}|^{4}+\omega_{e f f}^{4}-2|\vec{q}|^{2} \omega_{e f f}^{2}}{4 M^{2}|\vec{q}|^{2}} \\
=\int d \vec{k} f\left[\frac{\omega_{e f f}^{2}}{|\vec{q}|^{2}}\left(\frac{\epsilon}{M}\right)^{2}-\frac{\omega_{e f f}\left(|\vec{q}|^{2}-\omega_{e f f}^{2}\right)}{|\vec{q}|^{2} M}\left(\frac{\epsilon}{M}\right)+\frac{\left(|\vec{q}|^{2}-\omega_{e f f}^{2}\right)^{2}}{4 M^{2}|\vec{q}|^{2}}\right] \\
=c^{2} b_{0}+2 c d b_{1}+d^{2} b_{0},
\end{gathered}
$$

$$
\begin{aligned}
a_{4} & =\int d \vec{k} f \frac{\epsilon_{k}^{2}}{M^{2}}=\int d \vec{k} f\left(\frac{\epsilon^{2}}{M^{2}}-\frac{2 \epsilon \epsilon_{1}}{M^{2}}+\frac{\epsilon_{1}^{2}}{M^{2}}\right) \\
& =b_{2}^{2}-2 \frac{\epsilon_{1}}{M} b_{1}+\frac{\epsilon_{1}^{2}}{M^{2}} b_{0}
\end{aligned}
$$




$$
\begin{aligned}
a_{5}= & \int d \vec{k} f \frac{\epsilon_{k} k \cos \tau}{M^{2}}=\int d \vec{k} f\left(\epsilon-\epsilon_{1}\right)\left(\frac{|\vec{q}|^{2}-\omega_{\text {eff }}^{2}-2 \epsilon \omega_{\text {eff }}}{2|\vec{k}||\vec{q}|}\right) \\
= & \int d \vec{k} f\left[-\frac{\omega_{\text {eff }}}{|\vec{q}|}\left(\frac{\epsilon}{M}\right)^{2}+\left(\frac{q_{\text {eff }}^{2}}{2|\vec{q}| m}+\frac{\epsilon_{1} \omega_{\text {eff }}}{m|\vec{q}|}\right)\left(\frac{\epsilon}{M}\right)-\frac{\epsilon_{1} q_{\text {eff }}^{2}}{2 M^{2}|\vec{q}|}\right] \\
= & c b_{2}+\left(d-\frac{\epsilon_{1} c}{M}\right) b_{1}-\frac{\epsilon_{1} d}{M} b_{0}, \\
a_{6}= & \int d \vec{k} f \frac{k \cos \tau}{M}=\int d \vec{k} f \frac{k}{M}\left(f r|\vec{q}|^{2}-\omega_{\text {eff }}^{2}-2 \epsilon \omega_{e f f} 2|\vec{k}||\vec{q}|\right) \\
= & c b_{1}+d b_{0}, \\
& a_{7}=\int d \vec{k} f \frac{\epsilon_{k}}{M}=\int d \vec{k} f\left(\frac{\epsilon-\epsilon_{1}}{M}\right)=b_{1}-\frac{\epsilon_{1}}{M} b_{0} .
\end{aligned}
$$

\section{C.2.5 Hadronic nucleon functions, definitions}

All hadronic nucleon functions are described with combinations of nucleon form factors. We can find hadronic nucleon functions in Smith Moniz formula from Llewellyn-Smith formula.

We found the following Pais's nucleon functions (Eq. C.29, C.30, C.31, C.32, and C.33,

$$
\begin{aligned}
& w_{\mu \nu}=w_{1} g_{\mu \nu}+w_{2} P_{\mu} P_{\nu}+w_{3} i \epsilon_{\mu \nu \alpha \beta} q_{\alpha} P_{\beta}+w_{4} q_{\mu} q_{\nu}+w_{5}\left(P_{\mu} q_{\nu}+P_{\nu} q_{\mu}\right) \\
& w_{1}=-8 M^{2} g_{A}^{2}+2 q^{2}\left(g_{V}^{2}+g_{A}^{2}\right) \\
& w_{2}=2 g_{A}^{2}+2\left|g_{V}-2 M f_{V}\right|^{2}-2 q^{2}\left(f_{V}^{2}+f_{A}^{2}\right) \\
& w_{3}=-4 \operatorname{Re}\left(g_{A}{ }^{*} g_{V}\right) \\
& w_{4}=-2 g_{V}^{2}-2\left|g_{A}+2 M h_{A}\right|^{2}+2\left(-q^{2}+4 M^{2}\right)\left(h_{V}^{2}+h_{A}^{2}\right) \\
& w_{5}=2 \operatorname{Re}\left\{2 M\left[\left(g_{V}-2 M f_{V}\left(1-\frac{q^{2}}{4 M^{2}}\right)\right) h_{V}^{*}+\left(g_{A}{ }^{*}+\frac{q^{2}}{2 M} h_{A}^{*}\right) f_{A}\right]\right\}
\end{aligned}
$$

Since we already know that $w_{6}$ gives zero contribution to the cross section after taking contraction with leptonic tensor (Sec. C.1.4), we ignore it. Here nucleon functions $w_{i}$ are written as functions of $P=\left(p_{1}+p_{2}\right)$ and $q$. On the other hand, in Smith and Moniz use 
$p\left(=p_{1}\right)$ and $q$ for nucleon functions $T_{i}$ (Eq. C.61). Thus,

$$
\begin{aligned}
w_{\mu \nu}= & w_{1} g_{\mu \nu}+w_{2}(2 p+q)_{\mu}(2 p+q)_{\nu}+w_{3} i \epsilon_{\mu \nu \alpha \beta} q_{\alpha}(2 p+q)_{\beta} \\
& +w_{4} q_{\mu} q_{\nu}+w_{5}\left[(2 p+q)_{\mu} q_{\nu}+(2 p+q)_{\nu} q_{\mu}\right] \\
= & w_{1} g_{\mu \nu}+4 w_{2} p_{\mu} p_{\nu}-2 i w_{3} \epsilon_{\mu \nu \alpha \beta} p_{\alpha} q_{\beta}+\left(w_{2}+w_{4}+2 w_{5}\right) q_{\mu} q_{\nu} \\
& +2\left(w_{2}+w_{5}\right)\left(p_{\mu} q_{\nu}+p_{\nu} q_{\mu}\right) .
\end{aligned}
$$

Compare Eq. C.61 with Eq. C.103, one can get the relationships between Llewellyn-Smith and Smith Moniz nucleon tensors.

$$
\begin{aligned}
\text { Smith Moniz } & \leftrightarrow \text { Pais } \\
T_{1} & =-w_{1} \\
T_{2} & =4 M^{2} w_{2} \\
T_{\alpha} & =M^{2}\left(w_{2}+w_{4}+2 w_{5}\right) \\
T_{\beta} & =2 M^{2}\left(w_{2}+w_{5}\right) \\
T_{8} & =-2 M^{2} w_{3}
\end{aligned}
$$

The definition of nucleon current is secondary once you get nucleon tensors. Since we accept Pais's definitions for nucleon tensors, we can adjust Smith-Moniz's nucleon current definition to be consistent with Pais. In Smith-Moniz paper [16, the definition of nucleon current is given in Eq. (8). Comparing with the current definition by Pais (Eq. C.8), using Gordon's decomposition (Eq. C.9), we can modify Eq. (8) for our purpose.

$$
\begin{aligned}
\Gamma_{\mu} & =\gamma_{\mu} F_{1}+\sigma_{\mu \nu} q^{\nu} F_{2}+i F_{V}^{3} q_{\mu} \tau_{z}+\gamma_{\mu} \gamma_{5} F_{A}-i F_{P} \gamma_{5} q_{\mu}+F_{A}{ }^{3} \gamma_{5} \sigma_{\mu \nu} q^{\nu} \\
& \rightarrow \gamma_{\mu} F_{1}-i \sigma_{\mu \nu} q^{\nu} F_{2}+F_{V}{ }^{3} q_{\mu} \tau_{z}-\gamma_{\mu} \gamma_{5} F_{A}-F_{P} \gamma_{5} q_{\mu}+F_{A}{ }^{3} \gamma_{5} P_{\mu} \\
& =\gamma_{\mu}\left[\left(F_{1}+2 M F_{2}\right)-F_{A} \gamma_{5}\right]-P_{\mu}\left(F_{2}-F_{A}{ }^{3} \gamma_{5} \tau_{z}\right)+q_{\mu}\left(F_{V}{ }^{3} \tau_{z}-F_{P}\right)
\end{aligned}
$$




\section{C.2. NEUTRINO-NUCLEI LEPTON SCATTERING}

Now, Smith-Moniz's form factors and Pais's form factor are related.

$$
\begin{aligned}
\text { Smith Moniz } & \leftrightarrow \text { Pais } \\
F_{1}+2 M F_{2} & =g_{V} \\
F_{2} & =f_{V} \\
F_{V}^{3} & =h_{V} \\
F_{A} & =g_{A} \\
F_{A}^{3} & =f_{A} \\
F_{P} & =h_{A}
\end{aligned}
$$

Thus, with a little care about the expressions for each nucleon tensor in Smith Moniz papers, the expressions are the following,

$$
\begin{gathered}
T_{1}==-w_{1}=8 M^{2} g_{A}^{2}-2 q^{2}\left(g_{V}^{2}+g_{A}^{2}\right)=8 M^{2} F_{A}^{2}-2 q^{2}\left[\left(F_{1}+2 M F_{2}\right)^{2}+F_{A}^{2}\right] \\
=4\left[\frac{1}{2} Q^{2}\left(F_{1}+2 M F_{2}\right)^{2}+\left(2 M^{2}+\frac{1}{2} Q^{2}\right) F_{A}^{2}\right] \\
T_{2}=4 M^{2} w_{2}=4 M^{2}\left[2 g_{A}^{2}+2\left|g_{V}-2 M f_{V}\right|^{2}-2 q^{2}\left(f_{V}^{2}+f_{A}^{2}\right]\right. \\
=4\left[2 M^{2}\left[F_{1}^{2}+Q^{2} F_{2}^{2}+F_{A}^{2}+Q^{2} F_{A}^{3^{2}}\right]\right] \\
T_{\beta}=\frac{2 M^{2}\left[w_{2}+w_{5}\right]=\frac{1}{2} T_{2}+2 M^{2} w_{5}}{=} \quad-2 M^{2}\left[-4 R e\left(g_{A} \cdot g_{V}\right)\right]=4\left[2 M^{2} F_{A}\left(F_{1}+2 M F_{2}\right)\right] \\
=\frac{1}{2} T_{2}+2 M^{2} \cdot 2 R e\left\{2 M\left[\left(g_{V}-2 M f_{V}\left(1-\frac{q^{2}}{4 M^{2}}\right)\right) h_{V}^{*}+\left(g_{A}^{*}+\frac{q^{2}}{2 M} h_{A}^{*}\right) f_{A}\right]\right\} \\
=\frac{1}{2} T_{2}+8 M^{3}\left[\left(F_{1}+\frac{F_{2} q^{2}}{2 M}\right) F_{V}^{3}+\left(F_{A}+\frac{F_{P} q^{2}}{2 M}\right) F_{A}^{3}\right] \\
\left.=\frac{1}{2} T_{2}+4\left[\left(2 M F_{1}-F_{2} Q^{2}\right) M^{2} F_{V}^{3}+\left(2 M F_{A}-F_{P} Q^{2}\right) M^{2} F_{A}^{3}\right]\right]
\end{gathered}
$$




$$
\begin{aligned}
T_{\alpha}= & M^{2}\left(w_{2}+w_{4}+2 w_{5}\right)=\frac{1}{4} T_{2}+M^{2}\left(w_{4}+2 w_{5}\right) \\
= & \frac{1}{4} T_{2}+M^{2}\left\{-2 g_{V}^{2}-2\left|g_{A}+2 M h_{A}\right|^{2}+2\left(-q^{2}+4 M^{2}\right)\left(h_{V}^{2}+h_{A}^{2}\right)\right. \\
& \left.+8 M R e\left\{\left[\left(g_{V}-2 M f_{V}\left(1-\frac{q^{2}}{4 M^{2}}\right)\right) h_{V}^{*}+\left(g_{A}^{*}+\frac{q^{2}}{2 M} h_{A}^{*}\right) f_{A}\right]\right\}\right\} \\
= & \frac{1}{4} T_{2}+M^{2}\left\{-2\left(F_{1}+2 M F_{2}\right)^{2}-2\left(F_{A}+2 M F_{P}\right)^{2}+2\left(-q^{2}+4 M^{2}\right)\left(F_{V}^{3^{2}}+F_{P}^{2}\right)\right. \\
& \left.+8 M\left[\left(F_{1}+\frac{F_{2} q^{2}}{2 M}\right) F_{V}^{3}+\left(F_{A}+\frac{F_{P} q^{2}}{2 M}\right) F_{A}^{3}\right]\right\} \\
= & \frac{1}{4} T_{2}-\frac{M^{2}}{q^{2}} T_{1}-\frac{8 M^{4}}{q^{2}} F_{A}^{2}-8 M^{3} F_{A} F_{P}-8 M^{4} F_{P}^{2}+2 M^{2}\left(-q^{2}+4 M^{2}\right)\left(F_{V} 3^{2}+F_{P}^{2}\right) \\
& +4 M^{2} F_{V}^{3}\left(F_{1}+F_{2} q^{2}\right)+4 M^{2} F_{A}^{3}\left(2 M F_{A}+F_{P} q^{2}\right) \\
= & \frac{1}{4} T_{2}-\frac{M^{2}}{q^{2}} T_{1}-\frac{8 M^{4}}{q^{2}} F_{A}^{2}-8 M^{3} F_{A} F_{P}-8 M^{4} F_{P}^{2}+2 M^{2}\left(-q^{2}+4 M^{2}\right)\left(F_{V}^{3^{2}}+F_{P}^{2}\right) \\
& +4 M^{2} F_{V}^{3}\left(F_{1}+F_{2} q^{2}\right)+4 M^{2} F_{A}^{3}\left(2 M F_{A}+F_{P} q^{2}\right),
\end{aligned}
$$

using $T_{1}$ (Eq. C.105),

$$
\begin{aligned}
= & -\frac{M^{2}}{q^{2}} T_{1}+\frac{1}{4} T_{2}+4 M^{2} F_{V}^{3}\left[2 M F_{1}+F_{2} q^{2}+\left(2 M^{2}-\frac{1}{2} q^{2}\right) F_{V}^{3}\right] \\
& +4 M^{2}\left(2 M F_{A}+F_{P} q^{2}\right)\left[F_{A}^{3}-\frac{1}{2 q^{2}}\left(2 M F_{A}+F_{P} q^{2}\right)\right] \\
= & \frac{M^{2}}{Q^{2}} T_{1}+\frac{1}{4} T_{2}+4 M^{2} F_{V}^{3}\left[2 M F_{1}-F_{2} Q^{2}+\left(2 M^{2}+\frac{1}{2} Q^{2}\right) F_{V}{ }^{3}\right] \\
& +4 M^{2}\left(2 M F_{A}-F_{P} Q^{2}\right)\left[F_{A}^{3}+\frac{1}{2 Q^{2}}\left(2 M F_{A}-F_{P} Q^{2}\right)\right] .
\end{aligned}
$$

All terms are consistent with Smith and Moniz's paper except for a factor of 4 , but this factor cancels with the factor $\frac{1}{4}$ which we see in Eq. C.47 and C.60. Therefore, we finally find the double differential cross section for RFG model,

$$
\begin{aligned}
\frac{d \sigma^{2}}{d k_{2} d \Omega}= & \frac{G_{V}^{2} k_{2}^{2}}{2 \pi^{2} m_{T}}\left\{2 W_{1} \sin ^{2}\left(\frac{1}{2} \chi\right)+W_{2} \cos ^{2}\left(\frac{1}{2} \chi\right)+\sin ^{2}\left(\frac{1}{2} \chi\right) \frac{m^{2}}{m_{T}^{2}} W_{\alpha}+\frac{m^{2}\left(W_{\beta}+W_{8}\right)}{m_{T} \epsilon_{2}}\right. \\
& -\frac{2 W_{8}}{m_{T}} \sin \left(\frac{1}{2} \chi\right) \sqrt{\left.q^{2} \cos ^{2}\left(\frac{1}{2} \chi\right)+|q|^{2} \sin ^{2}\left(\frac{1}{2} \chi\right)+m^{2}\right\} .}
\end{aligned}
$$




\section{Appendix D}

\section{Table for Predicted $\nu_{\mu}$ flux for MiniBooNE}

Tab. D.1 shows $\nu_{\mu}$ flux (Fig. 7.5 for each bin. The unit is neutrinos/POT/50MeV and the integrated number of all energy region is $5.167 \times 10^{-10}$ neutrinos/POT. 
APPENDIX D. TABLE FOR PREDICTED $\nu_{\mu}$ FLUX FOR MINIBOONE

\begin{tabular}{|c|c|c|c|c|c|}
\hline bin & $\nu_{\mu}$ flux & bin & $\nu_{\mu}$ flux & bin & $\nu_{\mu}$ flux \\
\hline $0-50 \mathrm{MeV}$ & $2.276 \times 10^{12}$ & $1000-1050 \mathrm{MeV}$ & $1.681 \times 10^{11}$ & $2000-2050 \mathrm{MeV}$ & $9.607 \times 10^{13}$ \\
\hline $50-100 \mathrm{MeV}$ & $8.583 \times 10^{12}$ & $1050-1100 \mathrm{MeV}$ & $1.561 \times 10^{11}$ & $2050-2100 \mathrm{MeV}$ & $8.166 \times 10^{13}$ \\
\hline $100-150 \mathrm{MeV}$ & $1.114 \times 10^{11}$ & $1100-1150 \mathrm{MeV}$ & $1.442 \times 10^{11}$ & $2100-2150 \mathrm{MeV}$ & $6.942 \times 10^{13}$ \\
\hline $150-200 \mathrm{MeV}$ & $1.337 \times 10^{11}$ & $1150-1200 \mathrm{MeV}$ & $1.321 \times 10^{11}$ & $2150-2200 \mathrm{MeV}$ & $5.949 \times 10^{13}$ \\
\hline $200-250 \mathrm{MeV}$ & $1.661 \times 10^{11}$ & $1200-1250 \mathrm{MeV}$ & $1.195 \times 10^{11}$ & $2200-2250 \mathrm{MeV}$ & $5.157 \times 10^{13}$ \\
\hline $250-300 \mathrm{MeV}$ & $1.823 \times 10^{11}$ & $1250-1300 \mathrm{MeV}$ & $1.071 \times 10^{11}$ & $2250-2300 \mathrm{MeV}$ & $4.487 \times 10^{13}$ \\
\hline $300-350 \mathrm{MeV}$ & $1.950 \times 10^{11}$ & $1300-1350 \mathrm{MeV}$ & $9.522 \times 10^{12}$ & $2300-2350 \mathrm{MeV}$ & $3.942 \times 10^{13}$ \\
\hline $350-400 \mathrm{MeV}$ & $2.049 \times 10^{11}$ & $1350-1400 \mathrm{MeV}$ & $8.372 \times 10^{12}$ & $2350-2400 \mathrm{MeV}$ & $3.507 \times 10^{13}$ \\
\hline $400-450 \mathrm{MeV}$ & $2.165 \times 10^{11}$ & $1400-1450 \mathrm{MeV}$ & $7.292 \times 10^{12}$ & $2400-2450 \mathrm{MeV}$ & $3.156 \times 10^{13}$ \\
\hline $450-500 \mathrm{MeV}$ & $2.245 \times 10^{11}$ & $1450-1500 \mathrm{MeV}$ & $6.304 \times 10^{12}$ & $2450-2500 \mathrm{MeV}$ & $2.872 \times 10^{13}$ \\
\hline $500-550 \mathrm{MeV}$ & $2.284 \times 10^{11}$ & $1500-1550 \mathrm{MeV}$ & $5.407 \times 10^{12}$ & $2500-2550 \mathrm{MeV}$ & $2.620 \times 10^{13}$ \\
\hline $550-600 \mathrm{MeV}$ & $2.296 \times 10^{11}$ & $1550-1600 \mathrm{MeV}$ & $4.610 \times 10^{12}$ & $2550-2600 \mathrm{MeV}$ & $2.414 \times 10^{13}$ \\
\hline $600-650 \mathrm{MeV}$ & $2.279 \times 10^{11}$ & $1600-1650 \mathrm{MeV}$ & $3.909 \times 10^{12}$ & $2600-2650 \mathrm{MeV}$ & $2.278 \times 10^{13}$ \\
\hline $650-700 \mathrm{MeV}$ & $2.258 \times 10^{11}$ & $1650-1700 \mathrm{MeV}$ & $3.292 \times 10^{12}$ & $2650-2700 \mathrm{MeV}$ & $2.114 \times 10^{13}$ \\
\hline $700-750 \mathrm{MeV}$ & $2.218 \times 10^{11}$ & $1700-1750 \mathrm{MeV}$ & $2.765 \times 10^{12}$ & $2700-2750 \mathrm{MeV}$ & $1.999 \times 10^{13}$ \\
\hline $750-800 \mathrm{MeV}$ & $2.160 \times 10^{11}$ & $1750-1800 \mathrm{MeV}$ & $2.316 \times 10^{12}$ & $2750-2800 \mathrm{MeV}$ & $1.923 \times 10^{13}$ \\
\hline $800-850 \mathrm{MeV}$ & $2.082 \times 10^{11}$ & $1800-1850 \mathrm{MeV}$ & $1.936 \times 10^{12}$ & $2800-2850 \mathrm{MeV}$ & $1.819 \times 10^{13}$ \\
\hline $850-900 \mathrm{MeV}$ & $1.996 \times 10^{11}$ & $1850-1900 \mathrm{MeV}$ & $1.619 \times 10^{12}$ & $2850-2900 \mathrm{MeV}$ & $1.730 \times 10^{13}$ \\
\hline $900-950 \mathrm{MeV}$ & $1.898 \times 10^{11}$ & $1900-1950 \mathrm{MeV}$ & $1.358 \times 10^{12}$ & $2900-2950 \mathrm{MeV}$ & $1.669 \times 10^{13}$ \\
\hline $950-1000 \mathrm{MeV}$ & $1.793 \times 10^{11}$ & $1950-2000 \mathrm{MeV}$ & $1.141 \times 10^{12}$ & $2950-3000 \mathrm{MeV}$ & $1.604 \times 10^{13}$ \\
\hline
\end{tabular}

Table D.1: Predicted $\nu_{\mu}$ flux in MiniBooNE. The unit is neutrinos/POT/50MeV and integrated value for all energy region is $5.167 \times 10^{-10}$ neutrinos/POT. 


\section{Bibliography}

[1] [LSND collaboration], L. B. Auerbach et al., Phys. Rev. D 72:076002 (2005).

[2] T. Katori, V. A. Kostelecký, and R. Tayloe, Phys. Rev. D 74:105009 (2006).

[3] [MiniBooNE collaboration], T. Katori and R. Tayloe p. 79, in CPT and Lorentz symmetry IV, World Scientific (2008).

[4] [MiniBooNE collaboration], A. A. Aguilar-Arevalo et al., Phys. Rev. Lett. 100:032301 (2008).

[5] R. Tayloe et al., Nucl. Inst. \& Meth. A 562:198 (2006).

[6] B. Kayser, Phys. Rev. D 24:110 (1981).

[7] C. Giunti, C. W. Kim, and U. W. Lee, Phys. Rev. D 44:3635 (1991).

[8] [Particle Data Group collaboration], W.-M.Yao et al., J. Phys. G 33:1 (2006).

[9] M. Fukugita and T. Yanagida, Physics of Neutrinos and Application to Astrophysics, springer-Verlag (2003).

[10] R. N. Mohapatra and P. B. Pal, Massive Neutrinos in Physics and Astrophysics, world Scientific (2004).

[11] C. Giunti and C. W. Kim, Fundamentals of Neutrino Physics and Astrophysics, oxford university press (2007). 
[12] V. A. Kostelecký and N. Russell (2008). [arXiv:0801.0287 [hep-ph]]

[13] F. Canè et al., Phys. Rev. Lett. 93:230801 (2004).

[14] V. A. Kostelecký et al., CPT and Lorentz symmetry IV, world Scientific (2008).

[15] C. H. Llewellyn-Smith, Phys. Rep. 3:261 (1972).

[16] R. A. Smith and E. J. Moniz, Nucl. Phys. B 43:605, Erratum-ibid. B101:547 (1975) (1972).

[17] E. D. Commins and P. H. Bucksbaum, Weak interactions of leptons and quarks, cambridge university press (1983).

[18] F. Halzen and A. D. Martin, Quarks E Leptons, john Wiley \& Sons (1984).

[19] S. Ouedraogo, Limit on the muon neutrino magnetic moment and a measurement of the CCPIP to CCQE cross section ratio, Ph.D. thesis, Louisiana State University (2008).

[20] G. P. Zeller (2003). [arXiv:hep-ex/0312061]

[21] G. Garvey, W. C. Louis, and D. H. White, Phys. Rev. C 48:761 (1993).

[22] G. Garvey et al., Phys. Rev. C 48:1919 (1993).

[23] [G0 collaboration], D. Armstrong et al., Phys. Rev. Lett. 95:092001 (2005).

[24] L. Bugel et al., http://www-finesse.fnal.gov/ (2005).

[25] A. V. Belitsky, X. Ji, and F. Yuan, Phys. Rev. D 69:074014 (2004).

[26] R. F. Streater and A. S. Wightman, PCT, Spin and Statistics, and All That, the Benjamin/Commings Publishing Company Inc. (1964).

[27] A. V. Butkevich, (private communication). 
[28] R. N. Mohapatra et al. (2004). [arXiv:hep-ph/0412099]

[29] [T2K collaboration], Y. Itow et al. (2001). [arXiv:hep-ex/0106019]

[30] [NOvA collaboration], D. S. Ayres et al. (2005). [arXiv:hep-ex/0503053]

[31] [K2K collaboration], R. Gran et al., Phys. Rev. D 74:052002 (2006).

[32] [SciBooNE collaboration], K. Hiraide et al. (2008). [arXiv:0811.0369 [hep-ex]]

[33] [MINOS collaboration], D. G. Michael et al., Phys. Rev. Lett. 97:191801 (2006).

[34] [MINERvA collaboration], D. Drakoulakos et al. (2004). [arXiv:hep-ex/0405002]

[35] [NOMAD collaboration], V. V. Lyubushkin et al. (2008). [arXiv:0812:4543[hep-ex]]

[36] P. Lipari, Nucl. Phys. B (Proc. Suppl.) 112:274 (2002).

[37] [K2K collaboration], X. Espinal and F. Sanchez, AIP Conf. Proc. 967:117 (2007).

[38] V. Bernard et al., J. Phys. G 28:R1 (2002).

[39] [MiniBooNE collaboration], T. Katori, AIP Conf. Proc. 967:123 (2007).

[40] C. W. Walter, AIP Conf. Proc. 967:3 (2007).

[41] J. E. Amaro et al., Phys. Rev. C 71:015501 (2005).

[42] T. Leitner et al., Phys. Rev. C 73:065502 (2006).

[43] O. Benhar et al., Phys. Rev. D 72:053005 (2005).

[44] A. Ahmad et al., Phys. Rev. D 74:073008 (2006).

[45] A. V. Butkevich and S. P. Mikheyev, Phys. Rev. C 72:025501 (2005).

[46] V. A. Kostelecký and S. Samuel, Phys. Rev. D 39:683 (1989). 
[47] S. W. Hawking, Phys. Rev. D 14:2460 (1976).

[48] D. Colladay and V. A. Kostelecký, Phys. Rev. D 55:6760 (1997).

[49] D. Colladay and V. A. Kostelecký, Phys. Rev. D 58:116002 (1998).

[50] A. Kostelecký, Phys. Rev. D 69:105009 (2004).

[51] O. W. Greenberg, Phys. Rev. Lett. 89:231602 (2002).

[52] V. A. Kostelecký et al., CPT and Lorentz symmetry I, world Scientific (1999).

[53] V. A. Kostelecký et al., CPT and Lorentz symmetry II, world Scientific (2002).

[54] V. A. Kostelecký et al., CPT and Lorentz symmetry III, world Scientific (2005).

[55] B. R. Heckel et al., Phys. Rev. Lett. 97:021603 (2006).

[56] V. A. Kostelecký and M. Mewes, Phys. Rev. D 69:016005 (2004).

[57] S. Coleman and S. L. Glashow, Phys. Rev. D 59:116008 (2005).

[58] V. Barger, S. Pakvasa, T. J. Weiler, and K. Whisnant, Phys. Rev. Lett. 85:5055 (2000).

[59] G. Battistoni et al., Phys. Lett. B 615:14 (2005).

[60] J. N. Bahcall, V. Barger, and D. Marfatia, Phys. Lett. B 534:120 (2002).

[61] A. de Gouvêa and C. Pena-Garay, Phys. Rev. D 71:093002 (2005).

[62] M. D. Messier p. 84, in CPT and Lorentz symmetry III, World Scientific (2005).

[63] [MINOS collaboration], P. Adamson et al., Phys. Rev. Lett. 101:151601 (2008).

[64] J. Ahrens and J. L. Kelley p. 234, in CPT and Lorentz symmetry III, World Scientific (2008). 
[65] V. A. Kostelecký and M. Mewes, Phys. Rev. D 70:031902 (2004).

[66] V. A. Kostelecký and M. Mewes, Phys. Rev. D 70:076002 (2004).

[67] [MiniBooNE collaboration], A. A. Aguilar-Arevalo et al., Phys. Rev. Lett. 98:231801 (2007).

[68] G. Karagiorgi, http://theory.fnal.gov/jetp/talks/karagiorgi.pdf.

[69] [LSND collaboration], A. A. Aguilar-Arevalo et al., Phys. Rev. D 64:112007 (2001).

[70] [Homestake collaboration], B. T. Cleveland et al., Astrophys. J. 496:505 (1998).

[71] [Kamiokande collaboration], K. S. Hirata et al., Phys. Rev. Lett. 63:16 (1989).

[72] [Super-Kamiokande collaboration], J. P. Cravens et al., Phys. Rev. D 78:032002 (2008).

[73] [GALLEX collaboration], W. Hampel et al., Phys. Lett. B 447:127 (1999).

[74] [SAGE collaboration], J. N. Abdurashitov et al. 95:181 (2002).

[75] [GNO collaboration], M. Altmann et al., Phys. Lett. B 490:16 (2000).

[76] [SNO collaboration], Q. R. Ahmad et al., Phys. Rev. Lett. 87:071301 (2001).

[77] [Borexino collaboration], C. Arpesella et al., Phys. Lett. B 658:101 (2008).

[78] [Super-Kamiokande collaboration], Y. Fukuda et al., Phys. Rev. Lett. 81:1562 (1998).

[79] [MACRO collaboration], M. Ambrosio et al., Phys. Lett. B 566:35 (2003).

[80] [MINOS collaboration], P. Adamson et al., Phys. Rev. D 75:092003 (2007).

[81] [K2K collaboration], M. H. Ahn et al., Phys. Rev. Lett. 90:041801 (2003).

[82] [MINOS collaboration], D. G. Michael et al., Phys. Rev. Lett. 97:191801 (2006). 
[83] [KamLAND collaboration], K. Eguchi et al., Phys. Rev. Lett. 90:021802 (2003).

[84] M. Sorel, J. M. Conrad, and M. Shaevitz, Phys. Rev. D 70:073004 (2004).

[85] G. Karagiorgi et al., Phys. Rev. D 75:013011 (2007).

[86] V. A. Kostelecký and M. Mewes, Phys. Rev. D 66:056005 (2002).

[87] R. Bluhm et al., Phys. Rev. D 68:125008 (2003).

[88] Google Map, http://maps.google.com/.

[89] L. Lyons, Statistics for nuclear and particle physicist, cambridge university press (1992).

[90] A. G. Frodesen, O. Skjeggestad, and H. Tofte, Fundamentals of Neutrino Physics and Astrophysics, oxford university press (2007).

[91] W. H. Press, S. A. Teukolsky, W. T. Vetterling, and B. P. Flannery, Numerical Recipes in $C++$, cambridge university press (2002).

[92] K. Whisnant, (private communication).

[93] V. D. Barger, S. Pakvasa, T. J. Weiler, and K. Whisnant, Phys. Lett. B 437:107 (1998).

[94] [MINOS collaboration], P. Adamson et al., Phys. Rev. Lett. 101:131802 (2008).

[95] [Borexino collaboration], C. Arpesella et al., Phys. Rev. Lett. 101:091302 (2008).

[96] [KamLAND collaboration], S. Abe et al., Phys. Rev. Lett. 100:221803 (2008).

[97] V. Barger, D. Marfatia, and K. Whisnant, Phys. Lett. B 617:78 (2005).

[98] V. Barger, D. Marfatia, and K. Whisnant, Phys. Lett. B 653:267 (2007). 


\section{BIBLIOGRAPHY}

[99] [Bugey collaboration], Y. Declais et al., Nucl. Phys. B 434:503 (1995).

[100] [Gösgen collaboration], G. Zacek et al., Phys. Rev. D 34:2621 (1986).

[101] [Palo Verde collaboration], F. Boehm et al., Phys. Rev. D 64:112001 (2001).

[102] [CHOOZ collaboration], M. Apollonio et al., Eur. Phys. J. C 27:331 (2003).

[103] [KARMEN collaboration], B. Armbruster et al., Phys. Rev. D 65:112001 (2002).

[104] [OscSNS collaboration], G. T. Garvey et al., Phys. Rev. D 72:092001 (2005).

[105] MiniBooNE April 2007 Data Release, http://www-boone.fnal.gov/for_ physicists/april07datarelease/index.html.

[106] M. Sorel, Search for Sterile Neutrinos Using the MiniBooNE Beam, Ph.D. thesis, Columbia University (2005).

[107] [SNO collaboration], B. Aharmim et al., Phys. Rev. C 72:055502 (2005).

[108] [MiniBooNE collaboration], A. A. Aguilar-Arevalo et al. (2008). [arXiv:0812.2243 [hep-ex]]

[109] [MiniBooNE collaboration], T. Katori and R. Tayloe p. 296, in CPT and Lorentz symmetry IV, World Scientific (2008).

[110] D. C. Cox, A measurement of the neutral current neutrino-nucleon elastic cross sectnio at MiniBooNE, Ph.D. thesis, Indiana University (2008).

[111] J. L. Raaf, A Measurement of the Neutrino Neutral Current $\pi^{o}$ Cross Section at MiniBooNE, Ph.D. thesis, University of Cincinnati (2005).

[112] D. Schmitz, A measurement of hadron production cross-sections for the simulation of accelerator neutrino beams and a search for $\nu_{\mu} \rightarrow \nu_{e}$ oscillations in the $1 \mathrm{~m}^{2} \mathrm{eV}^{2}$ region, Ph.D. thesis, Columbia University (2008). 
[113] R. Patterson, A Search for Muon Neutrino to Electron Neutrino Oscillations at $\Delta m^{2}>1 e V^{2}$, Ph.D. thesis, Princeton University (2007).

[114] A. A. Aguilar-Arévalo, An Improved Neutrino Oscillations Analysis of the MiniBooNE Data, Ph.D. thesis, Columbia University (2008).

[115] J. Monroe, A Combined $\nu_{\mu}$ and $\nu_{e}$ Oscillation Search at MiniBooNE, Ph.D. thesis, Columbia University (2006).

[116] [MiniBooNE collaboration], A. A. Aguilar-Arevalo et al. (2008). [arXiv:0806.4201 [hep-ex]]

[117] [MiniBooNE collaboration], A. A. Aguilar-Arevalo et al. (2008). [arXiv:0806.1449 [hep-ex]]

[118] S. Y. Lee, Accelerator Physics, world Scientific (2004).

[119] B. Worthel et al., Booster Rookie Book, http://www-bdnew.fnal.gov/operations/ rookie_books/.

[120] [HARP collaboration], M. Catanesi et al., Eur. Phys. J. C 52:29 (2007).

[121] [MiniBooNE collaboration], A. A. Aguilar-Arevalo et al., Phys. Lett. B 664:41 (2008).

[122] J. R. Sanford and C. L. Wang, BNL AGS Internal Report p. 11299 (1967).

[123] I. Chemakin et al., Nucl. Phys. A 639:407 (1998).

[124] S. J. Brice et al., Nucl. Inst. \& Meth. A 562:97 (2006).

[125] B. C. Brown et al., IEEE Nucl. Sci. Symp. Conf. Rec. 1:652 (2004).

[126] D. Casper, Nucl. Phys. B (Proc. Suppl.) 112:161, http://nuint.ps.uci.edu/ nuance/ (2002). 
[127] D. Rein and L. M. Sehgal, Nucl. Phys. B 223:29 (1983).

[128] M. Gluck, E. Reya, and A. Vogt, Eur. Phys. J. C 5:461 (1998).

[129] A. Bodek and U. K. Yang, AIP Conf. Proc. 670:110 (2003).

[130] H. Budd, A. Bodek, and J. Arrington (2003). [arXiv:hep-ex/0308005]

[131] E. J. Moniz et al., Phys. Rev. Lett. 26:445 (1971).

[132] G. Garvey, (private communication).

[133] A. A. Aguilar-Arevalo et al., Phys. Rev. D 78:012007 (2008).

[134] K. B. M. Mahn, http://theory.fnal.gov/jetp/talks/mahn.pdf.

[135] [K2K collaboration], M. Hasegawa et al., Phys. Rev. Lett. 95:252301 (2005).

[136] W. Reuter, G. Fricke, K. Merle, and H. Miska, Phys. Rev. C 26:806 (1982).

[137] V. Flaminio et al. CERN-HERA-83-01 (1983).

[138] V. Flaminio et al. CERN-HERA-83-02 (1983).

[139] V. Flaminio et al. CERN-HERA-84-01 (1984).

[140] D. Casper, (private communication).

[141] B. Hahn, D. G. Ravenhall, and R. Hofstadter, Phys. Rev. 101:1131 (1956).

[142] E. A. Hawker, Nucl. Phys. B (Proc. Suppl.) 139:260 (2005).

[143] D. Ashery et al., Phys. Rev. C 23:2173 (1981).

[144] M. K. Jones et al., Phys. Rev. C 48:2800 (1993).

[145] I. Navon et al., Phys. Rev. C 28:2548 (1983). 
[146] R. D. Ransome et al., Phys. Rev. C 45:R509 (1992).

[147] R. Brun et al. CERN-DD/EE/84-1 (1987).

[148] A. Fasso et al., Proc. Workshop on Simulating Accelerator Radiation Environments, Santa Fe (1993).

[149] C. Zeitnitz and T. A. Gabriel, Nucl. Inst. \& Meth. 349:106 (1994).

[150] T. Suzuki et al., Phys. Rev. C 35:2212 (1987).

[151] D. Perevalov, (private communication).

[152] G. P. Zeller, (private communication).

[153] R. A. Jhonson, (private communication).

[154] M. Shaevitz, (private communication).

[155] K. S. Kuzmin, V. V. Lyubushkin, and V. A. Naumov, Eur. Phys. J. C 54:517 (2008).

[156] T. Katori (2008). [arXiv:0805.2476[hep-ex]]

[157] H. Chen et al., http://www-microboone.fnal.gov/Documents/ MicroBooNEProposal.pdf.

[158] [SAMPLE collaboration], D. Spayde et al., Phys. Lett. B 583:79 (2004).

[159] F. Maas et al., Phys. Rev. Lett. 94:152001 (2005).

[160] [HAPPEX collaboration], A. Acha et al., Phys. Rev. Lett. 98:032301 (2007).

[161] E. J. Beise, Nucl. Phys. A 737:93 (2004).

[162] [HERMES collaboration], A. Airapetian et al., Phys. Rev. Lett. 92:012005 (2004).

[163] L. A. Ahrens et al., Phys. Rev. D 35:785 (1987). 


\section{BIBLIOGRAPHY}

[164] [HERMES collaboration], A. Airapetian et al., Phys. Lett. B 666:446 (2008).

[165] [Spin Muon collaboration], D. Adams et al., Phys. Rev. D 56:5330 (1997).

[166] D. C. Cox, AIP Conf. Proc. 967:130 (2007).

[167] H. Takei, http://www-sciboone.fnal.gov/documents/talks/2008/takei_ aps2008.pdf, talk given at APS april meeting 08 (2008).

[168] H. Akaike, SIAM J. Appl. Math. 24:234 (1973).

[169] A. Pais, Annal. Phys. 63:361 (1971). 


\section{Curriculum Vitae}

\section{Teppei Katori}

\section{Affiliation}

Indiana University Cyclotron Facility (IUCF),

2401 Milo B. Sampson Lane,

Bloomington, IN 47408, USA

\section{Contact information}

Mail Stop 309,

Fermi National Accelerator Laboratory (Fermilab),

P. O. Box 500,

Batavia, IL 60510, USA

Phone (home) 1-773-320-0126

Phone (work) 1-630-840-2077 
E-mail:katori@fnal.gov

\section{Education}

2002-present $\mathrm{PhD}$ student at Indiana University, Bloomington

1998-2002 B.S. at Tokyo Institute of Technology

\section{Teaching Experience}

2004 P221 Physics Lab. (Mechanics)

2003 G530 Teaching in the U.S. Class Room

2002-2003 Tutor of Physics Forum

2002 P504 Practicum in Physics Laboratory Instruction

\section{Awards}

2008 William Koss memorial award for outstanding graduate research in physics

2005 COAS Graduate student travel grants

\section{Publications, Talks, and Posters}

\section{Major contributed publications}

1. A. A. Aguilar-Arevalo et al. [MiniBooNE Collaboration], "Measurement of Muon Neutrino Quasi-Elastic Scattering on Carbon in MiniBooNE”, Phys. Rev. Lett. 100, $032301(2008)$ 
2. T. Katori, V. A. Kostelecký and R. Tayloe, "Global three-parameter model for neutrino oscillations using Lorentz violation", Phys. Rev. D 74, 105009 (2006)

3. R. Tayloe et al., "A large-volume detector capable of charged-particle tracking", Nucl. Instrum. Meth. A 562, 198 (2006).

4. L. B. Auerbach et al. [LSND Collaboration], "Tests of Lorentz violation in $\bar{\nu}_{\mu} \rightarrow \bar{\nu}_{e}$ oscillations", Phys. Rev. D 72, 076004 (2005)

\section{Proceedings}

1. Teppei Katori, "Neutrino cross section measurements for long-baseline neutrino oscillation experiments," prepared for 43rd Rencontres de Moriond "Electroweak interactions and Unified theories",La Thuile, Italy, March 1-8, 2008, will be published(2008)

2. T. Katori and R. Tayloe [for the MiniBooNE Collaboration], "A Search for LorentzViolating Neutrino Oscillations in MiniBooNE," prepared for 4th Meeting on CPT and Lorentz Symmetry (CPT 07), Bloomington, Indiana, 8-11 Aug 2007, will be published in "CPT and Lorentz Symmetry", Proceedings of the Forth Meeting on CPT and Lorentz symmetry (V. A. Kostelecký ed.), World Scientific (2008)

3. T. Katori and R. Tayloe [for the MiniBooNE Collaboration], "Test for Lorentz violation in the MiniBooNE neutrino oscillation experiment," prepared for the poster session of 4th Meeting on CPT and Lorentz Symmetry (CPT 07), Bloomington, Indiana, 8-11 Aug 200\%, will be published in "CPT and Lorentz Symmetry", Proceedings of the Forth Meeting on CPT and Lorentz symmetry (V. A. Kostelecký ed.), World Scientific (2008)

4. Teppei Katori [for the MiniBooNE Collaboration], "Charged-Current Interaction Measurements in MiniBooNE," prepared for the Fifth International Workshop on NeutrinoNucleus Interactions in the Few-GeV Region (NuInt 07), Fermilab, Batavia, IL, May 
30-June 3, 2007, will be published in "NuInt 2007", Proceedings of Fifth International Workshop on Neutrino-Nucleus Interactions in the Few-GeV Region, Institute of Physics Publishing (2007)

5. Teppei Katori, V. Alan Kostelecký, and Rex Tayloe, "Global three-parameter model for neutrino oscillations using Lorentz violation ," prepared for the poster session of Neutrino 06, Lensic Theater, Santa Fe, NM, June 13-19, 2006, will be published in "Neutrino 2006", Proceedings of Neutrino 2006, Institute of Physics Publishing (2007)

6. T. Katori and R. Tayloe [for the LSND Collaboration], "Lorentz and CPT violation with LSND," prepared for 3rd Meeting on CPT and Lorentz Symmetry (CPT 04), Bloomington, Indiana, 4-7 Aug 2004, published in "CPT and Lorentz Symmetry", Proceedings of the Third Meeting on CPT and Lorentz symmetry (V. A. Kostelecký ed.), 150-158, World Scientific (2005)

\section{Other publications}

1. A. A. Aguilar-Arevalo et al. [MiniBooNE Collaboration], "Unexplained Excess of Electron-Like Events From a 1-GeV Neutrino Beam", arXiv:0812.2243 [hep-ex]

2. K. Hiraide et al. [SciBooNE Collaboration], "Search for Charged Current Coherent Pion Production on Carbon in a Few-GeV", arXiv:0811.0369 [hep-ex]

3. A. A. Aguilar-Arevalo et al. [MiniBooNE Collaboration], "First Measurement of $\nu_{\mu}$ and $\nu_{e}$ Events in an Off-Axis Horn-Focused Neutrino Beam", arXiv:0809.2447 [hep-ex]

4. A. A. Aguilar-Arevalo et al. [MiniBooNE Collaboration], "The MiniBooNE Detector", arXiv:0806.4201 [hep-ex]

5. A. A. Aguilar-Arevalo et al. [MiniBooNE Collaboration], "The Neutrino Flux prediction at MiniBooNE,",arXiv:0806.1449 [hep-ex] 
6. A. A. Aguilar-Arevalo et al. [MiniBooNE Collaboration], "Compatibility of high- $\Delta \mathrm{m}^{2}$ $\nu_{e}$ and $\bar{\nu}_{e}$ neutrino oscillation searches", Phys. Rev. D 78, 012007 (2008)

7. A. A. Aguilar-Arevalo et al. [MiniBooNE Collaboration], "First Observation of Coherent $\pi^{0}$ Production in Neutrino Nucleus Interactions with $E_{\nu}<2 \mathrm{GeV}$ ", arXiv:0803.3423 [hep-ex]

8. A. A. Aguilar-Arevalo et al. [MiniBooNE Collaboration], "Constraining Muon Internal Bremsstrahlung as a Contribution to the MiniBooNE Low Energy Excess", Phys. Lett. B 664, 41 (2008)

9. A. A. Aguilar-Arevalo et al. [MiniBooNE Collaboration], "A Search for Electron Neutrino Appearance at the $\Delta m^{2} \sim 1 e V^{2}$ Scale", Phys. Rev. Lett. 98, 231801 (2007)

10. L. Bugel et al. [FINeSSE Collaboration], "A Proposal for A Near Detector Experiment on The Booster Neutrino Beamline: FINeSSE: Fermilab Intense Neutrino Scattering Scintillator Experiment", arXiv:hep-ex/0402007v1

11. 2004 APS Neutrino Study, Neutrino Astrophysics and Cosmology Working Group, "The Neutrino Matrix", arXiv:physics/0411216v2

\section{Invited talks}

1. "Neutrino cross section measurements for long-baseline neutrino oscillation experiments", The 43rd Rencontres de Moriond "Electroweak interactions and Unified theories",La Thuile, Italy, March 06, 2008

2. "The first result of MiniBooNE oscillation experiment", Physics of Massive Neutrinos (PMN) 2007, Blaubeuren, Germany, July 03,2007

3. "Charged-Current Interaction Measurements in MiniBooNE", Neutrino-Nucleus Interactions (NuInt) 2007, Fermilab, Batavia, IL, June 31,2007 


\section{Seminars}

1. "A measurement of the muon neutrino charged current quasielastic (CCQE) interaction and a test of Lorentz violation with the MiniBooNE experiment", $\mathrm{PhD}$ defense seminar, Bloomington, IN, December 10, 2008

2. "Measurement of muon neutrino charged current quasielastic (CCQE) scattering on carbon in MiniBooNE ", University of Wisconsin, Madison, WI, May 23,2008

3. "Test for Lorentz and CPT violation using the neutrino oscillation", Columbia University Particle seminar, New York, NY, September 19,2007

4. "The first result of MiniBooNE oscillation experiment", McGill University HEP seminar, Montreal, QC, Canada, May 09,2007

5. " $\Delta \mathrm{s}$, Lorentz Violation and Neutrinos", Kyoto University spin physics group seminar, Kyoto, Japan, July 12, 2005

6. " $\Delta \mathrm{s}$, Lorentz Violation and Neutrinos ", PhD candidacy seminar, Bloomington, IN, April 26, 2005

\section{Other talks}

1. "SciBooNE experiment, the neutrino cross section measurement", Division of Nuclear Physics (DNP) 2007 Meeting, Newport News, VA, October 13,2007

2. "Charged-Current Interaction Measurements in MiniBooNE", Division of Nuclear Physics (DNP) 2007 Meeting, Newport News, VA, October 13,2007

3. "The neutrino interaction measurements in MiniBooNE experiment", Physics of Massive Neutrinos (PMN) 2007, Blaubeuren, Germany, July 03,2007 
4. " $\Delta$ measurement through the neutrino neutral current elastic scattering", International School of Physics "Enrico Fermi" course 167, Varenna, Italy, June 29,2007

5. "Charged-current cross section measurements in MiniBooNE", Division of Nuclear Physics (DNP) 2006 Meeting, Nashville, TN, October 26,2006

6. "Global 3 parameter model for neutrino oscillations with Lorentz Violation", Division of Nuclear Physics (DNP) 2006 Meeting, Nashville, TN, October 26,2006

7. "MiniBooNE, a neutrino oscillation search at Fermilab", National Nuclear Physics Summer School (NNPSS) 2006, Bloomington, IN, July 28,2006

8. "FINeSSE, $\Delta$ s measurement through neutrino scattering", Division of Nuclear Physics (DNP) 2005 Meeting, September 21, 2005, Maui, HI

9. "Search for Lorentz Violation in LSND", Division of Nuclear Physics (DNP) 2005 Meeting, September 22, 2005, Maui, HI

10. "FINeSSE, $\Delta \mathrm{s}$ measurement through the Neutrino-Nucleon Neutral Current Scattering", Pan Pacific spin 2005, July 5, 2005, Tokyo Institute of Technology, Tokyo, Japan

11. "FINeSSE, prototype beam test", Division of Nuclear Physics (DNP) 2004 Meeting, October 29, 2004, Chicago, IL

\section{Posters}

1. "Charged-Current Quasi-Elastic (CCQE) Interaction Measurements in MiniBooNE", NSF site visit at Indiana University Cyclotron Facility, December 5, 2007, IUCF, Bloomington, IN

2. "Test for Lorentz violation in the MiniBooNE Experiment", Fourth Meeting on CPT and Lorentz Symmetry Neutrino 2007, August 9, 2007, Bloomington, IN 
3. "Global three parameter model for Neutrino Oscillations using Lorentz Violation", Neutrino 2006, June 15, 2006, Lensic Theater, Santa Fe, NM

4. "A look inside the particle identification of MiniBooNE", DOE site visit at Fermi National Accelerator Laboratory, August, 2006, Fermilab, Batavia, IL

5. "Test of Lorentz Violation with LSND", Fermilab's Graduate Student Association (GSA) New Perspectives 2006, May 31, 2006, Fermilab, Batavia, IL

6. "FINeSSE, Fine-grained Intense Neutrino Scintillator Scattering Experiment", NSF site visit at Indiana University Cyclotron Facility, November 17, 2004, IUCF, Bloomington, IN

7. "FINeSSE, prototype detector beam test", Fermilab's Graduate Student Association (GSA) New Perspectives 2004, June 4, 2004, Fermilab, Batavia, IL

8. "FINeSSE, a neutrino scattering experiment", Neutrino 2004, June 13-19, 2004, College de France, Paris, France (absence from the poster session)

\section{Schools}

1. International School of Physics "Enrico Fermi" course 167, "Strangeness and Spin in Fundamental Physics", Varenna, Italia, June 19-29, 2007

2. 18th National Nuclear Physics Summer School, Bloomington, IN, July 23 - August 5, 2006

3. US Particle Accelerator School 05, "Accelerator Physics", San Francisco, CA, January $10-21,2005$ 


\section{Membership}

1. American Physical Society, Division of Astrophysics (DAP), Division of Nuclear Physics (DNP), and Division of Particles and Fields (DPF)

\section{Service work}

2007 SciBooNE experiment shift coordinator

2006-2007 MiniBooNE experiment shift coordinator

\section{Research}

2008 Currently, I am an active collaborator on the SciBooNE and MiniBooNE experiments. For MiniBooNE, I am working on 2 topics: a $\nu_{\mu}$ CCQE double differential cross section measurement and a test of Lorentz violation for MiniBooNE. Since the double differential cross section completely specifies the kinematics of $\nu_{\mu} \mathrm{CCQE}$, this measurement will provide rich information to understand $\nu_{\mu} \mathrm{CCQE}$ kinematics for neutrino scattering energy reconstruction, and this is one of the critical topics for the success of future long baseline oscillation experiments, such as the T2K experiment in Japan. For the latter, I am working from 2 different aspects of Lorentz violating neutrino oscillation model; one is the test of sidereal variation of MiniBooNE low energy excess, and the other is understanding of MiniBooNE low energy excess energy dependence in terms of our model which we developed in 2006.

2007 I worked on 2 experiments at Fermilab: SciBar Booster Neutrino Experiment (SciBooNE) and MiniBooNE. For SciBooNE, I was charged with the operation of the high voltage system and online environmental monitoring system. We have successfully in- 
stalled the high voltage system for both Scintillation bar (SciBar) detector and Muon Range Detector (MRD). We also equipped various transducers, to monitor electric rack temperatures and detector hall humidities, and their status are online and monitored from SciBooNE main control room. I also worked on the assembly of MRD. For MiniBooNE, I am continuing to work on the $\nu_{\mu} \mathrm{CCQE}$ analysis. I performed a grid search to find the best fit values of the $\nu_{\mu} \mathrm{CCQE}$ kinematics parameters in our $\mathrm{MC}$ to describe the data. A paper on this work (Phys. Rev. Lett. 100, 032301 (2008)) is presented at NuInt '07 at Fermilab. This result was used for MiniBooNE first $\nu_{e}$ appearance search analysis (Phys. Rev. Lett. 98, 231801 (2007)). We also tested the Lorentz violating model for neutrino oscillations including LSND with MiniBooNE data, and the result was presented at CPT and Lorentz symmetry '07 at Indiana University.

2006 I worked on 2 topics: a global model for neutrino oscillations with Lorentz violation and muon neutrino charged-current quasi-elastic scattering $\left(\nu_{\mu} \mathrm{CCQE}\right)$ analysis. For the former, we constructed a model which describes the world neutrino oscillation data, including the LSND result, with Lorentz violation. We succeeded to make a model the uses only 3 free parameters (Phys. Rev. D 74, 105009 (2006)). For the latter, I studied the $\nu_{\mu} \mathrm{CCQE}$ event kinematics, and estimated the cross section error for our neutrino oscillation analysis. I also performed the $\nu_{\mu} \mathrm{CCQE}$ analysis with full systematic errors.

2005 I worked on 2 topics: a Lorentz violation search with the LSND data and a "dirt" event analysis for MiniBooNE. For the former, I performed an unbinned likelihood fit to extract the model parameters for an LSND oscillation signal with sidereal time variation. We found the result is consistent with no Lorentz violation, however, a Lorentz violation model is not completely ruled out (Phys. Rev. D 72, 076004 (2005)). For the latter, I analyzed the dirt events, the background that originates outside of 
the detector and is an important background for MiniBooNE. We found that the dirt background is well-predicted with the MC simulation.

2004 I worked on 2 experiments: Mini-Booster Neutrino Experiment (MiniBooNE) at Fermilab and Fine-grained Intense Neutrino Scattering Scintillator Experiment (FINeSSE). For MiniBooNE, we tested mineral oil in a $200 \mathrm{MeV}$ proton beam (at Indiana University) for better understanding of the nature of scintillation light. For FINeSSE, we tested a pilot detector with improved scintillator and Wave Length Shifting (WLS) fiber using the $200 \mathrm{MeV}$ proton beam (Nucl. Instrum. Meth. A 562, 198 (2006)). I worked on most of part of this beam test, including calibration of the pilot detector, building the electronics, data taking, and data analysis. I studied the light propagation model in the liquid scintillator. In addition, I worked on an analysis of LSND signal to see a Lorentz violating signal.

2003 I studied the relationship of the light emission from WLS fiber end and its surface condition. Also I worked on a beam distribution calculation for Booster neutrino beamline. In this year, we did our first beam test of our FINeSSE pilot detector.

2002 We tested mineral oil of MiniBooNE using proton beam. Also I studied background neutrons for FINeSSE experiment, which will be produced by the dirt around the detector.

2001 In my undergraduate studies, I worked on neutrino phenomenology. In particular, I studied exotic neutrino oscillation models and supernova neutrino signals. 


\section{References}

\section{Associate professor Rex Tayloe}

Indiana University,

727 E. Third Street,

Bloomington, IN 47405, USA

Phone: 1-812-855-9365

E-mail:rtayloe@indiana.edu

\section{Doctor Bill Louis}

Los Alamos National Laboratory (LANL),

MS H846,

Los Alamos, NM 87545, USA

Phone: 1-505-667-6723

E-mail:louis@lanl.gov

\section{Lecturer Morgan Wascko}

Imperial College of London,

Blackett Lab 525,

Prince Consort Road,

London, SW7 2AZ, UK 
Phone: 44-(0)207-594-1607

E-mail:m.wasckop@imperial.ac.uk 